\title{
La lecture, moteur de changement
}

\section{PERFORMANCES ET ENGAGEMENT D'UN PAYS A L'AUTRE}

RÉSULTATS DE PISA 2000

\section{OCDE $《 O$}

Programme international pour le suivi des acquis des élèves 


\title{
LA LECTURE, MOTEUR DE CHANGEMENT
}

\section{PERFORMANCES ET ENGAGEMENT D'UN PAYS À L'AUTRE RÉSULTATS DU CYCLE D'ENQUÊTES DE PISA 2000}

\author{
Irwin Kirsch \\ John de Jong \\ Dominique Lafontaine \\ Joy McQueen \\ Juliette Mendelovits \\ Christian Monseur
}

\section{OCDE}

ORGANISATION DE COOPÉRATION ET DE DÉVELOPPEMENT ÉCONOMIQUES 


\section{ORGANISATION DE COOPÉRATION ET DE DÉVELOPPEMENT ÉCONOMIQUES}

En vertu de l'article $1^{\text {er }}$ de la Convention signée le 14 décembre 1960, à Paris, et entrée en vigueur le 30 septembre 1961, l'Organisation de Coopération et de Développement Économiques (OCDE) a pour objectif de promouvoir des politiques visant :

- à réaliser la plus forte expansion de l'économie et de l'emploi et une progression du niveau de vie dans les pays membres, tout en maintenant la stabilité financière, et à contribuer ainsi au développement de l'économie mondiale ;

- à contribuer à une saine expansion économique dans les pays membres, ainsi que les pays non membres, en voie de développement économique ;

- à contribuer à l'expansion du commerce mondial sur une base multilatérale et non discriminatoire conformément aux obligations internationales.

Les pays membres originaires de l'OCDE sont : l'Allemagne, l'Autriche, la Belgique, le Canada, le Danemark, l'Espagne, les États-Unis, la France, la Grèce, l'Irlande, l'Islande, l'Italie, le Luxembourg, la Norvège, les Pays-Bas, le Portugal, le Royaume-Uni, la Suède, la Suisse et la Turquie. Les pays suivants sont ultérieurement devenus membres par adhésion aux dates indiquées ci-après : le Japon (28 avril 1964), la Finlande (28 janvier 1969), l'Australie (7 juin 1971), la Nouvelle-Zélande (29 mai 1973), le Mexique (18 mai 1994), la République tchèque (21 décembre 1995), la Hongrie (7 mai 1996), la Pologne (22 novembre 1996), la Corée (12 décembre 1996) et la République slovaque (14 décembre 2000). La Commission des Communautés européennes participe aux travaux de l'OCDE (article 13 de la Convention de l'OCDE).

Le Centre pour la Recherche et l'Innovation dans l'Enseignement a été créé par le Conseil de l'Organisation de Coopération et de Développement Économiques en juin 1968 et tous les pays membres de l'OCDE y participent.

Les principaux objectifs $d u$ Centre sont les suivants :

- poursuivre les travaux de recherche et d'analyse sur les innovations et les indicateurs clés afin de mieux appréhender les problèmes d'enseignement et d'apprentissage existants ou qui se font jour, ainsi que leurs liens avec les autres domaines d'action ;

- explorer des stratégies d'enseignement et d'apprentissage cohérentes et prometteuses qui tiennent compte de l'évolution $d u$ contexte économique, social et culturel aux niveaux national et international; et

- faciliter la coopération pratique entre les pays membres et, si nécessaire avec les pays non membres, afin qu'ils recherchent des solutions à des problèmes éducatifs communs et échangent leurs points de vue sur ces problèmes.

Le Centre exerce son activité au sein de l'Organisation de Coopération et de Développement Économiques conformément aux décisions $d u$ Conseil de l'Organisation, sous l'autorité du Secrétaire général et le contrôle direct d'un Comité directeur composé d'experts nationaux dans le domaine de compétence du Centre, chaque pays participant étant représenté par un expert.

Crédidt photo : Photodisc

(C) OCDE 2003

Les permissions de reproduction partielle à usage non commercial ou destinée à une formation doivent être adressées au Centre français d'exploitation du droit de copie (CFC), 20, rue des Grands-Augustins, 75006 Paris, France, tél. (33-1) 440747 70, fax (33-1) 463467 19, pour tous les pays à l'exception des États-Unis. Aux États-Unis, l'autorisation doit être obtenue du Copyright Clearance Center, Service Client, (508)750-8400, 222 Rosewood Drive, Danvers, MA 01923 USA, or CCC Online: www.copyright.com. Toute autre demande d'autorisation de reproduction ou de traduction totale ou partielle de cette publication doit être adressée aux Éditions de l'OCDE, 2, rue André-Pascal, 75775 Paris Cedex 16, France. 


\section{AVANT-PROPOS}

Exhorter les individus, les économies et les sociétés à rehausser les niveaux de formation est l'objectif majeur des gouvernements, soucieux d'améliorer la qualité des systèmes d'éducation. Les pays de l'OCDE doivent en grande partie leur prospérité à leur capital humain et aux possibilités offertes à leurs citoyens d'acquérir des connaissances et des compétences qui leur permettront de continuer à apprendre tout au long de leur vie.

Le Programme international de l'OCDE pour le suivi des acquis des élèves (PISA) a été créé pour déterminer dans quelle mesure les jeunes sont préparés à relever les défis des sociétés modernes à l'approche de la fin de leur scolarité obligatoire. Tourné vers l'avenir, PISA se concentre sur la capacité des jeunes à réfléchir et à exploiter leurs connaissances et leurs compétences pour faire face aux exigences de la vie adulte dans le monde réel.

PISA évalue les connaissances et les compétences des élèves tous les trois ans dans trois domaines : la compréhension de l'écrit, la culture mathématique et la culture scientifique. Chaque cycle d'évaluation concentre le recueil de données dans l'un de ces trois domaines. Lors du cycle PISA 2000, le premier exercice de recueil de données du PISA, c'est la compréhension de l'écrit qui a été le domaine majeur et a fait l'objet de deux tiers environ de l'évaluation. La littératie n'est pas seulement l'un des fondements essentiels de la progression dans d'autres matières dans le cadre scolaire, elle est aussi la condition sine qua non de la réussite dans tous les domaines de la vie d'adulte. Le monde d'aujourd'hui impose aux citoyens de pratiquer l'apprentissage tout au long de la vie. Pour y parvenir, les élèves doivent être préparés à traiter l'éventail d'écrits qu'ils seront amenés à lire durant toute leur vie.

Les premiers résultats du cycle PISA 2000 ont été publiés en 2001 dans un rapport (OCDE, 2001b) qui englobe les trois domaines d'évaluation et étudie la relation entre la performance des élèves dans ces domaines et une série de caractéristiques individuelles, familiales et scolaires. Le présent rapport a pour objet d'approfondir ces analyses à propos des résultats en compréhension de l'écrit.

Nous avons intitulé ce rapport La lecture, moteur de changement pour insister sur deux messages qui revêtent une importance capitale. Le premier renvoie à la compétence en compréhension de l'écrit : les résultats du cycle PISA 2000 suggèrent que l'amélioration de la littératie des élèves peut avoir un grand impact sur les perspectives qui s'offriront à eux une fois adultes. Le second message concerne l'engagement à l'égard de la lecture : le degré d'intérêt que les élèves lui portent, leurs attitudes vis-à-vis de l'écrit, le temps qu'ils passent à lire pendant leurs loisirs et la diversité de leurs lectures sont étroitement associés à leur niveau de compétence en lecture. D'autre part, nos analyses montrent que bien que le degré d'engagement à l'égard de la lecture varie d'un pays à l'autre, les jeunes de 15 ans dont les parents accusent le plus faible indice de statut professionnel, mais qui s'investissent beaucoup dans la lecture, obtiennent de meilleurs résultats en compréhension de l'écrit que ceux dont les parents affichent un indice moyen ou élevé de statut professionnel, mais qui ne s'intéressent guère à la lecture. Ce constat suggère qu'amener les élèves à s'investir dans la lecture pourrait être l'un des meilleurs moyens de stimuler le progrès social.

Même dans les pays affichant un niveau élevé de compétence en compréhension de l'écrit, un nombre substantiel de jeunes de 15 ans présentent des lacunes en lecture qui sont susceptibles de limiter les choix et les perspectives qui s'offriront à eux plus tard. Dans ce rapport, nous ne pouvons définir en termes 
généraux le niveau de compétence ou le point précis sur l'échelle combinée en compréhension de l'écrit correspondant au degré de littératie qui garantit la réussite. Les conclusions politiques doivent donc être tirées au niveau national : chaque pays doit étudier attentivement ses caractéristiques particulières et leurs relations avec le niveau de compétence des élèves en compréhension de l'écrit. Les moyens à mettre en œuvre pour combler les lacunes varient selon les pays, en fonction de la structure de leur système d'enseignement et d'autres variables nationales. Les analyses exposées ici suggèrent toutefois que tous les pays seraient bien avisés de chercher à encourager les élèves, en particulier les garçons, à s'investir davantage dans la lecture.

Pour évaluer le niveau de compétence des élèves en compréhension de l'écrit, PISA leur demande de montrer qu'ils sont capables d'appréhender un large éventail de textes qui s'inscrivent dans des contextes différents et qui sont abordés selon diverses perspectives. Le présent rapport a notamment pour objet de présenter de manière assez détaillée le construct de compréhension de l'écrit qui sous-tend PISA et d'expliquer les relations qui existent entre ce construct et les résultats des pays. C'est la raison pour laquelle nous rendons compte des résultats en les exprimant sous la forme de moyennes et de performances réparties sur l'échelle combinée de compréhension de l'écrit, mais également en les rapportant sur cinq sous-échelles, dont deux portent sur le format des textes (les textes continus et non continus) et trois distinguent les aspects de la compréhension de l'écrit (trouver l'information, interpréter le texte, et réfléchir et évaluer).

Bien que la plupart de ceux qui consultent les enquêtes internationales cherchent avant tout à classer les différents pays sur une échelle de compétence globale, il y a lieu de souligner que les différences de performance sont significativement plus importantes au sein des pays qu'entre les pays. Sur l'échelle combinée de compréhension de l'écrit, l'écart de performance entre les élèves les plus forts et les plus faibles (c'està-dire ceux situés au $90^{\mathrm{e}}$ ou $10^{\mathrm{e}}$ percentile) se situe dans chaque pays entre deux niveaux et demi de compétence et quatre niveaux et demi de compétence, ce qui traduit un degré élevé et préoccupant d'inégalité dans quelques pays. Les pays ne sont pas tous logés à la même enseigne : les différences entre les sexes sont nettement plus marquées dans certains que dans d'autres. Il en va de même pour les élèves issus de milieux peu aisés, qui sont plus défavorisés dans certains pays que dans d'autres. Enfin, la structure éducative de plusieurs pays semble avoir un impact plus fort sur les différences entre élèves que celle d'autres pays.

Les pays se distinguent également par leurs points forts sur les sous-échelles de compréhension de l'écrit. Les élèves de certains pays obtiennent de meilleurs résultats lorsqu'il s'agit de trouver des informations dans des textes plutôt que de réfléchir à ces textes. Ces élèves comprennent également mieux les textes non continus que les textes continus. En revanche, dans les pays où les résultats sont meilleurs en réflexion qu'en localisation d'informations, les élèves comprennent mieux les textes continus que les textes non continus. Mais certains pays affichent aussi des tendances assez différentes de résultats sur les sous-échelles par aspect et par format de l'évaluation de la compréhension de l'écrit PISA.

Le programme PISA est le fruit d'un effort concerté qui met en synergie l'expertise scientifique des pays participants et que les gouvernements de ces pays dirigent conjointement en fonction de préoccupations communes touchant l'action des pouvoirs publics. Les pays participants assument la responsabilité politique du projet par l'entremise du Conseil des pays participants. Des experts des pays participants coopèrent au sein de groupes de travail visant à relier les objectifs d'action politique du programme aux meilleures compétences techniques disponibles dans le domaine de l'évaluation comparative internationale du rendement scolaire. En collaborant aux travaux de ces groupes d'experts, les pays veillent à ce que les instruments d'évaluation 
utilisés dans le cadre de PISA soient valides sur le plan international, prennent en compte le contexte culturel et éducatif des différents pays de l'OCDE, offrent une plate-forme de mesures réalistes et, enfin, mettent l'accent sur l'authenticité et la validité des données sur le plan éducatif. Le groupe fonctionnel d'experts qui a dirigé l'élaboration du cadre d'évaluation de la compréhension de l'écrit, a supervisé la préparation des tâches d'évaluation et a contribué à conceptualiser ce rapport, est constitué de membres de neuf pays qui ont été choisis pour leur spécialisation et leur expertise dans le domaine de la lecture et de l'évaluation. La liste des membres de ce groupe figure au dos de cette publication. Des développeurs de tests de l'Australian Council for Educational Research (ACER) et du CITO (Pays-Bas) ont sélectionné des textes en veillant à leur diversité culturelle et ont préparé une batterie d'items dans le strict respect des exigences définies dans le cadre d'évaluation.

Wolfram Schulz, d'ACER, a contribué à l'analyse des données présentées au chapitre 7. Kentaro Yamamoto, de l'Educational Testing Service (ETS), a réalisé les analyses qui ont permis de situer les élèves de PISA sur l'échelle de textes suivis de l'Enquête internationale sur la littératie des adultes, le thème du chapitre 8. Nous tenons également à remercier Marylou Lennon pour ses commentaires sur les premières versions des différents chapitres, ainsi que Lynn Jenkins, pour les travaux de mise en forme de grande qualité qu'elle a réalisés sur l'ensemble du rapport. Nos remerciements vont aussi à Aletta Grisay, à Eugene Johnson et aux membres du Conseil des pays participants qui nous ont fait part de leurs commentaires.

Ce rapport est le fruit d'une véritable collaboration entre les auteurs qui sont membres du groupe fonctionnel d'experts chargé de la lecture ou du consortium de PISA. Le mérite que cette publication pourrait valoir à ses auteurs revient aussi aux personnes et institutions citées.

Irwin Kirsch

John de Jong

Dominique Lafontaine

Joy McQueen

Juliette Mendelovits

Christian Monseur 


\section{TABLE DES MATIĒRES}

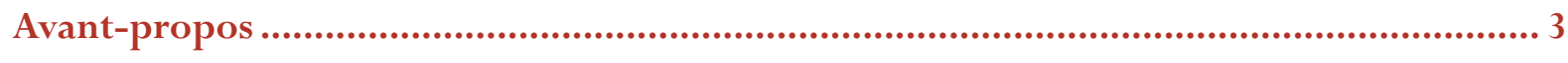

Chapitre $1:$ Présentation de PISA et du concept de compréhension de l'écrit ................ 11

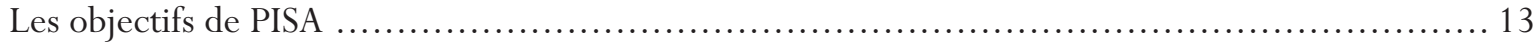

Originalité du programme PISA par rapport aux autres enquêtes internationales.................. 14

La compréhension de l'écrit, une compétence fondamentale ................................ 15

Contexte du cadre d'évaluation de la compréhension de l'écrit ............................... 17

Élaboration du cadre d'évaluation et des instruments de test pour la compréhension de l'écrit, ainsi

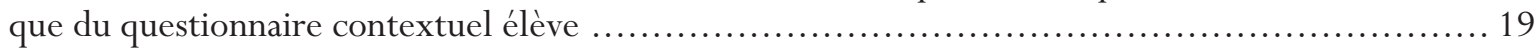

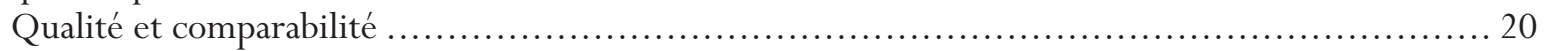



Chapitre 2 : Le construct de compréhension de l'écrit dans PISA................................. 25

Caractéristiques du cadre d'évaluation de la compréhension de l'écrit du programme PISA ............27

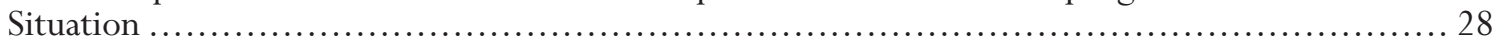

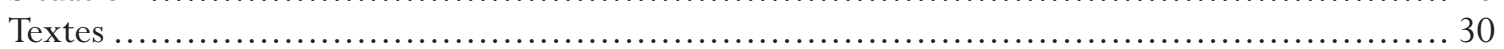

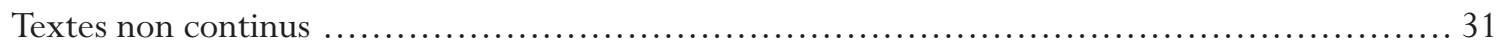

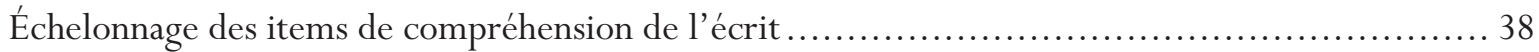

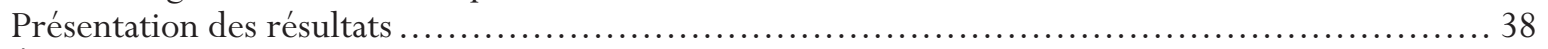

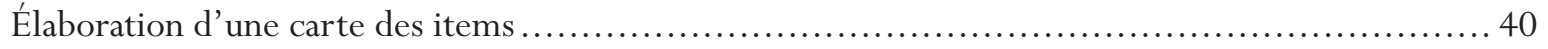

Niveaux de compétence en compréhension de l'écrit ...................................... 41

Interprétation des niveaux de compétence en compréhension de l'écrit .......................... 43

Chapitre 3 : Exemples d'items ................................................................................... 47

Sélection et organisation des exemples d'item ............................................... 48

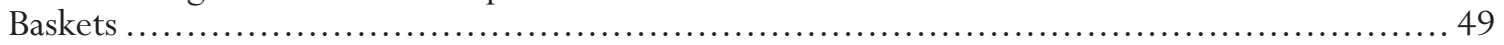







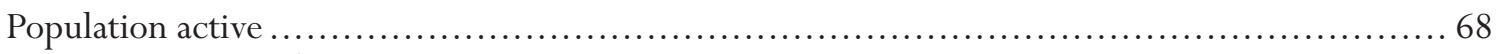



Guide du lecteur ............................................................................................... 78

Chapitre 4 : Performances en lecture des jeunes de 15 ans ............................................ 81

Performance sur l'échelle combinée de compréhension de l'écrit .............................. 85

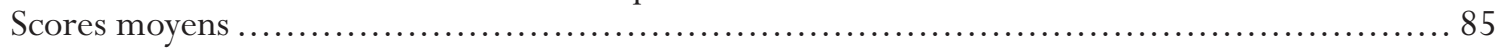

Répartition des scores : dispersion des scores pour différentes populations ..................... 90

Répartition des scores : Proportions d'élèves aux extrémités de l'échelle ...................... 92

Performances en compréhension de l'écrit sur les sous-échelles d'aspect ..........................99



Répartition des compétences en compréhension de l'écrit au sein des pays sur les trois sous-échel-

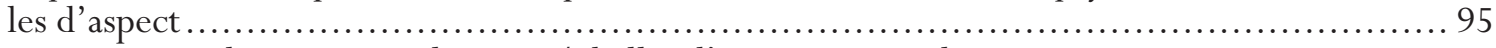

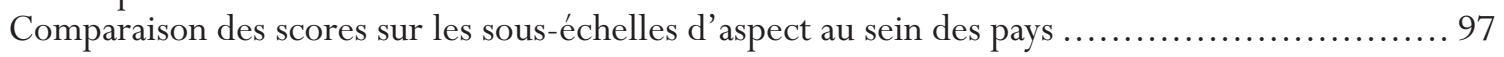

Performance en compréhension de l'écrit sur les sous-échelles de format de texte.................101

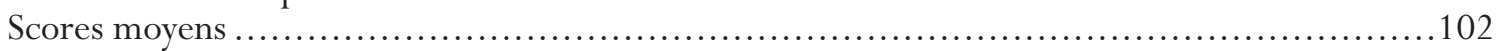

Répartition des compétences en compréhension de l'écrit au sein des pays sur les deux sous-

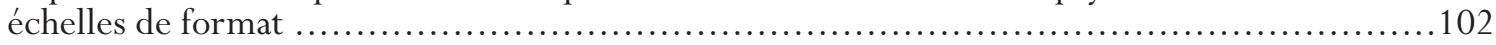

Comparaison des performances sur les sous-échelles de format de texte, au sein des pays ........103

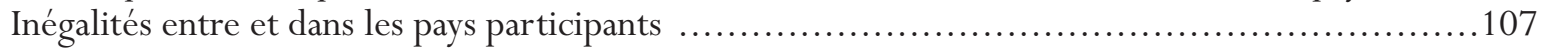


Chapitre 5 : L'engagement des jeunes de 15 ans à l'égard de la lecture ..........................113

L'évaluation de l'engagement à l'égard de la lecture dans PISA .................................. 116

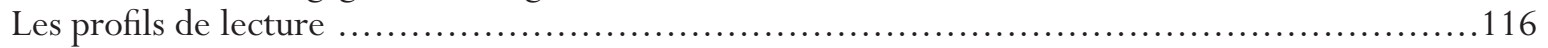

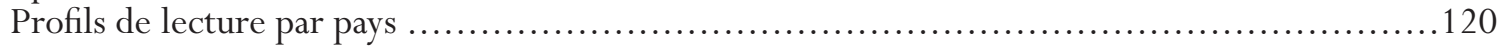

Profils de lecture et performances par pays ............................................... 120

Profils de lecture et niveaux de compétence ; liens avec les sous-échelles de compréhension de

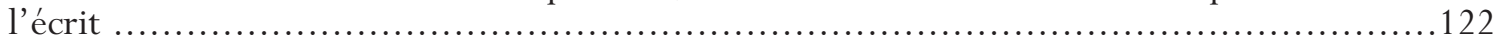

Relations entre les profils de lecture et certaines caractéristiques des élèves et de leur milieu.......124

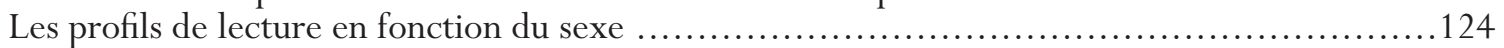

Les profils de lecture en fonction du milieu socio-économique ................................ 126

Les profils de lecture en fonction de l'accès à l'écrit ....................................... 126

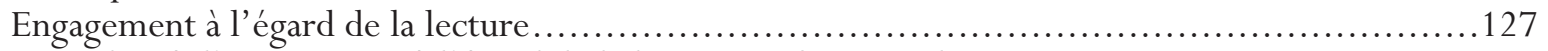

Le degré d'engagement à l'égard de la lecture en fonction du sexe ............................ 128

L'engagement à l'égard de la lecture peut-il atténuer les inégalités liées au milieu social ? ..........128

Chapitre 6 : Relations entre les caractéristiques contextuelles et les performances en lecture..133

La compréhension de l'écrit et les caractéristiques individuelles des élèves ......................135



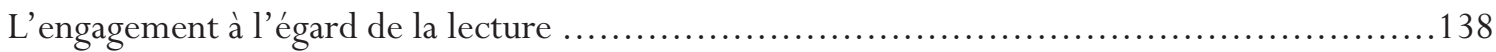

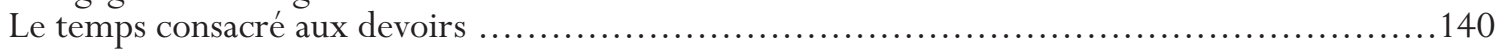

La compréhension de l'écrit et les caractéristiques familiales ...................................141

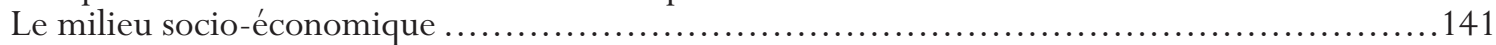

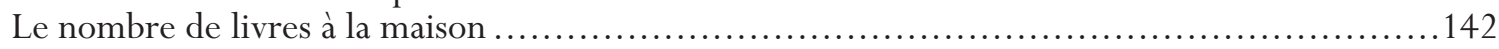



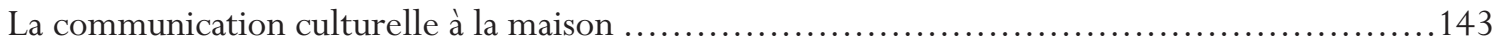

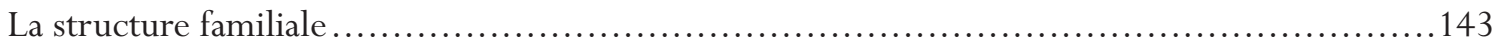

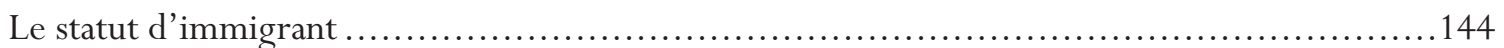

La compréhension de l'écrit et l'environnement scolaire ........................................ 145

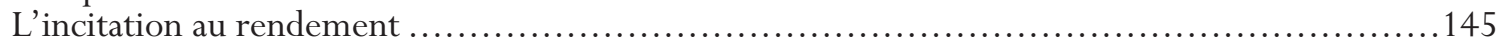

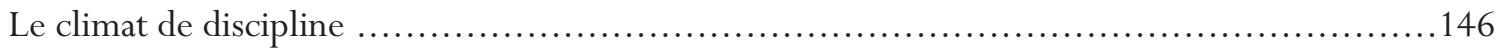

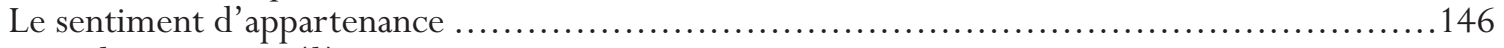

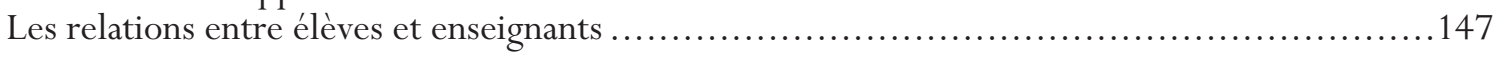

Chapitre 7 : L'interaction entre les caractéristiques individuelles et les facteurs scolaires . 149

Un modèle d'analyse à variables et niveaux multiples ....................................... 151

Variables individuelles et scolaires utilisées dans le modèle.....................................154

L'influence prépondérante du milieu socio-économique et de l'engagement à l'égard de la lecture.. 155



Les sous-échelles de compréhension de l'écrit ........................................ 157

Variation selon les pays de l'effet des variables individuelles et scolaires sur les performances en

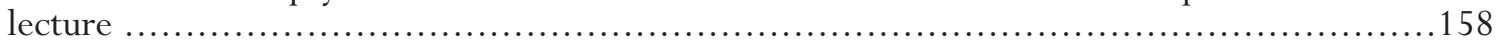

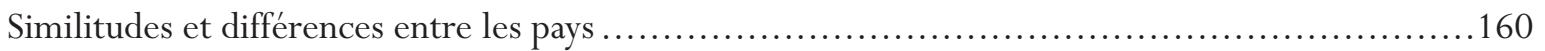

Variation des performances en lecture entre établissements ................................ 160

Influence du milieu socio-économique des élèves sur les performances en lecture ...............162

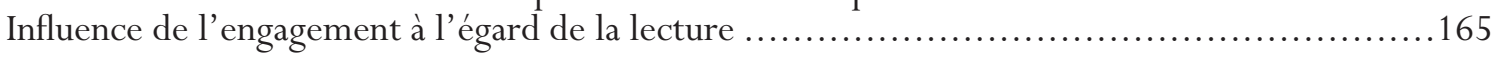

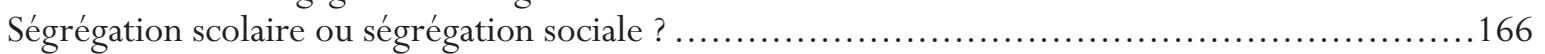

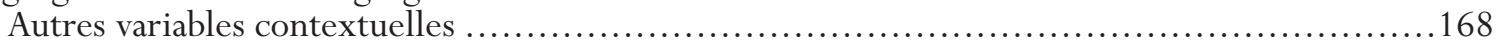

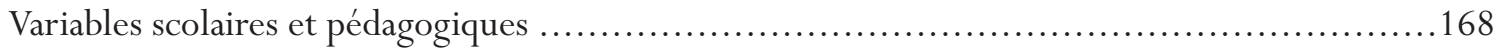

Variation de performances en lecture entre élèves au sein des établissements ..................169 
Chapitre 8 : Niveau de préparation des jeunes de 15 ans aux défis de l'avenir ...............173 L'influence de la littératie sur le plan social..................................................... 176 Comparaison de l'échelle de compréhension des textes suivis de IALS et de l'échelle combinée de

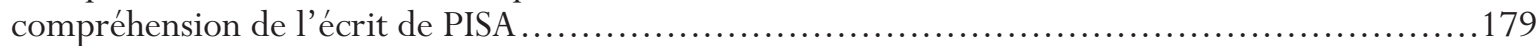

Situer les élèves de PISA sur l'échelle de compréhension de textes suivis de IALS ..................181

Performance des élèves de PISA sur l'échelle de compréhension de textes suivis de IALS...........182

Bibliographie ............................................................................................. 189

Annexe A: Notes techniques .....................................................................................195

Annexe A1 : Indices quantitatifs relatifs à l'équilibre culturel des items du cycle d'évaluation PISA 2000 . 196

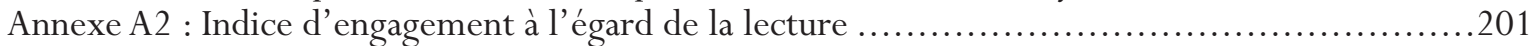

Annexe A3 : Notes techniques sur les analyses multivariées à plusieurs niveaux ....................203

Annexe A4 : Erreurs types, tests de signification et comparaisons multiples ......................208

Annexe B :Tableaux de données.....................................................................................209

Annexe C : Développement et mise en ouvre de PISA - une initiative concertée..........275 
2 


\begin{abstract}
This monograph is concerned with ... the interests, attitudes, and skills that enable young people and adults to meet effectively the reading demands of their current lives (and) ... to take full advantage of the many opportunities now available to make life richer and more satisfying through reading.

[Cette monographie traite [...] des intérêts, des attitudes et des compétences qui permettent aux jeunes et aux adultes de répondre totalement aux exigences de lecture que leur impose la vie [et] de tirer pleinement parti des nombreuses occasions qui leur sont offertes d'enrichir leur vie et de la rendre plus satisfaisante au travers de la lecture.]
\end{abstract}

William S. Gray et Bernice Rogers

Maturity in Reading, 1956

Bien qu'écrites voici déjà près d'un demi-siècle, ces lignes reflètent les principes fondamentaux du cadre d'évaluation de la compréhension de l'écrit adopté par le Programme international pour le suivi des acquis des élèves (PISA), une enquête internationale sur les compétences en lecture, en mathématiques et en sciences chez les jeunes âgés de 15 ans. Ce cadre d'évaluation ne met pas l'accent sur les mécanismes de la lecture mais sur les compétences en lecture, appliquées à une série de situations et à différentes fins. Dès lors, il reconnait à quel point la compréhension de l'écrit est importante dans la vie privée et professionnelle, à l'école et dans le monde du travail et aussi pour la participation citoyenne et l'apprentissage tout au long de la vie. Comprendre l'écrit permet à l'individu d'atteindre ses objectifs, qu'il s'agisse d'aspirations bien définies - par exemple terminer avec fruit l'enseignement initial ou trouver un emploi, - ou d'aspirations moins précises et moins immédiates, mais qui touchent néanmoins l'épanouissement personnel de l'individu. Il est facile à comprendre que la compréhension de l'écrit est une composante importante du concept de capital humain qui détermine l'avenir social et économique des individus et des nations (OCDE, 2001a).

Le niveau global de formation de la population mondiale ne cesse d'augmenter : de décennie en décennie, les jeunes adultes sont de plus en plus nombreux à atteindre des niveaux supérieurs de formation. Parallèlement, le renforcement progressif du rôle des connaissances dans les économies modernes a donné lieu à une élévation du niveau de compétence demandé sur le marché du travail. L'expansion des systèmes éducatifs s'est révélée insuffisante pour satisfaire à la demande croissante de travailleurs qualifiés, et d'autre part, les pays doivent faire face à une augmentation du chômage chez les personnes peu qualifiées.

Ce premier chapitre retrace la création de PISA et en présente le contexte. Il montre en quoi PISA se distingue d'autres enquêtes internationales de rendement scolaire, explique l'importance de la compréhension de l'écrit dans les sociétés modernes et expose les principes qui ont présidé à la définition de ce concept et à la rédaction du cadre d'évaluation. De plus, il décrit le processus mis en œuvre pour garantir la comparabilité des mesures malgré les différences linguistiques et culturelles. Enfin, il présente l'objet et la structure de ce rapport. 


\section{Les objectifs de PISA}

Améliorer la qualité de l'enseignement est un objectif politique majeur des pays Membres de l'Organisation de coopération et de développement économiques (OCDE). La priorité donnée à cet objectif s'explique notamment par le fait que l'on s'accorde de plus en plus à reconnaître que la prospérité des nations dépend dans une grande mesure de son capital humain et des possibilités qu' ont les individus d'apprendre et d'acquérir des savoirs tout au long de la vie.

Depuis 1985, l'OCDE recueille des données comparables dans divers domaines, notamment les ressources humaines et financières investies dans l'enseignement, l'accès et la participation à l'éducation et la progression dans les études et, enfin, le fonctionnement des systèmes éducatifs et d'apprentissage. L'OCDE publie ces indicateurs depuis le début des années quatre-vingt-dix dans des rapports annuels intitulés Regards sur l'éducation.

Le programme PISA, créé pour étayer ces informations, vise à déterminer dans quelle mesure les jeunes adultes de 15 ans sont préparés à relever les défis de la société de la connaissance à l'approche de la fin de leur scolarité obligatoire, sinon au terme de celle-ci. L'évaluation de PISA est prospective, dans le sens où elle ne cherche pas à déterminer dans quelle mesure les élèves ont assimilé une matière spécifique du programme d'enseignement, mais plutôt à estimer l'aptitude des jeunes à exploiter leurs connaissances et compétences pour faire face aux défis de la vie réelle, qu'ils envisagent de poursuivre des études - voire une carrière universitaire - ou qu'ils se préparent à entrer dans la vie active.

Le programme PISA est une initiative concertée des gouvernements des états Membres de l'OCDE et est dirigé par le Conseil des pays participants, constitué de représentants de ces pays. Le premier cycle d'évaluation de PISA a été réalisé en 2000 dans 32 pays (28 pays Membres de l'OCDE et quatre pays tiers), sous la forme d'épreuves écrites en lecture, en mathématiques et en sciences auxquelles les élèves ont répondu dans leurs écoles en situation de test. L'évaluation sera répétée tous les trois ans en mettant chaque fois l'accent sur un domaine de compétence différent. Elle vise essentiellement à permettre aux parties intéressées de suivre le développement des systèmes nationaux d'éducation en analysant précisément l'évolution des résultats au fil du temps.

Le rapport présentant les premiers résultats du cycle PISA 2000 en compréhension de l'écrit, en culture mathématique et en culture scientifique a été publié en 2001 (OCDE, 2001b). Il contient également des variables, telles que l'intérêt pour la lecture, qui sont considérées comme faisant partie des résultats de l'apprentissage. Le présent rapport porte sur le domaine majeur du premier cycle, à savoir la compréhension de l'écrit. D'autres rapports thématiques basés sur les données recueillies lors de ce premier cycle seront également publiés.

PISA a pour vocation d'informer les parents, les élèves, le grand public et les responsables des systèmes éducatifs, de leur montrer si les jeunes approchant de la fin de leur scolarité obligatoire ont acquis les connaissances et compétences nécessaires pour leur permettre de relever les défis de notre société moderne. Il vise plus particulièrement à déterminer si les élèves sont suffisamment armés pour continuer à enrichir leurs savoirs et savoir-faire et, ainsi, faire face aux changements qui ne manqueront pas de survenir. C'est pourquoi PISA définit les compétences par rapport aux situations de la vie réelle et les considère comme le bagage intellectuel indispensable que les jeunes doivent posséder pour entrer dans la vie active et se livrer à l'apprentissage tout au long de la vie. PISA se propose de fournir ces résultats régulièrement et de comparer leur évolution entre les pays, mais aussi au fil du temps. 


\section{Originalité du programme PISA par rapport aux autres enquêtes internationales}

Les compétences en lecture ont fait l'objet d'un certain nombre d'enquêtes internationales. Deux études ont notamment été réalisées par l'Association internationale pour l'évaluation du rendement scolaire (IEA) : la «Six Subject Study» de 1971 consacrée entre autres à la compréhension de l'écrit et à la littérature (Walker, Anderson et Wolf, 1976) et la «Reading Literacy Study » (Enquête IEA/RLS) de 1991 (Elley, 1992 et 1994). La population cible de la première était les élèves de 10 et 14 ans, et celle de la seconde, les élèves fréquentant des classes constituées en majorité d'enfants de 9 et 14 ans. L'Enquête internationale sur la littératie des adultes (IALS) a quant à elle évalué les compétences en lecture d'adultes âgés de 16 à 65 ans dans 23 pays (OCDE et Statistique Canada, 2000). La nouvelle enquête internationale sur la littératie et les compétences pour la vie chez les adultes(ALL) vise à mesurer les compétences en lecture, en calcul et en résolution de problèmes des adultes âgés de 16 à 65 ans dans les pays participants.

Une différence majeure entre PISA et les deux enquêtes de l'IEA sur la littératie est que, contrairement à ces deux enquêtes, PISA a commencé par préparer un cadre conceptuel détaillé, définissant les aspects théoriques, l'organisation des domaines et les méthodes d'évaluation, qui a servi de base à l'élaboration des instruments de test. Les cadres d'évaluation de la compréhension de l'écrit, de la culture mathématique et de la culture scientifique ont été soumis aux pays participants, puis modifiés en fonction des critiques et commentaires formulés et, enfin, adoptés par les gouvernements des pays de l'OCDE avant que les épreuves d'évaluation soient préparées (OCDE, 1999 et 2000). Aucun document de ce type n'a été diffusé lors de la réalisation des deux enquêtes de l'IEA citées ci-dessus, «même s'il est probable que des documents de travail de ce type, à usage interne, aient été rédigés lors d'études antérieures » (Lafontaine, 1999). Un cadre conceptuel a été préparé pour IALS et fait partie de la documentation relative à l'enquête ALL (Kirsch, 2001).

Le programme PISA se distingue nettement des deux enquêtes de l'IEA à un autre égard, à savoir le format des items. Les deux enquêtes de l'IEA « Six Subject Study » et IEA/RLS ont utilisé des items à choix multiple avec, pour la seconde, quelques items ouverts à réponse brève et objective. En revanche, dans PISA, plus de la moitié des items demandent aux élèves d'élaborer leur propre réponse, souvent en reliant le texte qui leur est soumis à leurs connaissances et à leurs expériences personnelles.

Une troisième enquête, «Progress in International Reading Literacy Study (PIRLS), a été réalisée en 2001 par l'IEA dans les classes fréquentées en majorité par des élèves de neuf ans en vue d'établir un lien avec l'IEA/RLS. À l'instar de PISA, l'enquête PIRLS a débuté par la préparation d'un cadre d'évaluation très élaboré (Campbell, Kelly, Mulis, Martin et Sainsbury, 2001) et a adopté une théorie interactive de la lecture. Il existe de grandes similitudes entre les deux évaluations mais également des différences significatives qui tiennent notamment à leur objectif d'échantillonner des populations différentes. Comme sa population cible se situe juste après l'enseignement initial de la lecture, l'enquête PIRLS s'est attachée à étudier la relation entre, d'une part, les compétences en lecture et, d'autre part, le programme d'enseignement et les pratiques pédagogiques dans ce domaine. Il n'en va pas de même pour PISA. En effet, la lecture ne figure pas en tant que telle dans le programme d'enseignement des jeunes de 15 ans qui constituent la population cible de PISA. Pour cette raison, les facteurs associés au niveau de compétences en lecture chez les élèves de 15 ans ne peuvent être mis aussi directement en relation avec le programme de cours et l'enseignement de la lecture. En outre, et c'est peut-être plus important encore, PISA part du principe que les jeunes de 15 ans doivent non seulement savoir lire, mais aussi savoir utiliser leurs connaissances et leurs compétences dans des contextes de la vie quotidienne, car ils entament le processus de transition 
vers la vie adulte. PISA met l'accent sur l'évaluation de la compréhension de l'écrit dans des situations de la vie de tous les jours.

\section{La compréhension de l'écrit, une compétence fondamentale}

La compréhension de l'écrit est une des exigences fondamentales du développement éducatif des citoyens. L'apparition du téléphone et de la télévision a un temps donné à croire que les modes oraux et visuels de communication allaient rapidement supplanter l'écrit (Birkerts, 1994 et 1998 ; Coover, 1992). Or, contrairement à ces prévisions, l'écrit a gagné du terrain en tant que moyen de communication.

Selon Halloway (1999), les compétences en lecture conditionnent la réussite scolaire des élèves de l'enseignement secondaire inférieur et supérieur mais nombre d'entre eux ne maîtrisent pas suffisamment la lecture après sept ou huit années d'enseignement fondamental et continuent à obtenir des résultats inacceptables.

Olson (1997a et 1997b) prétend même que dans nos sociétés modernes, la littératie introduit un biais dans le sens où elle privilégie ceux qui possèdent les compétences nécessaires au niveau requis. Elle donne accès aux institutions et aux ressources basées sur l'écrit et a un impact sur la cognition (Olson, 1994) ; elle influe sur notre mode de pensée. Dans le même ordre d'idée, Elwert (2001) évoque le concept de «littératie sociale » qui traduit l'importance fondamentale de la compréhension de l'écrit pour communiquer avec les institutions d'une société bureaucratique moderne. Le droit, le commerce et les sciences utilisent des procédures et des documents écrits, tels que la législation, les contrats et les publications, que l'individu doit pouvoir comprendre pour évoluer dans ces domaines. Quelques années avant, Elwert, Freire et Macedo (1987) ont écrit que la littératie est le pouvoir du peuple et ont expliqué qu'ils la considéraient comme un processus de « prise de conscience » qui implique de lire le monde (reading the world) plutôt que le mot (reading the word).

Dans nos sociétés basées sur l'écrit, la lecture est une condition sine qua non pour réussir dans la vie, mais elle ne suffit pas. Apprendre, travailler et vivre dépendent fortement de la compréhension de l'écrit. Moffett et Wagner (1983) soutiennent que la compréhension de l'écrit ne se distingue pas de la compréhension générale. Les compétences requises pour comprendre des textes - en identifier l'idée principale, se rappeler des détails, rapporter des faits, tirer des conclusions et prévoir des effets - sont importantes dans la vie de tous les jours. Pour résoudre les problèmes de la vie courante, il faut être capable d'identifier un schéma général, de se souvenir de détails, de voir des relations et de tirer des conclusions de ses expériences. La pratique de la lecture enrichit notre propre expérience et renforce la capacité d'apprendre à vivre dans notre société.

L'Enquête internationale sur la littératie des adultes (IALS : OCDE et Statistique Canada, 1997) montre que le niveau de littératie a - abstraction faite de l'effet connu des diplômes - un impact direct sur les revenus avant impôt, sur l'emploi, la santé et la participation à des activités de formation continue. Cette étude a également établi que les individus présentant des niveaux inférieurs de littératie étaient plus susceptibles que d'autres de dépendre de l'aide sociale et de se livrer à des activités criminelles. Kirsch, Jungeblut, Jenkins et Kolstad (1993) font état de résultats similaires après une étude sur la littératie des adultes menée aux États-Unis. Ils en concluent que la littératie peut être considérée comme une valeur monétaire dans notre société. Outre ses conséquences pour l'individu, notamment la probabilité accrue de ne pas travailler à plein temps ou de vivre dans la pauvreté, la faiblesse du niveau de littératie moyen des citoyens 
d'un pays diminue les ressources de celui-ci ainsi que sa capacité à «atteindre ses objectifs, qu'ils soient sociaux, politiques, civiques ou économiques » (p. xix).

Lewis (2002) prétend que dans certains états des États-Unis, les pouvoirs publics se basent sur les résultats en lecture des élèves de troisième année pour calculer le nombre de places à prévoir dix ans plus tard dans les prisons. Cette thèse peut certes paraître peu vraisemblable, mais il n'en reste pas moins vrai que selon certaines estimations, la moitié de la population adulte incarcérée dans les prisons fédérales des États-Unis ne sait ni lire, ni écrire. Le détenu typique âgé de 25 ans fonctionne à un niveau qui se situe à deux ou trois années d'études en dessous du niveau de la dernière année d'études qu'il a achevée (Bellarado, 1986).

L'Organisation des Nations Unies pour l'éducation, la science et la culture (UNESCO) considère que la littératie fournit une bonne mesure du rendement de l'éducation et donne une meilleure indication de l'enseignement que les chiffres relatifs aux effectifs scolarisés, en particulier pour les populations jeunes des régions en voie de développement, car elle reflète généralement le niveau minimum d'enseignement réussi avec fruit (PNUD, 2002). Dans la même publication, le PNUD souligne l'existence d'une étroite corrélation entre le niveau de littératie et la réussite sociale. Cette corrélation a également été observée dans l'Enquête internationale sur la littératie des adultes (OCDE et Statistique Canada, 2000).

La lecture est un prérequis pour obtenir de bons résultats dans toute matière scolaire. En intégrant les trois domaines de littératie (la Compréhension de l'écrit, la Culture mathématique et la Culture scientifique), le programme PISA 2000 génère des informations sur les relations entre ces domaines d'évaluation. La corrélation entre les résultats en lecture et en mathématiques dans PISA est de 0,81 et la corrélation entre les résultats en lecture et en sciences est de 0,86 . Ces deux corrélations se révèlent légèrement supérieures lorsqu'elles sont calculées séparément pour les filles et les garçons.

La lecture n'est donc pas seulement un objectif : elle est aussi un instrument important pour l'éducation et l'épanouissement personnel, que ce soit à l'école ou plus tard dans la vie. La Commission européenne (2001) reconnaît que les compétences en lecture jouent un rôle fondamental dans l'apprentissage des individus à l'école : "La faculté de lire et de comprendre des instructions et des textes est une condition de base pour la réussite dans l'ensemble des matières enseignées. L'importance des compétences en lecture et en écriture ne cesse toutefois pas au moment où les enfants quittent l'école. Ces compétences sont capitales dans tous les domaines de l'éducation; elles facilitent la participation au cadre plus large de l'apprentissage tout au long de la vie et contribuent au développement personnel et à l'intégration sociale des individus ».

Ce qui précède nous amène à conclure que la capacité de lire et de comprendre des informations complexes est importante pour réussir des études de niveau supérieur, pour réussir dans le monde du travail et dans la vie de tous les jours. Le niveau de littératie conditionne non seulement les résultats dans les autres matières enseignées à l'école mais également la réussite dans la plupart des domaines de la vie adulte (voir notamment Cunningham et Stanovich, 1998 ; Smith, Mikulecky, Kibby et Dreher, 2000). Tout le monde s'accorde désormais à reconnaître que l'apprentissage scolaire ne suffit pas. En effet, il faut amener les élèves à utiliser l'écrit - quelle que soit la forme sous laquelle il se présente - à bon escient et sans appréhension pour leur permettre de se livrer à l'apprentissage tout au long de la vie.

Combler le fossé de compétence en lecture qui existe entre des élèves issus de divers milieux défavorisés et leurs condisciples qui se fondent dans la norme est l'un des défis lancés aux systèmes éducatifs à l'aube de ce nouveau millénaire (Au et Raphael, 2000). L'étude de la répartition des niveaux de compétence entre les jeunes adultes et l'analyse du réseau de relations existant entre ces niveaux et les variables liées 
au milieu des élèves sont deux étapes préliminaires qu'il est indispensable de franchir pour s'attaquer au problème posé par l'insuffisance des compétences en lecture des jeunes adultes au terme de leur scolarité obligatoire.

\section{Contexte du cadre d'évaluation de la compréhension de l'écrit}

Comme le laisse transparaitre l'extrait de l'ouvrage de Gray et Rogers cité au début de ce chapitre, la compréhension de l'écrit est un processus dynamique, et non statique, qui doit suivre l'évolution sociétale et culturelle. Les compétences en lecture dont on avait besoin il y a vingt ans pour s'épanouir, participer à la vie économique et se conduire en citoyen étaient très différentes de celles requises aujourd'hui et, selon toute vraisemblance, de celles qui le seront dans vingt ans. Nous vivons dans un monde en pleine mutation, qui assiste à la multiplication incessante du nombre et des types d'écrits et qui attend d'un nombre croissant d'individus qu'ils se servent de ces documents en adoptant de nouvelles approches, parfois plus complexes.

La société industrielle avait seulement besoin de travailleurs qualifiés et instruits dont l'éducation consistait en la maîtrise de certains savoirs et savoir-faire assimilés pendant leur scolarité. À l'époque, on attendait uniquement des individus qu'ils puissent accomplir des tâches relativement simples et bien définies de manière itérative, en s'appuyant essentiellement sur les savoirs et savoir-faire acquis pendant leurs études. L'entrée dans l'ère de l'information demande aux entreprises d'innover et d'adapter leurs produits en permanence. Comme le travailleur moyen réoriente sa carrière tous les cinq à dix ans, l'apprentissage tout au long de la vie est devenu un facteur déterminant pour la réussite de chaque individu. L'objectif de l'enseignement a cessé d'être la simple assimilation d'informations statiques, pour être remplacé par la nécessité d'acquérir des compétences qui permettent de traiter, de synthétiser et d'évaluer l'information.

Aux États-Unis, la commission ministérielle sur l'acquisition des compétences fondamentales (Commission on Achieving Necessary Skills, SCANS) estime dans un rapport (1991) qu'à l'avenir, le marché de l'emploi exigera des travailleurs qu'ils soient capables «d'acquérir, d'organiser, d'interpréter et d'évaluer les informations et d'utiliser un ordinateur pour ce faire ». Dans le même ordre d'idée, elle définit le lecteur compétent comme une personne qui «situe, comprend et interprète l'information écrite par exemple dans des textes continus et des documents - des graphiques et des horaires - pour effectuer des tâches ; qui acquiert des connaissances en identifiant l'idée principale ou le message essentiel d'un texte ; qui identifie les consignes, les faits et les détails pertinents ; qui déduit la signification de termes inconnus ou techniques ou du moins les situe, et qui juge de la précision, de l'adéquation, du style et du caractère plausible de rapports, de propositions ou de théories d'autres auteurs ». Cette manière de voir les choses est très similaire à la définition de la compréhension de l'écrit adoptée par PISA, ainsi que le montre le chapitre 2.

La lecture fait partie de la vie depuis des siècles mais la série de compétences requises pour lire efficacement ne cesse d'évoluer. Rechercher des informations sur Internet devient pour un nombre croissant d'individus une activité de tous les jours. C'est aussi un exemple manifeste de contexte qui impose la maîtrise de compétences en lecture qui vont bien au-delà du simple déchiffrage. Traditionnellement, l'écrit passe par un processus de vérification et de mise en forme impliquant un certain nombre de personnes avant d'atteindre le lecteur. Il n'en va pas de même sur Internet : la qualité des informations qui y figurent est plus variable. Certaines de ces informations ont été vérifiées, certes, mais une partie importante d'entre elles ne l'ont pas été. Dans ce contexte, le lecteur doit pouvoir s'appuyer sur ses propres facultés critiques pour décider s'il convient d'utiliser ces informations ou non, et comment. De plus, le volume important 
d'informations disponibles impose la définition de critères de sélection. Pour recueillir des informations sur Internet, il faut être capable de parcourir et sonder de grandes quantités d'informations et de les soumettre à un jugement de valeur immédiat. Faire preuve de sens critique est donc devenu un aspect plus important que jamais de la compréhension de l'écrit (Halpern, 1989 ; Shetzer et Warschauer, 2000 ; Warschauer, 1999). Warschauer (sous presse) conclut que combler le «fossé technologique » n’est «pas seulement une question d'accès, mais relève aussi de la capacité des individus à s'adapter et à développer des connaissances à l'aide des TIC ». Il existe une corrélation de 0,53 entre la part de la population des pays de l'OCDE qui utilise Internet (CIA, 2001) et les scores moyens obtenus par les mêmes pays en compréhension de l'écrit lors du cycle PISA 2000. Il est probable que plus les individus consulteront Internet, plus ils en deviendront des adeptes. Nos systèmes éducatifs peuvent toutefois accélérer le processus en dotant les jeunes des compétences requises en compréhension de l'écrit.

Internet n'est qu'une source d'informations parmi tant d'autres. La capacité d'accéder à tous les types d'informations, de les comprendre et de réfléchir à leur propos est essentielle pour pouvoir participer pleinement à notre société de la connaissance. C'est la raison pour laquelle le cadre d'évaluation de la compréhension de l'écrit chez les élèves en fin de scolarité obligatoire se concentre sur les différents aspects de la lecture que sont la localisation, la sélection, l'interprétation et l'évaluation des informations provenant d'un large éventail de textes associés à des contextes scolaires et non scolaires.

Qu'il soit défini au niveau national ou local, le curriculum n'est pas le seul facteur qui détermine le niveau de compétence en lecture. L'engagement des élèves (Guthrie et Wigfield, 2000) et l'accès à des matériels écrits (Neuman et Celano, 2001) figurent parmi les variables dont il a été établi qu'elles étaient en relation avec les performances en lecture. Le temps que les élèves consacrent à la lecture est également un indicateur probant de la compréhension de l'écrit (Anderson, Wilson et Fielding, 1988). Si les élèves lisent bien, ils ont tendance à lire davantage et, donc, à acquérir plus de connaissances dans tous les domaines (Cunningham et Stanovich, 1998). En revanche, les élèves qui lisent peu trouvent souvent que les textes sont trop difficiles (Allington, 1984) et développent des attitudes négatives à l'égard de la lecture (Oka et Paris, 1986). Ils s'enferment dans un cercle vicieux car le fait de lire moins réduit leurs chances d'élaborer des stratégies en compréhension de l'écrit (Brown, Palincsar et Purcell, 1986). Ils restent à la traîne dans toutes les matières puisque la lecture est indispensable pour progresser dans toutes les disciplines scolaires (Chall, Jacobs et Baldwin, 1990).

Ce constat n'implique cependant pas qu'il est impossible de changer le cours des choses. Les parents, les enseignants et la société peuvent très fortement influer sur le volume d'écrits lus par les enfants (Gambrell, 1996). En outre, des études récentes donnent à penser que l'utilisation consciente des stratégies métacognitives est étroitement corrélée aux performances en lecture et, surtout, que cette utilisation consciente peut faire l'objet d'un apprentissage efficace (Cornoldi, 1990, Mokhtari et Sheorey, 2001 ; Paris et Winograd, 1990 ; Winn, 1994).

Les analyses multivariées des données recueillies nous permettent en outre de déterminer en quoi les facteurs liés au milieu et aux attitudes contribuent à expliquer les différences en compréhension de l'écrit observées au niveau des élèves. De plus, le présent rapport étudie la part de variance qui peut être imputée aux établissements et se penche sur les relations complexes entre le milieu des élèves et les caractéristiques de leur école. Il peut donc donner de nouvelles indications sur les différences de performances entre élèves qui permettront aux décideurs de définir des stratégies plus efficaces pour lutter contre ces effets. 
La définition de la compréhension de l'écrit adoptée par PISA s'inspire en partie de la «Reading Literacy Study » de l'IEA (Elley, 1992), mais aussi de l'Enquête internationale sur la littératie des adultes (IALS). Elle reflète la priorité donnée par cette dernière enquête à l'importance des compétences en lecture pour la participation active et critique à la vie en société. Elle est également influencée par des théories récentes sur la lecture qui insistent sur la nature interactive de la compréhension de l'écrit (Dechant, 1991 ; McCormick, 1988 ; Rumelhart, 1985), par les modèles de la compréhension du discours (Graesser, Millis et Zwaan, 1997 ; Kintsch et van Dijk, 1978 ; van Dijk et Kintsch, 1983) et par les théories relatives à la résolution de tâches de lecture (Kirsch, 2001 ; Kirsch et Mosenthal, 1990).

Outre ces aspects procéduraux, PISA utilise différents contextes de lecture, car il vise essentiellement à mesurer les compétences en lecture dont les jeunes de 15 ans auront besoin pour participer à la vie sociale en tant que citoyens, travailleurs et apprenants tout au long de la vie. Le Cadre européen de référence pour les langues (Conseil de l'Europe, 2001) distingue quatre grands domaines d'utilisation des compétences linguistiques, le domaines privé - ou personnel -, public, éducatif et, enfin, professionnel. Les tâches de lecture conçues pour PISA intègrent ces sphères, car elles font appel à différents types de lecture, notamment lire des textes éducatifs « pour apprendre » ou des textes professionnels « pour agir » (Sticht, 1975 ; Stiggins, 1982).

\section{Élaboration du cadre d'évaluation et des instruments de test pour la compréhension de l'écrit, ainsi que du questionnaire contextuel Élève}

En vertu de l'approche décrite dans les paragraphes précédents, la définition de la compréhension de l'écrit adoptée par PISA ne s'appuie pas sur une conception unidimensionnelle de la lecture qui pourrait être représentée fidèlement par une simple échelle ou un point donné sur une échelle. Des perspectives différentes sur le nombre et la composition des échelles définissant la compréhension de l'écrit ont été prises en considération lors de la phase de conception de PISA. Ce rapport donne des informations sur la performance des pays en la rapportant sur une échelle composite de compréhension de l'écrit ainsi que sur une série de sous-échelles qui traduisent des aspects importants du cadre d'évaluation. Il rend compte des résultats sur ces sous-échelles dans le but d'étudier le processus de lecture dans toute sa complexité et d'examiner l'hypothèse que les compétences en lecture puissent varier d'un pays à l'autre.

Le cadre d'évaluation a été rédigé collectivement par le groupe fonctionnel d'experts chargé de la compréhension de l'écrit (RFEG), un comité international de spécialistes de la lecture constitué dans le but de définir l'orientation théorique de l'enquête. Des versions préliminaires du cadre conceptuel ont été soumises aux pays Membres qui ont été invités à faire part de leurs commentaires. Les versions successives ont été remaniées compte tenu de leurs remarques. Comme le cadre d'évaluation a servi de base à l'élaboration des épreuves de lecture, les experts réunis au sein du groupe fonctionnel ont également joué un rôle majeur dans la conception des tests : ils ont supervisé la sélection des textes servant de support à l'évaluation, l'élaboration des questions associées à ces textes et, enfin, la préparation des guides de correction relatifs aux items à réponse construite afin de garantir leur adéquation avec l'objectif des questions ou des consignes.

Le Consortium PISA a invité les pays participants à soumettre des tâches de lecture opérationnalisant les spécifications du cadre d'évaluation préparé par le groupe d'experts chargé de la compréhension de l'écrit. Quelque 800 items à réponse construite et à choix multiple ont été sélectionnés parmi les items soumis par les pays et ceux élaborés par les développeurs de tests en Australie et aux Pays-Bas. Ces items ont été testés une première fois en Australie et aux Pays-Bas afin de vérifier l'adéquation des tâches de 
lecture. Ce test pilote a par ailleurs permis de recueillir des exemples de réponses d'élèves aux items à réponse construite. Ces exemples de réponses ont été utilisés pour affiner les guides de correction et lors de la formation des correcteurs. Après les feed-back successifs des panels nationaux, 372 items remaniés, y compris quelques items de l'Enquête internationale sur la littératie des adultes (IALS), ont fait l'objet d'un essai de terrain dans les pays participants. Au terme de l'analyse approfondie des résultats de cet essai de terrain et d'un nouveau processus de consultation des panels nationaux, 141 items, dont 15 tirés de l'IALS, ont été retenus pour la campagne de tests définitive.

Le programme PISA a également recueilli des informations sur les élèves par le biais de questionnaires conçus à cet effet. Le questionnaire Élève comportait notamment deux séries de questions visant à évaluer les pratiques de lecture des élèves et leurs attitudes à cet égard. Concernant les pratiques de lecture, les élèves ont été priés d'indiquer la fréquence à laquelle ils lisaient les différents types d'écrit énumérés dans une liste qui leur était proposée. Un jeu de 10 affirmations à propos de la lecture a été élaboré pour évaluer leurs attitudes à l'égard de la lecture. Les élèves ont indiqué s'ils étaient d'accord ou non avec chacune des affirmations. Des propositions négatives et positives à propos de la lecture ont été mélangées afin d'éviter les biais de désirabilité sociale. De plus, les élèves ont été invités à indiquer combien de temps ils consacraient par jour à lire pour le plaisir. Ces questions ont été combinées pour construire un indice d'engagement à l'égard de la lecture.

\section{Qualité et comparabilité}

Mesurer le niveau de littératie dans un large éventail de langues et de cultures différentes est une entreprise ambitieuse. L'équivalence culturelle et linguistique des instruments dans tous les groupes participants s'impose si l'on veut que l'évaluation ait du sens. Le Rapport technique de PISA 2000 (OCDE, 2002b) décrit de manière détaillée l'ensemble des stratégies qui ont été adoptées pour garantir cette équivalence. Nous présentons ci-dessous quelques-unes des principales dispositions qui ont été prises pour garantir la comparabilité des données.

Les différences de structures entre les systèmes éducatifs des divers pays empêchent de définir les années d'études d'une manière comparable au niveau international. Pour être valides, les comparaisons internationales du rendement entre systèmes éducatifs doivent se baser sur des populations définies en fonction de l'âge. La population cible de PISA est constituée des jeunes âgés de 15 ans au moment de l'évaluation, quels que soient leur année d'études, le type d'établissement qu'ils fréquentent et leur mode de scolarisation (à temps plein ou à temps partiel). Elle exclut toutefois les jeunes de 15 ans qui ne sont pas inscrits dans un établissement d'enseignement. Dans l'ensemble, 95 pour cent au moins de cette population cible a été couverte par les échantillons utilisés pour le cycle d'évaluation de PISA 2000. Des pourcentages supérieurs ont même été enregistrés dans la majorité des pays. Ce degré élevé de couverture contribue à la comparabilité des résultats de l'évaluation (consulter le Rapport technique de PISA 2000 pour plus de détails sur la définition de la population de PISA et la couverture des échantillons).

Plus de 250000 élèves, représentant près de 17 millions d'élèves de 15 ans inscrits dans les établissements d'enseignement des 32 pays participants, ont répondu à des tests papier-crayon d'une durée de deux heures. Lors de ce premier cycle, les élèves ont consacré en moyenne 85 minutes aux tâches de lecture. L'échantillonnage des élèves à partir de la population cible totale de chaque pays participant a été réalisé dans le respect de règles rigoureuses, élaborées pour garantir la représentativité des échantillons nationaux. 
Dans les études comparatives internationales, il est important que les tâches proviennent d'un large éventail de cultures et de langues. C'est la raison pour laquelle dès le début du processus de développement des tests du cycle PISA 2000, l'OCDE a invité les pays participants à soumettre des items en suivant des directives de soumission précises. Ces directives décrivaient notamment l'orientation à l'égard de la compréhension de l'écrit prise par PISA, et son objectif d'évaluer l'état de préparation des jeunes à la vie adulte. Les pays participants ont été instamment priés de soumettre des items authentiques. Près de deux tiers des pays ont fourni du matériel (voir l'annexe A1.1 pour la liste des pays et organisations ayant contribué à fournir des items pour PISA).

Peut-être le matériel de compréhension de l'écrit fourni par un pays donné risque-t-il d'être culturellement peu familier aux élèves d'autres pays. On pourrait toutefois avancer que des élèves de 15 ans devraient pouvoir lire du matériel provenant d'autres pays que le leur ou représentant une culture différente. L'élaboration des instruments a été réalisé dans le respect d'un principe général, à savoir l'équilibre entre trois composantes clés du cadre d'évaluation de la compréhension de l'écrit : la situation, la structure des textes et la nature de la question ou de la consigne associée à un texte (OCDE, 1999). Grâce à cette approche, PISA s'est concentré davantage sur le construct ${ }^{1}$ de compréhension de l'écrit, plutôt que sur les aspects liés au contenu et au caractère familier des instruments. Il n'en reste pas moins vrai que la collecte de matériels auprès des pays participants a grandement contribué à la présence dans les tests de compréhension de l'écrit d'autant de perspectives culturelles que possible.

D'autres moyens, notamment ceux décrits ci-dessous, ont été utilisés pour optimiser la pertinence et l'adéquation culturelle des tâches pour tous les pays et tous les sous-groupes participants (voir l'annexe A1 pour les indices quantifiables visant à garantir l'adéquation culturelle dans le cadre de PISA 2000).

- L'adéquation des matériels fournis par les pays participants a été vérifiée lors de la révision approfondie des items. Ce processus de révision mis en ouvre avant l'essai de terrain a notamment consisté en l'analyse des items dans chaque pays et en leur étude détaillée par l'équipe internationale de traducteurs. Les experts nationaux spécialisés dans les matières visées ont évalué tous les items et stimuli en fonction de l'exposition des élèves au contenu des items, de leur difficulté, de leur contexte culturel, des biais éventuels, des problèmes de traduction et de la priorité générale accordée aux items. Une banque de données a été créée pour garder la trace de tous les commentaires formulés à propos de chaque item de lecture. Cette banque de données s'est ajoutée aux autres sources d'information qui ont été utilisées lors de la révision et de la sélection des items en vue de la campagne de tests définitive.

- Les textes et les items ont été révisés par un panel culturel international. Ce panel a été chargé d'étudier l'ensemble des items de compréhension de l'écrit et les guides de correction utilisés lors de l'essai de terrain, ainsi que les analyses d'item réalisées pour chaque tâche. Le feedback du panel a été associé à d'autres résultats pour revoir et sélectionner la batterie définitive d'items en vue de la campagne de tests définitive.

- L'équivalence culturelle a été améliorée grâce aux données de l'essai de terrain, qui ont permis d'inclure dans les guides de correction un éventail plus large d'exemples de réponses recueillies dans les pays participants. Ces données ont également servi de critère pour décider de l'exclusion d'items présentant des niveaux inacceptables d'interaction entre le degré de difficulté et le pays, la langue, le sexe ou le milieu socio-économique. 
- Des procédures de traduction aussi détaillées que rigoureuses ont été mises en ouvre pour assurer la qualité et la comparabilité des instruments de tests. Deux versions sources de l'ensemble du matériel de test ont été préparées, l'une en anglais et l'autre en français. Grâce à l'élaboration de ces deux versions sources parallèles, des biais culturels et des problèmes de traduction potentiels ont pu être repérés à un stade précoce. Les deux versions ont ensuite été envoyées pour être traduites dans les pays participants, accompagnées de consignes de traduction détaillées (concernant tant les questions de psychométrie que le vocabulaire et les structures de phrase, comprenant une liste de pièges de traduction fréquents, des directives quant aux adaptations acceptables ou non, etc.). Les versions nationales, élaborées sur base de la traduction des deux langues sources et de leur conciliation, ont ensuite été soumises à la vérification d'une équipe de traducteurs engagée par le Consortium et formée à contrôler l'équivalence entre les versions sources et les versions nationales. Les pays ont été autorisés à procéder à des adaptations limitées du matériel de test afin d'en améliorer l'adéquation culturelle et linguistique à leur langue ou à leur contexte national, pour autant que le Consortium international ait jugé que l'adaptation n'affecterait pas ce qui est mesuré.

- Soucieux d'éviter que le processus de traduction ne modifie la difficulté des items, les développeurs de test ont accompagné certains items de notes de traduction destinées à attirer l'attention sur des problèmes potentiels de traduction et d'interprétation. Ils ont également préparé une synthèse de l'objectif de chaque question et des processus principaux que chaque item visait à évaluer pour s'assurer que l'objet des questions resterait inchangé. Au cours du processus de traduction, ils ont accepté ou rejeté les adaptations nationales proposées de sorte que celles-ci n'affectent pas l'objectif des items.

- Les correcteurs des pays participants ont suivi une formation commune avant l'essai de terrain et une autre en vue de la campagne de tests définitive. Pour tous les items nécessitant un codage manuel, des guides de correction ont été préparés et illustrés par de nombreux exemples de réponses, couvrant l'ensemble des scores pouvant être attribués à chaque item. Les pays participants ont largement contribué à l'élaboration de ces guides lors de la formation des correcteurs. Les rédacteurs de ces guides ont veillé à mettre en évidence l'objet de la mesure associée à chaque item et ont insisté sur le fait que les guides devaient être utilisés par les correcteurs pour «coder» les réponses, plutôt que de les «noter ». De la sorte, la subjectivité des correcteurs a pu être réduite, car la mission qui leur a été confiée n'était pas de juger de la qualité des réponses mais d'assigner à chaque réponse la meilleure classification parmi celles qui leur étaient proposées dans le guide de correction. La mise au point du codage définitif a été réalisée au terme des analyses des données de l'essai de terrain, qui ont permis de recueillir des informations sur le caractère adéquat des «scores » pour chaque catégorie de réponse.

- Le Consortium international a mis en place un service de messagerie électronique permettant de traiter les questions des correcteurs en ligne et de s'assurer que les correcteurs de tous les pays interprétaient et appliquaient d'une manière similaire les guides de correction. Des données ont été comparées entre et dans les pays afin de garantir la comparabilité du codage. Les résultats de ces analyses figurent dans le Rapport technique de PISA 2000 (OCDE, 2002b).

- La longueur de chaque test a été contrôlée, afin d'éviter de désavantager les élèves n'ayant pas l'habitude de passer des examens limités dans le temps (le nombre de mots maximum a été déterminé sur base des taux d'omission relevés dans les données de l'essai de terrain).

- Un indice statistique appelé fonctionnement différentiel des items (DIF, pour differential item functioning) a été utilisé pour identifier les items qui avaient fonctionné différemment dans certains pays. Les items « soup- 
çonnés » de présenter un biais culturel ont été revus en concertation avec les experts nationaux des pays concernés. Suite à cette analyse, certains items ont été exclus de l'échelle, comme s'ils n'avaient pas été administrés dans certains pays.

\section{Objet et structure du présent rapport}

Ce rapport analyse de manière approfondie le construct de la compréhension de l'écrit tel qu'il a été défini et opérationnalisé pour PISA. Il est illustré par des items utilisés lors du cycle PISA 2000 et représentatifs des principales composantes du cadre d'évaluation. Chaque item utilisé dans les tests est une source importante d'information mise à profit pour tirer des conclusions concernant les connaissances et les compétences des élèves dans les pays participants. Il est important de savoir comment ces items ont été élaborés et ce qu'ils mesurent pour comprendre les résultats et pour en examiner les implications à l'intention des décideurs et des professionnels de l'éducation. Le chapitre 2 présente le cadre d'évaluation de la compréhension de l'écrit et définit le principe d'échelle de compétence afin d'expliquer le niveau de performance par rapport à chaque échelle. Il décrit par ailleurs les sous-échelles de compétence utilisées pour rendre compte des résultats. Le chapitre 3 illustre ce qui précède par une série d'exemples d'items et les situe par rapport aux spécifications du cadre d'évaluation.

Si le rapport initial (OCDE, 2001b) documente abondamment le niveau de performance des pays sur l'échelle combinée de compréhension de l'écrit, il ne s'est guère étendu sur les sous-échelles qui ont été créées afin d'explorer les éventuelles interactions entre pays. Le chapitre 4 porte sur le classement des pays et sur la variation du niveau de compétence entre et dans les pays rapporté à deux groupes de souséchelles. Il met en évidence les différences significatives entre pays relevées sur ces sous-échelles. Enfin, il présente des statistiques sur l'importance des inégalités au sein même des pays, car les pays participants s'intéressent non seulement à la performance globale de leurs élèves, mais aussi à l'ampleur de la variation entre les élèves les plus performants et les plus faibles.

Ce rapport apporte une contribution originale par l'importance qu'il accorde au concept d'engagement dans la lecture. Le chapitre 5 présente la répartition du niveau d'engagement dans la lecture des élèves des pays participants et explique en quoi il est associé à la performance sur les échelles de compréhension de l'écrit. La relation entre le niveau d'engagement à l'égard de la lecture et certaines caractéristiques du milieu des élèves est également étudiée de manière approfondie dans ce chapitre.

Le chapitre 6 poursuit l'analyse de la relation entre le niveau de littératie et certaines caractéristiques contextuelles. Il étudie les différences relevées entre et dans les pays concernant la relation entre les caractéristiques individuelles, familiales et scolaires d'une part, la performance sur chacune des sous-échelles de compréhension de l'écrit ainsi que l'engagement dans la lecture d'autre part.

Le chapitre 7 étudie l'impact des caractéristiques contextuelles des élèves sur leur performance par le biais d'une analyse multivariée. Le modèle de régression multivariée multi-niveaux choisi correspond au meilleur ajustement possible pour les pays participants. Ce chapitre étudie l'importance de chaque caractéristique individuelle des élèves après avoir pris en compte toutes les autres variables. Ces analyses, qui ont été réalisées pour l'échelle combinée de compréhension de l'écrit ainsi que pour chacune des souséchelles qui la composent, révèlent des interactions intéressantes entre les sous-échelles.

Le chapitre 8 se penche sur la relation entre les résultats de PISA et ceux de l'Enquête internationale sur la littératie des adultes (IALS) à la faveur de l'analyse des 15 items tirés de l'IALS qui ont été utilisés lors du cycle d'évaluation PISA 2000. Le programme IALS a explicitement étudié les relations entre le niveau 
CHAPITRE 1 La lecture, moteur de changement: Performance et engagement d'un pays à l'autre

de littératie des adultes et leurs caractéristiques sociales, culturelles et économiques. Les analyses présentées au chapitre 8 peuvent donc fournir une occasion d'évaluer les perspectives qui s'offrent aux jeunes de 15 ans en fonction de leur degré de littératie.

1. NDT : Le terme « construct » a volontairement été laissé en anglais, car il est d'usage courant dans la terminologie technique. 


\section{Chapitre}

2

\section{LE CONSTRUCT DE \\ COMPRÉHENSION DE L'ÉCRIT \\ DANS PISA}

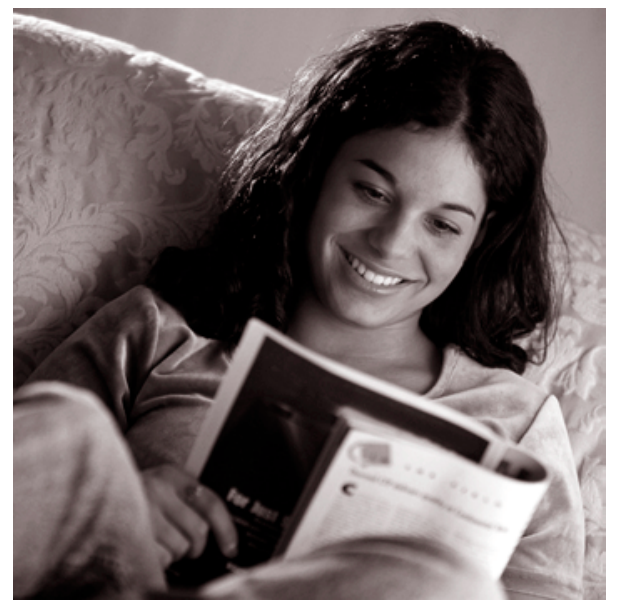




\section{SYNTHÈSE}

- Ce chapitre présente le cadre conceptuel sur lequel se fonde le choix des textes utilisés dans l'évaluation de PISA et l'élaboration des tâches qui y sont associées. En donnant des indications sur les connaissances et les compétences des élèves qui ont participé à l'évaluation PISA, chacun des items de lecture permet d'étayer les déductions relatives à leur capacité de comprendre et d'utiliser des informations figurant dans un large éventail de textes qui s'inscrivent à l'intérieur et à l'extérieur du cadre scolaire.

- La définition de la compréhension de l'écrit est un élément du cadre d'évaluation. La définition de la lecture et de la compréhension de l'écrit a progressé au fil du temps, parallèlement aux changements de société, d'économie et de culture. Le concept d'apprentissage et plus particulièrement celui d'apprentissage tout au long de la vie a élargi la notion de compréhension de l'écrit et a multiplié les exigences qui y sont associées. La littératie n'est plus considérée comme une faculté acquise uniquement pendant l'enfance et les premières années de scolarisation. Elle est désormais définie comme un ensemble de connaissances, de compétences et de stratégies que les individus étoffent tout au long de leur vie dans les diverses situations qui se présentent à eux et par les interactions avec leurs semblables et les communautés dans lesquelles ils vivent.

- Après la présentation du cadre conceptuel et des tâches qui ont été élaborées dans le but de mesurer le niveau de compétence en compréhension de l'écrit, ce chapitre explique l'approche adoptée pour rendre compte des résultats de PISA. La batterie de tests de compréhension de l'écrit constituée pour le cycle PISA 2000 est rapportée globalement sur une échelle composite de compréhension de l'écrit et sur cinq sous-échelles, dont trois dites d'aspect (trouver l'information, développer une interprétation, réfléchir et évaluer) et deux dites de format de texte (les textes continus et non continus).

- L'échelle de compréhension de l'écrit ne résume pas seulement la compétence mais elle illustre également la batterie de tests utilisée pour la mesurer en définissant chaque item en fonction de la même dimension. Tout comme l'échantillon des élèves soumis à l'évaluation est représentatif de la population d'élèves de 15 ans d'un pays, les items de compréhension de l'écrit sont représentatifs des catégories de tâches dans le domaine de la compréhension de l'écrit défini dans le cadre de PISA. Ce chapitre explique par ailleurs que la progression des items sur l'échelle composite de compréhension de l'écrit et sur les cinq sous-échelles représente une série de types de textes et d'exigences articulées selon un principe de complexité progressive. Cette progression est définie en fonction des processus que les élèves doivent appliquer et des compétences dont ils doivent faire preuve pour répondre correctement aux items qui leur sont soumis. 
Ce chapitre décrit les principales composantes du cadre conceptuel de compréhension de l'écrit qui ont été utilisées pour sélectionner les textes des tests et élaborer les tâches y afférentes. Les items de PISA ont tous été construits dans le but de fournir des informations sur le statut de la compréhension de l'écrit chez les élèves de 15 ans dans les pays participants. L'objectif essentiel qui a présidé à l'élaboration du cadre d'évaluation de la compréhension de l'écrit est d'améliorer la qualité des mesures en privilégiant une interprétation plus significative, fondée sur la détermination de niveaux de performance suffisamment généralisables pour permettre des comparaisons valides entre groupes de population et à long terme, plutôt qu'une interprétation des résultats d'enquête en termes de tâches distinctes ou de valeurs numériques uniques (par exemple le pourcentage moyen de réponses correctes). Cette approche vise à augmenter la mesurabilité (Messick, 1989). Si l'élaboration d'un cadre d'évaluation pour la compréhension de l'écrit doit bien permettre une meilleure mesurabilité, d'autres avantages potentiels ont été également jugés importants :

- le cadre d'évaluation a le mérite de proposer un langage commun et d'ouvrir le débat à propos de la définition de son objet et des hypothèses qui la sous-tendent ;

- ces débats offrent la possibilité d'élaborer un consensus à propos du cadre d'évaluation et des objectifs de mesure qu'il projette ;

- le processus qui consiste à élaborer un cadre conceptuel puis à relier ce cadre aux informations recueillies par le biais des épreuves d'évaluation permet d'arriver à une meilleure compréhension de l'objet des mesures ;

- cette compréhension des tenants et aboutissants et son rapport avec ce que nous disons des élèves constituent un lien important entre l'action publique, l'évaluation et la recherche, qui renforce encore l'utilité des données recueillies.

Outre la présentation des principales composantes du cadre d'évaluation de la compréhension de l'écrit, ce chapitre décrit également la manière dont le niveau de compétence des élèves dans un ou plusieurs groupes de tâches de lecture est résumé et présenté aux divers publics cibles de ce rapport.

\section{Caractéristiques du cadre d'évaluation de la compréhension de l'écrit du programme PISA'}

La définition de la lecture et de la compréhension de l'écrit a progressé au fil du temps, parallèlement aux changements de société, d'économie et de culture. Le concept d'apprentissage et plus particulièrement celui d'apprentissage tout au long de la vie a élargi la notion de compréhension de l'écrit et a multiplié les exigences qui y sont associées. La littératie n'est plus considérée comme une faculté acquise uniquement pendant l'enfance et les premières années de scolarisation. Elle est désormais considérée comme un ensemble en expansion de connaissances, de compétences et de stratégies, que les individus étoffent tout au long de leur vie, dans les diverses situations qui se présentent à eux et dans les interactions avec leurs semblables et les communautés dans lesquelles ils vivent.

Au fil d'un processus de recherche de consensus auquel ont participé le groupe fonctionnel d'experts chargé de la lecture (RFEG) et les groupes de conseillers de PISA, la définition suivante de la compréhension de l'écrit a été adoptée dans le cadre de l'enquête. 
"Comprendre l'écrit, c'est non seulement comprendre et utiliser des textes écrits, mais aussi réfléchir à leur propos. Cette capacité devrait permettre à chacun(e) de réaliser ses objectifs, de développer ses connaissances et son potentiel et de prendre une part active dans la société. »

Cette définition va au-delà du simple décodage et de la compréhension littérale. Elle implique que la compréhension de l'écrit est un concept qui intègre la compréhension et l'utilisation de l'écrit, mais aussi la réflexion à son propos à différentes fins. Elle prend donc en considération le rôle actif et interactif que joue le lecteur lorsqu'il dégage du sens de textes écrits. Elle reconnaît par ailleurs le large éventail de situations dans lesquelles la compréhension de l'écrit intervient pour les jeunes adultes : de la vie privée à la vie publique, de l'école au travail, au cours de toute une vie d'apprentissage et de citoyenneté active. Elle exprime l'idée que la compréhension de l'écrit permet l'accomplissement des aspirations individuelles, qu'il s'agisse d'aspirations bien définies - obtenir un diplôme ou trouver un emploi, par exemple - ou d'aspirations moins immédiates, qui concernent l'épanouissement personnel de l'individu. La compréhension de l'écrit fournit également au lecteur tout un ensemble d'outils linguistiques dont il a de plus en plus besoin pour répondre aux exigences de nos sociétés modernes et de leurs institutions officielles, de leurs grandes administrations et de leurs systèmes juridiques complexes.

Les lecteurs réagissent de différentes manières à un texte donné à mesure qu'ils cherchent à comprendre et utiliser ce qu'ils lisent. Ce processus dynamique implique de multiples facteurs, dont certains peuvent être opérationnalisés dans des évaluations à grande échelle telles que PISA. Parmi ces facteurs, citons la situation de lecture, la structure du texte et les caractéristiques des questions posées au sujet du texte. Ces facteurs sont tous considérés comme des éléments importants du processus de compréhension de l'écrit et ont été pris en compte lors de la conception des items utilisés.

Pour utiliser la situation de lecture, le texte et les caractéristiques des items de test lors de la conception des tâches d'évaluation et, plus tard, lors de l'interprétation des résultats, il a fallu opérationnaliser ces éléments. En d'autres termes, il a fallu spécifier la valeur et le champ d'application de chacune des composantes, ce qui a permis de classer les tâches par catégorie de sorte que la pondération de chaque composante puisse être prise en considération lors de l'assemblage définitif de l'évaluation.

\section{Situation}

La définition de la notion de situation s'est inspirée des travaux du Conseil de l'Europe (2001) sur le langage. Quatre variables de situation ont été identifiées en fonction du contexte dans lequel s'inscrit la lecture : la lecture à des fins privées, publiques, éducatives et, enfin, professionnelles. Certes, l'objectif de PISA est d'étudier les types de lecture qui se situent tant à l'intérieur qu'à l'extérieur du cadre scolaire ; cependant, la façon dont la situation a été définie ne peut se borner à indiquer l'endroit où se déroule l'activité de lecture. Par exemple, les manuels scolaires sont lus aussi bien à l'école qu'à la maison et les processus et objectifs de cette lecture varient très peu d'un endroit à l'autre. D'autres facteurs interviennent également dans le processus de lecture : l'intention de l'auteur, la diversité des types de contenu et aussi la possibilité que des tiers (enseignants ou employeurs, par exemple) imposent au lecteur ce qu'il doit lire et dans quel but.

Dans le cadre de cette évaluation, la notion de situation peut être considérée comme un critère de classification des textes sur la base de l'intention de l'auteur du texte, de la relation entre le texte et d'autres personnes auxquelles il est explicitement ou implicitement associé et de son contenu général. Les exemples de textes s'inscrivent dans un éventail de situations différentes afin d'optimiser la diversité des contenus 
dans l'évaluation de la compréhension de l'écrit. L'origine des textes sélectionnés a aussi fait l'objet d'une attention toute particulière. En effet, il fallait trouver un équilibre entre la définition globale de la compréhension de l'écrit utilisée dans le cadre de PISA et la diversité linguistique et culturelle des pays participants, pour garantir qu'aucun groupe ne soit avantagé ou désavantagé par le contenu de l'évaluation.

Les quatre situations tirées des travaux du Conseil de l'Europe peuvent être décrites comme suit :

- la lecture à des fins privées (personnelles) : on effectue ce type de lecture pour satisfaire ses intérêts personnels, qu'ils soient d'ordre pratique ou intellectuel. Ceci inclut également la lecture réalisée dans le but de maintenir ou de développer des liens personnels avec d'autres personnes. Parmi les contenus typiques de ce genre de lecture figurent les lettres personnelles, les textes de fiction, les biographies et les textes informatifs lus par curiosité, dans le cadre d'activités récréatives et de loisirs ;

- la lecture à des fins publiques : ce type de lecture est pratiqué pour prendre part à des activités sociales dans le sens large. Il comprend la lecture de documents officiels ainsi que d'informations concernant des événements publics. Généralement, ces tâches sont associées à des contacts avec les autres, qui demeurent relativement anonymes ;

- la lecture à des fins professionnelles : bien que seuls quelques jeunes de 15 ans aient réellement à lire dans un cadre professionnel, il est important de déterminer s'ils sont prêts à entrer dans le monde du travail puisque, dans la plupart des pays participants, plus de 50 pour cent d'entre eux rejoindront la population active un ou deux ans après la réalisation de l'évaluation. Les tâches prototypiques de ce genre sont souvent qualifiées de tâches de «lecture pour agir » (Sticht, 1975 ; Stiggins, 1982), dans la mesure où elles sont liées à l'accomplissement de certaines tâches immédiates ;

- la lecture à des fins éducatives : ce type de lecture, qui a pour finalité l'acquisition d'informations, s'inscrit normalement dans le cadre plus vaste d'une tâche d'apprentissage. Le texte n'a souvent pas été choisi par le lecteur mais donné à lire par un enseignant. Son contenu est habituellement conçu à des fins spécifiques d'instruction. Les tâches prototypiques de ce genre sont souvent qualifiées de tâches de «lecture pour apprendre » (Sticht, 1975 ; Stiggins, 1982).

Le graphique 2.1 présente la répartition des items de compréhension de l'écrit en fonction de ces quatre situations. Les items auraient pu être répartis uniformément entre les quatre situations mais la lecture à des fins professionnelles a été minimisée, sachant qu'il était peu probable que les jeunes de 15 ans soient relativement familiarisés avec ce genre de texte. Par ailleurs, il était important de réduire la dépendance potentielle à l'égard de connaissances professionnelles spécifiques, un danger inhérent à la sélection de textes à caractère professionnel.

\section{Graphique 2.1}

Répartition des items de compréhension de l'écrit en fonction de la situation

\begin{tabular}{lc}
\hline Contexte & Nombre d'items $^{\mathbf{1}}$ \\
\hline Scolaire & 39 \\
Professionnel & 22 \\
Personnel & 26 \\
Public & 54 \\
Total & $\mathbf{1 4 1}$
\end{tabular}

1. Inclus dans la batterie de test. 


\section{Textes}

La distinction entre les textes continus et non continus est l'une des dimensions essentielles sur lesquelles se fonde l'organisation de l'évaluation PISA :

- les textes continus sont conventionnellement constitués de phrases, elles-mêmes organisées en paragraphes. Ils peuvent s'inscrire dans des structures plus vastes, telles que des sections, des chapitres et des livres. La classification principale des textes continus se fonde sur leur objectif rhétorique, c'est-à-dire sur le type de texte ;

- les textes non continus ou documents, ainsi qu'on les désigne parfois, peuvent être classés de deux manières. La première est l'approche par la structure formelle qu'ont utilisée Kirsch et Mosenthal (1989$1991)^{2}$ dans leurs travaux. Ces auteurs distinguent les textes selon la manière dont sont agencées les listes sous-jacentes qui composent les différents types de textes non continus. Leur approche est utile pour comprendre les similitudes et les différences entre les types de textes non continus. L'autre approche utilise des descriptions ordinaires des formats de ces textes. C'est celle qu'a adoptée le PISA pour classer les textes non continus.

\section{Textes continus}

Les types de texte correspondent à des façons standardisées de classer des textes continus en fonction de leur contenu et de l'objectif de leur auteur. ${ }^{3}$

- Le texte narratif est un type de texte où l'information concerne les propriétés des objets dans le temps. La narration répond typiquement à la question « quand... ?» ou à la question « dans quel ordre...?».

- Le texte informatif est un type de texte dans lequel l'information se présente sous la forme d'un ensemble de concepts ou de constructs mentaux, ou encore sous la forme d'éléments constitutifs de ces concepts et constructs. Le texte fournit une explication sur la façon dont ces composantes s'organisent pour former un tout significatif et répondent souvent à la question « comment... ?».

- Le texte descriptif est un type de texte où l'information porte sur les propriétés physiques des objets dans l'espace. La question typique à laquelle les textes descriptifs apportent une réponse est « qu'est-ce que...? ».

- Le texte argumentatif est un type de texte qui présente des propositions relatives aux rapports entre des concepts ou d'autres propositions. Les textes argumentatifs répondent souvent à la question «pourquoi... ?». Une autre sous-catégorie importante des textes argumentatifs est celle des textes persuasifs.

- Le texte de directives (appelé parfois texte injonctif) est un type de texte qui fournit des directives sur ce qu'il faut faire. Parmi ces textes figurent les procédures, les règles, les règlements et les statuts qui spécifient les exigences concernant certains comportements.

- Le document, ou rapport, est un texte conçu pour standardiser et conserver l'information. Il se distingue souvent par des caractéristiques textuelles et structurelles extrêmement formalisées. 
- L'hypertexte est un ensemble de fragments de textes reliés les uns aux autres de sorte que les unités peuvent être lues dans des ordres séquentiels différents, ce qui permet au lecteur d'emprunter différentes voies pour prendre connaissance de l'information. ${ }^{4}$

\section{Textes non continus}

Les textes non continus sont organisés d'une autre manière que les textes continus et font dès lors appel à des démarches de lecture différentes. Il y a lieu de consulter les travaux de Kirsch et Mosenthal pour davantage d'informations sur l'approche structurelle adoptée. Selon ces travaux, les listes sont les textes non continus les plus fondamentaux. Elles sont constituées d'un certain nombre d'éléments qui ont une ou plusieurs propriétés en commun. Ces propriétés communes peuvent servir de titre ou d'en-tête aux listes. Les éléments d'une liste peuvent être classés dans un certain ordre (la liste des élèves d'une classe par ordre alphabétique, par exemple) ou non (la liste des achats à faire dans un magasin, par exemple). La classification des textes non continus en fonction de leur format, comme ci-dessous, permet de déterminer assez facilement le type de textes non continus qui peuvent être inclus dans l'évaluation.

- Les graphiques constituent des représentations iconiques de données. Ils sont utilisés dans les exposés scientifiques ainsi que dans des revues et des journaux pour présenter au public des informations chiffrées dans un format visuel.

- Les tableaux et matrices. Les tableaux sont des matrices de colonnes et de lignes. Les entrées de chaque colonne et de chaque ligne ont généralement toutes des propriétés communes, ce qui explique que les titres des colonnes et des lignes font partie intégrante de la structure informative du texte. Parmi les tableaux les plus courants, citons les horaires, les feuilles de calcul, les formulaires de commande et les index.

- Les diagrammes accompagnent souvent les descriptions techniques (par exemple, pour illustrer les éléments d'un appareil électroménager), les textes informatifs et injonctifs (par exemple, pour présenter la façon de monter un appareil électroménager). Il est souvent utile de faire la distinction entre les diagrammes de procédure (« comment faire ?») et les diagrammes de processus (« comment cela fonctionne-t-il ? »).

- Les cartes sont des textes non continus qui indiquent les relations géographiques entre des lieux. Il existe de nombreuses sortes de cartes. Les cartes routières indiquent les distances et les itinéraires entre des lieux donnés. Les cartes thématiques indiquent les rapports entre des endroits et des caractéristiques sociales ou physiques.

- Les formulaires sont des textes structurés et formatés qui demandent au lecteur de répondre à des questions spécifiques de manière spécifique. Ils sont utilisés par un grand nombre d'institutions pour recueillir des données. Ils comportent souvent des formats de réponse structurés ou pré-codés. Les déclarations d'impôt, dossiers d'immigration, demandes de visa, fiches d'inscriptions, questionnaires statistiques, etc. sont autant d'exemples de formulaires.

- Les feuilles d'information diffèrent des formulaires en cela qu'elles proposent une information plutôt que de la demander. Elles résument l'information d'une façon structurée et dans un format tel que le lecteur peut facilement et rapidement localiser des informations particulières. Les feuilles d'information peuvent contenir diverses formes de textes ainsi que des listes, des tableaux, des schémas, et présenter une typographie sophistiquée (titres, polices, retraits, encadrements, etc.) pour résumer ou mettre en 
évidence l'information. Les horaires, les tarifs, les catalogues et les programmes sont des exemples de ce type de texte non continu.

- Les annonces et les publicités sont des documents destinés à inviter le lecteur à faire quelque chose, par exemple, acheter des marchandises ou des services, participer à des réunions, des rassemblements, élire une personne pour une fonction officielle, etc. Le but de ces documents est de persuader le lecteur. Ils offrent quelque chose et, en même temps, requièrent de l'attention et appellent à une action. Les réclames, les invitations, les assignations, les avertissements et les notices sont des exemples de ce type de format.

- Les coupons servent à certifier que leur titulaire est autorisé à utiliser tel ou tel service. L'information que contient le coupon doit être suffisante pour déterminer s'il est valide ou non. Des exemples typiques de coupons sont les tickets, les factures, etc.

- Les certificats sont des écrits reconnaissant la validité d'un accord ou d'un contrat. C'est leur contenu qui est formalisé plutôt que leur format. Pour garantir la véracité de la déclaration contenue dans le document, ils requièrent en général la signature d'une ou de plusieurs personnes autorisées et qualifiées. Les garanties, les certificats scolaires, les diplômes, les contrats, etc., sont des documents qui présentent ces caractéristiques.

La répartition et la diversité des textes proposés aux élèves dans le cadre du programme PISA constituent une caractéristique essentielle de l'évaluation. Les graphiques 2.2a et 2.2b montrent la répartition recommandée entre les textes continus et non continus. On constate d'emblée que deux tiers environ des items de l'évaluation se rapportent à des textes continus. Dans cette catégorie, le plus grand pourcentage se rapporte à des textes informatifs (31 items), tandis que les items se rapportant à des textes injonctifs (9 items) représentent le pourcentage le plus faible. Les autres items fondés sur des textes continus sont répartis de manière assez uniforme entre des textes narratifs, argumentatifs et descriptifs. Les items se rapportant à des textes non continus représentent environ un tiers de l'évaluation de la compréhension de l'écrit. La grande majorité de ceux-ci demande aux élèves d'interpréter des tableaux, des diagrammes ou des graphiques. Les autres items fondés sur des textes non continus se réfèrent à des cartes géographiques, des messages publicitaires, des schémas ou des formulaires que des élèves âgés de 15 ans sont censés pouvoir lire et utiliser.

$$
\text { Graphique 2.2a }
$$

Répartition des items de compréhension de l'écrit en fonction du format de texte: textes continus

\begin{tabular}{lc}
\hline Format de texte & $\begin{array}{c}\text { Nombre d'items basés } \\
\text { sur des textes continus }\end{array}$ \\
\hline Narratif & 18 \\
Informatif & 31 \\
Descriptif & 13 \\
Argumentatif/persuasif & 18 \\
Injonctif & 9 \\
Total & $\mathbf{8 9}$
\end{tabular}

\section{Graphique $2.2 b$}

Répartition des items de compréhension de l'écrit en fonction du format de texte: textes non-continous.

\begin{tabular}{lc}
\hline Format de texte & $\begin{array}{c}\text { Nombre d'items basés } \\
\text { sur des textes continus }\end{array}$ \\
\hline Diagrammes et graphiques & 16 \\
Tableaux & 15 \\
Schémas & 5 \\
Cartes & 4 \\
Formulaires & 8 \\
Publicités & 4 \\
Total & $\mathbf{5 2}$
\end{tabular}




\section{Modalités de questions/réponses et caractéristiques des items}

Trois ensembles de variables sont concernés ici : les questions ou consignes, qui présentent aux élèves la tâche à effectuer, les formats de réponse, qui indiquent la façon dont les élèves sont appelés à montrer leur compétence à accomplir la tâche, et enfin, les consignes de correction, qui spécifient comment les réponses des élèves doivent être évaluées. Chacune de ces variables est présentée ci-dessous mais c'est la première d'entre elles qui requiert de loin le plus d'attention.

\section{Questions et consignes - Cinq aspects}

Pour tenter de simuler des situations authentiques de lecture, l'évaluation PISA mesure les cinq aspects suivants qui sont associés à une pleine compréhension d'un texte, qu'il soit de type continu ou non continu. Les élèves doivent montrer leur compétence pour chacun de ces aspects :

- comprendre globalement un texte ;

- trouver l'information;

- développer une interprétation ;

- réfléchir sur le contenu d'un texte et l'évaluer ;

- réfléchir sur la forme d'un texte et l'évaluer.

La bonne compréhension d'un texte implique la mise en ouvre de tous ces aspects. Tous les lecteurs, quel que soit leur niveau de compétence global, doivent être capables de faire preuve d'un certain niveau de compétence pour chacun d'eux (Langer, 1995). Bien qu'il y ait une interaction entre les divers aspects - chacun de ces aspects peut solliciter chez le lecteur plusieurs des mêmes savoir-faire sousjacents -, la maîtrise de l'un n'est pas nécessairement dépendante de la maîtrise de l'autre. Certains considèrent ces aspects comme faisant partie du répertoire de tout lecteur à chaque étape de son développement, plutôt que comme un ensemble hiérarchisé et séquentiel de compétences.

Le graphique 2.3 présente les caractéristiques fondamentales qui permettent de distinguer les cinq aspects de la lecture mesurés dans PISA. Bien que chaque aspect y apparaisse de manière simplifiée, ce schéma fournit un cadre utile pour organiser et rappeler les rapports existant entre ces différents aspects. Ainsi que le montre ce graphique, ces cinq aspects se distinguent en fonction de quatre caractéristiques différentes. La première est la mesure dans laquelle le lecteur devra utiliser des informations provenant essentiellement du texte ou devra aussi faire appel à des connaissances extérieures au texte. La seconde est la mesure dans laquelle le lecteur devra se concentrer sur des parties isolées du texte ou sur les relations inhérentes à l'information contenue dans le texte. En effet, le lecteur doit tantôt retrouver des informations isolées, tantôt montrer qu'il a compris les rapports unissant différentes parties de texte. La mesure dans laquelle le lecteur devra se concentrer sur l'ensemble du texte ou sur des relations entre certaines parties du texte constitue la troisième caractéristique distinctive. Enfin, la quatrième est la mesure dans laquelle on demande au lecteur de traiter le contenu ou la substance du texte ou alors sa forme, sa structure. Les cinq aspects de la lecture sont représentés à la dernière ligne du graphique 2.3, à l'extrémité des différentes branches. Il suffit de partir du haut du schéma et de suivre chaque branche pour voir quelles sont les caractéristiques qui sont associées à chacun des aspects. 


\section{Graphique 2.3}

Caractéristiques distinctives des cinq aspects de la compréhension de l'écrit

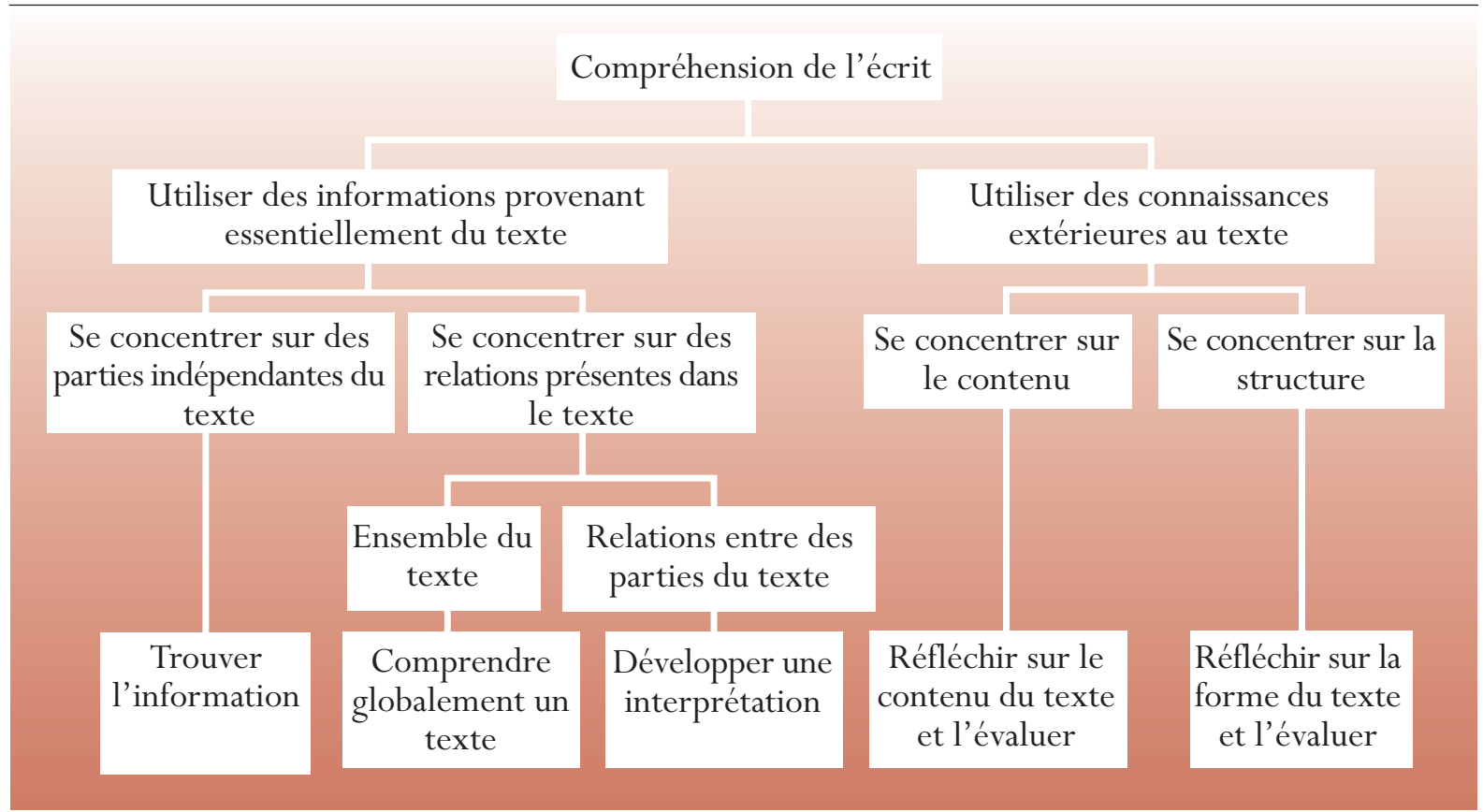

Ci-dessous figure une première ébauche de définition opérationnelle de ces aspects de la compréhension d'un texte visant notamment à les associer à des types particuliers de questions et de consignes. Même si chacun des aspects est présenté ci-après dans le cadre d'un texte unique, ils peuvent également s'appliquer à plusieurs textes lorsque ceux-ci constituent une seule unité au sein de l'évaluation. La description de chaque aspect comprend deux parties. La première partie consiste en une approche globale de l'aspect, tandis que la seconde décrit des manières spécifiques de l'évaluer.

Trouver l'information. Dans leur vie quotidienne, les lecteurs ont souvent besoin d'informations particulières. Il peut s'agir de trouver un numéro de téléphone, de vérifier l'heure de départ d'un autobus ou d'un train ou encore de découvrir un fait précis pour étayer ou réfuter une affirmation avancée par quelqu'un. Dans de telles situations, les lecteurs s'intéressent à la recherche d'une information isolée. Pour la découvrir, ils doivent parcourir des documents, chercher, localiser et sélectionner l'information qui leur sera utile. Le processus impliqué dans cet aspect de la lecture se situe le plus souvent au niveau de la phrase, bien que, dans certains cas, l'information puisse se situer dans deux ou plusieurs phrases ou être répartie dans différents paragraphes.

Dans les items de l'évaluation qui demandent de trouver une information, les élèves testés doivent établir entre l'information fournie dans la question et celle qui figure dans le texte une correspondance qui peut être littérale ou synonyme, et l'utiliser pour trouver la nouvelle information requise. Dans ce type de tâche, la localisation d'informations se fonde sur le texte lui-même et sur l'information explicite qu'il contient. Les tâches de localisation demandent à l'élève testé de trouver des informations en se fondant sur les conditions ou les éléments définis dans les questions ou les consignes. L'élève doit repérer ou identifier un ou plusieurs éléments essentiels du message (les personnages, le rythme ou le temps, le cadre, etc.) avant de rechercher un équivalent qui soit littéral ou synonyme. 
Les items de localisation peuvent également impliquer différents niveaux d'ambiguité. Ainsi, on peut demander à l'élève testé de sélectionner, dans un texte ou dans un tableau, une information explicite, une indication de temps ou de lieu par exemple. Une version plus difficile du même type d'item peut consister à trouver une information synonyme. Cette opération repose parfois sur une catégorisation ou sur une discrimination entre deux informations semblables. En faisant varier systématiquement les paramètres qui contribuent à la difficulté, on peut mesurer les différents niveaux de compétence associés à cet aspect de la compréhension.

Comprendre globalement un texte. Pour saisir globalement le texte qu'il a lu, le lecteur doit le considérer comme un tout, en avoir une vision d'ensemble. Divers types de tâches demandent une compréhension globale de la part du lecteur. Ainsi, les élèves testés peuvent faire preuve de leur compréhension initiale en identifiant le sujet traité ou le message principal ou en cernant l'objectif ou l'usage général du texte. Choisir ou imaginer le titre ou le thème du texte, expliquer l'ordre d'une série d'instructions simples ou identifier les dimensions principales d'un graphique ou d'un tableau constituent des exemples de ce type de tâche. Les élèves peuvent également être priés de décrire le personnage principal, le décor ou le contexte d'une histoire, d'identifier le thème ou le message d'un texte littéraire ou d'expliquer l'objectif ou l'usage d'une carte ou d'un schéma.

Certaines des tâches relatives à cet aspect peuvent demander aux élèves testés d'établir une correspondance entre un extrait donné du texte et la question. C'est notamment le cas lorsqu'un thème ou une idée maîtresse est formulé explicitement dans le texte. D'autres tâches peuvent demander aux élèves de se concentrer sur plus d'une référence spécifique du texte. C'est le cas, par exemple, lorsque le lecteur doit identifier le thème d'un texte à partir de la répétition d'une catégorie particulière d'informations. Sélectionner l'idée maîtresse d'un texte implique d'établir une hiérarchie parmi les idées et de choisir les plus générales et les plus globales. Une tâche de ce type permet de vérifier si les élèves testés sont capables d'établir une distinction entre les idées essentielles et les détails mineurs ou de reconnaître le thème principal résumé dans une phrase ou un titre.

Développer une interprétation. Cette opération demande aux lecteurs d'étendre leurs impressions initiales de façon à développer une compréhension plus spécifique ou plus complète de ce qu'ils ont lu. Les items de cette catégorie font appel à une compréhension logique. En effet, les lecteurs doivent se pencher sur l'organisation de l'information dans le texte. Pour ce faire, ils doivent montrer qu'ils ont compris la cohésion du texte, même s'ils sont incapables de la définir explicitement. Dans certains cas, développer une interprétation demande aux lecteurs de traiter une séquence de deux phrases seulement qui sont unies par une relation qui relève de la cohésion locale. Cette opération peut être facilitée par la présence de connecteurs textuels, tels que les expressions « en premier lieu » et « en second lieu » qui indiquent l'ordre de la séquence. Dans des cas plus difficiles (par exemple, dans le cas d'une relation de cause à effet), il se peut qu'aucun indicateur explicite ne soit présent.

Parmi les tâches utilisées pour évaluer cet aspect, citons celles qui demandent aux élèves de comparer et de confronter des informations, d'établir des inférences ou d'identifier et d'énumérer des éléments étayant l'information. Les tâches de comparaison et de confrontation d'informations requièrent de la part des élèves testés qu'ils intègrent deux informations, ou plus, présentes dans le texte. Pour traiter des informations explicites ou implicites à partir d'une ou de plusieurs sources, le lecteur doit souvent inférer les relations ou la catégorisation que l'auteur a voulu suggérer. Les tâches qui demandent à l'élève testé de 
faire des inférences sur les intentions de l'auteur et d'identifier les éléments lui permettant d'inférer ces intentions sont d'autres exemples de tâches qui évaluent cet aspect de la compréhension.

Réfléchir sur le contenu d'un texte et l'évaluer. Cette opération requiert que le lecteur relie des informations qu'il a trouvées dans le texte aux connaissances qu'il détient d'autres sources, ou qu'il évalue des affirmations énoncées dans le texte par rapport à sa propre connaissance du monde. Dans de nombreuses situations, les lecteurs sont appelés à justifier et défendre leurs propres points de vue. Pour ce faire, ils doivent être capables de développer une compréhension de ce qui est dit et sous-entendu dans un texte et de confronter cette représentation mentale à leurs propres connaissances et convictions, basées sur des informations antérieures ou des informations provenant d'autres textes. Ils doivent faire appel à des éléments de preuve contenus dans le texte et les confronter à d'autres sources d'information, en utilisant des connaissances générales et spécifiques tout autant que leur capacité à raisonner de manière abstraite.

Parmi les tâches représentatives de cet aspect de la compréhension de l'écrit, citons : fournir des éléments probants ou des arguments ne provenant pas du texte, évaluer la pertinence de certaines informations ou données ou encore établir des comparaisons avec des normes morales ou esthétiques (standards). On peut demander à l'élève testé de proposer ou d'identifier une autre information susceptible de confirmer le point de vue de l'auteur ou d'évaluer si les informations ou les faits fournis dans le texte sont des preuves suffisantes.

Les informations extérieures à mettre en rapport avec l'information textuelle peuvent provenir des connaissances des élèves, d'autres textes fournis dans l'évaluation ou encore d'idées explicitement fournies dans la question.

Réfléchir sur la forme du texte et l'évaluer. Les items de cette catégorie demandent au lecteur de se tenir à distance du texte, de le considérer avec objectivité et d'évaluer ses qualités et sa pertinence. La connaissance d'éléments comme la structure, le genre et le registre du texte revêt une grande importance dans ces tâches. Ces aspects, qui sont une partie essentielle du «métier» de l'auteur, jouent un rôle considérable dans la compréhension des normes, qui est inhérente aux tâches de cette nature. Pour évaluer le talent d'un auteur à décrire certaines caractéristiques ou à persuader le lecteur, ce dernier doit non seulement comprendre le contenu du texte, mais aussi détecter les nuances de langage - comprendre par exemple en quoi le choix d'un adjectif peut nuancer l'interprétation.

Parmi les tâches qui imposent aux élèves de réfléchir et d'émettre un jugement de valeur sur la forme et la structure d'un texte, citons celles qui leur demandent de déterminer l'utilité d'un texte donné pour atteindre un objectif spécifique ou d'évaluer l'usage fait par l'auteur de certaines techniques textuelles pour parvenir à un but précis. On peut aussi demander aux élèves de définir le style de l'auteur, de commenter l'usage qu'il en fait et d'identifier l'objectif qu'il poursuit ou l'attitude qu'il a adoptée.

Le graphique 2.4 montre la répartition des items de compréhension de l'écrit en fonction des cinq aspects décrits ci-dessus. La catégorie d'items la plus fournie, c'est-à-dire celle représentée par les deux branches du graphique 2.3, requiert des élèves

$$
\text { Graphique } 2.4
$$

Répartition des items en fonction des aspects de la compréhension de l'écrit

\begin{tabular}{lc}
\hline Aspect & Nombre d'items \\
\hline Localisation d'informations & 42 \\
$\begin{array}{l}\text { Interprétation (aspect combinant le } \\
\text { développement d'une interprétation } \\
\text { et la compréhension globale) }\end{array}$ & 70 \\
$\begin{array}{l}\text { Réflexion sur le contenu et la forme } \\
\text { et évaluation }\end{array}$ & 29 \\
Total & $\mathbf{1 4 1}$
\end{tabular}


qu'ils se focalisent sur les relations existant dans un texte. Ces 70 items imposent aux élèves soit de comprendre globalement un texte, soit de développer une interprétation à son propos. Pour les besoins de ce rapport, ils ont été regroupés sous un aspect unique appelé «interprétation de textes ». La deuxième plus grande catégorie comprend les 42 items qui demandent aux élèves de montrer qu'ils sont capables de trouver des informations isolées. Chacun de ces aspects, à savoir comprendre globalement un texte, trouver l'information et développer une interprétation, porte sur la mesure dans laquelle le lecteur est capable de comprendre et d'utiliser des informations provenant essentiellement du texte. Les 29 derniers items demandent aux élèves de réfléchir au contenu ou à des informations contenues dans le texte ou à la structure et à la forme du texte lui-même.

\section{Formats de réponse}

Le graphique 2.5 montre que 63 des 141 items de compréhension de l'écrit de l'évaluation PISA sont des items à réponse construite ouverte qui nécessitent un jugement de la part du correcteur (ou codeur). Les autres sont des items à réponse construite fermée dont la correction ne demande guère de jugement subjectif de la part du codeur, ainsi que des items simples à choix multiple - dans lesquels les élèves choisissent une réponse parmi plusieurs alternatives - et des items complexes à choix multiple - dans lesquels les élèves choisissent plus d'une réponse.

\section{Graphique 2.5}

Répartition des items à réponse construite et à choix multiple en fonction des aspects de la compréhension de l'écrit

\begin{tabular}{lcccc}
\hline Aspect & $\begin{array}{c}\text { Nombre d'items à } \\
\text { choix multiple }\end{array}$ & $\begin{array}{c}\text { Nombre d'items } \\
\text { complexes à choix } \\
\text { multiple }\end{array}$ & $\begin{array}{c}\text { Nombre d'items à } \\
\text { réponse construite } \\
\text { fermée }\end{array}$ & $\begin{array}{c}\text { Nombre d'items à } \\
\text { réponse construite } \\
\text { ouverte }\end{array}$ \\
\hline $\begin{array}{l}\text { Localisation } \\
\text { d'informations }\end{array}$ & 10 & 2 & 10 & 20 \\
$\begin{array}{l}\text { Interprétation } \\
\text { Réflexion et }\end{array}$ & 43 & 3 & 5 & 19 \\
évaluation & 3 & 2 & 0 & 24 \\
Total & 56 & 7 & $\mathbf{1 5}$ & $\mathbf{6 3}$ \\
\hline
\end{tabular}

Ce graphique montre également que les items à choix multiple et à réponse construite sont répartis entre les aspects de la compréhension de l'écrit, sans toutefois l'être de façon uniforme. En fait, un nombre plus important d'items à choix multiple est associé aux deux aspects qui portent sur l'interprétation des relations existant dans un texte, ainsi que l'indique la deuxième ligne du graphique 2.5. Par contraste, signalons que sur les 29 items de réflexion et d'évaluation, seuls 5 d'entre eux sont à choix multiple, les 24 autres étant des items à réponse construite demandant un jugement de la part du codeur.

\section{Codage}

Le codage est relativement simple pour les items à choix multiple, à corriger de façon dichotomique : soit l'élève testé a choisi la bonne réponse, soit il ne l'a pas choisie. Des modèles de crédit partiel permettent un codage plus complexe des items. Dans ce système, certaines mauvaises réponses sont plus complètes que d'autres : les élèves qui fournissent une «presque bonne réponse » reçoivent un crédit partiel. Les modèles psychométriques portant sur ce genre de codage à valeurs multiples sont maintenant bien établis et sont, à certains égards, préférables aux barèmes dichotomiques, dans la mesure où ils utilisent davantage 
l'information contenue dans les réponses. L'interprétation des barèmes à valeurs multiples est cependant plus complexe, car chaque tâche présente plusieurs niveaux de difficulté : un pour la réponse à crédit complet et d'autres pour chacune des réponses à crédit partiel. Dans PISA, le crédit partiel a été utilisé pour le codage des items à réponse construite plus complexes.

\section{Échelonnage des items de compréhension de l'écrit}

Au total, ce sont 141 items de compréhension de l'écrit qui ont été élaborés et soumis à des échantillons de jeunes de 15 ans représentatifs de la population des pays participants, dans le but de garantir que l'évaluation présente la plus grande couverture possible de la compréhension de l'écrit telle qu'elle est définie ici. Toutefois, il était impossible de demander aux élèves de répondre individuellement à la totalité de ces items. C'est pourquoi l'évaluation a été conçue de manière à soumettre un sous-ensemble de la batterie d'items à chaque élève participant, compte tenu cependant de la nécessité de veiller à ce que chaque item soit administré à des échantillons d'élèves qui soient représentatifs des populations nationales. Résumer les performances des élèves par rapport à la batterie complète des items représentait un défi considérable.

Conceptuellement, on peut organiser ces 141 items de compréhension de l'écrit selon un continuum basé tant sur la difficulté que présente chacun d'entre eux pour les élèves que sur le niveau de compétence requis pour y répondre correctement. L'approche adoptée par PISA pour cerner ce continuum de difficulté et de compétence repose sur la théorie de réponse à l'item (IRT: Item response theory). Il s'agit d'un modèle mathématique qui permet d'évaluer la probabilité qu'un individu réponde correctement à une tâche donnée tirée d'une batterie de tâches spécifique. Cette probabilité est modélisée le long d'un continuum qui résume à la fois la compétence de l'individu en termes d'aptitude et la complexité d'un item en termes de difficulté. Ce continuum de difficulté et de compétence est désigné par le terme «échelle».

\section{Présentation des résultats}

Les résultats de l'évaluation des compétences en compréhension de l'écrit ont été tout d'abord résumés sur une échelle composite unique, dont la moyenne a été fixée à 500 et l'écart type, à 100. Les résultats des élèves sont également présentés sur cinq sous-échelles ${ }^{5}$ : trois sous-échelles liées aux aspects de la compréhension de l'écrit (trouver l'information, développer une interprétation, réfléchir et évaluer) et deux sous-échelles liées aux types de texte (textes continus et non continus). Ces cinq sous-échelles permettent de comparer les scores moyens et leur répartition entre les sous-groupes et les pays en fonction des différentes composantes du construct de compréhension de l'écrit. Bien qu'il existe une forte corrélation entre ces cinq sous-échelles, rendre compte des résultats sur chacune d'entre elles offre la possibilité de révéler d'intéressantes interactions entre les pays participants. Lorsque des phénomènes de cet ordre apparaissent, ils peuvent être étudiés et mis en rapport avec les programmes de cours et les méthodes d'enseignement en vigueur. Ces résultats indiqueront pour certains pays qu'il y a lieu de chercher des moyens de mieux enseigner le programme de cours existant tandis que, pour d'autres, il ne faut pas seulement remettre en question la manière d'enseigner mais aussi les contenus d'enseignement.

Le graphique 2.6 donne, sur les deux sous-échelles de format, une vue synoptique des divers types de textes et des tâches qui y sont associées : l'une des sous-échelles de format est basée sur les 89 items portant sur des textes continus et l'autre, sur les 52 items correspondant à des textes non continus. Ce mode d'organisation des données permet de déterminer l'importance de la variation de compétence entre les pays en fonction de ces deux dimensions. 


\section{Graphique 2.6}

Relation entre le cadre d'évaluation de la compréhension de l'écrit et les sous-échelles de format

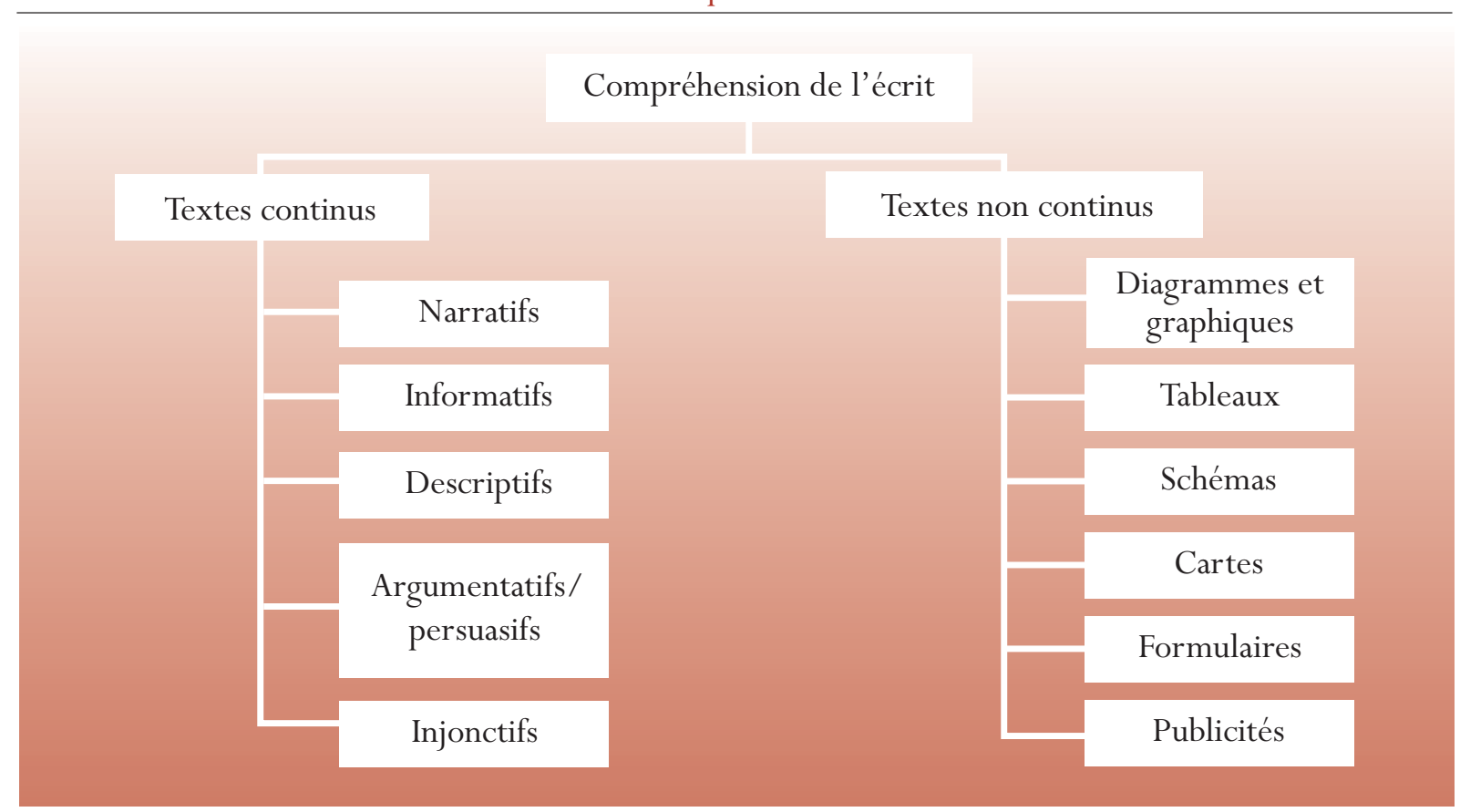

Le graphique 2.7 montre de façon schématique l'organisation des 141 items de lecture en fonction de trois aspects. Deux raisons ont conduit à réduire le nombre des sous-échelles d'aspect de cinq à trois. La première est pragmatique : en 2003 et en 2006, la compréhension de l'écrit sera un domaine mineur de l'évaluation PISA et les tests se limiteront à une trentaine d'items, au lieu des 141 utilisés lors du cycle PISA 2000. Le volume de données sera donc insuffisant pour rendre compte de l'évolution des performances sur cinq sous-échelles d'aspect. La seconde raison est d'ordre conceptuel. Les trois sous-échelles sont basées sur les cinq aspects de la compréhension de l'écrit qui sont décrits dans le graphique 2.3. Les échelles Comprendre globalement un texte et Développer une interprétation ont été fusionnées, car elles impliquent toutes deux pour le lecteur une opération de traitement des informations contenues dans le texte : l'ensemble du texte pour la première, une partie du texte par rapport à une autre pour la seconde. Les échelles Réfléchir sur le contenu du texte et l'évaluer et Réfléchir sur la forme du texte et l'évaluer ont également été réunies en une seule échelle «Réfléchir et évaluer », car la distinction établie entre la réflexion/évaluation sur le fond et la réflexion/évaluation sur la forme a, en pratique, été jugée quelque peu arbitraire.

Les scores sur l'échelle composite de compréhension de l'écrit ainsi que sur chacune des cinq sous-échelles représentent les différents niveaux de performance. Un score peu élevé indique que les connaissances et compétences de l'élève sont très limitées, et un score très élevé, qu'elles sont assez avancées. L'application de la théorie de réponse à l'item (IRT) permet non seulement de résumer les résultats de diverses sous-populations d'élèves mais également de déterminer la difficulté relative des items de compréhension de l'écrit inclus dans l'évaluation. En d'autres termes, tout comme une valeur spécifique est attribuée à l'élève sur une échelle de compétence en fonction de ses réponses aux items de l'évaluation, une valeur spécifique est accordée à chaque item sur une échelle de difficulté, le degré de difficulté des items étant déterminé par la performance des élèves des divers pays participants. 


\section{Graphique 2.7}

Caractéristiques distinctives des cinq aspects de la compréhension de l'écrit

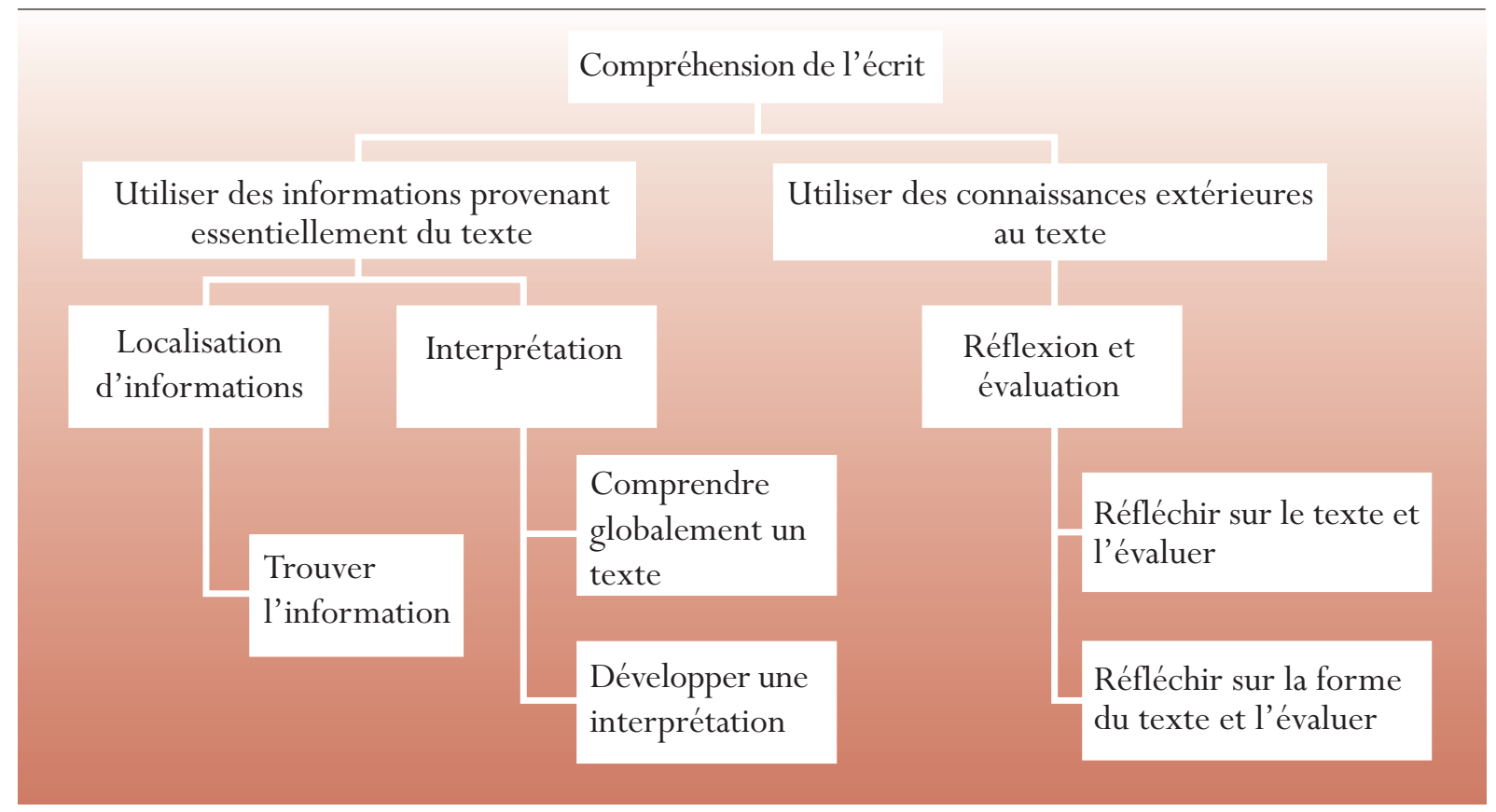

\section{Élaboration d'une carte des items}

La batterie de tests utilisée dans l'évaluation PISA de la compréhension de l'écrit représente un large éventail de types de texte, de situations, d'exigences et de degrés de difficulté. Le graphique 2.8 schématise cet éventail sous la forme d'une carte des items qui présente de manière visuelle sur l'échelle composite et les cinq sous-échelles les compétences en lecture des élèves. Cette carte décrit succinctement une série d'items rendus publics et indique leur valeur sur les échelles de compétence. Ces descriptions concernent les savoir-faire spécifiques que les items sont destinés à mesurer et, dans le cas des items à réponse ouverte, les critères appliqués pour déterminer si la réponse est correcte. L'analyse de ces descriptions permet de mieux cerner les divers processus que les élèves doivent appliquer et les compétences dont ils doivent faire preuve aux différents niveaux des échelles de compréhension de l'écrit.

Il semble utile d'expliquer comment procéder pour interpréter la carte des items. Prenons à titre d'exemple un item du graphique 2.8. qui vaut 421 sur l'échelle composite de compréhension de l'écrit. Cet item demande aux élèves d'identifier l'objectif que deux textes courts ont en commun, en comparant les idées maîtresses de ces deux textes. Le score attribué à chaque item se fonde sur l'hypothèse qu'un individu situé à un certain niveau de l'échelle est également capable de mener à bien toutes les tâches situées au même niveau de l'échelle. En vue de répondre aux objectifs déterminés par le programme PISA, il a été décidé qu'un niveau de compétence attribué à certains élèves indiquait qu'il y avait 62 pour cent de chances que ces élèves réussissent les items de ce niveau. En d'autres termes, les élèves dont le score est de 421 sur l'échelle composite de compréhension de l'écrit auront 62 pour cent de chances de répondre correctement aux items de valeur 421 sur l'échelle. Cela ne signifie pas pour autant que les élèves ayant obtenu un score inférieur à 421 répondront systématiquement de manière erronée à ces items. En fait, la probabilité que les élèves ont de répondre correctement variera en fonction de leur score sur l'échelle de compréhension de l'écrit. Les élèves ayant obtenu un score supérieur à 421 auront plus de 62 pour cent 
de chances de répondre correctement à des items de ce niveau de difficulté, tandis que ceux dont le score est inférieur à 421 auront moins de 62 pour cent de chances d'y parvenir. Il y a lieu de souligner enfin que l'item apparaît sur l'une des sous-échelles d'aspect et sur l'une des sous-échelles de format, en plus de l'échelle composite de compréhension de l'écrit. L'item situé à 421 et choisi à titre d'exemple est un item d'interprétation et figure donc tant sur l'échelle d'interprétation que sur l'échelle de texte continu. ${ }^{6}$

\section{Niveaux de compétence en compréhension de l'écrit}

Tout comme l'échantillon des élèves soumis à l'évaluation est représentatif de la population d'élèves de 15 ans d'un pays, chaque item de compréhension de l'écrit est représentatif d'une catégorie de tâches dans le domaine de la compréhension de l'écrit. Il illustre donc un type de texte et un type de traitement à la portée des élèves de 15 ans. Deux questions s'imposent : «En quoi les items situés au bas de l'échelle de compétence se distinguent-ils de ceux situés au milieu ou au sommet de l'échelle ?» et «Les questions situées au même niveau de l'échelle ont-elles des caractéristiques en commun qui concourent à leur donner un niveau de difficulté similaire ?» L'analyse superficielle de la carte d'items suffit pour constater que les items situés au bas de l'échelle diffèrent de ceux situés au sommet. Et l'analyse plus approfondie des items associés à chaque échelle donne des informations sur un ensemble hiérarchisé de stratégies et de savoir-faire en matière de traitement de l'information. Le groupe d'experts chargé de la compréhension de l'écrit a étudié les items un à un pour identifier le jeu de variables influençant la difficulté des items. Les membres de ce groupe sont arrivés à la conclusion que la difficulté était en partie déterminée par la longueur, la structure et la complexité du texte lui-même. Mais ils ont aussi constaté que dans la plupart des unités de lecture (qui sont constituées d'un texte et d'une série de questions ou de consignes), les questions ou consignes se répartissaient entre les divers niveaux de l'échelle de compréhension de l'écrit. En d'autres termes, la difficulté des items est déterminée non seulement par la structure des textes auxquels ils sont associés, mais également par la nature de la tâche qui est demandée aux élèves dans les questions ou les consignes y afférentes.

Un certain nombre de variables pouvant influencer la difficulté des items de lecture ont été identifiées. Le type de processus impliqué pour trouver une information, interpréter un texte ou réfléchir sur le fond et la forme d'un écrit est manifestement un facteur de difficulté. La complexité et la sophistication des processus varient. Cela va d'établir des liens simples entre plusieurs éléments d'information, à classer des idées en fonction de critères donnés, voire à évaluer de manière critique le passage d'un texte et de construire des hypothèses le concernant. La difficulté des items de localisation de l'information dépend, outre du type de processus, du nombre d'éléments d'information à inclure dans la réponse, du nombre de critères que les informations localisées doivent respecter et de la nécessité ou non de classer les informations dans un ordre particulier. En revanche, la difficulté des items de réflexion/d'évaluation et d'interprétation réside davantage dans le volume de texte qui doit être assimilé. Dans le cas d'items demandant au lecteur de réfléchir, la difficulté est également conditionnée par la familiarité ou la spécificité de connaissances extérieures au texte auxquelles il faut faire appel. Dans tous les aspects de la lecture, la difficulté des items dépend de la perceptibilité des informations qu'il faut exploiter, du nombre d'informations distractrices et du caractère explicite ou non des idées ou des informations nécessaires pour mener à bien les tâches. 


\section{Graphique 2.8}

Carte des items PISA

822 CONSTRUIRE une hypothèse à propos d'un phénomène contraire aux attentes sur la base de connaissances extérieures au texte et de toutes les informations pertinentes figurant dans un TABLEAU COMPLEXE consacré à un thème relativement peu familier (niveau 2).

727 ANALYSER plusieurs cas décrits et les CLASSER dans des catégories définies dans un DIAGRAMME EN ARBRE dont certaines mentions figurent en note de bas de page (niveau 2).

705 CONSTRUIRE une hypothèse à propos d'un phénomène contraire aux attentes sur la base de connaissances extérieures au texte et de toutes les informations pertinentes figurant dans un TABLEAU COMPLEXE consacré à un thème relativement peu familier (niveau 1).

652 ÉVALUER la fin d'un LONG RÉCIT compte tenu de son thème ou de son atmosphère implicite (niveau 2)

645 ÉTABLIR DES LIENS ENTRE DES NUANCES DE LANGAGE présentes dans un LONG RÉCIT et le thème principal de celui-ci malgré la présence d'idées conflictuelles (niveau 2).

631 LOCALISER des informations dans un DIAGRAMME EN ARBRE compte tenu des mentions figurant en note de bas de page (niveau 2).

603 TROUVER le sens d'une phrase en la replaçant dans le contexte plus large d'un LONG RÉCIT.

600 CONSTRUIRE une hypothèse à propos de la décision de l'auteur en établissant une relation entre des faits présentés dans un graphique et le thème principal d'un GROUPE DE REPRÉSENTATIONS GRAPHIQUES.

581 COMPARER ET ÉVALUER le style de deux LETTRES ouvertes.

567 ÉVALUER la fin d'un LONG RÉCIT par rapport à son intrigue.

542 ÉTABLIR UNE RELATION ANALOGIQUE entre deux phénomènes présentés dans une lettre ouverte.

540 IDENTIFIER la date implicite à laquelle commence un GRAPHIQUE.

539 TROUVER LE SENS de brèves citations extraites d'un LONG RÉCIT à propos de l'atmosphère ou d'une situation immédiate (niveau 1).

537 ÉTABLIR UNE RELATION entre des faits présentés dans un LONG RÉCIT et des concepts personnels pour justifier des points de vue contradictoires (niveau 2).

529 EXPLIQUER la motivation d'un personnage en établissant des liens entre des événements relatés dans un LONG RÉCIT.

508 ÉTABLIR UNE RELATION entre DEUX REPRÉSENTATIONS GRAPHIQUES basées sur des conventions différentes.

486 ÉVALUER l'adéquation d'un DIAGRAMME EN ARBRE à des fins particulières.

485 LOCALISER des informations numériques dans un DIAGRAMME EN ARBRE.

480 ÉTABLIR UNE RELATION entre des faits présentés dans un LONG RÉCIT et des concepts personnels pour justifier un point de vue unique (niveau 1).

478 LOCALISER ET COMBINER des informations dans un GRAPHIQUE LINÉAIRE et dans son introduction pour trouver une valeur manquante.

477 COMPRENDRE la structure d'un DIAGRAMME EN ARBRE.

473 CLASSER dans des catégories décrites dans un DIAGRAMME EN ARBRE des cas présentés alors que certaines des informations requises figurent en note de bas de page.

447 INTERPRÉTER les informations figurant dans un seul paragraphe pour comprendre le contexte d'un RÉCIT.

445 ÉTABLIR UNE DISTINCTION entre des variables et des CARACTÉRISTIQUES STRUCTURELLES d'U DIAGRAMME EN ARBRE.

421 IDENTIFIER L'OBJECTIF commun de textes courts.

405 LOCALISER des fragments d'information explicites dans un TEXTE très structuré.

397 TROUVER L'IDÉE PRINCIPALE d'un GRAPHIQUE EN BÂTONS sur la base de son titre.

392 LOCALISER un fragment d'information littéral dans un TEXTE bien structuré.

367 LOCALISER DES INFORMATIONS EXPLICITES DANS UN PASSAGE BREF ET BIEN SITUÉ d'un RÉCIT.

363 LOCALISER un fragment d'information explicitement défini dans un TEXTE structuré par des soustitres.

356 RECONNAÎTRE LETHÈME d'un article assez redondant et bien structuré par des sous-titres. 
L'échelle composite de compréhension de l'écrit et les cinq sous-échelles d'aspect et de format ont été divisées en cinq niveaux dans le but de rendre compte de la progression de la difficulté et de la complexité.

\begin{tabular}{c|c}
\hline Niveau & Résultats sur l'échelle PISA \\
\hline 1 & de 335 à 407 \\
2 & de 408 à 480 \\
3 & de 481 à 552 \\
4 & de 553 à 625 \\
5 & plus de 625 \\
\hline
\end{tabular}

Les panels d'experts ont découvert que les items correspondant à chaque niveau de compétence avaient en commun de nombreuses caractéristiques et exigences et qu'ils différaient par des aspects systématiques des items correspondant à des niveaux supérieurs ou inférieurs. Ils en ont déduit que ces niveaux permettent de refléter la progression des compétences requises sur chaque échelle. Cette progression est résumée dans le graphique 2.9.

\section{Interprétation des niveaux de compétence en compréhension de l'écrit}

Chaque niveau de compétence représente non seulement une série de tâches et de savoirs et savoir-faire y afférents, mais également une série de compétences démontrées par les élèves. Comme nous l'avons dit précédemment, les niveaux de compétence en compréhension de l'écrit ont initialement été définis par PISA pour représenter un jeu de tâches avec des caractéristiques communes. Ces niveaux ont également des propriétés statistiques communes. Ainsi, l'élève moyen d'un niveau de compétence est censé pouvoir répondre correctement dans 62 pour cent des cas à une question de difficulté moyenne correspondant à ce niveau. Par ailleurs, les bornes de chaque niveau ont été définies de manière à ce que, entre autres, les élèves situés au bas d'un niveau aient un taux attendu de réussite de 50 pour cent à tout test virtuel qui serait composé d'une sélection aléatoire d'items de ce niveau.

Comme chaque échelle de compréhension de l'écrit représente un continuum de connaissances et de savoir-faire, les élèves ayant atteint un certain niveau de l'échelle possèdent non seulement les connaissances et savoir-faire associés à ce niveau mais également ceux correspondant aux niveaux inférieurs. Les savoirs et savoir-faire attendus à un niveau donné se fondent sur ceux associés aux niveaux inférieurs et les intègrent. Ainsi, un élève situé au niveau 3 sur une échelle de compréhension de l'écrit est capable de mener à bien les tâches associées à ce niveau ainsi que celles associées aux niveaux 1 et 2. Les élèves situés aux deux premiers niveaux sont censés ne répondre correctement aux tâches typiques du niveau 3 que dans moins de 50 pour cent des cas. En d'autres termes, ces derniers obtiendraient moins de 50 pour cent à un test constitué d'items correspondant au niveau 3. 


\section{Graphique 2.9}

Carte des niveaux de compétence en compréhension de l'écrit

\begin{tabular}{l:l|l}
\hline \multicolumn{1}{c}{ Localisation d'informations } & \multicolumn{1}{c}{ Interprétation } & \multicolumn{1}{c}{ Réflexion } \\
\hline $\begin{array}{l}\text { Localiser et, parfois, classer ou combiner de } \\
\text { multiples fragments d'information profon- }\end{array}$ & $\begin{array}{l}\text { Dégager le sens d'un passage très nuancé ou } \\
\text { montrer qu'un texte est compris parfaitement et } \\
\text { dément enfouis, figurant parfois en dehors } \\
\text { du corps du texte. Identifier les informations }\end{array}$ & $\begin{array}{l}\text { Procéder à une évaluation critique ou } \\
\text { connaire une hypothèse, sur la base de } \\
\text { pertinentes pour la tâche à accomplir, malgré } \\
\text { la présence d'informations extrêmement } \\
\text { plausibles et/ou concurrentes. }\end{array}$ \\
des concepts contraires aux attentes \\
grâce à la compréhension approfondie \\
de textes longs ou complexes.
\end{tabular}

Textes continus : appréhender des textes dont la structure n'apparaît pas d'emblée ou n'est pas clairement établie, pour discerner des relations entre des passages spécifiques du texte et son thème ou intention implicite.

Textes non continus : identifier des tendances parmi de nombreux fragments d'information figurant dans des représentations qui peuvent être longues et détaillées, parfois sur la base d'informations mentionnées en dehors des représentations. Dans certains cas, le lecteur peut avoir à prendre conscience qu'il doit se référer à un passage séparé d'un même document, tel qu' une note de bas de page, pour comprendre une partie du texte de manière approfondie.

4 Localiser et, parfois, classer ou combiner, éventuellement sur la base de nombreux critères, de multiples éléments d'information profondément enfouis dans un texte familier en termes de contenu ou de forme. Identifier les informations du texte qui sont pertinentes pour la tâche à accomplir.

\begin{tabular}{|l|l|} 
Établir une inférence d'ordre supérieur sur la & Procéder à l'évaluation critique d'un \\
base du texte pour comprendre et appliquer des & texte ou construire des hypothèses à son \\
catégories dans un contexte peu familier et déga- & propos. Montrer que des textes longs \\
ger le sens d'un passage du texte compte tenu de & ou complexes sont compris de manière \\
l'ensemble du texte. Faire face à des ambiguïtés, & précise. \\
à des idées contraires aux attentes ou à des con- & \\
cepts exprimés de manière négative. &
\end{tabular}

Textes continus : suivre des liens linguistiques ou thématiques pendant plusieurs paragraphes, souvent en l'absence d'éléments organisant clairement le texte, pour localiser, interpréter ou évaluer des informations enfouies dans le texte ou dégager le sens psychologique ou métaphysique du texte.

Texte non continu : parcourir un texte long et détaillé pour trouver les informations pertinentes qu'il faut comparer ou combiner, sans l'aide ou presque d'éléments organisant clairement le texte, tels que des titres. existant entre des éléments d'informations qui doivent dans certains cas satisfaire à des critères multiples, en présence d'informations concurrentes explicites.

\begin{abstract}
Intégrer plusieurs parties d'un texte pour en identifier l'idée principale, comprendre une relation ou trouver le sens d'un terme ou d'une phrase. Comparer, confronter et classer des informations compte tenu de critères multiples, en présence d'informations concurrentes.
\end{abstract}

Établir des relations à propos d'un élément du texte ou comparer, expliquer ou évaluer une caractéristique du texte. Montrer que le texte est compris dans le détail grâce à des connaissances qui sont plus ou moins courantes.

Textes continus : utiliser les éventuelles conventions organisant le texte et suivre des liens logiques explicites ou implicites, tels que les relations de cause à effet, chevauchant des phrases ou des paragraphes pour localiser, interpréter ou évaluer des informations.

Textes non continus : étudier une représentation à la lumière d'une autre représentation, séparer un document ou une représentation éventuellement dans un format différent ou combiner plusieurs fragments d'informations circonstancielles, factuelles ou numériques figurant dans un graphique ou sur une carte pour tirer des conclusions à propos des informations données.

2 Localiser un ou plusieurs fragments d'information, parfois dans le respect de critères multiples, en présence d'informations concurrentes.
Identifier l'idée principale d'un texte, comprendre des relations, constituer ou appliquer des catégories simples ou trouver le sens d'un passage délimité d'un texte alors que les informations ne sont pas explicites et que des inférences de niveau inférieur sont requises.
Faire une comparaison ou établir des relations entre le texte et des connaissances extérieures ou expliquer une caractéristique du texte sur la base d'expériences ou d'attitudes personnelles.

Textes continus : suivre des relations logiques et linguistiques dans un paragraphe pour localiser ou interpréter des informations ; ou synthétiser des informations dans des textes ou fragments de textes pour inférer l'intention de l'auteur.

Textes non continus : démontrer une compréhension de la structure sous-jacente d'éléments visuels, tels un diagramme en arbre simple ou un tableau, ou combiner deux fragments d'informations d'un graphique ou d'un tableau.

Localiser un ou plusieurs éléments d'informa- Reconnaître le thème principal ou l'intention de tion indépendants et explicites habituellement l'auteur d'un texte consacré à un sujet famidans le respect d'un seul critère dans un texte lier dans lequel les informations requises sont ne comportant guère d'informations concur- saillantes. rentes, voire aucune.

Etablir une relation simple entre des informations figurant dans le texte et des connaissances courantes.

Textes continus : utiliser la redondance, les titres de paragraphes ou des conventions typographiques courantes pour se faire une idée de l'idée maîtresse du texte ou pour localiser des informations explicitement mentionnées dans un passage court du texte.

Textes non continus : se concentrer sur des éléments d'information discrets, figurant généralement dans une représentation simple telle qu'une carte, un graphique linéaire ou un diagramme en bâtons, qui présente un nombre limité d'informations de manière directe et des textes courts ne comptant que quelques termes ou phrases. 
Le graphique 2.10 chiffre la probabilité que les élèves situés à des niveaux donnés de l'échelle combinée de compréhension de l'écrit répondent correctement à des items de difficulté variée: les deux premiers items correspondent respectivement aux niveaux 1 et 3 et le troisième correspond aux niveaux 4 et 5 . Le tableau montre qu'un élève situé en deçà du niveau 1 avec un score de 298 n'a que 43 pour cent de chance de répondre correctement à la tâche du niveau 1 qui se situe à 367 sur l'échelle de compréhension de l'écrit, à peine 14 pour cent de chances d'y arriver dans le cas d'un item de niveau 3 et pratiquement aucune chance de mener à bien une tâche de niveau 5. Pour un élève situé au milieu du niveau 1 (soit un score de 371), la probabilité de réussite est de 63 pour cent dans le cas de l'item de 367, un peu plus de 25 pour cent dans le cas de l'item de 508 et 7 pour cent seulement dans le cas de l'item de niveau 5 . Par contraste, un élève situé au niveau 3 doit en principe répondre correctement à l'item de 367 dans 89 pour cent des cas et à des items de 508 , c'est-à-dire proches du milieu du niveau 3, dans 64 pour cent des cas. Cet élève n'a toutefois qu'une chance sur quatre environ (27 pour cent) de répondre correctement à des items situés au milieu du niveau 5. Enfin, un élève situé au niveau 5 est censé répondre correctement à pratiquement tous les items. Ainsi que le montre le graphique 2.10, un élève ayant obtenu un score de 662 sur l'échelle combinée de compréhension de l'écrit doit en principe répondre correctement à un item de 367 dans 98 pour cent des cas, à un item de 508 (niveau 3) dans 90 pour cent des cas et à un item de 652 (situé à peu près au milieu du niveau 5) dans 65 pour cent des cas.

$$
\text { Graphique } 2.10
$$

Probabilité de répondre correctement à des items sélectionnés de difficulté variable en fonction du niveau de compétence des élèves

\begin{tabular}{lcccc}
\hline & $\begin{array}{c}\text { Item de niveau 1 Item de niveau 3 } \\
\text { Item de niveau 4 Item de niveau } 5\end{array}$ \\
\hline Élèves situés en deçà du niveau 1 (298) & $\mathbf{( 3 6 7 )}$ & $\mathbf{( 5 0 8 )}$ & $\mathbf{( 5 6 7 )}$ & $\mathbf{( 6 5 2 )}$ \\
Élèves situés au niveau 1 (371) & 43 & 14 & 8 & 3 \\
Élèves situés au niveau 2 (444) & 63 & 27 & 16 & 7 \\
Élèves situés au niveau 3 (517) & 79 & 45 & 30 & 14 \\
Élèves situés au niveau 4 (589) & 89 & 64 & 48 & 27 \\
Élèves situés au niveau 5 (662) & 95 & 80 & 68 & 45 \\
\hline
\end{tabular}

Le graphique 2.10 nous amène par ailleurs à évoquer la question des niveaux maximum et minimum désignés. L'échelle de compréhension de l'écrit n'a pas de niveau maximum mais nous pouvons affirmer avec un certain degré de certitude que les élèves extrêmement compétents sont capables de répondre correctement à des items associés au niveau le plus élevé de compétence. Le problème est plus délicat pour les élèves qui se situent à l'extrémité inférieure de l'échelle. Comme le niveau 1 commence à un score de 335 , il y a dans tous les pays un certain pourcentage d'élèves que l'on estime situé en deçà de ce niveau. Même si aucun item de lecture inférieur à 335 n'a été inclus dans les tests, il serait faux de dire que ces élèves ne possèdent aucune compétence en compréhension de l'écrit ou sont « totalement illettrés ». Toutefois, leurs résultats à la batterie de tests utilisés dans l'évaluation permettent de supposer qu'ils auraient obtenu moins de 50 pour cent à un test constitué d'items de niveau 1. Ces élèves sont donc considérés comme situés en deçà du niveau 1.

Le cadre d'évaluation de PISA n'a pas prévu de déterminer si les élèves de 15 ans étaient capables de lire au sens technique du terme, parce que relativement peu de jeunes adultes dans nos sociétés sont totale- 
ment dénués de compétence en lecture. PISA n’a donc pas cherché à évaluer si les élèves de 15 ans lisaient couramment, étaient capables de reconnaître des mots ou avaient une bonne orthographe. En revanche, PISA reflète les théories contemporaines sur la lecture qui considèrent que les élèves sur le point de quitter l'enseignement secondaire doivent être capables de construire du sens, développer ce sens et y réfléchir à partir de ce qu'ils ont lu dans un grand éventail de textes continus et non continus généralement associés à une série de situations de lecture tant à l'intérieur qu'à l'extérieur du cadre scolaire. Nous ne sommes pas en mesure de définir les connaissances et compétences en lecture que les élèves situés en deçà du niveau 1 sont susceptibles de posséder, mais nous pouvons affirmer sur la base de leur niveau de compétence qu'ils ne sont sans doute guère capables d'utiliser la lecture comme un outil indépendant pour acquérir des connaissances et des savoir-faire dans d'autres domaines.

\section{Notes}

1. Ces paragraphes s'inspirent fortement du cadre d'évaluation PISA de la compréhension de l'écrit qui a été rédigé par le Groupe fonctionnel d'experts chargé de la lecture (RFEG) et approuvé par le Conseil des pays participants (BPC). Ce document peut être consulté sur le site web-de PISA. Le lecteur est invité à prendre connaissance de ce document s'il souhaite des informations plus détaillées sur certains des aspects présentés ici.

2. Le modèle de Kirsch et Mosenthal a été présenté en détail dans une série d'articles mensuels intitulée «Understanding Documents », parus entre 1989 et 1991 dans le Journal of Reading.

3. Cette section se base sur les travaux de Werlich (1976). Les appellations de catégorie placées entre parenthèses sont des dénominations alternatives.

4. Il y a lieu de noter que deux types de textes continus - les documents/enregistrements et l'hypertexte - et trois types de textes non continus - les feuilles d'information, les coupons et les certificats - n'étaient pas représentés dans l'évaluation PISA 2000.

5. Chaque sous-échelle a été construite en conservant des paramètres d'item fixes et en réévaluant la distribution des compétences pour chaque pays sur base de ce sous-ensemble précis d'items de compréhension de l'écrit.

6. Le graphique 2.8 présente chaque item de lecture en fonction de sa position sur l'échelle composite de compréhension de l'écrit ainsi que sur l'une des trois sous-échelles d'aspect et sur l'une des deux sous-échelles de format. 


\section{Chapitre}

3

EXEMPLES D'ITEMS

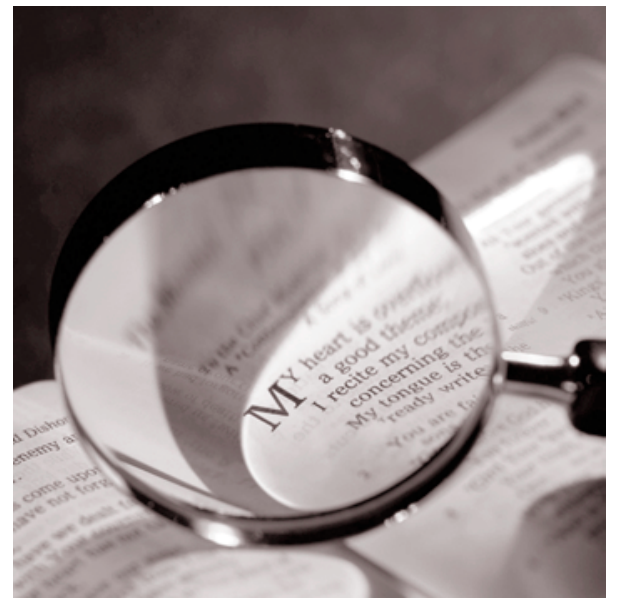


L'idée sous-jacente à la définition de la compréhension de l'écrit et au cadre conceptuel d'évaluation dans PISA est que les jeunes adultes vivant dans les pays développés doivent être capables, à l'approche de la fin de leurs études, de construire et de développer du sens à partir de ce qu'ils ont lu dans un large éventail de textes continus et non continus généralement associés à une série de situations s'inscrivant à l'intérieur et à l'extérieur du cadre scolaire. Les tâches élaborées selon le cadre conceptuel donnent toutes des informations sur la nature et le niveau des compétences acquises dans ce domaine par les élèves des pays participants.

Ce chapitre présente quelques items qui ont été sélectionnés dans la batterie de tests de compréhension de l'écrit du cycle PISA 2000. La plupart de ces 141 items n'ont pas été rendus publics par l'OCDÉ afin qu'ils puissent figurer dans les cycles ultérieurs et, ainsi, permettre de mesurer l'évolution des tendances au fil du temps dans le domaine des compétences en compréhension de l'écrit. Une série de 45 items a toutefois été publiée dans Sample tasks from the PISA 2000 Assessment - Reading, Mathematical and Scientific Literacy (OCDE, 2002a) et peut être consultée sur le site OCDE/PISA www.pisa.oecd.org. Les 19 items présentés dans ce chapitre sont tirés de cette publication et sont accompagnés de commentaires qui expliquent les divers types d'information que chacun d'entre eux permet de recueillir à propos des compétences en compréhension de l'écrit.

\section{Sélection et organisation des exemples d'item}

Les textes et les items choisis pour ce chapitre couvrent les divers degrés de difficulté associés à chacune des trois sous-échelles d'aspect et des deux sous-échelles de format qui ont été décrites dans le chapitre précédent. Parmi les 19 items repris ici, 13 sont dits « dichotomiques » : les réponses des élèves sont soit correctes, ce qui vaut un crédit complet (1 point), soit incorrectes, ce qui vaut un crédit nul ( 0 point). Les six items restants sont corrigés de façon dite «polytomique » : ils peuvent donner droit à un crédit complet ( 2 points), à un crédit partiel (1 point) ou à un crédit nul ( 0 point). Tant les crédits complets que partiels donnent des informations sur le degré de difficulté d'un item. Une série de « 25 degrés de difficulté » a été dérivée de ces 19 items : quatre d'entre eux correspondent au niveau 1, cinq au niveau 2, sept au niveau 3, trois au niveau 4 et six au niveau 5. Les items sélectionnés sont présentés dans le graphique 2.8 , au chapitre 2 .

Lors du cycle PISA 2000, la compréhension de l'écrit a été évaluée à l'aide d'une série de textes auxquels diverses tâches ont été associées. Le ou les textes et les tâches y afférentes constituent ce que l'on appelle une unité. Les items proposés dans ce chapitre sont tirés de six unités : les trois premières unités sont basées sur des textes continus et les trois dernières, sur des textes non continus.

La présentation des textes qui servent de stimulus à chaque unité est relativement proche de celle qui a été soumise aux élèves de l'échantillon PISA. Cette approche a été choisie afin de donner au lecteur du présent rapport une idée aussi précise que possible de ce qui était évalué. En effet, la présentation d'un texte - la mise en page, la taille de la police, les titres et les illustrations, par exemple - peut influencer son impact sur le lecteur et, donc, les réponses à toutes les questions qui y sont associées dans une évaluation. Les textes sont précédés d'une brève description qui explique en quoi ils reflètent les composantes du cadre d'évaluation et pourquoi ils ont été choisis pour figurer dans la batterie de tests de compréhension de l'écrit. 
Chaque item est précédé d'une description des caractéristiques dont ont pense qu'elles contribuent à sa difficulté globale. Ces caractéristiques permettent également de distinguer les items en fonction de leur niveau de difficulté et des sous-échelles auxquelles ils se rapportent.

Comme nous l'avons dit dans le chapitre 2, 55 pour cent environ des items de lecture de la batterie de tests du cycle PISA 2000 étaient des questions à choix multiple ou des tâches dont la réponse ne demandait guère de jugement de la part du correcteur. Les 45 pour cent restants étaient des items à réponse construite qui, eux, appelaient un jugement de la part du correcteur. Pour aider ce processus de codage, les items étaient assortis de guides de correction décrivant le type de réponse requise pour chaque catégorie de code et fournissant des exemples de réponses pour chacune d'entre elles. Ces exemples de réponses étaient principalement issus des réponses d'élèves recueillies lors de l'essai pilote international et des essais de terrain. Tous les correcteurs ont suivi un programme intensif de formation et le processus de codage a été strictement contrôlé du début à la fin. La méthodologie appliquée pour la correction des tests est décrite en détail dans le Rapport technique PISA 2000 (OCDE, 2002b).

Chaque item à réponse construite présenté dans ce chapitre est classé en fonction de la situation, du format du texte, de l'aspect étudié, du niveau et du score sur les échelles PISA. Ainsi, dans l'exemple qui suit, l'item s'inscrit dans un contexte scolaire et est basé sur un texte non continu. Il s'agit d'un item de localisation d'informations qui correspond à un score de 460 sur l'échelle PISA, ce qui le situe au niveau 2 en termes de difficulté.

\author{
Situation : scolaire \\ Format de texte : non continu \\ Aspect : Trouver l'information \\ Niveau : 2 \\ Score sur l'échelle PISA : 460
}

\title{
Baskets
}

Le premier texte est de type informatif et est extrait d'un magazine belge de langue française destiné aux adolescents. Il est considéré comme s'inscrivant dans le contexte scolaire. L'une des raisons qui ont dicté son inclusion dans la batterie de tests de compréhension de l'écrit du cycle PISA 2000 est son thème, jugé très intéressant pour la population cible de PISA, à savoir les jeunes de 15 ans. L'article en question est illustré par un dessin amusant qui s'inspire de la bande dessinée et est structuré par des sous-titres accrocheurs. Il appartient à la catégorie des textes continus et constitue un exemple d'écrit informatif dans la mesure où il développe un concept mental en énumérant une série de critères qui permettent de juger de la qualité de chaussures de sport du point de vue des jeunes sportifs.

Les items de cette unité concernent les trois aspects de la compréhension de l'écrit - trouver l'information ; développer une interprétation ; réfléchir et évaluer - mais ils sont tous relativement faciles puisqu'ils correspondent au niveau 1.

Deux des quatre items de l'unité Baskets sont reproduits ci-après. 


\section{Bien dans ses baskets}

Le Centre médical de Médecine Sportive de Lyon (France) a mené pendant 14 ans des recherches sur les lésions qui affectent les jeunes qui font du sport et les sportifs professionnels. D'après les conclusions, le mieux à faire est de prévenir... et de porter de bonnes chaussures.

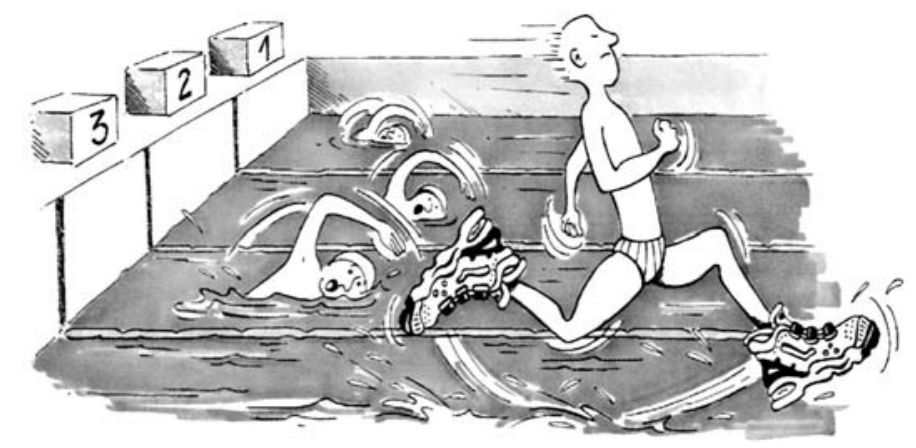

\section{Chocs, chutes, usure...}

Dix-huit pour cent des sportifs de 8 à 12 ans souffrent déjà de lésions au talon. Le cartilage de la cheville des footballeurs encaisse mal les chocs, et $25 \%$ des professionnels se découvrent là un vrai point faible. Le cartilage de la délicate articulation du genou s'abîme lui aussi de façon irréversible et, s'il n'est pas soigné dès l'enfance (10-12 ans), cela peut provoquer une arthrose précoce. La hanche n'est pas épargnée et, la fatigue aidant, les joueurs risquent des fractures, résultat de chutes ou de collisions.

Selon l'étude, les footballeurs de plus de dix ans de pratique présentent l'une ou l'autre excroissance osseuse au tibia ou au talon. C'est ce qu' on appelle « le pied du footballeur », une déformation provoquée par des chaussures aux semelles et tiges trop souples.

\section{Protéger, soutenir, stabiliser, amortir}

Trop rigide, la chaussure gêne les mouvements. Trop souple, elle augmente les risques de blessures et de foulures. Une bonne chaussure de sport doit répondre à quatre critères:

D'abord, protéger de l'extérieur : contre les chocs avec le ballon ou avec un autre joueur, résister aux inégalités du sol et garder le pied au chaud et au sec malgré le gel et la pluie.

Elle doit soutenir le pied et surtout l'articulation de la cheville, pour éviter les entorses, inflammations et autres maux, même au genou.
Elle assurera aussi une bonne stabilité aux joueurs, pour qu'ils ne glissent pas sur un sol mouillé ou ne dérapent pas sur un terrain trop sec.

Enfin, elle amortira les chocs, surtout ceux qu' encaissent les joueurs de volley et de basket, qui sautent sans arrêt.

\section{À pieds secs}

Pour éviter les ennuis de parcours mineurs, mais douloureux - cloques et ampoules, voire crevasses ou mycoses (champignons) - la chaussure doit permettre l'évaporation de la transpiration et empêcher l'humidité extérieure de pénétrer. La matière idéale pour cela est le cuir. Et il peut être imperméabilisé pour éviter que la chaussure ne soit détrempée par la première pluie. 


\section{Question 1 : BASKETS}

\section{Que veut montrer l'auteur de ce texte?}

A Que la qualité de beaucoup de chaussures de sport a été fortement améliorée.

B Qu'il vaut mieux ne pas jouer au football quand on a moins de 12 ans.

C Que les jeunes ont de plus en plus de blessures à cause de leur mauvaise condition physique.

D Qu'il est très important pour les jeunes sportifs de porter de bonnes chaussures de sport.

Situation : scolaire

Format de texte : continu

Aspect : développer une interprétation

Niveau : 1

Score sur l'échelle PISA : 356

La tâche la plus facile de cette unité [R110Q01]' est une tâche d'interprétation et se situe au niveau 1 avec un score de 356 sur l'échelle PISA. Elle demande au lecteur d'identifier l'idée principale d'un article qui porte sur un sujet familier.

Le message principal de l'auteur n'est pas exprimé directement, ni même de manière synonymique, ce qui classe cet item dans la catégorie des tâches d'interprétation du texte plutôt que dans celle de localisation d'informations. Deux caractéristiques au moins expliquent la facilité de cet item. Tout d'abord, les informations requises sont situées dans l'introduction, qui est un passage assez court du texte. Deuxièmement, on a une redondance marquée dans le texte puisque l'idée principale exprimée dans l'introduction est répétée à diverses reprises tout au long du texte. Les items de lecture sont relativement faciles lorsque l'information que le lecteur doit utiliser figure au début du texte ou est répétée, deux critères que l'on retrouve dans cet item.

La question a pour objectif de déterminer si les élèves sont capables de comprendre un texte globalement. Rares sont les élèves qui n'ont pas choisi la bonne réponse. Les réponses incorrectes se répartissent entre les trois distracteurs A, B et C. Les élèves les moins compétents, qui constituent le plus faible pourcentage, ont choisi la réponse B : «Qu'il vaut mieux ne pas jouer au football quand on a moins de 12 ans ». Ces élèves ont probablement tenté de trouver une équivalence entre des termes de la question et des éléments du texte en reliant le «12» du distracteur B aux deux références aux jeunes de 12 ans qui figurent au début de l'article.

\section{Question 2 : BASKETS}

D'après l'article, pourquoi les chaussures de sport ne doivent-elles pas être trop rigides ?

Situation : scolaire

Format de texte : continu

Aspect : trouver l'information

Niveau : 1

Score sur l'échelle PISA : 392 
Ce deuxième item [R110Q04] se situe également au niveau 1 avec un score de 392 sur l'échelle PISA et est classé en termes d'aspect dans la catégorie «Trouver l'information ». Il requiert du lecteur qu'il trouve une information explicitement exprimée qui répond à un seul critère.

Le lecteur peut directement associer le terme « rigide » figurant dans la question au passage adéquat du texte, ce qui rend l'information facile à trouver. Bien que l'information requise se trouve au milieu du texte - et non au début comme dans la tâche précédente -, elle est relativement perceptible, car proche du début de l'une des trois sections délimitées par un sous-titre.

Pour obtenir un crédit complet, les élèves doivent faire référence au fait que cela gêne les mouvements. Il s'agit pourtant d'une question assez facile puisque le crédit complet est accordé aux réponses citant directement un passage du texte : « ...la chaussure gêne les mouvements ». De nombreux élèves ont cependant choisi d'exprimer cette idée avec leurs propres mots, par exemple :

«Elles vous empêchent de courir facilement. »

ou

«Vous pouvez bouger. »

Aucun crédit n'est accordé aux réponses qui témoignent d'une compréhension incorrecte ou qui sont peu plausibles ou hors de propos. Voici une mauvaise réponse courante :

«Parce qu'il faut soutenir le pied. »

Cet exemple est à l'opposé de la réponse requise, bien qu'il s'agisse également d'une idée exprimée dans le texte. Les élèves qui ont répondu de la sorte ont probablement négligé la tournure négative de la question ("pourquoi les chaussures de sport ne doivent-elles pas être trop rigides ? )) ou ont associé les idées de «rigidité » et de «soutien», ce qui les a amenés à prendre en considération un passage du texte qui ne convenait pas pour la tâche. Exception faite de cet élément, il n'y a guère d'informations distractrices susceptibles de lancer le lecteur sur une mauvaise piste.

\section{Graffiti}

Le stimulus de cette unité est constitué de deux lettres mises en ligne sur Internet à l'origine en Finlande. Les tâches qui y sont associées simulent des activités typiques de compréhension de l'écrit, puisque les lecteurs sont souvent appelés à résumer, comparer et confronter des idées provenant de deux sources différentes, sinon davantage.

Comme ces lettres ont été publiées sur Internet, l'unité Graffiti s'inscrit dans une situation dite «publique ». Ces textes appartiennent à la catégorie générale des textes continus et sont argumentatifs, dans la mesure où ils avancent des propositions et tentent de convaincre le lecteur de partager un point de vue.

Comme pour l'unité précédente Baskets, on a estimé que le thème de Graffiti serait intéressant pour les jeunes de 15 ans. Le débat sous-jacent entre les auteurs des lettres, c'est-à-dire la question de savoir si les graffiteurs sont des artistes ou des vandales, doit en principe interpeller les élèves testés.

La difficulté des quatre items de l'unité Graffiti utilisés pour évaluer les compétences en compréhension de l'écrit lors du cycle PISA 2000 se situe entre le niveau 2 et le niveau 4. Les aspects évalués sont «Développer une interprétation » d'une part et, d'autre part, « Réfléchir et évaluer » d'autre part. Trois de ces quatre items sont présentés ci-après. 
Je bous de rage en voyant que le mur de l'école a été nettoyé et repeint pour la quatrième fois consécutive pour effacer des graffiti. La créativité est admirable, mais les gens devraient trouver le moyen de s'exprimer sans infliger des coûts supplémentaires à la société.

Pourquoi tenez-vous à ternir la réputation des jeunes en peignant des graffiti là où c'est interdit? Les artistes professionnels n'accrochent pourtant pas leurs tableaux dans la rue! Ils cherchent plutôt à obtenir des subventions et se font connaître à travers des expositions légales.

À mon sens, les bâtiments, les palissades et les bancs publics sont eux-mêmes déjà des œuvres d'art. C'est vraiment pitoyable de gâcher cette architecture par des graffiti et, de plus, la méthode utilisée détruit la couche d'ozone. Vraiment, je ne comprends pas pourquoi ces artistes criminels prennent tant de peine, alors que leurs « ouvres d'art» sont, chaque fois, simplement ôtées de la vue.

Helga

Source : Mari Hamkala.
On n'a pas à rendre compte de ses goûts. Notre société est envahie par la communication et la publicité. Logos d'entreprises, noms de boutiques. Immenses affiches s'imposant partout dans les rues. Sont-elles acceptables? Oui, pour la plupart. Les graffiti sont-ils acceptables? Certains disent que oui, d'autres disent que non.

Qui paie le prix des graffiti? Qui, en fin de compte, paie le prix de la publicité ? Bonne question. Le consommateur.

Les gens qui ont placé des panneaux publicitaires vous ont-ils demandé la permission ? Non. Les auteurs des graffiti devraient-ils le faire, dans ce cas ? N'est-ce pas simplement une question de communication - votre propre nom, les noms de bandes et de grandes œuvres d'art dans la rue?

Pensez aux vêtements à rayures et à carreaux qui ont fait leur apparition dans les magasins il y a quelques années. Et aux équipements de ski. Les motifs et les tons ont souvent été empruntés tout droit à ces murs de béton fleuris. Il est assez amusant de constater que ces motifs et ces tons sont acceptés et admirés, mais que les graffiti du même style sont considérés comme abominables.

Les temps sont durs pour l'art.

\section{Question 3 : GRAFFITI}

\section{Le but de ces deux lettres est :}

A d'expliquer ce que sont les graffiti.

B de présenter une opinion sur les graffiti.

C de démontrer la popularité des graffiti.

D de faire savoir aux gens ce que cela coûte d'effacer les graffiti. 


\author{
Situation : publique \\ Format de texte : continu \\ Aspect : développer une interprétation \\ Niveau : 2
}

Score sur l'échelle PISA : 421

Cet item [R081Q01] d'interprétation de niveau 2, avec un score de 421 sur l'échelle PISA, demande aux élèves d'identifier le but que deux textes courts ont en commun en comparant les idées principales de chacun d'eux. L'information n'est pas clairement perceptible et il y a lieu de faire une inférence de bas niveau. L'objectif de cette question est d'établir si l'élève peut comprendre globalement le texte et en identifier le but. Le lecteur doit suivre des liens logiques et synthétiser les informations issues des deux textes pour déduire les objectifs des auteurs. La nécessité de comparer et de confronter deux lettres rend la tâche plus difficile que celle qui demanderait par exemple d'identifier l'objet d'une seule lettre.

Parmi les élèves qui n’ont pas choisi la réponse correcte, la proposition B en l'occurrence, les plus nombreux ont opté pour la proposition D : «de faire savoir aux gens ce que cela coûte d'effacer les graffiti ». Bien que cet aspect ne soit l'idée principale d'aucun des deux textes, il se rapporte clairement aux premières lignes du premier texte. Ce choix peut refléter une difficulté caractéristique des lecteurs moins compétents, pouvoir aller au-delà de la première partie d'un texte.

\title{
Question 4 : GRAFFITI
}

\section{Pourquoi Sophie évoque-t-elle la publicité ?}

\section{Situation : publique}

Format de texte : continu

Aspect : développer une interprétation

Niveau : 3

\section{Score sur l'échelle PISA : 542}

Cet item d'interprétation [R081Q05] basé sur les textes de l'unité Graffiti est plus difficile que les précédents et se situe au niveau 3 avec un score de 542 sur l'échelle PISA.

La tâche requiert des élèves qu'ils suivent un lien logique implicite entre les phrases, en l'occurrence une comparaison entre la publicité et les graffiti. La difficulté relative de cette question peut être imputée au fait que la comparaison doit être construite à partir d'une série de questions. Pour répondre correctement, les élèves doivent se rendre compte qu'une comparaison est faite entre les graffiti et la publicité. La réponse doit être cohérente avec l'idée que la publicité est une forme légale de graffiti. Ou alors, il faut qu'ils comprennent que la référence à la publicité est une stratégie pour défendre les graffiti. Les réponses caractéristiques du crédit complet sont celles qui donnent une explication spécifique relativement détaillée, comme dans le premier exemple ci-dessous, ou celles qui font simplement état de la comparaison de l'auteur entre les graffiti et la publicité, comme dans le deuxième exemple ci-dessous :

«Parce que de nombreuses affiches publicitaires sont de la pollution visuelle, mais qu'elles sont légales. »

«Elle dit que la publicité, c'est comme les graffiti. »

Aucun crédit n'est attribué aux réponses vagues ou insuffisantes, ni à celles qui témoignent d'une compréhension incorrecte du texte ou qui sont peu plausibles ou hors de propos. 


\section{Question 5 : GRAFFITI}

On peut parler de ce que dit une lettre (son contenu).

On peut parler de la façon dont une lettre est écrite (son style).

En faisant abstraction de votre propre opinion, qui a écrit la meilleure lettre, d'après vous ? Justifiez votre réponse en vous référant à la façon dont la lettre choisie est écrite lou à la façon dont sont écrites les deux lettres).

\section{Situation : publique \\ Format de texte : continu \\ Aspect : réfléchir et évaluer \\ Niveau : 4 \\ Score sur l'échelle PISA : 581}

Parmi les items associés aux textes de l'unité Graffiti, le plus difficile [R081Q06B] se situe au niveau 4, avec un score de 581 sur l'échelle PISA. Il demande aux élèves d'exploiter des connaissances formelles pour évaluer le talent d'un auteur en comparant les deux lettres. En termes d'aspect, il se classe dans la catégorie des tâches de réflexion sur la forme d'un texte et d'évaluation car les élèves doivent se baser sur leur propre conception de la qualité du style pour y répondre.

On peut attribuer un crédit complet à de nombreuses réponses, notamment celles qui traitent du ton ou des stratégies d'argumentation d'un ou des deux auteurs ou encore de la structure du texte. Les élèves doivent expliquer leur point de vue en faisant référence au style ou à la forme d'une ou des deux lettres. La référence à des critères tels que le style, la structure argumentative, le bien-fondé des arguments, le ton, le registre et les stratégies de persuasion du lecteur vaut un crédit complet, mais des formulations du type « de meilleurs arguments » doivent être justifiées.

Citons en exemple quelques réponses donnant lieu à un crédit complet :

«La lettre d'Helga est efficace parce qu'elle s'adresse directement aux auteurs de graffiti. »

«À mon avis, la deuxième lettre est meilleure, car elle comprend des questions qui vous interpellent, ce qui donne l'impression de participer à un débat et pas d'assister à une conférence. »

Les réponses qui n'ont donné lieu à aucun crédit étaient vagues ou pouvaient s'appliquer indifféremment aux deux lettres, ainsi que le montrent les exemples suivants :

«La lettre d'Helga est la meilleure, car elle est plus digne de foi. »

«Celle de Sophie est mieux écrite.»

Aucun crédit n'a été accordé aux réponses qui traitaient du contenu plutôt que du style, comme les suivantes :

«Celle d'Helga. Je suis d'accord avec tout ce qu' elle dit.»

«Celle de Sophie, parce que le graffiti est une forme d'art.»

De même, aucun crédit n’a été attribué aux réponses qui témoignaient d'une mauvaise compréhension du ton rhétorique des lettres, en particulier de la seconde, comme celle-ci : 
«Celle d'Helga est la meilleure, parce que Sophie ne donne pas son opinion et qu'elle ne fait que poser des questions. »

La difficulté relative de cet item, à l'instar de celle d'autres items similaires proposés dans l'évaluation PISA de la compréhension de l'écrit, donne à penser que de nombreux jeunes de 15 ans ne sont pas habitués à se servir de leurs connaissances formelles à propos du style et de la structure pour procéder à des évaluations critiques de textes.

\section{Le cadeau}

Les items de cette unité s'inscrivent dans une situation personnelle et sont associés à un texte continu de type narratif.

Cette nouvelle, venue des États-Unis d'Amérique, reflète les qualités humaines, affectives et esthétiques de la littérature qui font de la lecture de ce genre de texte une composante importante de la vie personnelle de nombreux individus. Elle doit notamment son inclusion dans les tests d'évaluation PISA à sa qualité littéraire : l'usage mesuré et précis de la langue et la description puissante mais subtile de l'état d'esprit du personnage principal et de l'évolution de sa réaction face au puma.

Une des autres raisons qui expliquent l'inclusion du texte Le Cadeau dans l'évaluation PISA est sa longueur. Il s'agit d'une nouvelle relativement courte en comparaison de beaucoup d'autres nouvelles publiées, mais d'un texte assez long par rapport à ceux généralement proposés aux élèves dans ce type de tests. Le groupe international d'experts chargé de la lecture qui a rédigé le cadre conceptuel d'évaluation et supervisé l'élaboration des tests a considéré que la persévérance nécessaire pour lire des textes plus longs, était une facette importante des compétences en compréhension de l'écrit qu'il fallait prendre en considération dans l'évaluation PISA.

Dans les tests PISA, le nombre de tâches associées à chaque texte est plus ou moins proportionnel à la longueur des textes. C'est donc Le Cadeau, le texte le plus long de l'évaluation, qui a donné lieu au plus grand nombre d'items. Cinq des sept tâches de cette unité sont présentées et commentées ci-dessous. L'ensemble des tâches associées à cette unité couvre les trois aspects et les cinq niveaux de compétences en compréhension de l'écrit.

Combien de jours, se demandait-elle, était-elle restée assise ainsi, à regarder l'eau brune et froide monter peu à peu et engloutir le promontoire ? Elle se souvenait à peine quand la pluie s'était mise à tomber, arrivant par le sud sur le marais et frappant la charpente de sa maison. Ensuite, c'était la rivière qui avait commencé à monter, d'abord lentement, jusqu'à ce qu' enfin 5 elle s'arrête, pour repartir de plus belle. D'heure en heure, elle remplissait le lit des ruisseaux et les fossés et submergeait les basses terres. La nuit, pendant son sommeil, elle avait pris possession de la route et l'avait encerclée, la laissant assise seule, sa barque disparue, sa maison comme échouée sur son promontoire. À présent, les eaux venaient même lécher les planches goudronnées des piliers. Et elles montaient toujours.

10 Aussi loin qu'elle pouvait voir, jusqu'au sommet des arbres, où se trouvait auparavant la rive d'en face, le marais n'était plus qu'une vaste étendue d'eau déserte, balayée par des torrents de pluie, la rivière perdue quelque part au milieu de son immensité. Avec son rez-de-chaussée en 
forme de bateau, sa maison avait été conçue pour résister à une telle inondation, s'il en venait jamais une, mais aujourd'hui elle était vieille. Peut-être les planches du bas étaient-elles en 15 partie pourries. Peut-être le câble qui amarrait sa maison au très vieux chêne allait-il céder et la laisser dériver au fil du courant, tout comme sa barque, qui était partie ainsi.

Personne ne pouvait plus venir. Elle pouvait crier, mais cela ne servirait à rien, personne n'entendrait. Tout autour du marais, d'autres luttaient pour sauver le peu qu'ils pouvaient, peut-être même leur vie. Elle avait vu toute une maison partir à la dérive, tellement silencieuse

20 qu'elle s'était crue en train d'assister à des funérailles. En la voyant, elle avait pensé qu'elle savait à qui était la maison. Cela avait été pénible de la voir dériver ainsi, mais ses propriétaires avaient dû s'enfuir vers les hautes terres. Plus tard, alors que la pluie et l'obscurité se faisaient plus denses, elle avait entendu le cri d'un puma en amont.

À présent, la maison paraissait trembler autour d'elle comme quelque chose de vivant. Elle

25 se pencha pour attraper la lampe qui glissait de la table de nuit, et la cala entre ses pieds pour la maintenir fermement. Alors, craquant et grondant sous l'effort, la maison s'arracha de la terre argileuse et se mit à flotter, dansant sur l'eau comme un bouchon et se laissant ballotter par le courant de la rivière. Elle s'agrippa au bord de son lit. Balancée de tous les côtés, la maison parvint au bout de son amarre. Il y eut une secousse et une plainte provenant des vieilles

30 poutres, puis le silence. Doucement, le courant relâcha sa pression et laissa la maison revenir en arrière en grinçant, vers son point d'attache. Elle retint son souffle et resta assise un long moment à sentir les lents mouvements de balancier. L'obscurité tomba sur la pluie incessante. La tête reposant sur un bras, elle s'endormit cramponnée au lit.

Le hurlement la réveilla dans la nuit, un cri si angoissé qu'elle fut debout avant d'être éveillée.

35 Dans l'obscurité, elle se cogna contre le lit. Cela venait du dehors, de la rivière. Elle pouvait entendre quelque chose bouger, quelque chose de grand qui faisait un large bruit de raclement. C'était peut-être une autre maison. Puis cela vint frapper, non de plein fouet, mais obliquement, le long de sa maison. C'était un arbre. Elle entendit les branches et les feuilles se dégager pour s'en aller au fil du courant, faisant place à la pluie et aux clapotis de l'inondation, qui étaient

40 devenus des bruits tellement constants qu'ils semblaient faire partie du silence. Recroquevillée sur son lit, elle s'était presque endormie quand un second hurlement se produisit, si proche cette fois qu'il aurait pu provenir de la pièce. Les yeux grand ouverts dans le noir, elle recula sur le lit jusqu'à ce que sa main rencontre la forme froide de la carabine. Ensuite, tapie contre son oreiller, elle tint le fusil posé en travers de ses genoux. «Qui est là ? », cria-t-elle.

45 La réponse fut un autre hurlement, moins perçant celui-là, comme fatigué, avant qu'un profond silence ne retombe. Elle se recroquevilla sur son lit. Quoi que ce soit, elle pouvait l'entendre bouger autour de la véranda. Les planches grinçaient et elle pouvait distinguer le bruit d'objets renversés. Il y eut un grattement sur le mur, comme si on voulait le déchirer pour entrer. Elle sut alors de quoi il s'agissait : c'était un gros félin, déposé par l'arbre déraciné qui était passé 50 près d'elle. Il était arrivé avec l'inondation, un cadeau.

Inconsciemment, elle passa une main sur son visage et le long de sa gorge contractée. La carabine chancela sur ses genoux. Elle n'avait jamais vu de puma de sa vie. Elle avait entendu d'autres personnes en parler, elle les avait entendus pousser leurs cris au loin, comme des cris de souf- 
france. Le félin grattait le mur à nouveau, faisant bouger la fenêtre près de la porte. Tant qu'elle 55 surveillerait la fenêtre et que le félin resterait coincé entre le mur et l'eau, il ne pouvait pas lui arriver grand chose. Dehors, l'animal s'était arrêté pour faire ses griffes contre la moustiquaire extérieure rouillée. De temps en temps, il gémissait ou grondait.

Lorsque, enfin, la lumière perça au travers de la pluie, comme une autre sorte d'obscurité, elle était encore assise sur son lit, toute raide et glacée. Ses bras, habitués à tenir les rames sur la 60 rivière, étaient douloureux à force de rester immobiles à tenir la carabine. Elle avait à peine osé bouger, de peur qu'un bruit ranime le félin. Pétrifiée, elle oscillait au rythme de la maison. La pluie continuait de tomber comme si elle ne devait jamais s'arrêter. À travers la lumière grise, elle finit par entrevoir les eaux de l'inondation piquetées par la pluie et, au loin, la forme vague du sommet des arbres immergés. En ce moment, le félin ne bougeait pas. Peut-être était-il parti.

65 Laissant le fusil, elle se glissa hors du lit et s'approcha sans bruit de la fenêtre. Il était encore là, tapi près du bord de la véranda, observant le vieux chêne, point d'amarrage de la maison, comme pour évaluer ses chances d'atteindre en bondissant quelque branche saillante. Il ne semblait pas si effrayant, maintenant qu'elle pouvait le voir, sa fourrure épaisse aux poils collés en épis, ses flancs creusés laissant voir ses côtes. Il serait facile de tirer sur lui, là où il était assis, sa

70 longue queue balayant le sol. Elle reculait pour prendre le fusil, lorsqu'il se retourna. Sans avertissement, sans élan ou tension des muscles, il bondit vers la fenêtre, et brisa un carreau. Elle tomba en arrière, étouffant un cri, saisit le fusil et fit feu à travers la fenêtre. Elle ne voyait plus le puma à présent, mais elle avait manqué son coup. Il s'était remis à marcher de long en large. Elle pouvait apercevoir sa tête et la cambrure de son dos lorsqu'il passait devant la fenêtre.

75 Tremblante, elle revint vers le lit et se coucha. Le bruit berçant et régulier de la rivière et de la pluie, le froid pénétrant, entamèrent sa détermination. Elle fixa la fenêtre et garda le fusil prêt. Après un long moment, elle se leva à nouveau pour regarder. Le puma s'était endormi, la tête sur les pattes, comme un chat domestique. Pour la première fois depuis que la pluie s'était mise à tomber, elle eut envie de pleurer, sur elle-même, sur tous, sur tout ce qui était touché par 80 l'inondation. Elle se laissa glisser sur le lit et s'enveloppa dans l'édredon. Elle aurait dû s'en aller quand elle le pouvait, quand les routes étaient encore ouvertes, ou avant que sa barque ne soit emportée. Alors qu'elle se balançait d'avant en arrière au rythme des oscillations de la maison, une crampe à l' estomac lui rappela qu' elle n'avait pas mangé. Elle ne pouvait se souvenir depuis quand. Comme le félin, elle était affamée. Elle se faufila dans la cuisine, alluma un feu avec les 85 quelques morceaux de bois qui restaient. Si la crise se prolongeait, elle devrait brûler la chaise, peut-être même la table. S'emparant du dernier morceau de jambon fumé qui pendait au plafond, elle coupa d'épaisses tranches de la viande brun rouge et les mit dans une poêle. L'odeur de la viande en train de frire lui donna le vertige. Il restait quelques biscuits rassis de la dernière fois où elle avait cuisiné et elle pourrait faire du café. Ce n'était pas l'eau qui manquait.

90 Pendant qu'elle se préparait à manger, elle oublia presque le félin, jusqu'à ce qu'il émette un gémissement. Il avait faim, lui aussi. «Laisse-moi manger », lui lança-t-elle, « ensuite, je m’occuperai de toi ». Et elle rit sous cape. Alors qu' elle accrochait le reste du jambon à son clou, le puma poussa un profond rugissement qui fit trembler sa main.

Quand elle eut fini de manger, elle retourna à son lit et s'empara à nouveau de la carabine. La 95 maison était à présent montée si haut qu'elle ne raclait plus le promontoire lorsque le mouve- 
ment de la rivière l'y repoussait. La nourriture l'avait réchauffée. Elle pouvait se débarrasser de l'animal tant que la lumière traversait encore le rideau de pluie. Elle se glissa lentement vers la fenêtre. Il était toujours là, feulant, et s'était mis à tourner en rond autour de la véranda. Elle l'observa un long moment, sans crainte. Puis, sans réfléchir à ce qu'elle faisait, elle déposa le 100 fusil, contourna le lit et alla à la cuisine. Derrière elle, le puma s'agitait, indécis. Elle décrocha ce qui restait du jambon, traversa la pièce qui tanguait pour aller vers la fenêtre, où elle fit passer la viande par le carreau cassé. De l'autre côté, il y eut un grondement affamé, et ce fut comme si une onde de choc passait entre l'animal et elle. Stupéfaite de ce qu'elle venait de faire, elle retourna à son lit. Elle pouvait entendre le puma en train de déchirer la viande. La maison se 105 balançait autour d'elle.

Lorsqu' elle se réveilla à nouveau, elle sut immédiatement que tout avait changé. La pluie avait cessé. Elle tenta de sentir les mouvements de la maison, mais celle-ci ne se balançait plus sur l'eau. Elle ouvrit la porte et vit à travers la moustiquaire déchirée un monde tout différent. La maison reposait sur le promontoire, où elle avait toujours été. À quelques pas de là, la ri110 vière coulait toujours en torrent, mais elle ne recouvrait plus les quelques pas qui séparaient la maison du vieux chêne. Et le puma avait disparu. Partant de la terrasse en direction du chêne et s'enfonçant sans doute vers le marais, des traces de pas indistinctes disparaissaient déjà dans la boue molle. Et là, sur la terrasse, rongé jusqu'à l'os, se trouvait ce qui restait du jambon.

Source : Louis Dollarhide, «The gift» dans : Mississippi Writers: Reflections of Childhood and Youth, Volume 1. Publié par Dorothy Abbot, University Press of Mississippi, 1985.

\section{Question 6 : LE CADEAU}

Voici un extrait de dialogue entre deux personnes qui ont lu " Le cadeau ".

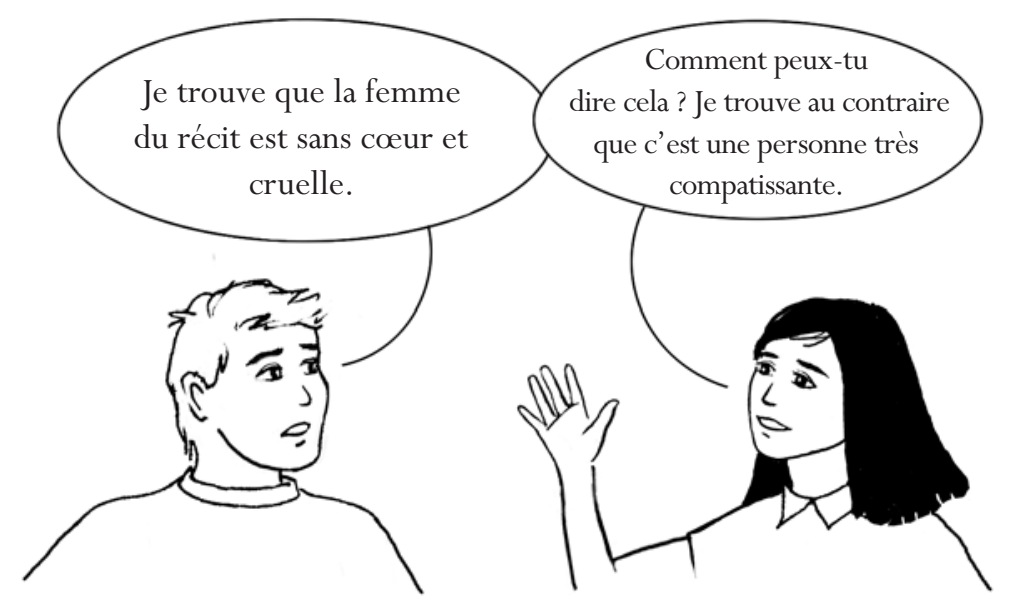

Trouvez dans le récit des éléments que chacun des interlocuteurs ci-dessus pourrait utiliser pour justifier son point de vue.

Interlocuteur 1

Interlocuteur 2 
Situation : personnelle

Format de texte : continu

Aspect : réfléchir et évaluer

Niveaux : niveau 2 et niveau 3

Score sur l'échelle PISA : 480 et 537

Parmi les tâches de réflexion et d'évaluation associées au texte Le cadeau, celle-ci [R119Q09] est la plus facile : elle demande aux élèves de faire des comparaisons et d'établir des liens entre le texte et des connaissances extérieures au texte en se basant sur leurs expériences et leurs attitudes personnelles. Pour obtenir un crédit complet, les élèves doivent faire le rapprochement entre le comportement d'un personnage de l'histoire et leurs valeurs personnelles, en s'appuyant sur leur conception de la compassion et de la cruauté et en utilisant des éléments d'information contenus dans le texte.

Au codage, cet item peut recevoir un crédit complet ou un crédit partiel et compte dès lors deux degrés de difficulté. Pour obtenir un crédit partiel (niveau 2, score de 480 sur l'échelle PISA), les élèves doivent trouver dans le texte des éléments qui démontrent soit la compassion, soit la cruauté, et pour obtenir un crédit complet (niveau 3, score de 537 sur l'échelle PISA), des éléments qui démontrent à la fois la compassion et la cruauté. Le crédit complet reflète la capacité de prendre en considération des concepts opposés ou ambigus, une compétence supérieure à celle généralement associée au niveau 2. Les réponses qui sont insuffisantes ou qui témoignent d'une compréhension incorrecte du texte ne reçoivent aucun crédit.

Le contenu de la réponse ne doit pas être très élaboré pour recevoir un crédit. La réponse typique d'un crédit complet est « parce qu'elle était sur le point de tirer sur le puma » pour la partie A et « parce qu' elle a finalement nourri le puma » pour la partie B.

Dans d'autres tâches, comme les deux que l'on trouve ci-après, plus la lecture du texte est élaborée, plus le crédit attribué est important.

\section{Question 7 : LE CADEAU}

Pensez-vous que la dernière phrase du récit " Le cadeau " est une fin appropriée ? Justifiez votre réponse en indiquant comment vous interprétez la relation entre cette dernière phrase et la signification du récit.

Situation : personnelle

Format de texte : continu

Aspect : réfléchir et évaluer

Niveaux : niveau 4 et niveau 5

Score sur l'échelle PISA : 567 et 647

Comme la précédente, cette deuxième tâche de réflexion et d'évaluation [R119Q05] reçoit au codage un crédit complet ou un crédit partiel. Avec le crédit partiel, elle se situe au niveau 4 avec un score de 567 sur l'échelle PISA, tandis qu'avec le crédit complet, elle se classe au niveau 5 avec un score de 647 sur l'échelle PISA.

Pour obtenir un crédit complet, le lecteur doit aller au-delà de l'interprétation littérale du récit et jeter un regard critique sur le texte en se basant sur ses connaissances spécialisées et sur sa compréhension approfondie de ce texte, long et complexe. Il doit commenter de manière critique le caractère approprié de la 
fin du récit en réfléchissant aux liens qui existent entre la fin et le thème général ou la tonalité du texte. Il doit établir des inférences en s'appuyant sur des idées qui ont surgi pendant la lecture, mais qui ne sont pas exprimées de manière explicite. Le lecteur doit implicitement baser sa réponse sur sa propre conception de ce qu'est une fin «appropriée », sachant qu'à ce niveau, les critères auxquels on fait appel sont profonds et abstraits plutôt que superficiels et littéraux. Ainsi, une réponse valant un crédit complet peut commenter la signification métaphorique de l'os ou encore la touche finale apportée au thème du récit. Ces concepts, qui se basent sur des notions formelles de la littérature, peuvent être considérés comme des connaissances spécialisées pour les jeunes de 15 ans. L'éventail d'interprétations auxquelles ce récit peut donner lieu est reflété dans les réponses ci-après, qui ont reçu un crédit complet. Citons quelques exemples :

«Oui. Je suppose que les restes du jambon laissés par le puma sont aussi un cadeau, dont le message est «Vivre et laisser vivre». »

«Je pense que la fin est appropriée, parce que je crois que le puma était le cadeau pour arrêter l'inondation. Comme la femme l'a nourri au lieu de le tuer, l'inondation a cessé et, assez mystérieusement, les restes de jambon sont sur la terrasse comme pour dire merci. »

«L'inondation a cessé, tout ce qui en reste sont les dégâts. En fait, c'est ce que dit la dernière ligne, avec la blancheur de l'os qui est tout ce qui reste du jambon.»

Pour obtenir un crédit partiel, les élèves doivent évaluer le caractère approprié de la fin à un niveau plus littéral en commentant sa cohérence par rapport au récit. À l'instar de la catégorie de réponses donnant lieu à un crédit complet, cette catégorie à crédit partiel demande aux élèves de procéder à une évaluation (positive ou négative) en se basant sur leur conception de ce qu'est le caractère approprié de la fin d'un récit mais, à la différence de la première, elle se réfère à des caractéristiques superficielles du récit, telles que la cohérence de l'intrigue. La difficulté relative de cette catégorie (niveau 4) s'explique par le fait que la réponse doit faire référence à certains critères formels d'adéquation et, plus important encore, qu'elle doit démontrer une compréhension précise d'un texte long et complexe. À titre d'illustration, voici quelques exemples de réponse ayant reçu un crédit partiel :

«Je trouve que c'est une bonne fin. Une fois qu'elle lui a donné de la nourriture, tout a été mieux. L'animal l'a laissée tranquille et tout a changé. »

« Oui, c'est fini, parce que le jambon est fini, comme l'histoire d'ailleurs. »

« Je pense que c'est une fin stupide, parfaite pour clôturer une histoire stupide! Bien sûr que le jambon va être mangé. Je le savais, mais je n’ai jamais imaginé que l'auteur serait assez idiot pour le mentionner. »

Comme le montrent ces exemples, un crédit peut être attribué aussi bien aux évaluations positives qu'aux évaluations négatives. Ce principe s'applique également à des tâches similaires. La pertinence de la réponse est jugée en fonction de la qualité de la compréhension du texte et de la sophistication des outils critiques mis en jeu, plutôt qu'en fonction d'une quelconque notion d'un point de vue, correct ou non, émis par le lecteur.

Certaines réponses n'ont donné lieu à aucun crédit. Parmi ces réponses, figurent celles qui étaient peu plausibles ou hors de propos, comme dans le premier exemple ci-dessous, ou celles qui étaient considérées comme trop vagues, comme dans le deuxième exemple ci-dessous. 
« Je pense que c'est une fin appropriée. Elle montre qu'il n’y a peut-être jamais eu de puma et le jambon qu'elle

a jeté par la fenêtre est là pour le prouver. »

«Oui, parce qu' elle explique ce qui s'est passé à la fin. »

À l'instar des deux premières tâches de réflexion et d'évaluation, la tâche d'interprétation suivante reçoit au codage un crédit complet / ou un crédit partiel. Le crédit complet la situe au niveau 5, avec un score de 645 sur l'échelle PISA et le crédit partiel, au niveau 3, avec un score de 539 sur l'échelle PISA. Les niveaux de difficulté de ces deux catégories de réponses sont donc séparés par un écart de plus de 100 , soit plus d'un écart type, sur l'échelle de compréhension de l'écrit.

\section{Question 8 : LE CADEAU}

Voici quelques-unes des premières références au puma dans le récit :

"le hurlement la réveilla dans la nuit, un cri si angoissé... " : (ligne 34).

"La réponse fut un autre hurlement, moins perçant celui-là, comme fatigué,... " (ligne 45).

"...elle les avait entendus pousser leurs cris au loin, comme des cris de souffrance. " (lignes 53-54).

En tenant compte de ce qui se passe dans la suite du récit, pourquoi pensez-vous que l'auteur a décidé d'introduire le puma par de telles descriptions ?

Situation : personnelle

Format de texte : continu

Aspect : développer une interprétation

Niveaux : niveau 3 et niveau 5

Score sur l'échelle PISA : 539 et 645

Pour obtenir un crédit complet à cette tâche [R119Q07], les élèves doivent construire du sens à partir d'un langage tout en nuances tout en étant confrontés à des notions contraires aux attentes. Ils doivent aborder un texte dont la structure rhétorique n'apparaît pas d'emblée, pour distinguer la relation entre des passages spécifiques du texte (indiqués dans la question) et son thème implicite.

Le texte est délibérément ambigu, car il contient des idées qui sont contraires aux attentes. Bien que la peur soit la réaction principale de la femme lorsqu' elle prend conscience de la présence du puma, le choix délibéré et subtil de décrire les cris de l'animal par les termes « angoissé », « comme fatigué » et « souffrance » implique le pathos, plutôt que la menace. Cette suggestion, qui figure au début de l'histoire, est importante pour comprendre parfaitement le comportement «inattendu » de la femme à la fin et, donc, le thème implicite du récit. Pour obtenir un crédit complet, les élèves doivent comprendre que ces descriptions ont pour but de susciter la pitié.

Un crédit partiel est attribué aux réponses qui traitent le texte à un niveau plus direct et qui relient à l'intrigue les phrases citées dans la question. A propos des descriptions citées dans la question, les élèves peuvent faire état d'intentions (ou d'effets) possibles autres que celles de susciter la pitié. À ce niveau, la question demande de suivre des liens logiques implicites qui unissent les phrases entre elles pour inférer que le puma crie parce qu'il a faim. Il existe une seconde catégorie de réponses qui donnent lieu à un crédit partiel, celles qui relient différents passages du texte pour en identifier l'idée maîtresse. Ce type de réponse 
identifie l'atmosphère qui règne à ce stade du récit. Les élèves peuvent faire référence à l'information littérale contenue dans les passages cités.

\section{Question 9 : LE CADEAU}

Lorsque la femme dit : "ensuite, je m'occuperai de toi " (lignes 91-92), elle veut dire :

A qu'elle est sûre que le félin ne lui fera pas de mal.

B qu'elle essaie d'effrayer le félin.

(C) qu'elle a l'intention de tirer sur le félin.

D qu'elle envisage de nourrir le félin.

Situation : personnelle

Format de texte : continu

Aspect : développer une interprétation

Niveau : niveau 4

Score sur l'échelle PISA : 603

Cette tâche [R119Q04] requiert des élèves qu'ils établissent une inférence de haut niveau en se basant sur le texte pour dégager le sens d'un passage en contexte et de traiter des ambiguïtés et des idées qui peuvent être contraires aux attentes. Pour désigner laquelle des quatre options constitue la meilleure réponse, le lecteur doit inférer une signification psychologique en suivant des liens thématiques situés dans plusieurs paragraphes.

Hors contexte, la phrase citée dans la question est ambiguë et même replacée dans son contexte, elle donne lieu à diverses interprétations alternatives, apparemment plausibles. Cet item a été spécialement conçu pour évaluer la capacité des élèves à aborder de telles ambiguïtés. Parmi les notes de traduction qui ont été envoyées aux équipes nationales en même temps que les deux versions sources du matériel de test (l'une en français et l'autre en anglais), il en est une qui concerne ce passage : «Veillez à faire en sorte que la phrase «ensuite, je m'occuperai de toi » puisse permettre les deux interprétations suivantes : «ensuite, je te nourrirai » et «ensuite, je te tirerai dessus ». Pourtant, une seule interprétation est cohérente avec la séquence psychologique du récit : la femme doit avoir l'intention de tuer le puma, puisqu'elle s'est saisie du fusil juste après en se disant qu' elle pouvait «se débarrasser de l'animal tant que la lumière traversait encore le rideau de pluie ». L'éventuelle compassion de la femme pour le puma est un distracteur puissant, contraire aux attentes suggérées ailleurs dans le récit. L'option de la question à choix multiple basée sur cette interprétation - «qu'elle envisage de nourrir le félin »-a convaincu près de la moitié des élèves. Ceux-ci ont de toute évidence suivi la trame du récit sans changer de niveau. Ils ont identifié un thème majeur et ont construit du sens dans une partie limitée du texte (des compétences relevant des niveaux 1 et 2), mais ils n'ont pas tenu compte des ambiguiités et des idées contraires aux attentes, une exigence associée aux tâches d'interprétation de niveau 4 .

Dans l'ensemble, les tâches basées sur ce texte long et relativement subtil sont difficiles. Néanmoins, cette unité contient également une tâche de niveau 1. 


\section{Question 10 : LE CADEAU}

"Alors, craquant et grondant sous l'effort, la maison s'arracha... " (lignes 26).

Qu'est-il arrivé à la maison à ce moment du récit ?

A Elle s'est écroulée.

(B) Elle s'est mise à flotter.

C Elle a heurté le chêne.

D Elle a coulé au fond de la rivière.

Situation : personnelle

Format de texte : continu

Aspect : trouver une information

Niveau : niveau 1

Score sur l'échelle PISA : 367

Cette tâche [R119Q06] demande aux élèves de localiser une information explicitement exprimée dans un passage court du texte et de la faire correspondre avec l'une des quatre options de réponse proposées.

Bien que le texte soit long, le passage du texte auquel les élèves doivent se référer est bref et très explicitement localisé dans la question, par sa citation directe ainsi que par la référence aux numéros de ligne. La réponse correcte - «Elle s'est mise à flotter »- contient un terme qui correspond directement à un terme figurant immédiatement après le passage cité : «Alors, craquant et grondant sous l'effort, la maison s'arracha de la terre argileuse et se mit à flotter... ».

Le lac Tchad

Les textes constituant le stimulus de cette unité appartiennent à la catégorie des textes non continus. L'unité Le lac Tchad propose deux graphiques extraits d'un atlas archéologique. La figure A de cette unité est un graphique linéaire et la figure $\mathrm{B}$, un histogramme horizontal. Cette unité comprend également un troisième type de texte non continu, à savoir une petite carte du lac insérée dans la figure A. Enfin, deux textes courts accompagnent ces illustrations.

En juxtaposant ces éléments d'information, l'auteur invite le lecteur à inférer un lien entre l'évolution du niveau du lac Tchad au fil du temps et les périodes au cours desquelles certaines espèces d'animaux peuplaient les environs.

Ce genre de texte est typique de ceux que les élèves peuvent rencontrer dans le cadre scolaire. Toutefois, la situation des textes de cette unité est considérée comme publique car l'atlas dont sont tirés les textes est destiné au grand public. Les cinq questions de cette unité couvrent les trois aspects de la compréhension de l'écrit et présentent une difficulté allant du niveau 1 au niveau 4.

Quatre des questions de l'unité Le lac Tchad sont présentées ci-après. 
La figure A présente les changements de niveau du lac Tchad, situé au Sahara, en Afrique du Nord. Le lac Tchad a complètement disparu vers 20000 av. J.-C., pendant la dernière ère glaciaire. Il a réapparu vers 11000 av. J.-C. À présent, son niveau est à peu près le même que celui qu'il avait en 1000 apr. J.-C.

Figure A

Lac Tchad : changements de niveau

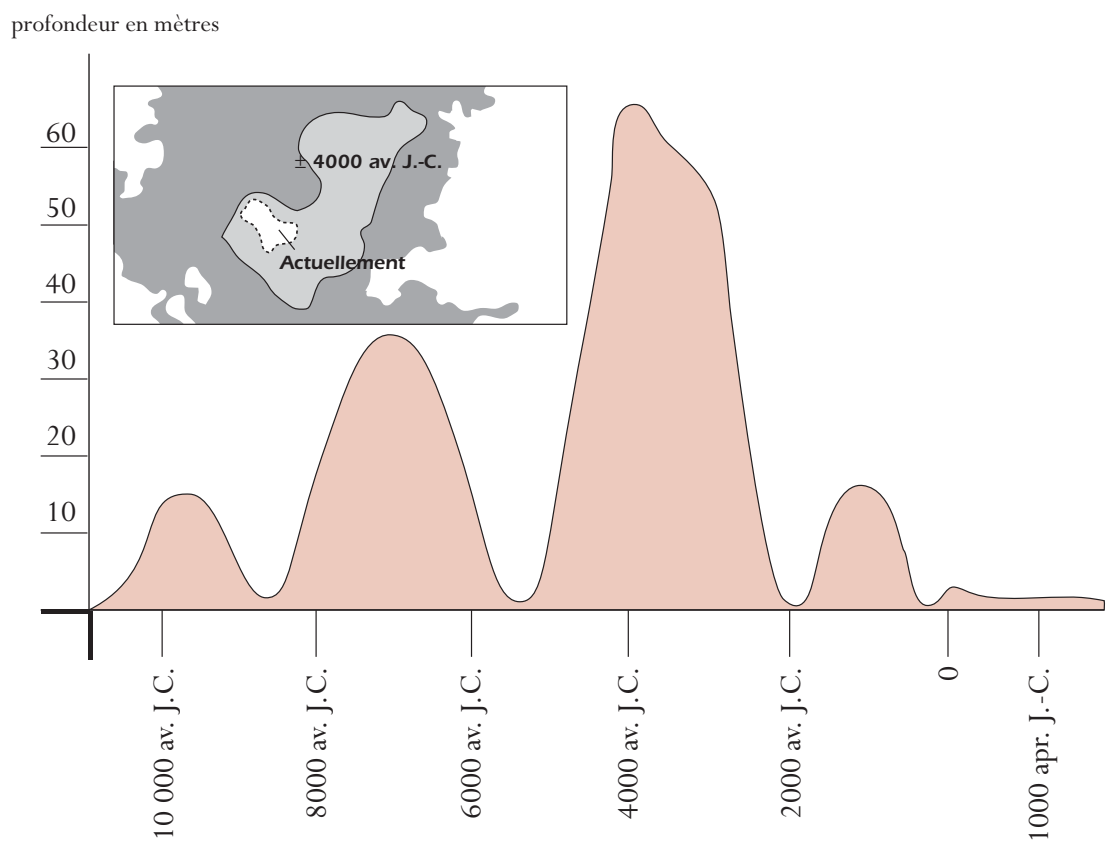

La figure B présente l'art rupestre saharien (c'est à dire les dessins et les peintures préhistoriques trouvés sur les parois des cavernes) et l'évolution de la faune.

Figure B

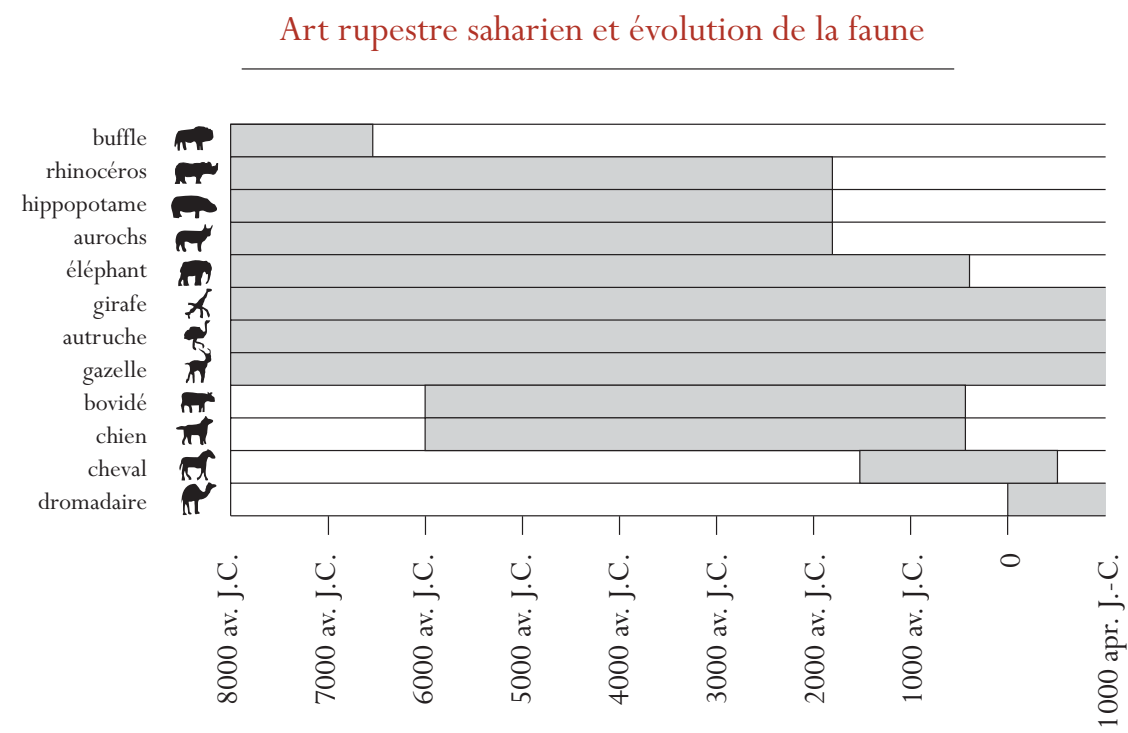

Source : Copyright Bartholomew Ltd. 1988. Extrait de The times Atlas of Archaeology et reproduit avec l'autorisation de Harper Collins Publishers. 


\title{
Question 11 : LE LAC TCHAD
}

\section{Quelle est la profondeur du lac Tchad à présent ?}

\section{A Environ deux mètres.}

B Environ quinze mètres.

C Environ cinquante mètres.

D Il a complètement disparu.

E L'information n'est pas donnée.

\section{Situation : publique}

Format de texte : non continu

Aspect : trouver l'information

Niveau : niveau 2

\section{Score sur l'échelle PISA : 478}

Cette première tâche [R040Q02] est une tâche de localisation d'informations de niveau 2 avec un score de 478 sur l'échelle PISA. Elle demande aux élèves de localiser et de combiner des éléments d'information situés dans un graphique linéaire et dans le texte d'introduction.

La locution adverbiale «à présent » qui figure dans la question peut être mise directement en correspondance avec la phrase adéquate de l'introduction qui explique qu' «à présent », le niveau du lac est à peu près le même que celui qu'il avait en 1000 de notre ère. Les élèves doivent combiner cette information avec l'information de la figure A en situant l'an 1000 sur le graphique, puis en lisant le niveau qu'avait alors le lac. Des informations distractrices sont présentes, sous forme de dates multiples dans la figure A et de la répétition de «1 000 Apr. J.-C. » dans la figure B. Cette question est néanmoins assez facile car l'information clé est exprimée explicitement dans l'introduction textuelle. La plupart des élèves qui n'ont pas choisi la bonne réponse, la proposition A «Environ deux mètres », ont opté pour la proposition E «L'information n'est pas donnée ». Leur erreur provient vraisemblablement du fait qu'ils se sont limités à la figure $\mathrm{A}$, sans combiner les informations qui s'y trouvaient avec le texte d'introduction. Les items de niveau 2 basés sur des textes non continus, tels que ceux-ci, demandent parfois de combiner des informations tirées de différentes représentations, alors que les items de niveau 1 portent en principe sur des éléments d'information distincts, qui figurent habituellement dans une seule représentation.

\section{Question 12 : LE LAC TCHAD}

À peu près en quelle année commence le graphique présenté par la figure A ?

\author{
Situation : publique \\ Format de texte : non continu \\ Aspect : trouver l'information \\ Niveau : niveau 3 \\ Score sur l'échelle PISA : 540
}


Cette deuxième tâche de localisation d'informations [R040Q03A] est plus difficile que la première et se situe au niveau 3 avec un score de 540 sur l'échelle PISA.

Pour répondre à cette question, les élèves doivent localiser et identifier les relations entre des informations situées dans le graphique linéaire et dans le texte d'introduction, tout en ayant affaire à des informations distractrices bien perceptibles.

Comme dans la tâche précédente, le lecteur doit localiser l'information adéquate dans le texte d'introduction («Il a réapparu vers 11000 av. J.-C. ») et la mettre en rapport avec la partie spécifiée du graphique (l'époque à laquelle il commence). Cet item peut sembler plus facile que le précédent dans la mesure où les élèves sont explicitement invités à consulter la figure A. Toutefois, les informations distractrices sont plus fortes. L'erreur courante qui s'est produite montre bien l'efficacité du leurre : on a pris à tort la première date inscrite en abscisse sur la figure A (10 000 av. J.-C.) pour le début du graphique linéaire représentant le niveau du lac Tchad vers 11000 av. J.-C.

Cette tâche est classée parmi celles de localisation d'informations car elle demande avant tout de trouver des informations dans un texte. Toutefois, elle nécessite également la mise en œuvre de stratégies d'interprétation pour inférer la bonne réponse du graphique. De plus, elle impose aux lecteurs de réfléchir à ce qu'ils savent sur les conventions en matière de dates et de se baser sur un concept contextuel connu, à savoir que les dates situées avant notre ère vont «à rebours». Ces éléments montrent le chevauchement considérable des trois aspects de la compréhension de l'écrit que sont «trouver une information ; développer une interprétation ; réfléchir et évaluer » : la plupart des tâches imposent un certain nombre d'exigences diverses aux lecteurs et chacun d'eux peut les aborder de différentes façons. Comme le souligne le cadre d'évaluation de la compréhension de l'écrit (OCDE, 1999), associer un item à une des échelles d'aspect revient souvent à porter des jugements sur ses caractéristiques principales et sur l'approche que les lecteurs vont le plus vraisemblablement adopter pour y répondre.

\section{Question 13 : LE LAC TCHAD}

La figure B se fonde sur I'hypothèse que :

A les animaux représentés dans l'art rupestre étaient présents dans la région à l'époque où ils ont été dessinés.

B les artistes qui ont dessiné les animaux étaient très doués.

C les artistes qui ont dessiné les animaux avaient la possibilité de voyager loin.

D il n'y eut aucune tentative de domestiquer les animaux représentés dans l'art rupestre.

Situation : publique

Format de texte : non continu

Aspect : développer une interprétation

Niveau : niveau 1

Score sur l'échelle PISA : 397

Cet item [R040Q04], le plus facile de l'unité Le lac Tchad, correspond à un score de 397 sur l'échelle PISA, c'est-à-dire au niveau 1. L'item demande aux élèves d'identifier l'idée principale d'un graphique où les informations ne sont pas perceptibles et se concentrent dans une seule représentation accompagnée d'un texte explicatif très bref. 
Le stimulus de l'unité Le lac Tchad comporte deux figures mais cette question invite les élèves à n'en consulter qu'une seule, la figure B. Cette figure ne comporte que peu d'indications (les dates et le nom des animaux) et les symboles qu'elle contient sont figuratifs, et non abstraits. En d'autres termes, un traitement d'un niveau relativement bas suffit pour interpréter la figure. Par contre, l'information requise dans le texte n'apparaît pas d'emblée, en l'absence de toute référence explicite au fait que les artistes peignaient ce qu'ils voyaient ; en fait, il n'y a même aucune référence directe aux artistes. Il ressort toutefois des résultats que les élèves n’ont eu aucun mal à faire cette inférence.

\section{Question 14 : LE LAC TCHAD}

Pour répondre à cette question, vous devez utiliser des informations provenant à la fois de la figure $A$ et de la figure $B$.

La disparition des rhinocéros, des hippopotames et des aurochs de l'art rupestre saharien s'est produite :

A au début de la période glaciaire la plus récente.

B au milieu de la période où le niveau du lac Tchad était le plus élevé.

(C) après que le niveau du lac Tchad a progressivement baissé pendant plus de mille ans.

D au début d’une période ininterrompue de sécheresse.

Situation : publique

Format de texte : non continu

Aspect : développer une interprétation

Niveau : niveau 3

Score sur l'échelle PISA :508

Cet item d'interprétation [R040Q06], le plus difficile de l'unité Le lac Tchad (niveau 3, avec un score de 508 sur l'échelle PISA), demande aux élèves de relier entre elles plusieurs parties de textes non continus pour comprendre une relation et de comparer les informations données dans les deux graphiques.

La nécessité de combiner des informations provenant de deux sources contribue modérément au niveau de difficulté de la tâche (niveau 3). Une autre de ses caractéristiques est qu'elle se base sur deux types de graphiques différents (un graphique linéaire et un histogramme) dont le lecteur doit interpréter les structures pour transposer les informations nécessaires de l'un à l'autre.

Parmi les élèves qui n'ont pas choisi la réponse correcte, les plus nombreux ont opté pour le distracteur D : « au début d'une période ininterrompue de sécheresse ». Si on néglige les textes, cette option est la plus plausible des réponses incorrectes. Sa sélection fréquente indique que ces élèves ont probablement traité cet item comme une question de réflexion et d'évaluation de niveau 2, pour laquelle il aurait été indiqué de construire des hypothèses pour expliquer un élément du texte en se basant sur des connaissances familières extérieures.

Population active

Les tâches de l'unité Population active se fondent sur des textes appartenant à la catégorie des textes non continus. Cette unité repose sur un diagramme en arbre qui présente la structure et la répartition de la population active d'un pays en 1995. Ce diagramme a été publié dans un manuel d'économie destiné 
à des élèves fréquentant le deuxième cycle de l'enseignement secondaire. C'est la raison pour laquelle cette unité s'inscrit dans un contexte (situation) scolaire. Les informations spécifiques figurant dans le diagramme se rapportent à la Nouvelle-Zélande, mais les définitions et les termes utilisés sont empruntés à la terminologie officielle de l'OCDE. Le stimulus peut donc être considéré comme présentant une dimension essentiellement internationale.

Cette unité n'est pas aussi attractive que d'autres qui ont été présentées ci-dessus. Son contenu ne risque guère de susciter un grand enthousiasme chez les jeunes de 15 ans et sa présentation est de toute évidence très académique. Il suffit pour s'en convaincre de la comparer au texte de la dernière unité de la présente sélection : celui-ci est accompagné de quelques petites illustrations qui donnent une touche plus gaie aux chiffres et aux informations figurant dans les graphiques. Toutefois, l'unité Population active est un type de texte que les adultes sont susceptibles de rencontrer et qu'ils doivent être capables d'interpréter pour participer pleinement à la vie économique et politique d'une société contemporaine.

L'unité Population active compte cinq tâches qui couvrent les trois aspects de la compréhension de l'écrit et dont les degrés de difficulté sont compris entre les niveaux 2 et 5 . Quatre de ces tâches sont présentées ci-dessous.

Le diagramme en arbre ci-dessous présente la structure de la population active d'un pays, c'est-àdire sa «population en âge de travailler ». En 1995, la population totale de ce pays était d'environ 3,4 millions d'habitants.

\section{La structure de la population active au 31 mars 1995 (x 1000$)^{1}$}

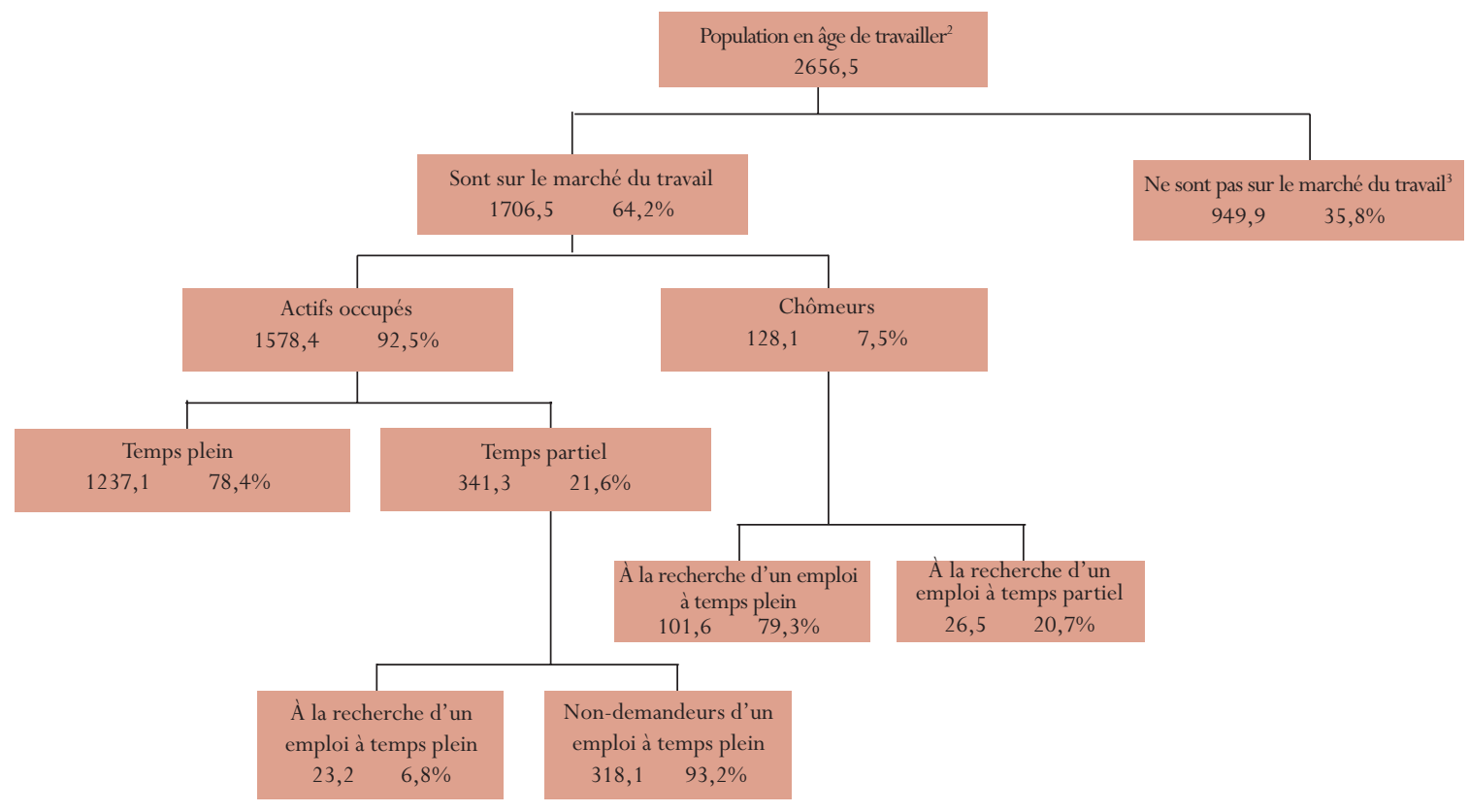

1. Le nombre de personnes est exprimé en milliers (x 1000 ).

2. La population en âge de travailler est définie comme l'ensemble des personnes âgées de 15 à 65 ans.

3. Les personnes qui «ne sont pas sur le marché du travail » sont celles qui ne sont pas activement à la recherche d'un emploi ou ne sont pas disponibles pour travailler.

Source : D. Miller, Form 6 Economics, ESA Publications, Box 9453, Newmarker, Auckland, NZ, p. 64. 


\section{Question 15 : POPULATION ACTIVE}

Combien de personnes en âge de travailler ne sont pas sur le marché du travail ? (Écrivez le nombre de personnes, non le pourcentage).

Situation : scolaire

Format de texte : non continu

Aspect : trouver l'information

Niveaux : niveau 3 et niveau 5

Score sur l'échelle PISA : 485 et 631

Cette première tâche [R088Q03] comporte deux niveaux de difficulté : l'un associé à la catégorie de réponses sanctionnées par un crédit partiel qui se situe au niveau 3 avec un score de 485 sur l'échelle PISA et l'autre, à la catégorie de réponses donnant droit à un crédit complet qui équivaut au niveau 5 avec un score de 631 sur l'échelle PISA. L'item de niveau 5 est le plus difficile des items de localisation d'informations de la batterie de tests de compréhension de l'écrit de l'évaluation PISA.

Pour obtenir un crédit complet (niveau 5), les élèves sont tenus de trouver une information numérique figurant dans le corps du texte (le diagramme en arbre) et de la combiner avec une mention située dans une note de bas de page, c'est-à-dire en dehors du corps du texte. De plus, ils doivent utiliser cette information mentionnée en note de bas de page pour calculer le nombre correct de personnes appartenant à la catégorie adéquate. Ces deux caractéristiques contribuent à rehausser le degré de difficulté de l'item.

Pour obtenir un crédit partiel (niveau 3), les élèves doivent uniquement localiser le nombre fourni dans la catégorie adéquate du diagramme en arbre. Ils ne sont pas tenus d'utiliser l'information figurant dans la note de bas de page pour se voir attribuer un crédit partiel. Même sans cette information importante, la tâche reste assez difficile.

En règle générale, la nécessité d'utiliser une information située en dehors du corps du texte accroît significativement la difficulté des items. Cet aspect est bien illustré par les deux catégories de réponses associées à cette question. En effet, la différence entre les réponses qui reçoivent un crédit complet et celles qui reçoivent un crédit partiel réside dans l'association ou non de l'information située en dehors du corps du texte à une information numérique correctement identifiée dans le corps du texte. L'écart de difficulté entre ces deux catégories de réponse représente plus de deux niveaux de compétence.

\section{Question 16 : POPULATION ACTIVE}

Faites une croix pour indiquer à quelle catégorie du diagramme en arbre appartiennent les personnes suivantes, pour autant qu'il y ait une catégorie qui convienne.

Indiquez votre réponse en faisant une croix dans la case appropriée.

La première croix a été faite à votre place, à titre d'exemple.

Situation : scolaire

Format de texte : non continu

Aspect : développer une interprétation

Niveaux : niveau 2 et niveau 5

Score sur l'échelle PISA : 473 et 727 


\begin{tabular}{|c|c|c|c|c|}
\hline & $\begin{array}{l}\text { «Sur le marché du } \\
\text { travail: actifs occupés » }\end{array}$ & $\begin{array}{l}\text { «Sur le marché du } \\
\text { travail : chômeurs » }\end{array}$ & $\begin{array}{l}\text { «Ne sont pas sur le } \\
\text { marché du travail » }\end{array}$ & $\begin{array}{l}\text { N'est compris dans } \\
\text { aucune catégorie }\end{array}$ \\
\hline \multicolumn{5}{|l|}{ Un serveur à mi-temps, âgé de 35 ans } \\
\hline \multicolumn{5}{|l|}{$\begin{array}{l}\text { Une femme d'affaires de } 43 \text { ans qui travaille } \\
60 \mathrm{~h} \text { par semaine }\end{array}$} \\
\hline \multicolumn{5}{|l|}{ Un étudiant à plein temps, âgé de 21 ans } \\
\hline \multicolumn{5}{|l|}{$\begin{array}{l}\text { Un homme, âgé de } 28 \text { ans, qui a cédé son com- } \\
\text { merce récemment et qui cherche du travail. }\end{array}$} \\
\hline \multicolumn{5}{|l|}{$\begin{array}{l}\text { Une femme de } 55 \text { ans qui n'a jamais travaillé ni } \\
\text { voulu travailler en dehors de son ménage }\end{array}$} \\
\hline $\begin{array}{l}\text { Une grand-mère âgée de } 80 \text { ans qui travaille } \\
\text { encore quelques heures par jour sur le stand } \\
\text { que la famille tient au marché. }\end{array}$ & & & & \\
\hline
\end{tabular}

Ce deuxième item [R088Q04] basé sur le diagramme en arbre est classé dans la catégorie des tâches d'interprétation d'un texte. Il présente également deux degrés de difficulté : l'un associé aux réponses sanctionnées par un crédit partiel, qui se situe au niveau 2 avec un score de 473 sur l'échelle PISA et l'autre, aux réponses donnant droit à un crédit complet qui équivaut au niveau 5 avec un score de 727 sur l'échelle PISA.

Cette tâche exige des élèves qu'ils analysent plusieurs cas décrits et classent chaque cas dans une des catégories du diagramme en arbre. Les cas décrits sont destinés à déterminer si les élèves ont compris, totalement et dans le détail, les distinctions et les définitions du diagramme. Comme dans l'item précédent, une partie des informations adéquates figure dans des notes de bas de page en dehors du corps du texte.

Pour se situer au niveau 5, les élèves doivent prouver qu'ils ont compris le texte totalement et dans ses moindres détails, en se référant parfois à des informations extérieures à la représentation principale. Pour obtenir un crédit complet, ils doivent répondre correctement aux cinq parties de la question.

Pour obtenir un crédit partiel équivalant au niveau 2 avec un score de 473 sur l'échelle PISA, les élèves doivent faire la preuve qu'ils ont compris certains aspects du texte - en reliant correctement trois ou quatre des cinq cas décrits aux catégories correspondantes de la population active. Les élèves participant à PISA 2000 ont le plus souvent choisi la bonne catégorie pour les deuxième et quatrième cas proposés, c'est-à-dire ceux qui ne nécessitent pas l'utilisation des informations situées dans les notes de bas de page $\mathrm{n}^{\text {os }} 2$ et 3 (la définition des personnes «en âge de travailler» et des personnes qui «ne sont pas sur le marché du travail »). Les cas les plus difficiles à classer correctement sont ceux situés en troisième, cinquième et sixième positions. Il s'agit de ceux qui imposent l'assimilation des informations figurant dans les notes de bas de page. À l'instar de la tâche précédente, le recours nécessaire à une information située en dehors du corps du texte contribue à rendre l'item plus difficile. Un autre facteur de difficulté réside dans la nécessité pour les élèves de fournir plusieurs réponses indépendantes. 


\section{Question 17 : POPULATION ACTIVE}

Supposez que des informations sur la structure de la population active soient présentées chaque année dans un diagramme comme celui de cet exercice.

Le tableau ci-dessous présente quatre données figurant dans le diagramme. Peut-on s'attendre à ce que ces données changent d'une année à l'autre ? Répondez en entourant "Changeront " ou "Ne changeront pas " pour chacune des quatre données. La première réponse a été entourée à votre place, à titre d'exemple.

\begin{tabular}{|l|r|}
\multicolumn{1}{|c|}{ Données du diagramme } & \multicolumn{2}{c|}{ Réponses } \\
\hline $\begin{array}{l}\text { Les intitulés de chaque cellule (par ex. : « sont sur le marché } \\
\text { du travail }) .\end{array}$ & Changeront / Ne changeront pas \\
\hline Les pourcentages (par ex. «64,2\%»). & Changeront/Ne changeront pas \\
\hline Les chiffres (par ex. «2656,5»). & Changeront /Ne changeront pas \\
\hline Les notes au bas du diagramme en arbre. & \\
\hline
\end{tabular}

Situation : scolaire

Format de texte : non continu

Aspect : réfléchir et évaluer

Niveaux : niveau 2

Score sur l'échelle PISA : 445

Ce troisième item [R088Q05] de l'unité Population active est une tâche de réflexion et d'évaluation relativement facile, qui se situe au niveau 2 avec un score de 445 sur l'échelle PISA.

Cette tâche requiert des élèves qu'ils identifient les caractéristiques du texte en démontrant qu'ils ont compris la structure sous-jacente d'un diagramme en arbre en établissant des distinctions entre variables et constantes. Bien qu'il ne soit pas nécessaire de connaître les termes techniques que sont «variable » et « constante », il faut pleinement comprendre la structure du diagramme en arbre pour répondre correctement à cette question. Cet item est classé parmi les tâches « réfléchir et évaluer », non pas parce qu'il demande une évaluation critique ou une réponse personnelle, mais parce qu'il impose au lecteur de considérer l'écrit comme un artefact, du moins en termes de forme et de structure. Pour obtenir un crédit complet, les élèves doivent répondre correctement aux trois parties de la question. Les élèves n'ayant repondu correctement qu'à une ou deux parties ne reçoivent pas de crédit

\section{Question 18 : POPULATION ACTIVE}

L'information sur la population active est présentée sous forme de diagramme en arbre, mais elle aurait pu être présentée de bien d'autres manières, par exemple une description écrite, un diagramme en " camembert", un graphique ou un tableau.

Le diagramme en arbre a vraisemblablement été choisi parce qu'il est particulièrement utile pour présenter :

A l'évolution dans le temps.

B la taille de la population totale du pays.

(C) les catégories au sein de chaque groupe.

D la taille de chacun des groupes. 
Situation : scolaire

Format de texte : non continu

Aspect : réfléchir et évaluer

Niveaux : niveau 3

Score sur l'échelle PISA : 486

Cette dernière tâche [R088Q07] basée sur le diagramme en arbre Population active requiert d'évaluer une caractéristique du texte. Elle a pour objet de comparer la pertinence du diagramme en arbre en regard de ses objectifs particuliers par rapport à d'autres formes de présentation. Les connaissances formelles sur les structures textuelles et leurs avantages et inconvénients respectifs appartiennent à un domaine relativement peu familier pour les jeunes de 15 ans, ce qui contribue à expliquer la difficulté moyenne de cet item (niveau 3). Contrairement à la question précédente de l'unité Population active qui ne demande qu'implicitement aux élèves de montrer qu'ils ont compris la structure de l'écrit, cette tâche impose cette exigence de manière explicite. Pour obtenir un crédit, les élèves doivent reconnaître la pertinence des diagrammes en arbre pour présenter des catégories dans des groupes. L'approche ouvertement abstraite de la question pourrait contribuer sa difficulté. Les deuxième et quatrième distracteurs, qui ont piégé un nombre significatif d'élèves, se concentrent sur des informations qui sont présentées dans le diagramme, mais la structure de celui-ci ne se prête pas particulièrement à leur mise en évidence. Les élèves qui ont choisi ces distracteurs semblent avoir abordé cette question comme si elle visait à trouver des informations (« Parmi ces types d'information, lesquels sont présentés dans le diagramme ? »), et non à évaluer la structure de la présentation.

\section{PLAN International}

Le troisième et dernier texte non continu présenté ici est un tableau contenant des informations sur les types d'interventions proposées par une organisation humanitaire internationale, PLAN International. Il est extrait d'un rapport public diffusé par cette organisation, ce qui explique le classement de cette unité dans la catégorie des situations publiques.

Le tableau énumère les pays situés dans une région d'intervention de PLAN International et les types d'interventions proposés (27 types d'interventions regroupés dans trois grandes catégories) ; il quantifie les travaux effectués dans chaque pays et dans chaque catégorie d'interventions. Le tableau contient un volume important d'informations présentées de manière plutôt dense, susceptible de dérouter les lecteurs moins compétents. Les lecteurs plus à l'aise vont vraisemblablement commencer par parcourir le tableau pour se faire une idée de sa structure et de son contenu, plutôt que de s'astreindre à lire chaque détail du tableau indifféremment. 
Une seule question de l'unité PLAN International a été incluse dans la construction de l'échelle de compréhension de l'écrit de PISA.

\section{PLAN International - Résultats des programmes pour l’année budgétaire 1996}

\section{Région : Afrique de l'Est et du Sud}

\section{RAES}

Dispensaires construits (4 chambres ou moins)
$\begin{aligned} & \text { Assistants en soins de santé formés pendant une journée } \\ & \text { Enfants ayant reçu un complément nutritionnel }>1 \text { semaine }\end{aligned} \quad 10195$
$\begin{aligned} & \text { Enfants ayant reçu une aide financière pour des } \\ & \text { soins de santé / des traitements dentaires }\end{aligned}$

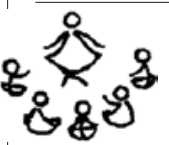

Enseignants formés pendant une semaine

Cahiers scolaires achetés / reçus en don

Manuels scolaires achetés / reçus en don

$\begin{array}{rrrrrrrrrr}0 & 0 & 367 & 0 & 970 & 115 & 565 & 0 & 303 & \mathbf{2 3 2 0} \\ 667 & 0 & 0 & 41200 & 0 & 69106 & 0 & 150 & 0 & \mathbf{1 1 1} 123 \\ 0 & 0 & 45650 & 9600 & 1182 & 8769 & 7285 & 150 & 58387 & \mathbf{1 3 1 0 2 3} \\ 8897 & 0 & 5761 & 0 & 2000 & 6040 & 0 & 0 & 434 & \mathbf{2 3 1 3 2} \\ 12321 & 0 & 1598 & 0 & 154 & 0 & 0 & 0 & 2014 & \mathbf{1 6 0 8 7} \\ 3200 & 0 & 3689 & 250 & 1564 & 1725 & 1794 & 0 & 4109 & \mathbf{1 6 3 3 1} \\ 44 & 0 & 50 & 8 & 93 & 31 & 45 & 0 & 82 & 353 \\ 0 & 0 & 34 & 0 & 0 & 14 & 0 & 0 & 33 & 81 \\ 1160 & 0 & 3000 & 568 & 3617 & 0 & 0 & 0 & 350 & \mathbf{8 6 9 5}\end{array}$

Uniformes scolaires achetés / confectionnés / reçus en don 8897

Enfants ayant bénéficié d'une aide pour les droits

d'inscription à l'école / ayant obtenu une bourse

Pupitres construits / achetés / reçus en don

Salles de classe permanentes construites

Salles de classe remises en état

Adultes ayant reçu des cours d'alphabétisation

$1160-0 \quad 3000$

au cours de cette année budgétaire

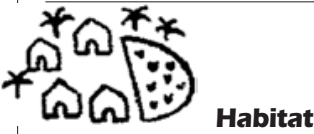

Latrines ou toilettes creusées / construites

Maisons raccordées à de nouveaux égouts

Puits creusés / remis en état (ou sources captées)

Nouveaux puits forés avec succès

Systèmes d'eau potable à alimentation par gravité installés

Systèmes d'eau potable réparés / améliorés

Maisons remises en état grâce à un projet PLAN

Nouvelles maisons construites pour les bénéficiaires

Salles publiques construites ou remises en état

Dirigeants de collectivités formés pendant un jour ou plus $2214 \quad 95$

Kilomètres de route remis en état

Ponts construits

Familles ayant bénéficié directement du contrôle de l'érosion $0-0$

Maisons nouvellement desservies par un projet d'électrification $448 \quad 0$

Source : adaptation du rapport financier de PLAN International pour l'exercice 1996, annexe du rapport trimestriel au

Conseil international, premier trimestre de 1997. 


\section{Question 19A : PLAN INTERNATIONAL}

D'après le tableau, comment se situait le taux d'interventions effectuées en 1996 par PLAN International en Éthiopie, comparativement aux autres pays de cette région ?

A Le taux d’interventions en Éthiopie a été comparativement élevé.

(B) Le taux d'interventions en Éthiopie a été comparativement bas.

C Il a été à peu près le même que dans les autres pays de la région.

D Il a été comparativement élevé pour la catégorie « Habitat », mais bas pour les autres catégories.

\section{Question 19B: PLAN INTERNATIONAL}

En 1996, I'Éthiopie était l'un des pays les plus pauvres du monde.

À votre avis, en tenant compte de ce fait et des informations fournies par le tableau, qu'estce qui pourrait expliquer le taux d'interventions de PLAN International en Éthiopie, comparé à celui de ses interventions dans d'autres pays ?

Situation : publique

Format de texte : non continu

Aspect : réfléchir et évaluer

Niveaux : niveau 5

Score sur l'échelle PISA : 705 et 822

Les consignes de correction pour cette tâche [R099Q04B] sont assez complexes. Bien que les élèves aient à répondre à deux questions dans cette tâche - l'une à choix multiple et l'autre à réponse construite -, seule la seconde est codée. Comme cette tâche est classée dans la catégorie «Réfléchir et évaluer », l'item à choix multiple qui consiste essentiellement en une localisation d'informations ne vaut en soi aucun crédit. Toutefois, il est pris en considération dans la mesure où les élèves doivent y répondre correctement pour obtenir un crédit pour la seconde question, qui demande une réponse construite.

La deuxième question peut donner lieu à un crédit partiel ou total mais les deux catégories de réponse la situent au niveau 5 (avec des scores respectifs de 705 et 872 sur l'échelle PISA). Elle requiert des élèves qu'ils formulent des hypothèses sur le contenu du texte en se basant sur des connaissances spécialisées et qu'ils appréhendent un concept contraire aux attentes. Les élèves doivent également identifier des schémas parmi les nombreux éléments d'information qui figurent dans cette présentation complexe et détaillée.

Les élèves doivent donc réfléchir à l'importance de l'aide apportée à l'Éthiopie par PLAN International et la comparer à celle dont bénéficient d'autres pays de la région. Pour ce faire, ils doivent formuler une hypothèse plutôt que de se borner à expliquer simplement un fait, étant donné que fort peu de jeunes de 15 ans sont susceptibles d'être au fait des raisons pour lesquelles cette organisation humanitaire a pu accorder à l'Éthiopie une quantité d'aide donnée. Les connaissances auxquelles cette question fait appel sont dites spécialisées, dans la mesure où le travail des organisations humanitaires n'est pas un domaine familier pour la majorité des adolescents, même si l'on peut supposer que des jeunes de cet âge ont une certaine idée des actions menées par ces organisations. En revanche, il est peu vraisemblable qu'ils possèdent des connaissances spécifiques à propos de la situation économique de tel ou tel pays; et c'est pourquoi cette 
unité fournit l'information relative à la pauvreté qui règne en Éthiopie. Cette tâche fait référence à un phénomène contraire aux attentes, à savoir qu'une organisation humanitaire apporte une aide relativement limitée à un pays très pauvre.

Pour obtenir un crédit complet, les élèves doivent répondre correctement à la question 19A, puis construire leur réponse en se fondant sur toutes les informations fournies par cette réponse. Ils doivent formuler une hypothèse à propos des raisons qui expliquent pourquoi l'Éthiopie n'a bénéficié que d'une aide relativement réduite de la part de PLAN International, compte tenu de toutes les informations pertinentes figurant dans le tableau - à la fois le volume d'aide et le type d'intervention - et des informations fournies dans la question. Plusieurs hypothèses qui s'appuient sur toutes les informations présentées dans le tableau ont été émises par les élèves. Parmi les réponses qui ont donné lieu à un crédit complet, citons les suivantes :

«PLAN a aidé les dirigeants de collectivités à devenir autonomes. Comme il s'agit d'une organisation humanitaire, cela semble être la meilleure idée. »

«La seule aide dont a bénéficié l'Éthiopie concerne la formation des dirigeants de collectivités. Peut-être que l'Éthiopie ne laisse pas PLAN International s'impliquer dans d'autres aspects de la vie du pays. »

Pour obtenir un crédit partiel, les élèves doivent répondre correctement à la question 19A, puis prendre en considération une partie, mais pas l'ensemble, des informations pertinentes figurant dans le tableau : le volume d'aide mais pas le type d'intervention. De plus, leur hypothèse doit être cohérente par rapport aux connaissances extérieures sur le travail des organisations humanitaires. Parmi les hypothèses le plus souvent avancées qui ont donné lieu à un crédit partiel, citons les exemples suivants :

«Il y a peut-être eu des inondations ou quelque chose d'autre dans le pays qui les ont amenés à arrêter leur aide. »

«Peut-être que PLAN International vient de commencer dans cette communauté et que c'est pour ça que leurs activités sont réduites. »

«Peut-être que d'autres organisations humanitaires travaillent déjà en Éthiopie et que les gens n'ont pas trop besoin de PLAN.»

«C'est tout simplement trop difficile d'aider là-bas. »

Plusieurs raisons, en plus de celles évoquées ci-dessus, expliquent pourquoi cette tâche est particulièrement difficile. En premier lieu, elle impose la synthèse d'un grand nombre d'informations qui se trouvent à la fois dans le texte et en dehors du texte. En second lieu, elle ne donne qu'une indication minimale sur la partie du texte qu'il faut consulter pour obtenir un crédit complet. Plus particulièrement, elle n'indique pas que le type d'aide dont bénéficie l'Éthiopie doit être mentionné dans la réponse pour obtenir un crédit complet. En d'autres termes, les informations requises ne sont pas du tout perceptibles, ni dans la question ni dans le texte lui-même. C'est probablement la conjonction de tous ces facteurs qui explique pourquoi cette tâche est l'une des plus difficiles de l'évaluation en compréhension de l'écrit du programme PISA. 


\section{Notes}

1. Dans PISA, chaque item a un identifiant unique. Cet identifiant est présenté entre crochets, comme [R110Q01] dans ce chapitre. Cet identifiant est destiné aux utilisateurs qui souhaitent consulter des réponses d'élèves à cet item dans la base de données en ligne de PISA 2000 (http://www.pisa.oecd.org/pisa/outcome.htm). 


\section{GUIDE DU LECTEUR}

\section{Données des graphiques}

Les données auxquelles les chapitres 4 à 8 font référence sont présentées dans l'annexe B à la fin du volume, et des détails supplémentaires sont disponibles sur www.pisa.oecd.org. Les symboles suivants indiquent que des données sont manquantes dans les tableaux et figures :

n.a. la catégorie ne s'applique pas au pays concerné, les données sont donc manquantes.

$m$ les données ne sont pas disponibles. Si aucune autre précision n’est donnée, ces données ont été recueillies mais retirées soit à la demande du pays concerné, soit en raison de considérations techniques.

$x \quad$ les données figurent dans une autre catégorie ou colonne du tableau.

\section{Calcul des moyennes internationales}

Une moyenne de l'OCDE a été calculée pour la plupart des indicateurs présentés dans ce rapport. La valeur totale, représentant l'ensemble du territoire de l'OCDE, a également été calculée pour certains indicateurs.

La moyenne de l'OCDE, aussi appelée moyenne des pays, est la moyenne des valeurs de tous les pays de l'OCDE pour lesquels des données sont disponibles ou peuvent être estimées. La moyenne de l'OCDE sert notamment à comparer la valeur d'un indicateur d'un pays donné à celle d'un pays typique de l'OCDE. Elle ne tient pas compte des effectifs absolus d'élèves dans les pays. En d'autres termes, chaque pays intervient de la même manière dans la moyenne.

Les valeurs de trois pays de l'OCDE, à savoir les Pays-Bas, la République slovaque (devenue membre de l'OCDE en 2000) et la Turquie sont exclues des calculs de moyenne ou d'autres estimations globales. Les Pays-Bas ont été exclus, car des estimations fiables des scores moyens n'ont pu être calculées en raison de taux de participation insuffisants. La République slovaque et la Turquie participeront à PISA à partir du cycle d'évaluation de 2003.

Par ailleurs, il arrive que les données de certains pays ne soient pas disponibles pour des indicateurs spécifiques, ou que des catégories particulières de données ne soient pas appliquées. Les lecteurs doivent donc garder à l'esprit que les termes Moyenne de l'OCDE et Total de l'OCDE font référence aux pays de l'OCDE qui font l'objet d'une comparaison.

\section{Indice de tendance centrale}

La moyenne ou la valeur médiane peuvent toutes deux être utilisées pour illustrer la tendance générale observée dans l'analyse des pays. Dans certains cas, il est préférable d'utiliser la valeur médiane pour éviter d'accorder trop de poids aux valeurs extrêmes qui ont été observées dans certains pays. La valeur médiane divise la distribution en deux parties égales et est équivalente au $50^{\mathrm{e}}$ percentile. 
Le règles suivantes ont été adoptées pour les besoins de ce rapport : les fréquences moyennes ou moyennes ont été utilisées pour les analyses descriptives ; pour les analyses bivariées ou multivariées (corrélations ou analyses de régression), les valeurs médianes ont été utilisées.

\section{Présentation des données relatives aux élèves}

Le rapport désigne souvent la population cible de PISA par l'expression «les jeunes de 15 ans ». Pratiquement, la population cible comprend les élèves dont l'âge se situait entre 15 ans et trois mois (accomplis) et 16 ans et deux mois (accomplis) au début de la période d'évaluation et qui étaient inscrits dans des établissements d'enseignement, quels que soient leur année d'études, le type d'établissement fréquenté et leur mode de scolarisation (à temps plein ou à temps partiel).

\section{Présentation des données relatives aux établissements}

Les chefs des établissements fréquentés par les élèves soumis à l'évaluation ont été priés de remplir un questionnaire portant sur les caractéristiques de leur école. Les réponses des chefs d'établissement présentées dans ce rapport ont été pondérées de manière à être proportionnelles au nombre de jeunes de 15 ans inscrits dans chaque établissement.

\section{Arrondis}

Dans certains tableaux, il arrive que la somme des chiffres ne corresponde pas exactement au total mentionné en raison des ajustements d'arrondis. Les totaux, les différences et les moyennes sont systématiquement calculés à partir des chiffres exacts. Ils ne sont arrondis qu'une fois calculés.

\section{Abréviations utilisées dans ce rapport}

Les abréviations suivantes ont été utilisées dans ce rapport :

Éc. t. Écart type

Er. t. Erreur type

\section{Documentation complémentaire}

Pour plus d'informations sur les instruments d'évaluation de PISA et les méthodes utilisées, voir Connaissances et compétences : des atouts pour la vie. Premiers résultats de PISA 2000 (OCDE, 2001b), le Rapport technique de PISA 2000 (OCDE, 2002b) et le site Internet de PISA (www.pisa.oecd.org). 


\section{Chapitre}

4

\section{PERFORMANCES EN LECTURE DES JEUNES DE 15 ANS}

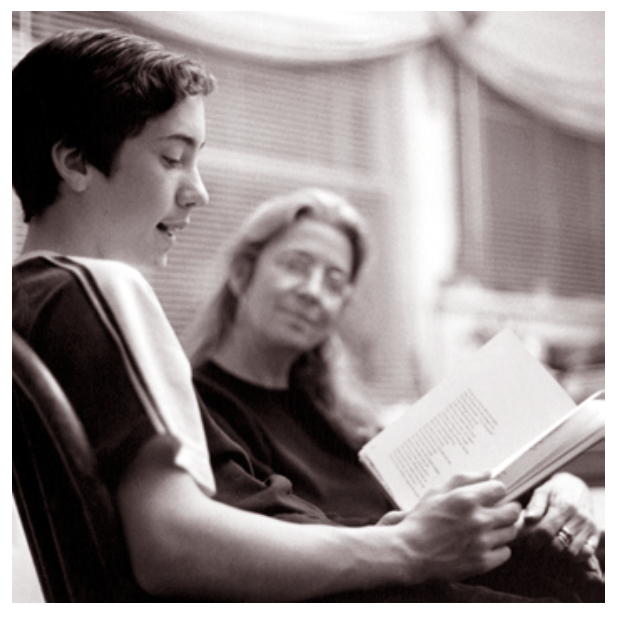




\section{SYNTHÈSE}

- Sur l'échelle combinée de compréhension de l'écrit, dont la moyenne est fixée à 500 et l'écart type, à 100, l'écart entre le score moyen du pays en tête du classement et celui du pays au bas du classement est de 150, soit l'équivalent de plus de deux niveaux de compétence.

- Le score moyen de la majorité des pays correspond au niveau 3. C'est également au niveau 3 que se situe la proportion la plus élevée d'élèves dans la plupart des pays, tant sur l'échelle combinée de compréhension de l'écrit que sur les sous-échelles. Tous pays de l'OCDE confondus, un peu plus d'un quart des élèves se situent au niveau 3 sur chaque échelle.

- La répartition des élèves entre les niveaux varie considérablement de pays à pays, même dans le cas de moyennes nationales semblables. La variation est particulièrement prononcée entre les proportions d'élèves situés aux deux extrémités des échelles. Ainsi, la proportion d'élèves ayant atteint le niveau 5 va de moins de 1 pour cent à un petit peu moins de 20 pour cent.

- Dans tous les pays, certains élèves se situent en deçà du niveau le plus bas sur l'échelle, tandis que d'autres élèves se situent au niveau le plus élevé de l'échelle.

- Dans de nombreux pays, un nombre élevé d'élèves font preuve de compétences limitées en compréhension de l'écrit, ainsi qu'en témoigne le fait que dans plus d'un tiers des pays participants, plus de 20 pour cent des élèves ne parviennent pas à atteindre le niveau 2 sur l'échelle de compétence en lecture de PISA.

- Les corrélations entre les trois sous-échelles d'aspect et entre les deux sous-échelles de format sont généralement importantes. Toutefois, certaines différences relevées dans le profil de compétence des pays selon les sous-échelles méritent d'être soulignées. Certains pays affichant une performance globale des plus faibles obtiennent de meilleurs résultats sur la sous-échelle Réfléchir et évaluer que sur la sous-échelle Trouver l'information. Les pays dont la performance globale est peu élevée obtiennent également un meilleur score sur la sous-échelle Textes continus que sur la sous-échelle Textes non continus.

- En ce qui concerne les trois sous-échelles d'aspect, c'est la sous-échelle Développer une interprétation qui affiche les plus faibles variations de compétence, et la sous-échelle Trouver l'information, les plus fortes variations.

- En termes de format, la variation de performance est sensiblement plus importante lorsque les items sont basés sur des textes non continus que lorsqu'ils sont associés à des textes continus. Pour chaque pays, l'écart entre les scores des élèves les plus compétents et les moins compétents (représentés par le $90^{\mathrm{e}}$ et le $10^{\mathrm{e}}$ percentile) sur l'échelle combinée de compréhension de l'écrit varie entre deux niveaux et demi et quatre niveaux et demi. Cela signifie que dans de nombreux pays, l'inégalité des résultats dans le domaine de la compréhension de l'écrit est importante et préoccupante. 
L'une des premières questions que suscite une enquête internationale sur le rendement scolaire est sans aucun doute : «Comment nous situons-nous par rapport à... ?». Ce chapitre propose des réponses à une série de questions sur le sujet : non seulement nous analyserons la performance moyenne des pays en compréhension de l'écrit, mais nous nous pencherons également sur la répartition des différents niveaux de compétence entre pays et dans les pays, ainsi que sur les différences relatives de performance relevées dans les diverses composantes de ce domaine d'évaluation.

Nous commencerons par présenter les scores moyens des pays et leur répartition sur l'échelle combinée de compréhension de l'écrit. Nous poursuivrons par l'analyse des performances sur chacune des trois sous-échelles d'aspect (Trouver l'information, Développer une interprétation, Réfléchir et évaluer) et des deux sous-échelles de format (textes continus et textes non continus). Nous conclurons par une analyse des différences d'accès à la compréhension de l'écrit et de leurs conséquences pour les individus, les sociétés et les pays.

Les résultats rapportés sur l'échelle combinée de compréhension de l'écrit et les trois sous-échelles d'aspect sont publiés dans le rapport initial du cycle PISA 2000 (OCDE, 2001b). Par comparaison avec d'autres enquêtes internationales sur la littératie, les sous-échelles d'aspect permettent d'envisager la compréhension de l'écrit sous un nouvel angle et fournissent de précieuses informations aux décideurs. Ces deux raisons nous ont amenés à inclure les résultats sur ces sous-échelles dans le rapport initial. L'analyse des résultats des élèves rapportés sur les deux sous-échelles de format de PISA, à savoir textes continus et textes non continus, est présentée pour la première fois dans ce rapport. La distinction établie en fonction du format de texte dans le domaine de la compréhension de l'écrit ne surprendra pas les lecteurs qui connaissent les enquêtes internationales antérieures. À titre d'exemple, citons la «Reading Literacy Study » mise en ouvre par l'IEA (Elley, $1992 ; 1994)$, qui différencie les textes narratifs et informatifs (similaires aux textes continus de PISA) des documents (comparables aux textes non continus de PISA) pour rendre compte de la performance en lecture des élèves de 10 et de 14 ans ; ou encore l'Enquête internationale sur la littératie des adultes (voir OCDE 1995, 1997, 2000) qui rend compte des résultats séparément pour les textes en prose (textes continus) et pour les documents (textes non continus). Présenter les résultats de PISA sur les deux sous-échelles de format fournit donc aux pays de nouveaux points de comparaison avec d'autres études et permet également, tout comme les trois sous-échelles d'aspect, d'alimenter des analyses qui peuvent avoir d'importantes implications pour les programmes de cours et l'enseignement de la lecture.

Ce chapitre est essentiellement descriptif. Son objectif est de communiquer aux pays participants les principales données sur les résultats, sous l'angle de la comparaison. Ces données ne manqueront pas de susciter des questions et des hypothèses, dont certaines seront étudiées de manière plus approfondie dans la suite de ce rapport. Ainsi, nous nous pencherons sur la relation entre les performances en lecture et l'engagement à l'égard de la lecture ou les profils de lecteur (chapitre 5), sur la relation entre les performances en lecture et les caractéristiques contextuelles des élèves, de leur famille et de leur environnement scolaire (chapitre 6) et, enfin, sur la relation entre les performances en lecture et certaines combinaisons de ces variables, les structures scolaires en particulier (chapitre 7). Bien que certaines observations, jugées utiles, soient émises ici à propos de pays particuliers ou de groupes de pays, un grand nombre d'analyses, qui permettraient d'expliquer les résultats de pays donnés à certains égards, sortent du cadre du présent chapitre, voire du présent rapport. Nous espérons toutefois que les informations contenues dans ce chapitre amèneront les pays à se poser de telles questions et à se livrer à des analyses plus approfondies, en particulier à l'échelon national. 
CHAPITRE 4 La lecture, moteur de changement: Performance et engagement d'un pays à l'autre

\section{Graphique 4.1}

Comparaisons multiples de la performance moyenne sur l'échelle combinée de compréhension de l'écrit

\begin{tabular}{|c|c|c|c|c|c|c|c|c|c|c|c|c|c|c|c|c|c|c|c|c|c|c|c|c|c|c|c|c|c|}
\hline & & & & & & & & & & & & & & & & & & & & & & & & & $5 \approx$ 实 & 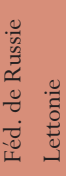 & 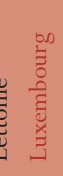 & & \\
\hline & \multicolumn{2}{|c|}{ Moyenne } & & & & & & in & & & & 合 & 空? & & & $\begin{array}{ll}0 \\
0 \\
0\end{array}$ & & $f^{\prime}$ & 7 & $=\sigma$ & & & & 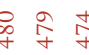 & 孛学我 & 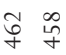 & 导 & & \\
\hline & & r. & & & & & & & & & & ક્ & & & & & & & & $\stackrel{\vec{d}}{\stackrel{f}{d}}$ & & & & \begin{tabular}{ll}
6 & \multicolumn{1}{c}{} \\
$\dot{ \pm}$ & \pm
\end{tabular} &  & $\begin{array}{ll}\widehat{T} & \hat{+} \\
\dot{ \pm} & \dot{v}\end{array}$ & $\stackrel{6}{=}$ & $\begin{array}{l}\hat{n} \\
\hat{n}\end{array}$ & $\stackrel{\overbrace{}}{\stackrel{\infty}{\infty}}$ \\
\hline alande & 46 & . & & $\Delta$ & & $\boldsymbol{\Delta}$ & $\Delta$ & $\boldsymbol{\Delta}$ & $\boldsymbol{\Delta}$ & $\Delta$ & $\Delta$ & $\Delta$ & $\Delta$ & $\Delta$ & $\Delta$ & $\Delta$ & $\Delta$ & & $\boldsymbol{\Delta}$ & $\Delta \Delta$ & $\Delta$ & $\Delta$ & $\boldsymbol{\Delta}$ & $\Delta \Delta<$ & $\Delta \Delta$ & $\Delta \Delta$ & $\Delta$ & $\boldsymbol{\Delta}$ &  \\
\hline Canada & 534 & . & $\nabla$ & & O & 0 & 0 & $\Delta$ & $\boldsymbol{\Delta}$ & O & $\Delta$ & $\Delta$ & $\Delta$ & $\Delta$ & $\Delta$ & $\Delta$ & $\boldsymbol{\Delta}$ & $\Delta$ & $\Delta$ & $\Delta \Delta$ & $\Delta$ & $\Delta$ & $\Delta$ & $\Delta \Delta$ & $\Delta \Delta$ & $\Delta \Delta$ & $\Delta$ & $\Delta$ &  \\
\hline Vouvelle-Zélande & 529 & (2. & V & 0 & & 0 & 0 & 0 & 0 & O & $\Delta$ & $\Delta$ & $\Delta$ & $\Delta$ & $\Delta$ & $\Delta$ & $\boldsymbol{\Delta}$ & $\boldsymbol{\Delta}$ & $\Delta$ & $\Delta \Delta$ & $\Delta$ & $\Delta$ & $\boldsymbol{\Delta}$ & $\Delta \boldsymbol{\Delta}$ & $\Delta \Delta$ & $\Delta \Delta$ & $\Delta$ & $\boldsymbol{\Delta}$ & $\boldsymbol{\Delta}$ \\
\hline Australie & 528 & (3. & $V$ & 0 & O & & 0 & 0 & O & o & 0 & $\Delta$ & $\Delta$ & $\boldsymbol{\Delta}$ & $\boldsymbol{\Delta}$ & $\Delta$ & $\mathrm{O}$ & $\boldsymbol{\Delta}$ & $\Delta$ & $\Delta \Delta$ & $\Delta$ & $\boldsymbol{\Delta}$ & $\boldsymbol{\Delta}$ & $\Delta \boldsymbol{\Delta}$ & $\Delta \boldsymbol{\Delta}$ & $\Delta \boldsymbol{\Delta}$ & $\Delta$ & $\boldsymbol{\Delta}$ & $\boldsymbol{\Delta}$ \\
\hline lande & 527 & (3.) & $\nabla$ & 0 & 0 & 0 & & 0 & 0 & o & 0 & $\Delta$ & $\Delta$ & $\boldsymbol{\Delta}$ & $\boldsymbol{\Delta}$ & $\boldsymbol{\Delta}$ & 0 & $\boldsymbol{\Delta}$ & $\Delta$ & $\Delta \Delta$ & $\Delta$ & $\Delta$ & $\boldsymbol{\Delta}$ & $\Delta \Delta$ & $\Delta \Delta$ & $\Delta \boldsymbol{\Delta}$ & $\Delta$ & $\boldsymbol{\Delta}$ & $\boldsymbol{\Delta}$ \\
\hline Corée & 525 & . & $\nabla$ & $\nabla$ & 0 & 0 & 0 & & 0 & 0 & 0 & $\Delta$ & $\Delta$ & $\boldsymbol{\Delta}$ & $\mathbf{\Delta}$ & $\boldsymbol{\Delta}$ & 0 & $\boldsymbol{\Delta}$ & $\Delta$ & $\boldsymbol{\Delta} \Delta$ & $\Delta$ & $\boldsymbol{\Delta}$ & $\boldsymbol{\Delta}$ & $\Delta \mathbf{\Delta}$ & $\Delta \Delta$ & $\boldsymbol{\Delta} \Delta$ & $\Delta$ & $\boldsymbol{\Delta}$ & $\boldsymbol{\Delta}$ \\
\hline Royaume-Uni & 523 &. & $\nabla$ & $\nabla$ & O & 0 & 0 & 0 & & 0 & 0 & $\Delta$ & $\Delta$ & $\Delta$ & $\Delta$ & $\Delta$ & 0 & $\boldsymbol{\Delta}$ & $\Delta$ & $\Delta \Delta$ & $\Delta$ & $\boldsymbol{\Delta}$ & $\boldsymbol{\Delta}$ & $\Delta \Delta$ & $\Delta \Delta$ & $\Delta \Delta$ & $\Delta$ & $\boldsymbol{\Delta}$ & $\Delta$ \\
\hline pon & 522 & (5. & V & 0 & 0 & 0 & 0 & 0 & O & & 10 & 0 & 0 & 0 & 0 & O & 0 & $\boldsymbol{\Delta}$ & $\Delta$ & $\Delta \Delta$ & $\Delta$ & $\Delta$ & $\Delta$ & $\Delta \Delta$ & $\Delta \Delta$ & $\Delta \Delta$ & $\Delta$ & $\boldsymbol{\Delta}$ & $\boldsymbol{\Delta}$ \\
\hline Suède & 516 & 2. & $\nabla$ & $\nabla$ & $\nabla$ & 0 & 0 & 0 & 0 & O & & 0 & 0. & $\boldsymbol{\Delta}$ & O & $\boldsymbol{\Delta}$ & 0 & $\boldsymbol{\Delta}$ & $\Delta$ & $\Delta \Delta$ & $\Delta$ & $\Delta$ & $\boldsymbol{\Delta}$ & $\Delta \mathbf{A}$ & $\Delta \Delta$ & $\Delta \boldsymbol{\Delta}$ & $\Delta$ & $\boldsymbol{\Delta}$ & $\boldsymbol{\Delta}$ \\
\hline Autriche & 507 & (2. & $\nabla$ & $\nabla$ & $\nabla$ & $\nabla$ & $\nabla$ & $\nabla$ & $\nabla$ & 0 & 0 & & 0 & 0 & 0 & O & 0 & O & 0 & $\Delta \Delta$ & $\Delta$ & $\Delta$ & $\Delta$ & $\Delta \Delta$ & $\Delta \Delta$ & $\Delta \Delta$ & $\Delta$ & $\Delta$ & 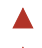 \\
\hline Belgique & 507 & (3.) & 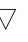 & $\nabla$ & $\nabla$ & $\nabla$ & $\nabla$ & $\nabla$ & $\nabla$ & $\mathrm{O}$ & 0 & 0 & L & 0 & 0 & O & 0 & 0 & 0 & $\Delta \Delta$ & $\Delta$ & $\Delta$ & $\boldsymbol{\Delta}$ & $\Delta \Delta$ & $\Delta \Delta$ & $\Delta \Delta$ & $\Delta$ & $\boldsymbol{\Delta}$ & $\boldsymbol{\Delta}$ \\
\hline Islande & 507 & . & $\nabla$ & $\nabla$ & $\nabla$ & $\nabla$ & $\nabla$ & $\nabla$ & $\nabla$ & $\mathrm{O}$ & $\nabla$ & 0 & 0 & & 0 & 0 & O & $\boldsymbol{\Delta}$ & 0 & $\Delta \Delta$ & $\Delta$ & $\boldsymbol{\Delta}$ & $\boldsymbol{\Delta}$ & $\Delta \mathbf{\Delta}$ & $\Delta \Delta$ & $\Delta \boldsymbol{\Delta}$ & $\Delta$ & $\boldsymbol{\Delta}$ & $\boldsymbol{\Delta}$ \\
\hline Vorvège & 505 & (2. & $\nabla$ & $\nabla$ & $\nabla$ & $\nabla$ & $\nabla$ & $\nabla$ & $\nabla$ & O & 0 & 0 & 0 & O & & 0 & 0 & O & 0 & $\Delta \Delta$ & $\Delta$ & $\Delta$ & $\Delta$ & $\Delta \Delta$ & $\Delta \Delta$ & $\Delta \Delta$ & $\Delta$ & $\boldsymbol{\Delta}$ & $\boldsymbol{A}$ \\
\hline France & 5 & (2. & 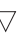 & $\nabla$ & $\nabla$ & $\nabla$ & $\nabla$ & $\nabla$ & V & & $\nabla$ & 0 & 0 & 0 & $O$ & & 0 & $\mathrm{O}$ & O & $\Delta \Delta$ & $\Delta$ & $\Delta$ & $\boldsymbol{\Delta}$ & $\Delta \boldsymbol{\Delta}$ & $\Delta \Delta$ & $\Delta \Delta$ & $\Delta$ & $\boldsymbol{\Delta}$ & $\boldsymbol{\Delta}$ \\
\hline États-Unis & 504 & 7. & $\nabla$ & $\nabla$ & $\nabla$ & O & 0 & 0 & 0 & 0 & 0 & 0 & 0 & 0 & 0 & O & & O & O & 00 & 0 & 0 & 0 & 00 & $\Delta \Delta$ & $\Delta \boldsymbol{\Delta}$ & $\Delta$ & $\Delta$ & $\boldsymbol{\Delta}$ \\
\hline anemark & 497 & (2. & $\nabla$ & $\nabla$ & $\nabla$ & $\nabla$ & $\nabla$ & $\nabla$ & $\nabla$ & $\nabla$ & $\nabla$ & 0 & 0 & $\nabla$ & O & 0 & $\mathrm{O}$ & & o & 00 & 0 & $\Delta$ & 0 & $\Delta \boldsymbol{\Delta}$ & $\Delta \Delta$ & $\Delta \Delta$ & $\Delta$ & $\boldsymbol{\Delta}$ & $\Delta$ \\
\hline Suisse & 494 & (4. & $\nabla$ & $\nabla$ & V & $V$ & $\nabla$ & $\nabla$ & $\nabla$ & $\nabla$ & $\nabla$ & 0 & 0 & 0 & 0 & O & 0 & $\mathrm{OL}$ & & 00 & 0 & 0 & 0 & 00 & $\Delta \boldsymbol{\Delta}$ & $\Delta \boldsymbol{\Delta}$ & $\Delta$ & $\boldsymbol{\Delta}$ & $\boldsymbol{\Delta}$ \\
\hline Espagne & 3 & (2. & $\nabla$ & $\nabla$ & $\nabla$ & $\nabla$ & $\nabla$ & $\nabla$ & $\nabla$ & $\nabla$ & $\nabla$ & $\nabla$ & $\nabla$ & $\nabla$ & $\nabla$ & $\nabla$ & 0 & 0 & 0 & 0 & 0 & 0 & 0 & 00 & $\Delta \Delta$ & $\Delta \Delta$ & $\Delta$ & $\boldsymbol{\Delta}$ & $\boldsymbol{\Delta}$ \\
\hline Rép. tchèque & 492 & . & $\nabla$ & $\nabla$ & $\nabla$ & $\nabla$ & $\nabla$ & $\nabla$ & $\nabla$ & $\nabla$ & $\nabla$ & $\nabla$ & $\nabla$ & $\nabla$ & $\nabla$ & $\nabla$ & 0 & O & $0^{2}$ & 0 & 0 & 0 & 0 & 001 & $\Delta \boldsymbol{\Delta}$ & $\Delta \Delta$ & $\Delta$ & $\boldsymbol{\Delta}$ & $\Delta$ \\
\hline alie & 487 & (2. & $\nabla$ & $\nabla$ & $\nabla$ & $\nabla$ & $\nabla$ & $\nabla$ & V & $\nabla$ & $\nabla$ & $\nabla$ & $\nabla$ & $\nabla$ & $\nabla$ & $\nabla$ & 0 & O & O & 00 & & 0 & O & 00 & $0 \Delta$ & $\Delta \boldsymbol{\Delta}$ & $\Delta$ & $\boldsymbol{\Delta}$ & $\boldsymbol{\Lambda}$ \\
\hline Allemagne & 484 & (2. & $\nabla$ & $\nabla$ & $\nabla$ & $\nabla$ & $\nabla$ & $\nabla$ & $\nabla$ & $\nabla$ & $\nabla$ & $\nabla$ & $\nabla$ & $\nabla$ & $\nabla$ & $\nabla$ & & $\nabla$ & O & 00 & 0 & & 0 & 00 & 001 & $\Delta \Delta$ & $\Delta$ & $\boldsymbol{\Delta}$ & $\boldsymbol{\Delta}$ \\
\hline Liechtenstein & 33 & (4. & $\nabla$ & $\nabla$ & $\nabla$ & $\nabla$ & $\nabla$ & $\nabla$ & $\nabla$ & $\nabla$ & $\nabla$ & $\nabla$ & $\nabla$ & $\nabla$ & $\nabla$ & $\nabla$ & 0 & O & 0 & 00 & 0 & 0 & & 00 & 001 & $\Delta \boldsymbol{\Delta}$ & $\Delta$ & $\boldsymbol{\Delta}$ & $\Delta$ \\
\hline Hongrie & 480 & (4. & $\nabla$ & $\nabla$ & $\nabla$ & $\nabla$ & $\nabla$ & $\nabla$ & $\nabla$ & $\nabla$ & $\nabla$ & $\nabla$ & $\nabla$ & $\nabla$ & $\nabla$ & $\nabla$ & ○ & $\nabla$ & O & 00 & 0 & 0 & 0 & 0 & 001 & $\Delta \boldsymbol{\Delta}$ & $\Delta \Delta$ & $\boldsymbol{\Delta}$ & $\Delta$ \\
\hline ologne & 479 & (4. & $V$ & $\nabla$ & $\nabla$ & $\nabla$ & $\nabla$ & $\nabla$ & $\nabla$ & $\nabla$ & $\nabla$ & $\nabla$ & $\nabla$ & $\nabla$ & $\nabla$ & $\nabla$ & 0 & $\nabla$ & 0 & 00 & 0 & 0 & 0 & $O L$ & 100 & 00 & $D$ & $\boldsymbol{\Delta}$ & $\boldsymbol{\Delta}$ \\
\hline Grèce & 4 & (5. & $\nabla$ & $\nabla$ & $\nabla$ & $\nabla$ & $\nabla$ & $\nabla$ & $\nabla$ & $\nabla$ & $\nabla$ & $\nabla$ & $\nabla$ & $\nabla$ & $\nabla$ & $\nabla$ & $\nabla$ & $\nabla$ & $\nabla$ & $\nabla \nabla$ & 70 & 0 & 0 & 00 & 10 & 00 & $\boldsymbol{\Delta}$ & $\Delta$ & $\Delta$ \\
\hline Portugal & 4 & & $\nabla$ & $\nabla$ & $\nabla$ & $\nabla$ & $\nabla$ & $\nabla$ & $\nabla$ & $\nabla$ & $\nabla$ & $\nabla$ & $\nabla$ & $\nabla$ & $\nabla$ & $\nabla$ & $\nabla$ & $\nabla$ & $\nabla$ & $\nabla \nabla$ & $7 \nabla$ & 0 & 0 & 00 & $O$ & 00 & $\Delta$ & $\Delta$ & $\boldsymbol{\Delta}$ \\
\hline Féd. de Russie & 462 & (4. & $\nabla$ & $\nabla$ & $\nabla$ & $\nabla$ & $\nabla$ & $\nabla$ & $\nabla$ & $\nabla$ & $\nabla$ & $\nabla$ & $\nabla$ & $\nabla$ & $\nabla$ & $\nabla$ & $\nabla$ & $\nabla$ & $\nabla$ & $\nabla \nabla$ & $7 \nabla$ & $\nabla$ & $\nabla$ & $\nabla \circ$ & $00 L$ & 0 & $\boldsymbol{\Delta}$ & $\boldsymbol{\Delta}$ & $\Delta$ \\
\hline Lettonie & 458 & (5. & V & $\nabla$ & $\nabla$ & $\checkmark$ & $\nabla$ & $\nabla$ & 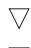 & $\nabla$ & $\nabla$ & $\nabla$ & $\nabla$ & $\nabla$ & $\nabla$ & $\nabla$ & $\nabla$ & $\nabla$ & $\nabla$ & $\nabla \nabla$ & $7 \nabla$ & $\nabla$ & $\nabla$ & $\nabla \circ$ & $00 c$ & 0 & 0 & $\boldsymbol{\Delta}$ & $\boldsymbol{\Delta}$ \\
\hline uxe & 441 & (1. & $V$ & $\nabla$ & V & V & $\nabla$ & $\nabla$ & V & V & $\nabla$ & $\nabla$ & $\nabla$ & $\nabla$ & $\nabla$ & $\nabla$ & $\nabla$ & 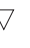 & $\nabla$ & $\nabla \nabla$ & $7 \nabla$ & $\nabla$ & $\nabla$ & $\nabla \nabla$ & $\nabla \nabla$ & $\nabla 0$ & & $\Delta$ & $\boldsymbol{A}$ \\
\hline Mexique & 422 & (3. & $\nabla$ & $\nabla$ & $V$ & & $\nabla$ & $\nabla$ & & $V$ & $\nabla$ & $\nabla$ & $\nabla$ & $\nabla$ & & $\nabla$ & $\nabla$ & $\nabla$ & $V$ & $\nabla \nabla$ & $7 \nabla$ & $\nabla$ & $\nabla$ & $\nabla \nabla$ & $\nabla \nabla$ & $\nabla \nabla$ & $\nabla \nabla$ & & $\Delta$ \\
\hline Brésil & 96 & (3. & $\nabla$ & $\nabla$ & $\nabla$ & & $\nabla$ & $\nabla$ & V & & $\nabla$ & $\nabla$ & $\nabla$ & $\nabla$ & & $\nabla$ & $\nabla$ & & $\nabla$ & & $\nabla$ & $\nabla$ & $\nabla$ & $\nabla \nabla$ & $\nabla \nabla$ & $\nabla \nabla$ & $\nabla \nabla$ & $\nabla$ & \\
\hline
\end{tabular}

Instructions:

Pour procéder à la comparaison, il suffit de choisir un pays en abscisse et de le comparer avec l'un des pays en ordonnée. Les symboles indiquent que statistiquement, la performance moyenne du pays en abscisse est significativement inférieure ou supérieure à celle du pays en ordonnée ou ne s'en écarte pas significativement.

\footnotetext{
A Performance moyenne significativement supérieure sur le plan statistique à celle du pays en ordonnée.

$\bigcirc$ Pas de différence statistiquement significative par rapport au pays en ordonnée.

$\nabla$ Performance moyenne significativement inférieure sur le plan statistique à celle du pays en ordonnée.
}

\section{Significativement supérieure sur le plan statistique à la moyenne de l'OCDE Pas d'écart statistiquement significatif par rapport à la moyenne de l'OCDE Significativement inférieure sur le plan statistique à la moyenne de l'OCDE} Source : Base de données PISA de l'OCDE, 2001, tableau 4.1. 


\section{Performance sur l'échelle combinée de compréhension de l'écrit}

\section{Scores moyens}

Une batterie de quelque 141 items de lecture a été utilisée pour recueillir, dans les pays participants, des informations sur les compétences des jeunes de 15 ans en compréhension de l'écrit, comme le définit le cadre d'évaluation de PISA. Les échelles de compétences en lecture décrites plus haut, basées sur le cadre d'évaluation, ont été développées en fonction des résultats obtenus par les élèves à ces items d'évaluation. Les scores obtenus par les élèves ont été transformés pour générer une échelle combinée numérique de compréhension de l'écrit qui se rapporte aux échelles décrites. L'échelle combinée de compréhension de l'écrit est construite en sorte qu'un score moyen vaille 500 et l'écart type, 100, tous pays de l'OCDE confondus.

Le graphique 4.1 montre le score moyen et l'écart type de l'échelle combinée de compréhension de l'écrit qui ont été calculés pour chaque pays. ${ }^{1}$ Elle compare également les performances des pays et indique si la performance d'un pays donné est équivalente ou significativement supérieure ou inférieure à celles de chacun des autres pays participants. ${ }^{2}$

\section{Encadré 4.1 : Les différences de scores sont-elles significatives?}

Lors de la comparaison des scores moyens, il y a lieu de garder présent à l'esprit le fait que les écarts de score ne sont pas tous statistiquement significatifs. Chaque mesure comprend une certaine marge d'erreur dont l'importance dépend de facteurs tels que la taille de l'échantillon, la méthodologie d'échantillonnage et les instruments d'évaluation utilisés. En raison de cette marge d'erreur, les différences entre les scores moyens des divers pays participants ne correspondent pas forcément aux différences de compétence entre les populations étudiées. Un test statistique est appliqué à la différence entre les moyennes des divers pays. Si la probabilité qu'une différence soit due au hasard est inférieure à cinq pour cent, cette différence est considérée comme significative.

Les scores moyens des pays sur l'échelle combinée de compréhension de l'écrit sont compris entre 396 (Brésil) et 546 (Finlande). Cet écart représente l'équivalent d'un peu plus de deux niveaux de compétence sur cette échelle qui en compte cinq : en Finlande, l'élève moyen se situe dans la partie supérieure du niveau 3, tandis qu'au Brésil, l'élève moyen se situe dans la partie supérieure du niveau $1 .^{3}$

Il ressort de l'analyse des moyennes nationales que 22 pays se situent au niveau 3, huit au niveau 2 et un au niveau 1. La moyenne de 5 pays (le Danemark, les États-Unis, la France, la Norvège et la Suisse) ne s'écarte pas significativement de la moyenne de l'OCDE. En revanche, celle de 14 pays (l'Allemagne, le Brésil, l’Espagne, la Fédération de Russie, la Grèce, la Hongrie, l’Italie, la Lettonie, le Liechtenstein, le Luxembourg, le Mexique, la Pologne, le Portugal et la République tchèque) est significativement inférieure à la moyenne de l'OCDE, tandis que celle de 12 pays (l'Australie, l'Autriche, la Belgique, le Canada, la Corée, la Finlande, l’Irlande, l’Islande, le Japon, la Nouvelle-Zélande, le Royaume-Uni et la Suède) lui est significativement supérieure. 
Pour avoir une idée plus précise du niveau global de compétence de chaque pays, il est utile également de connaître la position de la proportion la plus importante d'élèves de chaque pays sur l'échelle de compétence. Dans le cas de 23 pays, cette proportion se situe au niveau 3, ainsi que le montre le graphique 4.2.

En Belgique, en Finlande et en Nouvelle-Zélande, le niveau le plus souvent atteint est le niveau 4 ; en Lettonie, au Luxembourg, au Mexique et dans la Fédération de Russie, il s'agit du niveau 2 et, au Brésil, du niveau 1.

\section{Graphique 4.2}

Niveau de compétence sur l'échelle combinée de compréhension de l'écrit comptant la proportion la plus élevée d'élèves dans chaque pays

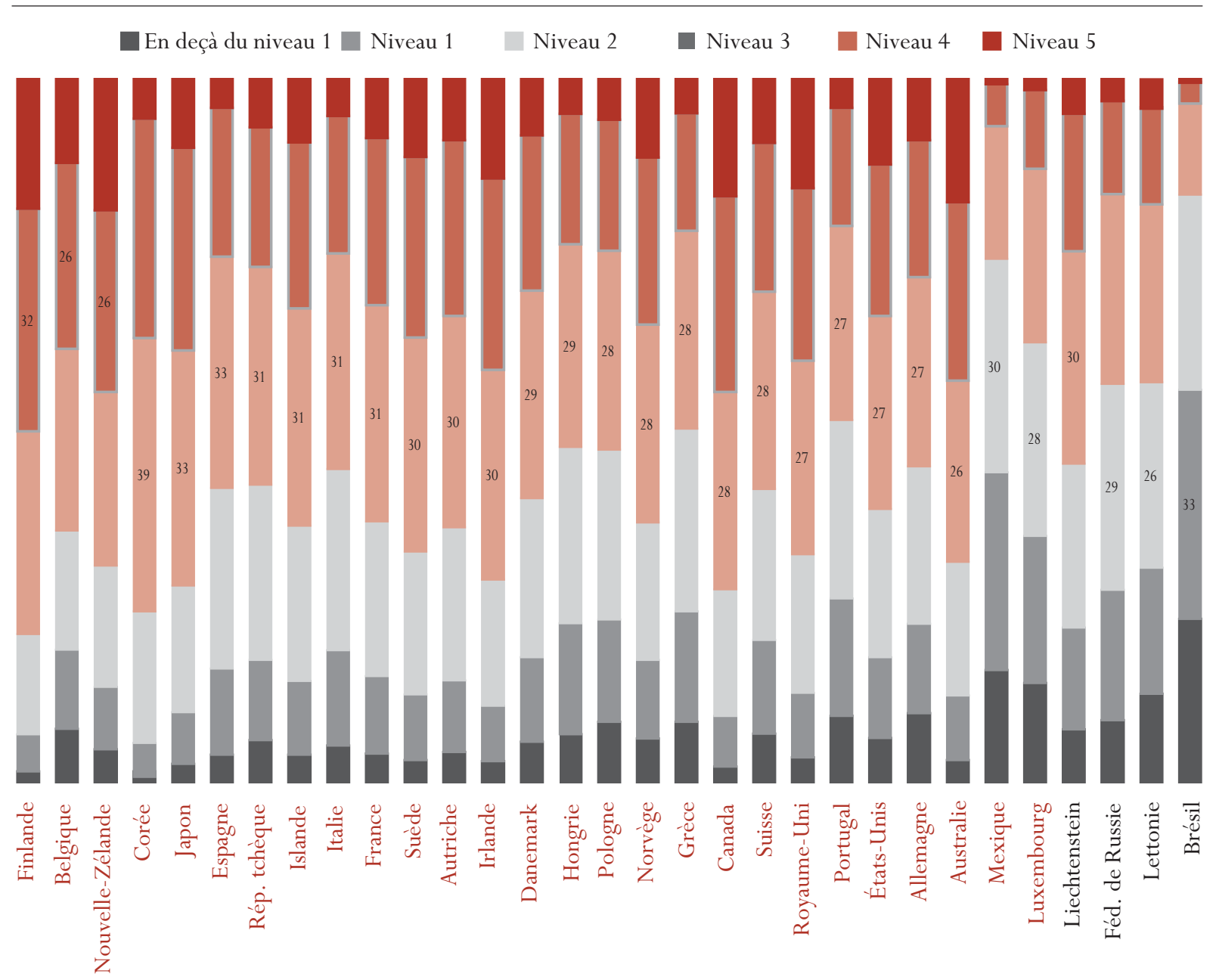

Source: Base de données PISA de l'OCDE, 2001, tableau 4.2. 


\section{Encadré 4.2 : Moyennes et répartition des scores}

Les scores moyens donnent une idée de la performance globale de chaque pays mais ne nous apprennent rien sur la variation et la répartition des niveaux de compétence au sein des pays. Or des pays affichant des moyennes similaires peuvent présenter des profils de compétence fort différents.

À titre d'exemple, étudions le graphique 4.3 qui présente la performance de trois pays hypothétiques sur l'échelle combinée de compréhension de l'écrit.

Le pays A affiche un score moyen nettement supérieur à la moyenne de l'OCDE et les résultats des élèves se concentrent sur une section limitée de l'échelle combinée de compréhension de l'écrit. Le score moyen du pays B est inférieur à celui du pays A mais reste supérieur à la moyenne de l'OCDE, avec des résultats se situant sur une section limitée de l'échelle de compréhension de l'écrit. Enfin, le score moyen du pays $\mathrm{C}$ est équivalent à celui du pays $\mathrm{B}$ mais les résultats des élèves y sont plus dispersés. Cela signifie qu'il y a davantage d'élèves «forts » à l'extrémité supérieure et davantage d'élèves faibles à l'extrémité inférieure de l'échelle.

\section{Graphique 4.3}

Performance de trois pays fictifs

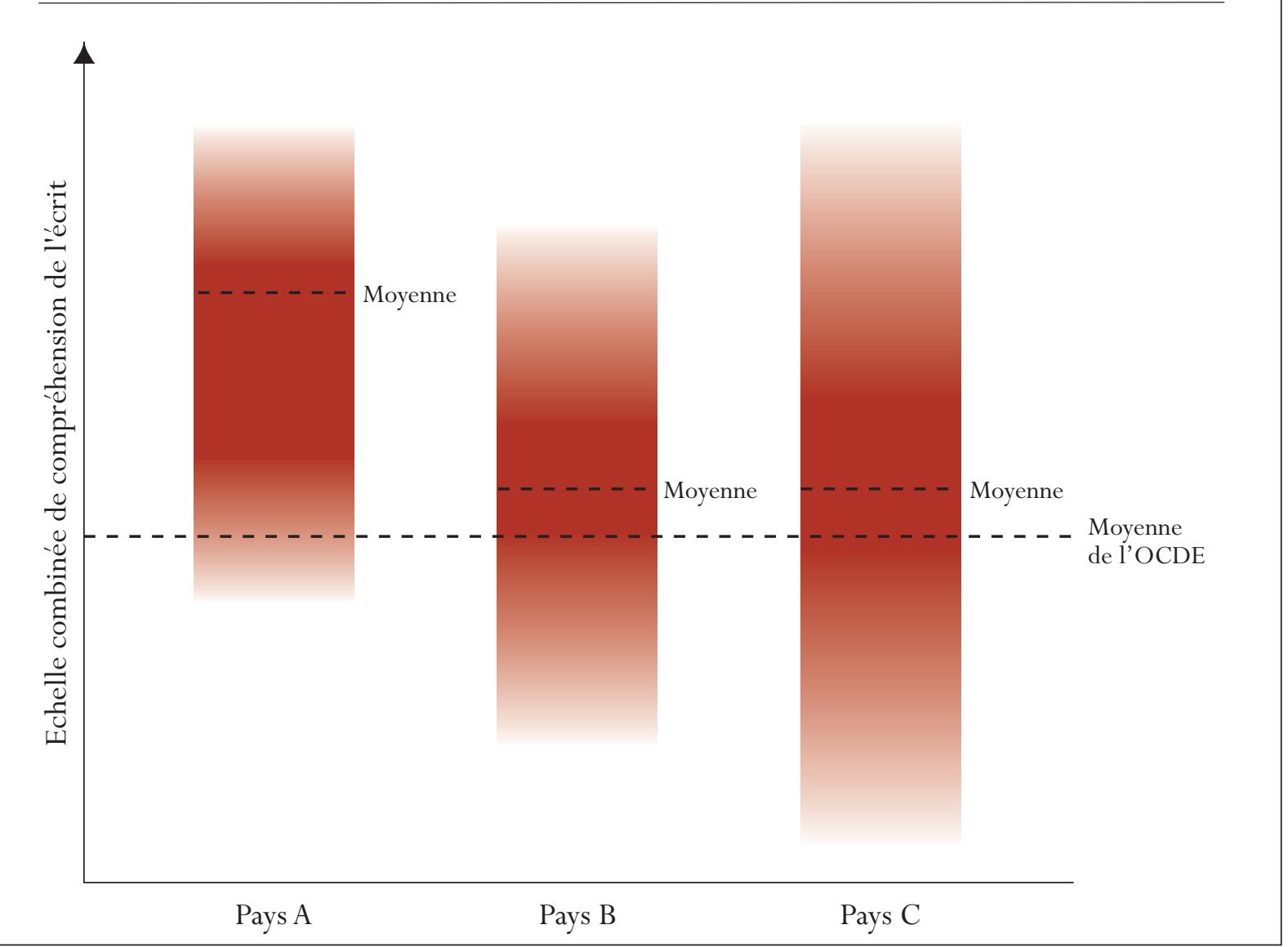


Les résultats du pays A sont assez incontestablement les plus enviables. En effet, dans ce pays, les scores des élèves sont relativement bons en moyenne, ceux des élèves les plus «forts » sont excellents et ceux des élèves les plus «faibles » s'écartent à peine de la moyenne internationale. On pourrait expliquer ces bons résultats par l'homogénéité de la population ou l'efficacité du système éducatif à réduire les inégalités. Quelle qu'en soit la raison, la combinaison d'un score moyen élevé et d'une concentration des scores sur l'échelle de compréhension de l'écrit indique que la plupart des élèves possèdent les compétences requises pour non seulement s'insérer dans la société et lui apporter leur contribution, mais aussi pour développer leur potentiel.

Passons maintenant aux deux autres pays. Dans lequel des deux la répartition des résultats est-elle la plus enviable ? Dans le pays B, même les élèves les moins performants se situent à des niveaux de compétence acceptables, ce qui indique que pratiquement tous les élèves sont capables de satisfaire aux exigences normales de lecture imposées aux adultes dans la vie courante, notamment se tenir au courant de l'actualité en lisant le journal, poser sa candidature pour un emploi, comprendre les déclarations politiques d'un candidat aux élections locales ou encore lire un roman pour le plaisir. Mais les résultats de ce pays montrent aussi que les élèves les plus compétents n'arrivent pas au même niveau que les élèves les plus compétents de par le monde. Ce constat suggère que le pays B manque probablement d'un nombre important de jeunes adultes à même de rivaliser avec l'élite internationale, une lacune qui pourrait désavantager toute la nation; ou encore, que les élèves les plus brillants n'ont pas la possibilité de tirer pleinement parti de leur potentiel, alors que des ressources relativement importantes sont consacrées aux élèves les moins compétents.

Dans le pays $\mathrm{C}$, les élèves les plus brillants sont au moins aussi compétents que ceux des deux autres pays, et sont donc susceptibles de gérer correctement leur pays dans un contexte international. En revanche, il n'est guère probable que les élèves les moins compétents de ce pays puissent satisfaire aux exigences de lecture de la vie adulte. Ce phénomène n'a probablement pas d'impact économique au sens strict du terme (il existe aujourd'hui encore des métiers qui demandent peu ou pas de compétence en lecture, sinon aucune). Certains iront même jusqu'à prétendre que prodiguer des niveaux élevés de formation à chacun contribue à provoquer une pénurie de travailleurs peu qualifiés. Mais les personnes ne sont pas uniquement des unités économiques : elles ont une famille, vivent dans une communauté et élisent leurs représentants au gouvernement. Une proportion élevée d'adultes incapables d'évoluer dans un contexte familial, culturel et politique n'est pas souhaitable. Dans une démocratie moderne, il faut que chacun puisse satisfaire aux exigences de lecture qu'imposent la vie familiale (lire un article à propos des soins à donner à un nouveau-né, par exemple), la vie sociale (lire un avis annonçant une séance publique d'information à propos du développement d'un centre commercial, par exemple) et la vie politique.

Des performances moyennes similaires dans deux pays différents peuvent de toute évidence masquer des répartitions très différentes de compétence. Quelle que soit la stratégie appliquée par les pays pour optimiser l'organisation et le financement de leur système d'éducation, c'est une question de justice sociale que de veiller à ce que les systèmes d'éducation visent à former tous les élèves pour leur permettre de tirer pleinement parti de leur potentiel. 


\section{Graphique 4.4}

Répartition des performances des élèves sur l'échelle combinée de compréhension de l'écrit



Source: Base de données PISA de l'OCDE, 2001, tableau 4.16. 


\section{Répartition des scores : dispersion des scores pour différentes populations}

Le graphique 4.4 montre la variation de la performance des élèves sur l'échelle combinée de compréhension de l'écrit. La dispersion des scores par rapport à la moyenne est exprimée par l'écart type. Pour interpréter l'écart type de chaque pays, il y a lieu de garder présent à l'esprit le fait que dans une répartition normale, 95 pour cent des performances se situent dans un intervalle égal à deux écarts types par rapport à la moyenne et 68 pour cent des performances, dans une plage égale à un écart type. Sur l'échelle de compréhension de l'écrit de PISA, un écart type correspond à 100.

C'est la Finlande qui présente la répartition de scores la plus proche de celle du pays A représenté dans le graphique 4.3 ci-dessus. La Finlande allie à une dispersion relativement limitée des scores une performance moyenne (546) nettement supérieure à celle de tout autre pays. Son écart type est de 89. Seuls quatre pays de l'OCDE affichent des écarts types plus faibles.

La Corée et la Nouvelle-Zélande ressemblent respectivement aux pays B et $\mathrm{C}$ du graphique 4.3. Ces deux pays affichent des moyennes supérieures à celle de l'OCDE et qui ne sont pas statistiquement différentes l'une de l'autre. En revanche, c'est en Corée que l'on observe la répartition la plus compacte de scores par rapport à tous les autres pays, avec un écart type de 70, alors que la Nouvelle Zélande obtient la deuxième plus grande dispersion de scores.

Le Brésil et le Mexique figurent également au nombre des pays qui affichent des dispersions relativement limitées des scores : tous deux ont un écart type de 86 . Il y a lieu de souligner toutefois que la faiblesse du taux de scolarisation peut expliquer en partie ce résultat. Ainsi, au Brésil, seulement 53 pour cent des jeunes de 15 ans fréquentent les classes du grade 7 au grade 10 et sont donc concernés par l'évaluation PISA. En conséquence, il est probable que les écarts types relativement réduits de ce pays s'expliquent par la définition de la population cible de PISA, puisqu'une une grande part de la population susceptible d'obtenir les moins bons résultats a été exclue de l'échantillon. Pour plus de détails sur la couverture de la population et les taux d'exclusion, voir le Rapport technique de PISA 2000 (OCDE, 2002b).

Le contraste entre les écarts types de l'Italie et de l'Allemagne est intéressant. En effet, les scores moyens de ces deux pays ne sont pas sensiblement différents (487 pour le premier et 484 pour le second), mais il n'en va pas de même pour leur écart type. L'Italie est le septième pays affichant l'écart type le plus faible (91), tandis que l'Allemagne accuse l'écart type le plus important (111). Ce contraste nous rappelle que, si les moyennes sont utiles pour résumer la performance globale des pays, elles ne rendent pas compte des différences dans la répartition des scores.

Nous pouvons aussi donner une idée de la répartition des scores en comparant les pourcentages d'élèves situés à chaque niveau de compétence. Le graphique 4.5 présente les pourcentages d'élèves situés à chacun des niveaux 1 à 5 et en deçà du niveau 1 sur l'échelle combinée de compréhension de l'écrit.

Les profils qui ressortent de la comparaison de la répartition des élèves de chaque pays entre les niveaux de compétence de PISA sont, sans surprise, similaires à ceux révélés par la comparaison des scores moyens et des écarts types de chacun d'entre eux. En Finlande, environ 60 pour cent des élèves atteignent les niveaux 3 et 4 et seuls 7 pour cent d'entre eux se situent en deçà du niveau 2. L'écart type de 108 enregistré en Nouvelle-Zélande se reflète dans la proportion plus faible d'élèves situés aux niveaux 3 et 4 (environ 50 pour cent) et dans la proportion plus élevée d'élèves au bas de l'échelle : 14 pour cent des élèves n'atteignent pas le niveau 2. Dans ces deux pays cependant, 19 pour cent des élèves parviennent au niveau 5. 
La Corée allie une performance moyenne élevée à un écart type limité : la majorité des élèves coréens -70 pour cent - se concentrent aux niveaux 3 et 4 mais ils ne sont que 6 pour cent à atteindre le niveau 5 ou à se situer en deçà du niveau 2. Cette proportion d'élèves en deçà du niveau 2 est la plus faible de tous les pays participants.

Au Japon, la répartition des scores révèle la concentration des élèves aux niveaux 3 et 4 (62 pour cent), même si elle est moins marquée qu'en Corée. Bien que le score moyen du Japon soit inférieur à celui de la Corée, la proportion d'élèves japonais situés au niveau 5 est supérieure (10 pour cent).

\section{Graphique 4.5}

Pourcentage d'élèves situés à chaque niveau de compétence sur l'échelle combinée de compréhension de l'écrit



Source: Base de données PISA de l'OCDE, 2001, tableau 4.2. 
L'écart type de l'Espagne (85) reflète une dispersion des scores similaire à celle du Japon, mais avec un niveau global de performance significativement plus bas. Dans ce pays, 33 pour cent des élèves se concentrent au niveau 3. Seuls la Corée et le Japon comptent plus d'élèves à ce niveau. Par ailleurs, le second groupe le plus important d'élèves espagnols (26 pour cent) se situe au niveau 2, tandis qu'en Corée et au Japon, le deuxième plus grande concentration d'élèves se situe au niveau 4.

\section{Répartition des scores : Proportions d'élèves aux extrémités de l'échelle}

L'analyse des proportions d'élèves de chaque pays qui peuvent être considérés comme très peu ou extrêmement compétents en compréhension de l'écrit révèle d'importantes disparités dans la distribution des scores. Le graphique 4.6 indique pour chaque pays le pourcentage des élèves situés au niveau 5 et en deçà du niveau 2 de l'échelle de compétence. La proportion d'élèves au niveau 5 varie de 1 à 19 pour cent selon le pays. La proportion moyenne d'élèves à ce niveau est de 9 pour cent pour les pays de l'OCDE. En Australie, au Canada, en Finlande, en Nouvelle-Zélande et au Royaume-Uni, cette proportion représente

Graphique 4.6

Pourcentage d'élèves situés au niveau 5 et en deçà du niveau 2 sur l'échelle combinée de compréhension de l'écrit

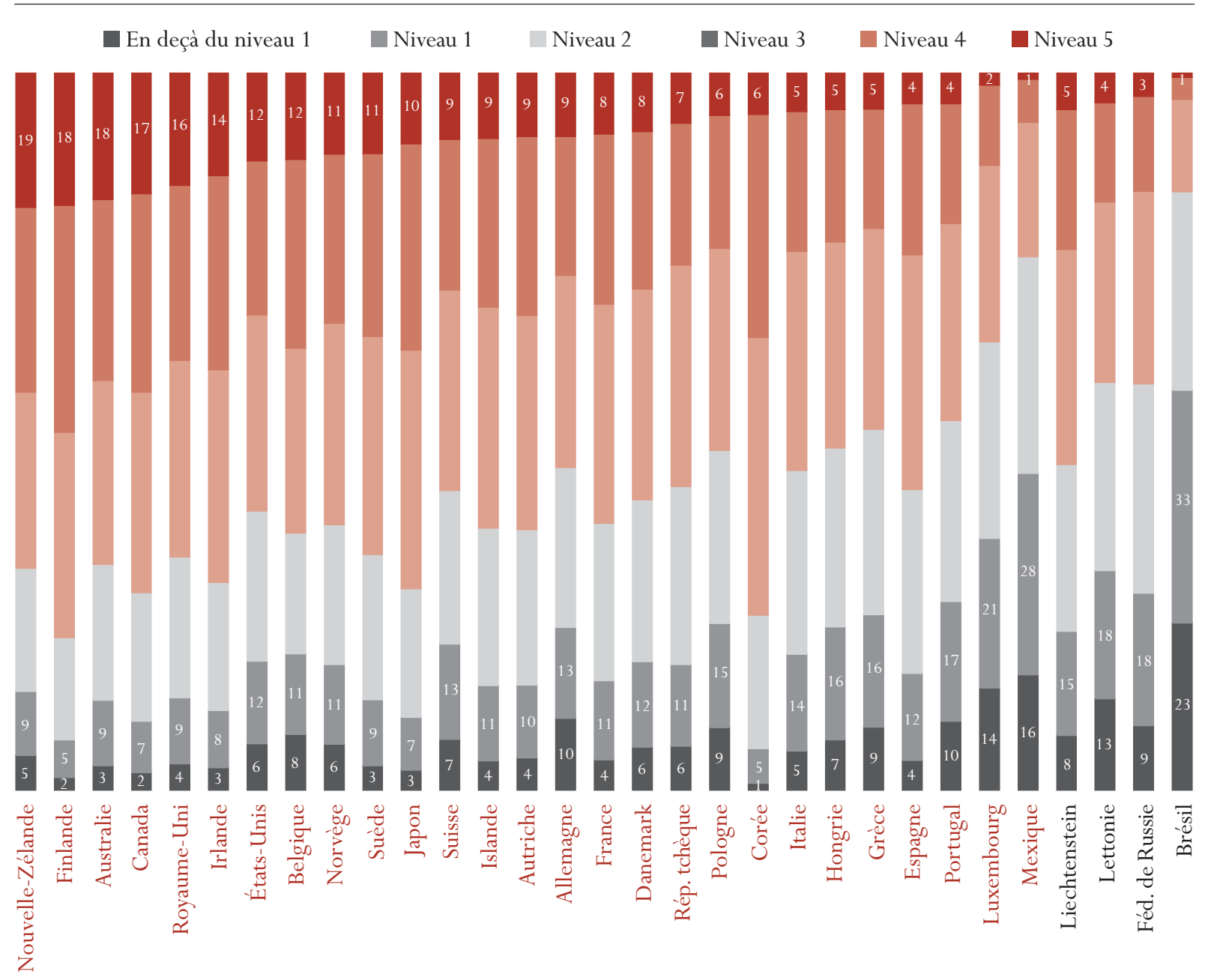

Source: Base de données PISA de l'OCDE, 2001, tableau 4.2. 
au moins 16 pour cent. Comme nous l'avons dit, ils ne sont que 6 pour cent au niveau 5 en Corée, un pays dont le score moyen n'est pourtant pas significativement différent de celui de l'Australie, de la NouvelleZélande et du Royaume-Uni.

Les pays où les proportions d'élèves situés au niveau 5 sont similaires se distinguent souvent par des proportions d'élèves différentes aux autres niveaux et, donc, par des scores moyens différents. À titre d'exemple, comparons la Pologne et la Corée. Dans ces deux pays, 6 pour cent des élèves se situent au niveau 5, mais seuls 47 pour cent des élèves se concentrent aux niveaux 3 et 4 en Pologne alors qu'ils sont 70 pour cent à ces niveaux en Corée. La Corée obtient dès lors un score moyen supérieur de 46 à celui de la Pologne. Quatorze pour cent des élèves testés se situent en deçà du niveau 2 en Nouvelle-Zélande contre 7 pour cent seulement en Finlande, alors que dans ces deux pays, 19 pour cent des élèves parviennent au niveau 5. En Allemagne et en Autriche, la proportion d'élèves situés au niveau 5 ( 8,8 pour cent dans chacun de ces deux pays) est proche de la moyenne de l'OCDE ( 9,5 pour cent). Mais en Allemagne, 23 pour cent d'élèves ne parviennent pas au niveau 2, une proportion qui est nettement supérieure à la moyenne de l'OCDE (18 pour cent) et qui contraste fortement avec celle de l'Autriche (15 pour cent). En fait, l'Allemagne présente le cinquième pourcentage le plus élevé d'élèves situés en deçà du niveau 1.

Chaque pays compte un certain nombre d'élèves dont les compétences sont inférieures à celles associées au niveau le plus élémentaire de l'échelle de compréhension de l'écrit. La proportion d'élèves appartenant à cette catégorie varie selon les pays participants : de 1 pour cent (en Corée) à 23 pour cent (au Brésil). De tous les pays de l'OCDE, c'est le Mexique qui accuse la plus forte proportion d'élèves en deçà du niveau 1 (16 pour cent). Dans 18 pays, plus de 5 pour cent des élèves ne parviennent pas au niveau 1. Les élèves dont les scores sont en deçà du niveau 1 sont incapables de mener à bien les tâches de lecture les plus élémentaires que PISA cherche à mesurer. Cela ne signifie pas qu'ils n'ont aucune compétence en lecture. Il est même probable que la plupart de ces élèves sachent lire au sens technique du terme. Toutefois, ils rencontrent de sérieuses difficultés lorsqu'il s'agit d'utiliser la compréhension de l'écrit comme un moyen efficace pour étendre et approfondir leurs connaissances et savoir-faire dans d'autres domaines. En conséquence, ils risquent non seulement de passer plus difficilement de leurs études au monde du travail, mais aussi de ne pouvoir accéder aux études supérieures ou bénéficier d'occasions de s'instruire tout au long de leur vie.

Il y a lieu de souligner que les proportions relatives d'élèves situés aux niveaux les plus bas de l'échelle de compétence ont pu être influencées dans une certaine mesure par des décisions différentes prises par les pays concernant l'identification des groupes d'élèves à exclure de l'évaluation. Les pays ont été autorisés à exclure de l'évaluation les élèves appartenant à un certain nombre de catégories, notamment les élèves dont les retards mentaux, les troubles émotionnels ou les handicaps physiques les rendraient trop peu performants face aux tests PISA, ainsi que les élèves allophones ayant suivi moins d'une année d'études dans la langue de l'évaluation. Tous les pays, sauf trois, ont atteint la couverture requise de 95 pour cent de la population cible souhaitée, et la moitié des pays ont couvert 98 pour cent ou plus de cette population. Le taux d'exclusion limité à un maximum de 5 pour cent de la population cible garantit que le biais potentiel résultant des exclusions ne dépasse pas une erreur type d'échantillonnage. Il est probable que les élèves susceptibles d'être exclus ne possèdent que de faibles compétences en compréhension de l'écrit. Les taux globaux d'exclusion pour PISA 2000 sont présentés dans le Rapport technique de PISA 2000 (OCDE, 2002b). 


\section{Performances en compréhension de l'écrit sur les sous-échelles d'aspect}

Après avoir examiné les performances sur l'échelle combinée de compréhension de l'écrit, nous allons maintenant passer à une analyse plus détaillée de la performance en lecture. Dans PISA, la compréhension de l'écrit est modélisée sous la forme d'un système complexe de relations entre perspectives, stratégies et approches, désignés par le terme «aspect »: trouver l'information, développer une interprétation, réfléchir et évaluer. Comme son nom l'indique, l'aspect Trouver l'information demande aux élèves d'identifier une ou plusieurs données ou idées distinctes figurant dans le texte. L'aspect Développer une interprétation les invite à montrer qu'ils sont capables de faire des inférences et de relier des passages du texte. Enfin, l'aspect Réfléchir et évaluer leur impose d'établir un lien entre le texte et leur propre conception du monde. Ces trois aspects sont essentiels pour satisfaire aux exigences de lecture du monde moderne et peuvent tous trois être associés à différents degrés de complexité et de difficulté.

Pour l'ensemble des pays participants, les corrélations entre les aspects sont généralement importantes mais elles ne sont pas uniformes. Les corrélations les plus fortes sont relevées entre la localisation d'informations et l'interprétation de textes, le coefficient médian s'établissant à 0,95 . Pour les pays considérés séparément, la corrélation la plus faible enregistrée entre ces deux aspects est de 0,90 et la plus élevée, de 0,96. Les coefficients de corrélation entre la localisation d'informations et la réflexion et l'évaluation sont généralement plus faibles que les précédents : ils sont compris entre 0,74 et 0,92 , avec une valeur médiane de 0,88. Entre l'interprétation de textes et la réflexion et l'évaluation, les coefficients de corrélation se situent entre 0,80 et 0,95, avec une médiane de 0,924. Malgré l'importance de ces corrélations et la similitude générale entre les résultats sur les sous-échelles d'aspect et ceux sur l'échelle combinée de compréhension de l'écrit, les profils de performance de certains pays sur ces trois sous-échelles présentent des différences intéressantes. Ces interactions peuvent traduire des différences dans les pratiques pédagogiques privilégiées par les pays ou encore suggérer aux décideurs d'envisager certains types d'améliorations. Ainsi, dans un pays donné, l'enregistrement d'une performance élevée sur la sous-échelle Trouver l'information mais faible sur la sous-échelle Réfléchir et évaluer peut indiquer que les futurs citoyens de ce pays sont à l'aise quand il s'agit d'informations purement factuelles mais le sont nettement moins quand il s'agit de poser des jugements critiques sur ce qu'ils ont lu. Les décideurs avisés de l'émergence de ce profil de performance dans leur pays peuvent envisager de placer davantage l'accent sur les compétences d'évaluation critique dans leur système d'éducation. À l'inverse, les pays qui affichent une performance relativement élevée sur la sous-échelle Réfléchir et évaluer mais faible sur la sous-échelle Trouver l'information peuvent envisager la possibilité de concentrer les efforts pédagogiques sur l'amélioration de la précision lors de la lecture et des capacités de recherche d'information. Nous allons à présent rendre compte des scores moyens et de la répartition des performances aspect par aspect dans chaque pays. Nous poursuivrons par l'analyse des écarts de performance relevés entre les aspects dans certains pays.

\section{Scores moyens}

Le tableau 4.3 présente le score moyen et l'écart type des pays pour chaque aspect et précise les erreurs types correspondantes.

Le score moyen de l'OCDE sur la sous-échelle Trouver l'information s'établit à 498. Les scores sont significativement supérieurs à la moyenne de l'OCDE dans 12 pays et significativement inférieurs dans 13 pays. Ils ne s'en écartent pas de manière significative dans six pays. Le score moyen des pays participants est compris entre 556 (en Finlande) et 365 (au Brésil). De tous les pays membres de l'OCDE, c'est le Mexique qui accuse le score moyen le plus faible (402). C'est pour cet aspect que l'intervalle de scores moyens, représentant l'équivalent de près de trois niveaux de compétence, est le plus étendu. Le tableau 4.4 compare la 
performance des pays sur la sous-échelle Trouver l'information et indique si la performance d'un pays donné est équivalente, significativement supérieure ou inférieure à celle de chacun des autres pays participants.

Sur la sous-échelle « Développer une interprétation », les scores moyens sont compris entre 555 (en Finlande) et 400 (au Brésil). Parmi les pays membres de l'OCDE, c'est le Mexique qui affiche le score moyen le plus faible (419). Douze pays présentent un score moyen significativement supérieur à la moyenne de l'OCDE (qui est de 501), 14 pays, un score significativement inférieur et cinq pays, un score qui ne s'en écarte pas significativement. L'intervalle des scores moyens représente l'équivalent d'un peu plus de deux niveaux de compétence. Le tableau 4.5 compare la performance des pays sur l'échelle Développer une interprétation et indique si la performance d'un pays donné est équivalente, significativement supérieure ou inférieure à celle de chacun des autres pays participants.

Sur la sous-échelle Réfléchir et évaluer, le score moyen le plus élevé est celui du Canada (542) et le plus faible, celui du Brésil (417). Parmi les pays membres de l'OCDE, c'est le Luxembourg qui obtient le score moyen le plus faible (442). L'intervalle des scores moyens représente l'équivalent d'un peu moins de deux niveaux de compétence et c'est pour cet aspect qu'il est le moins étendu. Dix pays ont un score nettement supérieur à la moyenne de l'OCDE, qui se situe à 502 . C'est un chiffre inférieur à celui enregistré pour les deux autres aspects. Le tableau 4.6 compare les résultats des pays sur l'échelle Réfléchir et évaluer, et indique si la performance d'un pays donné est équivalente, significativement supérieure ou inférieure à celle de chacun des autres pays participants.

Pour chacun des aspects, les scores moyens de 21 ou 22 pays se situent au niveau 3, et ceux de 7 à 9 pays au niveau 2. Seule la Finlande affiche des scores moyens qui atteignent de justesse le niveau 4 sur les échelles Trouver l'information et Développer une interprétation. Les scores moyens du Brésil et du Mexique se situent au niveau 1 sur l'échelle Trouver l'information. Le score du Brésil correspond également au niveau 1 sur l'échelle Développer une interprétation.

Répartition des compétences en compréhension de l'écrit au sein des pays sur les trois sous-échelles d'aspect

Trouver l'information

L'écart type moyen de l'OCDE sur l'échelle Trouver l'information s'établit à 111 , ainsi que le montre le tableau 4.3. La valeur de cet écart indique que la dispersion des scores est plus marquée dans cet aspect que dans les deux autres (100 sur la sous échelle Développer une interprétation et 106 sur celle Réfléchir et évaluer). Les écarts types des pays considérés isolément sont compris entre 82 (pour la Corée) et 120 (pour la Belgique). La dispersion des scores enregistrée en Belgique (120) contraste avec celle de l'Autriche (96), alors que les scores moyens de ces deux pays ne sont pas statistiquement différents. En comparaison avec l'Autriche, la Belgique compte davantage d'élèves situés en deçà du niveau 1 et au niveau 5, et moins d'élèves aux niveaux 2 et 3. La variance importante observée en Belgique peut être liée aux différences entre ses deux principales communautés linguistiques (la Communauté flamande et la Communauté française).

Le tableau 4.7 présente pour chaque pays le pourcentage de jeunes de 15 ans qui se situent à chaque niveau de compétence PISA sur la sous-échelle Trouver l'information.

Dans 22 des 31 pays, c'est le niveau 3 qui rassemble le plus d'élèves sur l'échelle Trouver l'information. Cette proportion est certes importante, mais elle est légèrement inférieure à celle enregistrée sur les deux autres sous-échelles d'aspect. 
De nombreux pays affichent de fortes proportions d'élèves situés au bas de la sous-échelle Trouver l'information. Ainsi, dans 16 pays, il y a au moins $16 \%$ d'élèves situés en deçà du niveau 2, et dans 7 autres pays, ce nombre s'élève à plus de $30 \%$ (respectivement 68 et 52 pour cent).

Certains des pays qui présentent des proportions similaires d'élèves en deçà du niveau 2 se différencient fortement par leurs proportions aux niveaux supérieurs de l'échelle. Ainsi, 8 pour cent des élèves n'atteignent pas le niveau 2 en Corée et en Finlande mais 54 pour cent se situent aux niveaux 4 et 5 en Finlande, contre 41 pour cent seulement en Corée. De même, tant en Belgique qu'au Danemark, 19 pour cent des élèves ne parviennent pas au niveau 2 sur l'échelle Trouver l'information alors qu'ils sont respectivement 43 et 32 pour cent à se situer aux niveaux 4 et 5 .

\section{Développer une interprétation}

Sur la sous-échelle Développer une interprétation comme sur celles des deux autres aspects, la proportion la plus importante d'élèves se situe au niveau 3 dans la plupart des pays ( 23 pays). C'est pour l'aspect Interprétation que le nombre de pays présentant au moins 20 pour cent d'élèves en deçà du niveau 2 est le plus faible : ils sont 11 pays à figurer dans cette catégorie, alors qu'ils sont respectivement 16 et 15 pays sur l'échelle Trouver l'information et sur l'échelle Réfléchir et évaluer.

Dans plusieurs pays, notamment en Allemagne, en Belgique et en Nouvelle-Zélande, une proportion modérée à élevée d'élèves incapables de dépasser le niveau 1 est associée à une proportion considérable d'élèves situés à l'extrémité supérieure de l'échelle. La grande dispersion des scores enregistrée dans ces pays se reflète dans leur écart type, qui est compris entre 105 et 111, ainsi que le montre le tableau 4.3. La Nouvelle-Zélande présente l'écart type le plus élevé de tous les pays (111). Elle contraste fortement avec la Corée, dont l'écart type n'est que de 69 mais dont le score moyen (525) ne s'écarte du sien que d'un seul point. L'écart type international moyen de la sous-échelle Développer une interprétation représente l'équivalent de 100, une valeur inférieure à celle de l'écart type international moyen des deux autres souséchelles (111 sur la sous-échelle Trouver une information et 106 sur la sous-échelle Réfléchir et évaluer).

Le tableau 4.8 présente pour chaque pays les pourcentages de jeunes de 15 ans qui se situent à chaque niveau de compétence PISA sur la sous-échelle Développer une interprétation.

\section{Réfléchir et évaluer}

Comme le montre le tableau 4.9, dans la plupart des pays, 24 en l'occurrence, les élèves se situent à nouveau en majorité au niveau 3 sur la sous-échelle Réfléchir et évaluer. Plus de 90 pour cent des élèves dépassent le niveau 1 dans cinq pays - au Canada, en Corée, en Finlande, en Irlande et au Royaume-Uni. Dans 15 pays, plus de 20 pour cent des élèves ne parviennent pas au niveau 2.

Avec un écart type de 124 (voir le tableau 4.3), l'Allemagne est de loin le pays qui présente la plus grande dispersion des scores sur l'échelle Réfléchir et évaluer, ainsi, d'ailleurs, que sur l'échelle combinée de compréhension de l'écrit et sur les autres sous-échelles. Si l'on compare la répartition des résultats de l'Allemagne avec celles de la Hongrie et du Portugal, dont les scores moyens ne s'écartent pas significativement du sien, on voit clairement que l'Allemagne présente une proportion nettement plus élevée d'élèves situés soit au niveau 5, soit en deçà du niveau 1, et une proportion nettement plus faible d'élèves aux niveaux 2 et 3 . 


\section{Comparaison des scores sur les sous-échelles d'aspect au sein des pays}

\section{Scores moyens}

Les moyennes de l'OCDE sur les trois sous-échelles d'aspect sont pratiquement identiques (498 pour Trouver l'information, 501 pour Développer une interprétation et 502 pour Réfléchir et évaluer), mais chacun des aspects s'est révélé plus facile dans certains pays et plus difficile dans d'autres.

\section{Graphique 4.7a}

Différence de performance entre les sous-échelles Trouver l'information et Réfléchir et évaluer

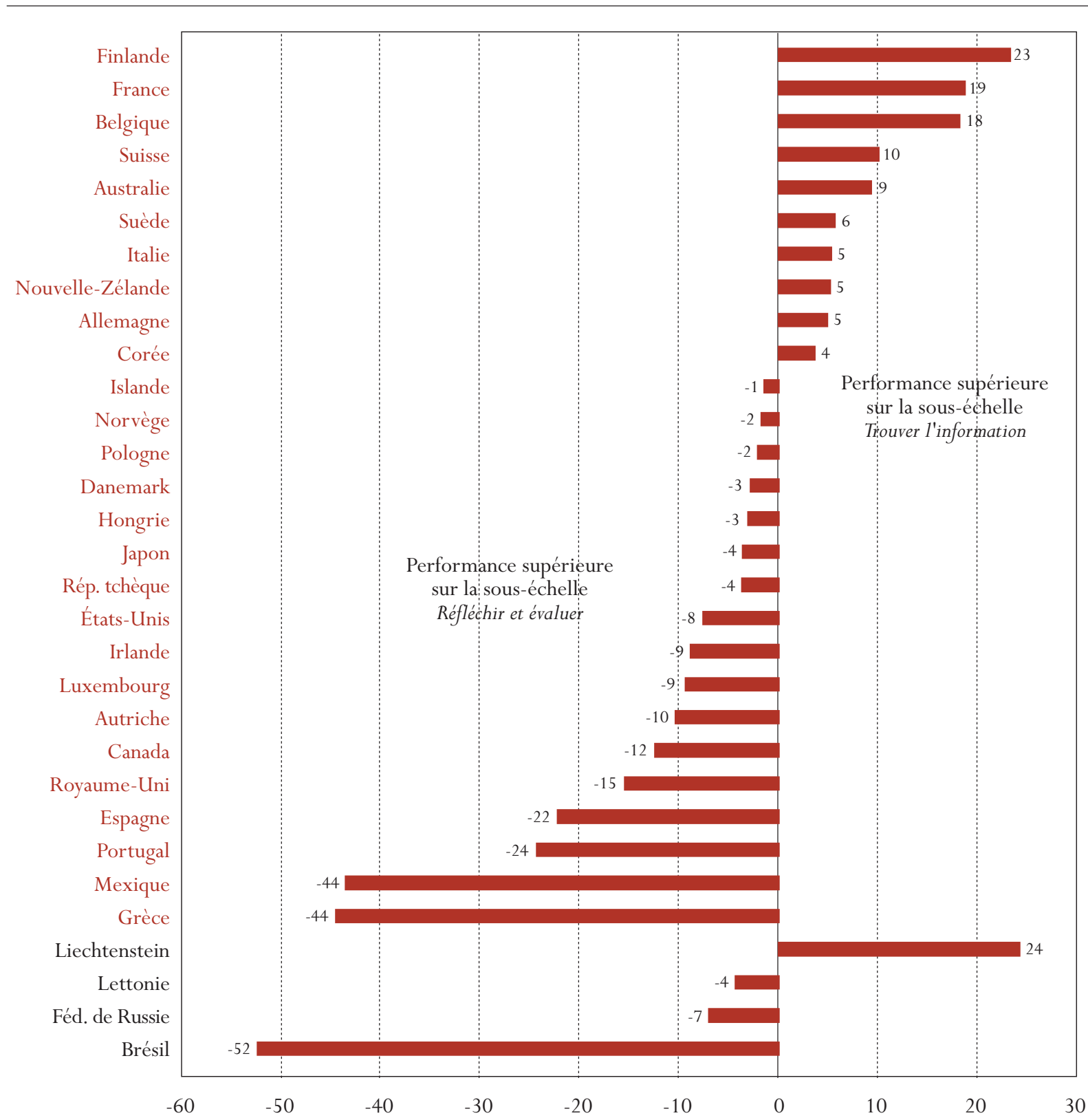

Source : Base de données PISA de l'OCDE, 2001, tableau 4.3. 


\section{Graphique 4.7b}

Différence de performance entre les sous-échelles Trouver l'information et Développer une interprétation

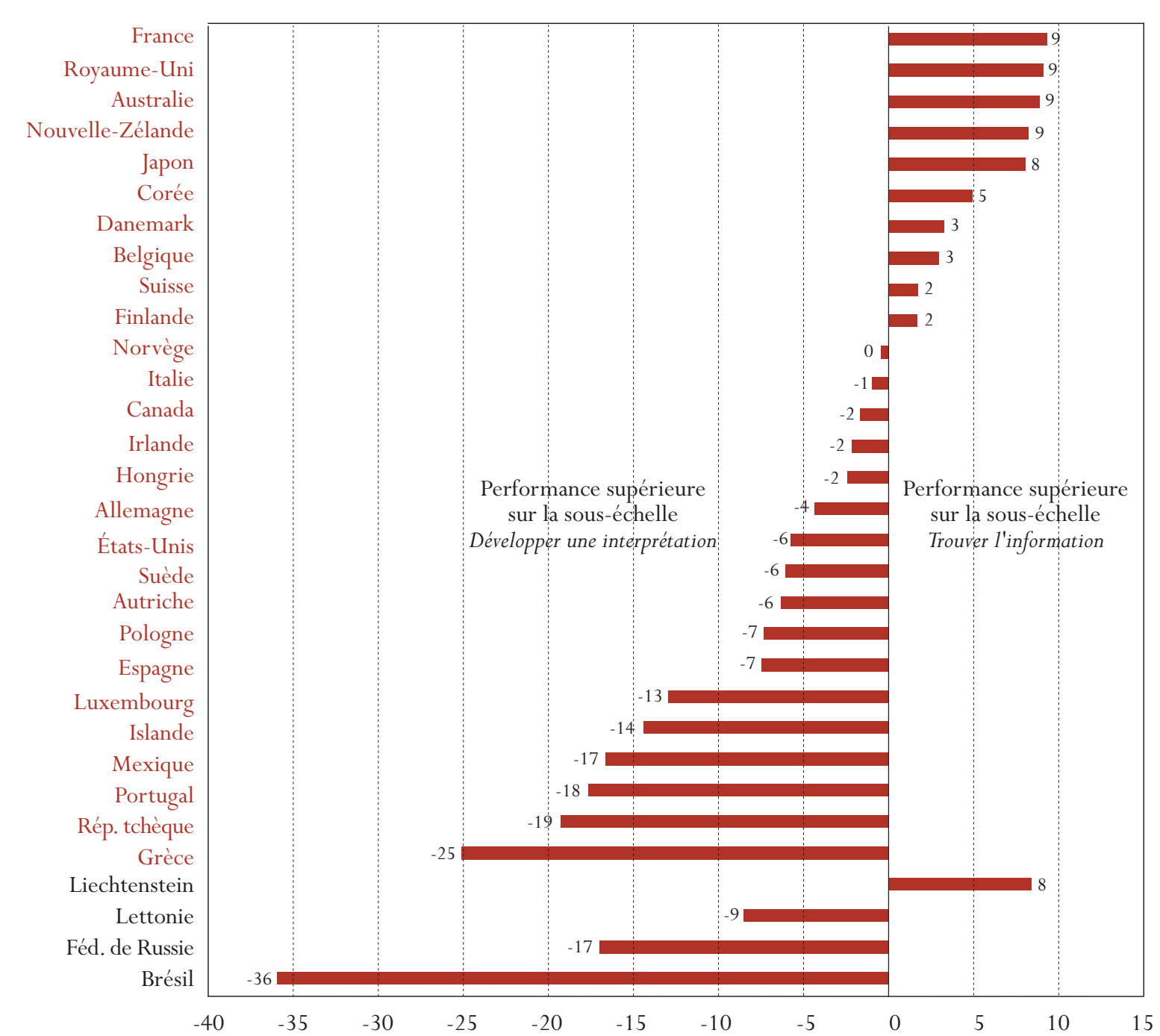

Source : Base de données PISA de l'OCDE, 2001, tableau 4.3.

Trois pays - la Finlande, la France et le Liechtenstein - affichent des performances relativement élevées sur la sous-échelle Trouver l'information, supérieures de 20 points environ à leur score moyen sur les souséchelles Réfléchir et évaluer et Développer une interprétation (voir le graphique 4.7a). La Fédération de Russie, l'Islande, le Luxembourg et la République tchèque obtiennent les meilleurs scores sur la sous-échelle Développer une interprétation par rapport aux deux autres sous-échelles, même si les écarts sont parfois minimes. Au Brésil, en Espagne, en Grèce, au Mexique et au Portugal, les écarts sont très marqués, les scores les plus élevés étant enregistrés sur la sous-échelle Réfléchir et évaluer et les moins élevés sur la sous-échelle Trouver l'information. Dans ces cinq pays, les scores moyens sur ces deux sous-échelles diffèrent de plus de 20 points. Il est intéressant de noter la présence des quatre pays hispanophones et lusophones dans ce groupe, ce qui peut suggérer un effet linguistique, culturel ou pédagogique. 


\section{Graphique 4.8}

Pourcentage d'élèves situés à chaque niveau de compétence sur les sous-échelles d'aspect

Réfléchir et évaluer $\square$ En deçà du niveau $1 \square$ Niveau $1 \square$ Niveau $2 \square$ Niveau $3 \square$ Niveau $4 \square$ Niveau 5 Développer une interprétation En deçà du niveau $1 \square$ Niveau $1 \square$ Niveau $2 \square$ Niveau $3 \square$ Niveau $4 \square$ Niveau 5
Trouver l'information
En deçà du niveau 1
$\square$ Niveau $1 \square$ Niveau 2
$\square$ Niveau $3 \quad \square$ Niveau $4 \quad \square$ Niveau 5

$\begin{array}{llllll}0 \% & 20 \% & 40 \% & 60 \% & 80 \% & 100 \%\end{array}$

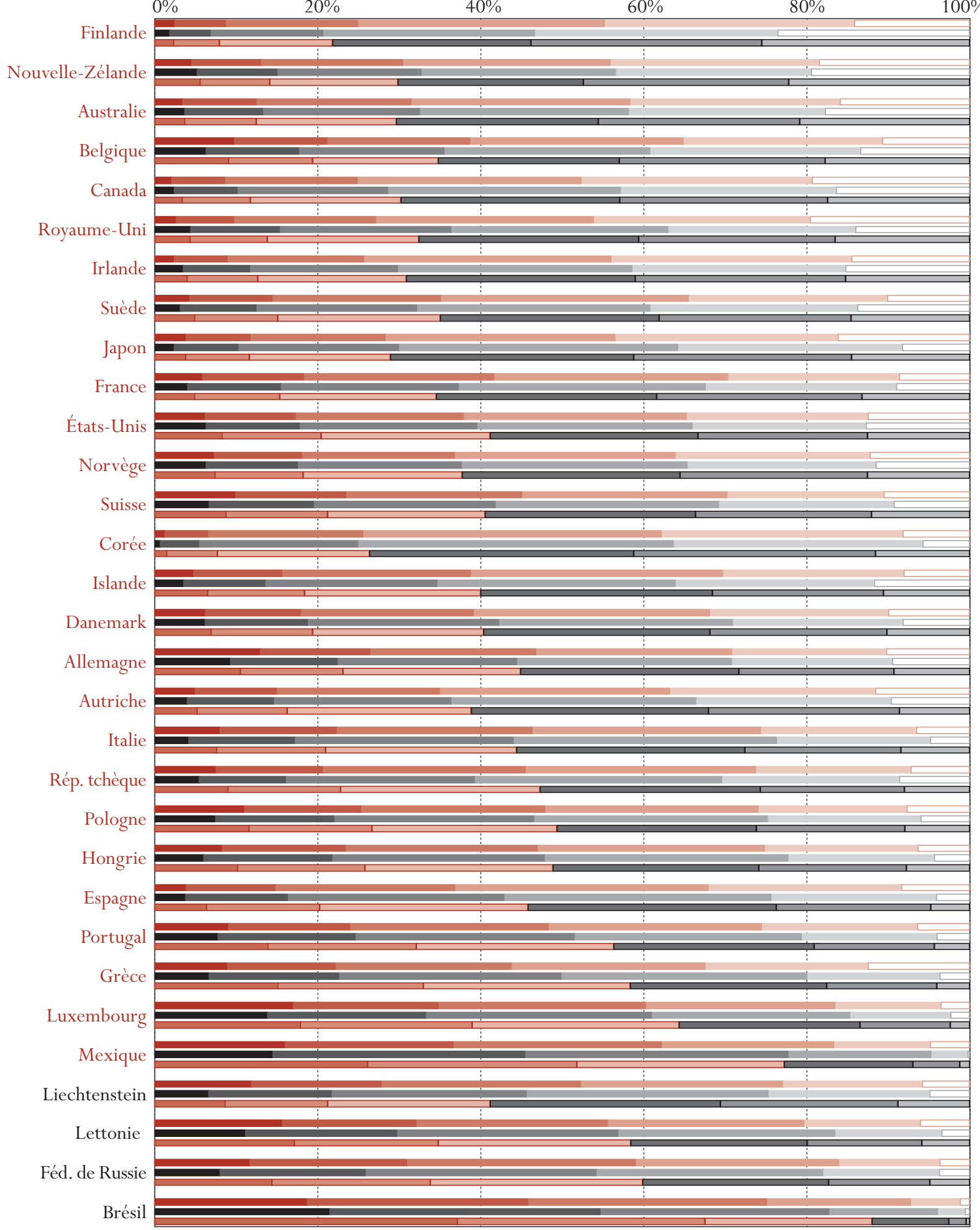




\section{Encadré 4.3 : Évaluation des différences de performance sur les sous-échelles au sein des pays}

Les résultats rapportés sur les sous-échelles Trouver l'information, Développer une interprétation et Réfléchir et évaluer ne sont pas le fruit de mesures indépendantes, puisque les niveaux de difficulté de toutes les tâches ont été estimés lors de la même analyse. Cette remarque s'applique également aux sous-échelles de textes continus et non continus. Les tests classiques de signification ne sont donc pas appropriés dans ce cas. La présentation de la variation des profils nationaux de performance sur les trois sous-échelles d'aspect et sur les deux sous-échelles de format se base sur l'identification de tendances dans les données et non sur des tests statistiques de signification.

Aucun autre profil manifeste ne caractérise les groupes de pays unis par des liens linguistiques ou culturels. Ainsi, parmi les pays essentiellement anglophones, le Canada, les États-Unis, l'Irlande et le RoyaumeUni obtiennent les meilleurs scores sur la sous-échelle Réfléchir et évaluer alors que l'Australie et la Nouvelle-Zélande enregistrent les meilleures performances sur la sous-échelle Trouver l'information. En ce qui concerne le groupe des pays nordiques, les résultats de la Finlande sont comparativement bons sur les sous-échelles Trouver l'information et Développer une interprétation, alors que c'est en interprétation que l'Islande et la Suède obtiennent les meilleurs scores. Enfin, il n'existe pratiquement aucun écart de performance entre les trois sous-échelles au Danemark et en Norvège. Dans les pays où vit une population majoritairement germanophone, l'Autriche présente d'assez bons résultats sur la sous-échelle Réfléchir et évaluer, contrairement à l'Allemagne, au Liechtenstein et à la Suisse, dont les scores sont relativement faibles sur cette sous-échelle.

\section{Répartition}

Le graphique 4.8 montre la proportion de jeunes de 15 ans qui se situent aux cinq niveaux de compétence et en deçà du niveau le plus faible sur les trois sous-échelles d'aspect dans chaque pays.

L'écart important enregistré en Finlande entre les scores moyens sur les sous-échelles de Trouver l'information et Réfléchir et évaluer correspond à des différences dans la répartition entre les niveaux 3, 4 et 5. L'écart le plus grand concerne le niveau 5, que seuls 14 pour cent des élèves finnois atteignent sur la sous-échelle Réfléchir et évaluer alors qu'ils sont respectivement 24 et 26 pour cent à y parvenir sur les sous-échelles Développer une interprétation et Trouver l'information.

Plusieurs pays affichent des proportions relativement élevées d'élèves aux deux niveaux supérieurs de l'une des sous-échelles d'aspect : celle de localisation dans le cas du Canada, de la France, du Liechtenstein et du Royaume-Uni et celle de réflexion et d'évaluation dans le cas de l'Espagne. Au Japon, en revanche, la proportion d'élèves au niveau 5 est nettement plus faible en interprétation de textes qu'en localisation d'informations ou en réflexion et évaluation, ce qui est compensé par de plus fortes proportions d'élèves aux niveaux 2 et 3. En Fédération de Russie, la proportion assez élevée d'élèves situés au niveau 3 de la sous-échelle Développer une interprétation est compensée par une proportion plus faible d'élèves situés, non pas au niveau 5 comme au Japon, mais au niveau 1. 
Au Brésil, c'est en deçà du niveau 1 que se situe l'écart le plus important entre les proportions d'élèves selon les trois aspects, puisqu' on y trouve 37 pour cent des élèves sur l'échelle Trouver l'information contre respectivement 22 et 19 pour cent sur les échelles Développer une interprétation et Réfléchir et évaluer.

Le profil du Mexique est similaire, quoique moins prononcé : 26 pour cent des élèves se situent en deçà du niveau 1 sur la sous-échelle Trouver l'information, contre 15 et 16 pour cent sur les deux autres souséchelles.

Les élèves grecs se concentrent davantage en deçà du niveau 2 sur la sous-échelle Trouver l'information que sous le niveau 2 des deux autres sous-échelles (33 pour cent, contre 23 pour cent sur celle d'interprétation de textes et 22 pour cent sur celle de réflexion et d'évaluation), et aux niveaux 4 et 5 sur la sous-échelle Réfléchir et évaluer (33 pour cent, contre 20 pour cent sur celle d'interprétation de textes et 18 pour cent sur celle de localisation d'informations). Les proportions plus faibles d'élèves situés aux extrémités de la sous-échelle Développer une interprétation se reflètent dans un écart type peu important, de seulement 89 points, contre 109 sur la sous-échelle Trouver l'information et 115 sur la sous-échelle Réfléchir et évaluer.

\section{Performance en compréhension de l'écrit sur les sous-échelles de format de texte}

Nous allons à présent aborder les résultats obtenus par les élèves en fonction du format des textes auxquels les items sont associés. Comme nous l'avons dit au chapitre précédent, les textes sélectionnés pour les épreuves d'évaluation de la compréhension de l'écrit de PISA représentent un grand éventail de types de textes qui se classent dans deux grandes catégories de format, les textes continus et non continus. Les textes continus sont des textes en prose, constitués de paragraphes qui sont eux-mêmes composés de phrases complètes. Cette catégorie comprend des textes narratifs, informatifs, argumentatifs et injonctifs. Les textes non continus (parfois appelés «documents») peuvent contenir des phrases mais ils ne sont pas structurés en paragraphes. Ils peuvent inclure des graphiques de types différents, ainsi que des mots. Généralement, leur sens dépend dans une large mesure de la disposition des informations sur la page. Les tableaux, les graphiques, les cartes et les formulaires sont autant d'exemples de textes non continus.

Les résultats plus ou moins élevés obtenus aux items de compréhension de l'écrit portant sur des textes continus et non continus donnent une idée des pratiques pédagogiques et du contenu des curriculum en cours dans les différents pays. Il est de tradition d'associer essentiellement la lecture à la prose. Dans les cours de langue d'enseignement, les systèmes éducatifs et les enseignants se préoccupent surtout d'apprendre aux élèves à lire des textes littéraires ou informatifs en prose. Toutefois, la lecture et la compréhension d'autres textes que ceux en prose sont au moins aussi importantes pour réussir dans d'autres matières du curriculum. Ainsi, les élèves doivent être capables de comprendre et d'interpréter des cartes et des tableaux pour suivre les cours de sciences sociales, et des graphiques et des diagrammes pour suivre ceux de physique ou de chimie. De plus, en dehors de l'établissement scolaire, les documents constituent une large part des textes que les adultes doivent impérativement lire : les déclarations fiscales, les horaires des transports publics, les graphiques de consommation d'énergie domestique, etc. Dans les sociétés modernes, il est important que les citoyens puissent comprendre des textes non continus, que ce soit dans la vie familiale, professionnelle ou sociale. Toutes ces raisons expliquent pourquoi il est intéressant, dans une étude qui évalue la capacité des jeunes gens à satisfaire aux exigences de lecture de la vie adulte, de mesurer leur aptitude à appréhender tant des textes continus que des textes non continus. Les systèmes éducatifs peuvent alors s'attaquer aux faiblesses épinglées dans la compréhension de textes de l'un ou l'autre format en consacrant du temps d'enseignement à leurs structures et à leurs usages respectifs. 


\section{Scores moyens}

Le tableau 4.10 présente le score et l'écart type de chaque pays sur les deux sous-échelles de format et précise les erreurs types correspondantes.

Le score moyen de l'OCDE sur la sous-échelle de format de texte continu se situe à 501. Les scores moyens sur cette sous-échelle sont compris entre 408 (Brésil) et 544 (Finlande). En d'autres termes, au Brésil, l'élève moyen se situe juste entre les niveaux 1 et 2, tandis qu'en Finlande, l'élève moyen atteint pratiquement le sommet du niveau 3 , ce qui représente un écart de près de deux niveaux de compétence. Le score moyen dans la plupart des pays se situe au niveau 3, sauf au Brésil, en Fédération de Russie, en Lettonie, au Luxembourg, au Mexique et au Portugal, où il ne dépasse pas le niveau 2.

Sur la sous-échelle de format de textes non continus, les scores des pays sont compris entre 366 (Brésil) et 554 (Finlande), la moyenne de l'OCDE se situant à 500. Ils se situent entre le niveau 1 (au Brésil et au Mexique) et le niveau 4 (en Finlande). Comme dans le cas de la sous-échelle de format de textes continus, dans la majorité des pays, le score moyen correspond toutefois au niveau 3. Huit pays - la Fédération de Russie, la Grèce, la Hongrie, l'Italie, la Lettonie, le Luxembourg, la Pologne et le Portugal - affichent un score moyen de niveau 2.

Les tableaux 4.11 et 4.12 comparent les performances moyennes des pays sur les sous-échelles de textes continus et non continus et indiquent si elles sont équivalentes, significativement supérieures ou inférieures à celles des autres pays participants et à la moyenne de l'OCDE.

Répartition des compétences en compréhension de l'écrit au sein des pays sur les deux sous-échelles de format

\section{Textes continus}

Sur la sous-échelle de textes continus, l'écart type moyen de l'OCDE s'établit à 101, le plus faible étant celui de la Corée (69) et le plus élevé, celui de l'Allemagne (115), ainsi que le montre le tableau 4.10. Ces deux valeurs sont relativement éloignées de celles des deuxièmes écarts types plus faible et plus élevé, qui sont respectivement celui de l'Espagne (84) et de la Nouvelle-Zélande (110). Il n'existe pas de différences significatives entre le score moyen de la Corée, dont l'écart type est le plus faible de l'OCDE, et celui de la Nouvelle-Zélande, dont l'écart type est le deuxième plus important.

Parmi les autres pays, certains de ceux qui ne présentent pas d'écart significatif en termes de scores moyens affichent parfois des dispersions de scores très différentes, ainsi que le montrent les écarts types. À titre d'exemple, comparons les écarts types des pays suivants, groupés deux par deux en raison de leurs scores moyens assez proches : l'Allemagne (dont le score moyen est de 484 et l'écart type de 115) et la Hongrie (481 et 92), la Suisse (494 et 104) et l’Espagne (493 et 84), la Belgique (503 et 108) et la France (500 et 94) et, enfin, la Nouvelle-Zélande (526 et 110) et la Corée (530 et 69).

L'analyse des pourcentages d'élèves situés à chaque niveau de compétence, présentés dans le tableau 4.13, donne un autre éclairage sur la répartition des performances en compréhension de l'écrit au sein des pays. Sur la sous-échelle des textes continus, plus de 40 pour cent des élèves se situent au moins au niveau 4 en Australie, au Canada, en Finlande, en Irlande et en Nouvelle-Zélande. Cette proportion avoisine même les 50 pour cent en Finlande. À l'autre extrême, 50 pour cent au moins des élèves ne dépassent pas le niveau 2 dans six pays, le Brésil, la Fédération de Russie, la Lettonie, le Luxembourg, le Mexique et le Portugal. Et dans ce même groupe de pays, au moins 25 pour cent des élèves se situent au niveau 1, voire en deçà. 


\section{Textes non continus}

Sur la sous-échelle de format de textes non continus, l'écart type varie entre 81 et 114 points selon les pays (voir le tableau 4.10). L'écart type moyen de l'OCDE s'établit à 109. Tous les pays sauf trois (l'Allemagne, l'Australie et le Royaume-Uni) affichent une variation de compétence plus importante sur la sous-échelle de textes non continus que sur celle de textes continus.

Comme sur la sous-échelle de textes continus, certains des pays qui affichent des moyennes similaires sur la sous-échelle de textes non continus présentent des profils de dispersion quelque peu différents, même si ce phénomène est moins prononcé sur cette sous-échelle. Parmi ces pays dont les scores moyens sont similaires mais dont les dispersions sont différentes sur la sous-échelle de format de textes non continus, le contraste le plus frappant est celui que révèle la comparaison des deux pays présentant l'écart type le plus faible et le plus élevé, à savoir la Corée, dont le score moyen est de 517 et l'écart type, de 81, et la Belgique (respectivement 516 et 114).

Le tableau 4.14 permet de constater que les pourcentages d'élèves situés à chaque niveau de compétence sur la sous-échelle de textes non continus sont similaires à ceux enregistrés sur la sous-échelle de textes continus. Plus de 40 pour cent des élèves parviennent aux niveaux 4 et 5 de la sous-échelle de textes non continus en Australie, en Belgique, au Canada, en Finlande, en Irlande, en Nouvelle-Zélande, au RoyaumeUni et en Suède. Ils sont même plus de 50 pour cent en Finlande. En revanche, plus de 50 pour cent des élèves ne dépassent pas le niveau 2 sur cette sous-échelle dans huit pays, à savoir au Brésil, dans la Fédération de Russie, en Grèce, en Lettonie, au Luxembourg, au Mexique, en Pologne et au Portugal.

\section{Comparaison des performances sur les sous-échelles de format de texte, au sein des pays}

Les instruments d'évaluation ont été conçus de manière à ce que les items basés sur les deux formats varient en termes de difficulté, de format d'item (items à choix multiple et à réponse construite) et d'aspect et soient associés à de nombreux types de texte. Cela signifie que les différences de performance entre les deux sous-échelles de format peuvent être imputées à la variable de format, et non aux autres variables.

\section{Scores moyens}

Le score moyen de l'OCDE est pratiquement identique sur les deux sous-échelles de format: il est de 501 sur celle de textes continus et de 500 sur celle de textes non continus (voir le tableau 4.10). La corrélation entre les performances sur les deux sous-échelles de format de texte varie entre 0,83 et 0,94 , la valeur médiane étant de 0,90 . Ces similitudes pourraient donner à penser que la performance des élèves d'un pays sur l'une des sous-échelles est très proche de sa performance sur l'autre sous-échelle. Force est de constater pourtant que dans de nombreux pays, il existe des écarts importants entre les sous-échelles de format tant en termes de performance moyenne que de dispersion des scores, comme le montre le graphique 4.9. 


\section{Graphique 4.9}

Différence de performance entre les sous-échelles Textes continus et Textes non continus

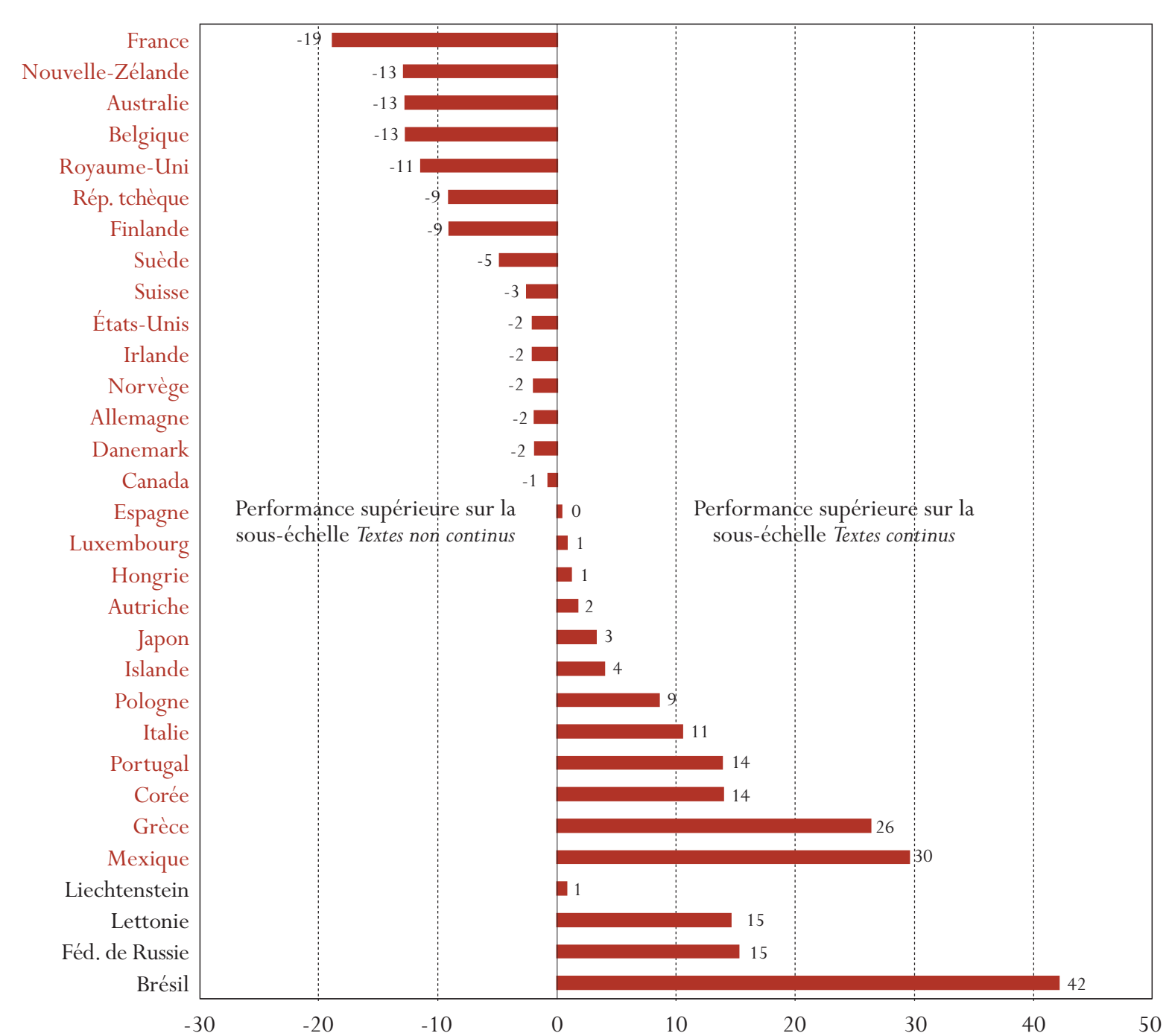

Source : Base de données PISA de l'OCDE, 2001, tableau 4.10.

Dans 16 des pays participants, dont tous les pays majoritairement anglophones, tous les pays d'Europe septentrionale (à l'exception de l'Islande) et tous les pays comptant une population francophone (à l'exception du Luxembourg), les scores sur la sous-échelle de textes non continus sont au moins équivalents à ceux enregistrés sur la sous-échelle de textes continus. C'est en France que l'écart le plus important (19 sur l'échelle PISA) est observé en faveur de la sous-échelle de textes non continus. Des écarts de 10 points environ, favorables au format non continu, sont enregistrés en Australie, en Belgique, en Finlande, en Nouvelle-Zélande, en République tchèque et au Royaume-Uni. Dans la plupart des pays, les scores moyens sur les deux sous-échelles se situent au même niveau de compétence, à savoir le niveau 3. La Finlande fait figure d'exception : son score moyen sur la sous-échelle de textes continus correspond au niveau 3, mais au niveau 4 sur celle de textes non continus. 
Dans huit pays, l'écart moyen entre les scores sur la sous-échelle de textes continus et sur celle de textes non continus dépasse dix points. Ce groupe comprend plusieurs des pays qui affichent les performances moyennes les moins élevées, mais aussi la Corée, qui affiche une performance globale parmi les plus élevées. Dans cinq pays, le score moyen sur la sous-échelle de textes continus se situe à un niveau supérieur à celui du score moyen sur la sous-échelle de textes non continus. Il s'agit de la Grèce, de l'Italie et de la Pologne (le niveau 3 pour le format continu et le niveau 2 pour le format non continu) ainsi que du Brésil et du Mexique (les niveaux 2 et 1 respectivement). Comme nous l'avons dit précédemment, ce phénomène peut être dû au caractère plus traditionnel des programmes de cours de langue dans ces pays, plus axés sur la littérature et qui privilégient les textes littéraires et informatifs au détriment des textes qui ne sont pas en prose, qu'ils s'inscrivent dans le cadre scolaire ou celui de la vie courante. Ce constat pourrait également expliquer la performance relativement plus faible de la Corée et du Japon sur la sous-échelle de textes non continus. Toutefois, il convient de ne pas exclure l'hypothèse de différences culturelles qui pourraient contribuer à rendre certains formats non continus peu familiers, un phénomène susceptible d'être aggravé par l'utilisation de caractères romains lors de la traduction de plusieurs textes non continus.

\section{Répartition}

Comme nous l'avons souligné ci-dessus, la moyenne de l'OCDE sur la sous-échelle de textes continus (501) est pratiquement identique à celle enregistrée sur la sous-échelle de textes non continus (500) mais il n'en va pas de même pour l'écart type moyen de l'OCDE, qui s'établit respectivement à 101 et 109. Ce phénomène indique qu'en moyenne, les écarts de scores sont plus étendus sur la sous-échelle de textes non continus que sur celle de textes continus. Le graphique 4.10 montre la répartition des élèves entre les cinq niveaux de compétence des deux sous-échelles de format dans chaque pays. La répartition des performances de chaque pays est symbolisée par le trait supérieur pour la sous-échelle de format de textes continus et par le trait inférieur pour la sous-échelle de format de textes non continus.

Le profil de répartition entre les niveaux des deux sous-échelles est assez similaire pour les pays suivants : l'Allemagne, l'Australie, l'Autriche, le Canada, le Danemark, les États-Unis, l'Irlande, l'Islande, l'Italie, le Liechtenstein, le Luxembourg, la Norvège, la Pologne, le Portugal, le Royaume-Uni et la Suisse.

Dans d'autres pays, les scores divergent selon les deux sous-échelles de format. En Corée, les élèves sont nettement plus nombreux à atteindre le niveau 4 sur la sous-échelle de textes continus que sur celle des textes non continus et nettement moins nombreux aux niveaux 1 et 2. Globalement, en Corée comme dans la plupart des autres pays, les scores sont plus dispersés sur la sous-échelle de textes non continus que sur celle de textes continus. L'écart type de la Corée est toutefois inférieur à celui de tout autre pays, tant sur la sous-échelle de textes non continus que sur celle de textes continus.

La répartition des scores enregistrée au Brésil et au Mexique est relativement similaire à celle observée en Corée : les élèves se concentrent davantage aux niveaux supérieurs sur la sous-échelle de textes continus que sur celle de textes non continus. Toutefois, leurs scores sont généralement inférieurs à ceux de la Corée sur les deux sous-échelles. Au Brésil et au Mexique, les proportions d'élèves situés aux niveaux 2 et 3 sont plus importantes sur la sous-échelle de textes continus que sur celle de textes non continus. De plus, la proportion d'élèves en deçà du niveau 1 est nettement supérieure sur l'échelle de textes non continus que sur celle de textes continus. En Fédération de Russie, en Grèce et en Lettonie, la proportion d'élèves situés aux niveaux inférieurs est plus importante sur la sous-échelle de textes non continus que sur celle de textes continus. 


\section{Graphique 4.10}

Pourcentage d'élèves situés à chaque niveau de compétence sur les sous-échelles

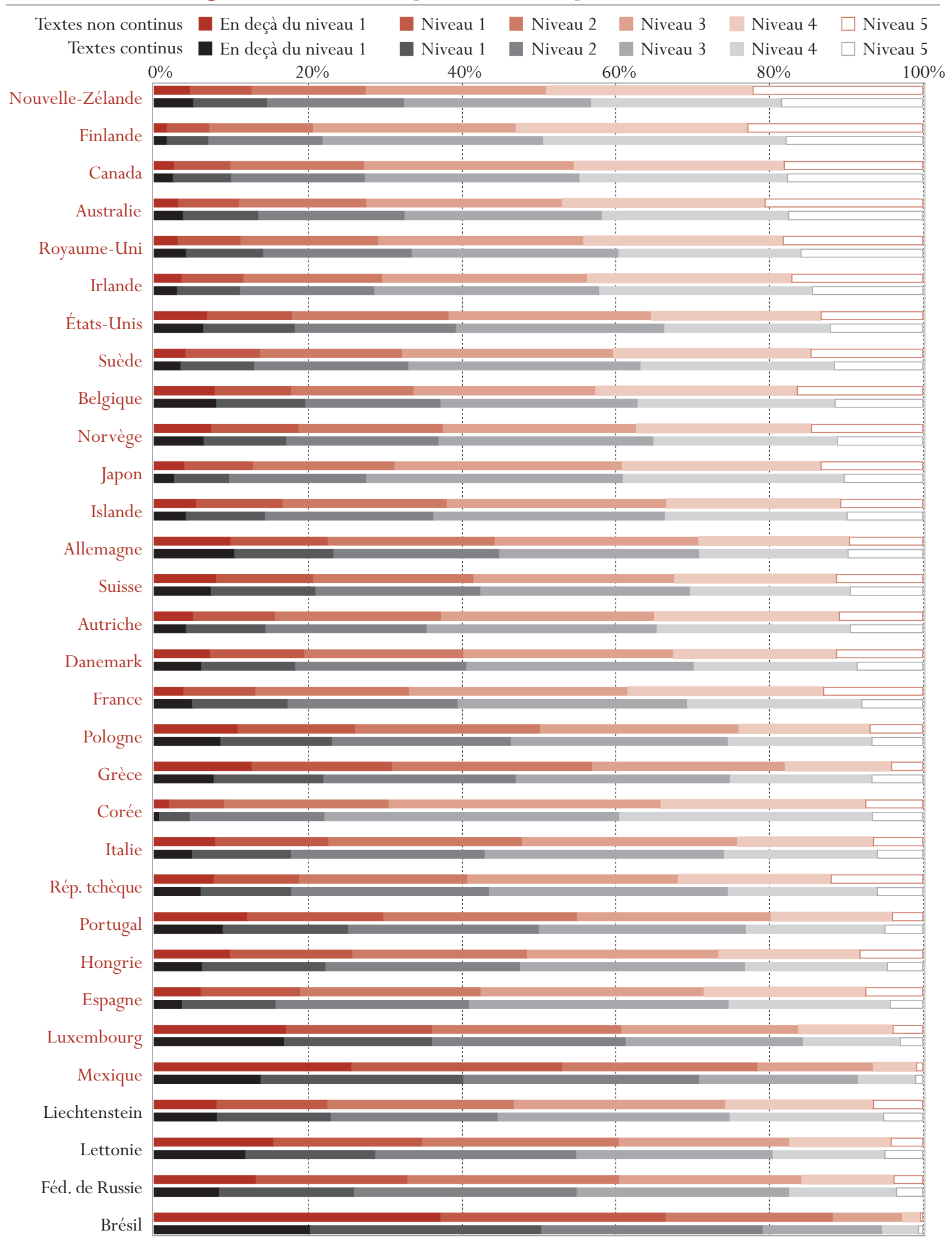

Source : Base de données PISA de l'OCDE, 2001, tableaux 4.13 et 4.14 
D'autres pays présentent un profil de répartition inverse. Dans ces pays, les élèves se concentrent davantage aux niveaux supérieurs de la sous-échelle de textes non continus que sur celle de textes continus. Ainsi, en Belgique, en Finlande, en France et en République tchèque, les proportions d'élèves situés au niveau 5 sont nettement plus importantes sur la sous-échelle de textes non continus que sur la sous-échelle de textes continus, et les proportions d'élèves situés aux niveaux inférieurs sont nettement plus importantes sur la sous-échelle de textes continus. En République tchèque, la proportion d'élèves situés au niveau 3 est particulièrement plus élevée sur la sous-échelle de textes continus que sur celle de textes non continus. Cette concentration d'élèves autour du milieu de la sous-échelle de texts continus est reflétée par un écart type de 93, nettement inférieur à celui de la sous-échelle de textes non continus (112). Seuls quatre pays affichent un écart type supérieur sur la sous-échelle de textes non continus, alors que 19 pays présentent une dispersion plus étendue que la République tchèque sur la sous-échelle de textes continus.

La tendance à une concentration accrue d'élèves aux extrémités des sous-échelles de format s'observe uniquement dans un sens : aucun pays ne compte davantage d'élèves aux extrémités de la sous-échelle de textes continus qu'aux extrémités de la sous-échelle de textes non continus. Il y a lieu de souligner toutefois que deux pays échappent à la tendance générale, à savoir une dispersion plus importante des scores sur la sous-échelle de textes non continus. Le Royaume-Uni présente un écart type de 104 sur la sous-échelle de textes continus et de 101 sur celle de textes non continus et l'Allemagne, des écarts types de 115 et 113 respectivement.

\section{Inégalités entre et dans les pays participants}

La question de l'inégalité surgit inévitablement lors de l'étude de la compréhension de l'écrit, car le niveau de littératie a un impact considérable sur le bien-être personnel de l'individu, sur l'état de la nation et sur l'importance économique des pays dans le monde. L'association entre le niveau de littératie et l'épanouissement personnel de l'individu est étudiée de manière plus approfondie dans le chapitre 8. Dans le présent chapitre en revanche, c'est pour attirer l'attention sur les différences potentielles d'accès aux opportunités éducatives, sociales et économiques entre les deux groupes que nous analysons l'écart entre les lecteurs les plus forts et les plus faibles.

Un des moyens de mesurer les inégalités consiste à mesurer l'ampleur de l'écart de performance séparant les groupes d'élèves les plus «forts » et les plus «faibles » d'un pays. Pour cela, nous avons utilisé ici la différence de performance entre le $90^{\mathrm{e}}$ et le $10^{\mathrm{e}}$ percentile d'un pays donné. Dans un pays, le $90^{\mathrm{e}}$ percentile correspond au score en dessous duquel se trouve 90 pour cent de l'échantillon national et le $10^{\mathrm{e}}$ percentile, au score en dessous duquel se trouve 10 pour cent seulement de l'échantillon national. La différence entre ces scores représente l'intervalle des scores obtenus par les 80 pour cent médians de l'échantillon d'un pays. Plus cet intervalle est étendu, plus l'écart est important entre les élèves situés aux deux extrêmes et plus les inégalités sont grandes.

Le graphique 4.11 montre l'écart entre les scores du $90^{\mathrm{e}}$ et du $10^{\mathrm{e}}$ percentile sur l'échelle combinée de compréhension de l'écrit. Les pays sont classés dans le graphique en fonction de l'importance de l'écart sur l'échelle combinée de compréhension de l'écrit. Les tableaux 4.16 à 4.21 indiquent l'écart type et les scores des $10^{\mathrm{e}}$ et $90^{\mathrm{e}}$ percentiles sur l'échelle combinée de compréhension de l'écrit et sur les cinq souséchelles. 


\section{Graphique 4.11}

Différences de score entre le $10^{\mathrm{e}}$ et le $90^{\mathrm{e}}$ percentile sur l'échelle combinée de compréhension de l'écrit

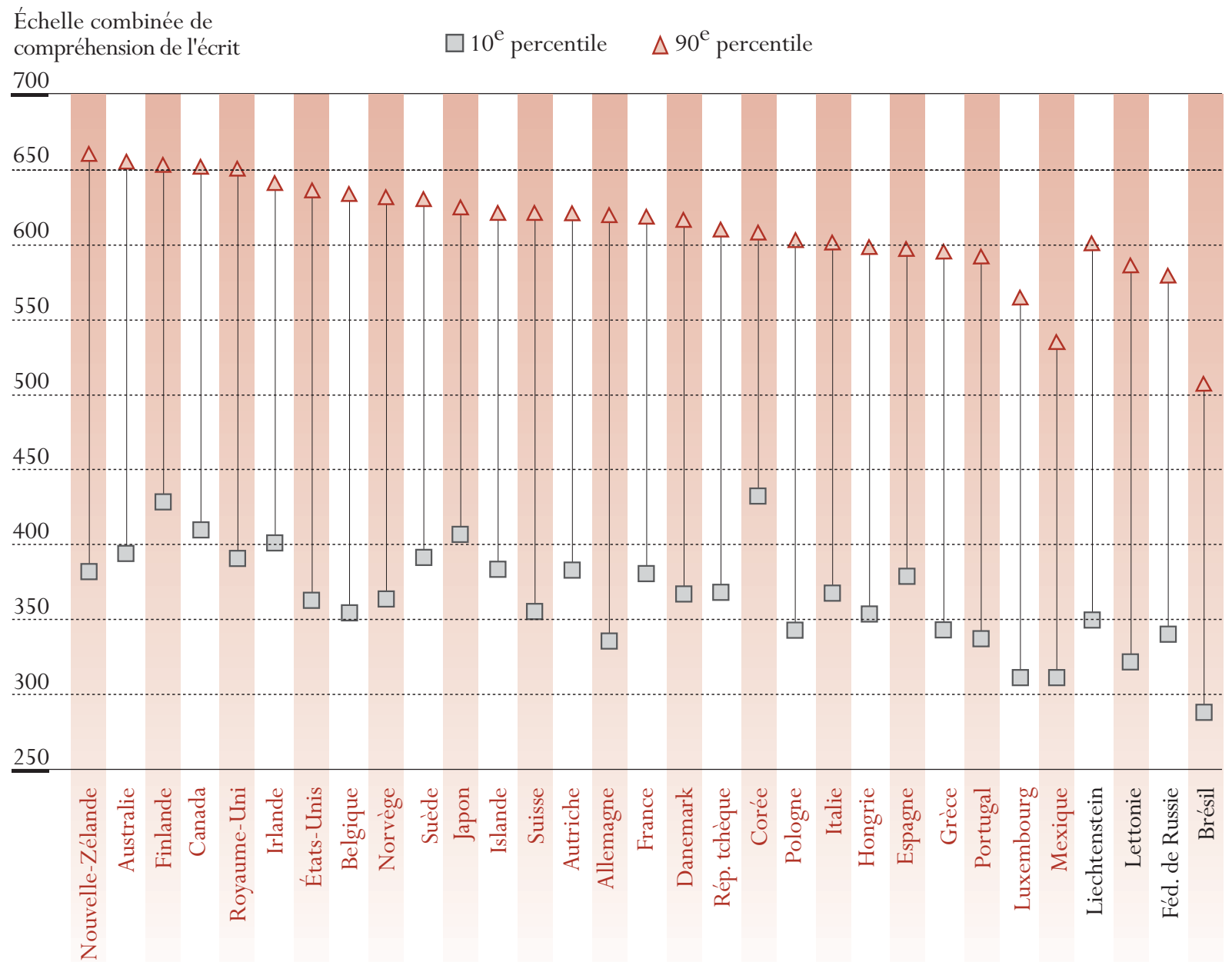

Source : Base de données PISA de l'OCDE, 2001, tableau 4.16.

En Corée, l'intervalle de scores compris entre le $90^{\mathrm{e}}$ et le $10^{\mathrm{e}}$ percentile sur l'échelle combinée de compréhension de l'écrit est de 175, soit l'équivalent d'un petit peu moins de deux niveaux et demi de compétence. C'est de loin l'écart le plus faible de tous les pays de l'OCDE. Dans ce classement, la Corée est suivie par l'Espagne et le Japon qui affichent un écart de 218 , soit l'équivalent de trois niveaux de compétence.

À l'autre extrême se trouve l'Allemagne, qui enregistre un écart de 284 entre le $90^{\mathrm{e}}$ et le $10^{\mathrm{e}}$ percentile, soit l'équivalent de près de quatre niveaux de compétence. L'intervalle représente plus de 270 en Belgique, aux États-Unis et en Nouvelle-Zélande.

En Finlande et au Mexique, l'écart entre le $90^{\mathrm{e}}$ et le $10^{\mathrm{e}}$ percentile est similaire (respectivement 225 et 223). Par contraste avec quelques autres pays, aucun de ces deux pays ne présente d'écart sérieux entre les élèves les plus compétents et les moins compétents. Mais au Mexique, avec 44 pour cent des élèves de 15 ans en deçà du niveau 2, une grande proportion de la population mexicaine ne semble pas armée pour faire face à un monde en mutation, tandis qu'en Finlande, tous les élèves, sauf 7 pour cent, se situent à des niveaux supérieurs à ceux des 44 pour cent d'élèves mexicains. 
Des trois aspects, c'est celui qui consiste à trouver une information qui donne lieu à la dispersion des scores la plus étendue chez les élèves appartenant au groupe des 80 pour cent médians. Dans les pays de l'OCDE, l'écart moyen entre les scores du $90^{\mathrm{e}}$ et du $10^{\mathrm{e}}$ percentile représente 284 , soit l'équivalent de quatre niveaux de compétence. La dispersion la plus importante s'observe en Belgique, où un écart de 313 sépare les élèves situés au sommet de l'échelle de ceux au bas de l'échelle, soit l'équivalent de près de quatre niveaux et demi de compétence.

Pour l'aspect Développer une interprétation, l'écart entre les scores du $90^{\mathrm{e}}$ et du $10^{\mathrm{e}}$ percentile est moindre que pour les deux autres aspects. L'écart moyen de l'OCDE entre ces deux percentiles représente 257, soit 12 points de moins que pour Réfléchir et évaluer et 27 points de moins que pour Trouver une information. L'écart des scores de la Corée ne représente que 176, soit 26 points de moins par rapport au pays qui la suit dans ce classement. L'écart le plus important est enregistré en Nouvelle-Zélande (289).

Pour l'aspect Réfléchir et évaluer, l'intervalle des scores obtenus en Allemagne par les élèves appartenant au groupe des 80 pour cent médians représente 316, soit l'équivalent de quatre niveaux et demi de compétence environ. Cet écart est supérieur de 20 points à celui de la Grèce, qui arrive en deuxième position dans le classement par importance de l'intervalle des scores entre le $90^{\mathrm{e}}$ et le $10^{\mathrm{e}}$ percentile sur la souséchelle Réfléchir et évaluer. Aucun pays sur aucune autre sous-échelle n'affiche d'écart plus important entre les scores des $10^{\mathrm{e}}$ et $90^{\mathrm{e}}$ percentiles.

Sur les sous-échelles de format de texte, les écarts entre le $90^{\mathrm{e}}$ et le $10^{\mathrm{e}}$ percentiles sont plus importants sur la sous-échelle de textes non continus que sur celle de textes continus dans tous les pays, sauf en Allemagne, en Australie, au Liechtenstein et au Royaume-Uni. L'écart le plus faible est enregistré en Corée (172) sur la sous-échelle de textes continus, tandis que le plus important est observé en Belgique (296) sur la sous-échelle de textes non continus.

Tant en Allemagne qu'en Belgique, des écarts considérables entre le $90^{\mathrm{e}}$ et le $10^{\mathrm{e}}$ percentiles s'observent systématiquement sur toutes les sous-échelles. L'Allemagne accuse l'écart le plus élevé sur la sous-échelle Réfléchir et évaluer et celle de textes continus et la Belgique, l'écart le plus important sur la sous-échelle Trouver l'information. Ces écarts représentent quatre niveaux et demi sur l'échelle de compétence. De plus, il n'y a jamais plus de cinq pays qui présentent des écarts plus importants sur une sous-échelle que ceux de l'Allemagne ou de la Belgique.

Par contraste, quelle que soit la sous-échelle, c'est la Corée qui affiche l'écart de performance le plus faible entre élèves se situant au $90^{\mathrm{e}}$ et au $10^{\mathrm{e}}$ percentiles.

Jusqu'ici, nous avons présenté les tendances qui correspondaient à des écarts uniformément élevés ou faibles sur les sous-échelles. Il ne faut toutefois pas en conclure que des différences considérables sur l'une des sous-échelles en impliquent nécessairement d'autres sur les autres sous-échelles. Plusieurs pays présentent des profils très différents de dispersion selon les sous-échelles. Le Mexique en est l'exemple le plus frappant. Ce pays arrive en deuxième position dans le classement des pays qui présentent le plus faible écart de performance sur la sous-échelle Développer une interprétation. Les 80 pour cent médians d'élèves se répartissent sur un intervalle de 202, soit moins de trois niveaux de compétence sur cette sous-échelle. En revanche, l'intervalle des scores des élèves mexicains entre les $10^{\mathrm{e}}$ et $90^{\mathrm{e}}$ percentiles représente 283 points sur la sous-échelle Réfléchir et évaluer, soit l'équivalent de pratiquement quatre niveaux de compétence. Seuls sept pays accusent une dispersion plus étendue. D'autres pays affichent des profils de dispersion assez 
différents selon les sous-échelles, que ce soit en termes absolus ou par rapport à d'autres pays. Il s'agit de la Grèce, de la Hongrie, de l'Irlande, de la Lettonie, de la République tchèque et du Royaume-Uni.

Certains profils de répartition semblent aller de pair avec des groupes linguistiques différents. Ainsi, sur la sous-échelle Développer une interprétation, la dispersion des scores des 80 pour cent médians d'élèves tend à être plus étendue dans les pays anglophones que dans les autres pays. Quatre pays anglophones figurent parmi les sept pays qui affichent les écarts les plus importants sur cette sous-échelle d'aspect. La dispersion des scores est systématiquement moins importante en réflexion et évaluation qu'en interprétation des textes dans ces pays. En Nouvelle-Zélande par exemple, l'intervalle des scores entre le $90^{\mathrm{e}}$ et le $10^{\mathrm{e}}$ percentile est plus étendu que dans tous les autres pays sur la sous-échelle Développer une interprétation, alors que sur celle Réfléchir et évaluer, dix pays affichent des intervalles de scores plus étendus. Les pays où l'anglais n'est pas la langue d'enseignement présentent un tout autre profil : l'intervalle de scores des 80 pour cent médians est plus étendu en réflexion et évaluation qu'en interprétation de textes dans tous les pays sauf la Finlande, l'Islande et la Suède. La variation de ces profils de performance peut s'expliquer par des pratiques pédagogiques différentes. Une des hypothèses qu'il convient d'envisager est la suivante : privilégier un aspect de la lecture au détriment d'autres aspects peut avoir pour effet de rehausser le niveau de compétence des élèves pour cet aspect.

Dans ce rapport, nous avons systématiquement épinglé les différences importantes de performance entre les pays sur l'échelle combinée de compréhension de l'écrit ainsi que sur les sous-échelles. L'écart entre le score moyen le plus élevé et le plus faible de deux pays quels qu'ils soient représente 150 points sur l'échelle combinée de compréhension de l'écrit, soit l'équivalent de plus de deux niveaux de performance. La proportion d'élèves situés aux deux niveaux supérieurs de cette échelle va de 4 pour cent à plus de 50 pour cent selon les pays. Ces écarts peuvent être lourds de conséquences pour les pays qui cherchent à renforcer leur rôle économique et à affirmer leur présence politique dans des contextes internationaux. À l'heure de la mondialisation, les niveaux de compétence en compréhension de l'écrit sur lesquels les pays peuvent compter ont assurément un impact considérable sur leur capacité à se distinguer sur la scène internationale. Les pays qui accusent un faible niveau général de compétence en compréhension de l'écrit courent le risque d'être à la traîne dans d'autres domaines.

Les différences relevées au sein des pays sont au moins aussi importantes que celles observées entre les pays. C'est un fait établi que la présence de niveaux inégaux de compétence en compréhension de l'écrit est associée à de nombreuses formes de malaise social et est lourde de conséquences tant pour l'individu que pour la société dans son ensemble. Il existe une relation positive entre la littératie et l'emploi, la satisfaction professionnelle, les revenus, la longévité et la santé. Cette relation s'explique peut-être en partie par le renforcement de la capacité à prendre des décisions. Il est établi que la littératie a un effet important non seulement sur le produit intérieur brut mais aussi sur la cohésion sociale et la richesse culturelle (OCDE et Statistique Canada, 2000, pp. 80-81). Les individus qui possèdent de bonnes compétences en compréhension de l'écrit sont les mieux armés pour développer leurs savoirs et savoir-faire, en particulier par le biais de l'auto-apprentissage. Ils sont moins exposés au chômage provoqué par l'évolution rapide du monde qui les entoure. Ils sont moins susceptibles de se livrer à des activités criminelles et plus susceptibles de participer à la vie publique. La société dans son ensemble bénéficie de multiples façons de ce que peuvent lui apporter des niveaux de littératie élevés et équitablement répartis. 


\section{Notes}

1. Le taux de participation initial n'était que de 27 pour cent aux Pays-Bas. C'est pourquoi la performance moyenne des Pays-Bas ne peut pas être comparée à celles des autres pays et les données relatives aux Pays-Bas ne sont pas incluses dans les analyses de ce chapitre. Les données néerlandaises ont toutefois été considérées suffisamment fiables pour être incluses dans les analyses présentées aux chapitres 5, 6 et 7. Pour davantage de détails, consulter le rapport initial de PISA (OCDE 2001b), p. 236.

2. Une moyenne des pays de l'OCDE a été calculée pour un grand nombre des indicateurs présentés dans ce chapitre. Le rapport initial de PISA 2000 (OECD 2001b) définit la moyenne de l'OCDE comme suit : « la moyenne des valeurs de tous les pays de l'OCDE pour lesquels des données sont disponibles ou peuvent être estimées. La moyenne de l'OCDE sert notamment à comparer la valeur d'un indicateur d'un pays donné à celle d'un pays type de l'OCDE. Elle ne tient pas compte des effectifs absolus d'élèves dans les pays. En d'autres termes, chaque pays intervient de la même manière dans la moyenne. »

3. Comme nous l'avons expliqué au chapitre 2, l'échelle de compréhension de l'écrit a été divisée en niveaux sur la base de critères conceptuels et statistiques. Il y a lieu de se reporter à ce chapitre pour des informations détaillées à propos de ces critères.

4. À l'instar des autres coefficients de corrélation présentés dans ce chapitre, ces coefficients sont des estimations de corrélations latentes. 


\section{Chapitre}

5

L'ENGAGEMENT DES JEUNES DE

15 ANS À L'ÉGARD DE LA LECTURE

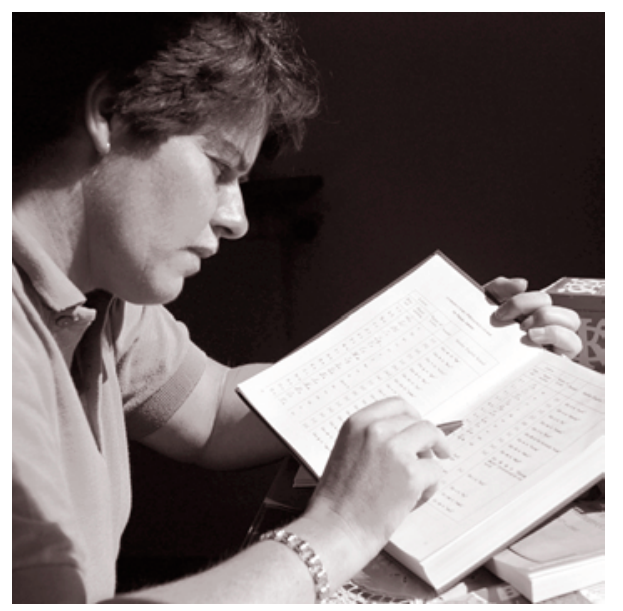




\section{SYNTHÈSE}

- Plusieurs profils de lecteur ont été identifiés. Certains jeunes de 15 ans centrent leurs lectures sur un éventail limité d'écrits : les revues uniquement, ou les revues et les journaux. D’autres diversifient davantage leurs lectures : certains lisent des bandes dessinées en plus des revues et des journaux, tandis que d'autres préfèrent les livres, de fiction ou non, aux bandes dessinées.

- Les profils de lecteur varient sensiblement d'un pays à l'autre. Dans certains pays, en Finlande et au Japon par exemple, une forte proportion des élèves qui diversifient leurs lectures lisent essentiellement des journaux, des revues et des bandes dessinées. Dans d'autres pays, notamment en Australie, en Nouvelle-Zélande et au Royaume-Uni, ces élèves tendent à lire des journaux, des revues et des livres (de fiction ou documentaires).

- Les filles et les garçons présentent de toute évidence des profils différents en matière de lecture. La répartition entre les sexes est équivalente chez les élèves associés aux deux premiers profils de lecteurs, c' est-à-dire ceux qui ne diversifient guère leurs lectures et qui lisent essentiellement des revues et des journaux. Pour le troisième profil, les garçons sont majoritaires parmi les élèves qui préfèrent les bandes dessinées et les filles sont plus nombreuses à correspondre au profil des lecteurs qui préfèrent les livres (de fiction surtout).

- En toute logique, les élèves de 15 ans qui diversifient leurs lectures sont plus performants que ceux qui limitent leurs lectures. Mais l'écart de performance entre ceux qui lisent des bandes dessinées ou des livres de fiction n'est pas énorme. Il semble que la lecture quotidienne de revues, de journaux et de bandes dessinées - un type d'écrit peut-être moins valorisé par l'école que les livres de fiction - soit un bon moyen de devenir un lecteur compétent, du moins dans certains contextes culturels.

- Il n'existe qu'une faible corrélation entre les profils de lecteur et le milieu socio-économique des élèves. Il n'en va pas de même pour l'accès à l'écrit à la maison, qui est déterminant pour les profils de lecteur. Les jeunes de 15 ans qui n'ont accès qu'à un nombre limité de livres à la maison ont, en moyenne, des lectures peu diversifiées. Ils lisent essentiellement des revues et des journaux. En revanche, ceux qui ont accès à un plus grand nombre de livres diversifient davantage leurs lectures et s'intéressent à d'autres types d'écrits, tels que les livres (de fiction ou documentaires) ou la bande dessinée.

- L'engagement à l'égard de la lecture tel qu'il est défini ici (le temps passé à lire pour le plaisir, la diversité des lectures, une motivation et un intérêt élevé pour la lecture) varie considérablement d'un pays à l'autre. En moyenne, les filles sont plus engagées que les garçons dans la lecture.

- Les jeunes de 15 ans dont les parents présentent le statut professionnel le plus faible mais qui sont très engagés dans la lecture obtiennent en compréhension de l'écrit des scores supérieurs à ceux des élèves dont les parents affichent un statut professionnel moyen à élevé mais qui ne sont guère engagés dans la lecture. 
Dans ce chapitre, nous commencerons par présenter des «profils de lecteurs » établis sur la base d'une analyse typologique des types d'écrit que les jeunes de 15 ans déclarent lire. Les élèves sont répartis dans des groupes correspondant à des profils de lecteurs différents, définis par la diversification et la fréquence de leurs lectures. Nous poursuivrons en comparant les performances de ces quatre groupes d'élèves sur l'échelle combinée de compréhension de l'écrit ainsi que sur les trois sous-échelles d'aspect et les deux sous-échelles de format. Pour conclure cette analyse, nous déterminerons dans quelle mesure les profils des lecteurs peuvent être associés à certaines de leurs caractéristiques. Nous passerons ensuite au concept plus vaste d'«engagement à l'égard de la lecture » qui intègre les trois variables décrites plus haut dans un indice composite unique $^{1}$. Nous comparerons les pays en fonction des valeurs de cet indice pour l'ensemble des élèves et par sexe. Enfin, nous clôturerons ce chapitre en abordant une question fondamentale : l'engagement à l'égard de la lecture peut-il atténuer l'effet du statut professionnel des parents ? La réponse à cette question est cruciale pour orienter l'action publique dans le domaine de l'enseignement de la lecture.

Les analyses exposées dans le présent chapitre sont univariées. Elles sont réalisées sans tenir compte d'éventuelles interactions plus complexes entre plusieurs variables. Les analyses multivariées sont présentées au chapitre 7 .

La lecture n'est pas seulement un acte cognitif, elle comporte aussi des aspects non cognitifs, tels que les attitudes à l'égard de la lecture ou les pratiques de lecture. Qu'ils portent sur l'acquisition des compétences ou sur les résultats de l'apprentissage, la plupart des modèles contemporains de littératie considèrent les attitudes, ou la motivation, et les pratiques comme des facteurs clés de la compréhension de l'écrit (pour une synthèse, voir Kamil et al., 2000 ; McKenna et al., 1995). Guthrie et Wigfield (2000) estiment que la motivation fait le lien entre la fréquence des lectures et les résultats en compréhension de l'écrit. La motivation pour la lecture génère l' « effet Matthieu » (Stanovich, 1986) qui désigne la relation de causalité circulaire entre les pratiques et les compétences. Comme ils sont motivés, les lecteurs compétents ont tendance à lire davantage et, en conséquence, à enrichir leur vocabulaire et à améliorer leurs compétences. Il s'ensuit que le fossé entre les «bons » et les «mauvais » lecteurs ne cesse de se creuser au fil du temps.

Aux États-Unis, le « National Assessment of Educational Progress » rapporte des résultats intéressants (Campbell, Voelkl et Donahue, 1997) qui démontrent le lien entre les performances et l'engagement à l'égard de la lecture. Cette étude a confirmé que les lecteurs qui s'investissaient davantage dans la lecture étaient plus compétents que ceux qui s'y investissaient moins et ce, dans les trois classes d'âge cibles ( 9,13 et 17 ans). De plus, elle a montré que les scores moyens des élèves de 13 ans affichant un degré important d'engagement à l'égard de la lecture étaient supérieurs à ceux des élèves de 17 ans qui étaient peu motivés. Enfin, il est apparu lors de cette même étude réalisée à l'échelle nationale que les lecteurs « engagés » issus de familles plus défavorisées tant en termes de moyens financiers que de niveau de formation obtenaient de meilleurs résultats que les jeunes moins engagés vivant dans des familles plus privilégiées. Selon Guthrie etWigfield, si les élèves deviennent des lecteurs engagés, ils s'offrent des occasions de se livrer à certaines formes d'apprentissage autonome qui équivalent à plusieurs années d'études. L'engagement à l'égard de la lecture peut atténuer dans une large mesure l'impact d'un milieu familial pauvre ou peu instruit (2000, p. 404).

Des études empiriques documentent également le lien entre la motivation pour la lecture, les pratiques de lecture et les compétences en compréhension de l'écrit chez les adultes. Le rapport final de IALS (OCDE et Statistique Canada, 2000) fait état d'une corrélation positive entre le niveau de littératie et l'importance de l'activité de lecture sur le lieu de travail et à la maison. Si ces variables semblent jouer un rôle moins déterminant que le niveau de formation dans une population adulte, il n'en ressort pas moins que, dans 
certains pays (au Chili et en Communauté flamande de Belgique, par exemple), les pratiques de lecture figurent parmi les quatre facteurs qui ont le plus d'impact sur le niveau de littératie.

\section{L'évaluation de l'engagement à l'égard de la lecture dans PISA}

L'engagement à l'égard de la lecture est mesuré via les réponses des élèves aux questions concernant le temps consacré à la lecture, l'intérêt et les attitudes à l'égard de la lecture ainsi que la diversité et les contenus de lecture. Cette mesure est une extension de l'indice d'engagement à l'égard de la lecture présenté dans Connaissances et compétences: des atouts pour la vie. Premiers résultats de PISA (OCDE, 2001b). L'indice d'engagement à l'égard de la lecture utilisé dans le présent rapport a été construit de manière à saisir le concept d'une façon plus large et davantage en conformité avec la théorie, tout en restant cohérent avec la mesure antérieure de l'engagement à l'égard de la lecture, essentiellement axée sur les attitudes des élèves vis-à-vis de la lecture.

Lors du cycle PISA 2000, les élèves ont été priés de répondre à plusieurs questions conçues pour évaluer des composantes de leur engagement à l'égard de la lecture 2 . Dans la littérature, le concept d' «engagement à l'égard de la lecture » englobe deux aspects : les pratiques et les attitudes. En effet, les lecteurs « engagés » lisent régulièrement différents types d'écrits, ils adoptent des attitudes positives à l'égard de la lecture, ils portent un grand intérêt à cette activité et ils sont très motivés pour la lecture. Ils estiment que la lecture est une activité intéressante, source de plaisir et de connaissance.

Trois séries de questions relatives à la notion d'engagement dans la lecture ont été posées dans le questionnaire Élève de PISA.

- Temps consacré à la lecture : les élèves ont indiqué combien de temps ils consacraient chaque jour à la lecture pour le plaisir. Ils ont été priés de répondre à cette question en choisissant l'option décrivant le mieux le temps passé à lire parmi les cinq proposées, allant de «Je ne lis pas pour mon plaisir » à «Plus de deux heures par jour ».

- Diversité et contenu des lectures : les élèves ont été priés d'identifier les types de textes lus par plaisir dans la liste proposée, qui comprenait des journaux, des revues, des bandes dessinées, des livres de fiction, des ouvrages documentaires, du courrier électronique ou des pages web. Il leur a été demandé de préciser la fréquence à laquelle ils lisaient chacun des types d'écrit en choisissant parmi des options allant de « Jamais » à « Plusieurs fois par semaine».

- Intérêt et goût pour la lecture : une échelle d'attitudes comportant neuf propositions, positives ou négatives, à propos de la lecture a été incluse dans le questionnaire Élève. Les élèves ont été priés d'indiquer s'ils étaient d'accord ou non avec ces propositions en choisissant une option parmi les quatre proposées, allant de «Pas du tout d'accord » à «Tout à fait d'accord».

\section{Les profils de lecture}

On sait qu'il existe une corrélation positive entre le temps consacré à la lecture et le niveau de compétence en compréhension de l'écrit. Mais qu'en est-il de la manière dont ce temps est mis à profit ? Est-il préférable de lire certains textes plutôt que d'autres ? Smith (1996) livre une étude intéressante à ce sujet sur la base des résultats de la « National Adult Literacy Survey » (NALS) réalisée aux États-Unis. Smith a élaboré des profils de lecture sur la base de la fréquence à laquelle plusieurs types d'écrit sont lus ${ }^{3}$. Les adultes qui lisent beaucoup un type d'écrit, quel qu'il soit, obtiennent en moyenne de meilleurs résultats que ceux qui ont déclaré ne pas lire du tout. Les gains de performance sont significatifs à chaque fois qu'un type d'écrit 


\section{Graphique 5.1}

Pourcentages moyens d'élèves déclarant lire chaque type d'écrit souvent, occasionnellement ou jamais, par profil de lecteur, tous pays de l'OCDE confondus
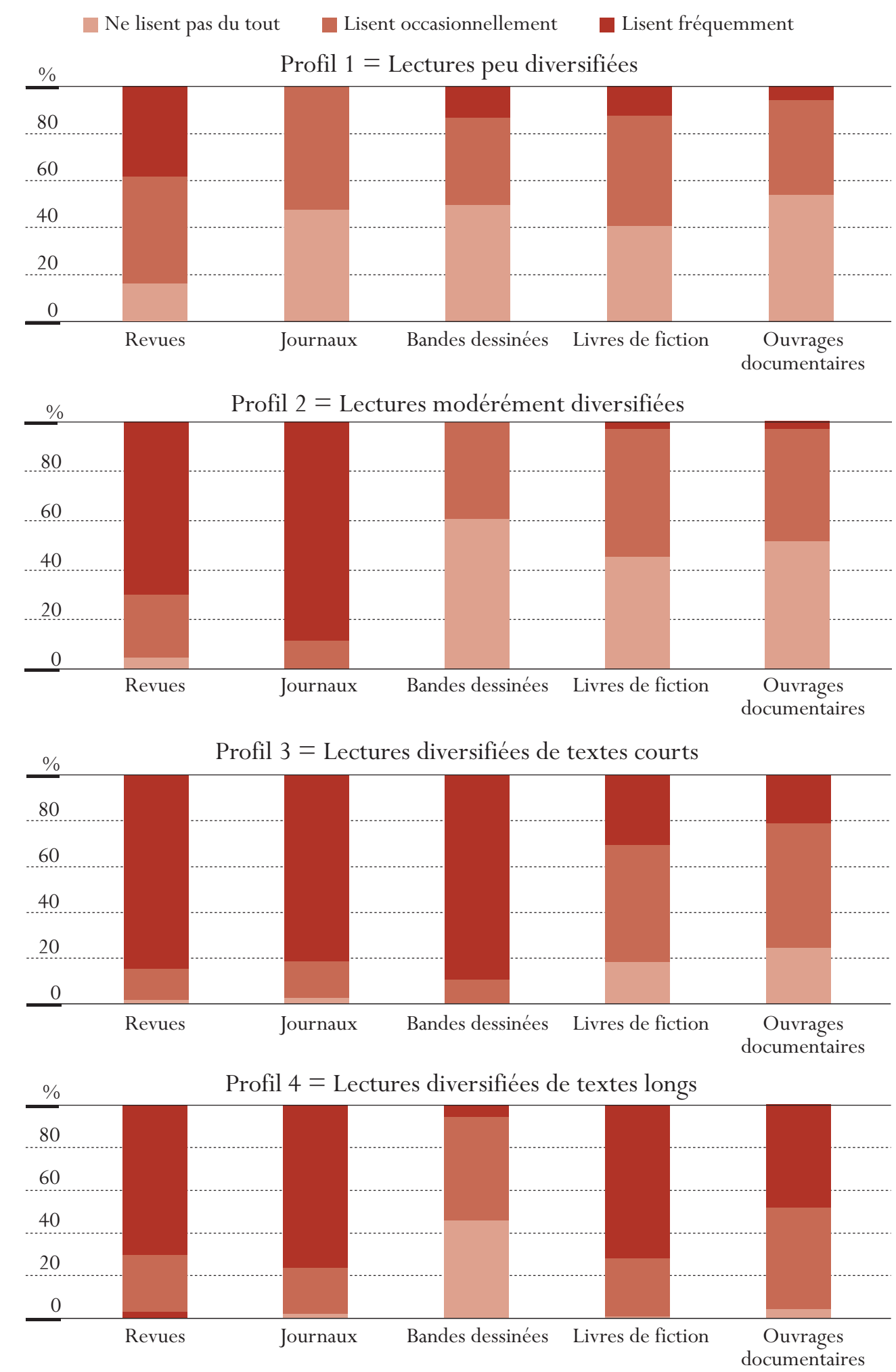

Source: Base de données PISA de l'OCDE, 2001, tableau 5.1. 
est ajouté, même s'ils perdent de l'importance à partir de quatre types d'écrit. Deux types de matériel en particulier - les livres et les documents professionnels - sont fortement corrélés à l'augmentation du niveau de performance. Smith a établi qu'il existait un lien entre les pratiques de lecture et la performance en compréhension de l'écrit même quand l'âge et le niveau d'éducation sont tenus sous contrôle.

Lors du cycle PISA 2000, les élèves ont été priés d'indiquer la fréquence à laquelle ils lisaient différents types d'écrit. Les analyses statistiques révèlent que le pattern de réponse à cette question est multidimensionnel, et que les élèves tendent à s'investir davantage dans certains types d'écrit que dans d'autres. Pour identifier ces patterns ou profils différents, les données ont été soumises à une analyse typologique ${ }^{4}$. Quatre grands profils de lecteurs ressortent de l'analyse.

Le graphique 5.1 montre les quatre grands profils de lecteur et indique les pourcentages d'élèves qui ont déclaré lire fréquemment, occasionnellement ou jamais chaque type de matériel ${ }^{5}$. Des chiffres plus détaillés sont présentés dans le tableau 5.1.

Le premier profil (groupe 1) est celui que présentent les élèves qui ont les lectures les moins diversifiées Le seul type de texte que ces élèves déclarent lire sont les revues (38 pour cent d'entre eux disent en lire souvent). Un petit pourcentage affirme lire souvent des livres de fiction (12 pour cent) ou des bandes dessinées (13 pour cent), et le pourcentage d'élèves déclarant lire des ouvrages documentaires est encore plus faible (6 pour cent).

Selon toute vraisemblance, ces élèves ne lisent pas pour le plaisir mais se servent probablement de certains matériels à des fins utilitaires limitées. La lecture de magazines peut correspondre à une forme restreinte de lecture informative (prendre connaissance des programmes de télévision ou de cinéma, par exemple) ou thématique (à propos d'un hobby, par exemple). Selon nos estimations, 22 pour cent des élèves présentent ce profil dans les pays de l'OCDE.

La majorité des élèves qui présentent le deuxième profil (groupe 2) déclarent lire souvent des revues (70 pour cent en moyenne) et des journaux ( 89 pour cent) mais rarement des livres (seuls 3 pour cent d'entre eux affirment lire souvent des livres de fiction ou des ouvrages documentaires) et presque jamais des bandes dessinées ( 0,5 pour cent seulement d'entre eux reconnaissent en lire souvent). Les élèves de ce groupe ne diversifient que modérément leurs lectures et ne lisent probablement que pour obtenir certaines informations dans des revues et dans des journaux. Malheureusement, nous ne connaissons pas la nature des magazines qu'ils lisent, ni des rubriques des journaux qui retiennent leur attention. Comme le montre le tableau 5.1, quelque 27 pour cent des élèves de PISA présentent ce profil.

La grande majorité des élèves qui présentent le troisième profil (groupe 3) affirment lire souvent des revues ( 85 pour cent) et des journaux ( 81 pour cent), comme ceux du groupe 2, mais aussi des bandes dessinées (89 pour cent). Ils peuvent être considérés comme des lecteurs occasionnels de livres de fiction (ce type d'écrit est lu fréquemment par 30 pour cent environ d'entre eux et occasionnellement par 51 pour cent d'entre eux) et d'ouvrages documentaires (21 pour cent des élèves affirment en lire souvent et 54 pour cent, occasionnellement). Par comparaison avec les élèves qui présentent les deux premiers profils, ces élèves diversifient davantage leurs lectures et s'engagent plus dans la lecture, mais ils préfèrent des textes qui sont assez courts et qui ne sont pas trop exigeants (ils lisent tous les types d'écrit, sauf les livres). Environ 28 pour cent des élèves de PISA présentent ce profil. 
Les élèves qui présentent le quatrième profil (groupe 4) diversifient également leurs lectures mais préfèrent les textes plus longs et plus exigeants (les livres). La majorité d'entre eux déclarent lire souvent des revues (70 pour cent), des journaux (76 pour cent), des livres de fiction (72 pour cent) et, dans une moindre mesure, des ouvrages documentaires (48 pour cent). Ils sont relativement peu nombreux à affirmer lire souvent des bandes dessinées ( 6 pour cent). Le contraste le plus frappant entre les élèves de ce groupe et ceux du groupe précédent porte sur la fréquence de la lecture de livres, par opposition à celle de journaux, de revues et de bandes dessinées. Près de 22 pour cent des élèves de PISA présentent ce dernier profil.

\section{Graphique 5.2}

Pourcentage d'élèves par profil de lecteur et score moyen par pays sur l'échelle combinée de compréhension de l'écrit

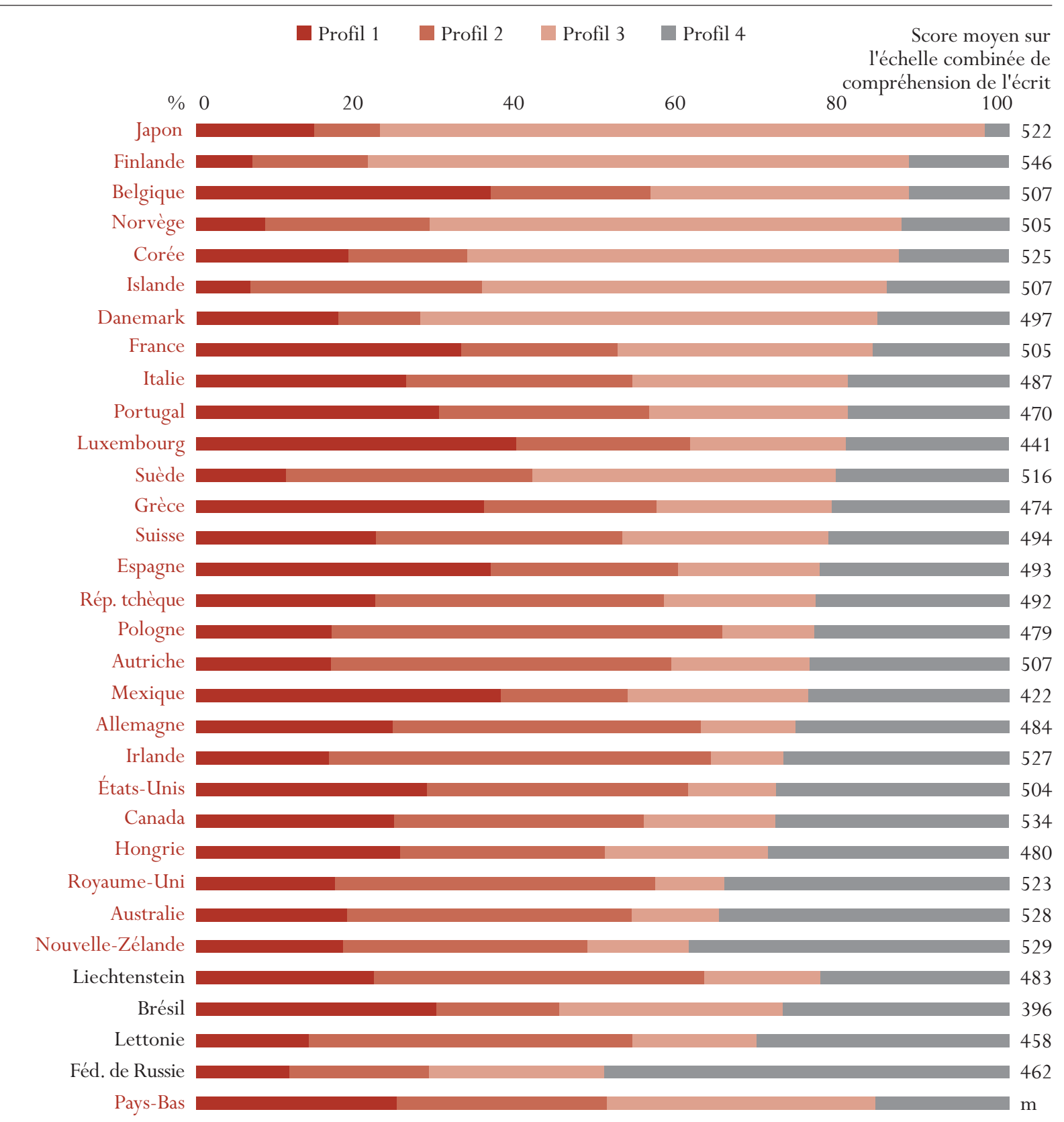

Source: Base de données PISA de l'OCDE, 2001, tableau 5.2. 


\section{Profils de lecture par pays}

Sachant que les quatre profils de lecteurs identifiés révèlent des différences dans les types d'écrit que les élèves déclarent lire, il est intéressant d'étudier la répartition de ces profils dans les pays. Le tableau 5.2 présente les chiffres à ce propos. En général, ce sont les pays d'Europe du Nord qui comptent les plus petits pourcentages d'élèves dans le groupe 1 (lectures les moins diversifiées) : 6,6 pour cent des élèves en Islande, 6,9 pour cent en Finlande, 8,5 pour cent en Norvège et 11,1 pour cent en Suède. À l'autre extrême, plusieurs pays affichent des proportions d'élèves supérieures à 30 pour cent dans ce groupe. Il s'agit de la Belgique (36,3 pour cent), de l'Espagne (36,2 pour cent), de la France (32,6 pour cent), de la Grèce (35,4 pour cent), du Luxembourg (39,4 pour cent) et du Mexique (37,5 pour cent). Quatre de ces pays accusent un score moyen significativement inférieur à la moyenne de l'OCDE (500) en compréhension de l'écrit, tandis que deux pays, la Belgique et la France, affichent un score moyen très légèrement supérieur à la moyenne, de 507 et 505 respectivement.

La proportion d'élèves qui ont des lectures diversifiées et lisent de longs textes (c'est-à-dire ceux qui présentent le quatrième profil) varie également selon les pays (voir le tableau 5.2). Elle ne représente que quelques pour cent au Japon ( 3 pour cent), se situe entre 10 et 20 pour cent dans des pays tels que la Belgique et la Finlande (12,3 pour cent), la Norvège (13,3 pour cent) et le Danemark (16,2 pour cent), mais dépasse les 30 pour cent en Australie (35,7 pour cent), en Nouvelle-Zélande (39,4 pour cent) et au Royaume-Uni (35,1 pour cent). De toute évidence, les pays dans lesquels de nombreux élèves lisent souvent tous les types de texte y compris des livres affichent des scores moyens nettement supérieurs à la moyenne de l'OCDE. La réciproque n'est toutefois pas vraie. Parmi les pays où cette proportion d'élèves est faible figurent la Finlande et le Japon, deux pays dont le score moyen est largement supérieur à la moyenne de l'OCDE. Ce phénomène s'explique en partie par le fait que dans ces deux pays, entre deux tiers et trois quarts des élèves présentent le troisième profil : ils diversifient leurs lectures mais préfèrent les textes plus courts et déclarent lire souvent des journaux, des revues et des bandes dessinées et, dans une moindre mesure, des livres de fiction.

\section{Profils de lecture et performances par pays}

Quels profils de lecteurs paraissent être le plus étroitement associés à de meilleures performances en compréhension de l'écrit? Dans les pays de l'OCDE, il existe une forte corrélation entre le niveau de littératie et la fréquence de lecture de divers écrits, ainsi que le montre l'analyse typologique. Les élèves présentant le premier profil, c'est-à-dire ceux qui diversifient le moins leurs lectures, obtiennent le score moyen le plus faible sur l'échelle combinée de compréhension de l'écrit (486). Ce score est significativement inférieur à la moyenne de l'OCDE (voir le tableau 5.2). Dans ce classement, ils sont suivis par les élèves qui diversifient modérément leurs lectures (le deuxième profil). Ces élèves affichent un score moyen de 498, proche de la moyenne de l'OCDE. Les élèves qui diversifient leurs lectures mais préfèrent les textes courts (le troisième profil) obtiennent un score moyen de 514 sur l'échelle combinée de compréhension de l'écrit, ce qui est significativement supérieur à la moyenne de l'OCDE. Enfin, les élèves qui s'investissent dans des lectures diversifiées - ceux qui déclarent lire un grand éventail d'écrits, y compris les textes plus exigeants (tels que des livres de fiction et des ouvrages documentaires) - le quatrième profil - obtiennent un score de 539. Leur score est significativement supérieur à la moyenne de l'OCDE et à celui des élèves qui présentent le troisième profil. La différence moyenne entre les élèves qui diversifient le plus (quatrième profil) et le moins (premier profil) leurs lectures est de 71, soit l'équivalent de près d'un niveau de compétence ${ }^{7}$. L'écart moyen entre ceux qui diversifient le moins leurs lectures (premier 


\section{Graphique 5.3}

Performance des élèves sur l'échelle combinée de compréhension de l'écrit par profil de lecteur

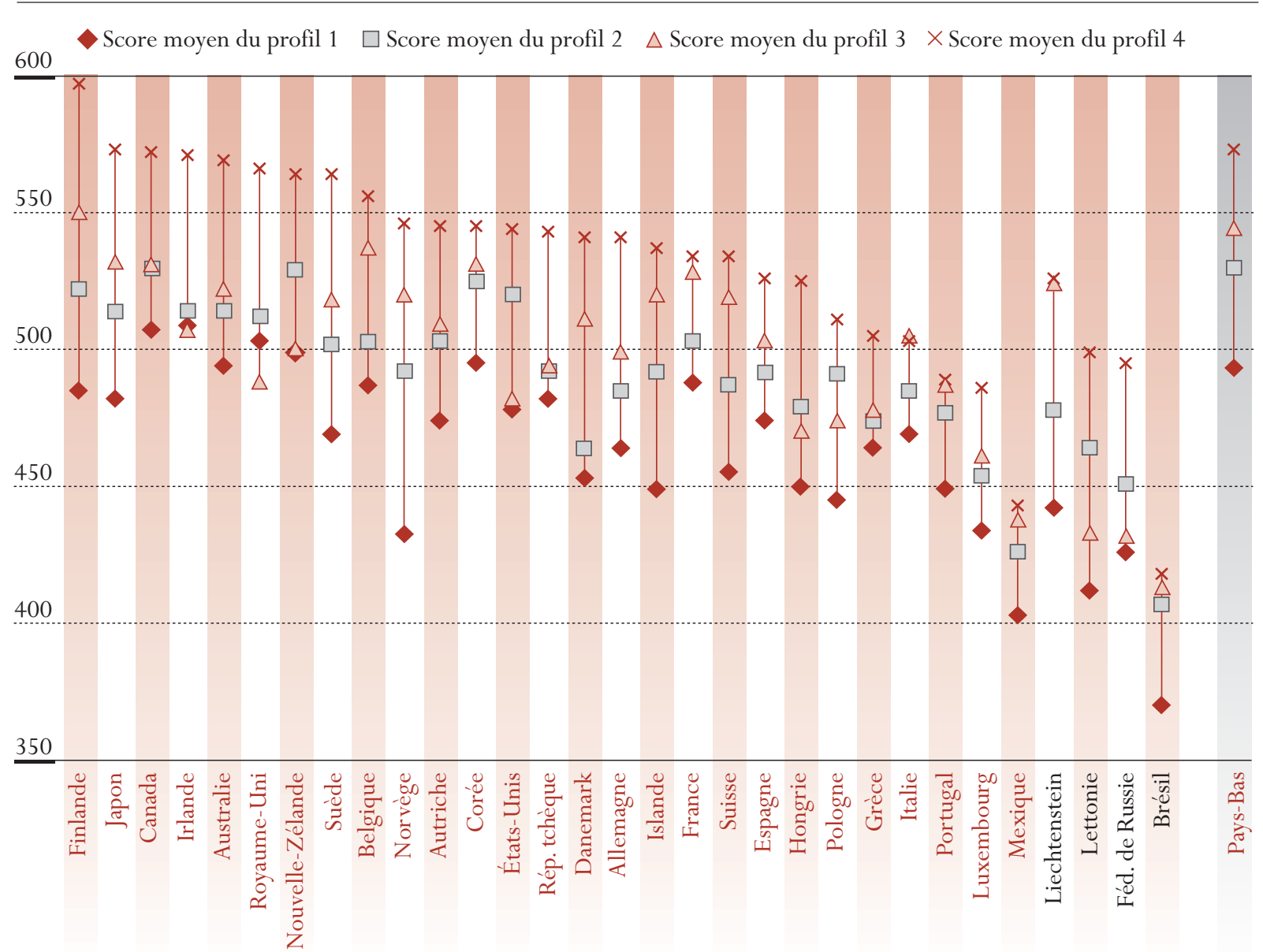

Source : Base de données PISA de l'OCDE, 2001, tableau 5.2.

profil) et ceux qui les diversifient modérément (deuxième profil) correspond déjà à 30, soit plus du tiers d'un niveau de compétence.

À quelques exceptions près, la tendance observée dans l'ensemble de l'OCDE se vérifie dans chaque pays. Dans tous les pays, sauf en Italie, les élèves qui diversifient leurs lectures et lisent de longs textes obtiennent le meilleur score moyen en compréhension de l'écrit. En Italie, deux points seulement, soit un écart non significatif, séparent les élèves présentant le troisième et le quatrième profil, deux profils qui se distinguent par la longueur des textes lus.

À l'autre extrême, les élèves qui diversifient le moins leurs lectures obtiennent en moyenne les scores les plus faibles. Deux pays, l'Irlande et le Royaume-Uni, font toutefois figure d'exception. Dans ces deux pays, ce sont les élèves présentant le troisième profil (lectures diversifiées de textes courts) qui obtiennent les scores les plus faibles en moyenne. En Irlande, il n'existe pas d'écart significatif entre les scores des élèves qui présentent les trois premiers profils. Et pourtant, les élèves qui diversifient le plus leurs lectures et qui lisent des textes longs (quatrième profil) atteignent un score moyen supérieur de près d'un niveau de compétence à celui des élèves qui présentent les trois premiers profils. Il est intéressant de souligner par ailleurs que dans ces deux pays, les proportions d'élèves qui présentent le troisième profil sont assez 
faibles ( 8,9 pour cent en Irlande et 8,4 pour cent au Royaume-Uni), ce qui pourrait expliquer les résultats légèrement différents de ces pays.

Dans plusieurs pays anglophones (les États-Unis, l'Irlande, la Nouvelle-Zélande et le Royaume-Uni) ainsi que dans plusieurs pays d'Europe centrale et de l'Est (la Fédération de Russie, la Hongrie, la Lettonie et la Pologne), les élèves qui présentent le deuxième profil obtiennent de meilleurs scores sur l'échelle combinée de compréhension de l'écrit que ceux présentant le troisième profil, qui diversifient pourtant davantage leurs lectures. Ce constat est frappant et, somme toute, inattendu : les élèves qui déclarent lire divers types d'écrit, surtout des bandes dessinées et, dans une moindre mesure, des livres, sont moins brillants que ceux qui disent ne lire que des revues et des journaux. Le plus surprenant est que ce modèle est associé à une caractéristique linguistique ou culturelle, dans la mesure où il est spécifiquement observé dans des pays anglophones et dans des pays d'Europe centrale et de l'Est.

Profils de lecture et niveaux de compétence ; liens avec les sous-échelles de compréhension de l'écrit

L'analyse de la distribution des quatre profils de lecteurs sur les niveaux de compétence en compréhension de l'écrit permet d'étudier sous un autre angle la relation entre la littératie et la fréquence des lectures et la diversité des écrits. Le graphique 5.4. met en évidence quelques différences intéressantes chez les

\section{Graphique 5.4}

Pourcentage d'élèves par profil de lecteur et par niveau de compétence en compréhension de l'écrit, tous pays de l'OCDE confondus

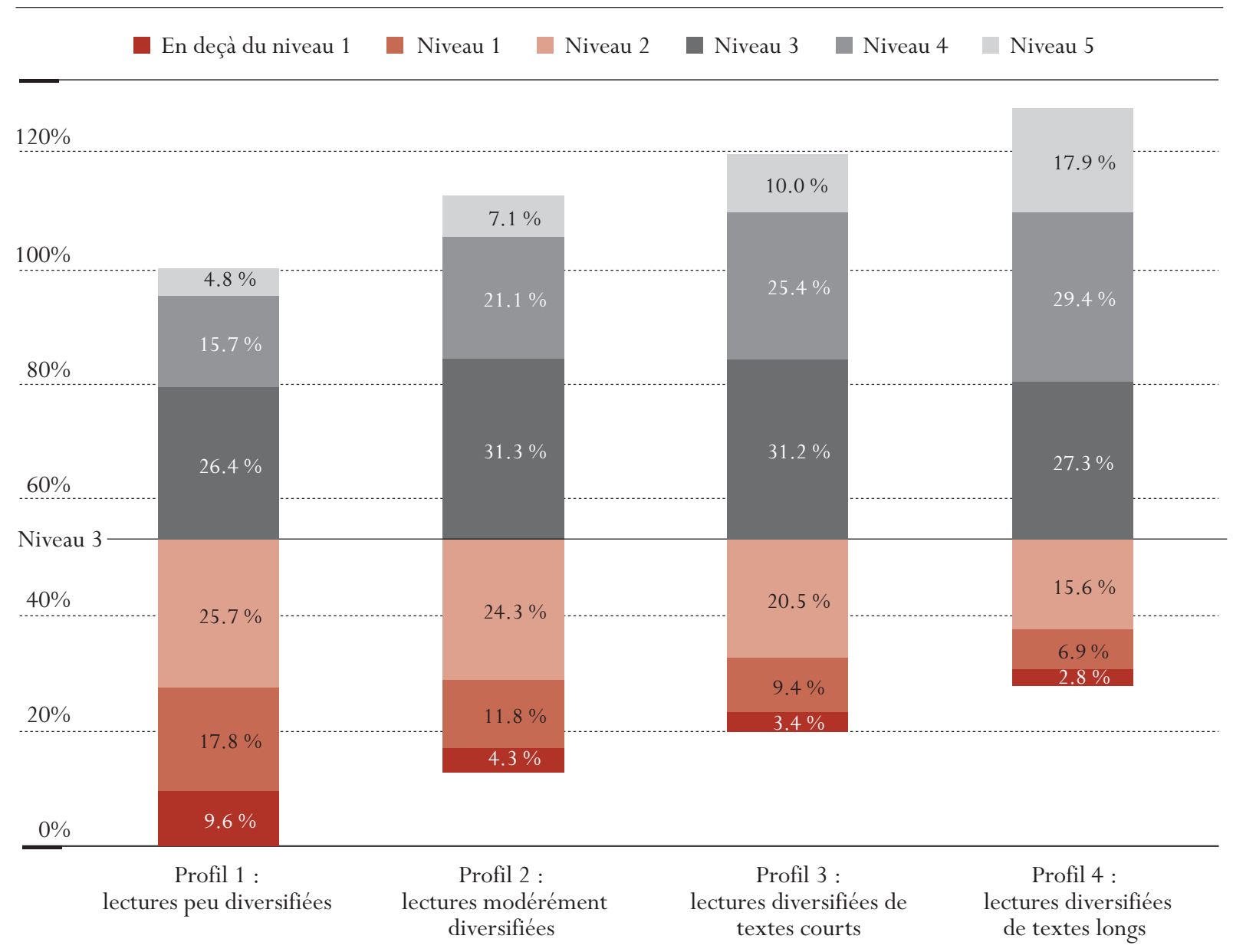


élèves qui présentent le premier profil. C'est le groupe de lecteurs dont les lectures sont les moins diversifiées qui compte la proportion la plus importante ( 9,6 pour cent) d'élèves situés en deçà du niveau 1 (voir le tableau 5.3). Dans les trois autres groupes de lecteurs, on trouve une proportion d'élèves en deçà du niveau 1 deux fois moindre. Bien qu'il n'existe pas de différences importantes entre les proportions d'élèves situés en deçà du niveau 1 dans les trois autres profils, la tendance est à une diminution du nombre de ces élèves quand on passe du deuxième au quatrième profil. Cette tendance s'observe également chez les élèves qui se situent au niveau 1 de compétence en compréhension de l'écrit. La proportion la plus importante d'élèves de ce niveau se retrouve dans le groupe des lecteurs qui diversifient le moins leurs lectures (premier profil). Ces élèves situés au niveau 1 sont de moins en moins nombreux à mesure que la diversification des lectures s'accentue (du deuxième au quatrième profil).

La tendance s'inverse aux niveaux 4 et 5 de compétence. C'est dans le groupe des lecteurs qui diversifient le moins leurs lectures (premier profil) que la proportion d'élèves situés à ces niveaux de compétence est la plus faible. Ces élèves sont de plus en plus nombreux dans les groupes correspondant aux autres profils qui représentent un accroissement progressif de la diversification des types d'écrits et de l'investissement dans la lecture. Il est intéressant de constater que près de $50 \%$ des élèves qui ont des lectures diversifiées de textes longs se situent aux niveaux 4 et 5 de compétences en compréhension de l'écrit, alors qu'ils sont moins de $20 \%$ dans ce cas pour le profil le moins diversifié.

Comme nous l'avons dit dans les chapitres précédents, des sous-échelles distinctes de compétence ont été élaborées pour trois aspects de la compréhension de l'écrit (la localisation d'informations, l'interprétation de textes, et la réflexion et l'évaluation) et deux formats de texte (les textes continus et non continus). Des analyses ont été effectuées pour déterminer si les différences de compétence associées aux profils de lecteurs étaient plus ou moins prononcées sur les trois sous-échelles d'aspect et les deux sous-échelles de format. La tendance générale observée sur l'échelle combinée de compréhension de l'écrit se confirme sur chacune des cinq sous-échelles, ainsi que le montre le graphique 5.5. En effet, les élèves qui diversifient le moins leurs lectures (premier profil) obtiennent le score moyen le plus faible sur chacune des souséchelles. Le score moyen augmente progressivement chez les élèves qui diversifient modérément leurs lectures (deuxième profil), qui diversifient leurs lectures mais préfèrent les textes courts (troisième profil) et, enfin, qui diversifient leurs lectures et lisent de longs textes (quatrième profil). Sur les sous-échelles, les écarts moyens entre les élèves présentant le premier et le quatrième profil - c'est-à-dire entre ceux qui diversifient le moins leurs lectures et ceux qui les diversifient le plus et lisent de longs textes - sont compris entre 69 sur l'échelle d'interprétation de textes et 74 sur celle de localisation d'informations. Ces écarts moyens sont plus ou moins équivalents à un niveau de compétence, soit trois-quarts environ de la valeur d'un écart type (voir le tableau 5.4).

La comparaison des scores sur les sous-échelles révèle quelques différences modestes mais intéressantes. Il existe par exemple des différences chez les élèves qui diversifient le moins leurs lectures (premier profil). Bien que ces élèves obtiennent les scores moyens les plus faibles sur chacune des sous-échelles de compréhension de l'écrit, le score moyen de ces élèves sur la sous-échelle de localisation d'informations est inférieur à leur score moyen sur les sous-échelles d'interprétation de textes et de réflexion et d'évaluation. De même, leur score moyen sur la sous-échelle de textes non continus est inférieur à celui enregistré sur la sous-échelle des textes continus. Une autre différence s'observe chez les élèves qui diversifient le plus leurs lectures et lisent de longs textes (quatrième profil). Ces élèves ont tendance à obtenir de meilleurs scores sur la sous-échelle de réflexion et d'évaluation ainsi que sur celle des textes continus. Cette tendance pourrait s'expliquer par le fait que ces élèves s'investissent souvent dans la lecture de textes plus exigeants tels que des livres. 


\section{Graphique 5.5}

Scores moyens par profil de lecteur sur l'échelle combinée de compréhension de l'écrit et sur les sous-échelles, tous pays de l'OCDE confondus

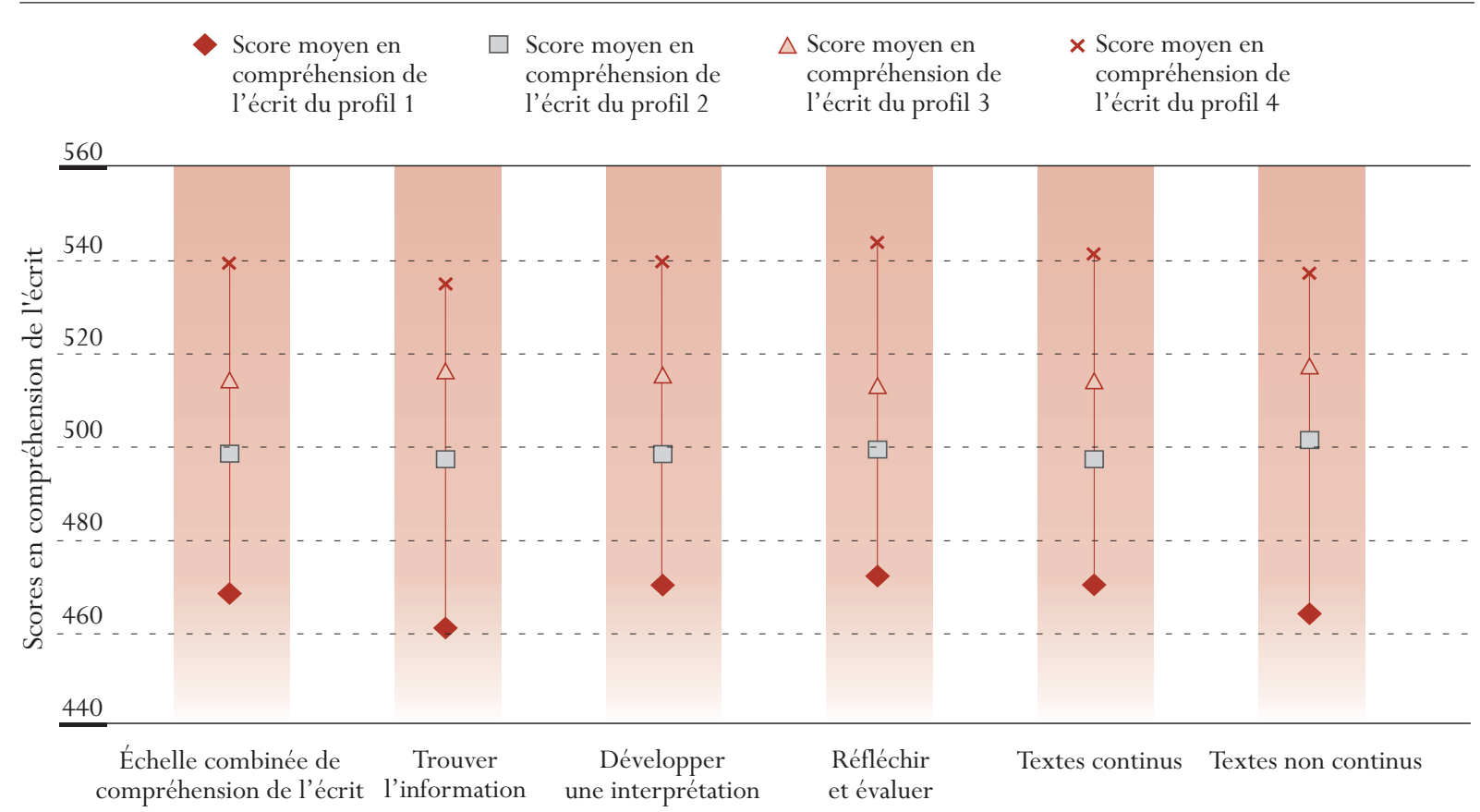

Source: Base de données PISA de l'OCDE, 2001, tableau 5.4.

\section{Relations entre les profils de lecture et certaines caractéristiques des élèves et de leur milieu}

Nous venons de présenter les profils de lecture et leur répartition dans les pays et d'étudier la relation entre ce construct et le niveau de compétence en compréhension de l'écrit. Nous allons maintenant analyser la relation entre ces profils et plusieurs variables de contexte. Seules des variables clés sont abordées ici : le sexe, le milieu socio-économique, les caractéristiques culturelles familiales et l'accès des élèves à l'écrit (le nombre de livres disponibles à la maison et la fréquence des emprunts de livres). Nous nous limiterons ici aux analyses bivariées. Certaines des variables envisagées peuvent être corrélées entre elles, comme le nombre de livres à la maison et le milieu socio-économique, par exemple. Les interactions plus complexes entre les variables seront présentées dans le chapitre 7.

\section{Les profils de lecture en fonction du sexe}

Le sexe est incontestablement un facteur crucial. De nombreuses études menées dans divers pays ont montré que les filles lisent davantage que les garçons. En moyenne, il apparaît qu'elles passent plus de temps à lire mais aussi qu'elles diversifient davantage leurs lectures. Le graphique 5.6 indique les pourcentages de filles et de garçons qui présentent chacun des quatre profils de lecteurs.

Le graphique 5.6 montre qu'il existe une différence manifeste dans les profils de lecteurs des filles et des garçons. Les garçons déclarent plus souvent que les filles qu'ils lisent principalement des journaux, des magazines et des bandes dessinées, et non des livres (surtout de fiction). Cette différence se marque bien chez les élèves qui présentent le troisième profil : dans les pays de l'OCDE, ce groupe accueille en moyenne 34 pour cent des garçons, contre 23 pour cent des filles (voir tableau 5.5). À l'inverse, tous pays 


\section{Graphique 5.6}

Pourcentage d'élèves présentant chaque profil de lecteur, en fonction du sexe

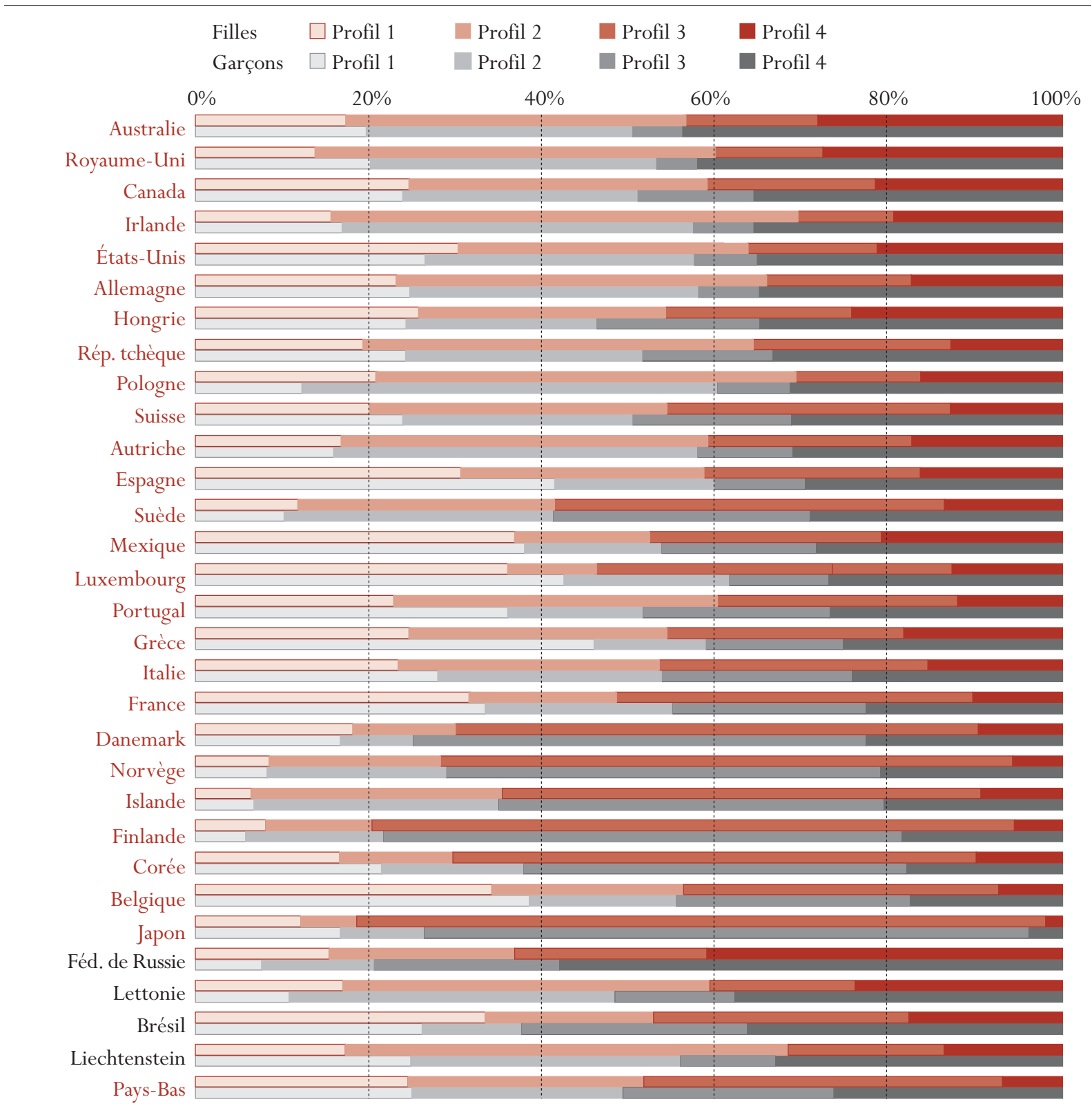

Source : Base de données PISA de l'OCDE, 2001, tableau 5.5.

confondus, les filles (29 pour cent en moyenne) sont plus nombreuses que les garçons (16 pour cent) dans le groupe correspondant au quatrième profil. Elles déclarent lire des journaux, des revues et des livres (surtout de fiction), mais pas de bandes dessinées. Il est utile de rappeler ici la différence entre le troisième et le quatrième profil : les lecteurs qui présentent ces deux profils diversifient leurs lectures, mais les premiers lisent de préférence des textes courts et les seconds, essentiellement des livres. Les filles sont de toute évidence plus attirées par la fiction. Cette tendance s'observe dans tous les pays participant à PISA. 
Les différences entre les filles et les garçons sont moins marquées dans les groupes correspondant aux profils 1 et 2, même si elles varient selon les pays (voir le tableau 5.5). Commençons par les élèves qui diversifient modérément leurs lectures et s'intéressent aux revues et aux journaux (deuxième profil). En moyenne dans l'OCDE, les garçons (30 pour cent) sont légèrement plus nombreux dans ce groupe que les filles (25 pour cent). Dans plusieurs pays, le Mexique, la Norvège, la Pologne et la Suède, les proportions de garçons et de filles sont identiques dans le groupe correspondant à ce profil, tandis que dans quatre autres pays, en Corée, en Finlande, en France et au Japon, les filles sont plus nombreuses que les garçons dans ce groupe. Pour le premier profil, les différences entre filles et garçons sont généralement peu prononcées. Dans la moitié des pays, les garçons sont plus nombreux que les filles, tandis que la situation inverse prévaut dans l'autre moitié des pays. Tous pays de l'OCDE confondus, les filles (24 pour cent) sont légèrement plus nombreuses que les garçons (21 pour cent) dans ce premier profil. La répartition moins stable entre les sexes observée dans les deux premiers profils s'explique probablement par la lecture fréquente de revues, commune à ces deux types de lecteurs. La catégorie « revues » couvre un vaste éventail d'écrits, présente une hétérogénéité extrême et comprend des magazines typiquement masculins ou féminins et des magazines assez neutres.

\section{Les profils de lecture en fonction du milieu socio-économique}

Dans l'ensemble, la corrélation entre les profils de lecture et le milieu socio-économique des élèves est faible ${ }^{8}$. Comme l'indique le tableau 5.6, le milieu socio-économique moyen est légèrement supérieur chez les élèves qui présentent le troisième et le quatrième profils, à savoir ceux qui déclarent lire souvent des revues, des journaux et des bandes dessinées (groupe $3: 49,7$ ) et ceux qui affirment lire fréquemment divers types d'écrits dont des livres (groupe $4: 51,6$ ). Il est légèrement inférieur chez les élèves associés au premier et au deuxième profils, c'est-à-dire ceux qui lisent uniquement des revues (groupe $1: 46,0$ ) et ceux qui lisent principalement des revues et des journaux (groupe $2: 48,6$ ). Mais les différences moyennes sont faibles dans tous les pays. Il est rassurant de constater que le milieu socio-économique, même s'il joue un rôle à cet égard, n'est pas un facteur prépondérant pour la diversification des écrits et l'engagement dans la lecture.

\section{Les profils de lecture en fonction de l'accès à l'écrit}

Par comparaison avec l'influence du milieu socio-économique, la relation entre le profil des lecteurs et l'accès à l'écrit à la maison' (l'accès à des livres, à des recueils de poésie et à des oeuvres de littérature classique à la maison) est plus forte (voir le tableau 5.7). Les élèves qui présentent le quatrième profil, c'est-à-dire ceux qui diversifient le plus leurs lectures et lisent des livres, jouissent d'un meilleur accès à l'écrit à la maison (la valeur moyenne de l'indice est de 0,36 ). Ils sont suivis dans ce classement par les élèves associés au troisième profil, à savoir ceux qui lisent souvent des revues, des journaux et des bandes dessinées (valeur moyenne de 0,11). Les élèves qui lisent principalement des revues et des journaux (deuxième profil) ou uniquement des revues (premier profil) ont moins accès à l'écrit chez eux (la valeur moyenne de l'indice est respectivement de - 0,13 et de -0,30). Il existe de toute évidence une forte corrélation entre l'accès à l'écrit à la maison et les profils des lecteurs. Les élèves qui n'ont accès qu'à un nombre limité de livres chez eux tendent à s'investir peu ou modérément dans des lectures diversifiées et lisent essentiellement des revues et/ou des journaux. En revanche, les élèves qui ont accès à un plus grand nombre de livres ont tendance à s'intéresser davantage à la lecture et à lire un éventail plus large d'écrits. L'accès à l'écrit à la maison semble donc jouer un rôle plus important que le milieu socio-économique, qui s'est avéré peu déterminant pour le profil de lecture. 


\section{Engagement à l'égard de la lecture}

Le graphique 5.7 indique la valeur moyenne de l'indice d'engagement à l'égard de la lecture dans chaque pays. La valeur moyenne de cet indice est fixée à 0 et l'écart type, à 1 pour l'ensemble des élèves de l'OCDE. Une valeur négative ne signifie pas forcément que les élèves ont répondu aux questions par la négative, mais indique que les élèves d'un pays donné ont dans l'ensemble répondu de manière moins positive que ne l'ont fait en moyenne les autres élèves de l'OCDE. À l'inverse, une valeur positive signifie que les élèves d'un pays donné ont répondu plus favorablement que ne l'ont fait en moyenne les autres élèves de l'OCDE.

Graphique 5.7

Différence d'engagement à l'égard de la lecture entre les filles et les garçons

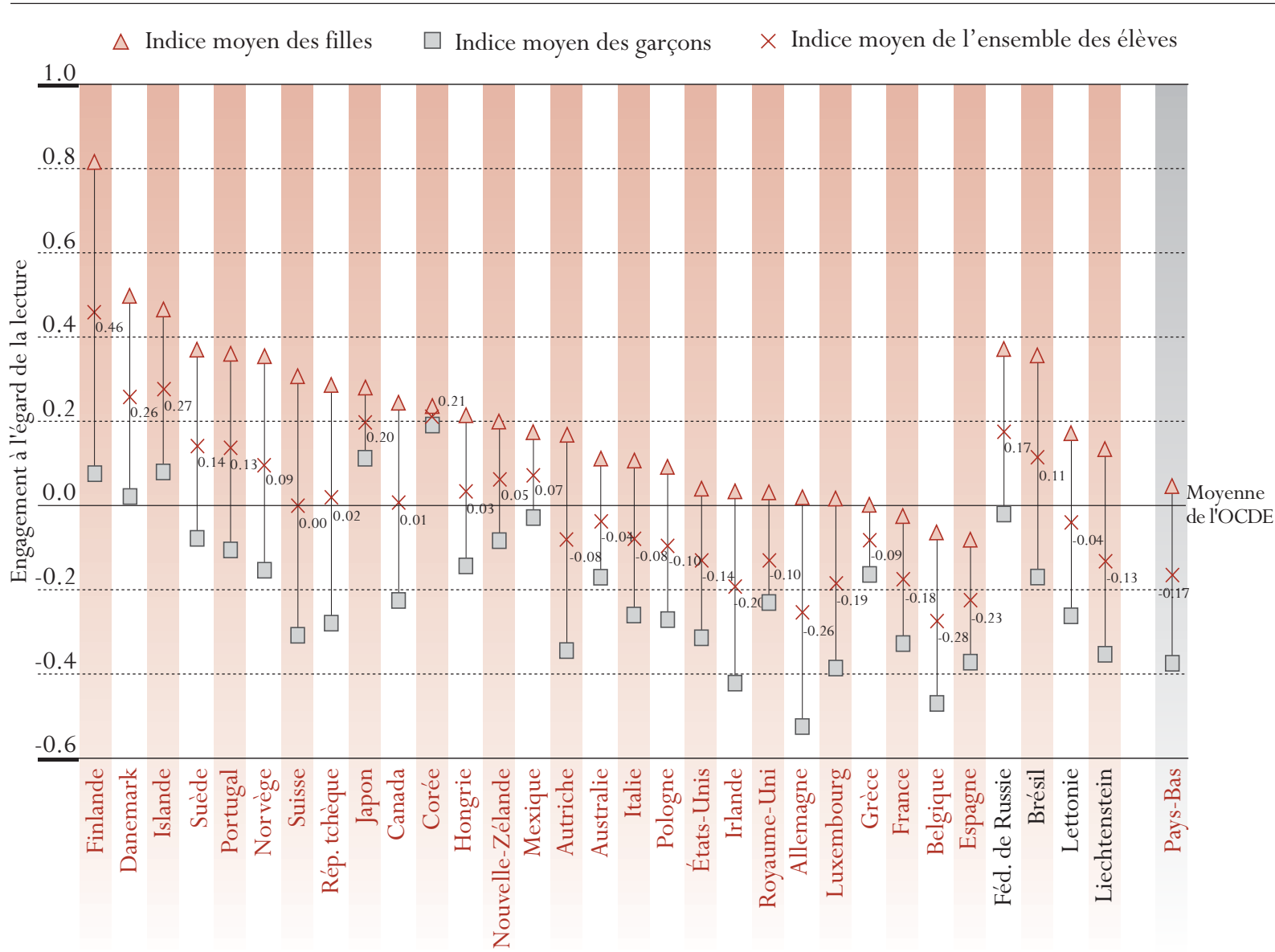

Source : Base de données PISA de l'OCDE, 2001, tableau 5.8.

Le pays qui affiche de loin le degré le plus élevé d'engagement à l'égard de la lecture est la Finlande $(0,46)$. Le degré d'engagement est important en Corée $(0,21)$, au Danemark $(0,26)$, en Islande $(0,27)$ et au Japon $(0,20)$, mais relativement faible en Allemagne $(-0,26)$, en Belgique $(-0,28)$, en Espagne $(-0,23)$, en Irlande $(-0,20)$ et au Luxembourg $(-0,19)$. 


\section{Le degré d'engagement à l'égard de la lecture en fonction du sexe}

Il existe une différence significative entre les filles et les garçons à cet égard. L'écart moyen entre les filles et les garçons est de 0,38 tous pays de l'OCDE confondus (voir le tableau 5.8). Tous les pays ont donc dans l'ensemble le même défi à relever. S'ils veulent renforcer l'engagement à l'égard de la lecture, il faut imaginer des mesures à l'intention des garçons. Les raisons qui expliquent leur degré plus faible d'engagement et les solutions qui permettraient de le rehausser sont abondamment discutées. Certains experts estiment que les garçons font l'objet de discriminations à l'école et que c'est essentiellement aux textes stéréotypés utilisés dans le cadre scolaire qu'il faut imputer le fait qu'ils ne développent pas leur littératie. D'autres soutiennent que les garçons qui décident de ne pas lire sont fortement influencés dans cette attitude par leurs condisciples et les normes sociales de masculinité (Young et Brozo, 2001). Le manque d'engagement à l'égard de la lecture constaté chez les garçons n'est qu'un symptôme d'une attitude plus générale à l'égard du travail scolaire. Selon Jackson (1998), les garçons qui réussissent bien à l'école doivent habilement gérer leur vie scolaire pour éviter d'afficher tout engagement manifeste vis-à-vis des études, qui les exposerait aux railleries de leurs condisciples (Mac An Ghaill, 1994, cité par Young et Brozo).

Le degré le plus élevé d'engagement à l'égard de la lecture s'observe chez les filles au Danemark $(0,50)$, en Finlande $(0,82)$, en Islande $(0,46)$, en Norvège $(0,35)$, au Portugal $(0,36)$ et en Suède $(0,37)$, tandis que le degré le plus faible s'observe chez les garçons en Allemagne (- 0,53), en Belgique (- 0,48), en Espagne $(-0,38)$, en Irlande $(0,43)$ et au Luxembourg $(-0,39)$. L'écart entre les filles et les garçons est particulièrement prononcé dans des pays comme la Finlande $(0,75)$ et la Suisse $(0,61)$. Il est relativement faible dans quelques pays, comme la Corée $(0,04)$, la Grèce $(0,17)$, le Japon $(0,17)$ et le Mexique $(0,20)$. Aucun pays ne présente d'écart en faveur des garçons.

Si le degré d'engagement des filles à l'égard de la lecture est plus élevé que celui des garçons, la comparaison des chiffres entre divers pays révèle des différences intéressantes. Ainsi, il apparaît que le degré d'engagement des garçons de certains pays est plus élevé que celui des filles d'autres pays. Le degré d'engagement des garçons en Corée, au Danemark, en Finlande, en Islande et au Japon est équivalent, sinon supérieur à celui des filles en Belgique, en Espagne ou en France.

\section{L'engagement à l'égard de la lecture peut-il atténuer les inégalités liées au milieu social ?}

Selon la littérature, l'engagement à l'égard de la lecture peut atténuer l'effet d'un milieu familial peu aisé et peu instruit (Guthrie et Wigfield, 2000) ou d'autres facteurs contextuels (Campbell, Voelkl et Donahue, 1997). Les données de PISA ont été analysées afin de déterminer si ce phénomène, principalement observé aux États-Unis, présentait une dimension internationale.

Pour étudier ce phénomène, les élèves ont été répartis en neuf groupes en fonction de deux variables : le niveau d'engagement à l'égard de la lecture et le statut professionnel des parents. Trois groupes ont été créés en fonction de ces deux indices : le groupe inférieur (en-dessous du $25^{\mathrm{e}}$ percentile), le groupe médian (entre le $25^{\mathrm{e}}$ et le $75^{\mathrm{e}}$ percentile) et le groupe supérieur (au-dessus du $75^{\mathrm{e}}$ percentile). Cette classification ${ }^{10}$ a été utilisée pour répartir les élèves en neuf catégories. Le graphique 5.8 compare les pourcentages attendus et observés pour chacune des neuf catégories dans les pays de l'OCDE.

Parmi les élèves dont les parents ont le statut professionnel le moins élevé, les élèves faiblement engagés à l'égard de la lecture sont légèrement plus nombreux qu'ils ne le seraient si les deux variables avaient été complètement indépendantes. De même, les élèves très engagés à l'égard de la lecture sont plus nombreux qu'attendu dans le groupe d'élèves dont l'indice de statut professionnel est le plus élevé. Il est toutefois 


\section{Graphique 5.8}

Pourcentages d'élèves attendus et observés en fonction du degré d'engagement

à l'égard de la lecture et du milieu socio-économique

\begin{tabular}{|c|c|c|c|c|c|c|}
\hline & \multicolumn{2}{|c|}{$\begin{array}{c}\text { Engagement faible dans } \\
\text { la lecture }\end{array}$} & \multicolumn{2}{|c|}{$\begin{array}{c}\text { Engagement moyen dans la } \\
\text { lecture }\end{array}$} & \multicolumn{2}{|c|}{$\begin{array}{c}\text { Engagement important dans } \\
\text { la lecture }\end{array}$} \\
\hline & Attendu (\%) & Observé (\%) & Attendu (\%) & Observé (\%) & Attendu (\%) & Observé (\%) \\
\hline $\begin{array}{l}\text { Milieu socio- } \\
\text { économique inférieur }\end{array}$ & 6.25 & 7.60 & 12.50 & 12.56 & 6.25 & 4.85 \\
\hline $\begin{array}{l}\text { Milieu socio- } \\
\text { économique moyen }\end{array}$ & 12.25 & 12.90 & 25.00 & 25.14 & 12.25 & 11.96 \\
\hline $\begin{array}{l}\text { Milieu socio- } \\
\text { économique supérieur }\end{array}$ & 6.25 & 4.50 & 12.50 & 12.30 & 6.25 & 8.19 \\
\hline
\end{tabular}

intéressant de constater que l'engagement à l'égard de la lecture n'est que partiellement déterminé par le statut professionnel des parents. Le graphique 5.9 montre en effet que certains élèves issus de milieux socio-économiques peu favorisés sont très engagés à l'égard de la lecture et que d'autres vivant dans des milieux plus privilégiés le sont peu. Ce constat nous amène naturellement à étudier la répartition de ces élèves en fonction de leur niveau de compétence en compréhension de l'écrit.

Les élèves dont les parents présentent l'indice de statut professionnel le plus élevé et qui sont très engagés dans la lecture obtiennent les meilleurs scores moyens sur l'échelle combinée de compréhension de l'écrit (583). Leur score est supérieur de plus d'un niveau de compétence, soit 0,83 fraction d'écart type, à la moyenne de l'OCDE. À l'inverse, les élèves dont les parents accusent l'indice de statut professionnel le plus faible et qui sont le moins engagés à l'égard de la lecture obtiennent le score moyen le plus faible (423). Leur score est inférieur d'un niveau de compétence à la moyenne de l'OCDE et s'écarte de plus d'un écart type et demi du score obtenu par les élèves qui affichent des valeurs élevées pour les deux indices. Une autre conclusion, plus importante encore à nos yeux, s'impose : les élèves de 15 ans qui s'investissent beaucoup dans la lecture et dont les parents présentent le statut professionnel le plus faible obtiennent en compréhension de l'écrit des scores moyens (540) supérieurs à ceux dont les parents affichent le statut professionnel le plus élevé mais qui ne s'investissent guère dans la lecture (491). L'écart entre les scores de ces deux groupes représente plus de la moitié d'un écart type. Et ces élèves très engagés dont les parents présentent un statut professionnel peu élevé obtiennent des scores équivalents à ceux des élèves dont l'indice d'engagement est moyen, mais dont les parents affichent un statut professionnel élevé.

En compréhension de l'écrit, tous les élèves très engagés dans la lecture obtiennent des scores significativement supérieurs à la moyenne internationale, quel que soit leur milieu familial. En revanche, les scores des élèves peu engagés à l'égard de la lecture sont inférieurs à cette moyenne, quel que soit le statut professionnel de leurs parents. Dans chaque groupe constitué sur base du statut professionnel, les élèves les moins engagés à l'égard de la lecture obtiennent des scores moyens inférieurs de 85 à 117 sur l'échelle combinée de compréhension de l'écrit à ceux des élèves très engagés à l'égard de la lecture. L'écart le plus élevé s'observe parmi les élèves appartenant au groupe de statut professionnel faible. Ces résultats revêtent une importance cruciale pour le monde de l'éducation et sont peut-être les plus intéressants de ce rapport. 
CHAPITRE 5 La lecture, moteur de changement: Performance et engagement d'un pays à l'autre

\section{Graphique 5.9}

Performance en compréhension de l'écrit en fonction du milieu socio-économique et du degré d'engagement à l'égard de la lecture

Milieu socio-économique inférieur $\square$ Milieu socio-économique moyen $\triangle$ Milieu socio-économique supérieur $\underline{600}$

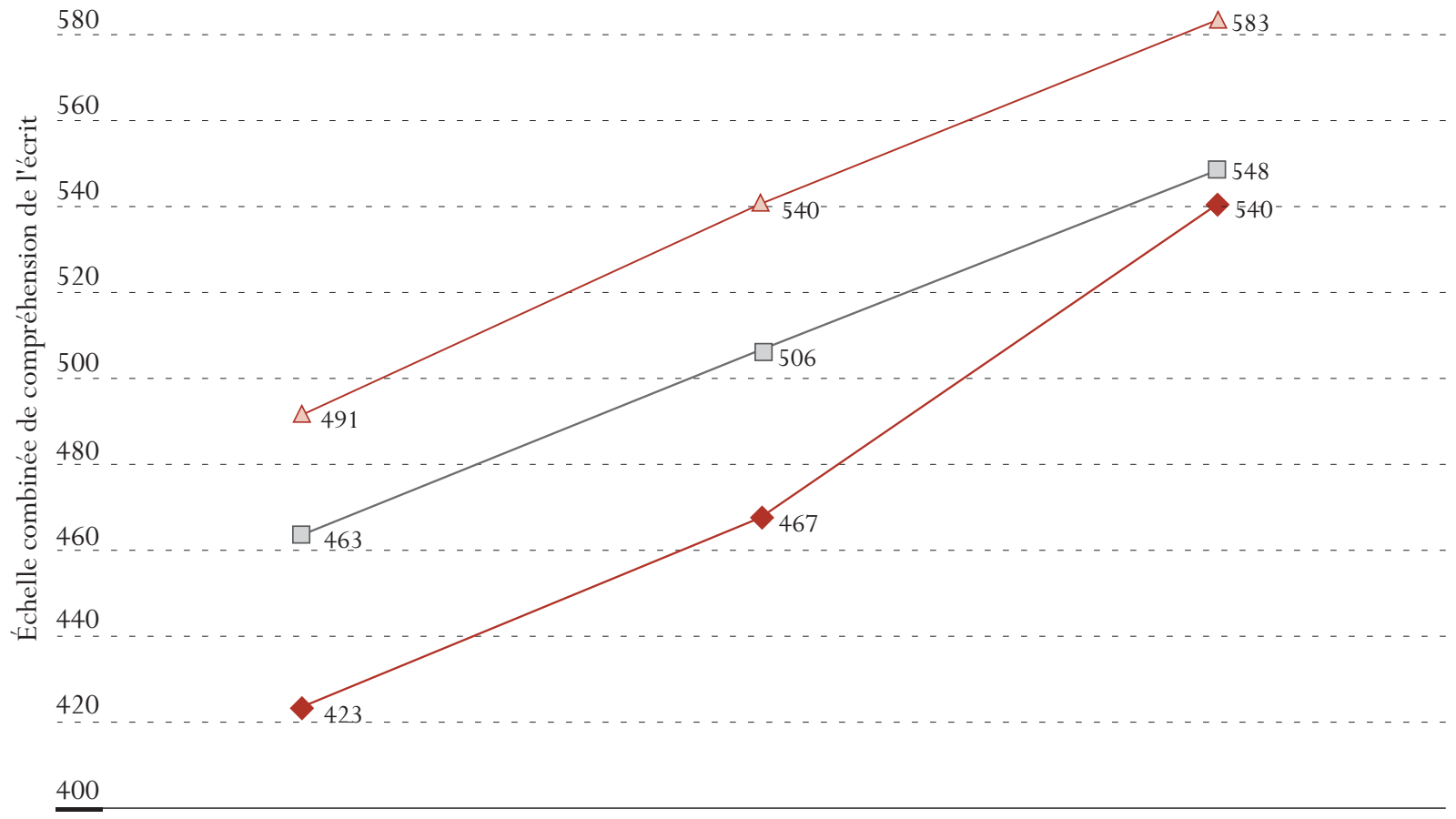

Degré faible d'engagement

Degré moyen d'engagement

Degré élevé d'engagement

Source: Base de données PISA de l'OCDE, 2001, tableau 5.9

Les pratiques de lecture peuvent jouer un rôle important pour réduire les écarts entre les scores de compréhension de l'écrit des élèves issus de milieux socio-économiques différents, voire les écarts observés entre les filles et les garçons. Il est établi que certaines pratiques pédagogiques sont très efficaces pour renforcer l'engagement des élèves à l'égard de la lecture (Burns, 1998 ; Ivey, 1999). Guthrie et Davis (2002) ont élaboré un modèle d'engagement au travers des pratiques pédagogiques en classe qui visent à motiver les élèves en difficulté lors du premier cycle de l'enseignement secondaire.

Les lecteurs en difficulté ont besoin non seulement d'un soutien sur le plan cognitif mais aussi sur le plan socio-affectif. Ce soutien est davantage bénéfique lorsqu'il passe par des interactions avec des textes intéressants et authentiques, par l'autonomie et la collaboration (avec d'autres élèves). Ces qualités d'ordre socio-affectif ne garantissent toutefois pas les gains sur le plan cognitif. L'amélioration des compétences cognitives demande aussi de longues périodes d'enseignement des stratégies de lecture. Il est établi que l'enseignement de stratégies n'est guère efficace s'il ne porte pas sur des contenus « motivants » (Guthrie, Schafer, Vonsecker et Alban, 2000). L'enseignement stratégique direct devient performant quand il est assorti des composantes motivationnelles évoquées ci-avant (Guthrie et Davis, 2002). 
Les composantes cognitives et non cognitives de l'engagement à l'égard de la lecture vont clairement de pair. Nous avons montré dans ce chapitre à quel point elles étaient indissociables. La cognition et la motivation, la littératie et l'engagement à l'égard de la lecture sont tous liés. Il est impossible de discerner la cause de l'effet. Les lecteurs performants s'investissent davantage dans la lecture, ce qui leur permet d'acquérir de nouvelles connaissances et compétences. En revanche, les élèves peu engagés dans la lecture estiment souvent que les textes sont trop difficiles (Allington, 1984) et développent des attitudes négatives à l'égard de la lecture (Oka et Paris, 1986). Ils entrent dès lors dans un cercle vicieux, car le fait de lire moins réduit leurs chances d'élaborer des stratégies en compréhension de l'écrit (Brown, Palincsar et Purcell, 1986), et restent à la traîne dans toutes les matières puisque la lecture est indispensable pour progresser dans les autres disciplines (Chall, Jacobs et Baldwin, 1990). Les modèles pédagogiques contemporains visant à améliorer la littératie ont bien mis cela en évidence et soulignent que le niveau de compétence ne peut être rehaussé qu'à condition de tenir compte des composantes non cognitives de l'engagement à l'égard de la lecture : la motivation pour la lecture et l'accès à des écrits intéressants et porteurs de sens. Les résultats des analyses de régression présentées dans les chapitres suivants de ce rapport viendront corroborer cette manière de voir. 
CHAPITRE 5 La lecture, moteur de changement: Performance et engagement d'un pays à l'autre

\section{Notes}

1. L'indice élargi d'« engagement à l'égard de la lecture » est corrélé à 0,88 avec l'indice utilisé dans le rapport initial et son indice de fidélité de 0,83 est plus élevé que celui qui caractérisait la mesure antérieure d'engagement à l'égard de la lecture $(0,72)$.

2. La plupart des indices présentés dans ce chapitre sont basés sur les déclarations des élèves à propos de leurs comportements et préférences, et non sur des observations directes. Pour cette raison, la désirabilité sociale ou des différences culturelles dans la manière de répondre pourraient avoir influencé les réponses des élèves. Il convient de faire preuve de prudence lors des comparaisons, même si les instruments sont basés sur des recherches reconnues et ont été abondamment testés avant d'être utilisés dans le cycle PISA 2000.

3. Dans l'étude de Smith, la liste d'écrits comprend les journaux, les magazines, les livres, les documents personnels et les documents professionnels.

4. Les courriers électroniques et les pages web n'ont pas été inclus dans l'analyse typologique, car il ne semble pas exister de relation entre ces écrits et les autres matériels.

5. Pour cette question, la lecture d'un type de texte est considérée comme fréquente lorsqu'elle est pratiquée «plusieurs fois par mois » ou « plusieurs fois par semaine », occasionnelle lorsqu' elle l'est « quelques fois par an » ou « environ une fois par mois » et inexistante lorsqu' elle ne l'est « jamais ou presque jamais ».

6. La distinction entre les quatre profils repose sur deux variables : la fréquence de la lecture et la diversité des écrits. L'expression « engagé dans la lecture d'écrits divers » traduit bien cette double dimension. Les noms donnés aux profils seront simplifiées dans les pages suivantes par souci de pragmatisme et de clarté. Ils mentionneront explicitement la notion de diversité, mais intégreront implicitement celle d'intensité.

7. Un niveau de compétence équivaut à un intervalle de 72 sur l'échelle combinée de compréhension de l'écrit.

8. Le milieu socio-économique des élèves est mesuré au moyen de l'indice socio-économique international de statut professionnel (ISEI). L'indice retenu dans le PISA pour chaque élève est soit la profession du père, soit celle de la mère, suivant lequel des deux parents a le statut le plus élevé. Les valeurs de cet indice sont comprises entre 0 et 90 . Les valeurs faibles de cet indice indiquent un statut socio-économique modeste et les valeurs élevées de cet indice, un statut socio-économique élevé. La valeur moyenne de l'OCDE est de 49.

9. Un indice portant sur l'accès à l'écrit à la maison a été élaboré sur la base d'une série de trois questions relatives au nombre de livres à la maison et à la présence d'un dictionnaire et de manuels à la maison. Les sept catégories de réponse à la question sur le nombre de livres à la maison ont été recodées pour constituer une variable dichotomique. Une valeur de zéro a été attribuée aux catégories 1 à 4 (de zéro à 100 livres) et une valeur de 1 a été attribuée aux catégories 5 à 7 (de 101 à plus de 500 livres).

10. Cette répartition en trois groupes a été adoptée pour permettre les comparaisons entre pays. 
Chapitre

6

RELATIONS ENTRE LES

CARACTÉRISTIQUES

CONTEXTUELLES ET LES

PERFORMANCES EN LECTURE

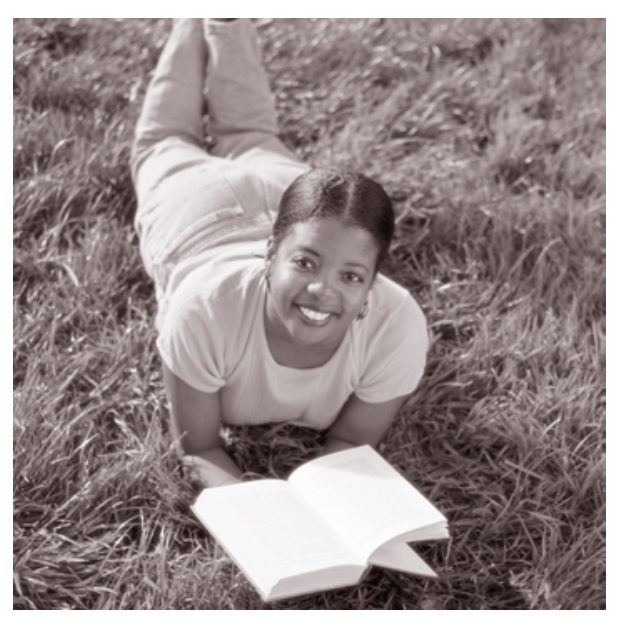




\section{SYNTHÈSE}

- Le statut professionnel des parents, le nombre de livres et les ressources éducatives à la maison ainsi que la communication culturelle sont des variables qui influent sur la performance en compréhension de l'écrit. Les corrélations sont positives dans tous les pays qui ont participé à PISA. Il est intéressant de constater que la corrélation entre l'engagement à l'égard de la lecture et le milieu socio-économique ne représente qu'un tiers de la corrélation avec la performance en compréhension de l'écrit : 0,12 contre 0,34. Par contraste, l'engagement à l'égard de la lecture est nettement plus corrélé au nombre de livres à la maison et à la communication culturelle.

- Parmi les caractéristiques personnelles des élèves, c'est l'engagement à l'égard de la lecture qui présente la plus forte corrélation médiane avec la performance en compréhension de l'écrit. La corrélation médiane entre le sexe et les performances en lecture et celle entre le temps consacré aux devoirs et ces performances sont du même ordre. En revanche, le temps consacré aux devoirs est plus étroitement corrélé à l'engagement à l'égard de la lecture que ne l'est le sexe. Par ailleurs, le temps consacré aux devoirs est plus étroitement corrélé à l'engagement à l'égard de la lecture qu'aux performances en lecture. Tous ces résultats indiquent que, dans chaque pays, l'engagement à l'égard de la lecture peut dans une large mesure contribuer à combler les écarts de performance observés entre les divers sous-groupes.

- Il existe une interaction intéressante entre le sexe et les sous-échelles de compréhension de l'écrit. Dans tous les pays, c'est sur la sous-échelle de textes non continus que l'écart le plus faible s'observe entre les sexes. La corrélation médiane est de 0,19 sur la sous-échelle de textes continus, soit plus du double de celle enregistrée sur l'échelle de textes non continus $(0,09)$.

- Les proportions d'élèves de 15 ans allochtones sont assez faibles dans tous les pays mais, de toute évidence, les élèves de cette catégorie sont pénalisés par leur statut en termes de performance sur l'échelle combinée de compréhension de l'écrit. Par contre, il semble que la relation entre le statut d'allochtone et l'engagement à l'égard de la lecture soit nettement moins marquée et moins uniforme.

- Il existe une corrélation positive entre la relation des élèves avec les enseignants et l'engagement à l'égard de la lecture. Le pattern de cette relation est homogène dans tous les pays, qu'ils soient membres de l'OCDE ou non. Le climat de discipline est une autre caractéristique propre à l'environnement scolaire, qui est associée de façon positive aux performances et à l'engagement. En revanche, il semble que le lien soit moins net entre le sentiment d'appartenance et l'incitation au rendement scolaire d'une part, et la performance et l'engagement à l'égard de la lecture d'autre part. 
Dans les deux chapitres précédents, nous nous sommes surtout intéressés aux scores moyens des élèves de 15 ans des pays participants et à leur répartition ainsi qu'à leur engagement à l'égard de la lecture. Ces aspects sont essentiels dans la mesure où ils sont considérés comme des produits importants de l'éducation et donnent une idée de ce que l'on attend des élèves au sortir de l'enseignement secondaire. Comme le soulignent Gray et Rogers, en littératie, la maturité en lecture se caractérise par «l'intérêt, les attitudes et les savoir-faire qui permettent à chaque personne de se livrer, de manière indépendante et efficace, aux activités de lecture qui sont essentielles pour vivre pleinement une vie riche et productive » (Gray et Rogers, 1956, p. 56). Dans ce chapitre, nous étudions la relation entre une série de caractéristiques propres aux élèves, à leur famille et à leur environnement d'apprentissage et les résultats en compréhension de l'écrit et l'engagement à l'égard de la lecture'. Nous savons que ces variables n'agissent pas indépendamment l'une de l'autre, mais nous estimons utile de les traiter d'abord une par une pour donner au lecteur une idée de leur impact propre tant sur la compréhension de l'écrit que sur l'engagement à l'égard de la lecture. Dans le chapitre 7, nous poursuivrons cette analyse en examinant l' «importance » relative de chaque variable après avoir pris en compte leurs interactions respectives.

Les élèves ont été invités à répondre à un questionnaire qui comportait une série de questions sur leur milieu familial, leurs activités extrascolaires et leur perception de certains aspects de leur environnement d'apprentissage en classe. Les réponses des élèves à ces questions ont permis d'étudier l'impact de ces caractéristiques sur leurs résultats en compréhension de l'écrit et leur engagement à l'égard de la lecture. Dans quelle mesure les scores des filles sont-ils supérieurs à ceux des garçons sur les sous-échelles de compréhension de l'écrit ? Dans quelle mesure les filles s'investissent-elles plus que les garçons dans la lecture ? En quoi l'engagement à l'égard de la lecture influence-t-il les scores sur les sous-échelles de compréhension de l'écrit ? L'impact du statut d'immigré ou de la structure familiale varie-t-il selon les pays de l'OCDE ? Quelle est la nature de la relation entre, d'une part, les ressources éducatives et les formes de communication familiale et, d'autre part, l'engagement à l'égard de la lecture et le niveau de compétence en compréhension de l'écrit ? Le climat de discipline et l'incitation au rendement scolaire ont-t-ils un impact sur ces deux variables ? Autant de questions auxquelles nous allons tenter de répondre pour chacun des 28 pays participants de l'OCDE et des 4 pays non membres de l'OCDE.

\section{La compréhension de l'écrit et les caractéristiques individuelles des élèves}

Nous allons commencer notre analyse par trois variables ou indices - le sexe, l'engagement à l'égard de la lecture et le temps consacré aux devoirs. Ce sont des caractéristiques propres aux élèves qui influent sur leurs résultats et se démarquent de la structure familiale et de l'environnement d'apprentissage en classe.

Le sexe

Par le passé, ce sont les faibles résultats des filles et des femmes qui inquiétaient la quasi-totalité de ceux qui se préoccupaient des différences entre les sexes. Au fil du temps, les filles ont réduit certains de ces retards et ont même surpassé les garçons dans maints domaines de l'enseignement. Cette évolution explique pourquoi les résultats trop faibles des garçons sont maintenant devenus un sujet de préoccupation. Selon un rapport récent de l'OCDE (2001b), le taux d'obtention d'un diplôme universitaire chez les femmes est équivalent, voire supérieur à celui des hommes dans 17 des 25 pays de l'OCDE pour lesquels des données comparables sont disponibles. De plus, un rapport sur la littératie aux États-Unis avance cette hypothèse : l'écart significatif en matière de lecture entre les filles et les garçons en âge d'être scolarisés pourrait contribuer dans une large mesure à creuser le fossé qui existe entre les deux sexes en termes de fréquentation de l'enseignement supérieur et de l'obtention d'un diplôme (Sum, Kirsch et Taggert, 2002). 
Les résultats de PISA révèlent que, dans tous les pays participants, les filles obtiennent de meilleurs scores que les garçons sur l'échelle combinée de compréhension de l'écrit. Le graphique 6.1 montre que la corrélation médiane entre le sexe et la compréhension de l'écrit est de 0,16 . Le coefficient de corrélation le plus faible est enregistré en Corée $(0,10)$. En fait, dans 21 des 28 pays de l'OCDE, ce coefficient se situe entre 0,10 et 0,19. Cette corrélation a tendance à être légèrement supérieure dans les pays d'Europe du Nord où elle dépasse 0,20 partout, si ce n'est au Danemark où elle est de 0,13. Le coefficient le plus élevé de corrélation entre le sexe et le niveau de compétence est enregistré en Finlande $(0,29)^{2}$.

\section{Graphique 6.1}

Relation entre le sexe et la performance en compréhension de l'écrit d'une part, le degré d'engagement à l'égard de la lecture d'autre part

\begin{tabular}{|c|c|c|}
\hline 0.40 & $\begin{array}{l}\text { Corrélation entre le sexe et la performance sur l'échelle } \\
\text { combinée de compréhension de l'écrit }\end{array}$ & $\begin{array}{l}\square \text { Corrélation entre le sexe et l'engagement } \\
\text { à l'égard de la lecture }\end{array}$ \\
\hline
\end{tabular}

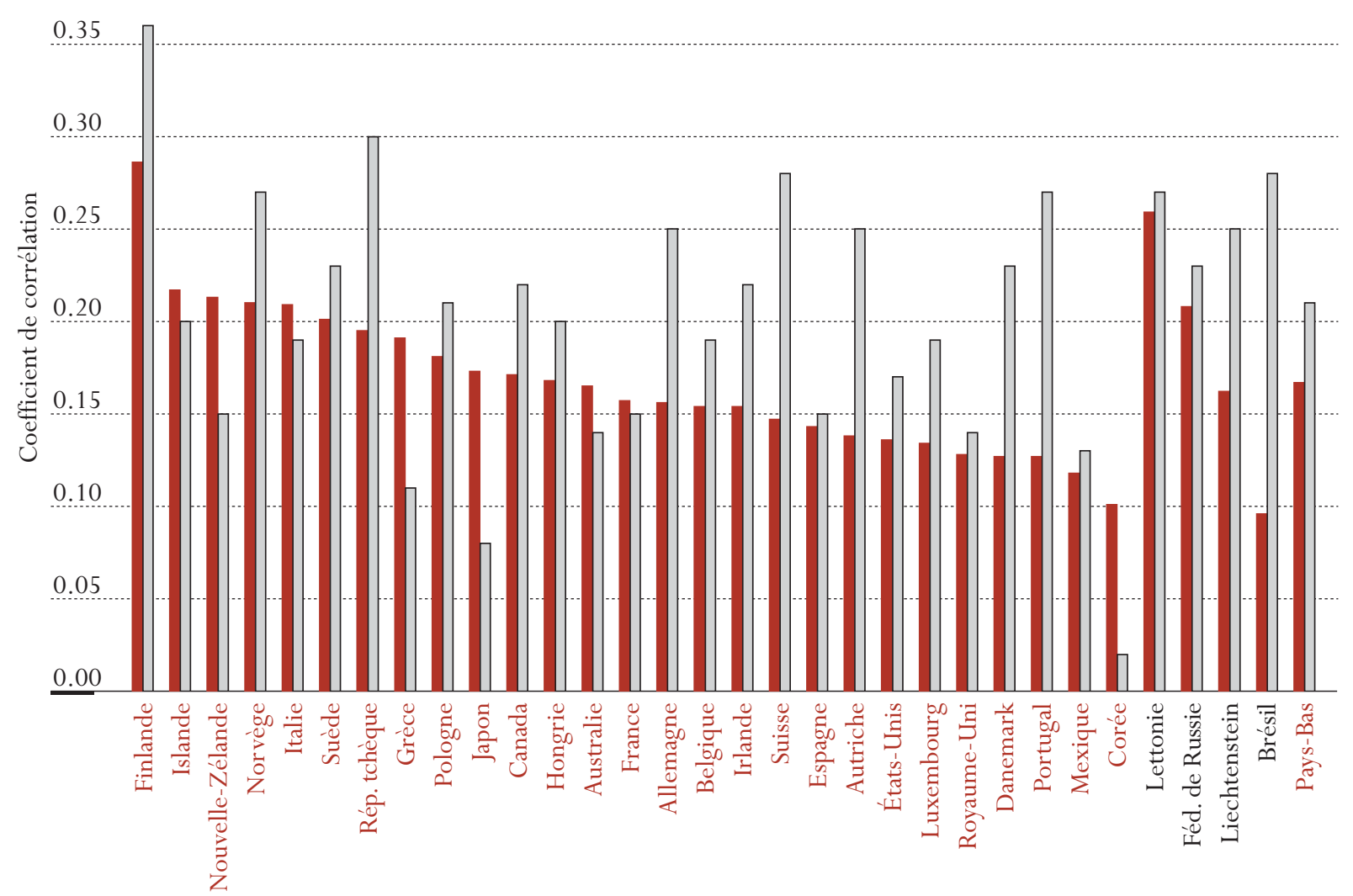

Source : Base de données PISA de l'OCDE, 2001, tableau 6.1. 


\section{Encadré 6.1 : Interprétation des données chiffrées de ce chapitre}

Toutes les données de ce chapitre sont présentées sous la forme de coefficients de corrélation. L'annexe B du rapport décrit aussi des effets de régression. Les variables et indices présentés ici sont tous intéressants en soi et leurs implications peuvent être d'importance pour l'action publique. Toutefois, les particularités de leur incidence sur la compréhension de l'écrit et l'engagement dans la lecture sont incomplètes, dans la mesure où ces variables n'agissent pas indépendamment les unes des autres. En fait, les interactions de ces variables sont complexes, car elles portent sur le développement des compétences en compréhension de l'écrit.

Toutes les données étudiées dans ce rapport sont basées sur les déclarations personnelles des élèves. Elles ont donc pu être influencées par des différences culturelles dans les comportements de réponse ou encore par la désirabilité sociale de certaines réponses. Plusieurs variables de ce chapitre (l'engagement à l'égard de la lecture, par exemple) sont présentées comme des indices qui résument les réponses des élèves à une série de questions. Ces questions ont été sélectionnées sur la base de considérations théoriques. La modélisation par équation structurelle a été utilisée pour confirmer le comportement théorique des indices et pour valider leur comparabilité entre pays. À cet effet, le modèle a été estimé séparément pour chaque pays, puis collectivement pour l'ensemble des pays de l'OCDE. Les indices de ce chapitre ont été développés de sorte que leur valeur moyenne équivale à 0 et leur écart type à 1 . En d'autres termes, les valeurs des indices de deux-tiers de l'ensemble des élèves de 15 ans de l'OCDE sont comprises entre 1 et - 1 . Il y a lieu de souligner qu' une valeur négative ne signifie pas forcément que les élèves ont répondu aux questions par la négative; elle indique simplement qu'un groupe d'élèves a répondu de manière moins positive que ne l'ont fait en moyenne les autres élèves de l'OCDE. À l'inverse, une valeur positive signifie qu'un groupe d'élèves a répondu plus favorablement que ne l'ont fait en moyenne les autres élèves de l'OCDE. D'autres variables de ce chapitre sont basées sur des échelles de réponses dichotomiques (réponse affirmative ou négative) ou à 3, 4, 5 ou 6 options décrivant une fréquence ou une probabilité. L'indice relatif au milieu social a été créé comme une échelle continue allant de 0 à 90. Il mesure les attributs professionnels en convertissant le niveau de formation en revenus. Plus la valeur de cet indice est élevée, plus le statut professionnel des parents d'élèves est élevé.

Le fait que l'analyse selon les sexes révèle certaines tendances ou interactions remarquables sur les souséchelles de compréhension de l'écrit est peut-être plus intéressant encore. Dans tous les pays participants, l'écart moyen le plus faible entre les filles et les garçons s'observe sur la sous-échelle de textes non continus, l'une des deux sous-échelles de format (voir le tableau 6.2a en annexe). La corrélation médiane des pays de l'OCDE est de 0,19 sur la sous-échelle de textes continus, soit plus du double du coefficient médian sur la sous-échelle de textes non continus $(0,9)$. Cette tendance s'observe dans tous les pays participants. La corrélation est nettement plus prononcée sur la sous-échelle de textes continus que sur la sous-échelle de textes non continus. Il ressort de la régression présentée au tableau 6.2a que dans 17 des 28 pays de l'OCDE, l'écart moyen entre les filles et les garçons sur la sous-échelle de textes non continus représente moins de 20 points, soit 20 pour cent de la valeur d'un écart type. En revanche, sur la souséchelle de textes continus, seule la Corée (19) affiche un écart moyen inférieur à 20. 
La tendance est similaire pour les trois sous-échelles d'aspect (voir le tableau 6.2a). Tous pays de l'OCDE confondus, c' est sur la sous-échelle Trouver l'information qu' on observe la corrélation médiane la plus faible entre le sexe et la performance en compréhension de l'écrit $(0,12)$. Ce coefficient augmente légèrement pour atteindre 0,15 sur la sous-échelle Développer une interprétation et passe à 0,21 sur la sous-échelle Réfléchir et évaluer. Dans tous les pays de l'OCDE, l'écart moyen entre les filles et les garçons sur la sous-échelle Réfléchir et évaluer représente plus de 25 points (le quart d'un écart type).

Comme le décrit le chapitre précédent sur l'engagement à l'égard de la lecture, les filles s'investissent généralement plus que les garçons dans la lecture. La valeur moyenne de l'indice d'engagement à l'égard de la lecture est de 0,19 chez les filles et de - 0,19 chez les garçons. De plus, même chez les garçons qui ont au moins des lectures relativement diversifiées, on observe des différences importantes dans les types d'écrit qu'ils déclarent lire par rapport aux filles. Ainsi, dans le troisième profil, on observe quelque 34 pour cent des garçons, contre 23 pour cent des filles. Par contraste, seuls 15 pour cent des garçons présentent le quatrième profil, contre 29 pour cent des filles. Les profils 3 et 4 se distinguent notamment l'un de l'autre par la quantité et le type de livres et de magazines que les élèves déclarent lire. Le troisième profil se caractérise par une proportion élevée d'élèves déclarant lire souvent des magazines (85 pour cent) et des journaux (81 pour cent), mais une proportion plus faible d'élèves reconnaissant lire souvent des livres de fiction (30 pour cent) et des ouvrages documentaires (21 pour cent seulement). En revanche, le quatrième profil présente des proportions légèrement plus faibles d'élèves qui déclarent lire fréquemment des journaux et des revues, mais une proportion nettement plus élevée d'élèves qui disent lire souvent des livres de fiction (plus de 70 pour cent) et des ouvrages documentaires (environ 50 pour cent).

Dans les pays de l'OCDE, le coefficient médian de corrélation entre le sexe et l'engagement à l'égard de la lecture est légèrement supérieur $(0,20)$ à celui observé entre le sexe et le niveau de compétence $(0,16)$. Il est toutefois intéressant de souligner que cela peut varier d'un pays de l'OCDE à l'autre. Le tableau 6.1 montre que l'écart type des corrélations entre le sexe et l'engagement à l'égard de la lecture représente près du double de celui enregistré entre le sexe et le niveau de compétence $(0,07$ contre 0,04$)$. Dans six des 28 pays de l'OCDE (la Corée, la Grèce, l'Islande, l'Italie, le Japon et la Nouvelle-Zélande), la corrélation entre le sexe et l'engagement à l'égard de la lecture est inférieure à celle entre le sexe et le niveau de compétence, alors que dans quatre pays (l'Espagne, la France, le Mexique et le Royaume-Uni), l'écart entre les deux corrélations n'est que de 0,01. Dans les 18 autres pays de l'OCDE, la corrélation entre le sexe et l'engagement à l'égard de la lecture est plus importante que celle entre le sexe et le niveau de compétence.

\section{L'engagement à l'égard de la lecture}

La motivation et le temps consacré à la lecture sont deux facteurs qui contribuent largement à creuser le fossé entre les « bons » et les « mauvais » lecteurs. La relation est sans doute réciproque, dans la mesure où les élèves plus compétents en lecture sont aussi susceptibles d'être plus motivés à lire et, donc, de passer plus de temps à lire ; en tout cas, certaines études ont établi l'existence d'une relation entre l'engagement à l'égard de la lecture et les performances en compréhension de l'écrit. Ces résultats s'appliquent aussi bien aux jeunes en âge d'être scolarisés qu'aux adultes. En fait, l'Enquête internationale sur la littératie des adultes (OCDE et Statistique Canada, 1995) a montré l'importance de la pratique et du type d'écrit que lisent les adultes pour entretenir les compétences en compréhension de l'écrit à l'âge adulte.

Le graphique 6.2 compare la corrélation entre l'engagement à l'égard de la lecture et la performance en lecture dans les pays qui ont participé à PISA. Il existe une corrélation modérément forte et significative 
entre l'engagement à l'égard de la lecture et la performance en compréhension de l'écrit dans tous les pays participants. La corrélation médiane de 0,38 indique qu'en moyenne, les élèves qui s'investissent davantage dans la lecture obtiennent des scores supérieurs sur l'échelle combinée de compréhension de l'écrit. Cette corrélation varie de 0,23 en Fédération de Russie à 0,48 en Finlande. La corrélation entre l'engagement à l'égard de la lecture et la performance est supérieure à 0,40 dans 12 des pays qui ont participé au cycle PISA 2000 : en Allemagne, en Australie, en Autriche, au Danemark, en Finlande, en Hongrie, en Islande, au Liechtenstein, en Norvège, en République tchèque, en Suède et en Suisse. Cette corrélation est sensiblement plus faible (inférieure à 0,30 ) dans plusieurs pays dont le Brésil, la Fédération de Russie, la Grèce, la Lettonie, le Luxembourg, le Mexique et la Pologne. Ces pays affichent un score moyen très inférieur à la moyenne de l'OCDE en compréhension de l'écrit. En fait, ce sont ceux qui obtenu les scores moyens les plus faibles en compréhension de l'écrit.

\section{Graphique 6.2}

Relation entre la performance en compréhension de l'écrit et le degré d'engagement à l'égard de la lecture

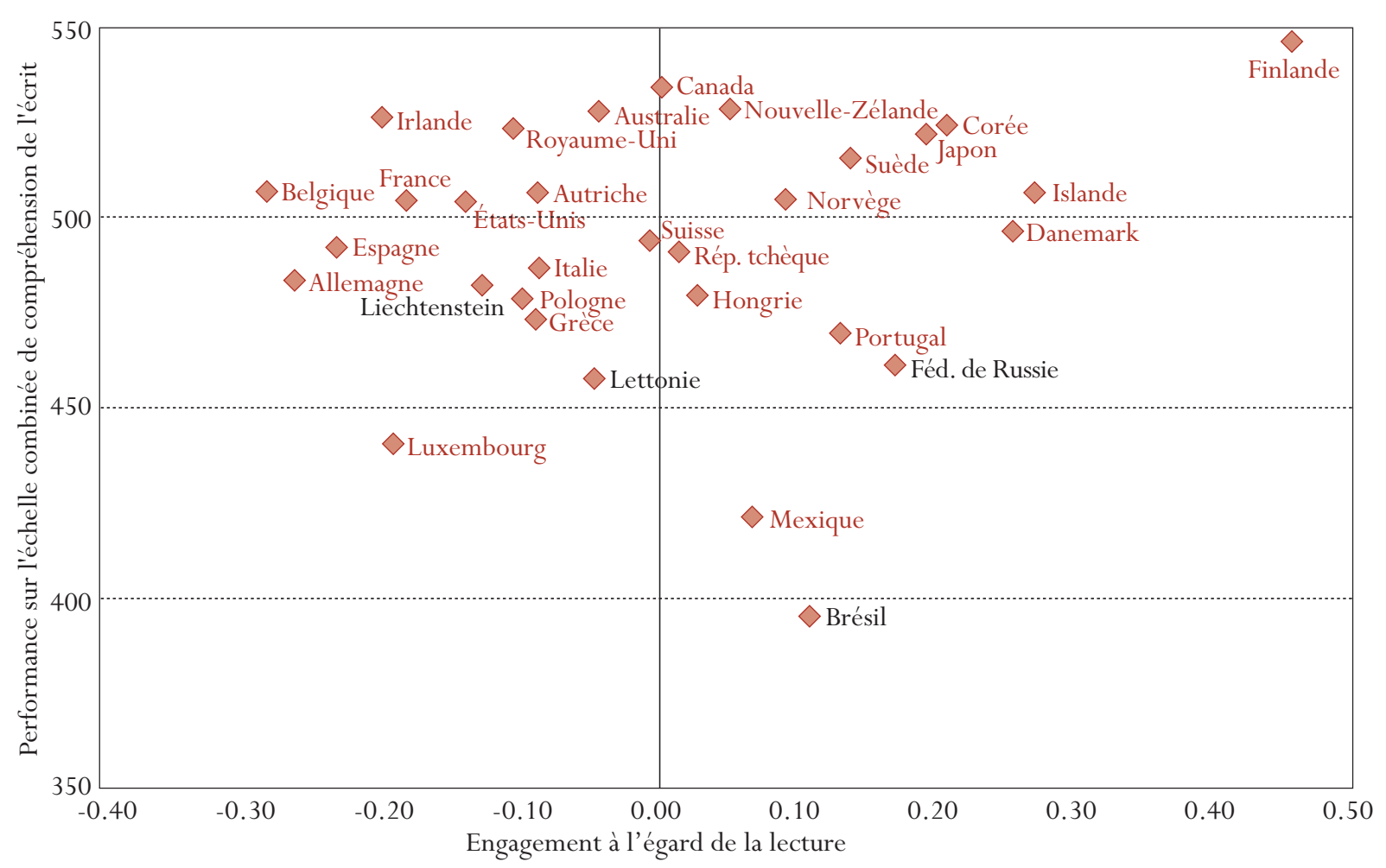

Source: Base de données PISA de l'OCDE, 2001, tableaux 4.1 et 6.1.

La corrélation entre l'engagement à l'égard de la lecture et la performance varie selon les deux souséchelles de format (voir tableau 6.2c), à l'instar de la corrélation précédente concernant le sexe. Tous pays de l'OCDE confondus, la corrélation médiane s'établit à 0,38 sur la sous-échelle de textes continus et à 0,34 sur celle de textes non continus. Cette tendance est assez uniforme dans tous les pays de l'OCDE. Il n'en va pas de même pour les sous-échelles d'aspect : bien que la corrélation médiane soit légèrement plus élevée entre l'engagement à l'égard de la lecture et la performance sur les sous-échelles Développer une interprétation $(0,38)$ et Réfléchir et évaluer $(0,37)$ que sur celle Trouver l'information $(0,35)$, la tendance est faible et varie selon le pays. 


\section{Le temps consacré aux devoirs}

Les élèves ont été priés d'indiquer le temps consacré chaque semaine à faire les devoirs donnés aux cours de langue d'enseignement, de mathématiques et de sciences. Pour répondre à cette question, ils avaient le choix entre quatre options : «Pas de temps du tout», «Moins d'une heure », «Entre une et trois heures » et «Trois heures ou plus ». Le graphique 6.3 montre qu'il existe une corrélation modérée entre le temps consacré aux devoirs et le niveau de compétence en compréhension de l'écrit. Le coefficient médian de corrélation s'établit à 0,18 dans les pays de l'OCDE (voir le tableau 6,1). Ce tableau révèle également une corrélation de 0,28 entre le temps consacré aux devoirs et l'engagement à l'égard de la lecture, soit près du double du coefficient de corrélation de la même variable avec le niveau de compétence. Le temps consacré aux devoirs est plus fortement associé à l'engagement à l'égard de la lecture qu'au niveau de compétence dans 21 des 28 pays de l'OCDE et dans les quatre pays non membres de l'OCDE, mais il l'est moins dans quatre pays de l'OCDE. La différence entre les deux corrélations est inférieure ou égale à 0,01 dans les trois pays restants.

\section{Graphique 6.3}

Relation entre le temps consacré aux devoirs et la performance en compréhension de l'écrit d'une part, le degré d'engagement à l'égard de la lecture d'autre part

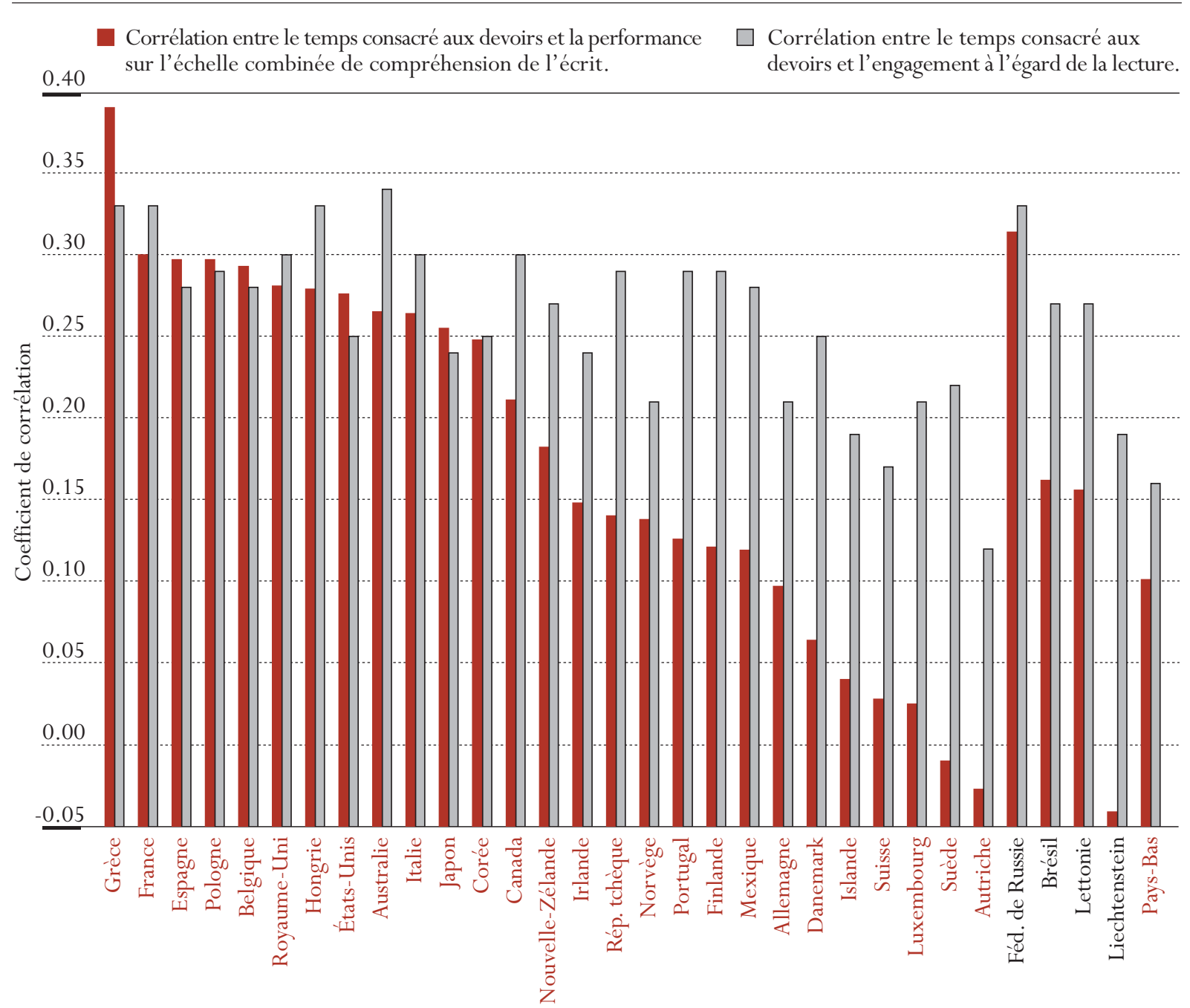

Source: Base de données PISA de l'OCDE, 2001, tableau 6.1. 
La corrélation entre le temps consacré aux devoirs et l'engagement à l'égard de la lecture est supérieure à 0,15 dans 31 des 32 pays qui ont participé à PISA (voir le tableau 6.2b). L'Autriche fait exception avec une corrélation de 0,12 . Dans huit pays, cette corrélation dépasse même 0,30 , tandis qu'elle est égale ou supérieure à 0,20 dans 19 autres pays. Par comparaison, la corrélation entre le temps consacré aux devoirs et le niveau de compétence est inférieure à 0,15 dans 14 pays et elle n'est égale ou supérieure à 0,30 que dans cinq pays. Dans les 13 autres pays qui ont participé à PISA, elle est comprise entre 0,15 et 0,29.

Comme nous l'avons dit, la corrélation entre le temps consacré aux devoirs et l'engagement à l'égard de la lecture tend à être supérieure dans la majorité des pays de l' OCDE et moins variable partout. L'écart type moyen de cette corrélation est de 0,06 alors que celui de la corrélation entre l'engagement et le niveau de compétence n'est que de 0,11. Les élèves qui s'investissent plus dans la lecture ont de toute évidence tendance à consacrer davantage de temps à faire leurs devoirs et à se préparer en vue des cours.

\section{La compréhension de l'écrit et les caractéristiques familiales}

La famille est une source importante de capital social pour les élèves. En combinaison avec le soutien apporté aux élèves par la collectivité et l'école, le soutien matériel et social que leur offre leur famille conditionne leur attitude à l'égard de l'enseignement et leurs aspirations à réussir leurs études. Nous allons à présent étudier la relation entre la compréhension de l'écrit et une série de caractéristiques propres à la famille et à l'environnement personnel des élèves, notamment la culture, le milieu socio-économique, la structure familiale et le pays d'origine.

Ces facteurs sont déterminants pour les premières expériences des élèves, leur préparation en vue d'entrer à l'école, leurs aspirations concernant les études, la valeur qu'ils accordent à l'éducation et leur degré de familiarité avec le type de langage scolaire auquel ils seront confrontés tout au long de leur parcours scolaire.

\section{Le milieu socio-économique}

Dans PISA, le milieu socio-économique des élèves est mesuré au moyen de l'indice socio-économique international de statut professionnel des parents (consulter l'annexe A3 pour les définitions). Le tableau 6.4a quantifie l'impact du statut professionnel des parents sur les compétences en lecture dans les pays membres et non membres de l'OCDE.

La corrélation médiane est de 0,33 en moyenne dans les pays de l'OCDE (voir le tableau 6.4a). Elle est relativement uniforme sur les cinq sous-échelles mais varie quelque peu selon les pays. Elle est sensiblement plus importante dans certains pays que dans d'autres. Dans 24 des 32 pays qui ont participé au cycle d'évaluation PISA, la corrélation entre le milieu socio-économique et la performance en compréhension de l'écrit est égale ou supérieure à 0,30 . Elle est supérieure à 0,20 dans tous les pays sauf en Corée, où elle est de 0,19 sur l'échelle combinée de compréhension de l'écrit.

La conclusion la plus importante - à laquelle le chapitre 5 a déjà fait référence - est l'existence d'une relation entre le milieu socio-économique et l'engagement à l'égard de la lecture. La corrélation médiane n'est que de 0,15 , soit moins de la moitié du coefficient de corrélation entre le milieu socio-économique et la performance en compréhension de l'écrit. La corrélation entre le milieu socio-économique et l'engagement à l'égard de la lecture est positive et supérieure à 0,10 dans tous les pays de l'OCDE, mais égale ou inférieure à 0,20 dans tous ces pays sauf deux. Les deux pays qui font exception sont l'Allemagne $(0,23)$ et la Suisse $(0,22)$. Comme nous l'avons dit au chapitre précédent, l'engagement à l'égard de la lecture, 
qui englobe les attitudes, la motivation et les pratiques, peut réduire les écarts entre les scores des élèves issus de milieux sociaux différents. Toute la question est bien entendu de trouver un moyen efficace pour améliorer l'engagement à l'égard de la lecture.

Le nombre de livres à la maison

Le statut socio-économique peut avantager des élèves en termes de valeur accordée à l'enseignement mais aussi en termes de volume de biens familiaux liés à l'éducation. Les élèves ont été priés d'indiquer le nombre de livres disponibles à la maison. Pour répondre à cette question, ils ont choisi une option parmi les sept proposées : «De 1 à 10 livres », «De 11 à 50 livres », «De 51 à 100 livres », «De 101 à 250 livres », « De 251 à 500 livres » et «Plus de 500 livres ». La corrélation médiane entre cette variable et le niveau de compétence en compréhension de l'écrit est de 0,35 (voir le tableau 6.3). La corrélation entre le nombre de livres disponibles à la maison et le score sur l'échelle combinée de compréhension de l'écrit est égale ou supérieure à 0,40 dans huit des 28 pays membres de l'OCDE et atteint même 0,52 en Hongrie. Elle est supérieure à 0,20 dans tous les autres pays membres et non membres de l'OCDE.

Le nombre de livres à la maison présente pratiquement la même corrélation avec le niveau de compétence qu'avec l'engagement à l'égard de la lecture. La corrélation médiane entre le nombre de livres à la maison et l'engagement à l'égard de la lecture est de 0,31 , c'est-à-dire pratiquement équivalente à la corrélation avec le niveau de compétence, de 0,35 . Elle est égale ou supérieure à 0,30 dans 20 des 28 pays de l'OCDE et à 0,20 dans tous ces pays, sauf au Mexique où elle est de 0,18 .

Il ne semble pas y avoir d'interaction manifeste entre le nombre de livres à la maison et les sous-échelles de compréhension de l'écrit (voir le tableau 6.4b).

\section{Les ressources éducatives à la maison}

Dans quelle mesure le fait de disposer d'un endroit calme pour étudier, d'une calculatrice et d'autres ressources éducatives est-il associé à des gains de performance et à un degré plus élevé d'engagement à l'égard de la lecture ? Cette variable a été mesurée dans le PISA par le biais d'une question qui demandait aux élèves d'indiquer s'ils avaient à leur disposition à la maison des ressources éducatives : un dictionnaire, un bureau, un endroit calme pour étudier, des manuels et des calculatrices. Leurs réponses à cette question ont été combinées pour constituer un indice dont la moyenne a été fixée à zéro et l'écart type à 1 .

Dans les pays de l'OCDE, l'impact des ressources éducatives à la maison est similaire à celui du milieu socio-économique. La corrélation médiane de cette variable est de 0,26 avec le niveau de compétence et de 0,16 seulement avec l'engagement à l'égard de la lecture (voir le tableau 6.3). En moyenne, les élèves qui disposent de davantage de ressources à la maison obtiennent des scores supérieurs à ceux des élèves qui n'en disposent pas. La corrélation entre les ressources éducatives disponibles à la maison et la performance est positive et excède 0,10 dans tous les pays de l'OCDE (voir le tableau 6.4c). C'est au Mexique que cette corrélation est la plus forte $(0,37)$, mais d'autres pays enregistrent également une corrélation supérieure à 0,30, à savoir la Belgique, les États-Unis, la Hongrie, le Luxembourg, la Norvège, la Nouvelle-Zélande et la Suisse. Même si les ressources éducatives disponibles à la maison sont moins corrélées à l'engagement à l'égard de la lecture que le nombre de livres à la maison, la corrélation est positive dans les 32 pays participants. Parmi les pays de l'OCDE, elle varie de 0,08 au Luxembourg à 0,24 au Royaume-Uni. 


\section{La communication culturelle à la maison}

C'est notamment en s'intéressant aux progrès des enfants et en s'impliquant dans leur éducation que les parents jouent un rôle primordial dans leur réussite scolaire. Lorsqu'ils communiquent avec leurs enfants, les parents manifestent l'intérêt qu'ils leur portent et, en outre, façonnent leurs comportements linguistiques et de questionnement, ce qui les aide dans les activités réalisées en classe. Les élèves ont été priés d'indiquer à quelle fréquence leurs parents discutaient de l'actualité sociale ou politique, de livres, d'émissions télévisées ou de films ou encore à quelle fréquence ils écoutaient de la musique classique avec eux. Leurs réponses à cette question ont été combinées pour constituer un indice dont la moyenne a été fixée à zéro et l'écart type à 1 .

Cette caractéristique familiale est corrélée à la fois à la performance et à l'engagement à l'égard de la lecture. La corrélation médiane entre cette variable et la performance en lecture est de 0,24 dans les pays de l'OCDE (voir le tableau 6.3). Elle atteint au moins 0,10 dans tous les pays, si ce n'est en Grèce où elle est de 0,09. Elle est égale ou supérieure à 0,30 en Allemagne, en Belgique, en Espagne, au Mexique, aux Pays-Bas et au Royaume-Uni (voir le tableau 6.4d). Par comparaison, la corrélation médiane entre la communication culturelle et l'engagement à l'égard de la lecture est de 0,35. Elle est donc supérieure de 50 pour cent à la corrélation précédente avec la performance. Elle dépasse 0,30 dans tous les pays de l'OCDE, à l' exception de la Corée $(0,27)$. L'écart type des corrélations entre la communication culturelle et l'engagement à l'égard de la lecture est de 0,03 , ce qui dénote une très faible variation entre les pays de l'OCDE. De toute évidence, le temps que les parents passent à parler avec leurs enfants de thèmes pertinents sur le plan culturel est associé à des gains de performance et à un accroissement de l'engagement à l'égard de la lecture.

\section{La structure familiale}

La structure familiale est un autre aspect du milieu personnel des élèves qui peut influencer leurs résultats. Comme nous l'avons dit, les parents jouent un grand rôle dans la réussite globale de leurs enfants, non seulement par l'environnement matériel et les biens qu'ils mettent à leur disposition, mais également par le soutien et les encouragements qu'ils leur prodiguent. Les élèves qui vivent avec un seul parent ou tuteur sont peut-être désavantagés à cet égard, dans la mesure où cette personne doit assumer seule les responsabilités liées aux activités professionnelles, familiales et sociales.

Les élèves ont été priés d'indiquer avec qui ils vivaient en général à la maison. Leurs réponses ont été classées en deux catégories, la première correspondant aux familles nucléaires (deux parents ou tuteurs) et la seconde, à toutes les autres structures familiales. Elles ont servi à élaborer une variable et à comparer les scores d'élèves vivant dans des structures familiales différentes. Le tableau 6.3 montre la relation entre la structure familiale et la performance et l'engagement à l'égard de la lecture.

En général, les élèves qui vivent avec leurs deux parents ou tuteurs obtiennent des scores légèrement plus élevés que ceux qui vivent dans d'autres structures familiales. La corrélation médiane est de 0,10 dans les pays de l'OCDE. Il n'est guère surprenant que l'impact de cette variable varie considérablement selon les pays participants (qu'ils soient membres ou non de l'OCDE).

Comme le montre le tableau 6.4e, c'est aux États-Unis que la corrélation la plus importante est enregistrée $(0,27)$. Elle est comprise entre 0,10 et 0,15 dans 14 autres pays membres de l'OCDE, à savoir en Australie, en Belgique, au Canada, au Danemark, en Finlande, en France, en Hongrie, en Irlande, au Luxembourg, en Norvège, en Nouvelle-Zélande, aux Pays-Bas, au Royaume-Uni et en Suède. Elle est positive, mais inférieure à 0,10 dans les autres pays. Comme précédemment, il n'existe pas d'interactions 
probantes entre les sous-échelles de compréhension de l'écrit. Le Liechtenstein $(0,15)$ est le seul pays non membre de l'OCDE à enregistrer une corrélation positive supérieure à 0,10 entre la structure familiale et le niveau de compétence.

Il est intéressant de souligner l'absence de lien entre la structure familiale et l'engagement à l'égard de la lecture, qui reste sensiblement la même dans les pays de l'OCDE. La corrélation médiane est de 0,04 et l'écart type entre les corrélations, de 0,03. La corrélation entre la structure familiale et l'engagement à l'égard de la lecture est pratiquement nulle dans tous les pays de l'OCDE. Elle est négative et comprise entre 0,01 et 0,03 dans trois pays. Elle est positive dans les 25 autres pays mais ne dépasse pas 0,08. Parmi les pays non membres de l'OCDE, seul le Liechtenstein $(0,15)$ enregistre une corrélation positive supérieure à 0,10 . Force est donc de conclure, en dépit de l'importance des familles et de leurs structures, à l'absence de lien avec l'engagement des élèves à l'égard de la lecture.

\section{Le statut d'immigrant}

Les facteurs sociaux, politiques et économiques ont un impact significatif sur les tendances de migration entre les pays et, donc, sur les environnements dans lesquels les élèves doivent souvent étudier. De plus en plus d'élèves doivent s'adapter à de nouvelles cultures et coutumes, mais aussi à de nouvelles langues d'enseignement. Bien qu'ils puissent très bien maîtriser leur langue maternelle, beaucoup d'entre eux, en raison de leur statut d'immigrant récent, découvrent une nouvelle langue d'enseignement et d'évaluation. Lors du cycle d'évaluation PISA, les élèves ont été priés d'indiquer si eux-mêmes et leurs deux parents étaient nés dans le pays où ils étaient soumis à l'évaluation PISA, ou dans un autre pays. Leurs réponses ont été codées de façon à déterminer dans quelle mesure chaque élève était issu d'une famille allochtone : les élèves qui ont déclaré qu'eux-mêmes et leurs deux parents étaient nés dans un autre pays se sont vus attribuer le code 1. Il y a lieu de souligner qu'il ne leur a pas été demandé d'indiquer depuis combien de temps eux ou leurs parents vivaient dans le pays de l'évaluation ou dans quelle mesure leur langue maternelle était similaire à la langue d'enseignement.

La comparaison des scores des élèves allochtones et de leurs condisciples révèle une corrélation assez faible du statut d'immigrant avec la performance $(-0,09)$ et aucune corrélation avec l'engagement à l'égard de la lecture $(0,00)$. Ces coefficients de corrélation sont trompeurs dans la mesure où ils sont influencés par le nombre relativement limité de jeunes de 15 ans qui ont déclaré être des élèves allochtones. Il reste que cette caractéristique a un impact important sur leur performance : l'effet de régression médian représente deux tiers de la valeur d'un écart type, soit 66, en moyenne dans les pays de l'OCDE (voir le tableau 6.4f). Les chiffres négatifs du tableau 6.3 indiquent que les élèves allochtones obtiennent des scores significativement inférieurs à ceux des autres élèves sur l'échelle combinée de compréhension de l'écrit. Dans 15 pays de l'OCDE, l'écart est supérieur à 65 : il va de 66 au Danemark à 103 en Suisse. Au Canada, au Portugal, en Nouvelle-Zélande et en République tchèque, il est compris entre 20 et 25.

L'Australie, la Hongrie et l'Irlande échappent à la tendance observée dans l'ensemble des pays de l'OCDE. L'Irlande est le seul pays où il existe une différence significative en faveur des élèves allochtones. L'écart moyen sur l'échelle combinée de compréhension de l'écrit y représente 47 points. Parmi les autres pays de l'OCDE, seules l'Australie (- 16) et la Hongrie (6) affichent des effets qui représentent moins de 20 pour cent de la valeur d'un écart type et qui sont jugés non pertinents.

La corrélation entre le statut d'immigrant et l'engagement à l'égard de la lecture est pratiquement nulle dans tous les pays de l'OCDE. Elle peut être aussi trompeuse que la corrélation précédente en raison des 
proportions relativement minimes d'élèves assimilés à des allochtones. Elle est négative et comprise entre 0,01 et 0,07 dans une douzaine de pays. Dans quelques-uns de ces pays, le statut d'immigrant semble avoir un impact négatif sur l'engagement à l'égard de la lecture. Comme le montre le tableau 6.7, l'effet représente environ un quart de la valeur d'un écart type en Islande, en République tchèque et en Suisse, et l'équivalent de près d'un écart type au Japon et en Pologne. Dans les autres pays de l'OCDE, le statut d'immigrant a un impact positif sur l'engagement à l'égard de la lecture. Ce constat est particulièrement vrai dans les pays anglophones. En moyenne, l'effet de régression calculé pour ces deux variables représente entre 20 et 50 pour cent de la valeur d'un écart type. Il est de 0,18 au Canada et de 0,19 en Australie, mais atteint 0,23 en Nouvelle-Zélande, 0,32 au Royaume-Uni et 0,43 en Irlande. Le Portugal présente une tendance similaire $(0,26)$.

\section{La compréhension de l'écrit et l'environnement scolaire}

Si les parents et le milieu familial dotent les élèves de caractéristiques importantes, la classe est un autre lieu d'apprentissage déterminant. Nous allons à présent nous pencher sur plusieurs caractéristiques de l'environnement scolaire - dérivées du questionnaire Élève - qui peuvent avoir un impact sur la performance des élèves. Ces variables portent sur la manière dont les élèves perçoivent l'incitation au rendement scolaire dont ils font l'objet, leur sentiment d'appartenance à l'école, leurs relations avec leurs professeurs et le climat de discipline en classe. La plus grande prudence s'impose lors de l'analyse de ces données, en particulier parce que les questions ont été posées aux élèves à un moment précis. Si le questionnaire fait référence à l'enseignant de la « langue de l'évaluation » du moment, il est possible que d'autres enseignants aient fortement influencé leurs attitudes et leur performance.

\section{L'incitation au rendement}

Les jeunes des divers pays sont-ils conscients du travail et des résultats que leurs professeurs attendent d'eux ? Dans quelle mesure cette perception influe-t-elle sur leur performance en compréhension de l'écrit et sur leur engagement à l'égard de la lecture ? Les élèves ont été priés de répondre à des questions portant sur la mesure dans laquelle : «Le professeur demande aux élèves de travailler beaucoup », «Les élèves ont beaucoup à étudier », «Le professeur est mécontent lorsque les élèves rendent un travail mal fait » et «Le professeur dit aux élèves qu'ils pourraient mieux faire ». Leurs réponses à ces questions ont été combinées pour construire un indice dont la moyenne a été fixée à zéro et l'écart type à 1 . Une valeur positive de l'indice indique que les professeurs donnent plus souvent l'impression à leurs élèves qu'ils veulent les voir réussir que la moyenne de l'OCDE pour cet indice.

Dans l'ensemble, il semble qu'il n' existe pas de relation tangible entre l'indice qui mesure l'incitation au rendement scolaire et les résultats des élèves en compréhension de l'écrit (voir le tableau 6.5). Tous pays de l'OCDE confondus, la corrélation médiane entre ces deux variables est de - 0,06 et l'effet médian de régression, de - 6. Il n'existe pas d'interactions probantes entre les sous-échelles de compréhension de l'écrit (voir le tableau 6.6a). De même, l'incitation au rendement scolaire n'est pas corrélée à l'engagement à l'égard de la lecture. La corrélation médiane entre ces deux variables s'établit à - 0,01.

Comme on peut s'y attendre, la comparaison de la corrélation de l'incitation au rendement avec la performance et l'engagement à l'égard de la lecture dans les divers pays révèle quelques différences intéressantes. Dans 19 des 28 pays de l'OCDE et dans deux des quatre pays non membres de l'OCDE, la corrélation entre l'incitation au rendement et la performance en compréhension de l'écrit est négative. Elle est supérieure à - 0,10 dans 9 de ces 19 pays, ce qui donne à penser que redoubler les incitations pourrait avoir un 
impact négatif sur la performance des élèves. Les effets négatifs les plus importants sont observés en Finlande, en Nouvelle-Zélande et en Suède $(-0,15)$. La corrélation entre ces deux variables est positive dans huit des 28 pays de l'OCDE et est nulle dans un pays (au Mexique). Deux pays seulement enregistrent une corrélation positive supérieure à 0,10 , à savoir la Grèce $(0,12)$ et la Corée $(0,19)$.

La corrélation avec l'engagement à l'égard de la lecture est similaire, mais avec encore moins de pays qui présentent une corrélation, positive ou négative, supérieure à 0,10 . C'est la Finlande qui enregistre la corrélation négative la plus importante entre l'encouragement à réussir et l'engagement à l'égard de la lecture $(-0,13)$. Seuls deux pays présentent une corrélation positive égale ou supérieure à 0,10 . Il s'agit de la Corée $(0,12)$ et des États-Unis $(0,10)$.

\section{Le climat de discipline}

Les élèves ont été priés d'indiquer la fréquence de situations et comportements susceptibles de perturber le climat d'apprentissage en classe : «Au début du cours, plus de cinq minutes se passent sans qu'on ne fasse rien », «Les élèves ne commencent à travailler que bien après le début des cours », «Les élèves n'écoutent pas ce que dit le professeur », «Les élèves ne peuvent pas bien travailler », « Il y a du bruit et de l'agitation » et «Le professeur doit attendre un long moment avant que les élèves se calment». Comme pour les indices précédents, les réponses des élèves à ces six questions ont été combinées pour construire un indice dont la moyenne a été fixée à 0 et l'écart type à 1 . Les valeurs positives de cet indice indiquent un climat plus favorable à l'apprentissage que la moyenne de l'OCDE.

À l'instar de la corrélation précédente avec l'incitation au rendement scolaire, la corrélation entre le climat de discipline en classe et les performances en lecture, et entre le climat de discipline et l'engagement à l'égard de la lecture, est faible mais légèrement positive dans l'ensemble des pays de l'OCDE et dans deux pays non membres. La corrélation médiane est de 0,10 dans les deux cas et pour l'ensemble des pays de l'OCDE (voir le tableau 6.5). La corrélation positive entre le climat de discipline et le niveau de performance en lecture atteint ou dépasse 0,10 dans 17 pays de l'OCDE et un des pays non membres de l'OCDE. La corrélation entre le climat de discipline et l'engagement à l'égard de la lecture est très similaire : elle est égale ou supérieure à 0,10 dans 17 des 28 pays de l'OCDE et dans deux des quatre pays non membres de l'OCDE. Il ne semble pas y avoir d'interaction manifeste entre le climat de discipline et les performances sur les sous-échelles de compréhension de l'écrit (voir le tableau 6.6b).

S'il ressort de ce qui précède qu'évoluer dans un climat considéré comme étant moins perturbé a un léger impact sur la performance ou l'engagement à l'égard de la lecture, il est impossible de distinguer les causes des effets à partir de ces données. Il se peut que les élèves moins compétents et moins motivés soient groupés dans des classes plus dissipées et plus perturbées. Il est possible également que les élèves finissent par apprendre moins et par perdre leur motivation s'ils passent plus de temps dans des classes perturbées.

\section{Le sentiment d'appartenance}

Dans quelle mesure les élèves des pays participants se sentent-ils à l'aise à l'école ? Peut-on établir un lien entre leur perception à cet égard et leurs résultats ? Les élèves ont été priés de livrer leur sentiment sur les cinq propositions suivantes : «Mon école est un endroit où : - je me sens comme étranger (ou hors du coup) ; - je me fais facilement des amis ; - je me sens chez moi ; - je me sens souvent mal à l'aise ; - je ne me sens pas à ma place ; - les autres élèves ont l'air de m’apprécier». Un indice dont la moyenne a été fixée à 0 et l'écart type à 1 a été construit sur la base de leurs réponses. Des valeurs positives indiquent un sentiment d'appartenance plus profond. La corrélation de cette variable est pratiquement nulle avec 
le niveau de compétence en compréhension de l'écrit $(0,07)$ et avec l'engagement à l'égard de la lecture $(0,04)$. Les chiffres relatifs à cette corrélation sont présentés dans le tableau 6.5.

À l'instar des autres corrélations étudiées ici, cette corrélation présente des différences intéressantes entre les pays. La corrélation entre le sentiment d'appartenance et le niveau de compétence et l'engagement à l'égard de la lecture est positive dans pratiquement tous les pays. Dans trois des pays de l'OCDE (le Luxembourg, le Mexique et le Portugal), la corrélation entre le sentiment d'appartenance et le niveau de compétence dépasse 0,20. La corrélation entre cette variable et l'engagement à l'égard de la lecture est de 0,22 au Brésil, de 0,21 au Mexique et de 0,19 au Portugal. Dans quelques pays, il existe une corrélation négative entre le sentiment d'appartenance et la performance ou l'engagement à l'égard de la lecture, mais elle ne dépasse pas - 0,03. Comme c'était le cas pour le climat de discipline, il ne semble pas y avoir d'interaction manifeste entre le sentiment d'appartenance et les performances sur les sous-échelles de compréhension de l'écrit (voir le tableau 6.6c).

\section{Les relations entre élèves et enseignants}

Comment les élèves des différents pays jugent-ils leurs relations avec leurs enseignants ? Dans quelle mesure sont-ils conscients de l'importance que leurs professeurs accordent à leur bien-être et à leur réussite ? Et dans quelle mesure ces perceptions sont-elles liées à leur performance ou à leur engagement à l'égard de la lecture ? Les élèves ont été priés de livrer leur sentiment sur les cinq propositions suivantes : «Les élèves s'entendent bien avec les professeurs », «Les professeurs s'intéressent au bien-être de leurs élèves », « Mes professeurs sont réellement à l'écoute de ce que j'ai à dire », «Si j'ai besoin d'aide supplémentaire, mes professeurs me l'apporteront» et « Mes professeurs me traitent avec justice ». Leurs réponses ont été combinées pour construire un indice dont la moyenne a été fixée à zéro et l'écart type à 1. Des valeurs positives indiquent une perception des relations entre élèves et enseignants plus favorable que la moyenne de l'OCDE.

À l'instar des autres corrélations présentées ici, la corrélation entre les relations élèves-enseignants telles qu'elles sont perçues par les élèves et le niveau de compétence en compréhension de l'écrit est relativement faible. Elle est de 0,08 en moyenne dans les pays de l'OCDE, ainsi que le montre le tableau 6.5. Elle est positive et supérieure à 0,10 dans 12 des 28 pays de l'OCDE; elle est négative dans sept d'entre eux ; toutefois elle n'atteint 0,10 qu'en Hongrie.

Par ailleurs, la corrélation médiane entre cette variable et l'engagement à l'égard de la lecture s'établit à 0,17 et est positive dans tous les pays. De plus, elle est systématiquement plus marquée que celle portant sur la performance. A cet égard, et peut-être de façon encore plus significative, cette corrélation varie moins que la précédente tant dans les pays de l'OCDE que dans les pays non membres. Elle est supérieure à 0,10 dans les 32 pays participants et atteint ou dépasse la barre des 0,20 dans 11 d'entre eux. Il y a lieu de souligner que tous ces pays sont soit des pays anglophones, soit des pays nordiques, exception faite de la Pologne où la corrélation des relations entre élèves et enseignants est de 0,04 avec la performance et de 0,22 avec l'engagement. Il ne semble pas y avoir d'interaction manifeste entre les relations élèves/enseignants et les performances sur les sous-échelles de compréhension de l'écrit. 
CHAPITRE 6 La lecture, moteur de changement: Performance et engagement d'un pays à l'autre

\section{Notes}

1. Ces caractéristiques contextuelles ont été sélectionnées parce qu'elles sont jugées importantes et vraisemblablement associées aux performances et à l'engagement à l'égard de la lecture et, d'autre part, parce que des données relativement exhaustives les concernant ont été recueillies dans chaque pays.

2. Dans ce chapitre, nous distinguons les coefficients de corrélation qui sont égaux ou supérieurs à 0,10 de ceux qui sont inférieurs à cette valeur. Un coefficient égal ou supérieur à 0,10 signifie que 1 pour cent au moins de la variance peut être imputé à la corrélation. 


\section{Chapitre}

7

L'INTERACTION ENTRE LES CARACTÉRISTIQUES INDIVIDUELLES ET LES FACTEURS SCOLAIRES

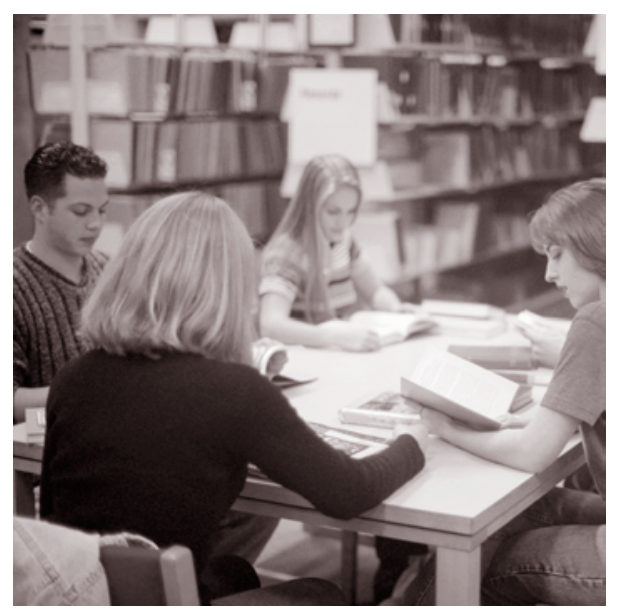




\section{SYNTHÈSE}

- La relation entre le niveau de compétence en compréhension de l'écrit et les caractéristiques des élèves et des établissements varie d'un pays à l'autre. Toutefois, il est possible d'identifier des groupes de pays qui présentent des similitudes dans leurs interactions entre ces variables de contexte personnelles et scolaires et les compétences en lecture.

- Certains pays, notamment le Danemark, la Finlande, l'Islande, la Norvège et la Suède, ont mis en place des structures éducatives qui limitent les différences entre établissements, tant en termes de fréquentation des écoles que de résultats des élèves. Ces pays présentent des profils d'interaction similaires : les caractéristiques personnelles des élèves semblent avoir un impact statistique au niveau individuel mais aucun impact au niveau des établissements.

- Les pays à dominante anglophone ont, à certains égards, un comportement analogue à celui du Danemark, de la Finlande, de l'Islande, de la Norvège et de la Suède. Toutefois, certains d'entre eux pratiquent le regroupement des élèves par aptitude, ce qui modifie le profil des interactions. De plus, dans les systèmes où coexistent des établissements gratuits et payants, il apparaît que la ségrégation sociale l'emporte sur la ségrégation scolaire.

- Les autres pays européens présentent des profils moins tranchés, qui varient essentiellement en fonction de l'échelle à laquelle sont pratiqués la différenciation des filières et le regroupement par aptitude. Dans certains pays, la ségrégation sociale l'emporte sur la ségrégation scolaire, alors que dans d'autres, la situation inverse prévaut.

- Il est peu vraisemblable que les systèmes éducatifs réussissent à abolir complètement les inégalités, qu'elles soient de nature sociale ou autre. Dans certains pays, les inégalités se situent essentiellement au niveau des établissements tandis que dans d'autres, les inégalités semblent concentrées au niveau familial. Certains pays combinent même ces deux formes d'inégalités. Tenter d'atténuer les inégalités à un niveau peut revenir à les accentuer à un autre niveau.

- Toutefois, les inégalités semblent moins marquées dans certains systèmes éducatifs. Il est donc indéniable que les structures éducatives jouent un rôle non négligeable dans cette problématique. 
Dans ce chapitre, nous nous proposons d'identifier et d'interpréter les relations entre le niveau de compétence des élèves en compréhension de l'écrit et une série de variables contextuelles dans le but de clarifier les interactions complexes entre des facteurs qui peuvent influer sur la performance des élèves, notamment les caractéristiques personnelles des élèves, leurs attitudes à l'égard de l'école et des études et leur environnement d'apprentissage.

Nous chercherons essentiellement à identifier des patterns de relations entre les variables contextuelles et la compréhension de l'écrit. Chaque pays présente certes un profil unique à cet égard; il existe cependant des similitudes marquées entre certains pays, comme en a déjà fait état le rapport initial de l'OCDE sur le cycle d'évaluation PISA 2000 (OCDE, 2001). Ainsi, on peut grouper les pays en différentes catégories selon la relation entre le niveau de compétence d'élèves en compréhension de l'écrit et l'homogénéité, ou l'hétérogénéité, de la population des élèves dans des établissements. Dans ce chapitre, nous privilégierons l'étude des grandes tendances observées dans des groupes de pays plutôt que l'analyse des particularités de chaque pays. Nous donnerons toutefois un bref aperçu des résultats individuels des pays pour les variables les plus importantes.

\section{Un modèle d'analyse à variables et niveaux multiples}

Dans le chapitre 6, nous avons décrit les relations linéaires entre une série de variables contextuelles et le niveau de compétence en compréhension de l'écrit en guise d'introduction à l'analyse des variables modélisées ici. Les analyses statistiques du présent chapitre se distinguent de celles exposées dans le chapitre précédent à deux égards.

En premier lieu, les analyses du chapitre précédent portaient sur la relation entre chacune des variables contextuelles prise individuellement et le niveau de compétence en compréhension de l'écrit, alors qu'ici toutes les variables contextuelles sont intégrées dans un même modèle. Ce type d'analyse peut se révéler très utile, car, comme les différentes variables de contexte interagissent entre elles, leurs effets peuvent être confondus. Ainsi, nous savons que les filles s'investissent généralement plus que les garçons dans la lecture. L'écart de compétence entre les filles et les garçons est donc dans une certaine mesure biaisé par cette variation du degré d'engagement à l'égard de la lecture entre les deux sexes. Faire abstraction des différences d'engagement entre les sexes revient à répondre à la question : quel serait l'écart de performance entre les filles et les garçons si leur degré d'engagement à l'égard de la lecture était identique ? L'intégration de nombreuses variables contextuelles dans un modèle unique nous permet d'estimer plus précisément l'impact de chaque variable, tout en contrôlant les effets respectifs des autres variables.

En second lieu, les modèles de régression linéaire tels que ceux présentés au chapitre 6 sont parfois utilisés pour tenter de démêler les relations complexes entre plusieurs variables. Cette méthode peut toutefois donner une idée tronquée, voire trompeuse du fonctionnement des systèmes éducatifs, car elle ne tient pas compte d'autres effets éventuels, qu'ils soient liés à la façon dont les élèves sont répartis entre les établissements ou dont ils sont groupés par classe dans chaque établissement. Considérons le milieu social des élèves à titre d'exemple. Dans certains pays, le milieu socio-économique intervient dans le choix de l'établissement, ce qui explique pourquoi il arrive que le milieu socio-économique ne varie guère au sein de chaque établissement. Dans d'autres pays en revanche, les établissements accueillent des élèves issus de milieux sociaux très différents mais, au sein de l'établissement, le milieu social des élèves aura une influence sur le type de cours vers lesquels ils seront orientés et, partant, sur la variance à l'intérieur de l'établissement. Les modèles de régression linéaire qui ne tiennent pas compte de la structure hiérarchique des données ne permettent pas d'établir de distinction entre ces deux systèmes. 
Les modèles de régression à niveaux multiples (Bryk et Raudenbush, 1992 ; Goldstein, 1995) permettent de tenir compte du fait que les élèves sont regroupés dans des établissements et dans des classes. La contribution relative de la classe et de l'établissement peut donc être prise en considération lors de l'évaluation de l'impact de chaque facteur sur les résultats des élèves.

\section{Graphique 7.1}

Modélisation de la relation entre le milieu socio-économique et la performance des élèves au niveau de l'individu et de l'établissement

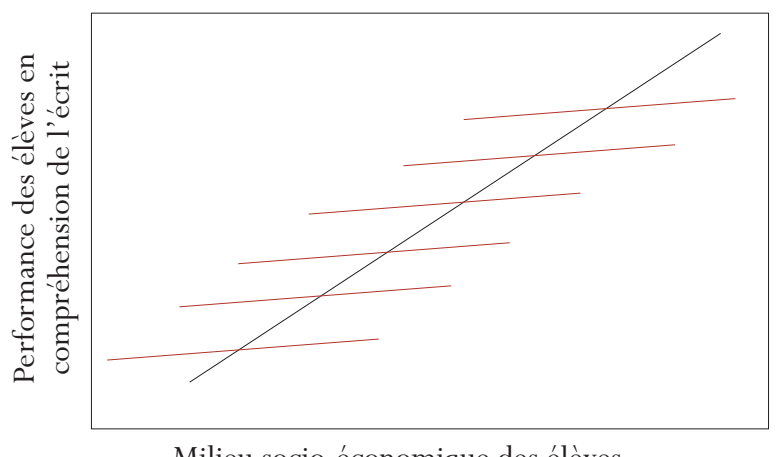

Milieu socio-économique des élèves
Le graphique 7.1 montre la différence entre un modèle de régression linéaire simple et un modèle de régression linéaire multi-niveaux. Supposons que ce graphique porte sur la relation entre le milieu socio-économique d'un élève et son niveau de performance théorique en lecture. La ligne noire représente la ligne de régression lorsque la structure hiérarchique des données n'est pas prise en considération. Elle montre que le score théorique d'un élève issu d'un milieu socio-économique plus privilégié est nettement supérieur à celui d'un élève vivant dans un milieu socio-économique moins favorisé.

Les lignes rouges montrent la relation entre ces deux variables dans plusieurs établissements particuliers. Chaque établissement est représenté par une ligne de régression (rouge en l'occurrence). La comparaison des deux modèles montre que le milieu social moyen varie d'un établissement à l'autre (certaines lignes de régression se situent plus à gauche que d'autres sur le graphique) et que l'effet du milieu socio-économique sur le niveau de performance est moins significatif au sein d'un même établissement qu'entre des établissements différents.

Le graphique 7.2 présente les trois types de coefficients de régression de l'impact du milieu socio-économique des élèves sur le score enregistré sur l'échelle combinée de compréhension de l'écrit, calculés selon trois modèles différents. Il montre aussi le coefficient de régression du milieu social moyen des établissements sur les performances en lecture. Pour chaque pays, trois barres verticales au-dessus de l'axe horizontal et une barre verticale en dessous de l'axe horizontal indiquent l'effet du milieu socio-économique des élèves sur la performance en lecture d'après un modèle de régression simple, un modèle de régression multiple et modèle hiérarchique à niveaux multiples.

La première barre montre les coefficients de régression selon le modèle de régression simple, qui ont été calculés sans tenir compte des autres variables. Prenons le cas de l'Australie à titre d'exemple : son coefficient de régression est de 31,7, ce qui signifie que l'augmentation du milieu socio-économique individuel d'un écart type donne lieu à un gain de performance théorique de 31,7 sur l'échelle de compétence PISA. Le milieu socio-économique est mesuré sur une échelle continue dont la moyenne est d'environ 45 et l'écart type d'environ 16 . En d'autres termes, un élève issu d'un milieu socio-économique estimé à 61 est censé obtenir un score supérieur de 31,7 à celui d'un élève issu d'un milieu qui correspond à 45 sur l'échelle du milieu socio-économique. 
Effets du milieu socio-économique sur la performance en compréhension de l'écrit selon trois modèles différents de régression

Effets du milieu socio-économique
Comme facteur unique

Sur la variance entre élèves à l'intérieur des écoles
Comme un facteur parmi d'autres facteurs Sur la variance entre écoles

$\underline{60}$

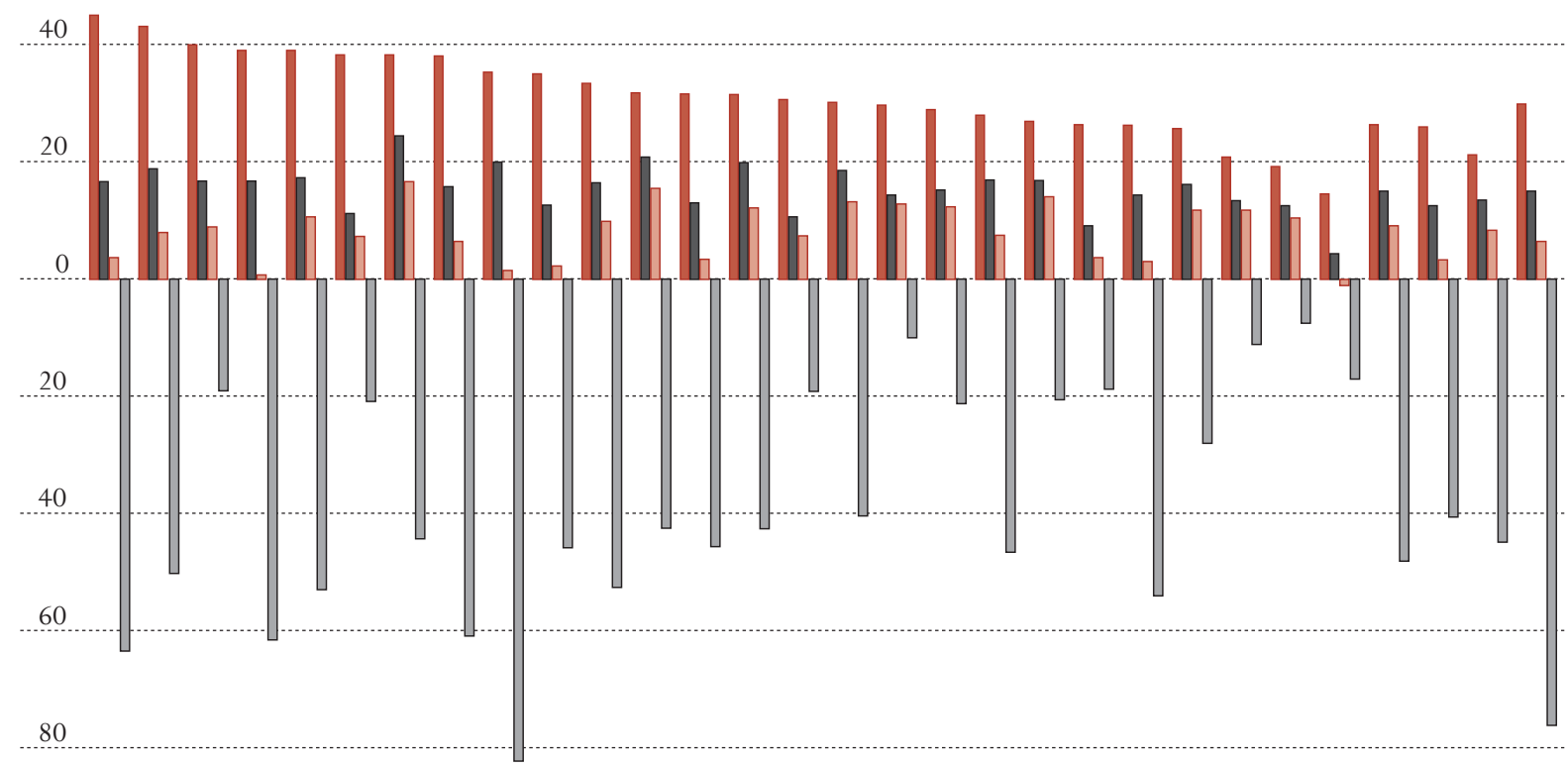

100



Source: Base de données PISA de l'OCDE, 2001, tableau 7.1.

La deuxième barre correspond aux coefficients de régression de l'impact du milieu socio-économique compte tenu de l'effet statistique des autres variables. Elle représente donc l'effet statistique net du milieu socio-économique. Dans ce cas, le coefficient de régression de l'Australie représente maintenant l'équivalent de 20,0, ce qui signifie qu'il existe un écart théorique de 20 entre les scores de deux élèves qui présentent les mêmes caractéristiques, si ce n'est qu'ils sont séparés par un écart type sur l'échelle de milieu socio-économique.

La comparaison de la première et de la deuxième barre du graphique 7.2 montre qu'une grande partie de l'effet apparent du milieu socio-économique est imputable à d'autres variables, telles que le degré d'engagement à l'égard de la lecture, la communication culturelle au sein de la famille, etc. Il y a lieu de souligner qu'en moyenne, le coefficient de régression de l'impact du milieu socio-économique de l'élève diminue en moyenne de moitié si d'autres variables sont incluses dans la régression linéaire. 
La troisième barre correspond aux coefficients de régression de l'impact du milieu socio-économique au sein des établissements, représentés par les lignes rouges dans le graphique 7.1. Ce coefficient de régression représente à présent pour l'Australie l'équivalent de 12,2, ce qui signifie qu'il existe un écart moyen de 12,2 entre les scores en compréhension de l'écrit de deux élèves qui fréquentent le même établissement et qui présentent les mêmes caractéristiques, mais qui sont séparés par un écart type sur l'échelle de milieu socio-économique. L'utilisation du modèle de régression à niveaux multiples réduit à nouveau à peu près de moitié le coefficient de régression de l'impact du milieu socio-économique calculé sur la base d'un modèle de régression à variables multiples. Cette réduction va de 10 pour cent en Norvège à près de 95 pour cent en Hongrie.

Enfin, la barre verticale en dessous de la ligne horizontale présente les coefficients de régression de l'impact du milieu socio-économique moyen des établissements, une variable correspondant à la moyenne des indices de milieu socio-économique des élèves participants inscrits dans chaque établissement. Le milieu socio-économique moyen varie selon les établissements. Certains d'entre eux accueillent des élèves issus de milieux plus privilégiés, tandis que d'autres - notamment ceux situés dans des zones industrielles - sont essentiellement fréquentés par des élèves vivant dans des milieux plus défavorisés. La variation du milieu socio-économique est plus importante entre les élèves qu'entre les établissements. En moyenne, l'indice de milieu socio-économique de 68 pour cent des élèves (correspondant à un écart type de part et d'autre de la moyenne) est compris entre 29 et 61, alors que celui de 68 pour cent des établissements est compris entre 37,5 et 52,5. En d'autres termes, l'écart type du milieu socio-économique des établissements est d'environ 7,5. Le coefficient de régression de l'impact du milieu socio-économique des établissements représente l'équivalent de 42,7 en Australie, ce qui signifie que dans ce pays, il existe un écart théorique de 42,7 entre les scores en compréhension de l'écrit de deux élèves qui présentent le même profil en termes de milieu socio-économique, de sexe, d'engagement à l'égard de la lecture, etc., mais qui fréquentent des établissements dont les indices moyens de milieu socio-économique diffèrent d'un écart type, soit 7,5.

Comme le montre le graphique 7.2, l'effet statistique d'une variable donnée peut considérablement varier selon le modèle choisi et les variables qui y sont incluses. Dans ce chapitre, nous présentons les résultats d'une analyse de régression à niveaux et variables multiples qui permet de distinguer les effets observés entre établissements et à l'intérieur des établissements. Tous les résultats correspondent à des effets nets, c'est-à-dire compte tenu de l'effet des autres variables.

\section{Variables individuelles et scolaires utilisées dans le modèle}

Les groupes de variables suivants ont été inclus dans l'analyse pour expliquer le niveau de compétence en compréhension de l'écrit ${ }^{1}$ :

- variables personnelles des élèves : sexe et engagement à l'égard de la lecture ;

- variables socio-économiques : structure familiale, statut professionnel des parents, nombre de livres à la maison, communication culturelle avec les parents, ressources éducatives à la maison et statut d'immigrant ;

- variables liées à l'enseignement et à l'apprentissage : temps consacré aux devoirs, encouragement à l'école et année d'études ;

- variables liées à la perception du climat dans l'établissement et dans la salle de classe : climat de discipline, relations entre élèves et enseignants et sens d'appartenance de l'élève à son école ; 
- variables collectives des établissements : le statut professionnel moyen des parents et le degré moyen d'engagement à l'égard de la lecture.

L'annexe A3 décrit de manière détaillée les variables incluses dans le modèle. D’autres variables dérivées du questionnaire Établissement ont dans un premier temps été prises en considération dans l'analyse, mais il est apparu que leur impact était minime et généralement non significatif ${ }^{2}$. De plus, l'inclusion de ces variables aurait donné lieu à un accroissement sensible du volume de données manquantes ${ }^{3}$. Pour toutes ces raisons, il a été jugé plus pertinent d'exclure ces variables du modèle d'analyse.

\section{L'influence prépondérante du milieu socio-économique et de l'engagement à l'égard de la lecture}

Le tableau 7.2 présente les moyennes des coefficients de régression nationaux de plusieurs variables sur l'échelle combinée de compréhension de l'écrit, les trois sous-échelles d'aspect (la localisation d'informations, l'interprétation de textes, et la réflexion et l'évaluation) et les deux sous-échelles de format (les textes continus et non continus). Les 14 premières variables de la première colonne sont basées sur le modèle de régression à niveaux multiples intra-établissement (voir la troisième colonne du tableau 7.1) et les deux dernières variables, sur le modèle de régression à niveaux multiples inter-établissement (voir la dernière colonne du tableau 7.1).

\section{Échelle combinée de compréhension de l'écrit}

Cinq variables semblent avoir un effet statistique important à l'intérieur de l'établissement : l'engagement à l'égard de la lecture, le statut d'immigrant, le milieu socio-économique, l'année d'études et le sexe.

C'est l'année d'études qui produit l'effet le plus important : 37,3 en moyenne. Comme nous l'avons dit, la population cible de PISA est constituée des jeunes de 15 ans qui sont inscrits dans un établissement d'enseignement. Dans la plupart des pays - mais pas dans tous -, les élèves de cette population fréquentent différentes années d'études. En général, l'écart entre les scores en compréhension de l'écrit des élèves de deux années d'études consécutives représente entre 50 et 80 points sur l'échelle de compétence PISA, selon les années d'études concernées. Toutefois, ces sous-groupes ne peuvent faire l'objet d'une comparaison directe, sachant qu'en général, les élèves qui redoublent sont issus de milieux sociaux plus défavorisés, s'investissent moins dans la lecture, etc. ${ }^{4}$ Le coefficient de régression de 37,3 est un effet net, c'est-à-dire calculé en contrôlant l'effet des autres variables. Il correspond à l'écart théorique de score entre des élèves qui fréquentent deux années d'études qui se suivent dans un établissement quel qu'il soit, toutes leurs autres caractéristiques étant identiques.

Le statut d'immigrant est la seconde variable par importance de l'effet. Si un élève et ses deux parents sont nés dans un autre pays, son score sur l'échelle de compréhension de l'écrit est en moyenne inférieur de 21,8 à celui d'un élève dont les autres caractéristiques seraient identiques.

L'engagement à l'égard de la lecture arrive en troisième position dans ce classement. Il existe un écart de performance en compréhension de l'écrit de 18 environ entre les élèves qui présentent le même profil, toutes variables confondues, et qui fréquentent le même établissement mais dont le degré d'engagement à l'égard de la lecture varie d'un écart type. Cet impact représente environ la moitié de celui lié à l'année d'études, mais - constat intéressant s'il en est - le double de celui lié au milieu socio-économique. En d'autres termes, le degré d'engagement à l'égard de la lecture d'un élève est plus déterminant que son milieu socio-économique pour estimer son score théorique en compréhension de l'écrit, quel que soit l'établissement fréquenté. 
Dans les chapitres précédents, nous avons déjà évoqué la grande différence de performance entre les filles et les garçons en compréhension de l'écrit. Cet écart peut en partie s'expliquer par d'autres différences, notamment celles relevées dans le degré d'engagement à l'égard de la lecture. Lorsque l'effet de cette variable est contrôlé, l'écart entre les filles et les garçons inscrits dans un établissement donné ne représente plus qu'un score de 12 environ sur l'échelle de compétence PISA, soit approximativement un tiers de l'écart absolu.

Enfin, le milieu socio-économique des élèves a en moyenne un impact de 8 , au sein d'un même établissement. Il serait hâtif de conclure à la lumière de ce coefficient de régression plutôt faible que le milieu socio-économique n'a qu'un effet minime sur le niveau de compétence des élèves en compréhension de l'écrit. À l'instar d'autres coefficients évoqués ici, ce coefficient correspond aux écarts de performance observés au sein des établissements. Or, dans de nombreux pays, le milieu socio-économique ou les résultats antérieurs des élèves déterminent en partie l'établissement dans lequel les élèves sont inscrits. Le coefficient de régression de l'impact du milieu socio-économique moyen de l'école correspond au gain de performance moyen de l'établissement en compréhension de l'écrit si la moyenne de l'indice de milieu socio-économique de l'école augmente de 7,5 (soit la valeur d'un écart type sur l'échelle de milieu socio-économique). Ce coefficient de régression représente l'équivalent de 40 en moyenne dans les pays participants. Aux États-Unis par exemple, les cinq établissements en queue de classement en termes de milieu socio-économique moyen présentent en moyenne un indice de milieu socio-économique moyen de 35 et un score de 387 en compréhension de l'écrit. En revanche, ces deux valeurs sont respectivement de 66 et de 596 pour les cinq établissements en tête de ce même classement. En d'autres termes, un écart de milieu socio-économique moyen de 31 - soit l'équivalent de quatre écarts types de l'indice socio-économique - est associé à un écart de performance de 210 environ en compréhension de l'écrit, soit le quadruple du coefficient de régression de l'impact du milieu socio-économique moyen aux ÉtatsUnis $(52,8)$. Force est de conclure qu'au sein de l'OCDE, les établissements se distinguent par leur milieu socio-économique moyen et que ces différences donnent lieu à des écarts importants de performance en compréhension de l'écrit.

Les établissements se distinguent également par le degré d'engagement de leurs élèves à l'égard de la lecture, ainsi qu'en atteste le coefficient de régression de cet indice à l'échelon des établissements. Les élèves semblent en moyenne s'investir davantage dans la lecture dans certains établissements, et moins dans d'autres. Ces différences sont associées à des écarts de performance de 23 en compréhension de l'écrit. Ce coefficient est important, certes, mais il apparaît qu'au niveau des établissements, les différences de milieu socio-économique ont plus d'impact sur la performance que celles liées à l'engagement à l'égard de la lecture.

Les autres variables incluses dans le modèle ont de toute évidence un effet plus limité. En toute logique, le niveau de compétence en compréhension de l'écrit est influencé par le nombre de livres disponibles à la maison $(5,0)$. De même, les élèves dont les parents passent du temps à discuter avec eux de questions culturelles tendent à obtenir de meilleurs résultats en compréhension de l'écrit $(2,9)$. Le volume de ressources éducatives disponibles à la maison produit également un effet positif, quoique faible $(3,0)$. Enfin, les élèves qui vivent dans des familles nucléaires obtiennent généralement des résultats supérieurs à ceux des élèves qui vivent dans des familles monoparentales ou mixtes $(2,3)$.

Les cinq variables scolaires qui ont un impact sont, par ordre décroissant d'effet : l'incitation au rendement $(-3,1)$, le climat de discipline $(1,4)$, les relations entre élèves et enseignants $(1,0)$, le sentiment d'appartenance de l'élève $(1,0)$ et, enfin, le temps consacré aux devoirs $(0,2)$. En d'autres termes, dans 
un établissement donné, les élèves qui ont l'impression que leurs professeurs les pressent davantage de réussir obtiennent généralement des résultats inférieurs en compréhension de l'écrit. Ce phénomène peut s'expliquer par le fait que les enseignants ont tendance à encourager davantage les élèves qui rencontrent des difficultés scolaires. Les établissements ou les classes où règne un climat de discipline moins favorable aux dires des élèves, sont également associés une performance légèrement moindre. Assez logiquement, les élèves qui estiment entretenir de bonnes relations avec leurs professeurs et qui sont mieux disposés à l'égard de l'école obtiennent de meilleurs résultats en moyenne. Enfin, le fait de consacrer davantage de temps à ses devoirs n'entraîne qu'une différence minime de performance en compréhension de l'écrit.

En résumé, les effets agrégés du degré d'engagement à l'égard de la lecture et du milieu socio-économique des élèves, y compris le statut d'immigrant, et le nombre de livres disponibles à la maison, sont associés aux plus grandes différences de scores en compréhension de l'écrit au sein d'un établissement donné, et il s'agit vraisemblablement des variables les plus déterminantes. Le temps consacré aux devoirs et les variables touchant aux perceptions par les élèves de leur école ont, en moyenne, un impact plus faible.

\section{Les sous-échelles de compréhension de l'écrit}

Les analyses qui portent sur l'échelle combinée de compréhension de l'écrit ont également été réalisées pour les trois sous-échelles d'aspect (la localisation d'informations, l'interprétation de textes, et la réflexion et l'évaluation) et les deux sous-échelles de format (les textes continus et non continus). Le tableau 7.2 indique les moyennes nationales des coefficients de régression sur chacune des cinq sous-échelles. Ces coefficients expriment le pouvoir prédictif «unique » de chaque variable prise séparément, les autres variables incluses dans l'analyse étant tenues sous contrôle.

Les coefficients de régression ne présentent pas de différences majeures sur les cinq sous-échelles, si ce n'est ceux liés au sexe. Les différences entre les sexes varient grandement selon les aspects et les formats, même quand les autres variables sont tenues sous contrôle. Si pour la sous-échelle de localisation d'informations et celle des textes non continus, elles s'expliquent en grande partie par les autres variables incluses dans le modèle, des différences importantes subsistent entre les sexes sur les deux autres sous-échelles d'aspect (l'interprétation de textes et la réflexion et l'évaluation) et l'autre sous-échelle de format (les textes continus).

Quelques autres différences entre les sous-échelles méritent d'être mentionnées, même si elles sont minimes en valeur absolue. Ainsi, dans presque tous les pays, l'effet produit par la communication culturelle est le plus faible en localisation d'informations mais le plus élevé en réflexion et évaluation. Il est aussi plus faible sur la sous-échelle des textes non continus que sur celle des textes continus.

La comparaison de l'indice de climat de discipline sur les cinq sous-échelles révèle également des différences intéressantes. Plus les problèmes de discipline sont nombreux dans les établissements, plus les résultats de leurs élèves sont faibles sur la sous-échelle d'interprétation de textes et celle de réflexion et d'évaluation, par comparaison avec la sous-échelle de localisation d'informations. L'impact du climat de discipline est également plus faible sur la sous-échelle des textes non continus que sur celle des textes continus. Ces différences sont minimes, certes, mais elles s'observent dans tous les pays ou presque. 


\section{Variation selon les pays de l'effet des variables individuelles et scolaires sur les performances en lecture}

Si de faibles différences sont relevées entre les sous-échelles, d'autres, nettement plus importantes, sont observées dans les valeurs numériques des coefficients de régression des divers pays. Les tableaux 7.3 à 7.8 indiquent pour chaque pays les coefficients de régression des variables incluses dans les analyses. Un astérisque signale les coefficients de régression qui s'écartent significativement de zéro (alpha de 0,05).

Le tableau 7.3 présente les coefficients de régression pour le sexe et l'engagement à l'égard de la lecture à l'intérieur des établissements. Au sein des établissements, les différences entre les sexes par pays sont en moyenne comprises entre -0,5 en Pologne et 28,6 en Nouvelle-Zélande. Dans cinq pays - l'Allemagne, le Brésil, l'Irlande, les Pays-Bas, la Pologne et le Portugal - ces différences ne sont pas statistiquement significatives.

La variation des coefficients de régression pour l'engagement à l'égard de la lecture est également sensible. Ces coefficients sont compris entre 8,9 en Grèce et 31 en Islande et s'écartent significativement de zéro dans tous les pays.

Le tableau 7.4 montre la relation qui existe à l'intérieur des établissements entre le score en compréhension de l'écrit et trois variables familiales : la structure familiale, le statut professionnel des parents et le statut d'immigrant.

On associe généralement le fait de vivre dans une famille nucléaire à de meilleures performances scolaires. Cette relation est confirmée dans un certain nombre de pays. C'est aux États-Unis que le coefficient de régression le plus élevé $(21,5)$ est enregistré. Vivre dans une famille monoparentale ou mixte entrâne un gain de performance dans certains pays, tels que la Belgique et le Mexique. Il y a lieu de souligner que dans la moitié environ des pays l'impact de la structure familiale ne s'écarte pas significativement de zéro.

Les coefficients de régression pour le milieu socio-économique individuel - dérivé de l'indice socioéconomique international de statut professionnel - à l'intérieur des établissements sont compris entre -1,1 en Corée et 16,7 au Royaume-Uni. Ils ne s'écartent pas significativement de zéro dans quatre pays, l' Autriche, la Corée, la Hongrie et la Pologne, ce qui indique que les élèves qui fréquentent le même établissement mais qui sont issus d'un milieu socio-économique différent sont susceptibles d'obtenir des scores identiques en compréhension de l'écrit.

Les coefficients de régression de l'impact du statut d'immigrant varient également fortement d'un pays à l'autre. Ils sont compris entre -61,8 en Finlande et 8,7 en Irlande. Ce phénomène peut vraisemblablement s'expliquer par la variation des profils d'immigrant selon les pays. Il est très probable que les pays affichant un coefficient de régression positif accueillent des immigrants issus de milieux socio-économiques plus privilégiés. La langue parlée par les immigrants - qu'elle soit ou non identique à celle parlée dans le pays de destination - joue aussi un rôle important dans l'interaction entre le statut d'immigrant et le niveau de compétence en compréhension de l'écrit. ${ }^{5}$

Le tableau 7.5 montre les coefficients de régression au niveau intra-établissement de la communication culturelle avec les parents et du volume de ressources éducatives et de livres disponibles à la maison.

La communication culturelle entre un élève et ses parents - une variable dérivée de la fréquence à laquelle les parents discutent avec leur enfant de l'actualité politique ou sociale, de livres, d'émissions télévisées 
ou de films ou encore écoutent de la musique classique avec eux - est associée à des gains de performance en compréhension de l'écrit dans certains pays, parmi lesquels l'Australie $(9,2)$ et le Danemark $(10,9)$. Dans la moitié des pays toutefois, le coefficient de régression de l'impact de la communication culturelle ne s'écarte pas significativement de zéro.

De même, les ressources éducatives disponibles à la maison - telles que des manuels, un bureau ou un endroit calme pour étudier - ont un impact significatif sur la performance dans quelques pays, dont la Norvège $(10,3)$ et la Nouvelle-Zélande $(9,6)$, mais n'en ont aucun dans un nombre considérable de pays.

Les coefficients de régression pour le nombre de livres à la maison sont compris entre 0,6 au Brésil et 9,3 aux États-Unis. Ils s'écartent significativement de zéro dans tous les pays, si ce n'est au Brésil, en Italie et en Pologne.

Lors du cycle d'évaluation PISA 2000, des informations ont été recueillies auprès des élèves à propos d'un certain nombre d'aspects liés à l'enseignement et à l'apprentissage. Les coefficients de régression pour l'incitation au rendement telle qu'elle est perçue par les élèves, du temps que ceux-ci consacrent à leurs devoirs et de leur année d'études sont présentés dans le tableau 7.6, tandis que les coefficients de régression pour les relations entre élèves et enseignants, le sentiment d'appartenance à l'école et le climat de discipline à l'école tels qu'ils sont perçus par les élèves sont présentés dans le tableau 7.7.

Les coefficients de régression de l'année d'études sont compris entre 70,7 en Espagne et 10,5 au RoyaumeUni. Ils s'écartent significativement de zéro dans tous les pays, si ce n’est en Corée et en Norvège.

Exception faite de l'année d'études, les variables scolaires sont généralement associées à des écarts plus faibles de compétence en compréhension de l'écrit, même s'il existe certaines différences selon les pays. Pour chacune des variables, dans certains pays, elles ne semblent avoir aucun effet significatif sur le niveau de compétence en compréhension de l'écrit, alors que dans d'autres, ces variables ont un impact significatif, soit positif soit négatif, sur le niveau de compétence.

Enfin, deux variables entre établissements sont à l'origine de différences importantes de compétence et varient considérablement selon les pays (voir le tableau 7.8), à savoir le niveau social moyen et le degré moyen d'engagement à l'égard de la lecture par établissement.

Les coefficients de régression du milieu socio-économique moyen des établissements sont compris entre 7,5 en Islande et 82,6 en Pologne. En Islande, les différences de milieu socio-économique moyen n'entraînent pas d'écarts de performance en compréhension de l'écrit, contrairement à la Pologne, où une différence d'un écart type sur l'échelle de milieu socio-économique moyen donne lieu à un écart de performance de plus de 80 sur l'échelle de compétence de PISA.

Les coefficients de régression de l'engagement à l'égard de la lecture varient également fortement d'un pays à l'autre. Ils vont de -1 au Brésil à 65 en Grèce. Ils s'écartent significativement de zéro dans la majorité des pays, mais pas dans tous.

La variation sensible des coefficients de régression des variables individuelles et scolaires dans les différents pays ne doit pas nous amener à conclure que les pays présentent tous un profil unique et qu'il serait vain de tenter d'identifier des similitudes entre des pays ou des groupes de pays. Nous savons que certains pays présentent des structures ou des caractéristiques éducatives similaires. Dans des pays tels que l'Allemagne, 
l'Autriche et la Belgique, par exemple, les élèves sont généralement regroupés en fonction de leurs aptitudes scolaires par le biais de divers mécanismes : l'enseignement spécial, la différenciation des filières, le regroupement par aptitude, le redoublement ou encore la concurrence entre établissements, pour n'en citer que les principaux. Il n'en va pas de même dans des pays comme le Danemark, la Finlande, la Norvège et la Suède : soucieux de minimiser autant que faire se peut les différences entre établissements, ces pays appliquent des politiques qui limitent l'éventail d'établissements parmi lesquels les parents peuvent choisir en les cantonnant à des secteurs géographiques bien définis. De plus, ces pays ne pratiquent pas la différenciation des filières ou le regroupement par aptitude. Ils utilisent un système automatique de progression qui régule le passage à la classe supérieure. Ces différences de structure éducative ont selon toute vraisemblance un impact sur la relation entre les variables individuelles et scolaires et le niveau de compétence en compréhension de l'écrit. Nous allons à présent tenter d'identifier ces profils communs.

\section{Similitudes et différences entre les pays}

Nous allons à présent nous pencher sur les relations entre les variables explicatives et la performance des élèves sur l'échelle combinée de compréhension de l'écrit. Notre intention n'est pas de décrire les pays un par un mais plutôt de tenter d'identifier des points communs entre plusieurs pays concernant les relations entre les variables explicatives modélisées ci-dessus et le niveau de compétence en compréhension de l'écrit. Nous classerons ensuite les pays dans diverses catégories sur la base des similitudes et des différences que nous aurons décelées.

Cet exercice ne va pas sans difficultés. Classer ou grouper les pays en fonction des tendances qui se dégagent dans les relations entre performances en lecture et d'autres variables n'est pas une opération à caractère strictement objectif : les analyses de ces relations sont, en effet, basées sur des variables continues. Or, pour construire un système de classification, il faut découper ces variables continues en un certain nombre de catégories, avec un risque d'arbitraire.

\section{Variation des performances en lecture entre établissements}

La performance en lecture d'un élève peut être exprimée non seulement par le score obtenu au test, mais aussi par la différence qui existe entre son score et le score moyen de son pays. Dans les recherches pédagogiques, cette différence entre le score de l'élève et le score moyen de son pays est souvent divisée en deux parties : (i) la distance entre l'estimation de la performance de l'élève et le score moyen de l'établissement qu'il fréquente et (ii) la distance entre le score moyen de son établissement et celui de son pays. La première différence renvoie à ce que l'on appelle la variance à l'intérieur de l'établissement. Elle indique dans quelle mesure les scores des élèves peuvent varier au sein du même établissement. La seconde différence, c'est-à-dire celle entre le score moyen de l'établissement et le score moyen du pays, correspond à la variance entre établissements. Elle indique dans quelle mesure les scores des élèves peuvent varier d'un établissement à l'autre. Il est d'usage d'exprimer l'importance relative de ces deux variances par le truchement de la corrélation intra-classe. 


\section{Encadré 7.1 : La corrélation intra-classe}

Ce coefficient est égal à la variance entre établissements divisée par la somme des variances inter-et intra-établissement. Il est égal à zéro si les estimations des scores moyens des établissements sont tous identiques et augmente à mesure que les estimations des scores moyens des établissements s'écartent les unes des autres. Un coefficient de 1 indique que tous les établissements affichent un score différent mais que tous les élèves de chaque établissement obtiennent le même score ou, en d'autres termes, que le score des élèves ne varie pas au sein des établissements une fois que la variation entre établissements est prise en considération.

Le graphique 7.3 présente le coefficient de corrélation intra-classe, c'est-à-dire le pourcentage de la variance totale du score en compréhension de l'écrit qui peut être imputé aux établissements dans chaque pays.

\section{Graphique 7.3}

Part de la variance entre établissements dans la variance totale des performances en compréhension de l'écrit

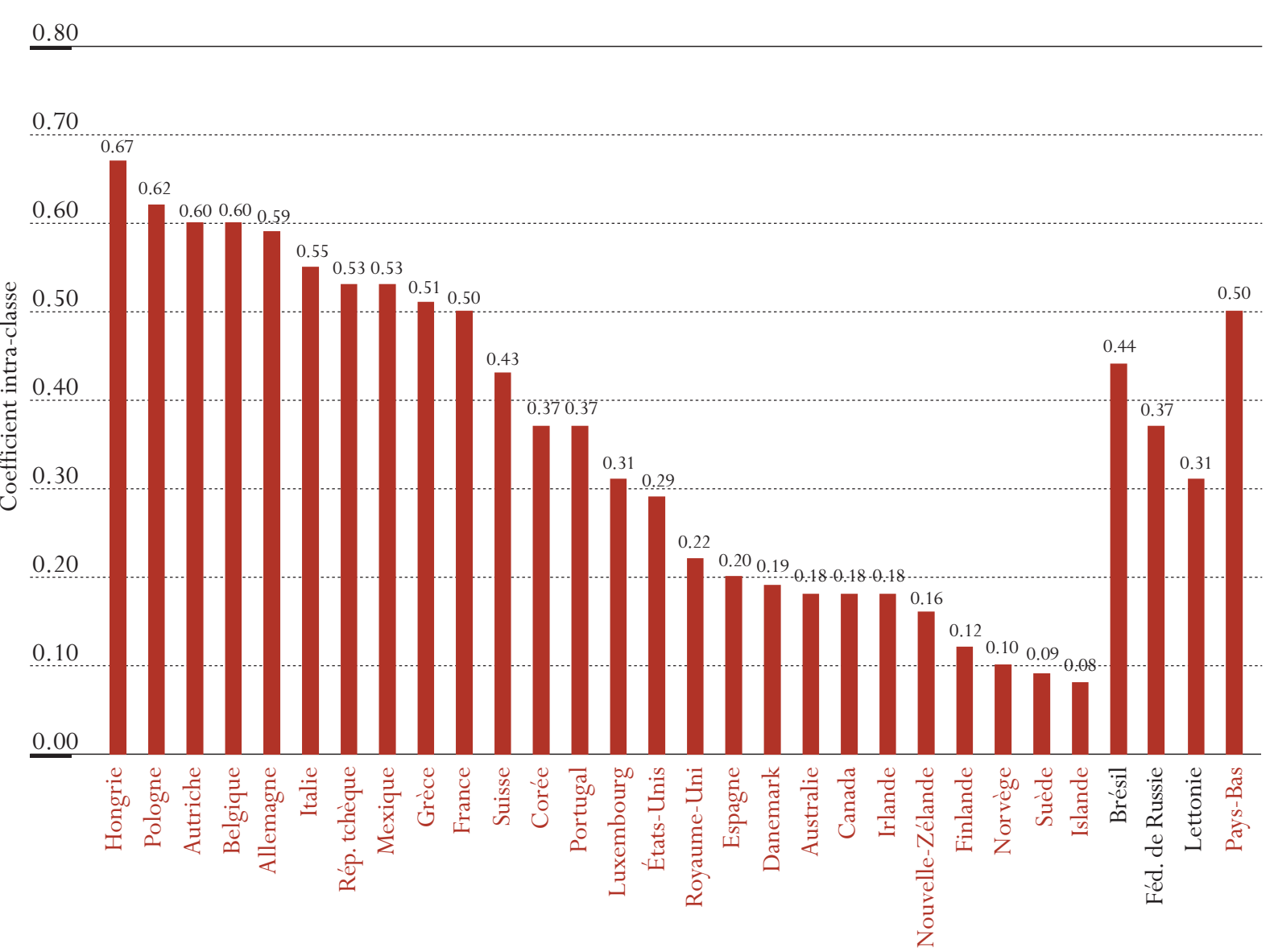

Source : Base de données PISA de l'OCDE, 2001, tableau 7.9. 
Dans les pays qui ont participé à PISA, les corrélations intra-classe sont comprises entre 0,08 et 0,67, ce qui signifie que la part de la variance totale imputable aux différences entre établissements représente 8 pour cent seulement dans le premier cas, mais plus des deux tiers dans le second cas. Plusieurs tendances se profilent à cet égard : dans tous les pays nordiques à l'exception du Danemark, c'est-à-dire en Finlande, en Islande, en Norvège et en Suède, les coefficients de corrélation intra-classe sont systématiquement inférieurs à 0,15 (ils sont compris entre 0,08 et 0,13 ). Les corrélations intra-classe vont de 0,15 à 0,30 dans les six pays essentiellement anglophones et sont supérieures à 0,30 dans tous les autres pays d'Europe, sauf en Espagne. Ces résultats montrent que des pays unis par des liens géographiques, politiques ou historiques ont certaines caractéristiques en commun dans le domaine de l'éducation. Selon toute vraisemblance, ces tendances sont en partie façonnées par les structures éducatives.

\section{Influence du milieu socio-économique des élèves sur les performances en lecture}

Comme nous l'avons dit, la relation entre le milieu socio-économique et les performances scolaires des élèves peut passer par des structures scolaires qui pratiquent la ségrégation sociale. Par exemple, dans certains pays, le choix de l'établissement dépend du milieu socio-économique et économique des élèves. En effet, dans les systèmes où coexistent des établissements payants et gratuits, certains élèves ont un choix restreint car leurs parents ne peuvent se permettre de payer des droits d'inscription. La ségrégation sociale peut également être induite par d'autres mécanismes. À titre d'exemple, citons les pays où l'enseignement général et professionnel est dispensé par des établissements différents. Dans ce cas, le milieu socio-économique moyen des établissements peut varier considérablement car les élèves issus de milieux socio-économiques moins privilégiés ont tendance à se diriger vers des filières professionnelles.

Le graphique 7.4 compare dans les différents pays les coefficients de régression du milieu socio-économique individuel des élèves et ceux du milieu socio-économique moyen des établissements (c'est-à-dire la valeur moyenne de l'indice du milieu socio-économique des élèves). Les coefficients de régression quantifient l'impact des variables explicatives sur le niveau de compétence en compréhension de l'écrit. Dans les pays situés dans la partie inférieure droite du graphique, le milieu socio-économique individuel influe davantage sur le niveau de compétence en compréhension de l'écrit que le milieu socio-économique moyen, c'est-à-dire celui calculé à l'échelle des établissements. À l'inverse, dans les pays situés dans la partie supérieure gauche du graphique, le milieu socio-économique moyen a un impact plus important sur le niveau de compétence en compréhension de l'écrit que le milieu socio-économique individuel.

Comme le montre le graphique 7.4, les pays anglophones et les pays nordiques comme le Danemark, la Finlande, l'Islande et la Norvège se démarquent des autres pays par un impact relativement important du niveau social individuel, ce qui signifie que la performance des élèves dépend de leur milieu socio-économique, quel que soit l'établissement fréquenté. L'analyse de ce résultat à la lumière des corrélations intra-classe présentées dans le graphique 7.3 montre que l'effet minime du milieu socio-économique moyen par établissement enregistré dans les pays nordiques n'a guère d'impact sur les écarts relativement faibles observés entre les établissements en compréhension de l'écrit. Dans les pays anglophones en revanche, l'impact du milieu socio-économique moyen est plus important, ce qui signifie que l'établissement que fréquente un élève est déterminé en partie par son milieu socio-économique et que la performance moyenne des établissements en lecture s'en trouve affectée.

L'impact du milieu socio-économique individuel est moins important dans la plupart des autres pays européens ainsi qu'au Brésil, en Corée et au Mexique. En revanche, les différences de milieu socio-économique moyen entre établissements sont étroitement associées aux écarts de performance observés entre eux. 
Effets du milieu socio-économique des élèves et des établissements sur les performances en compréhension de l'écrit

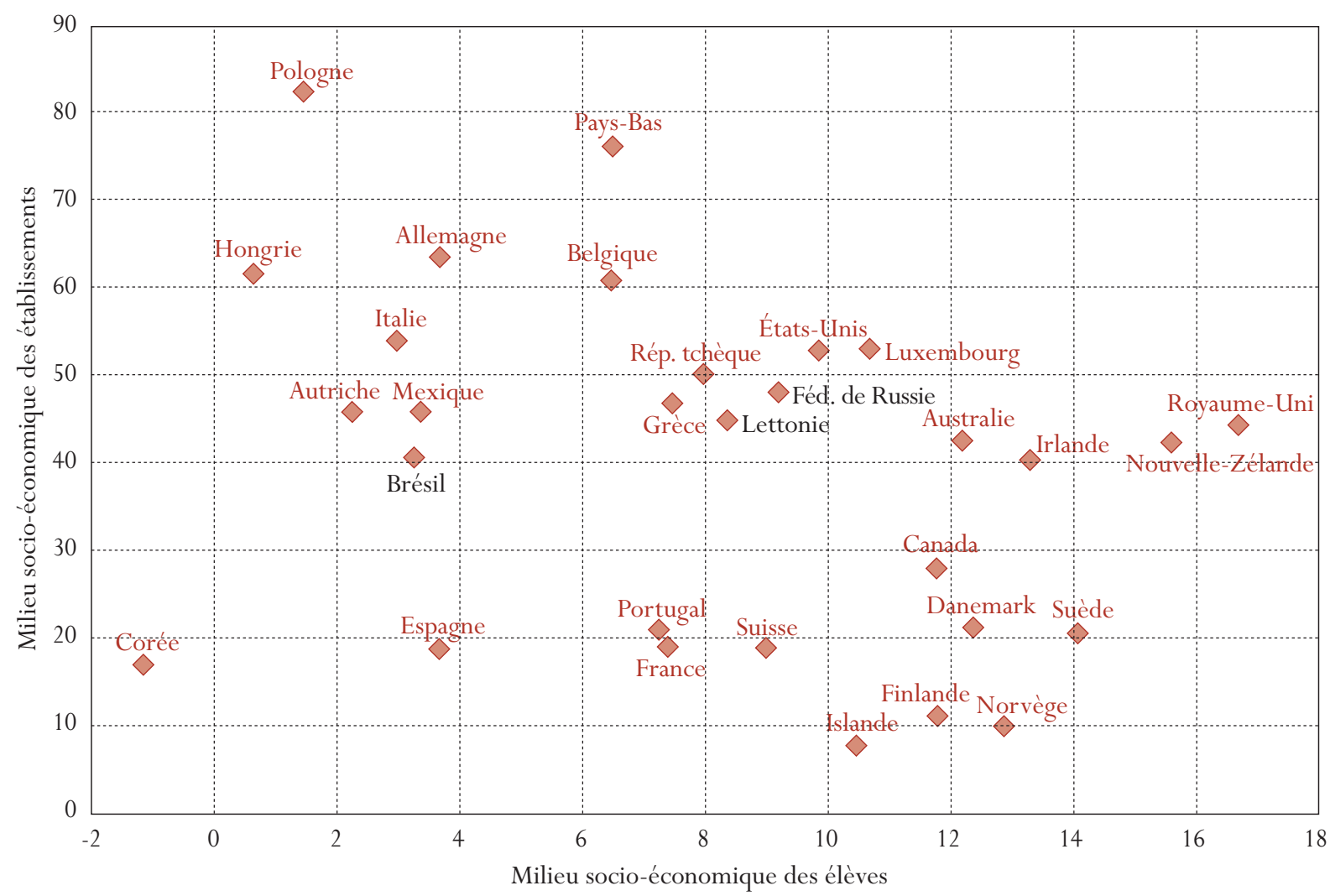

Source: Base de données PISA de l'OCDE, 2001, tableau 7.8.

Dans des pays tels que l'Allemagne, l'Autriche, la Belgique et les Pays-Bas, la grande différenciation des filières d'enseignement contribue largement à la ségrégation sociale.

En Corée, en Espagne, en France, au Portugal et en Suisse, le milieu socio-économique moyen ne semble pas être associé à des écarts de performance entre établissements.

Le graphique 7.4 montre également la complexité de la relation entre le milieu socio-économique des élèves et leurs résultats scolaires. Dans des pays tels que l'Islande et la Suède, l'établissement fréquenté par les élèves n'est pas déterminant mais la performance attendue au sein d'un établissement donné dépend du milieu économique et social des élèves.

Par contraste, en Autriche, en Corée, en Hongrie et au Portugal, la performance théorique d'un élève inscrit dans un établissement donné est indépendante de son milieu économique et social (les coefficients de régression de ces quatre pays ne s'écartent pas significativement de zéro). L'impact du milieu socioéconomique sur la performance individuelle des élèves est aussi relativement faible, mais significatif, en Allemagne, au Brésil, en Italie et au Mexique. Pourtant, l'influence de l'établissement est déterminante dans tous ces pays sauf en Corée, ainsi que le montrent les coefficients de régression du milieu socioéconomique moyen. En d'autres termes, dans ces pays, il semblerait que le milieu socio-économique 


\section{Graphique 7.5}

Droite de régression multi-niveaux du milieu socio-économique des élèves en Islande

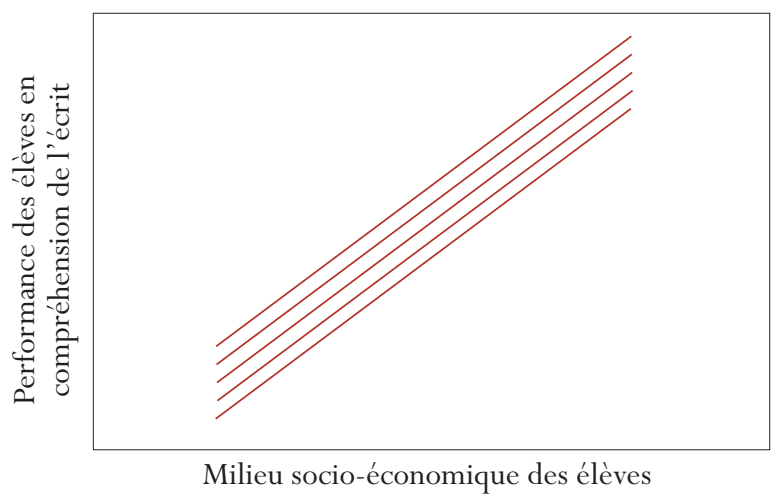

Graphique 7.6

Droite de régression multi-niveaux du milieu socio-économique des élèves en Grèce

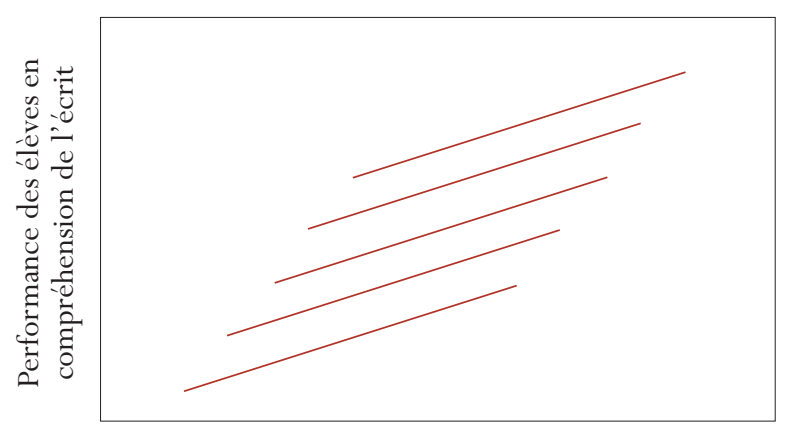

Milieu socio-économique des élèves

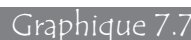

Droite de régression multi-niveaux du milieu socio-économique des élèves en Pologne

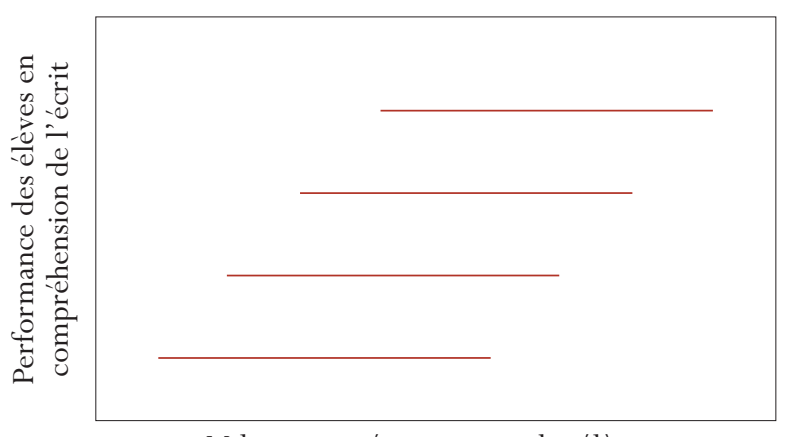

Milieu socio-économique des élèves individuel ne soit pas le facteur prépondérant, mais plutôt l'établissement fréquenté. Il y a lieu de rappeler toutefois que le milieu socio-économique des élèves peut également intervenir dans le choix de l'établissement.

Les graphiques 7.5 à 7.7 représentent d'une autre façon les différences relevées entre les systèmes d'éducation concernant l'impact de la composition socio-économique moyenne des établissements et du milieu socio-économique individuel des élèves. Comme au graphique 7.1 , chaque ligne correspond à la régression du milieu socioéconomique sur le niveau de compétence en compréhension de l'écrit dans un établissement donné. L'abscisse représente le milieu socioéconomique individuel des élèves et l'ordonnée, le niveau de compétence en compréhension de l'écrit.

Le graphique 7.5 montre à quoi ressembleraient les courbes de régression par établissement en Islande. La proximité des lignes de régression dénote une faible variance inter-établissement du niveau de compétence en compréhension de l'écrit. Quant à la pente des lignes de régression, elle indique que les élèves qui fréquentent le même établissement mais qui sont issus de milieux sociaux différents obtiennent des résultats différents en compréhension de l'écrit.

Le graphique 7.6 illustre le cas de la Grèce. Les lignes de régression débutent et se terminent à des niveaux différents de l'abscisse, ce qui traduit la variation de la composition socio-économique moyenne de l'établissement. La dispersion plus prononcée des lignes sur l'ordonnée montre la présence d'écarts de performance en compréhension de l'écrit entre les établissements. Toutefois, la pente de chacune des lignes de régression est moins forte, ce qui indique qu'au sein des établissements, le milieu socio-économique a un impact statistique plus faible sur le niveau de compétence en compréhension de l'écrit. 
Enfin, le graphique 7.7 présente les lignes de régression de la Pologne. Ces lignes sont parallèles à l'abscisse, ce qui indique que le milieu socio-économique individuel au sein des établissements n'a pas d'impact statistique. En revanche, la grande dispersion des lignes de régression le long des deux axes traduit la présence d'écarts importants de milieu socio-économique moyen et de niveau de compétence en compréhension de l'écrit entre les établissements.

\section{Influence de l'engagement à l'égard de la lecture}

Le degré d'engagement à l'égard de la lecture est une deuxième variable intéressante fortement corrélée au niveau de compétence en compréhension de l'écrit. De plus, contrairement au sexe ou au milieu socioéconomique, il s'agit d'une caractéristique qu'il est possible de faire évoluer.

À l'instar du milieu socio-économique, l'engagement à l'égard de la lecture a été introduit dans l'analyse tant au niveau de l'élève que de l'établissement (degré d'engagement moyen). Il s'agit d'une indication composite du temps consacré à la lecture par les élèves d'un établissement et de leurs attitudes à l'égard de la lecture. Au niveau individuel, une valeur élevée de cet indice signifie que l'élève a déclaré lire beaucoup et aimer la lecture. Au niveau collectif, l'indice indique dans quelle mesure les élèves fréquentant l'établissement s'investissent dans la lecture, toujours selon leurs propres déclarations.

\section{Graphique 7.8}

Effets de l'engagement des élèves à l'égard de la lecture et de l'engagement moyen des établissements sur les performances en compréhension de l'écrit



Source : Base de données PISA de l'OCDE, 2001, tableaux 7.3 et 7.8 . 
Le graphique 7.8 compare, par pays, les coefficients de régression de l'engagement à l'égard de la lecture calculés au niveau de l'élève et au niveau de l'établissement.

À l'instar de ce que nous avons observé concernant le milieu socio-économique, des pays comme le Danemark, la Finlande, l'Islande et la Norvège présentent un coefficient de régression élevé de l'indice individuel d'engagement à l'égard de la lecture et les pays anglophones, un coefficient moyen. Ces deux groupes de pays affichent un impact limité du degré moyen d'engagement à l'égard de la lecture, c'est-àdire l'indice calculé au niveau des établissements. Tous les autres pays se caractérisent par un impact faible ou modéré au niveau individuel mais généralement plus élevé que celui enregistré au Danemark, en Finlande, en Islande, en Norvège et dans les pays anglophones au niveau des établissements.

\section{Ségrégation scolaire ou ségrégation sociale?}

Le graphique 7.9 compare les coefficients de régression du degré moyen d'engagement à l'égard de la lecture à ceux du milieu socio-économique moyen. Elle met en évidence des différences entre pays similaires à celles évoquées ci-avant.

\section{Graphique 7.9}

Effets du milieu socio-économique opposés au degré d'engagement des établissements

à l'égard de la lecture sur les performances en compréhension de l'écrit

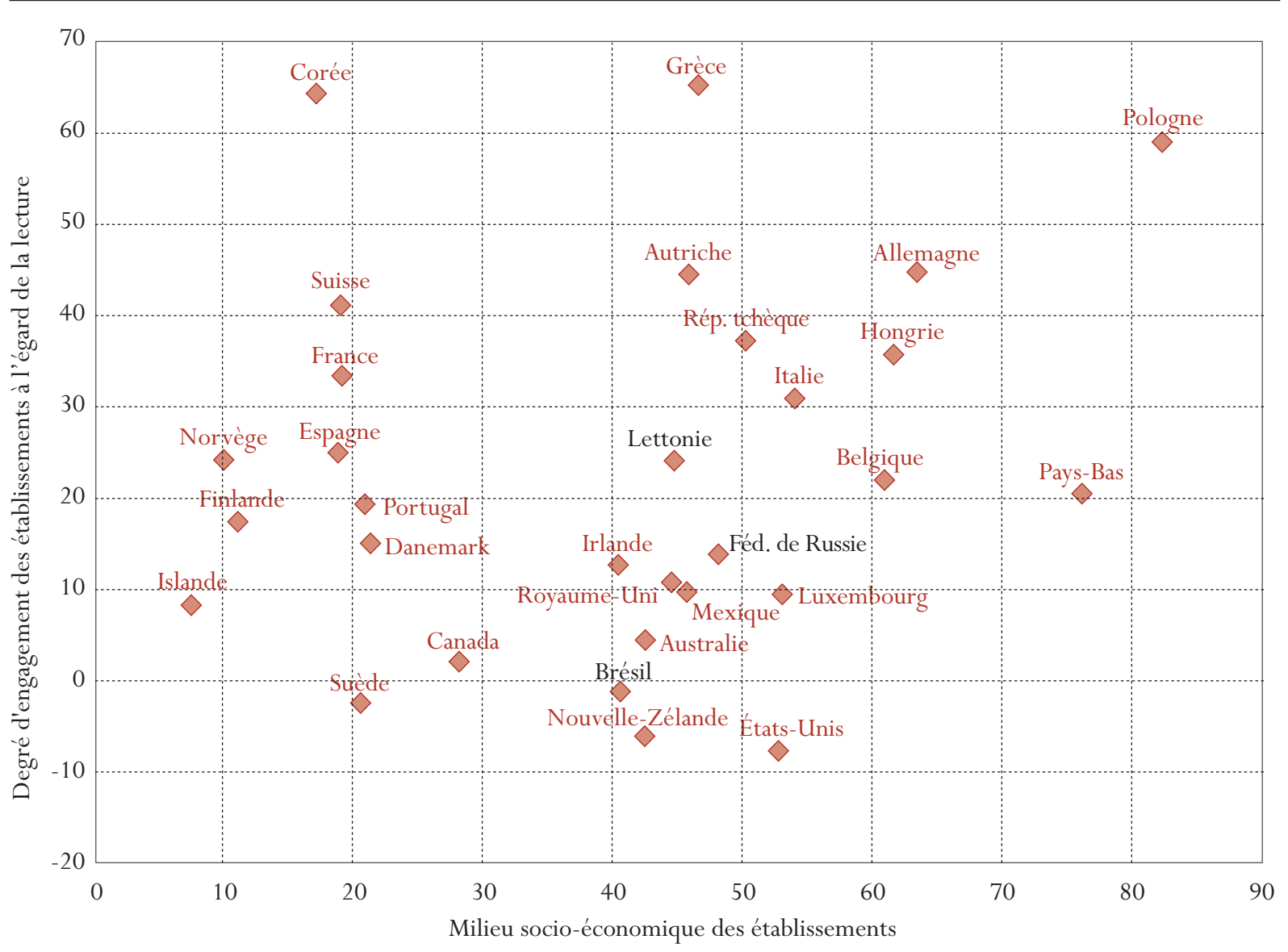

Source: Base de données PISA de l'OCDE, 2001, tableaux 7.4 et 7.8. 
Au Danemark, en Finlande, en Islande et en Suède, l'impact de ces deux indices composites est relativement limité au niveau des établissements.

Les pays anglophones enregistrent un impact plus faible du degré moyen d'engagement à l'égard de la lecture mais un impact plus important du milieu socio-économique moyen.

Le Brésil, la Fédération de Russie, le Luxembourg et le Mexique ressemblent aux pays anglophones dans la mesure où le coefficient de régression de l'impact du milieu socio-économique moyen y est plus élevé que celui de l'impact du degré moyen d'engagement à l'égard de la lecture.

Dans d'autres pays, dont l'Espagne, la France, le Portugal, la Suisse et surtout la Corée, le coefficient de régression de l'impact du milieu socio-économique moyen est plus faible que celui de l'impact du degré moyen d'engagement à l'égard de la lecture.

Comme nous l'avons dit, le coefficient de régression de l'impact du milieu socio-économique moyen traduit l'importance de la ségrégation sociale dans les structures éducatives de chaque pays. Les établissements peuvent recruter leurs élèves en fonction de leur milieu socio-économique, que ce soit directement ou indirectement. Une sélection directe s'opère par exemple dans les systèmes éducatifs où coexistent des établissements payants - qui imposent des droits d'inscription - et des établissements gratuits, car tous les parents ne peuvent se permettre de payer ces droits. Quant à la sélection indirecte, elle peut se fonder sur des critères géographiques, par exemple si le milieu socio-économique varie d'une région à l'autre et que les élèves fréquentent l'établissement situé à proximité de chez eux. Même gratuit, un établissement situé dans une banlieue huppée accueillera surtout des élèves issus de milieux sociaux privilégiés, générant de la sorte une forme indirecte de ségrégation sociale.

Contrairement au milieu socio-économique, l'engagement à l'égard de la lecture n'est pas un critère appliqué par les établissements pour recruter leurs élèves. Aucun établissement n'accepte ou ne refuse un élève sur la base du nombre de livres qu'il lit par an ou sur la façon dont il dit apprécier la lecture. Et pourtant, cette variable scolaire produit un impact suffisamment important dans plusieurs pays pour être assimilé à un effet de ségrégation supplémentaire. Comment peut-on expliquer cela ? Le premier critère qui vient à l'esprit est le parcours scolaire antérieur des élèves. Les parents des élèves «brillants » sont plus susceptibles de les inscrire dans des établissements très exigeants et dans des filières générales, contrairement aux parents des élèves «faibles» qui ont plutôt tendance à choisir des établissements moins exigeants. À la lumière de la corrélation importante des performances en lecture, en mathématiques et en sciences et de l'impact du degré d'engagement à l'égard de la lecture sur le niveau de compétence en compréhension de l'écrit, il n'est pas déraisonnable de considérer l'effet du degré moyen d'engagement à l'égard de la lecture comme un indicateur de sélection scolaire.

Les données représentées au graphique 7.9 étayent cette interprétation. Dans des pays tels que l'Australie et les États-Unis où le regroupement par filière n'est guère pratiqué par les établissements mais où un nombre considérable d'élèves fréquentent des établissements payants, les critères sociaux l'emportent sur les critères scolaires. Ainsi, un élève «brillant » issu d'un milieu socio-économique peu favorisé n'a guère de chances de fréquenter un établissement payant. En revanche, les parents qui en ont les moyens sont plus susceptibles d'inscrire leur enfant dans un établissement payant, quels que soient ses résultats scolaires.

Dans des pays tels que l'Allemagne, la Belgique et la Grèce où les établissements dispensent différents types de programmes d'enseignement (général, technique ou professionnel), les élèves sont groupés avant 
tout en fonction de leurs résultats scolaires. Comme les élèves inscrits dans les filières professionnelles sont souvent issus de milieux moins favorisés que ceux inscrits dans les filières générales, la différenciation des filières pratiquée dans ces pays perpétue la ségrégation sociale.

En Corée, en Espagne, en France, au Portugal et en Suisse, les écarts entre les établissements semblent provenir essentiellement du regroupement des élèves en fonction de leurs aptitudes scolaires.

Il y a lieu de souligner qu'en France et en Grèce, où le premier et le deuxième cycle du secondaire sont dispensés par deux types d'établissements différents, la sélection scolaire peut avoir été surestimée et la ségrégation sociale, sous-estimée. Enfin, il semble ne pas exister de système de sélection dans des pays tels le Danemark, la Finlande, l'Islande et la Norvège. L'impact de la corrélation intra-classe y est similaire à celui des pays anglophones (quoique plus faible), mais il ne s'explique pas par les mêmes raisons.

\section{Autres variables contextuelles}

Les autres variables individuelles incluses dans ce modèle épousent les mêmes tendances.

Ainsi, le coefficient moyen de régression de la communication culturelle est de 4,89 dans des pays nordiques et les pays anglophones, mais de 1,71 dans les pays accusant une forte corrélation intra-classe. Par ailleurs, l'influence de la structure familiale, du nombre de livres disponibles à la maison et du sexe semble supérieure dans les pays présentant une faible corrélation intra-classe. Dans ces pays, le fait de vivre dans une famille monoparentale ou nucléaire ou d'être de sexe masculin ou féminin est associé à des écarts de performance plus importants, quel que soit l'établissement fréquenté. Il ne faut pas en conclure que ces différences n'existent pas dans d'autres pays mais plutôt que l'effet de ces variables passe par l'établissement scolaire. Ainsi, dans les systèmes qui comportent des filières très différenciées, l'enseignement technique et professionnel accueille davantage de garçons et l'enseignement général, davantage de filles. De même, les élèves issus de milieux socio-économiques plus privilégiés ont aussi en général plus de livres à leur disposition chez eux et sont plus susceptibles de fréquenter des établissements d'enseignement général, plutôt que de se diriger vers des filières techniques ou professionnelles.

\section{Variables scolaires et pédagogiques}

L'effet intra-établissement de la perception par les élèves du degré d'incitation au rendement, du sentiment d'appartenance à l'école et de la qualité des relations entre élèves et enseignants sur les performances en lecture varie selon que les pays présentent une corrélation intra-classe plus élevée ou plus faible.

Dans les pays à dominante anglophone et certains pays nordiques cités plus haut, les élèves qui ont l'impression que leurs professeurs les pressent de réussir obtiennent en moyenne des scores en compréhension de l'écrit inférieurs à ceux des élèves qui n'ont pas cette impression. Cette impression provient peut-être des efforts déployés par les enseignants pour aider les élèves en difficulté. Dans d'autres pays, la relation à l'intérieur de l'établissement entre le niveau de compétence en compréhension de l'écrit et l'encouragement est faible, voire pratiquement nulle. En moyenne, les élèves perçoivent légèrement plus de pression de leurs professeurs au Danemark, en Finlande, en Norvège, en Suède et dans les pays à dominante anglophone que dans les autres pays.

Par ailleurs, on pourrait s'attendre à ce que l'indice de pression varie au niveau école, et non au niveau individuel, dans les systèmes d'éducation comportant des filières très différenciées ou encore que les professeurs des élèves inscrits dans l'enseignement général les encouragent davantage à réussir que ceux des élèves inscrits dans les filières professionnelles. La décomposition de la variance de l'incitation au rende- 
ment par école ou par élève infirme ces hypothèses. Dans tous les pays, sauf la République tchèque, la part de variance imputable aux établissements ne dépasse pas la barre des 10 pour cent. Ce phénomène peut s'expliquer par le fait que la perception de la pression à réussir par les élèves est une valeur relative, et non une valeur absolue : les élèves ressentent plus ou moins les encouragements de leurs professeurs par rapport à leurs condisciples, ce qui masque les différences au niveau des établissements. L'impact limité de l'incitation au rendement dans des systèmes aux filières très différenciées peut également provenir du regroupement par aptitude pratiqué au sein même des établissements. Dans cette hypothèse, les élèves qui sont incités à mieux travailler ne sont pas nécessairement ceux qui obtiennent des résultats médiocres par rapport à l'ensemble de la cohorte.

Les coefficients de régression du sentiment d'appartenance des élèves à leur établissement révèlent également une tendance intéressante. Ces coefficients sont compris entre -4,3 et 6,3. Ils ne s'écartent significativement de zéro qu'en Belgique, au Canada, en Finlande, en Grèce, en Hongrie, au Luxembourg, au Mexique, en Pologne, au Portugal, en République tchèque, en Suède et en Suisse. Même si les coefficients de régression ne sont pas significatifs ou sont pratiquement nuls dans la plupart des pays qui ont participé à PISA, force est de constater que les pays qui affichent le coefficient de régression le plus faible constituent un groupe familier : dans l'ordre croissant à partir du coefficient le plus négatif, ce sont la Finlande, la Norvège, la Suède, l'Australie, la Nouvelle-Zélande, l'Irlande, le Canada, le Royaume-Uni et l'Islande - c'est-à-dire des pays nordiques et les pays à dominante anglophone. Dans les autres pays, les coefficients de régression sont légèrement positifs, ce qui indique que les élèves qui ont un sentiment d'appartenance plus fort par rapport à leur école obtiennent en moyenne de meilleurs résultats que les autres.

Les coefficients de régression de la relation entre élèves et enseignants ont une influence similaire à ceux calculés pour le sentiment d'appartenance des élèves à leur école. Les pays qui affichent le coefficient le plus élevé sont, dans l'ordre décroissant, la Norvège, l'Islande, la Finlande, le Canada, la Suède, la Nouvelle-Zélande, le Danemark et le Royaume-Uni. Dans ces pays, les élèves qui déclarent entretenir de bonnes relations avec leurs enseignants obtiennent en moyenne de meilleurs résultats. Dans les autres pays d'Europe, les coefficients de régression ne sont pas significatifs et tendent à être négatifs.

\section{Variation de performances en lecture entre élèves au sein des établissements}

Les systèmes d'éducation des pays à dominante anglophone et le Danemark, la Finlande, l'Islande, la Norvège et la Suède présentent certains points communs, à l'exception toutefois du processus d'agrégation dû au milieu socio-économique. Il y a lieu de rappeler que la structure du PISA ne permet pas forcément d'identifier toutes les différences. Théoriquement, la variance totale peut être divisée en trois composantes : la variance entre les établissements, la variance entre les classes au sein des établissements et la variance au sein des classes. Comme PISA a adopté une méthode d'échantillonnage simple et aléatoire des élèves dans les établissements, la variance entre les classes au sein des établissements et la variance au sein des classes se confondent. Contrairement à PISA, l'IEA procède généralement à l'échantillonnage aléatoire d'une classe entière dans un établissement; dans ce cas, ce sont la variance entre établissements et la variance entre les classes au sein des établissements qui se confondent. La comparaison des coefficients de corrélation intra-classe calculés sur la base des données de PISA et de l'IEA permet de donner une idée générale de l'ampleur de la variance entre les classes au sein des établissements.

Le tableau 7.10 compare les corrélations intra-classe basées sur les données de PISA et celles de deux enquêtes de l'IEA, à savoir la « Reading Literacy Study » (IEA/RLS, 1991) et la Troisième étude internationale sur les mathématiques et les sciences (TIMSS, 1995). 
Le Danemark, la Finlande, l'Islande, la Norvège et la Suède ont participé à l'enquête IEA/RLS au début des années 1990. La part de la variance entre classes, exprimée en pourcentage de la variance totale, y est inférieure à 0,10 (Postlethwaite, 1995, voir le graphique 7.17.). En d'autres termes, les variances calculées par PISA, imputables aux établissements, ne diffèrent pas des variances de l'IEA/RLS, imputables aux établissements et aux classes. Par ailleurs, dans les pays à dominante anglophone, la variance entre classes estimée par l'IEA/RLS est différente de la variance entre établissements calculée dans PISA. Ainsi, en Irlande, la part de variance imputable aux classes et aux établissements estimée par l'IEA/RLS, exprimée en pourcentage de la variance totale, est de 0,48 alors que dans PISA, la variance imputable aux établissements y est de 0,18. Les corrélations intra-classe calculées dans l'IEA/RLS sont de 0,41 en Nouvelle-Zélande et de 0,42 aux États-Unis, alors que celles de PISA sont respectivement de 0,16 et 0,29. Des disparités du même ordre s'observent entre les corrélations intra-classe de PISA et de l'étude TIMSS en Australie, aux États-Unis, en Irlande et en Nouvelle-Zélande. Rappelons à cet égard que l'étude TIMSS - comme l'IEA/RLS - a échantillonné des classes entières. L'écart important observé dans la majorité des pays anglophones reflète le fait que leur système d'éducation regroupe les élèves dans des classes en fonction de leurs aptitudes ou d'autres critères touchant à leur rendement scolaire (au Canada et aux États-Unis toutefois, les corrélations intra-classe en mathématiques ne suggèrent pas d'éventuel regroupement par aptitude, du moins dans les cours de mathématiques).

Ce processus de regroupement par aptitude mis en œuvre au sein des établissements dans certains pays anglophones peut expliquer les écarts observés dans les coefficients de régression de la variable composite « devoirs ». Au Danemark, en Finlande, en Islande, en Norvège et en Suède, il existe une corrélation négative entre cette variable et le niveau de compétence. Les élèves qui obtiennent de piètres résultats ont tendance à consacrer davantage de temps à leurs devoirs. Il est possible que ces élèves aient besoin de plus de temps que les autres pour faire leurs devoirs ou que leurs professeurs leur donnent plus de devoirs pour les aider à rattraper leur retard. En revanche, dans les pays anglophones, il existe une corrélation positive entre la variable composite des devoirs et le niveau de compétence. Il n'est guère probable que les élèves «brillants » aient besoin de davantage de temps pour terminer leurs devoirs. Une hypothèse plausible peut être avancée sachant que le regroupement par aptitude est pratiqué dans ces pays : les professeurs donnent plus de devoirs à faire dans les classes très performantes.

La comparaison des coefficients de corrélation intra-classe de PISA et des études TIMSS et IEA/RLS nous rappelle que le choix de la population cible (définie soit en fonction de l'année d'études, soit de l'âge) peut affecter les résultats, en particulier dans les pays où les jeunes de 15 ans sont inscrits dans un large éventail d'années d'études. En conséquence, il convient de prendre systématiquement la population cible en considération lors de l'interprétation des résultats.

Nous avons expliqué dans l'introduction que, même s'il est indéniable que chaque pays présente - dans une certaine mesure - un profil unique dans le domaine de l'éducation, certains pays ont en commun des caractéristiques qui affectent le mode d'interaction entre les caractéristiques des établissements, le milieu socio-économique des élèves et leur comportement, qui se combinent pour influer sur les performances scolaires.

Dans ce chapitre, nous avons identifié des points communs dans le fonctionnement des systèmes d'éducation. Nous avons également expliqué à quel point les tendances qui ressortent des relations entre variables étaient complexes. Le temps que les élèves passent à faire leurs devoirs chez eux en est un bon exemple. Consacrer plus de temps à ses devoirs est associé à un léger gain de performance dans certains pays, à une 
légère perte de performance dans d'autres. Ce contraste s'explique de toute évidence par les politiques différentes menées par les pays en matière de devoirs.

Tous les pays s'inquiètent des inégalités dans l'éducation et plusieurs réformes ont déjà été mises en œuvre pour tenter de les atténuer. Toutefois, il est établi que les inégalités sont différentes selon les pays; il faut donc prendre ces différences en considération pour élaborer des réformes qui soient réellement efficaces. Ainsi, les pays qui cherchent à réduire les inégalités entre les sexes devraient s'intéresser à l'impact du sexe et du degré d'engagement à l'égard de la lecture sur le niveau de compétence tant au niveau individuel qu'au niveau des établissements. Les pays qui affichent de faibles corrélations intra-classe doivent trouver le moyen d'améliorer le degré d'engagement des garçons à l'égard de la lecture, quel que soit l'établissement fréquenté. En revanche, ceux qui présentent une forte corrélation intra-classe doivent plutôt concentrer leurs efforts en la matière sur certains types d'établissements, tels que ceux qui dispensent l'enseignement technique ou professionnel.

Il n'est guère probable que les systèmes d'éducation réussissent à supprimer totalement les inégalités d'origine sociale ou autre. Selon les pays, les inégalités se concentrent au sein même des établissements ou entre les établissements. Certains pays combinent même ces deux formes d'inégalités. Tenter d'atténuer les inégalités à un niveau peut revenir à les accentuer à un autre niveau.

Toutefois, les inégalités semblent moins marquées dans certains systèmes d'éducation. Les cinq pays d'Europe du Nord figurent tous parmi les pays qui enregistrent l'association la plus faible entre le milieu socioéconomique et le niveau de compétence en compréhension de l'écrit et affichent en outre en moyenne une variation plus faible des performances des élèves. 
CHAPITRE 7 La lecture, moteur de changement: Performance et engagement d'un pays à l'autre

\section{Notes}

1. La compréhension de l'écrit dépend selon nous de multiples facteurs. Le modèle utilisé ici se concentre toutefois sur des variables choisies par souci de concision et de clarté. Les variables incluses dans le modèle ont été sélectionnées à la lumière de considérations théoriques et de recherches.

2. Les variables telles que les ressources matérielles et éducatives des établissements, le comportement des enseignants et des élèves, la pénurie d'enseignants, le moral des enseignants, le type d'établissements et les filières proposées dans les établissements ont dans un premier temps été incluses dans le modèle mais elles en ont finalement été exclues en raison de leur impact minime ou du pourcentage de données manquantes.

3. Les analyses de régression multiplient les variables incluses dans le modèle, ce qui provoque une augmentation du nombre d'élèves exclus de ces analyses pour cause de données manquantes. Le tableau A3.1 en annexe indique le nombre d'élèves inclus dans la base de données internationale du cycle PISA 2000 et le nombre d'élèves qui ont été pris en considération dans les analyses de régression à niveaux multiples présentées ici, ainsi que le rapport entre ces deux chiffres. Près de 90 pour cent des élèves sont en moyenne inclus dans les analyses. Le tableau A3.1 montre clairement pourquoi le Japon a été exclu des analyses de ce chapitre : les variables incluses dans les analyses n'étaient complètes que pour 43 pour cent des élèves seulement. De plus, la méthode d'échantillonnage appliquée dans ce pays ne permet pas de décomposer la variance entre établissements et la variance à l'intérieur des établissements.

4. Les élèves inscrits dans l'année d'études inférieure à leur année d'études théorique n'ont pas nécessairement redoublé une année. Ils peuvent avoir entamé leur scolarité tardivement pour des raisons personnelles ou de santé.

5. Consulter l'annexe A3 pour une note explicative sur le statut d'immigrant. 


\section{Chapitre}

8

\section{NIVEAU DE PRÉPARATION DES JEUNES DE 15 ANS AUX DÉFIS DE L'AVENIR}

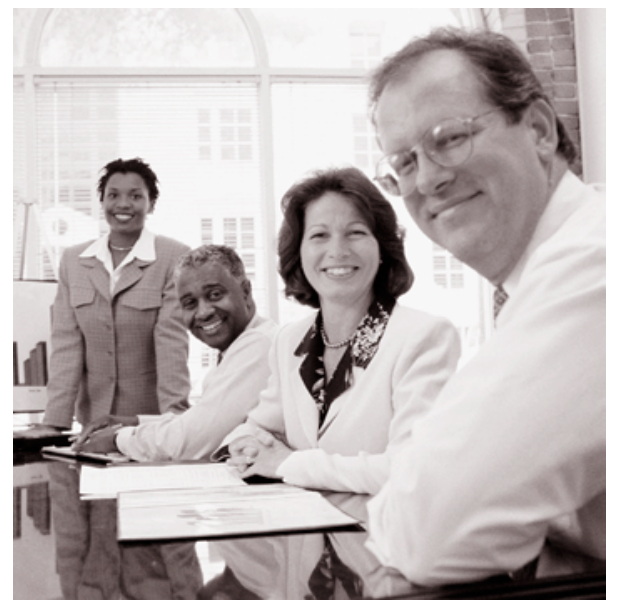




\section{SYNTHÈSE}

- Ce chapitre commence par étudier l'impact des compétences en lecture sur la vie sociale et économique à l'âge adulte en s'appuyant sur les résultats de l'Enquête internationale sur la littératie des adultes (IALS). Il y est expliqué combien la relation entre les compétences en compréhension de l'écrit et les perspectives d'avenir est significative et ne cesse de se renforcer. Des considérations tirées de la littérature récente et les résultats de IALS sont présentés pour mettre en lumière les liens entre la littératie et les résultats sur le plan éducatif, social et professionnel. Ces conséquences soulignent l'importance de connaître le degré de préparation des jeunes de 15 ans pour relever les défis de l'avenir.

- Ensuite, nous tenterons d'estimer quelle serait la performance des élèves ayant participé à PISA sur l'échelle de compréhension des textes suivis de IALS au moyen d'une analyse statistique des items fondés sur des textes suivis qui ont été intégrés à l'évaluation PISA. L'échelle de compréhension des textes suivis de IALS permet de se faire une idée de la mesure dans laquelle les élèves de 15 ans possèdent déjà les savoirs et savoir-faire dont ils auront besoin à l'âge adulte.

- Les résultats montrent une forte variation entre pays dans les pourcentages d'élèves de PISA se situant aux différents niveaux de compétence de l'échelle de compréhension des textes suivis de IALS. Au niveau 1, ces pourcentages varient d'environ 3 pour cent en Corée et au Japon à 33 pour cent au Luxembourg. Aux niveaux 4 et 5, les pourcentages varient de 30 pour cent en Finlande à seulement 1,4 pour cent au Brésil.

- Les chiffres présentés dans ce chapitre et tout au long de ce rapport fournissent aux pays une occasion de se demander si le niveau global de compétence des jeunes de 15 ans est suffisant pour leur permettre d'évoluer dans des sociétés qui se caractérisent par une dépendance accrue vis-à-vis de la technologie, la complexité de leurs systèmes juridiques et leurs administrations en expansion. Ils nous amènent à nous demander si la variabilité observée entre les pays ou entre des sous-groupes au sein des pays est un motif de préoccupation. 
Les chapitres précédents de ce rapport se sont attachés à décrire les compétences en compréhension de l'écrit des jeunes de 15 ans dans les principaux pays industrialisés. Dans de nombreux pays, force est de constater qu'une minorité non négligeable de jeunes âgés de 15 ans se situent à des niveaux de compétence fort bas. Dans quelle mesure un retard en lecture à l'âge de 15 ans constitue-t-il un obstacle à la réussite d'études ultérieures et à la transition vers le monde du travail chez les jeunes adultes ? La composante longitudinale de PISA, à travers laquelle certains pays suivront les jeunes de 15 ans évalués dans PISA sur une période de 15 ans, pourrait fournir des réponses concluantes à cette question.

De son côte, l'Enquête internationale sur la littératie des adultes (IALS) menée dans plus de 20 pays par Statistique Canada et l'OCDE entre 1994 and 1999, avait pour objectif de mieux saisir la distribution des compétences en lecture dans la population adulte, et elle a déjà fourni des éléments d'information sur les liens qui unissent la littératie à un ensemble de résultats sociaux, éducatifs et économiques (OCDE et Statistique Canada, 2000). Étant donné que les pays membres de l'OCDE ont conçu PISA de façon à permettre des comparaisons entre les performances dans PISA et dans IALS, il est possible de tirer certaines conclusions sur les conséquences sociales et économiques probables des faibles niveaux de compétence dans PISA. Ce chapitre développera ces résultats en détail.

Les données que IALS nous a fournies à propos de la littératie ont permis de mieux cerner son importance sociale et économique et de confirmer l'existence d'une relation de plus en plus forte entre les compétences en lecture des individus et les perspectives qui s'offrent à eux. Les piètres lecteurs n'ont guère d'atouts à faire valoir, ainsi qu'en témoigne le fait que dans la plupart des pays qui ont participé à IALS, ils ont tendance à exercer des professions peu rémunératrices et à éprouver des difficultés à participer pleinement dans des sociétés de plus en plus complexes. C'est en partie à cause de l'importance désormais établie du rôle de la littératie qu'il a été décidé d'axer PISA sur l'évaluation des connaissances et compétences des élèves de 15 ans tant à l'intérieur qu'à l'extérieur du cadre scolaire. C'est également la raison pour laquelle le Conseil des pays participants, l'instance chargée de superviser PISA, a recommandé de tenter de situer les élèves soumis à l'évaluation PISA sur l'échelle de compréhension de textes suivis de IALS. Cela nous permet d'extrapoler des mesures indirectes de la relation entre le niveau de compétence en compréhension de l'écrit et des résultats sociétaux en utilisant une méthode statistique qui permet d'estimer la performance théorique des élèves de PISA sur l'échelle de compréhension de textes suivis de IALS.

IALS a soumis des adultes âgés de 16 à 65 ans à une évaluation basée sur trois types d'écrit : les textes suivis (similaires à ceux de la sous-échelle des textes continus dans PISA), les textes schématiques (similaires à ceux de la sous-échelle des textes non continus dans PISA) et les textes au contenu quantitatif. Un groupe de 15 items de IALS, se rapportant à des textes suivis, a été inclus dans la batterie de tests de PISA. Les réponses des élèves de 15 ans à ces items nous ont permis de situer leurs performances sur l'échelle de compétence de IALS. Une extrapolation - vers la population évaluée dans PISA- des relations entre les compétences en lecture et les résultats sur le plan social et économique permet de mieux comprendre, à terme, les conséquences possibles de faibles niveaux de compétence en compréhension de l'écrit à l'âge de 15 ans.

Dans ce chapitre, nous allons commencer par présenter certains aspects de la relation qui existe entre la littératie et les résultats sur le plan éducatif, social et professionnel. Nous poursuivrons par une brève description des compétences mesurées sur l'échelle de compréhension de textes suivis de IALS, nous comparerons et contrasterons cette échelle avec celle de compréhension de l'écrit de PISA. Ensuite, nous 
résumerons la méthodologie utilisée pour étudier et estimer les compétences en littératie de la population sur l'échelle de compétence de IALS. Nous terminerons ce chapitre par une discussion de ce que pourraient être les résultats des élèves de PISA sur l'échelle de compréhension de textes suivis de IALS.

\section{L'influence de la littératie sur le plan social}

Dans le passé, il était d'usage de considérer que la littératie était essentiellement le fruit de l'instruction formelle à l'école. Aujourd'hui, il est établi que la capacité à comprendre l'écrit relève d'une série de connaissances et de compétences qui sont acquises tout au long de la vie, tant à l'intérieur qu'à l'extérieur de cadres institutionnels. De surcroît, nombreux sont ceux qui estiment que ces compétences sont indispensables pour prendre pleinement part à la vie de nos sociétés modernes qui se caractérisent par leur dépendance accrue vis-à-vis de la technologie et par la complexité de leurs processus sociaux. Il ressort des résultats de IALS et d'autres enquêtes réalisées auprès des adultes que la littératie intervient de toute évidence dans le fonctionnement du marché du travail. Ces résultats montrent en particulier que la littératie a un impact significatif sur l'accès au marché du travail, déterminé en fonction du taux d'activité et de la stabilité de l'emploi. La littératie est également associée aux revenus et à l'accès à la formation.

\section{Encadré 8.1 :}

Un niveau de compétence en lecture plus élevé améliore-t-il les perspectives d'emploi ?

L'Enquête internationale sur la littératie des adultes (IALS) a constaté que les personnes possédant un niveau de littératie plus élevé avaient de meilleures chances de trouver un emploi et de toucher un salaire moyen plus élevé que ceux dont le niveau de compétence est plus faible (OCDE et Statistique Canada, 2000). Est-ce uniquement parce qu'ils ont un meilleur niveau de formation, de meilleurs diplômes ? Si c'était le cas, IALS (et PISA) mesureraient - dans le meilleur des cas - des compétences qui aident les individus à suivre de meilleures études et, à travers elles, obtenir un meilleur emploi. Dans IALS, les adultes qui avaient terminé une forme d'enseignement tertiaire ont obtenu des scores moyens qui dépassaient de un à deux niveaux de compétence ceux des individus qui n'avaient pas terminé l'enseignement secondaire. Cependant, nombreux étaient les adultes qui, dans les 22 pays participants, ont fait preuve d'un niveau de littératie élevé et d'un faible niveau de scolarité, ou vice versa. Le plus important est que les niveaux de compétence en lecture contribuent à prédire les perspectives d'emploi des personnes au-delà de ce que leurs seuls diplômes permettent de prévoir.

Le graphique 8.1 illustre cela en montrant l'incidence du niveau de scolarité et des capacités de lecture et d'écriture sur la probabilité d'occuper un poste dans la catégorie des cadres hautement spécialisés. Les écarts entre les courbes montrent l'effet de l'accroissement du niveau de scolarité, tandis que la pente des courbes montre l'effet d'un niveau de littératie plus élevé à un niveau d'enseignement donné. Pour une personne de 26 à 35 ans qui travaille dans le secteur des entreprises, plus le niveau de littératie est élevé, plus les probabilités d'occuper un poste dans la catégorie des cadres hautement spécialisés augmentent à un rythme rapide. L'effet indépendant du niveau de littératie sur les perspectives d'emploi est comparable à l'effet indépendant du niveau de scolarité. Quelqu'un de moyennement qualifié (deuxième cycle de l'enseignement secondaire) a deux chances sur cinq d'occuper un emploi de haut niveau s'il atteint un niveau de littératie de 200 (au bas de l'échelle) 




Les adultes âgés de 25 à 65 ans qui se situent au moins au niveau 3 de l'échelle de compréhension de textes suivis de IALS ${ }^{1}$ sont plus susceptibles d'être actifs que les adultes situés aux niveaux 1 et 2 de cette même échelle (voir le tableau 8.1). Les inactifs ont tendance à se situer à un niveau de littératie en moyenne inférieur à celui des actifs, même si l'ampleur de l'écart varie selon les pays.

Les résultats de IALS laissent apparaître une autre tendance peut-être plus importante encore : les actifs situés aux niveaux 1 et 2 sur l'échelle de compréhension de textes suivis de IALS sont plus exposés au risque du chômage. Le tableau 8.2 compare les pourcentages d'adultes ayant déclaré être demandeurs d'emploi au moment de l'évaluation et qui sont situés aux deux premiers niveaux avec ceux aux trois derniers niveaux (3-5) de l'échelle de compréhension de textes suivis de IALS. Les demandeurs d'emploi sont quatre fois plus nombreux aux deux premiers niveaux qu'aux trois derniers niveaux de l'échelle de com- 
pétence de IALS en Nouvelle-Zélande, et presque deux fois plus nombreux dans la moitié des pays - l'Allemagne, l'Australie, la Belgique (Communauté flamande), le Canada, le Danemark, la Finlande, l'Irlande, le Royaume-Uni et la Slovénie. Dans quelques pays, les États-Unis, la Norvège et la Suisse, le taux global de chômage était si faible dans les années 1990 que les adultes peu qualifiés n'étaient guère exposés au risque du chômage. Pourtant, aux États-Unis et en Norvège, les demandeurs d'emploi situés aux deux premiers niveaux étaient environ deux fois plus nombreux que ceux situés aux trois derniers niveaux de compétence. Les résultats de IALS donnent également à penser que la littératie joue un rôle important dans le processus de détermination des salaires. La part de la variance salariale qui est imputable à la littératie atteint jusqu'à 33 pour cent dans plusieurs pays (Osberg, 2000 ; Green et Riddell, 2001). De plus, la littératie est également à l'origine d'inégalités salariales et intervient dans le choix des professions dans l'ensemble des pays (Blau et Kahn, 2001). À titre d'exemple, le tableau 8.3 montre la relation entre le niveau de compétence en compréhension de l'écrit et les revenus. Il indique les pourcentages d'adultes âgés de 25 à 65 ans situés dans les 60 pour cent dont les revenus annuels sont les plus élevés à chaque niveau de l'échelle de compréhension de textes suivis de IALS. Ces chiffres montrent que le pourcentage de ceux qui déclarent percevoir des revenus annuels élevés augmente avec le niveau de littératie. D'autres résultats de IALS non repris ici indiquent que la progression sur l'échelle de textes quantitatifs est légèrement plus déterminante dans certains pays, un constat corroboré par la « National Adult Literacy Survey » réalisée aux États-Unis (Sum, 1999).

La relation entre la littératie et les revenus n'est pas simple. Elle est rendue plus complexe par la relation entre la littératie et le niveau de formation. Force est de constater que dans tous les pays qui ont participé à IALS, les adultes qui ont un niveau de formation plus élevé obtiennent en moyenne des scores supérieurs, même si l'ampleur des écarts moyens varie selon les pays et selon les niveaux de formation dans les pays. ${ }^{2}$

L'éducation améliore de toute évidence la littératie qui, à son tour, est déterminante pour la réussite des études. Aux États-Unis par exemple, la littératie figure au nombre des facteurs décisifs à plusieurs égards : elle intervient dans le choix des cours suivis dans l'enseignement secondaire et détermine si les élèves obtiennent ou non leur diplôme de fin d'études secondaires ou un certificat alternatif', et s'ils poursuivent des études de niveau tertiaire. Elle influe également sur le type de diplômes obtenus (Berlin et Sum, 1988 ; Sum et Fogg, 1991).

La littératie a également un impact sur l'accès aux systèmes de formation pour adultes, car elle influe considérablement sur la fréquence et la durée des activités de formation et sur la prise en charge de ces activités de formation par les employeurs (Statistique Canada, 2000 et Sum, 1999 ; OCDE). Le tableau 8.4 indique le taux de participation à des activités de formation pour adultes par pays et par niveau de compétence sur l'échelle de compréhension de textes schématiques.

Comme le montre ce tableau, la participation à des programmes de formation pour adultes au cours de l'année précédant l'enquête varie considérablement selon les pays qui ont participé à IALS. Le Chili, la Hongrie, la Pologne et le Portugal font état de taux de participation inférieurs à 20 pour cent, tandis que le Danemark, la Finlande et la Suède déclarent des taux supérieurs à 50 pour cent. Ces taux de participation sont compris entre 20 et 50 pour cent dans la majorité des pays.

Si les taux de participation varient entre les pays, l'analyse des chiffres au sein même des pays révèle une tendance intéressante. Ceux qui sont les moins susceptibles de suivre des activités de formation pour adultes sont également ceux qui ont le plus besoin d'améliorer leurs compétences. Dans les pays qui ont 
participé à IALS, le pourcentage moyen d'adultes situés au niveau 1 de l'échelle de compétence qui ont déclaré participer à des activités de formation était de 14 pour cent. Le taux moyen de participation à ces activités est presque deux fois plus élevé parmi les adultes situés au niveau 2 ( 29 pour cent). Il est proche de 50 pour cent (46 pour cent) chez les adultes situés au niveau 3 et approche de 60 pour cent ( 59 pour cent) chez les adultes situés aux niveaux 4 et 5 . Les résultats de IALS montrent également que c'est l'employeur qui est le plus souvent susceptible de dispenser ces formations.

Les compétences en compréhension de l'écrit n'influencent pas seulement la participation à l'enseignement formel. Certains éléments de IALS donnent à penser qu'elles ont également un impact sur l'apprentissage informel (Desjardins, 2002). Les activités qui sortent du cadre professionnel, telles que le bénévolat et les activités citoyennes, constituent un capital social important qui offre aux individus l'occasion d'apprendre et d'évoluer, car elles stimulent leur intellect. Dans un rapport récent intitulé $D u$ bien-être des nations (OCDE, 2001a), l'OCDE affirme que le développement du capital humain procure des avantages sociétaux, dont l'amélioration de la santé publique, la diminution du taux de criminalité et une participation accrue aux activités de la collectivité.

\section{Comparaison de l'échelle de compréhension des textes suivis de IALS et de l'échelle combinée de compréhension de l'écrit de PISA}

Aussi bien dans IALS que dans PISA des cadres conceptuels ont servi de base à l'élaboration de leurs évaluations respectives ${ }^{4}$ Pour chaque évaluation, un panel d'experts a élaboré une définition fonctionnelle de la littératie, et cette définition a été opérationnalisée en déterminant trois caractéristiques de tâches. Ces caractéristiques de tâches circonscrivent une série limitée de caractéristiques qui peuvent être manipulées lors de l'élaboration des épreuves destinées à recueillir des informations sur le niveau de littératie de chaque population.

L'objectif de IALS et de PISA était de concevoir une batterie de tests fondés sur les cadres d'évaluation respectifs des deux enquêtes. La sélection d'items s'inscrivant dans un large éventail de contextes, ou de situations, a été dictée par le souci d'étoffer la diversité culturelle des tâches et de varier le vocabulaire utilisé. Cette approche a permis à son tour de garantir qu'aucun groupe ne soit avantagé ou pénalisé par le contenu des items de l'évaluation. Dans la catégorie des textes suivis, ou continus, IALS a privilégié les textes informatifs, argumentatifs et injonctifs ${ }^{5}$, car la plupart des écrits que les adultes doivent lire dans leur vie professionnelle, sociale et familiale sont associés à ce type de textes. En ajoutant un nombre significatif de textes narratifs aux textes informatifs, injonctifs et argumentatifs, PISA a proposé un ensemble de textes correspondant mieux aux lectures typiques des jeunes de 15 ans qui fréquentent encore l'école. PISA a suivi le même principe que IALS lors de la sélection des textes non continus, si ce n'est que IALS a accordé la même importance aux textes continus et non continus, tandis que PISA a mis davantage l'accent sur les textes continus.

Quant aux processus et stratégies, les deux enquêtes ont cherché à engager les personnes soumises à l'évaluation dans des processus généralement associés à chaque type d'écrit. Pour ce faire, le cadre conceptuel est utilisé pour construire des questions et des consignes jugées authentiques compte tenu du type d'informations qu'un individu est censé vouloir comprendre ou utiliser lorsqu'il lit un texte donné. IALS s'est concentrée sur la compréhension et la manipulation des conditions qui influent sur la difficulté globale des tâches en fonction de ses trois échelles de compétence (les textes suivis, les textes schématiques et les textes au contenu quantitatif). PISA a adopté une approche similaire lors de l'élaboration des items mais a privilégié la notion d'aspect, comme la localisation d'informations, l'interprétation de textes ou la réflexion 
et l'évaluation. PISA a, de façon plus particulière, élargi la notion de littératie définie par IALS en intégrant des items demandant aux élèves de réfléchir tant au contenu qu'à la structure des textes qu'ils ont lus.

\section{Encadré 8.2 : Distinctions entre PISA et IALS}

Dans IALS, le niveau de littératie est présenté sur des échelles de compétence dont la moyenne était initialement fixée à 250 et l'écart type à 50. Comme dans PISA, chaque échelle de compétence est divisée en cinq niveaux pour rendre compte de la progression des compétences et des pourcentages d'individus qui possèdent ces compétences. Il y a lieu de souligner toutefois que ces cinq niveaux ne mesurent pas exactement les mêmes compétences dans les deux enquêtes. Rappelons que PISA et IALS ont adopté un concept similaire de la compréhension de l'écrit mais ont mis l'accent sur des aspects différents de ce construct.

De plus, l'échelle de compréhension des textes suivis de IALS et l'échelle combinée de compréhension de l'écrit de PISA ainsi que les niveaux de chacune d'elles présentent des caractéristiques différentes. Ainsi, IALS compte cinq niveaux mais ne cherche pas à identifier les sujets les moins compétents parmi ceux situés au niveau 1, alors que PISA identifie un groupe d'élèves situés «en deçà du niveau 1 ». Ce classement permet d'identifier les élèves dont on estime qu'ils ne possèdent pas les compétences requises pour mener à bien des tâches de niveau 1 .

Les probabilités de réponse utilisées pour caractériser les compétences et les items sur les échelles de IALS et de PISA sont différentes. La probabilité de réponse indique la probabilité de voir un lecteur situé à un point donné de l'échelle répondre correctement à un item situé au même point de l'échelle. La probabilité de réponse est fixée à 0,62 dans PISA et à 0,80 dans IALS. Cela signifie que, dans PISA, un élève situé au milieu d'un niveau est censé répondre correctement à 62 pour cent des items d'un test hypothétique composé d'items de ce niveau, tandis qu'un élève situé au bas d'un niveau est censé répondre correctement à 50 pour cent des items de ce niveau. Dans IALS par contre, un adulte situé au milieu d'un niveau est censé répondre correctement à 80 pour cent des items d'un test hypothétique constitué d'items de ce niveau, alors qu'un adulte situé au bas d'un niveau n'est censé répondre correctement qu'à 60 pour cent des items du même niveau. ${ }^{6}$

Bien qu'il existe une forte corrélation $(0,85)$ entre l'échelle de compréhension de textes suivis de IALS et l'échelle combinée de compréhension de l'écrit de PISA après correction pour atténuation, il serait abusif de conclure que les compétences requises pour atteindre le niveau 2 de PISA par exemple sont identiques à celles qu'il faut mettre en ouvre pour répondre correctement aux items associés au niveau 2 de IALS. Toutefois, les procédures méthodologiques appliquées aux items de IALS basés sur des textes suivis qui ont été intégrés dans la batterie de tests de PISA nous permettent d'estimer à quel niveau de l'échelle de compréhension de textes suivis de IALS les élèves qui ont participé au PISA se situent.

Tous les items de IALS sont des items à réponse ouverte qui demandent aux lecteurs de sélectionner, de souligner, de transférer ou de produire une réponse. Dans le PISA en revanche, la batterie de tests de compréhension de l'écrit est constituée pour moitié d'items à réponse ouverte et pour moitié d'items à choix multiple. 


\section{Situer les élèves de PISA sur l'échelle de compréhension de textes suivis de IALS}

Les paramètres des items cognitifs de la batterie de tests de IALS ont été estimés en 1994 sur la base des réponses de plus de 25000 adultes. Ils ont ensuite été validés à deux reprises, la première fois en 1996, lors du deuxième cycle d'évaluation, et la seconde fois en 1998, lors du troisième cycle d'évaluation. Pour situer les élèves de PISA sur l'échelle de compréhension de textes suivis de IALS, il convient de déterminer dans quelle mesure les paramètres d’items estimés par IALS correspondent au pattern de réponses obtenu dans PISA. Plusieurs analyses ont été effectuées pour évaluer dans quelle mesure les paramètres originaux se maintiennent. Yamamoto (2002) a rédigé un rapport technique décrivant en détail ce qui a été fait ${ }^{7}$. Nous en proposons une synthèse succincte ci-dessous.

En premier lieu, nous avons comparé pour chaque pays le pourcentage moyen de réponses correctes aux 15 items de IALS se rapportant à des textes suivis intégrés dans la batterie de tests de PISA et le pourcentage moyen de réponses correctes à l'ensemble des items de PISA. Cette simple opération devait nous permettre de déceler d'éventuels facteurs susceptibles de compromettre la comparabilité des données d'un pays à l'autre. Un ou plusieurs pays pourrait par exemple avoir traduit les items d'évaluation différemment ou appliqué systématiquement une ou plusieurs consignes de correction de manière différente. Ces analyses n'ont pas conduit à détecter ce type de problème. Nous avons calculé que, dans les 32 pays participant à PISA, la corrélation entre les deux pourcentages moyens de réponses correctes était de 0,89 , ainsi que le montre le graphique 8.2.

En second lieu, nous avons procédé à des analyses IRT et à plusieurs traitements statistiques pour vérifier l'indice d'ajustement de tous les items dans chaque pays. Nous avons notamment évalué l'ajustement global du modèle à l'aide du khi carré, ainsi que l'écart moyen pondéré et la racine carrée de l'écart quadratique moyen pondéré. Ces deux analyses statistiques basées sur des écarts comparent en 31 points différents de l'échelle de compétence la proportion théorique de réponses correctes estimée par le modèle et la proportion réelle de réponses correctes.

\section{Graphique 8.2}

Corrélation entre le pourcentage moyen de réponses correctes aux items de compréhension de l'écrit dans PISA et le pourcentage moyen de réponses correctes aux items basés sur des textes suivis dans IALS

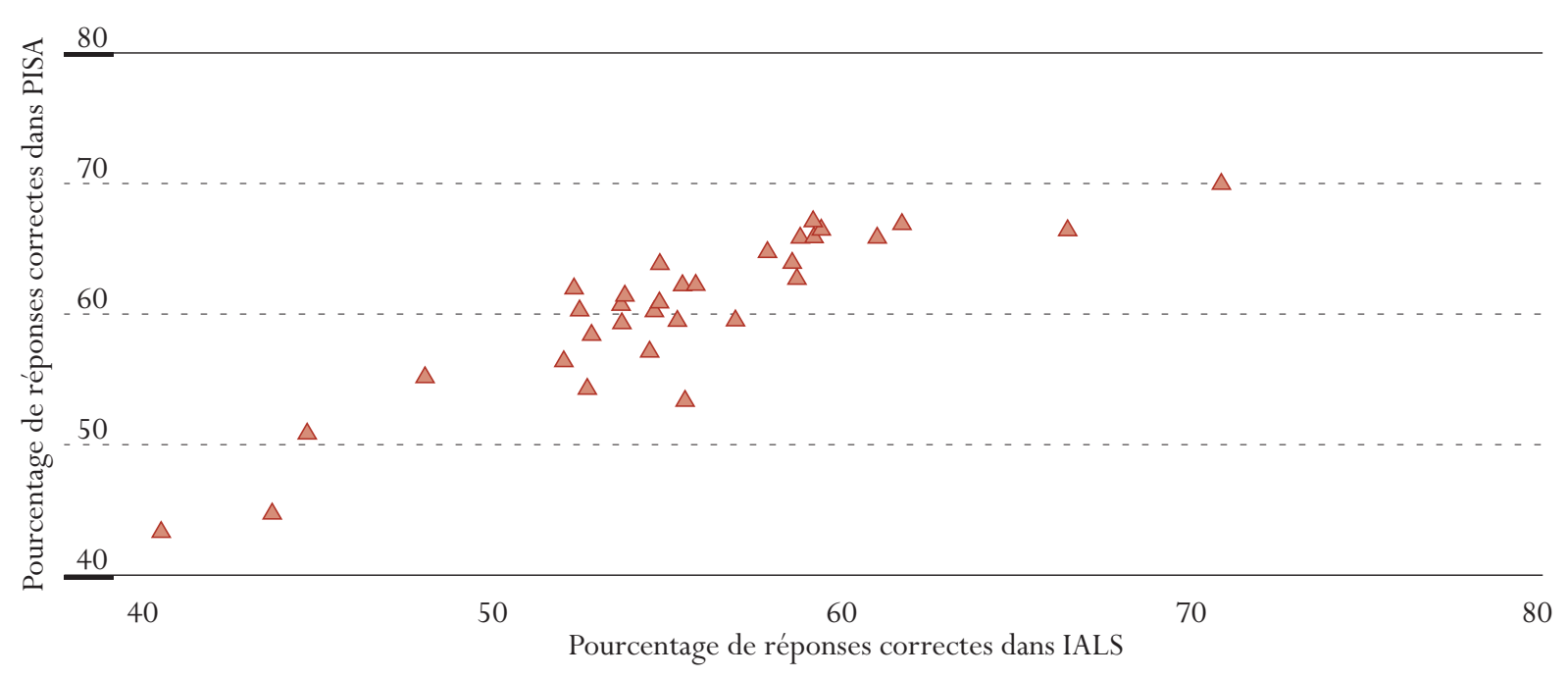

Source: Base de données PISA de l'OCDE, 2001; OCDE et Statistique Canada, 2000. 
L'idéal aurait été que chacun des paramètres des 15 items de IALS ait un bon indice d'ajustement dans les 32 pays participants, ce qui est rarement le cas dans des enquêtes de cette nature. Le défi a donc consisté à résoudre au mieux les problèmes d'ajustement des paramètres d'items dans les pays participants. Plusieurs approches peuvent être adoptées à cet égard. La première consiste à ignorer les problèmes d'ajustement mais cela entraîne un accroissement de la marge d'erreur, biaisant ainsi l'estimation globale. La deuxième est d'exclure l'item mal ajusté de l'analyse. L'inconvénient majeur de cette méthode est de réduire le nombre d'items qui servent à estimer les compétences. La troisième méthode, celle que nous avons choisie en l'occurrence, consiste à estimer de nouveaux paramètres pour les items qui ne sont pas ajustés dans un pays donné. Quelques items présentaient des problèmes d'ajustement très similaires d'un pays à l'autre, tant en termes de direction que d'amplitude. Pour ces items, un jeu de paramètres d'item a été estimé pour l'ensemble des pays concernés. Cette opération a permis de réduire le nombre global des nouveaux paramètres d'item qui ont dû être estimés tout en améliorant considérablement l'ajustement du modèle.

Selon Yamamoto (2002), l'indice d'ajustement global du modèle est relativement satisfaisant pour les 15 items de IALS inclus dans la batterie de tests de PISA. En moyenne, seuls 4,4 items présentaient des écarts quadratiques moyens absolus pondérés supérieurs à 0,10 nécessitant l'estimation de nouveaux paramètres. La majorité des items - 11 sur 15 -n'ont demandé aucune ou une seule nouvelle estimation d'une paire de paramètres, sinon aucune, en plus des paramètres d'items initiaux de IALS. Le fait qu'une seule série de paramètres ait dû être estimée pour ces items montre que l'erreur était similaire dans les pays concernés, tant en termes de direction que d'amplitude. Deux séries de paramètres d'items ont dû être estimées pour les quatre items restants.

Après avoir établi que l'ajustement du modèle était bon, c'est-à-dire que les items de IALS inclus dans la batterie de tests du PISA pouvaient être ancrés au niveau des items, nous sommes passés à l'étape suivante consistant à estimer les seuils de compétence et à les transposer sur l'échelle existante de compréhension des textes suivis de IALS. Nous avons appliqué la méthodologie des valeurs plausibles (Mislevy, 1991 ; Rubin, 1987) pour situer les élèves de PISA sur l'échelle de compréhension des textes suivis de IALS, une opération décrite par Yamamoto (2002). Rappelons à cet égard que les items de la batterie de tests de PISA ont été élaborés sur la base d'un cadre d'évaluation proche mais légèrement différent de celui de IALS. Il était dès lors logique de voir cette différence se refléter dans la relation statistique entre les scores sur l'échelle combinée de compréhension de l'écrit de PISA et les scores sur l'échelle de compréhension des textes suivis de IALS. La corrélation entre ces deux échelles est de 0,85 après correction de l'atténuation et de 0,73 avant correction. Elle a été estimée à partir de l'ensemble de l'échantillon de PISA qui a répondu aux items de PISA et de IALS, et est très similaire à celle qui existe entre les échelles de compréhension de textes suivis et schématiques de IALS $(0,89)$ après correction de l'atténuation.

\section{Performance des élèves de PISA sur l'échelle de compréhension de textes suivis de IALS}

À la lumière des relations établies entre la littératie et les résultats sur le plan social, éducatif et professionnel, le rapport de IALS a conclu que les adultes qui ne parvenaient pas atteindre le niveau 3 de l'échelle de compréhension de textes suivis étaient fragilisés quant à leurs perspectives d'avenir (OCDE et Statistique Canada, 1995). Si les adultes qui ont atteint les niveaux 1 et 2 de l'échelle de compétence de IALS ont été estimés capables de mener à bien un large éventail de tâches de lecture, ils n'en évoluent pas moins dans un monde en constante mutation où l'information et la technologie se généralisent et où les savoirs et savoir-faire ont un impact significatif sur les perspectives qui s'offrent à eux. Bien qu'il soit toujours délicat de choisir un point précis, le niveau 3 de IALS est considéré comme un objectif que les décideurs devraient se fixer lorsqu'ils s'interrogent sur le niveau de littératie dont les citoyens ont besoin pour participer pleinement à la vie de la société. 


\section{Encadré 8.3 :}

Description des niveaux de l'échelle de compréhension des textes suivis de IALS

\section{Niveau 1}

De 0 à 225

La plupart des tâches de ce niveau exigent du lecteur qu'il repère un élément d'information contenu dans le texte qui est identique ou synonymique à l'information donnée dans la consigne. Si un élément distracteur, est présent dans le texte, il est habituellement loin de la réponse correcte.

\section{Niveau 2}

De 226 à 275

À ce niveau, les tâches exigent habituellement du lecteur qu'il repère un ou plusieurs éléments d'information dans un texte ; ce dernier peut contenir plusieurs éléments distracteurs, ou le lecteur peut devoir faire des inférences de faible niveau. Les tâches de ce niveau commencent aussi à demander au lecteur d'intégrer deux éléments d’information ou plus, ou de comparer des données et de les mettre en opposition.

\section{Niveau 3}

De 276 à 325

Les tâches de ce niveau exigent habituellement du lecteur qu'il trouve des renseignements dans un texte en faisant des inférences de faible niveau ou en respectant des conditions précises. On demande parfois au lecteur de repérer plusieurs éléments d'information situés dans des phrases ou des paragraphes différents plutôt que dans une seule phrase. On peut également demander au lecteur d'intégrer ou de comparer et de mettre en opposition des renseignements trouvés dans différents paragraphes ou sections de texte.

\section{Niveau 4}

De 326 à 375

Ces tâches demandent au lecteur de faire des inférences de haut niveau. Certaines tâches nécessitent d'établir plusieurs correspondances ou de fournir plusieurs réponses pour lesquelles il est nécessaire de faire des inférences fondées sur le texte. Ces textes contiennent habituellement plus d'éléments distracteurs et abstraits.

\section{Niveau 5}

De 376 à 500

Certaines tâches de ce niveau exigent du lecteur qu'il recherche des informations dans un texte dense qui contient un certain nombre d'éléments distracteurs. D'autres exigent du lecteur qu'il fasse des inférences de haut niveau, qu'il traite des informations conditionnelles ou qu'il se serve de connaissances spécialisées.

Source : Littératie, économie et société, OCDE et Statistique Canada, 1995. 
Le graphique 8.3 indique le score moyen global de chaque pays participant à PISA sur l'échelle de compréhension de textes suivis de IALS ainsi que l'estimation du pourcentage d'élèves de 15 ans situés au niveau 1, 2, 3 et 4/5 . Il ressort de toute évidence de l'analyse de ces chiffres que les proportions de jeunes de 15 ans situés aux niveaux 1 et 2 de l'échelle de compréhension de textes suivis de IALS varient considérablement selon les pays, quels que soient les scores moyens de ceux-ci. La variation entre pays est également considérable en ce qui concerne les pourcentages d'élèves estimés se situer aux niveaux 4 and 5 .

\section{Graphique 8.3}

Performance moyenne et pourcentage d'élèves de PISA situés à chaque niveau de l'échelle de textes suivis de IALS

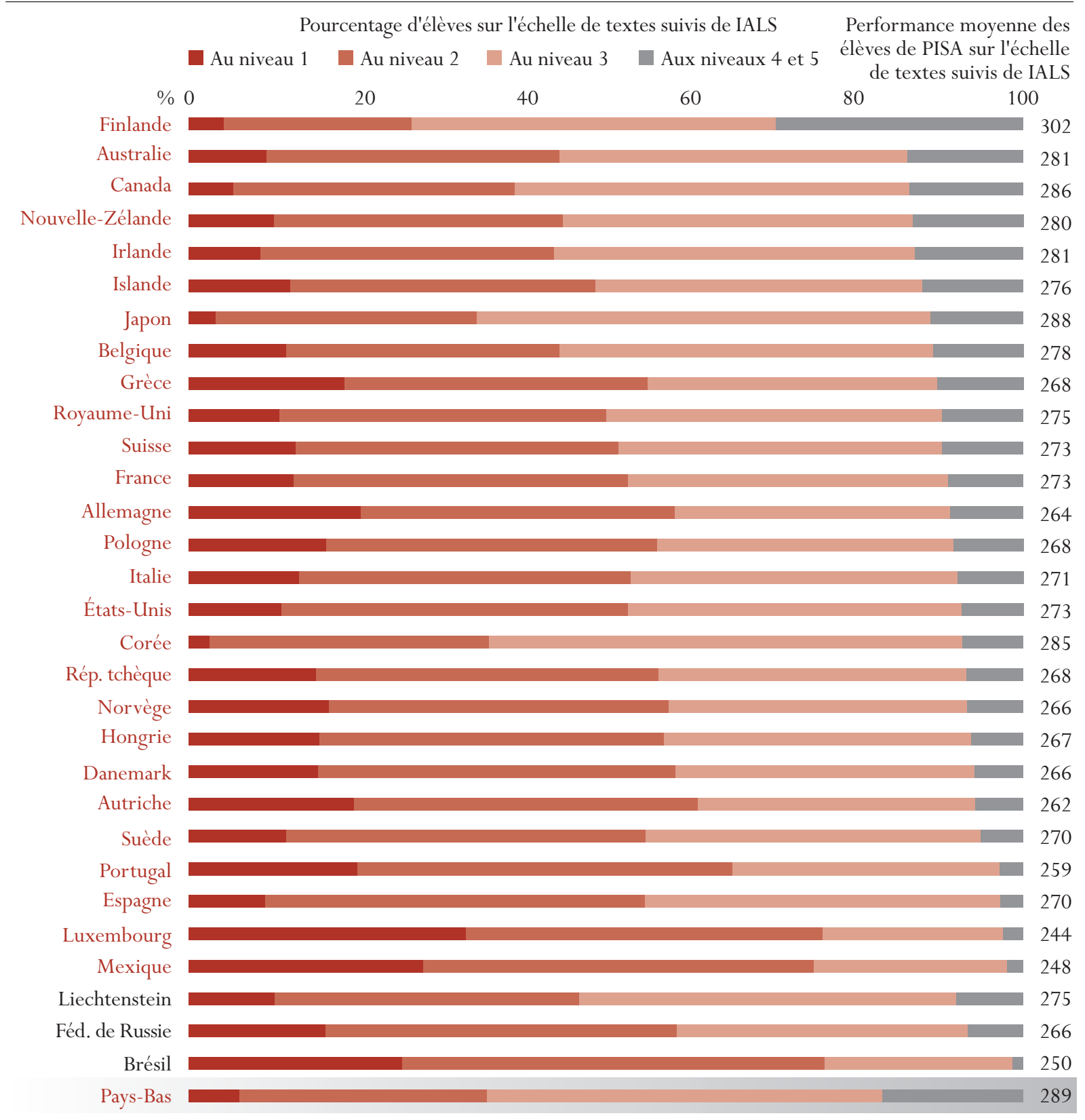

Source: Base de données PISA de l'OCDE, 2001 et Enquête internationale sur la littératie des adultes, 1994-1998, tableau 8.5. 
Les tableaux 8.5 à 8.8 montrent que les pourcentages de jeunes de 15 ans situés au niveau 1 de l'échelle de IALS varient de 3 pour cent seulement en Corée et au Japon à 33 pour cent au Luxembourg. Pour l'ensemble des pays participants, une moyenne de 15 pour cent des jeunes de 15 ans ont un niveau de littératie de niveau 1 sur l'échelle de compréhension de textes suivis de IALS. Ils seraient 42 pour cent au niveau 2 et 36 pour cent au niveau 3. On estime que seuls 7 pour cent des jeunes de 15 ans de l'ensemble des pays participant à PISA atteignent les deux niveaux de littératie les plus élevés (les niveaux 4 et 5). Ici encore, les proportions varient fortement d'un pays à l'autre : le pourcentage moyen des jeunes de 15 ans dont on estime qu'ils atteignent les niveaux 4 et 5 de IALS dépasse à peine 1 pour cent au Brésil mais s'élève à presque 30 pour cent en Finlande.

Les filles obtiennent des scores légèrement supérieurs à ceux des garçons sur l'échelle de compréhension de textes suivis de IALS puisque leur score moyen est de 275, alors que celui des garçons est de 261, une différence qui représente près du tiers d'un écart type (voir les tableaux 8.6 et 8.7). Tous pays participant à PISA confondus, la proportion de filles situées au niveau 1 est de 11 pour cent, alors que la proportion de garçons à ce même niveau est de 19 pour cent. En outre, il a été estimé que 9 pour cent des filles des pays participant à PISA atteignent les niveaux 4 et 5 , contre moins de 5 pour cent des garçons seulement.

Le graphique 8.4 montre les différences entre scores moyens des filles et des garçons, par pays. L'écart le plus important entre les deux sexes s'observe en Finlande, où il atteint 26, soit deux tiers d'écart type. L'écart le plus faible est enregistré en Corée, où la différence entre les scores moyens des filles et des garçons ne représente que 12 pour cent de la valeur d'un écart type (voir le tableau 8.8).

Ces résultats ne mesurent la performance des élèves qu'à un moment précis, celui où les élèves de la plupart des pays participants arrivent au terme de la scolarité obligatoire. On peut raisonnablement espérer que leurs compétences en compréhension de l'écrit continueront à se développer grâce à leur participation à des activités de formation, dans l'enseignement post-secondaire et/ou dans des programmes de formation liés à l'emploi, ou encore dans des activités plus informelles. Le tableau 8.9 compare les scores moyens en compréhension de textes suivis des jeunes de 15 ans ayant participé à PISA et ceux des personnes âgées de 16 à 25 ans ayant participé à IALS, par sexe et par pays. Ces chiffres laissent apparaître une tendance : le score moyen des femmes est supérieur à celui des hommes, mais ceux-ci se distinguent des femmes par une progression moyenne supérieure à celle des femmes âgées de 16 à 25 ans. ${ }^{9}$

Ces données constituent une première projection de la littératie des élèves de 15 ans quand ils deviendront adultes. C'est pourquoi il est important de ne pas interpréter ces chiffres hors contexte. Il convient plutôt de considérer cette projection comme une tentative préliminaire d'évaluer quel pourrait être le lien entre PISA et la littératie des adultes. Les données de la composante longitudinale de PISA fourniront des réponses plus complètes à la question du degré de préparation des élèves participant à PISA à la vie adulte.

Nous ne pouvons guère attendre des jeunes de 15 ans qu'ils aient appris tout ce qu' ils auront besoin de savoir et de faire à l'âge adulte, mais ils devraient disposer de bases solides en compréhension de l'écrit pour continuer à apprendre et participer pleinement à la vie d'un monde en mutation où les informations se multiplient à un rythme vertigineux, rendant de plus en plus importante la capacité d'accéder à l'information, de la traiter et de l'évaluer. Selon certains économistes, c'est avant tout l'évolution technologique qui accentue les inégalités en matière de revenus. Il ressort d'une étude récente réalisée par l'Institute for International Economics que c'est probablement cinq fois plus sous l'effet de l'évolution technologique que des échanges commerciaux que les écarts de revenus se sont creusés aux États-Unis entre 1973 et 1993 (Cline, 1997). 


\section{Graphique 8.4}

Différence entre les scores moyens des garçons et des filles sur l'échelle de textes suivis de IALS

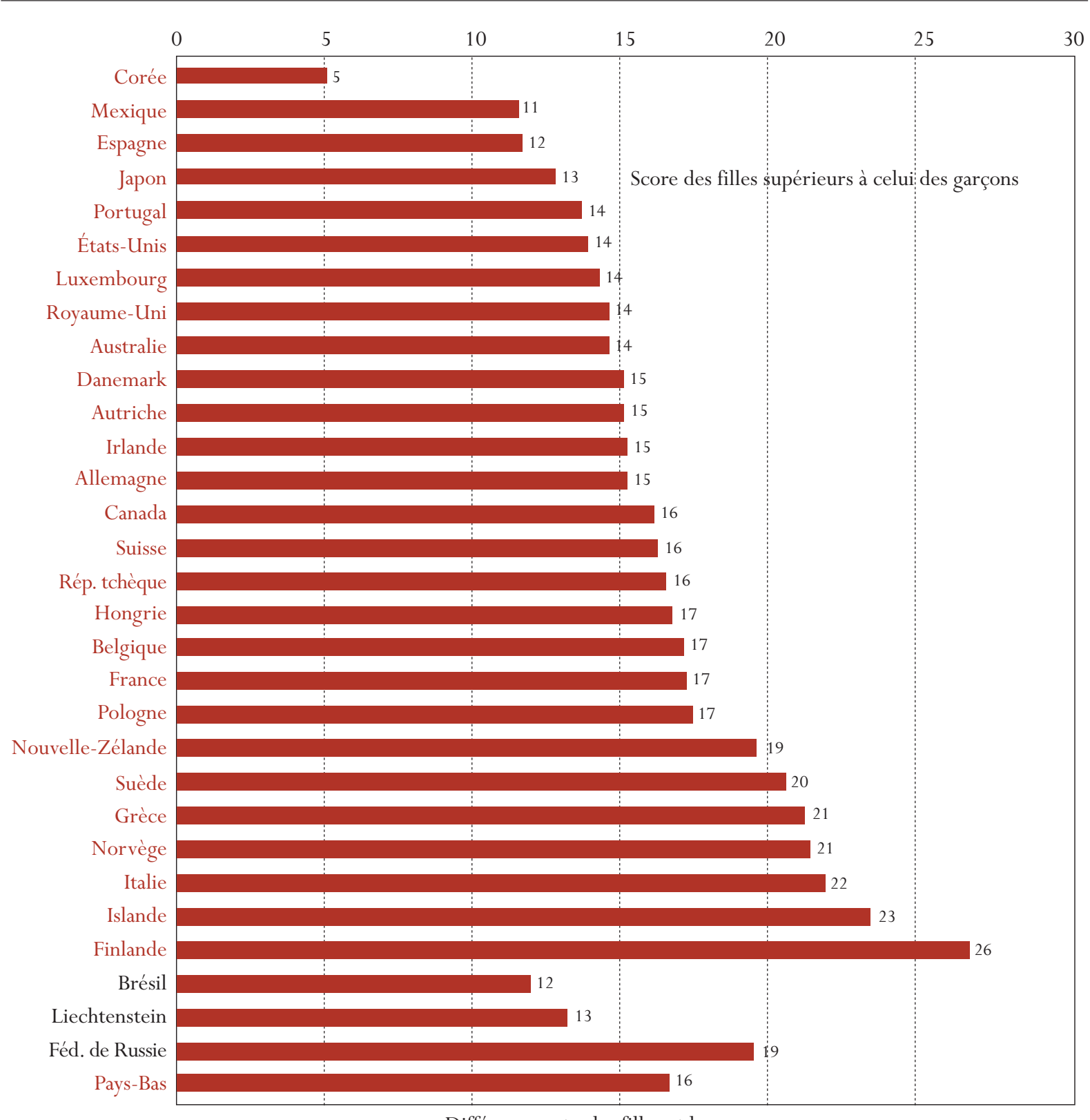

Différence entre les filles et les garçons

Source: Base de données PISA de l'OCDE, 2001, tableau 8.7

Dans quelle mesure les jeunes de 15 ans possèdent-ils les compétences en compréhension de l'écrit dont ils auront besoin à l'âge adulte ? La réponse à cette question variera d'un pays à l'autre. Les chiffres présentés ici nous amènent inévitablement à nous demander si le niveau global de compétence des élèves des pays participants est suffisant et si la variabilité observée entre des pays ou entre des sous-groupes au sein des pays est préoccupante. Comme l'affirme l'OCDE dans un rapport récent (2001a), le développement du capital humain est associé non seulement à des résultats éducatifs et professionnels, mais aussi à une 
série d'avantages sociétaux, dont l'amélioration de la santé publique, la diminution du taux de criminalité et l'accroissement de la participation à la vie sociale et politique de la société. L'impact économique de la littératie est donc peut-être aussi important que son impact social pour les individus, les communautés et les pays. 
CHAPITRE 8 La lecture, moteur de changement: Performance et engagement d'un pays à l'autre

\section{Notes}

1. Il y a lieu de souligner que les niveaux de compétence de IALS diffèrent des niveaux de PISA. Il convient de se reporter à l'encadré 8.2 pour davantage d'informations à ce sujet.

2. Dans IALS, les écarts moyens les plus importants s'observent entre ceux qui ont terminé le deuxième cycle de l'enseignement secondaire et ceux qui n'y sont pas arrivés. Selon IALS, la dispersion des scores moyens des adultes des pays participants présentant le niveau le plus faible d'éducation formelle est une fois et demie plus étendue que celle de ceux présentant le niveau le plus élevé d'éducation formelle (OCDE et Statistique Canada, 2000, p. 25).

3. Aux États-Unis, un certificat (d'études secondaires) alternatif sanctionne la réussite de l'examen de General Educational Development (GED).

4. Le cadre d'évaluation de PISA est résumé dans le chapitre 2 du présent rapport. Pour de plus amples informations sur le cadre de IALS, le lecteur consultera Kirsch, I. «The Framework Used in Developing and Interpreting the International Adult Literacy Survey », European Journal of Psychology of Education, vol. XVI (3), pp. 335-362.

5. Ces types de textes sont définis dans les cadres conceptuels de PISA et de IALS. Les textes informatifs y sont définis comme des textes qui présentent des concepts composites ou des constructs mentaux ; les textes argumentatifs, comme des textes qui défendent un point de vue et émettent des hypothèses concernant les relations entre des concepts ou d'autres hypothèses ; les textes injonctifs, comme des textes qui fournissent des consignes ou des instructions.

6. PISA et IALS ont appliqué des modèles différents de la théorie de la réponse à l'item (IRT), ce qui explique que leur système de mesure est associé à des origines et des logit différents (avant toute transformation logit).

7. Ce rapport est disponible sur le site web du PISA : www.oecd.pisa.org.

8. Il a été décidé, pour IALS, de confondre les pourcentages d'adultes situés à ces deux niveaux de compétence en raison du nombre relativement réduit de sujets interrogés dans certains pays. De la sorte, il est possible de donner des estimations précises de l'erreur type dans cette cellule. En effet, la simple addition des pourcentages des deux cellules ne permet pas d'estimer précisément l'erreur type correcte.

9. Il est difficile d'estimer l'ampleur globale de cet impact à partir des données, car celles-ci ont été recueillies auprès de deux types d'échantillons différents dans des cohortes d'âge différent. PISA a échantillonné les établissements d'enseignement tandis que IALS a échantillonné les ménages. IALS est donc susceptible de représenter un échantillon plus large des compétences au sein de chaque pays. 


\section{BIBLIOGRAPHIE}

Allington, R. L., «Content, coverage, and contextual reading in reading groups », Journal of Reading Behavior, 16, 1984, pp. 85-96.

Anderson, R. C., Wilson, P.T. et Fielding, L. G., « Growth in reading and how children spend their time outside school », Reading Research Quarterly, 23, 1988, pp. 285-303.

Au, K. H. et Raphael,T. E., «Equity and literacy in the next millennium », Reading Research Quarterly, 35, 1, 2000, pp. 170-188.

Bellorado, D., Making Literacy ProgramsWork : A Practical Guide for Correctional, ministère de la Justice, États-Unis, National Institute of Corrections, numéro d'acquisition NIC-006253, 1986.

Berlin, G. et Sum, A., Toward A More Perfect Union : Basic Skills, Poor Families and Our Economic Future, Fondation Ford, New York, 1988.

Birkerts, S., Readings, St. Paul, Minnesota, Greywolf Press, 1998.

Birkerts, S., The Gutenberg Elegies :The Fate of Reading in an Electronic Age, Boston et Londres, Faber and Faber, 1994.

Blau, F. D. et Kahn, L. M., Do cognitive test scores explain higher US wage inequality ?, document de travail $n^{\circ} 8210$, National Bureau of Economic Research, Cambridge, Massachussets, 2001.

Brown, A. L., Palincsar, A. S. et Purcell, L., « Poor readers : Teach, don't label », in U. Neisser (éd.), The school achievement of minority children : New perspectives, Hillsdale, New Jersey, Lawrence Erlbaum Associates, 1986, pp. 10543.

Bryk, A. S. et Raudenbush, S.W., Hierarchical Linear Models, and Behavioral Research : Applications and Data Analysis Methods, Beverly Hills, Sage Publications, 1992.

Burns, B., « Changing the classroom climate with literature circles », Journal of Adolescent and Adult Literacy, 42/2, 1998, pp. 124-129.

Campbell, J. R., Kelly, D. L., Mullis, I.V. S., Martin, M. O. et Sainsbury, M., Progress International Reading Literacy Study (PIRLS), Association internationale pour l'évaluation du rendement scolaire (IEA), deuxième édition, Chestnut Hill, Massachussets, PIRLS International Study Center, 2001.

Campbell, J. R., Voelkl, K. E. et Donahue, P. L., NAEP 1996 trends in academic progress, publication n 97-985, NCES, ministère de l'Éducation, Washington, D.C., 1997.

Central Intelligence Agency, TheWorld Factbook 2001, www.cia.gov/cia/publications/factbook, 2001.

Chall, J., Jacobs, V. et Baldwin, L., The reading crisis : Why poor children fall behind, Cambridge, Massachussets, Harvard University Press, 1990.

Cline, W., Trade and income distribution, Institute for International Economics, Washington, DC, 1997.

Commission européenne, Rapport européen sur la qualité de l'éducation scolaire - Seize indicateurs de qualité, Luxembourg, Office des publications officielles des Communautés européennes, 2001.

Conseil de l'Europe, Cadre européen de référence pour les langues : apprendre, enseigner, évaluer, Cambridge, Cambridge University Press, 2001.

Cunningham, A. E. et Stanovich, K. E., «Early reading acquisition and its relation to reading experience and ability 10 years later », Developmental Psychology, 33, 1998, pp. 934-945.

Dechant, E., Understanding and teaching reading: An interactive model, Hillsdale, New Jersey, Lawrence Erlbaum, 1991.

Desjardins, R., «The effect of learning on economic and social well-being : A comparative analysis », Peabody Journal of Education, vol. $75, \mathrm{n}^{\circ} 3 / 4$, sous presse.

Elley, W. B. (éd.), The IEA study of reading literacy : achievement and instruction in thirty-two school systems, Oxford, Pergamon, 1994. 
Elley, W. B., How in theWorld Do Students Read?, Association internationale pour l'évaluation du rendement scolaire (IEA), La Haye, 1992.

Elwert, G., « Societal literacy : Writing Culture and Development », in Olson, D. et Torrance, N. (éd.), The making of literate societies, Oxford, Blackwell, 2001, pp. 54-67.

Freire, P et Macedo, D., Literacy : Reading theWord and theWorld, Bergin \& Garvey, Massachussets, 1987.

Gambrell, L. B., «Creating classroom cultures that foster reading motivation », The Reading Teacher, 50, 1996, pp. 14-25.

Ganzeboom, H. B. G., de Graaf, P. M. et Treiman, D. J., «A standard international socio-economic index of occupational status », Social Science Research, 21, 1992, pp. 1-56.

Goldstein, H., Multilevel Statistical Models, Londres, Arnold, 1995.

Graesser, A. C., Millis, K. K. et Zwaan, R. A, «Discourse comprehension », Annual Review of Psychology, 48, 1997, pp. 163-189.

Gray, W. S. et Rogers, B., Maturity in reading, Chicago, University of Chicago Press, 1956.

Green, D. A. et Riddel, W. C., Les capacités de lecture et de calcul et la situation sur le marché du travail au Canada, Statistique Canada (STATCAN), Ottawa, 2001.

Guthrie, J et Davis, M. H., « Motivating struggling readers in middle school through an engagement model of classroom practice », Reading andWriting Quarterly, sous presse, 2002.

Guthrie, J.T., Schafer, W.D., Von Secker, C. and Alban, T. Contribution of reading instruction and text resources to achievement and engagement in a statewide school improvement program. Journal of Educational Research, 93, 2000, 211-226.

Guthrie, J. et Wigfield, A., «Engagement and motivation in reading », in Kamil, M. L. Mosenthal, P. B, Pearson, P. D. et Barr, R. (éd.), Handbook of reading research, vol. III, LEA, 2000, pp. 403-425.

Halpern, D. F., Thought and knowledge : An introduction to critical thinking, Hillsdale, New Jersey, Lawrence Erlbaum Associates, 1989.

Ivey, G. «Reflections on teaching struggling middle school readers », Journal of Adolescent and Adult Literacy, 42/5, 1999, pp. 372-381.

Jackson, D., «Breaking out of the binary trap : boy's underachievement, schooling and gender relations », in Epstein, D., Elwood, J., Hey, V. et Maw, J., (éd.), Failing boys? Issues in gender and achievement, Philadelphie, Open University Press, 1998, pp. 77-95.

Kamil, M.L., Mosenthal, P.B., Pearson, P. D. and Barr, R. (Eds.), Handbook of reading research. Vol III. Lawrence Erlbaum Associates, 2000.

Kintsch, W. et van Dijk, T., «Toward a Model of Text Comprehension and Production », Psychological Review, 85, 1978, pp. 363-394.

Kirsch, I. S., The International Adult Literacy Survey : UnderstandingWhat Was Measured, Princeton, New Jersey, Educational Testing Service, RR-01-25, 2001.

Kirsch, I. S. et Mosenthal, P. B., «Exploring document literacy : Variables underlying the performance of young adults », Reading Research Quarterly 25, 1, 1990, pp. 5-30.

Kirsch, I. S. et Mosenthal, P. B., «Understanding documents », chronique mensuelle publiée dans Journal of Reading, Newark, Delaware, International Reading Association, 1989-1991

Kirsch, I. S., Jungeblut, A., Jenkins, L. et Kolstad, A., Adult literacy in America : A first look at the results of the National Adult Literacy Survey, ministère de l’Éducation, Washington, DC, États-Unis, 1993.

Lafontaine, D., From Comprehension to Literacy :ThirtyYears of Reading Assessment, exposé non publié présenté lors de l'Assemblée générale de l'INES, 1999. 
Langer, J., Envisioning literature, Newark, Delaware, International Reading Association, 1995.

Lewis, B., Guide Picks - Top 5 Professional Books about Reading, http://k-6educators.about.com/libary/products/ aatp111201.htm?PM=ss03_k-6educator, About.com., 2002.

Littell, R. C., Milliken, G. A., Stroup,W.W. et Wolfinger, R. D., SAS System for Mixed Models, Cary, Caroline du Nord, SAS Publications, 1996.

Mac An Ghaill, M., The making of men : masculinities, sexualities and schooling, Philadelphie, Open University Press, 1994.

McCormick, Th.W., Theories of reading in dialogue : An interdisciplinary study, New York, University Press of America, 1988.

McKenna, M., Kear, D. J. et Ellsworth, R. A., « Children's attitudes toward reading : a national survey », Reading Research Quarterly, 30/4, 1995, pp. 934-956.

Messick, S., «Validity », in Linn, R. (éd.), Educational measurement (3éd.), New York, Macmillan, 1989, pp. 13-103.

Mislevy, R.J. Randomization-based inference about latent variables from complex samples. Psychometrika, 56, 1991, 177-1.

Moffett, J. et Wagner, B. J., Student-Centered Language Arts and Reading K-13 : A Handbook for Teachers, Boston, Houghton Mifflin, 1983.

Mokhtari, K. et Sheory, P., « Differences in the meta-cognitive awareness of reading strategies among native and non-native readers », System, 29, 4, 2001, pp. 431-450.

Neuman, S. B. et Celano, D., «Access to print in low-income and middle-income communities : An ecological study of four neighborhoods », Reading Research Quarterly, 36 (1), 2001, pp. 8-26.

Oka, E. et Paris, S., «Patterns of motivation and reading skills in underachieving children », in Ceci, S. (éd.), Handbook of cognitive, social, and neuropsychological aspects of learning disabilities, (Vol. 2), Hillsdale, New Jersey, Erlbaum, 1986.

Olson, D. R., «From Utterance to Text : The Bias of Language in Speech and Writing », Harvard Educational Review, 47, 1977a, pp. 257-81.

Olson, D. R., «The language of instruction : The literate bias of schooling », in Anderson, R., Spiro, R. et Montague, W. (éd.), Schooling and the acquisition of knowledge, Hillsdale, New Jersey, Erlbaum, 1977b, pp. 65-89.

Olson, D. R., TheWorld on Paper, Cambridge, Cambridge University Press, 1994.

Organisation de coopération et de développement économique (OCDE) et Statistique Canada (STATCAN), La littératie à l'ère de l'information : Rapport final de l'enquête internationale sur la littératie des adultes, OCDE, Paris, et STATCAN, Ottawa, 2000.

Organisation de coopération et de développement économique (OCDE) et Statistique Canada (STATCAN), Littératie, économie et société : Résultats de la première enquête sur l'alphabétisation des adultes, OCDE, Paris, et STATCAN, Ottawa, 1995.

Organisation de coopération et de développement économique (OCDE), L'illetrisme des adultes et les résultats économiques, OCDE, Paris, 1992.

Organisation de coopération et de développement économique (OCDE), Mesurer les connaissances et compétences des élèves : Un nouveau cadre d'évaluation, OCDE, Paris, 1999.

Organisation de coopération et de développement économique (OCDE), Connaissances et compétences : des atouts pour la vie-Premiers résultats de PISA 2000, OCDE, Paris, $2001 \mathrm{~b}$.

Organisation de coopération et de développement économique (OCDE), Le bien-être des nations, OCDE, Paris, $2001 a$.

Organisation de coopération et de développement économique (OCDE), Mesurer les connaissances et les compétences des élèves : Lecture, mathématiques et science - L'évaluation de PISA 2000, OCDE, Paris, 2000.

Organisation de coopération et de développement économique (OCDE), Connaissances et compétences : des atouts pour la vie - Premiers résultats de PISA 2000, OCDE, Paris, $2001 \mathrm{~b}$. 
Organisation de coopération et de développement économique (OCDE) et Statistique Canada (STATCAN), La littératie à l'ère de l'information : Rapport final de l'enquête internationale sur la littératie des adultes, OCDE, Paris, et STATCAN, Ottawa, 2000.

Organisation de coopération et de développement économique (OCDE), PISA 2000 Technical Report, OCDE, 2002.

Organisation de coopération et de développement économique (OCDE) et Statistique Canada (STATCAN), Littératie et société du savoir : Nouveaux résultats de l'Enquête internationale sur les capacités de lecture et d'écriture des adultes, OCDE, Paris, et STATCAN, Ottawa, 1997.

Organisation de coopération et de développement économique (OCDE) et Statistique Canada (STATCAN), Littératie, économie et société : Résultats de la première enquête sur l'alphabétisation des adultes, OCDE, Paris, et STATCAN, Ottawa, 1995.

Osberg, L., Scolarité, alphabétisme et gains personnels, Statistique Canada (STATCAN), Ottawa, et Développement des ressources humaines Canada, 2000.

Paris, S. G. et Winograd, P., « How metacognition can promote academic learning and instruction », in Jones, B. F. et Idol, L. (éd.), Dimensions of thinking and cognitive instruction, Hillsdale, New-Jersey, Erlbaum, 1990, pp. 15-52.

Postlethwaite, T. N., Calculation and Interpretation of Between-School and Within-School Variation in Achievement (rho) in Organisation de coopération et de développement économique (OCDE), Indicateurs des systèmes d'enseignement - Mesurer les résultats scolaires, CERI, Paris, 1995.

Programme des Nations Unies pour le développement (PNUD), Rapport mondial sur le développement humain 2002 - Approfondir la démocratie dans un monde fragmenté, New York, Oxford, Oxford University Press, 2002.

Rubin, D.B. Multiple imputation for nonresponse in surveys. New York: John Wiley and Sons, 1987.

Rumelhart, D. E., «Toward an interactive model of reading », in Singer, H. et Ruddell, R. B., (éd.), Theoretical models and the processes of reading, $3^{\mathrm{e}}$ édition, Newark, Delaware, International, 1985.

Secretary's Commission on Achieving Necessary Skills (SCANS), What Work Requires of Schools: A SCANS Report for America 2000, ministère du Travail, États-Unis, 1991.

Shetzer, H. et Warschauer, M., «An electronic literacy approach to network-based language teaching», in Warschauer, M. et Kern, R (éd.), Network-based language teaching : Concepts and practice, New York, Cambridge University Press, 2000, pp. 171-185.

Smith, C. M., «Differences in adults' reading practices and literacy proficiencies », Reading Research Quarterly, 31/2, 1996, pp. 196-219.

Smith, M. C., Mikulecky, L., Kibby, M.W et Dreher, M. J., «What will be the demands of literacy in the workplace in the next millennium ?», Reading Research Quarterly, 35 (3), 2000, pp. 378-383.

Stanovich, K. Matthew effects in reading: some consequences of individual differences in the acquisition of literacy. Reading Research Quarterly, 21, 1986, 360-407.

Sticht, T. G., (éd.), Reading for working : A functional literacy anthology, Alexandria, Virginie, Human Resources Research Organization, 1975.

Sticht, T. G., « Comprehending reading at work », in Just, M. et Carpenter, P. (éd.), Cognitive Processes in Comprehension, Hillsdale, New Jersey, Erlbaum, Reading Association, 1976.

Stiggins, R. J, «An analysis of the dimensions of job-related reading », ReadingWorld, 82, 1982, pp. 237-247.

Sum, A et Fogg, W. N., «The Adolescent Poor and the Transition to Early Adulthood », in Edelman, P. B. et Ladner, J. (éd.), Adolescence and Poverty : Challenge for the 1990s, Center for National Policy Press, Washington, D.C., 1991.

Sum, A., Kirsch, I. S. et Taggert, R., The Twin Challenges of Mediocrity and Inequality : Literacy in the United States from an International Perspective, Policy Information Center Report, Princeton, New Jersey, Educational Testing Service, février 2002.

Sum, A., Literacy in the Labor Force, National Center for Education Statistics, Washington, D.C, 1999. 
Van Dijk, T. A. et Kintsch, W., Strategies of Discourse Comprehension, Orlando, Academic Press, 1983.

Walker, D. A., Anderson, C. A. et Wolf, R. M., IEA Six Subject Survey : An Empirical Study of Education in Twenty-one Countries, New York, John Wiley and Sons Ltd, 1976.

Warschauer, M., Electronic Literacies : Language Culture and Power in Online Education, Mahwah, New Jersey, Lawrence Erlbaum Associates, 1999.

Warschauer, M., Technology and social inclusion : Rethinking the digital divide, Cambridge, MIT Press, sous presse.

Werlich, E., A text grammar of English, Heidelberg, Quelle \& Meyer, 1976.

Winn, J. A., «Promises and challenges of scaffolded instruction », Learning Disability Quarterly, 17, 1994, pp. 89-104.

Yamamoto, K., Estimating PISA Students on the IALS Prose Literacy Scale, Princeton, New Jersey, Educational Testing Service, sous presse.

Young, P.J. et Brozo, W. G., « Boys will be boys, or will they ? Literacy and masculinities », Reading Research Quarterly, 36/3, 2001, pp. 316-325. 


\section{ANNEXE \\ A \\ NOTES TECHNIQUES}

Annexe A1 : indices quantitatifs relatifs à l'équilibre culturel dans les items d'évaluation de PISA 2000

Annexe A2 : indice d'engagement à l'égard de la lecture

Annexe A3 : notes techniques sur les analyses multivariées à plusieurs niveaux

Annexe A4 : erreurs types, tests de signification et comparaisons multiples 


\section{Annexe A1 : indices quantitatifs relatifs à l'équilibre culturel des items du cycle d'évaluation PISA 2000}

Lors du processus d'élaboration des items, PISA a demandé aux pays participants de soumettre des items dans le souci de couvrir un large éventail de cultures et de langues. Vingt pays ont accédé à cette demande (voir le tableau A1.1). PISA a également intégré des items de l'Enquête internationale sur la littératie des adultes (IALS) dans sa batterie de tests à des fins d'ancrage entre ses résultats et ceux de IALS.

Tableau A1.1

Contribution des pays et organismes internationaux aux items de l'évaluation PISA 2000

\begin{tabular}{|c|c|c|c|c|}
\hline Domaine / Pays & Lecture & Mathématiques & Sciences & Total \\
\hline ACER & 123 & 3 & 29 & 155 \\
\hline Australie & & & 4 & 4 \\
\hline Autriche & 7 & 5 & & 12 \\
\hline Belgique & 13 & & & 13 \\
\hline groupe CITO & 51 & 34 & 20 & 130 \\
\hline République tchèque & & 2 & & 2 \\
\hline Danemark & 4 & & & 4 \\
\hline Finlande & 21 & & & 21 \\
\hline France & 14 & 7 & 11 & 32 \\
\hline Allemagne & & 2 & & 2 \\
\hline Grèce & 6 & 3 & & 9 \\
\hline IALS & 48 & & & 48 \\
\hline Irlande & 7 & & & 7 \\
\hline Italie & 8 & & & 8 \\
\hline Japon & 8 & & & 8 \\
\hline Corée & 6 & & 8 & 14 \\
\hline Nouvelle-Zélande & 13 & & & 13 \\
\hline Norvège & 7 & & 6 & 13 \\
\hline Suède & 8 & 6 & 6 & 20 \\
\hline Suisse & 6 & & & 6 \\
\hline Fédération de Russie & & & 4 & 4 \\
\hline TIMSS & & 5 & 5 & 10 \\
\hline Royaume-Uni & 6 & & & 6 \\
\hline États-Unis & 20 & 10 & 15 & 45 \\
\hline Total & 376 & 77 & 108 & 586 \\
\hline
\end{tabular}

PISA a demandé à chaque pays de juger de l'adéquation des items et des stimuli, en termes d'exposition des élèves à leur contenu et de difficulté des items, d'identifier d'éventuels biais d'ordre culturel ou autre, d'épingler d'éventuels problèmes de traduction et de se prononcer sur le bien-fondé de leur inclusion dans la batterie de tests de PISA. Les résultats de ces évaluations nationales sont présentés dans le tableau A1.2. Le système de notation utilisé pour ces évaluations est une échelle allant de 1 (inadéquation) à 4 (adéquation totale). Les évaluations et les commentaires qualitatifs des experts nationaux ont servi de base à la sélection des items de la batterie de tests du cycle PISA 2000 et à leur révision. 
Tableau A1.2

Évaluations nationales de l'adéquation des items d'évaluation PISA 2000

\begin{tabular}{|c|c|c|c|c|c|c|c|}
\hline \multirow{2}{*}{$\begin{array}{l}\text { Identification } \\
\text { de l'item }\end{array}$} & \multirow[b]{2}{*}{ Titre de l'unité } & \multirow{2}{*}{$\begin{array}{c}\text { Nombre de pays } \\
\text { participant à } \\
\text { l'évaluation }\end{array}$} & \multicolumn{4}{|c|}{$\begin{array}{l}\text { Nombre de pays donnant } \\
\text { l'évaluation ci-dessous : }\end{array}$} & \multirow{2}{*}{$\begin{array}{c}\text { Adéquation de l'item } \\
\text { sur la base des notes } \\
\text { moyennes }\end{array}$} \\
\hline & & & 1 & 2 & 3 & 4 & \\
\hline R040Q02 & Le lac Tchad & 25 & 0 & 3 & 12 & 6 & 2.64 \\
\hline R040Q03A & Le lac Tchad & 25 & 2 & 4 & 7 & 9 & 2.68 \\
\hline R040Q03B & Le lac Tchad & 25 & 2 & 6 & 6 & 8 & 2.56 \\
\hline R040Q04 & Le lac Tchad & 25 & 2 & 5 & 7 & 8 & 2.60 \\
\hline R040Q06 & Le lac Tchad & 25 & & 4 & 9 & 8 & 2.68 \\
\hline R055Q01 & Araignées droguées & 26 & 1 & 2 & 8 & 11 & 2.92 \\
\hline R055Q02 & Araignées droguées & 26 & & 4 & 7 & 10 & 2.76 \\
\hline R055Q03 & Araignées droguées & 26 & 2 & 1 & 10 & 9 & 2.80 \\
\hline R055Q05 & Araignées droguées & 26 & & 3 & 7 & 12 & 3.00 \\
\hline R061Q01 & Macondo & 25 & 3 & 3 & 9 & 7 & 2.56 \\
\hline R061Q03 & Macondo & 25 & 2 & 4 & 9 & 7 & 2.60 \\
\hline R061Q04 & Macondo & 25 & 3 & 4 & 9 & 6 & 2.48 \\
\hline R061Q05 & Macondo & 25 & 6 & 6 & 6 & 3 & 1.92 \\
\hline R067Q01 & Ésope & 25 & 1 & 3 & 5 & 12 & 2.80 \\
\hline R067Q04 & Ésope & 25 & 2 & 7 & 5 & 8 & 2.52 \\
\hline R067Q05 & Ésope & 25 & 3 & 6 & 6 & 7 & 2.44 \\
\hline R070Q02 & Plage & 25 & 1 & 6 & 9 & 5 & 2.40 \\
\hline R070Q03 & Plage & 25 & 2 & 6 & 7 & 6 & 2.36 \\
\hline R070Q04 & Plage & 25 & 4 & 11 & 5 & 2 & 1.96 \\
\hline R070Q07 & Plage & 25 & 3 & 5 & 7 & 5 & 2.16 \\
\hline R076Q03 & Iran Air & 26 & 5 & 3 & 8 & 6 & 2.36 \\
\hline R076Q04 & Iran Air & 26 & 5 & 3 & 10 & 4 & 2.28 \\
\hline R076Q05 & Iran Air & 26 & 6 & 1 & 10 & 5 & 2.32 \\
\hline R076Q06 & Iran Air & 26 & 3 & 3 & 10 & 6 & 2.52 \\
\hline R077Q02 & Grippe & 25 & & 3 & 12 & 6 & 2.64 \\
\hline R077Q03 & Grippe & 25 & 4 & 6 & 8 & 3 & 2.08 \\
\hline R077Q04 & Grippe & 25 & 1 & 5 & 9 & 5 & 2.32 \\
\hline R077Q05 & Grippe & 25 & 4 & 6 & 9 & 2 & 2.04 \\
\hline R077Q06 & Grippe & 25 & 1 & 4 & 13 & 2 & 2.24 \\
\hline R081Q01 & Graffiti & 25 & 2 & 6 & 5 & 8 & 2.44 \\
\hline R081Q02 & Graffiti & 25 & 4 & 6 & 8 & 4 & 2.24 \\
\hline R081Q05 & Graffiti & 25 & 3 & 6 & 6 & 7 & 2.44 \\
\hline R081Q06A & Graffiti & 25 & 6 & 6 & 5 & 5 & 2.12 \\
\hline R081Q06B & Graffiti & 25 & 4 & 10 & 3 & 4 & 1.96 \\
\hline R083Q01 & Travaux ménagers & 25 & 5 & 4 & 7 & 6 & 2.32 \\
\hline R083Q02 & Travaux ménagers & 25 & 3 & 4 & 11 & 3 & 2.24 \\
\hline R083Q03 & Travaux ménagers & 25 & 3 & 6 & 9 & 3 & 2.16 \\
\hline R083Q04 & Travaux ménagers & 25 & 4 & 5 & 9 & 4 & 2.28 \\
\hline R083Q06 & Travaux ménagers & 25 & 5 & 6 & 7 & 3 & 2.00 \\
\hline R086Q04 & $\mathrm{Si}$ & 25 & 4 & 7 & 4 & 6 & 2.16 \\
\hline R086Q05 & $\mathrm{Si}$ & 25 & 3 & 5 & 5 & 9 & 2.56 \\
\hline R086Q07 & $\mathrm{Si}$ & 25 & 5 & 6 & 2 & 8 & 2.20 \\
\hline R088Q01 & Population active & 26 & 1 & 7 & 7 & 6 & 2.40 \\
\hline R088Q03 & Population active & 26 & 2 & 3 & 12 & 6 & 2.72 \\
\hline R088Q04 & Population active & 26 & 1 & 6 & 11 & 5 & 2.64 \\
\hline R088Q05 & Population active & 26 & 3 & 4 & 11 & 4 & 2.40 \\
\hline R088Q07 & Population active & 26 & 4 & 4 & 9 & 5 & 2.36 \\
\hline R091Q05 & Bibliothèque & 25 & 4 & 2 & 7 & 9 & 2.60 \\
\hline R091Q06 & Bibliothèque & 25 & 2 & 6 & 5 & 8 & 2.44 \\
\hline R091Q07 & Bibliothèque & 25 & 4 & 6 & 2 & 10 & 2.48 \\
\hline R093Q03 & Agences de Presse & 25 & 4 & 7 & 7 & 2 & 1.88 \\
\hline R093Q04 & Agences de Presse & 25 & 4 & 8 & 5 & 4 & 2.04 \\
\hline R099Q02 & Plan international & 25 & 1 & 4 & 8 & 9 & 2.76 \\
\hline R099Q03 & Plan international & 25 & 3 & 4 & 7 & 8 & 2.56 \\
\hline R099Q04A & Plan international & 25 & & 5 & 9 & 7 & 2.60 \\
\hline
\end{tabular}




\begin{tabular}{|c|c|c|c|c|c|c|c|}
\hline \multirow{2}{*}{$\begin{array}{l}\text { Identification } \\
\text { de l'item }\end{array}$} & \multirow[b]{2}{*}{ Titre de l'unité } & \multirow{2}{*}{$\begin{array}{l}\text { Nombre de pays } \\
\text { participant a } \\
\text { l'évaluation }\end{array}$} & \multicolumn{4}{|c|}{$\begin{array}{l}\text { Nombre de pays donnant } \\
\text { l'évaluation ci-dessous : }\end{array}$} & \multirow{2}{*}{$\begin{array}{c}\text { Adéquation de l'item } \\
\text { sur la base des notes } \\
\text { moyennes }\end{array}$} \\
\hline & & & 1 & 2 & 3 & 4 & \\
\hline R099Q04B & Plan international & 25 & 4 & 3 & 7 & 7 & 2.36 \\
\hline R100Q04 & Police & 28 & 1 & 5 & 8 & 9 & 2.84 \\
\hline R100Q05 & Police & 28 & 1 & 3 & 8 & 10 & 2.84 \\
\hline R100Q06 & Police & 28 & 3 & 3 & 8 & 8 & 2.60 \\
\hline R100Q07 & Police & 28 & 1 & 4 & 8 & 10 & 2.92 \\
\hline R101Q01 & Rhinocéros & 28 & 3 & 3 & 7 & 10 & 2.80 \\
\hline R101Q02 & Rhinocéros & 27 & 3 & 6 & 5 & 6 & 2.16 \\
\hline R101Q03 & Rhinocéros & 25 & 1 & 3 & 6 & 10 & 2.60 \\
\hline R101Q04 & Rhinocéros & 25 & 1 & 3 & 7 & 9 & 2.56 \\
\hline R101Q05 & Rhinocéros & 28 & 3 & 4 & 7 & 8 & 2.56 \\
\hline R101Q08 & Rhinocéros & 28 & 3 & 5 & 6 & 9 & 2.68 \\
\hline R102Q01 & Chemises & 28 & 3 & 6 & 6 & 7 & 2.44 \\
\hline R102Q04A & Chemises & 28 & 5 & 9 & 7 & 2 & 2.08 \\
\hline R102Q05 & Chemises & 28 & 4 & 3 & 10 & 5 & 2.40 \\
\hline R102Q06 & Chemises & 28 & 8 & 6 & 6 & 2 & 1.84 \\
\hline R102Q07 & Chemises & 28 & 2 & 8 & 8 & 4 & 2.32 \\
\hline R104Q01 & Téléphone & 25 & 2 & 4 & 11 & 3 & 2.20 \\
\hline R104Q02 & Téléphone & 25 & 2 & 3 & 10 & 5 & 2.32 \\
\hline R104Q05 & Téléphone & 25 & 2 & 3 & 10 & 5 & 2.32 \\
\hline R104Q06 & Téléphone & 25 & 3 & 2 & 10 & 5 & 2.28 \\
\hline R110Q01 & Baskets & 25 & 1 & 5 & 9 & 7 & 2.64 \\
\hline R110Q04 & Baskets & 25 & & 6 & 10 & 5 & 2.48 \\
\hline R110Q05 & Baskets & 25 & 1 & 4 & 11 & 6 & 2.64 \\
\hline R110Q06 & Baskets & 25 & 1 & 6 & 7 & 7 & 2.48 \\
\hline R111Q01 & Échanges & 25 & 2 & 4 & 7 & 8 & 2.52 \\
\hline R111Q02B & Échanges & 12 & 1 & 5 & 2 & 4 & 1.32 \\
\hline R111Q04 & Échanges & 25 & 1 & 5 & 5 & 10 & 2.64 \\
\hline R111Q06A & Échanges & 25 & & 5 & 9 & 7 & 2.60 \\
\hline R111Q06B & Échanges & 25 & 1 & 6 & 7 & 8 & 2.64 \\
\hline R119Q01 & Le cadeau & 26 & 2 & 5 & 5 & 10 & 2.68 \\
\hline R119Q04 & Le cadeau & 26 & 5 & 4 & 6 & 7 & 2.36 \\
\hline R119Q05 & Le cadeau & 26 & 6 & 9 & 4 & 4 & 2.08 \\
\hline R119Q06 & Le cadeau & 25 & 1 & 7 & 5 & 8 & 2.48 \\
\hline R119Q07 & Le cadeau & 25 & 4 & 6 & 6 & 6 & 2.32 \\
\hline R119Q08 & Le cadeau & 25 & & 8 & 7 & 7 & 2.60 \\
\hline R119Q09 & Le cadeau & 25 & 4 & 6 & 5 & 6 & 2.20 \\
\hline R120Q01 & Avis d'élèves & 28 & 1 & 4 & 5 & 12 & 2.88 \\
\hline R120Q03 & Avis d'élèves & 28 & 1 & 6 & 7 & 8 & 2.64 \\
\hline R120Q06 & Avis d'élèves & 28 & 6 & 1 & 9 & 6 & 2.36 \\
\hline R120Q07 & Avis d'élèves & 28 & 1 & 5 & 8 & 9 & 2.84 \\
\hline R122Q01 & Just art & 25 & 4 & 8 & 5 & 4 & 2.04 \\
\hline R122Q02 & Just art & 25 & 6 & 7 & 6 & 2 & 1.84 \\
\hline R122Q03 & Just art & 25 & 4 & 11 & 4 & 2 & 1.84 \\
\hline R216Q01 & Amanda et la duchesse & 26 & 2 & 5 & 11 & 4 & 2.44 \\
\hline R216Q02 & Amanda et la duchesse & 26 & 2 & 6 & 9 & 5 & 2.44 \\
\hline R216Q03 & Amanda et la duchesse & 26 & 3 & 5 & 8 & 6 & 2.44 \\
\hline R216Q04 & Amanda et la duchesse & 26 & 4 & 8 & 6 & 5 & 2.32 \\
\hline R216Q06 & Amanda et la duchesse & 26 & 3 & 5 & 8 & 6 & 2.44 \\
\hline R219Q01 & Emploi & 26 & 4 & 6 & 3 & 9 & 2.44 \\
\hline R219Q02 & Emploi & 26 & 5 & 7 & 5 & 5 & 2.16 \\
\hline R220Q01 & Pôle Sud & 26 & & 6 & 11 & 5 & 2.60 \\
\hline R220Q02B & Pôle Sud & 26 & & 3 & 13 & 6 & 2.76 \\
\hline R220Q04 & Pôle Sud & 26 & 1 & 4 & 8 & 10 & 2.92 \\
\hline R220Q05 & Pôle Sud & 26 & 3 & 3 & 8 & 8 & 2.60 \\
\hline R220Q06 & Pôle Sud & 26 & 1 & 3 & 8 & 11 & 3.00 \\
\hline R225Q01 & Nucléaire & 25 & & 8 & 7 & 5 & 2.28 \\
\hline R225Q02 & Nucléaire & 25 & & 8 & 7 & 5 & 2.28 \\
\hline
\end{tabular}




\begin{tabular}{|c|c|c|c|c|c|c|c|}
\hline \multirow{2}{*}{$\begin{array}{l}\text { Identification } \\
\text { de l'item }\end{array}$} & \multirow[b]{2}{*}{ Titre de l'unité } & \multirow{2}{*}{$\begin{array}{l}\text { Nombre de pays } \\
\text { participant à } \\
\text { l'évaluation }\end{array}$} & \multicolumn{4}{|c|}{$\begin{array}{l}\text { Nombre de pays donnant } \\
\text { l'évaluation ci-dessous : }\end{array}$} & \multirow{2}{*}{$\begin{array}{c}\text { Adéquation de l'item } \\
\text { sur la base des notes } \\
\text { moyennes }\end{array}$} \\
\hline & & & 1 & 2 & 3 & 4 & \\
\hline R225Q03 & Nucléaire & 25 & & 4 & 12 & 4 & 2.40 \\
\hline R225Q04 & Nucléaire & 25 & 1 & 5 & 8 & 6 & 2.36 \\
\hline R227Q01 & Opticien & 25 & 2 & 10 & 7 & 3 & 2.20 \\
\hline R227Q02 & Opticien & 25 & 4 & 6 & 8 & 2 & 1.92 \\
\hline R227Q03 & Opticien & 25 & 4 & 8 & 6 & 3 & 2.00 \\
\hline R227Q04 & Opticien & 25 & 1 & 8 & 8 & 5 & 2.44 \\
\hline R227Q06 & Opticien & 25 & 1 & 9 & 5 & 6 & 2.32 \\
\hline R228Q01 & Guide & 26 & 2 & 3 & 7 & 10 & 2.76 \\
\hline R228Q02 & Guide & 26 & 1 & 4 & 7 & 10 & 2.80 \\
\hline R228Q04 & Guide & 25 & 3 & 3 & 5 & 10 & 2.56 \\
\hline R234Q01 & Personnel & 25 & 5 & 9 & 3 & 4 & 1.92 \\
\hline R234Q02 & Personnel & 25 & 6 & 7 & 4 & 3 & 1.76 \\
\hline R236Q01 & Nouvelles règles & 25 & 7 & 5 & 6 & 3 & 1.88 \\
\hline R236Q02 & Nouvelles règles & 25 & 4 & 8 & 5 & 4 & 2.04 \\
\hline R237Q01 & Entretien d'embauche & 25 & & 6 & 10 & 6 & 2.64 \\
\hline R237Q03 & Entretien d'embauche & 25 & 1 & 7 & 7 & 6 & 2.40 \\
\hline R238Q01 & Bicyclette (IALS) & 25 & 1 & 11 & 6 & 4 & 2.28 \\
\hline R238Q02 & Bicyclette (IALS) & 25 & 3 & 8 & 6 & 5 & 2.28 \\
\hline R239Q01 & Allergies & 25 & 4 & 9 & 6 & 1 & 1.76 \\
\hline R239Q02 & Allergies & 25 & 4 & 9 & 5 & 3 & 1.96 \\
\hline R241Q01 & Garantie Hotpoint & 25 & 6 & 9 & 4 & 1 & 1.60 \\
\hline R241Q02 & Garantie Hotpoint & 25 & 5 & 7 & 6 & 3 & 1.96 \\
\hline R245Q01 & Comptes rendus de films & 25 & 3 & 7 & 6 & 5 & 2.20 \\
\hline R245Q02 & Comptes rendus de films & 25 & 2 & 9 & 6 & 3 & 2.00 \\
\hline R246Q01 & Contact avec un employeur & 25 & 4 & 4 & 7 & 5 & 2.12 \\
\hline R246Q02 & Contact avec un employeur & 25 & 2 & 5 & 9 & 5 & 2.36 \\
\hline Total & & & & & & & 2.38 \\
\hline
\end{tabular}

Un panel international d'évaluation culturelle a été chargé d'étudier les éventuels biais culturels et linguistiques des items et des stimuli inclus dans la batterie de tests utilisée lors de l'essai de terrain de PISA. Les membres de ce panel se sont réunis en octobre 1999 pour analyser la batterie d'items de compréhension de l'écrit et les guides de correction utilisés lors de l'essai de terrain. Leurs conclusions ont été prises en considération pour réviser et sélectionner la batterie d'items en vue de la campagne de tests définitive. Le tableau A1.3 présente la couverture géographique et culturelle du panel international d'évaluation culturelle.

Tableau A1.3

Composition géographique et culturelle du panel d'évaluation culturelle (CRP)

\begin{tabular}{lclc}
\hline Pays & Nombre de participants & Pays & Nombre de participants \\
\hline Autriche & 1 & Pays-Bas & 3 \\
Belgique & 1 & Nouvelle- Zélande & 1 \\
France & 1 & Suisse & 1 \\
Allemagne & 1 & Espagne & 1 \\
Irlande & 1 & Suède & 1 \\
Italie & 2 & Royaume-Uni & 2 \\
Corée & 1 & États-Unis & 2 \\
Luxembourg & 1 & & 20
\end{tabular}


Un indice statistique appelé fonctionnement différentiel des items (DIF) a été utilisé pour déceler les items qui ne se comportaient pas de la même manière dans certains pays. Les items suspects, c'est-à-dire ceux susceptibles de présenter un biais culturel, ont été analysés en collaboration avec les experts nationaux. Au terme de ces travaux, certains items ont été exclus de l'échelonnage comme s'ils n'avaient pas été utilisés dans le pays concerné. Le tableau A1.4 dresse la liste des items qui ont été exclus l'échelonnage national dans chaque pays.

Tableau A1.4

Items exclus de l'échelonnage à cause d'un possible biais culturel

\begin{tabular}{l|rl|r}
\hline Pays & Identification de l'item & Pays & Identification de l'item \\
\hline Autriche & M155Q03 & Corée & R237Q03 \\
Autriche & R055Q03 & Corée & R246Q02 \\
Autriche & S133Q04T & Mexique & R040Q02 \\
Belgique version fla- & R076Q05 & Pays-Bas & R076Q05 \\
mande & R100Q05 & Pays-Bas & R100Q05 \\
Belgique version fla- & M033Q01 & Pays-Bas & S268Q02T \\
mande & R101Q08 & Pologne & R099Q04B \\
Canada version fran- & R076Q03 & Fédération de Russie & R091Q05 \\
çaise & R076Q04 & Espagne & R227Q01 \\
Angleterre & R055Q03 & Suède & R091Q07B \\
Angleterre & S133Q04T & Suisse, version allemande & M155Q01 \\
Allemagne & R040Q02 & Suisse, version allemande & M155Q03 \\
Allemagne & R119Q04 & Suisse, version allemande & M155Q04 \\
Grèce & R236Q01 & Suisse, version allemande & R055Q03 \\
Hongrie & S268Q02T & Suisse, version allemande & R076Q03 \\
Islande & R040Q06 & Suisse, version allemande & R091Q05 \\
Islande & R219Q01T & Suisse, version allemande & R111Q06B \\
Italie & M155Q01 & Suisse, version allemande & R239Q02 \\
Italie & R102Q04A & Suisse, version allemande & S133Q04T \\
Japon & R216Q02 & Suisse, version italienne & S268Q06 \\
Corée & &
\end{tabular}

Source : Rapport technique de PISA 2000 (OECD, 2002b) 


\section{Annexe A2 : Indice d'engagement à l'égard de la lecture}

\section{Extension de l'indice initial d'engagement à l'égard de la lecture}

Un indice d'engagement à l'égard de la lecture a été élaboré pour le rapport initial PISA sur la seule base des attitudes des élèves à l'égard de la lecture (OCDE, 2001b).

Des recherches ultérieures ont toutefois montré que l'engagement à l'égard de la lecture englobait de multiples aspects, telles que les pratiques de lecture, les attitudes à l'égard de cette activité et l'intérêt qui lui est porté. En conséquence, nous avons tenté d'étoffer l'indice initial d'engagement à l'égard de la lecture en combinant les deux aspects de cette activité dans le cadre du présent rapport.

Ce nouvel indice d'engagement à l'égard de la lecture est basé sur trois variables : la fréquence de la lecture, la diversité des écrits et l'intérêt porté à cette activité. Les deux premières variables renvoient aux pratiques de lecture, tandis que la troisième porte sur les attitudes à l'égard de la lecture.

Cet indice a été standardisé en sorte que les valeurs de l'indice d'environ deux tiers de l'ensemble des élèves de l'OCDE soient comprises entre 1 et - 1 et que sa valeur moyenne soit de zéro (la valeur moyenne de la population d'élèves des pays participants est fixée à zéro et l'écart type, à 1). Il y a lieu de souligner qu'une valeur négative ne signifie pas forcément que les élèves ont répondu aux questions par la négative, mais qu'elle indique simplement qu'un groupe d'élèves (ou tous les élèves d'un pays, collectivement) a dans l'ensemble répondu de manière moins positive que ne l'ont fait en moyenne les autres élèves de l'OCDE. À l'inverse, une valeur positive signifie qu'un groupe d'élèves a répondu plus favorablement, ou plus positivement, que ne l'ont fait en moyenne les autres élèves de l'OCDE.

La modélisation par équation structurelle a été utilisée pour confirmer le comportement théorique de l'indice et pour valider sa comparabilité entre pays. La fidélité de ce nouvel indice d'engagement à l'égard de la lecture est de 0,83 . Elle est passée de 0,72 à 0,83 après l'inclusion de la fréquence de la lecture et de la diversité des lectures dans le concept initial d'engagement à l'égard de la lecture. Le nouvel indice est également fortement corrélé à l'indice initial d'engagement à l'égard de la lecture basé sur les attitudes $(0,88)$.

\section{Relation avec le sexe et avec les performances en lecture}

Le tableau A2.1 compare les deux indices d'engagement à l'égard de la lecture à plusieurs niveaux, à savoir la moyenne des pays, les différences entre les sexes et leur impact sur les performances en lecture. Les valeurs des deux indices d'engagement à l'égard de la lecture sont relativement cohérentes par rapport à la moyenne de l'OCDE. En d'autres termes, les pays qui affichent une valeur supérieure à la moyenne de l'OCDE sur l'indice initial d'engagement à l'égard de la lecture présentent également une valeur supérieure à la moyenne sur le nouvel indice. La Norvège et la Suède sont les deux pays qui font exception à la règle. En effet, leur valeur est supérieure à la moyenne de l'OCDE selon l'indice initial, mais elle est y inférieure selon le nouvel indice. Il est probable que les élèves de ces deux pays ont déclaré lire moins souvent et diversifier moins leurs lectures que les élèves des autres pays de l'OCDE. Il y a lieu de souligner qu'en dépit de l'écart d'engagement moyen à l'égard de la lecture entre l'indice initial et le nouvel indice, les différences d'engagement entre les sexes présentent un profil cohérent, les disparités entre sexes restant relativement stables.

Il ressort également du tableau A2.1 que le nouvel indice d'engagement à l'égard de la lecture a des effets plus importants sur la performance en compréhension de l'écrit que l'indice initial. Signalons toutefois que les effets associés au nouvel indice sont sensiblement moindres en Fédération de Russie, en Irlande, en Lettonie, en NouvelleZélande et en Pologne. 
Table A2.1

Comparaison des moyennes nationales basée sur les deux indices PISA d'engagement à l'égard de la lecture

\begin{tabular}{|c|c|c|c|c|c|c|c|c|c|c|}
\hline \multirow[b]{3}{*}{ Australie } & \multicolumn{4}{|c|}{ Moyennes nationales } & \multicolumn{4}{|c|}{ Différence entre sexes } & \multicolumn{2}{|c|}{$\begin{array}{l}\text { Effets sur la performance en } \\
\text { compréhension de l'écrit }{ }^{2}\end{array}$} \\
\hline & $\begin{array}{l}\text { Nouvel } \\
\text { indice }\end{array}$ & E.S. & $\begin{array}{l}\text { Indice } \\
\text { initial }\end{array}$ & E.S. & $\begin{array}{l}\text { Nouvel } \\
\text { indice }\end{array}$ & Rang $^{1}$ & $\begin{array}{l}\text { Indice } \\
\text { initial }\end{array}$ & Rang $^{1}$ & $\begin{array}{l}\text { Nouvel } \\
\text { indice }\end{array}$ & $\begin{array}{l}\text { Indice } \\
\text { initial }\end{array}$ \\
\hline & -0.04 & $(0.03)$ & -0.07 & $(0.03)$ & 0.28 & 26 & 0.45 & 22 & 42.52 & 41.8 \\
\hline Autriche & -0.08 & $(0.03)$ & -0.04 & $(0.03)$ & 0.52 & 6 & 0.82 & 4 & 36.76 & 29.3 \\
\hline Belgique & -0.28 & $(0.02)$ & -0.25 & $(0.02)$ & 0.41 & 15 & 0.56 & 15 & 35.83 & 30.8 \\
\hline Canada & 0.01 & $(0.01)$ & 0.00 & $(0.01)$ & 0.47 & 10 & 0.61 & 11 & 35.14 & 36.2 \\
\hline République tchèque & 0.02 & $(0.02)$ & 0.17 & $(0.02)$ & 0.57 & 3 & 0.78 & 5 & 41.23 & 33.8 \\
\hline Danemark & 0.26 & $(0.02)$ & 0.00 & $(0.02)$ & 0.48 & 9 & 0.63 & 10 & 40.99 & 40.0 \\
\hline Finlande & 0.46 & $(0.02)$ & 0.20 & $(0.02)$ & 0.74 & 1 & 0.92 & 1 & 42.48 & 40.0 \\
\hline France & -0.18 & $(0.02)$ & -0.06 & $(0.02)$ & 0.30 & 23 & 0.53 & 16 & 31.18 & 27.5 \\
\hline Allemagne & -0.26 & $(0.02)$ & -0.08 & $(0.03)$ & 0.55 & 4 & 0.82 & 3 & 37.57 & 33.3 \\
\hline Grèce & -0.09 & $(0.02)$ & -0.01 & $(0.02)$ & 0.17 & 30 & 0.41 & 26 & 33.31 & 35.7 \\
\hline Hongrie & 0.03 & $(0.02)$ & 0.07 & $(0.02)$ & 0.36 & 21 & 0.44 & 24 & 43.71 & 43.0 \\
\hline Islande & 0.27 & $(0.01)$ & 0.02 & $(0.02)$ & 0.39 & 18 & 0.51 & 17 & 42.11 & 43.7 \\
\hline Irlande & -0.20 & $(0.02)$ & -0.07 & $(0.02)$ & 0.46 & 12 & 0.57 & 14 & 35.18 & 40.1 \\
\hline Italie & -0.08 & $(0.02)$ & 0.00 & $(0.03)$ & 0.37 & 19 & 0.57 & 13 & 28.54 & 28.0 \\
\hline Japon & 0.20 & $(0.03)$ & 0.09 & $(0.03)$ & 0.17 & 29 & 0.31 & 30 & 26.87 & 23.8 \\
\hline Corée & 0.21 & $(0.02)$ & 0.02 & $(0.02)$ & 0.04 & 31 & 0.22 & 31 & 24.96 & 25.9 \\
\hline Luxembourg & -0.19 & $(0.02)$ & -0.10 & $(0.02)$ & 0.40 & 16 & 0.66 & 8 & 22.02 & 19.4 \\
\hline Mexique & 0.07 & $(0.01)$ & 0.29 & $(0.02)$ & 0.20 & 28 & 0.34 & 28 & 26.93 & 14.6 \\
\hline Nouvelle-Zélande & 0.05 & $(0.02)$ & 0.01 & $(0.02)$ & 0.29 & 25 & 0.43 & 25 & 39.75 & 43.7 \\
\hline Norvège & 0.09 & $(0.02)$ & -0.22 & $(0.02)$ & 0.51 & 7 & 0.66 & 7 & 47.25 & 42.5 \\
\hline Pologne & -0.10 & $(0.02)$ & -0.01 & $(0.03)$ & 0.36 & 20 & 0.45 & 21 & 30.84 & 34.1 \\
\hline Portugal & 0.13 & $(0.02)$ & 0.31 & $(0.02)$ & 0.47 & 11 & 0.65 & 9 & 35.14 & 33.5 \\
\hline Espagne & -0.23 & $(0.02)$ & -0.04 & $(0.02)$ & 0.29 & 24 & 0.50 & 19 & 32.38 & 31.3 \\
\hline Suède & 0.14 & $(0.02)$ & -0.06 & $(0.02)$ & 0.45 & 13 & 0.59 & 12 & 41.53 & 39.5 \\
\hline Suisse & 0.00 & $(0.01)$ & 0.06 & $(0.03)$ & 0.62 & 2 & 0.90 & 2 & 38.10 & 34.8 \\
\hline Royaume-Uni & -0.10 & $(0.02)$ & -0.10 & $(0.02)$ & 0.26 & 27 & 0.44 & 23 & 37.95 & 40.4 \\
\hline États-Unis & -0.14 & $(0.03)$ & -0.13 & $(0.03)$ & 0.36 & 22 & 0.40 & 27 & 29.32 & 33.0 \\
\hline Moyenne de l'OCDE & 0.00 & $(0.00)$ & 0.00 & $(0.00)$ & 0.38 & & 0.56 & & & \\
\hline Brésil & 0.11 & $(0.02)$ & 0.15 & $(0.02)$ & 0.53 & 5 & 0.50 & 18 & 23.76 & 20.6 \\
\hline Lettonie & -0.04 & $(0.02)$ & -0.04 & $(0.02)$ & 0.44 & 14 & 0.48 & 20 & 36.19 & 42.3 \\
\hline Liechtenstein & -0.13 & $(0.05)$ & -0.08 & $(0.06)$ & 0.49 & 8 & 0.77 & 6 & 43.81 & 35.7 \\
\hline Fédération de Russie & 0.17 & $(0.02)$ & 0.05 & $(0.01)$ & 0.39 & 17 & 0.34 & 29 & 24.82 & 34.9 \\
\hline
\end{tabular}

1. Les pays sont rangés par ordre décroissant d'importance de la différence entre garçons et filles.

2. Ces chiffres indiquent de combien varie la performance en compréhension de l'écrit si on augmente d'une unité l'indice d'engagement à l'égard de la lecture.

Performance moyenne significativement supérieure à la Performance moyenne ne s'écartant pas significativement de la moyenne
de l'OCDE

Performance moyenne significativement inférieure à la moyenne de l'OCDE 


\section{Annexe A3 : Notes techniques sur les analyses multivariées à plusieurs niveaux}

\section{Description des variables utilisées dans les analyses multivariées à plusieurs niveaux}

Caractéristiques personnelles des élèves

- Sexe : la valeur 1 a été attribuée au sexe féminin et la valeur zéro, au sexe masculin.

- Engagement à l'égard de la lecture : cet indice indique dans quelle mesure les élèves s'investissent dans la lecture. Il est basé sur deux variables, à savoir le temps consacré à la lecture en général et la diversité des lectures (les revues, les journaux, les bandes dessinées, les livres de fiction et les ouvrages documentaires) et les réponses des élèves à neuf propositions concernant leurs attitudes à l'égard de la lecture. Il s'agit d'un indicateur qui donne une idée de l'engagement des élèves dans la lecture, de leur intérêt pour cette activité et de leur motivation à lire.

\section{Variables familiales et contextuelles}

- Structure familiale : une valeur de 1 a été attribuée aux élèves qui ont déclaré vivre avec leurs deux parents ou tuteurs et une valeur de zéro, à tous les autres élèves. Cet indice indique donc si les élèves vivent dans une famille nucléaire.

- Statut professionnel des parents : les élèves ont été priés de communiquer la profession de leurs père et mère et de choisir parmi les options suivantes celle qui s'appliquait à leur père et à leur mère : il/elle exerce « un travail rémunéré à temps plein », il/elle exerce « un travail rémunéré à temps partiel », il/elle «ne travaille pas, mais cherche un emploi » et «autres». Les réponses à cette question ouverte ont ensuite été recodées en fonction de la Classification internationale type des professions (CITP, 1988). L'indice socio-économique international de statut professionnel de PISA (ISEI) est basé sur les réponses des élèves aux questions portant sur la profession de leurs parents. Cet indice regroupe les attributs des professions qui permettent de convertir en revenus le niveau de formation des parents. Il a été calculé sur la base de la hiérarchisation optimale des groupes de professions afin de maximiser l'effet indirect du niveau de formation sur les revenus par l'intermédiaire de la profession et de minimiser l'effet direct du niveau de formation sur les revenus, abstraction faite de la profession (le tout sans prise en considération de l'âge). Il convient de consulter l'ouvrage de Ganzeboom, Draaf et Treiman (1992) pour plus d'informations sur la méthodologie utilisée. L'indice retenu dans PISA est basé soit sur la profession du père, soit sur celle de la mère, selon celle qui a le statut le plus élevé.

- Nombre de livres à la maison : cette variable donne une idée des ressources culturelles à la maison et du milieu socio-économique et éducatif de la famille. Elle a servi d'indicateur du milieu socio-économique et éducatif des élèves dans la plupart des enquêtes internationales sur les résultats scolaires (TIMSS, Éducation à la citoyenneté démocratique et Reading Literacy Study de l'IEA).

- Communication culturelle avec les parents : cet indice est dérivé des réponses des élèves à des questions concernant la fréquence à laquelle leurs parents discutent de l'actualité sociale ou politique, de livres, de films ou d'émissions télévisées ou encore écoutent de la musique classique avec eux. Il donne une idée du temps que les parents passent à parler avec leur enfant de thèmes socioculturels.

- Ressources éducatives à la maison : cet indice est dérivé des réponses des élèves à des questions portant sur la présence chez eux de ressources susceptibles d'avoir un impact favorable sur leur environnement d'apprentissage (un endroit calme et un bureau pour étudier, des manuels et des calculatrices). 
- Statut d'immigrant : une valeur de 1 a été attribuée aux élèves qui ont déclaré être nés dans un pays autre que celui de l'évaluation tout comme leurs deux parents et une valeur de zéro a été attribuée à tous les autres élèves. Cette variable indique si les élèves sont des immigrants de la première génération. Il ressort des analyses préliminaires que la langue parlée à la maison a un impact important dans de nombreux pays, surtout dans ceux où vivent des proportions plus fortes d'immigrants. En effet, les élèves qui parlent le plus souvent une langue autre que la langue d'enseignement à la maison obtiennent en moyenne des scores inférieurs en compréhension de l'écrit. Cependant, il a été décidé d'exclure cette variable du modèle définitif, car la question à ce propos n’a pas été incluse dans le questionnaire proposé dans deux pays et a donné lieu à un pourcentage élevé de non-réponses dans de nombreux pays.

\section{Enseignement et apprentissage}

- Incitation au rendement scolaire : cet indice est dérivé des réponses des élèves à la question portant sur la fréquence des situations suivantes dans les cours de <langue de l'évaluation $>$ : « Le professeur demande aux élèves de travailler beaucoup », «Le professeur dit aux élèves qu'ils pourraient mieux faire », « Le professeur est mécontent lorsque les élèves rendent un travail mal fait» et «Les élèves ont beaucoup à étudier ».

- Temps consacré aux devoirs : cet indice est dérivé des réponses des élèves à une question portant sur le temps qu'ils consacrent par semaine à leurs devoirs en <langue de l'évaluation>, en mathématiques et en sciences.

- Année d'études : cette variable correspond à l'année d'études fréquentée par les élèves. Les réponses des élèves ont été recodées pour montrer la différence entre l'année d'études de l'élève et l'année d'études modale des jeunes de 15 ans dans chaque pays. Cette variable a essentiellement été incluse dans l'analyse pour contrôler l'effet de différents niveaux d'enseignement. En effet, l'impact que semblent avoir certains indicateurs prédictifs (scolaires) lorsque l'année d'études est exclue de l'analyse est en fait imputable aux différences d'années d'études.

\section{Perception du climat dans l'établissement et la salle de classe}

- Sens d'appartenance à l'établissement : cet indice traduit les sentiments que les élèves éprouvent à l'égard de leur école. Des valeurs positives indiquent que les élèves perçoivent leur établissement de manière positive.

- Climat de discipline : cet indice est dérivé des réponses des élèves aux questions portant sur la fréquence des situations suivantes dans les cours de <langue de l'évaluation> : « Le professeur doit attendre un long moment avant que les élèves < se calment> », «Les élèves ne peuvent pas bien travailler », « Les élèves n'écoutent pas ce que dit le professeur», «Les élèves ne commencent à travailler que bien après le début des cours », « Il y a du bruit et de l'agitation » et «Au début du cours, plus de cinq minutes se passent sans qu' on ne fasse rien ». L'indice a été inversé, de manière à ce que les valeurs les plus faibles indiquent un climat de discipline moins favorable.

- Relations entre élèves et enseignants : cet indice est dérivé des réactions des élèves aux propositions suivantes : «Les élèves s'entendent bien avec les professeurs », «Les professeurs s'intéressent au bien-être de leurs élèves », « Mes professeurs sont réellement à l'écoute de ce que j'ai à dire », «Si j'ai besoin d'aide supplémentaire, mes professeurs me l'apporteront » et « Mes professeurs me traitent avec justice ». 


\section{Caractéristiques des établissements}

- Moyenne pour l'établissement du statut professionnel des parents : cette variable correspond à la valeur moyenne du statut professionnel des parents des élèves fréquentant l'établissement. C'est la profession du père ou de la mère qui est prise en considération, selon celle qui a le statut le plus élevé. Cette variable caractérise le public des établissements en indiquant si les élèves sont dans l'ensemble issus de familles dont le statut socioéconomique est plus élevé ou plus faible.

- Degré moyen d'engagement à l'égard de la lecture pour l'établissement : cette variable correspond à la valeur moyenne de l'indice d'engagement à l'égard de la lecture dans chaque établissement, calculée sur la base des indices des élèves fréquentant l'établissement. 


\section{Remarques techniques à propos du modèle statistique}

Dans les modèles de régression linéaire à niveaux multiples, les variables explicatives propres aux élèves peuvent être considérées comme :

- fixes, si les lignes de régression (de couleur rouge) sont identiques pour tous les établissements (les lignes rouges sont parallèles) ;

- aléatoires, si l'impact du milieu socio-économique sur la performance des élèves varie d'un établissement à l'autre (les lignes rouges ne sont pas parallèles).

Dans les analyses présentées dans ce chapitre, les variables explicatives propres aux élèves ont été considérées comme fixes. Il serait intéressant d'identifier les variables scolaires susceptibles d'accentuer ou d'atténuer la relation entre le milieu socio-économique et le score des élèves en compréhension de l'écrit dans un pays donné, mais cette opération serait difficile à mener à bien au niveau international sans une connaissance approfondie du contexte local.

L'estimation des modèles de régression a été effectuée par le biais de la procédure SAS pour modèles mixtes (Littell, Milliken, Stroup et Wolfinger, 1996). Cette méthode permet aux chercheurs de manipuler un grand nombre de jeux de données de niveau élève et facilite l'estimation d'erreurs types correctes à l'aide de méthodes de répétitions. Dans PISA, nous avons utilisé la méthode de Fay pour calculer les erreurs types.

Par nature, les coefficients de régression des analyses multivariées ne sont pas normalisés. En effet, ils montrent dans quelle mesure la variable dépendante augmente ou diminue selon la modification de la variable explicative par incrément d'une unité. Toutes les variables continues ont été normalisées pour que leur moyenne soit égale à zéro et leur écart type, à 1 dans chaque pays afin de permettre la comparaison de l'ampleur des effets entre pays. Les agrégats des variables des élèves au niveau des établissements n’ont pas été normalisés.

Le tableau 7.11 indique le pourcentage de variance imputable aux établissements et aux élèves. En moyenne, la part de la variance imputable aux élèves est de 23 pour cent et celle imputable aux établissements, de 72 pour cent.

Le nombre d'élèves n'est pas le même dans le modèle multivarié à plusieurs niveaux présenté dans le chapitre 7 et dans la base de données internationale de PISA, parce que des données étaient manquantes pour certaines des variables utilisées dans cette analyse. Le tableau A3.1 indique le nombre d'élèves dans la banque de données internationale, le nombre d'élèves inclus dans l'analyse et le rapport entre ces deux nombres. 
ANNEXE A3

Table A3.1

Nombre d'élèves inclus dans la base de données internationale PISA 2000, nombre d'élèves inclus dans l'analyse et proportion d'élèves inclus dans les analyses multi-niveaux

\begin{tabular}{|c|c|c|c|}
\hline & $\begin{array}{l}\text { Nombre d'élèves inclus } \\
\text { dans l'analyse (1) }\end{array}$ & $\begin{array}{c}\text { Nombre d'élèves dans la base de } \\
\text { données PISA (2) }\end{array}$ & $\begin{array}{c}\text { Proportion d'élèves : } \\
\text { (1) sur (2) }\end{array}$ \\
\hline Australie & 4795 & 5176 & 0.93 \\
\hline Autriche & 4181 & 4745 & 0.88 \\
\hline Belgique & 5968 & 6670 & 0.90 \\
\hline Canada & 26859 & 29687 & 0.91 \\
\hline République tchèque & 5007 & 5365 & 0.93 \\
\hline Danemark & 3667 & 4235 & 0.87 \\
\hline Finlande & 4570 & 4864 & 0.94 \\
\hline France & 4087 & 4673 & 0.88 \\
\hline Allemagne & 4675 & 5073 & 0.92 \\
\hline Grèce & 4170 & 4672 & 0.89 \\
\hline Hongrie & 4523 & 4887 & 0.93 \\
\hline Islande & 3157 & 3372 & 0.94 \\
\hline Irlande & 3642 & 3854 & 0.95 \\
\hline Italie & 4622 & 4984 & 0.93 \\
\hline Japon & 1992 & 5256 & 0.38 \\
\hline Corée & 4528 & 4982 & 0.91 \\
\hline Luxembourg & 2874 & 3528 & 0.82 \\
\hline Mexique & 3848 & 4600 & 0.84 \\
\hline Nouvelle-Zélande & 3353 & 3667 & 0.91 \\
\hline Norvège & 3779 & 4147 & 0.91 \\
\hline Pologne & 3165 & 3654 & 0.87 \\
\hline Portugal & 4172 & 4585 & 0.91 \\
\hline Espagne & 5473 & 6214 & 0.88 \\
\hline Suède & 4186 & 4416 & 0.95 \\
\hline Suisse & 5516 & 6100 & 0.90 \\
\hline Royaume-Uni & 8524 & 9340 & 0.91 \\
\hline États-Unis & 2990 & 3846 & 0.78 \\
\hline Brésil & 4065 & 4893 & 0.83 \\
\hline Lettonie & 3562 & 3893 & 0.92 \\
\hline Fédération de Russie & 6072 & 6701 & 0.91 \\
\hline Pays-Bas ${ }^{1}$ & 2346 & 2503 & 0.94 \\
\hline
\end{tabular}

1. Taux de participation insuffisant pour permettre les comparaisons. 


\section{Annexe A4 : Erreurs types, tests de signification et comparaisons multiples}

Les données statistiques présentées dans ce rapport correspondent à des estimations de la performance des pays sur la base d'échantillons d'élèves, et non à des valeurs qui auraient pu être obtenues si chaque élève de chaque pays avait répondu à chaque question. Par conséquent, il importe de connaître la marge d'erreur de ces estimations. Dans les résultats du cycle PISA 2000, chaque estimation est associée à un degré d'incertitude exprimé sous la forme d'une erreur type. Le recours aux intervalles de confiance est un moyen de faire des inférences relatives aux moyennes et proportions d'une population qui reflètent l'incertitude associée aux estimations fondées sur des échantillons. À partir d'une donnée statistique obtenue au moyen d'un échantillon et dans l'hypothèse d'une distribution normale, il est possible d'inférer que le résultat correspondant à l'échelle de la population se situerait dans l'intervalle de confiance dans 95 répétitions sur 100 de la même mesure, appliquée à différents échantillons tirés de la même population.

Très souvent, le lecteur s'intéressera principalement aux écarts entre des valeurs différentes au sein d'un même pays ou par rapport à un autre pays (la comparaison du rendement des élèves de sexe féminin et masculin dans le même pays, par exemple). Dans les tableaux et graphiques présentés dans ce rapport, les écarts sont considérés comme statistiquement significatifs à partir du moment où de tels écarts (ou des écarts encore plus grands) s'observeraient dans moins de 5 pour cent des cas s'il n'y avait effectivement aucune différence dans les valeurs de population correspondantes. De même, le risque de faire état d'un écart statistiquement significatif en l'absence de corrélation entre deux mesures est limité à 5 pour cent.

Bien que la probabilité de voir une différence déclarée « statistiquement significative » à tort soit faible (5 pour cent) dans chaque comparaison simple, la probabilité de commettre ce type d'erreur s'accroît lorsque plusieurs comparaisons sont effectuées simultanément.

Il est possible de réaliser un ajustement pour réduire à 5 pour cent la probabilité maximale de qualifier à tort des différences de «statistiquement significatives » dans au moins une comparaison parmi toutes celles qui sont faites. Cet ajustement, qui est basé sur la méthode de Bonferroni, a déjà été réalisé dans les graphiques de comparaisons multiples présentés aux chapitres 2 et 3, car leur objectif est de comparer la performance d'un pays à celle de tous les autres pays.

Pour tous les autres tableaux et graphiques, le lecteur est invité à garder à l'esprit qu'à supposer qu'il n'y ait pas de différences réelles pour une mesure donnée, une comparaison multiple associée à un seuil de signification de 5 pour cent conduirait à constater des différences significatives inexistantes à $[0,05$ fois le nombre de comparaisons effectuées] reprises. Par exemple, bien que les tests de signification appliqués dans PISA pour déceler les différences entre les sexes garantissent une probabilité inférieure à 5 pour cent de constater à tort une différence entre sexes, une comparaison qui indiquerait ces différences pour 27 pays identifierait en moyenne 1,4 cas $(0,05$ fois 27) de différences significatives entre sexes même s'il n'y avait aucune différence réelle dans un aucun pays. Ce principe s'applique aux autres données statistiques pour lesquelles des tests de signification ont été effectués dans cette publication, notamment les corrélations et les coefficients de régression. 


\section{ANNEXE}

B

TABLEAUX DE DONNÉES 
ANNEXE B

Tableau 4.1

Score moyen des élèves et écart type sur l'échelle combinée de compréhension de l'écrit

\begin{tabular}{|c|c|c|c|c|}
\hline & Score moyen & Er.T & Écart type & Er.T \\
\hline Australie & 528 & $(3.5)$ & 102 & $(1.6)$ \\
\hline Autriche & 507 & $(2.4)$ & 93 & (1.6) \\
\hline Belgique & 507 & $(3.6)$ & 107 & (2.4) \\
\hline Canada & 534 & $(1.6)$ & 95 & (1.1) \\
\hline République tchèque & 492 & $(2.4)$ & 96 & (1.9) \\
\hline Danemark & 497 & $(2.4)$ & 98 & (1.8) \\
\hline Finlande & 546 & $(2.6)$ & 89 & $(2.6)$ \\
\hline France & 505 & $(2.7)$ & 92 & (1.7) \\
\hline Allemagne & 484 & $(2.5)$ & 111 & (1.9) \\
\hline Grèce & 474 & $(5.0)$ & 97 & $(2.7)$ \\
\hline Hongrie & 480 & $(4.0)$ & 94 & (2.1) \\
\hline Islande & 507 & $(1.5)$ & 92 & (1.4) \\
\hline Irlande & 527 & $(3.2)$ & 94 & (1.7) \\
\hline Italie & 487 & $(2.9)$ & 91 & $(2.7)$ \\
\hline Japon & 522 & $(5.2)$ & 86 & (3.0) \\
\hline Corée & 525 & $(2.4)$ & 70 & (1.6) \\
\hline Luxembourg & 441 & $(1.6)$ & 100 & $(1.5)$ \\
\hline Mexique & 422 & $(3.3)$ & 86 & (2.1) \\
\hline Nouvelle-Zélande & 529 & $(2.8)$ & 108 & $(2.0)$ \\
\hline Norvège & 505 & $(2.8)$ & 104 & (1.7) \\
\hline Pologne & 479 & $(4.5)$ & 100 & $(3.1)$ \\
\hline Portugal & 470 & $(4.5)$ & 97 & $(1.8)$ \\
\hline Espagne & 493 & $(2.7)$ & 85 & $(1.2)$ \\
\hline Suède & 516 & $(2.2)$ & 92 & $(1.2)$ \\
\hline Suisse & 494 & $(4.3)$ & 102 & $(2.0)$ \\
\hline Royaume-Uni & 523 & $(2.6)$ & 100 & $(1.5)$ \\
\hline États-Unis & 504 & $(7.0)$ & 105 & (2.7) \\
\hline Moyenne de l'OCDE & 500 & $(0.6)$ & 100 & $(0.4)$ \\
\hline Brésil & 396 & $(3.1)$ & 86 & (1.9) \\
\hline Lettonie & 458 & $(5.3)$ & 102 & $(2.3)$ \\
\hline Liechtenstein & 483 & $(4.1)$ & 96 & (3.9) \\
\hline Fédération de Russie & 462 & $(4.2)$ & 92 & (1.8) \\
\hline
\end{tabular}


Tableau 4.2

Pourcentage d'élèves situés à chaque niveau de compétence sur l'échelle combinée de compréhension de l'écrit

\begin{tabular}{|c|c|c|c|c|c|c|c|c|c|c|c|c|}
\hline & \multicolumn{12}{|c|}{ Niveaux de compétence } \\
\hline & \multicolumn{2}{|c|}{$\begin{array}{c}\text { En deçà du niveau } 1 \\
\text { (moins de 335) }\end{array}$} & \multicolumn{2}{|c|}{$\begin{array}{c}\text { Niveau } 1 \\
\text { (entre } 335 \text { et 407) }\end{array}$} & \multicolumn{2}{|c|}{$\begin{array}{c}\text { Niveau } 2 \\
\text { (entre } 408 \text { et 480) }\end{array}$} & \multicolumn{2}{|c|}{$\begin{array}{c}\text { Niveau } 3 \\
\text { (entre } 481 \text { et } 552 \text { ) }\end{array}$} & \multicolumn{2}{|c|}{$\begin{array}{c}\text { Niveau } 4 \\
\text { (entre } 553 \text { et } 625 \text { ) }\end{array}$} & \multicolumn{2}{|c|}{$\begin{array}{l}\text { Niveau 5 } \\
\text { (plus de 625) }\end{array}$} \\
\hline & $\%$ & Er.T & $\%$ & Er.T & $\%$ & Er.T & $\%$ & Er.T & $\%$ & Er.T & $\%$ & Er.T \\
\hline Australie & 3.3 & $(0.5)$ & 9.1 & $(0.8)$ & 19.0 & $(1.1)$ & 25.7 & $(1.1)$ & 25.3 & $(0.9)$ & 17.6 & $(1.2)$ \\
\hline Autriche & 4.4 & $(0.4)$ & 10.2 & $(0.6)$ & 21.7 & $(0.9)$ & 29.9 & $(1.2)$ & 24.9 & $(1.0)$ & 8.8 & $(0.8)$ \\
\hline Belgique & 7.7 & $(1.0)$ & 11.3 & $(0.7)$ & 16.8 & $(0.7)$ & 25.8 & $(0.9)$ & 26.3 & $(0.9)$ & 12.0 & $(0.7)$ \\
\hline Canada & 2.4 & $(0.3)$ & 7.2 & $(0.3)$ & 18.0 & $(0.4)$ & 28.0 & $(0.5)$ & 27.7 & $(0.6)$ & 16.8 & $(0.5)$ \\
\hline Rép. tchèque & 6.1 & $(0.6)$ & 11.4 & $(0.7)$ & 24.8 & $(1.2)$ & 30.9 & $(1.1)$ & 19.8 & $(0.8)$ & 7.0 & $(0.6)$ \\
\hline Danemark & 5.9 & $(0.6)$ & 12.0 & $(0.7)$ & 22.5 & $(0.9)$ & 29.5 & $(1.0)$ & 22.0 & $(0.9)$ & 8.1 & $(0.5)$ \\
\hline Finlande & 1.7 & $(0.5)$ & 5.2 & $(0.4)$ & 14.3 & $(0.7)$ & 28.7 & $(0.8)$ & 31.6 & $(0.9)$ & 18.5 & $(0.9)$ \\
\hline France & 4.2 & $(0.6)$ & 11.0 & $(0.8)$ & 22.0 & $(0.8)$ & 30.6 & $(1.0)$ & 23.7 & $(0.9)$ & 8.5 & $(0.6)$ \\
\hline Allemagne & 9.9 & $(0.7)$ & 12.7 & $(0.6)$ & 22.3 & $(0.8)$ & 26.8 & $(1.0)$ & 19.4 & $(1.0)$ & 8.8 & $(0.5)$ \\
\hline Grèce & 8.7 & $(1.2)$ & 15.7 & $(1.4)$ & 25.9 & $(1.4)$ & 28.1 & $(1.7)$ & 16.7 & $(1.4)$ & 5.0 & $(0.7)$ \\
\hline Hongrie & 6.9 & $(0.7)$ & 15.8 & $(1.2)$ & 25.0 & $(1.1)$ & 28.8 & $(1.3)$ & 18.5 & $(1.1)$ & 5.1 & $(0.8)$ \\
\hline Islande & 4.0 & $(0.3)$ & 10.5 & $(0.6)$ & 22.0 & $(0.8)$ & 30.8 & $(0.9)$ & 23.6 & $(1.1)$ & 9.1 & $(0.7)$ \\
\hline Irlande & 3.1 & $(0.5)$ & 7.9 & $(0.8)$ & 17.9 & $(0.9)$ & 29.7 & $(1.1)$ & 27.1 & $(1.1)$ & 14.2 & $(0.8)$ \\
\hline Italie & 5.4 & $(0.9)$ & 13.5 & $(0.9)$ & 25.6 & $(1.0)$ & 30.6 & $(1.0)$ & 19.5 & $(1.1)$ & 5.3 & $(0.5)$ \\
\hline Japon & 2.7 & $(0.6)$ & 7.3 & $(1.1)$ & 18.0 & $(1.3)$ & 33.3 & $(1.3)$ & 28.8 & $(1.7)$ & 9.9 & (1.1) \\
\hline Corée & 0.9 & $(0.2)$ & 4.8 & $(0.6)$ & 18.6 & $(0.9)$ & 38.8 & $(1.1)$ & 31.1 & $(1.2)$ & 5.7 & $(0.6)$ \\
\hline Luxembourg & 14.2 & $(0.7)$ & 20.9 & $(0.8)$ & 27.5 & $(1.3)$ & 24.6 & $(1.1)$ & 11.2 & $(0.5)$ & 1.7 & $(0.3)$ \\
\hline Mexique & 16.1 & $(1.2)$ & 28.1 & $(1.4)$ & 30.3 & $(1.1)$ & 18.8 & $(1.2)$ & 6.0 & $(0.7)$ & 0.9 & $(0.2)$ \\
\hline Nouvelle-Zélande & 4.8 & $(0.5)$ & 8.9 & $(0.5)$ & 17.2 & $(0.9)$ & 24.6 & $(1.1)$ & 25.8 & $(1.1)$ & 18.7 & (1.0) \\
\hline Norvège & 6.3 & $(0.6)$ & 11.2 & $(0.8)$ & 19.5 & $(0.8)$ & 28.1 & $(0.8)$ & 23.7 & $(0.9)$ & 11.2 & $(0.7)$ \\
\hline Pologne & 8.7 & $(1.0)$ & 14.6 & $(1.0)$ & 24.1 & $(1.4)$ & 28.2 & $(1.3)$ & 18.6 & $(1.3)$ & 5.9 & $(1.0)$ \\
\hline Portugal & 9.6 & $(1.0)$ & 16.7 & $(1.2)$ & 25.3 & $(1.0)$ & 27.5 & $(1.2)$ & 16.8 & $(1.1)$ & 4.2 & $(0.5)$ \\
\hline Espagne & 4.1 & $(0.5)$ & 12.2 & $(0.9)$ & 25.7 & $(0.7)$ & 32.8 & $(1.0)$ & 21.1 & $(0.9)$ & 4.2 & $(0.5)$ \\
\hline Suède & 3.3 & $(0.4)$ & 9.3 & $(0.6)$ & 20.3 & $(0.7)$ & 30.4 & $(1.0)$ & 25.6 & $(1.0)$ & 11.2 & $(0.7)$ \\
\hline Suisse & 7.0 & $(0.7)$ & 13.3 & $(0.9)$ & 21.4 & $(1.0)$ & 28.0 & $(1.0)$ & 21.0 & $(1.0)$ & 9.2 & (1.0) \\
\hline Royaume-Uni & 3.6 & $(0.4)$ & 9.2 & $(0.5)$ & 19.6 & $(0.7)$ & 27.5 & $(0.9)$ & 24.4 & $(0.9)$ & 15.6 & (1.0) \\
\hline États-Unis & 6.4 & $(1.2)$ & 11.5 & $(1.2)$ & 21.0 & $(1.2)$ & 27.4 & $(1.3)$ & 21.5 & $(1.4)$ & 12.2 & (1.4) \\
\hline Moyenne de l'OCDE & 6.0 & $(0.1)$ & 11.9 & $(0.2)$ & 21.7 & $(0.2)$ & 28.7 & $(0.2)$ & 22.3 & $(0.2)$ & 9.5 & $(0.1)$ \\
\hline Brésil & 23.3 & $(1.4)$ & 32.5 & $(1.2)$ & 27.7 & $(1.3)$ & 12.9 & $(1.1)$ & 3.1 & $(0.5)$ & 0.6 & $(0.2)$ \\
\hline Lettonie & 12.7 & $(1.3)$ & 17.9 & $(1.3)$ & 26.3 & $(1.1)$ & 25.2 & $(1.3)$ & 13.8 & $(1.1)$ & 4.1 & $(0.6)$ \\
\hline Liechtenstein & 7.6 & $(1.5)$ & 14.5 & $(2.1)$ & 23.2 & $(2.9)$ & 30.1 & $(3.4)$ & 19.5 & $(2.2)$ & 5.1 & $(1.6)$ \\
\hline Fédération de Russie & 9.0 & $(1.0)$ & 18.5 & $(1.1)$ & 29.2 & $(0.8)$ & 26.9 & $(1.1)$ & 13.3 & $(1.0)$ & 3.2 & $(0.5)$ \\
\hline
\end{tabular}


Tableau 4.3

Scores moyens sur les sous-échelles d'aspect par pays

\begin{tabular}{|c|c|c|c|c|c|c|c|c|c|c|c|c|}
\hline & \multicolumn{4}{|c|}{ Trouver l'information } & \multicolumn{4}{|c|}{ Développer une interprétation } & \multicolumn{4}{|c|}{ Réfléchir et évaluer } \\
\hline & $\begin{array}{c}\text { Score } \\
\text { moyen }\end{array}$ & Er.T & Éc.T. & Er.T & $\begin{array}{c}\text { Score } \\
\text { moyen }\end{array}$ & Er.T & Éc.T. & Er.T & $\begin{array}{c}\text { Score } \\
\text { moyen }\end{array}$ & Er.T & Éc.T. & Er.T \\
\hline Australie & 536 & $(3.7)$ & 108 & $(1.6)$ & 527 & (3.5) & 104 & $(1.5)$ & 526 & $(3.5)$ & 100 & $(1.5)$ \\
\hline Autriche & 502 & $(2.3)$ & 96 & $(1.5)$ & 508 & (2.4) & 93 & $(1.6)$ & 512 & $(2.7)$ & 100 & $(1.8)$ \\
\hline Belgique & 515 & $(3.9)$ & 120 & $(2.7)$ & 512 & $(3.2)$ & 105 & $(2.0)$ & 497 & $(4.3)$ & 114 & $(4.1)$ \\
\hline Canada & 530 & $(1.7)$ & 102 & $(1.2)$ & 532 & $(1.6)$ & 95 & $(1.0)$ & 542 & $(1.6)$ & 96 & $(1.0)$ \\
\hline République tchèque & 481 & $(2.7)$ & 107 & $(1.9)$ & 500 & (2.4) & 96 & $(1.6)$ & 485 & $(2.6)$ & 103 & (1.8) \\
\hline Danemark & 498 & $(2.8)$ & 105 & (1.9) & 494 & (2.4) & 99 & $(1.7)$ & 500 & $(2.6)$ & 102 & (2.1) \\
\hline Finlande & 556 & $(2.8)$ & 102 & $(2.1)$ & 555 & (2.9) & 97 & (3.3) & 533 & $(2.7)$ & 91 & (3.9) \\
\hline France & 515 & $(3.0)$ & 101 & $(2.1)$ & 506 & (2.7) & 92 & $(1.7)$ & 496 & $(2.9)$ & 98 & (1.8) \\
\hline Allemagne & 483 & $(2.4)$ & 114 & $(2.0)$ & 488 & (2.5) & 109 & $(1.8)$ & 478 & $(2.9)$ & 124 & (1.8) \\
\hline Grèce & 450 & $(5.4)$ & 109 & $(3.0)$ & 475 & $(4.5)$ & 89 & $(2.4)$ & 495 & $(5.6)$ & 115 & (3.1) \\
\hline Hongrie & 478 & $(4.4)$ & 107 & $(2.2)$ & 480 & (3.8) & 90 & (1.9) & 481 & $(4.3)$ & 100 & (2.2) \\
\hline Islande & 500 & $(1.6)$ & 103 & $(1.3)$ & 514 & (1.4) & 95 & $(1.4)$ & 501 & $(1.3)$ & 93 & (1.3) \\
\hline Irlande & 524 & (3.3) & 100 & $(1.7)$ & 526 & (3.3) & 97 & $(1.7)$ & 533 & (3.1) & 90 & (1.7) \\
\hline Italie & 488 & $(3.1)$ & 104 & $(3.0)$ & 489 & (2.6) & 86 & $(2.4)$ & 483 & $(3.1)$ & 101 & (2.9) \\
\hline Japon & 526 & $(5.5)$ & 97 & $(3.1)$ & 518 & $(5.0)$ & 83 & $(2.9)$ & 530 & $(5.5)$ & 100 & (3.3) \\
\hline Corée & 530 & $(2.5)$ & 82 & $(1.6)$ & 525 & (2.3) & 69 & $(1.5)$ & 526 & (2.6) & 76 & (1.7) \\
\hline Luxembourg & 433 & $(1.6)$ & 109 & $(1.4)$ & 446 & (1.6) & 101 & $(1.3)$ & 442 & $(1.9)$ & 115 & (1.8) \\
\hline Mexique & 402 & (3.9) & 101 & $(2.2)$ & 419 & (2.9) & 78 & $(1.7)$ & 446 & $(3.7)$ & 109 & (2.2) \\
\hline Nouvelle-Zélande & 535 & $(2.8)$ & 116 & $(2.1)$ & 526 & $(2.7)$ & 111 & $(2.0)$ & 529 & $(2.9)$ & 107 & (1.8) \\
\hline Norvège & 505 & $(2.9)$ & 110 & (1.9) & 505 & $(2.8)$ & 104 & $(1.6)$ & 506 & $(3.0)$ & 108 & (1.8) \\
\hline Pologne & 475 & $(5.0)$ & 112 & (3.3) & 482 & $(4.3)$ & 97 & $(2.7)$ & 477 & $(4.7)$ & 110 & (3.2) \\
\hline Portugal & 455 & $(4.9)$ & 107 & $(2.2)$ & 473 & (4.3) & 93 & $(1.6)$ & 480 & $(4.5)$ & 101 & (1.7) \\
\hline Espagne & 483 & $(3.0)$ & 92 & $(1.2)$ & 491 & (2.6) & 84 & $(1.1)$ & 506 & $(2.8)$ & 91 & $(1.2)$ \\
\hline Suède & 516 & $(2.4)$ & 104 & $(1.5)$ & 522 & $(2.1)$ & 96 & $(1.3)$ & 510 & $(2.3)$ & 95 & $(1.2)$ \\
\hline Suisse & 498 & $(4.4)$ & 113 & $(2.1)$ & 496 & $(4.2)$ & 101 & $(2.0)$ & 488 & $(4.8)$ & 113 & $(2.2)$ \\
\hline Royaume-Uni & 523 & $(2.5)$ & 105 & $(1.5)$ & 514 & $(2.5)$ & 102 & $(1.4)$ & 539 & $(2.5)$ & 99 & (1.6) \\
\hline États-Unis & 499 & $(7.4)$ & 112 & $(2.7)$ & 505 & $(7.1)$ & 106 & $(2.6)$ & 507 & $(7.1)$ & 105 & $(2.7)$ \\
\hline Moyenne de l'OCDE & 498 & $(0.7)$ & 111 & $(0.4)$ & 501 & $(0.6)$ & 100 & $(0.4)$ & 502 & $(0.7)$ & 106 & $(0.4)$ \\
\hline Brésil & 365 & $(3.4)$ & 97 & $(2.1)$ & 400 & $(3.0)$ & 84 & $(1.8)$ & 417 & $(3.3)$ & 93 & $(2.2)$ \\
\hline Lettonie & 451 & $(5.7)$ & 117 & $(2.4)$ & 459 & $(4.9)$ & 95 & $(2.0)$ & 458 & $(5.9)$ & 113 & (2.3) \\
\hline Liechtenstein & 492 & $(4.9)$ & 106 & $(4.7)$ & 484 & $(4.5)$ & 94 & $(3.6)$ & 468 & $(5.7)$ & 108 & $(4.3)$ \\
\hline Fédération de Russie & 451 & $(4.9)$ & 108 & $(2.1)$ & 468 & $(4.0)$ & 92 & $(1.8)$ & 455 & $(4.0)$ & 98 & (1.7) \\
\hline
\end{tabular}


Tableau 4.4

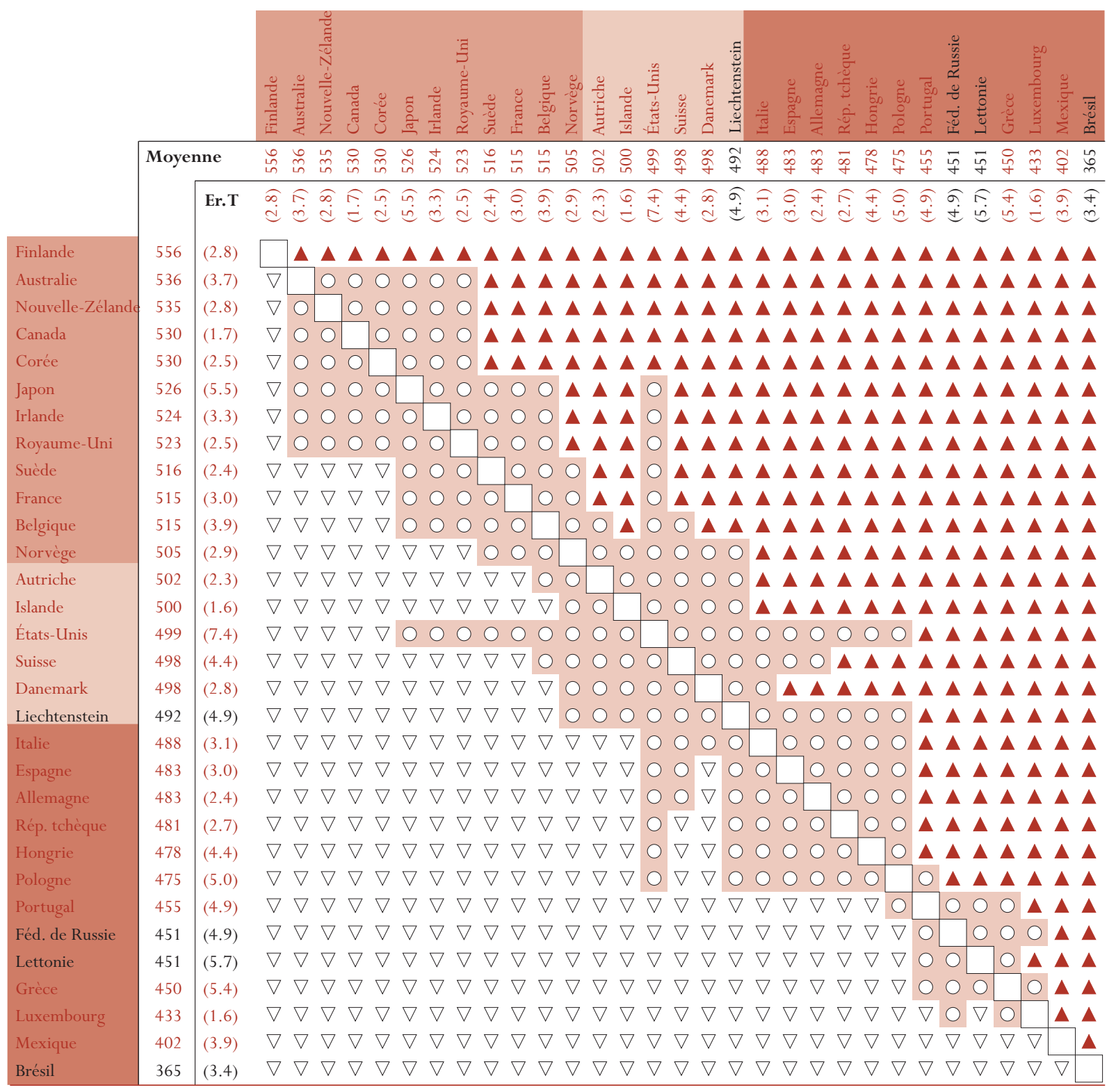

Instructions

Pour procéder à la comparaison, il suffit de choisir un pays en abscisse et de le comparer avec l'un des pays en ordonnée. Les symboles indiquent que statistiquement, la performance moyenne du pays en abscisse est significativement inférieure ou supérieure à celle du pays en ordonnée ou ne s'en écarte pas significativement.

- Performance moyenne significativement supérieure sur le plan statistique à celle du pays en ordonnée

Pas d'écart statistiquement significatif par rapport au pays en ordonnée

$\nabla$ Performance moyenne significativement inférieure sur le plan statistique à celle du pays en ordonnée

Significativement supérieure sur le plan statistique à la moyenne de l'OCDE

Pas d'écart statistiquement significatif par rapport à la moyenne de l'OCDE

Significativement inférieure sur le plan statistique à la moyenne de l'OCDE 




Instructions

Pour procéder à la comparaison, il suffit de choisir un pays en abscisse et de le comparer avec l'un des pays en ordonnée. Les symboles indiquent que statistiquement, la performance moyenne du pays en abscisse est significativement inférieure ou supérieure à celle du pays en ordonnée ou ne s'en écarte pas significativement.

A Performance moyenne significativement supérieure sur le plan statistique à celle du pays en ordonnée

Pas d'écart statistiquement significatif par rapport au pays en ordonnée

$\nabla$ Performance moyenne significativement inférieure sur le plan statistique à celle du pays en ordonnée

Significativement supérieure sur le plan statistique à la moyenne de l'OCDE Pas d'écart statistiquement significatif par rapport à la moyenne de l'OCDE Significativement inférieure sur le plan statistique à la moyenne de l'OCDE 


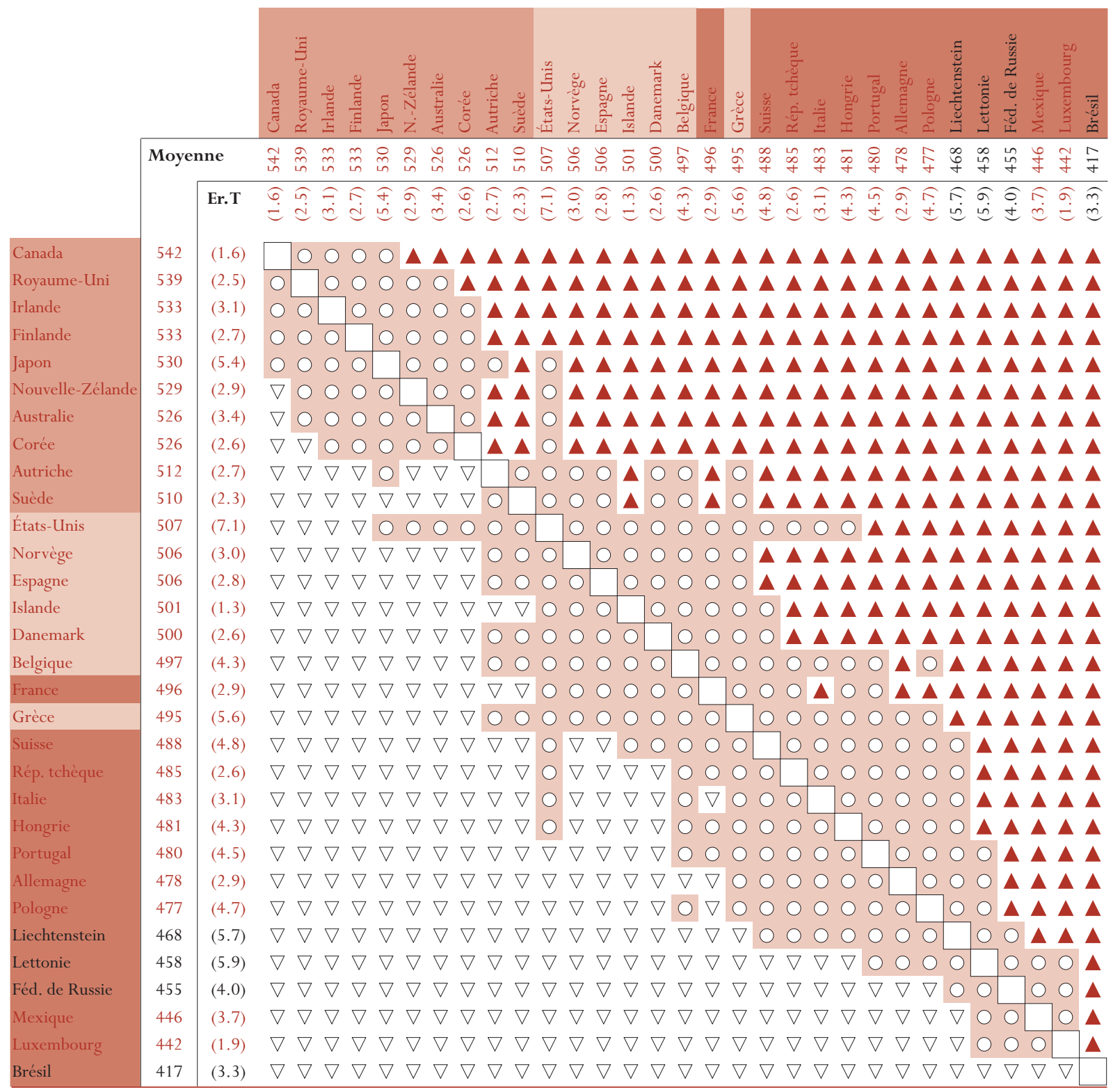

Instructions

Pour procéder à la comparaison, il suffit de choisir un pays en abscisse et de le comparer avec l'un des pays en ordonnée. Les symboles indiquent que statistiquement, la performance moyenne du pays en abscisse est significativement inférieure ou supérieure à celle du pays en ordonnée ou ne s'en écarte pas significativement.

A Performance moyenne significativement supérieure sur le plan statistique à celle du pays en ordonnée

Pas d'écart statistiquement significatif par rapport au pays en ordonnée

$\nabla$ Performance moyenne significativement inférieure sur le plan statistique à celle du pays en ordonnée

Significativement supérieure sur le plan statistique à la moyenne de l'OCDE Pas d'écart statistiquement significatif par rapport à la moyenne de l'OCDE Significativement inférieure sur le plan statistique à la moyenne de l'OCDE 
Tableau 4.7

Pourcentage d'élèves situés à chaque niveau de compétence sur la sous-échelle «Trouver l'information »

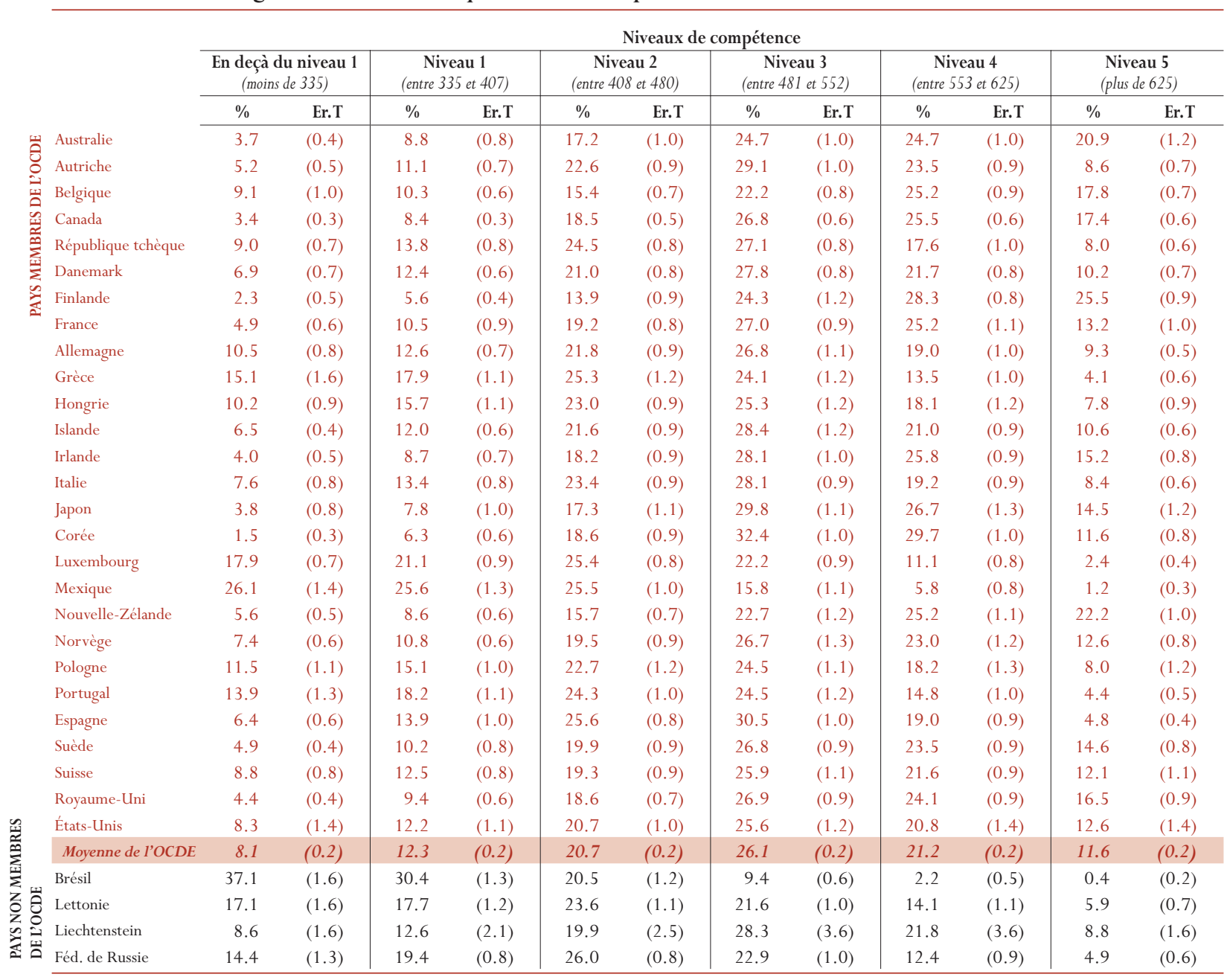


Tableau 4.8

Pourcentage d'élèves situés à chaque niveau de compétence sur la sous-échelle « Développer une interprétation »

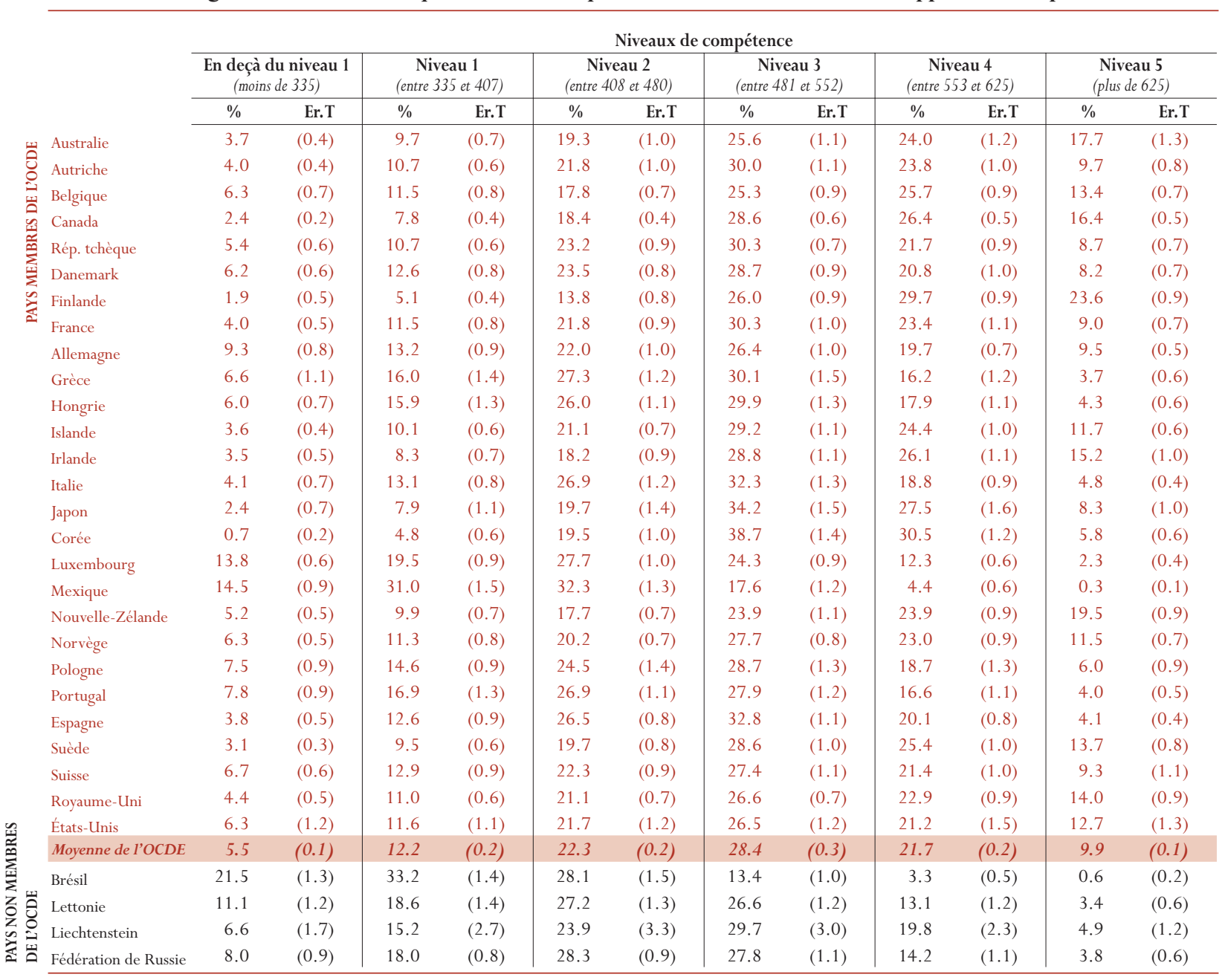


Tableau 4.9

Pourcentage d'élèves situés à chaque niveau de compétence sur la sous-échelle « Réfléchir et évaluer»

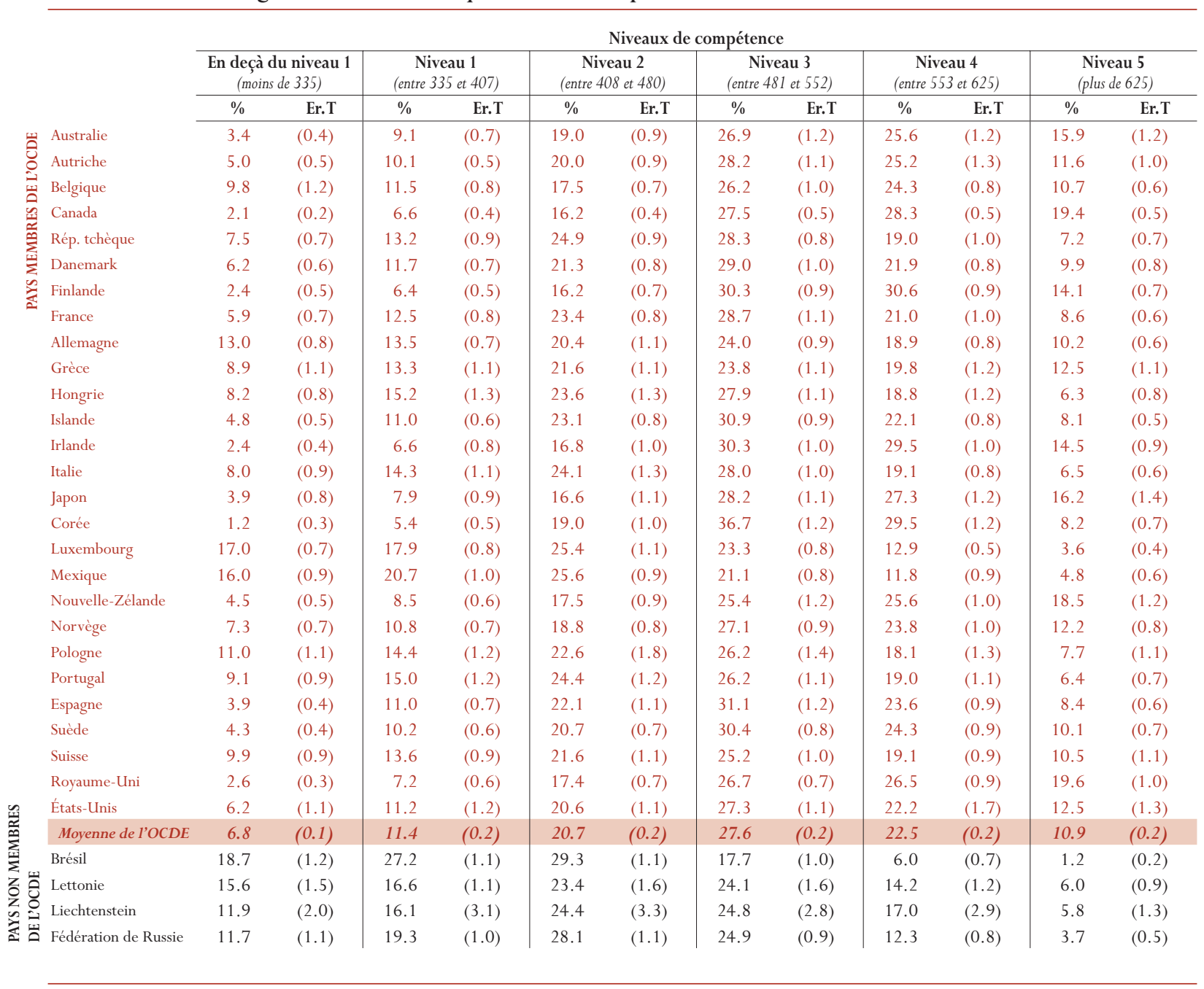


Tableau 4.10

Scores moyens sur les sous-échelles par format de texte

\begin{tabular}{|c|c|c|c|c|c|c|c|c|}
\hline & \multicolumn{4}{|c|}{ Textes continus } & \multicolumn{4}{|c|}{ Textes non continus } \\
\hline & Score moyen & Er.T & Éc.T. & Er.T & Score moyen & Er.T & Éc.T. & Er.T \\
\hline Australie & 526 & (3.5) & 104 & (1.6) & 539 & 3.3 & 104 & (1.6) \\
\hline Autriche & 509 & $(2.5)$ & 94 & (1.5) & 507 & 2.4 & 99 & (1.5) \\
\hline Belgique & 503 & $(4.0)$ & 108 & (3.5) & 516 & 3.5 & 114 & (2.4) \\
\hline Canada & 536 & (1.6) & 98 & (1.1) & 536 & 1.6 & 99 & (1.1) \\
\hline Rép. tchèque & 489 & $(2.3)$ & 93 & (1.8) & 498 & 2.9 & 112 & (2.3) \\
\hline Danemark & 497 & (2.4) & 99 & (1.9) & 499 & 2.6 & 109 & (1.8) \\
\hline Finlande & 544 & (2.7) & 90 & $(2.8)$ & 554 & 2.7 & 95 & $(2.0)$ \\
\hline France & 500 & $(2.8)$ & 94 & $(1.8)$ & 518 & 2.7 & 97 & (1.8) \\
\hline Allemagne & 484 & $(2.6)$ & 115 & (1.9) & 486 & 2.4 & 113 & $(2.2)$ \\
\hline Grèce & 483 & $(5.0)$ & 99 & (2.8) & 456 & 4.9 & 103 & (2.5) \\
\hline Hongrie & 481 & (3.9) & 92 & (1.9) & 479 & 4.4 & 108 & (2.1) \\
\hline Islande & 509 & (1.4) & 93 & $(1.2)$ & 505 & 1.5 & 100 & (1.4) \\
\hline Irlande & 528 & (3.2) & 94 & (1.7) & 530 & 3.3 & 100 & (1.8) \\
\hline Italie & 491 & (2.8) & 91 & (1.5) & 480 & 3.0 & 100 & $(2.8)$ \\
\hline Japon & 524 & (5.1) & 85 & (2.9) & 521 & 5.6 & 98 & (3.2) \\
\hline Corée & 530 & (2.4) & 69 & (1.8) & 517 & 2.5 & 81 & (1.7) \\
\hline Luxembourg & 442 & (1.7) & 108 & (1.3) & 441 & 1.6 & 113 & (1.5) \\
\hline Mexique & 431 & (3.2) & 86 & (1.8) & 401 & 3.7 & 97 & (2.4) \\
\hline Nouvelle-Zélande & 526 & (2.9) & 110 & (1.7) & 539 & 2.9 & 110 & $(1.9)$ \\
\hline Norvège & 506 & $(2.8)$ & 103 & (1.6) & 508 & 2.8 & 114 & (1.7) \\
\hline Pologne & 482 & (4.4) & 101 & (2.9) & 473 & 4.8 & 107 & (3.2) \\
\hline Portugal & 474 & $(4.5)$ & 98 & (1.8) & 460 & 4.5 & 102 & $(1.8)$ \\
\hline Espagne & 493 & $(2.6)$ & 84 & (1.1) & 493 & 3.0 & 96 & $(1.3)$ \\
\hline Suède & 516 & $(2.2)$ & 93 & $(1.2)$ & 521 & 2.4 & 100 & (1.3) \\
\hline Suisse & 494 & $(4.3)$ & 104 & $(2.0)$ & 496 & 4.4 & 109 & $(2.0)$ \\
\hline Royaume-Uni & 521 & $(2.5)$ & 104 & $(1.5)$ & 533 & 2.4 & 101 & $(1.5)$ \\
\hline États-Unis & 504 & (7.1) & 105 & $(2.5)$ & 506 & 7.2 & 109 & $(2.8)$ \\
\hline $\begin{array}{l}\text { Moyenne de } \\
\text { l'OCDE }\end{array}$ & 501 & $(0.6)$ & 101 & $(0.4)$ & 500 & 0.7 & 109 & (0.4) \\
\hline Brésil & 408 & (3.1) & 88 & $(1.8)$ & 366 & 3.4 & 97 & $(1.9)$ \\
\hline Lettonie & 462 & $(5.2)$ & 104 & $(2.3)$ & 447 & 5.3 & 109 & $(2.5)$ \\
\hline Liechtenstein & 483 & $(4.5)$ & 97 & $(4.0)$ & 482 & 4.3 & 99 & (3.9) \\
\hline Féd. de Russie & 465 & $(4.1)$ & 92 & (1.8) & 450 & 4.5 & 102 & $(2.0)$ \\
\hline
\end{tabular}




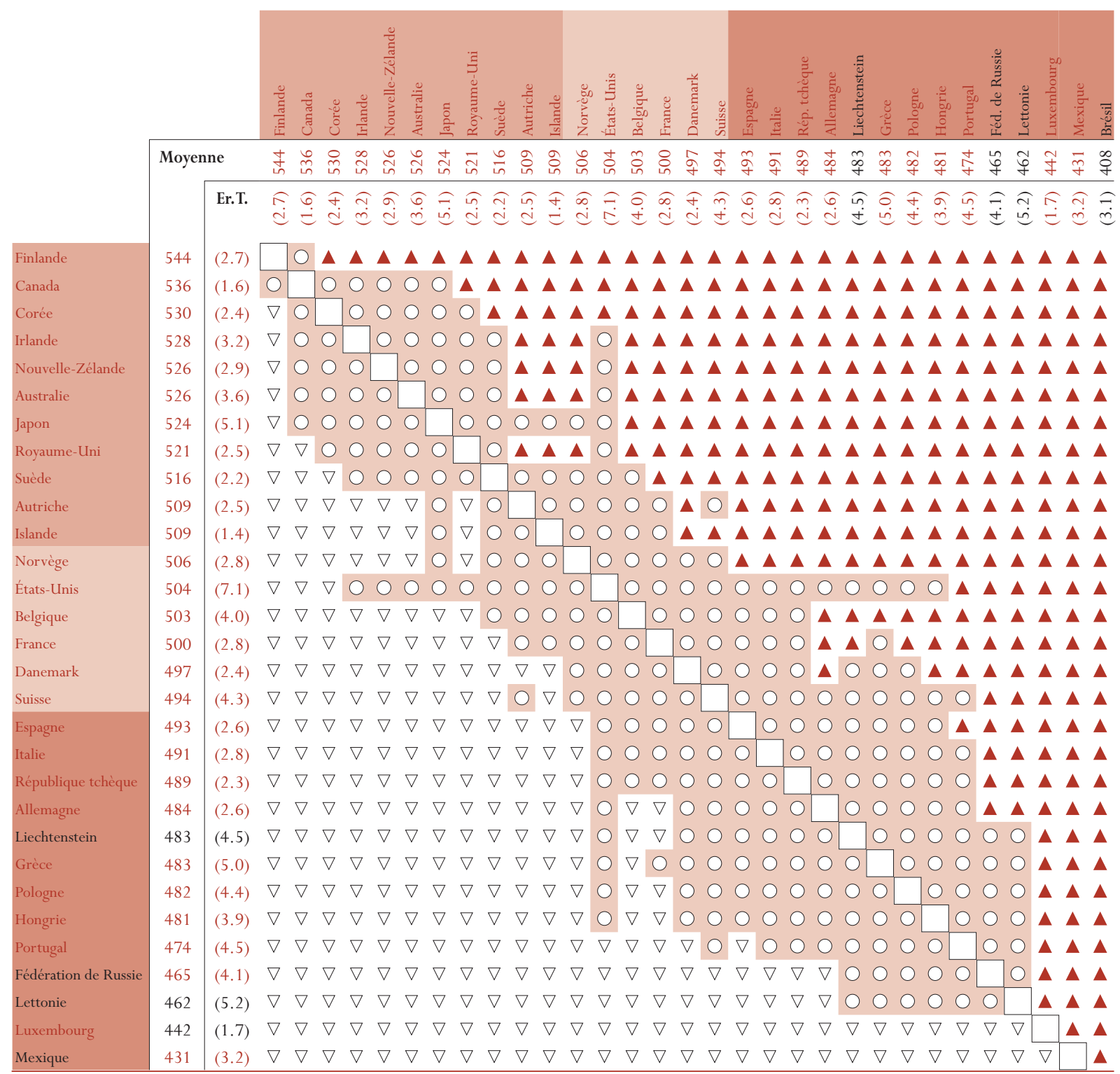

Instructions

Pour procéder à la comparaison, il suffit de choisir un pays en abscisse et de le comparer avec l'un des pays en ordonnée. Les symboles indiquent que statistiquement, la performance moyenne du pays en abscisse est significativement inférieure ou supérieure à celle du pays en ordonnée ou ne s'en écarte pas significativement.

$\Delta$ Performance moyenne significativement supérieure sur le plan statistique à celle du pays en ordonnée

Pas d'écart statistiquement significatif par rapport au pays en ordonnée

$\nabla$ Performance moyenne significativement inférieure sur le plan statistique à celle du pays en ordonnée

Significativement supérieure sur le plan statistique à la moyenne de l'OCDE Pas d'écart statistiquement significatif par rapport à la moyenne de l'OCDE Significativement inférieure sur le plan statistique à la moyenne de l'OCDE 


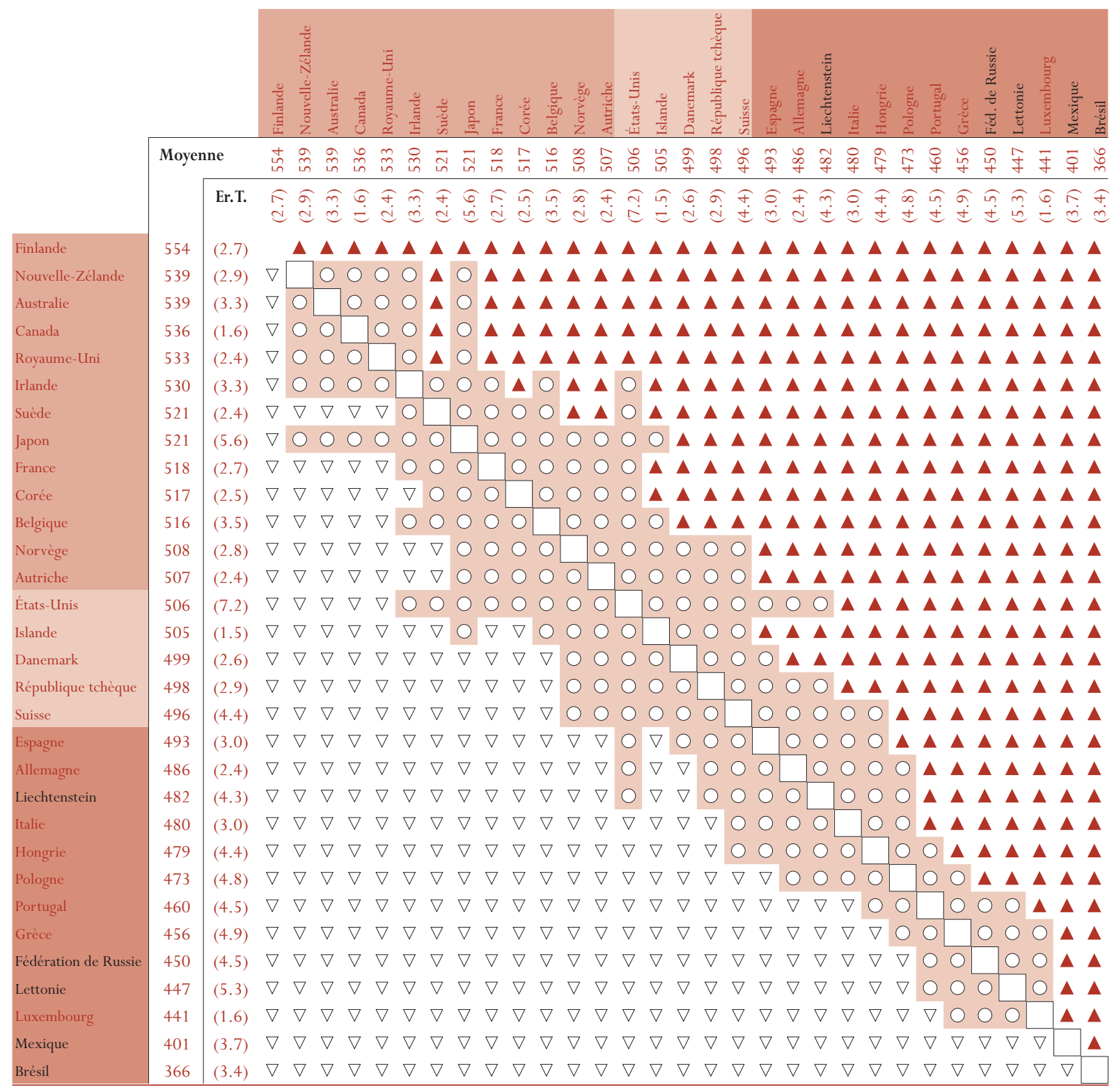

Instructions

Pour procéder à la comparaison, il suffit de choisir un pays en abscisse et de le comparer avec l'un des pays en ordonnée. Les symboles indiquent que statistiquement, la performance moyenne du pays en abscisse est significativement inférieure ou supérieure à celle du pays en ordonnée ou ne s'en écarte pas significativement.

$\Delta$ Performance moyenne significativement supérieure sur le plan statistique à celle du pays en ordonnée
$\bigcirc$ Pas d'écart statistiquement significatif par rapport au pays en ordonnée
$\nabla$ Performance moyenne significativement inférieure sur le plan statistique à celle du pays en ordonnée

Significativement supérieure sur le plan statistique à la moyenne de l'OCDE Pas d'écart statistiquement significatif par rapport à la moyenne de l'OCDE Significativement inférieure sur le plan statistique à la moyenne de l'OCDE 
Tableau 4.13

Pourcentage d'élèves situés à chaque niveau de compétence sur la sous-échelle «Textes continus »

\begin{tabular}{|c|c|c|c|c|c|c|c|c|c|c|c|c|}
\hline & \multicolumn{12}{|c|}{ Niveaux de compétence } \\
\hline & \multicolumn{2}{|c|}{$\begin{array}{c}\text { En deçà du niveau } 1 \\
\text { (moins de 335) }\end{array}$} & \multicolumn{2}{|c|}{$\begin{array}{c}\text { Niveau } 1 \\
\text { (entre } 335 \text { et } 407 \text { ) }\end{array}$} & \multicolumn{2}{|c|}{$\begin{array}{c}\text { Niveau } 2 \\
\text { (entre } 408 \text { et } 480 \text { ) }\end{array}$} & \multicolumn{2}{|c|}{$\begin{array}{c}\text { Niveau } 3 \\
\text { (entre } 481 \text { et } 552 \text { ) }\end{array}$} & \multicolumn{2}{|c|}{$\begin{array}{c}\text { Niveau } 4 \\
\text { (entre } 553 \text { et } 626 \text { ) }\end{array}$} & \multicolumn{2}{|c|}{$\begin{array}{l}\text { Niveau } 5 \\
\text { (plus de 626) }\end{array}$} \\
\hline & $\%$ & Er.T & $\%$ & Er.T & $\%$ & Er.T & $\%$ & Er.T & $\%$ & Er.T & $\%$ & Er.T \\
\hline Australie & 3.8 & $(0.4)$ & 9.8 & 0.8 & 19.0 & 1.1 & 25.7 & 1.0 & 24.2 & 0.9 & 17.5 & 1.1 \\
\hline Autriche & 4.2 & $(0.4)$ & 10.3 & 0.7 & 21.0 & 0.9 & 29.9 & 0.9 & 25.1 & 1.0 & 9.5 & 0.8 \\
\hline Belgique & 8.1 & $(1.1)$ & 11.6 & 0.8 & 17.6 & 0.9 & 25.6 & 1.0 & 25.6 & 0.9 & 11.5 & 0.6 \\
\hline Canada & 2.5 & $(0.2)$ & 7.5 & 0.3 & 17.4 & 0.4 & 27.9 & 0.6 & 27.0 & 0.5 & 17.6 & 0.5 \\
\hline Rép. tchèque & 6.1 & $(0.6)$ & 11.8 & 0.8 & 25.7 & 1.0 & 31.0 & 1.0 & 19.4 & 1.0 & 6.0 & 0.5 \\
\hline Danemark & 6.2 & $(0.6)$ & 12.2 & 0.7 & 22.3 & 1.0 & 29.6 & 1.1 & 21.2 & 1.0 & 8.6 & 0.6 \\
\hline Finlande & 1.7 & $(0.5)$ & 5.4 & 0.5 & 14.8 & 0.7 & 28.7 & 0.9 & 31.5 & 0.9 & 17.8 & 0.9 \\
\hline France & 5.0 & $(0.7)$ & 12.4 & 0.8 & 22.1 & 0.9 & 29.8 & 1.2 & 22.7 & 1.0 & 8.0 & 0.6 \\
\hline Allemagne & 10.5 & $(0.8)$ & 12.9 & 0.7 & 21.5 & 1.0 & 26.0 & 1.1 & 19.3 & 0.9 & 9.8 & 0.6 \\
\hline Grèce & 7.8 & $(1.2)$ & 14.3 & 1.4 & 25.0 & 1.4 & 27.9 & 1.4 & 18.4 & 1.2 & 6.7 & 0.8 \\
\hline Hongrie & 6.3 & $(0.8)$ & 16.0 & 1.2 & 25.3 & 1.1 & 29.3 & 1.4 & 18.4 & 1.1 & 4.7 & 0.7 \\
\hline Islande & 4.2 & $(0.4)$ & 10.3 & 0.6 & 21.9 & 0.8 & 30.1 & 1.0 & 23.7 & 0.9 & 9.9 & 0.6 \\
\hline Irlande & 3.0 & $(0.5)$ & 8.2 & 0.8 & 17.5 & 0.8 & 29.2 & 0.9 & 27.7 & 1.0 & 14.4 & 0.9 \\
\hline Italie & 5.0 & $(0.8)$ & 12.8 & 0.8 & 25.2 & 1.2 & 31.1 & 1.1 & 19.9 & 0.8 & 6.0 & 0.5 \\
\hline Japon & 2.6 & $(0.6)$ & 7.2 & 1.1 & 17.8 & 1.3 & 33.4 & 1.3 & 28.7 & 1.6 & 10.3 & 1.3 \\
\hline Corée & 0.7 & $(0.2)$ & 4.0 & 0.5 & 17.5 & 0.9 & 38.4 & 1.5 & 32.9 & 1.4 & 6.6 & 0.7 \\
\hline Luxembourg & 17.0 & $(0.7)$ & 19.2 & 0.8 & 25.2 & 0.9 & 23.1 & 0.9 & 12.6 & 0.6 & 3.0 & 0.3 \\
\hline Mexique & 13.9 & $(0.9)$ & 26.4 & 1.2 & 30.6 & 1.3 & 20.6 & 1.2 & 7.5 & 0.8 & 1.0 & 0.3 \\
\hline Nouvelle-Zélande & 5.1 & $(0.5)$ & 9.6 & 0.6 & 17.9 & 1.0 & 24.3 & 1.0 & 24.7 & 0.9 & 18.5 & 1.0 \\
\hline Norvège & 6.5 & $(0.6)$ & 10.7 & 0.7 & 19.9 & 0.8 & 27.9 & 0.8 & 23.9 & 1.1 & 11.2 & 0.9 \\
\hline Pologne & 8.7 & $(1.1)$ & 14.5 & 1.1 & 23.3 & 1.2 & 28.2 & 1.3 & 18.7 & 1.3 & 6.7 & 1.0 \\
\hline Portugal & 9.0 & $(1.0)$ & 16.3 & 1.2 & 24.8 & 1.0 & 27.0 & 1.0 & 18.0 & 1.1 & 5.0 & 0.6 \\
\hline Espagne & 3.7 & $(0.4)$ & 12.1 & 0.8 & 25.2 & 0.8 & 33.7 & 0.8 & 20.9 & 0.9 & 4.3 & 0.4 \\
\hline Suède & 3.5 & $(0.4)$ & 9.5 & 0.6 & 20.1 & 0.9 & 30.1 & 1.0 & 25.2 & 1.0 & 11.5 & 0.7 \\
\hline Suisse & 7.4 & $(0.7)$ & 13.6 & 1.0 & 21.5 & 1.2 & 27.3 & 1.2 & 20.8 & 1.0 & 9.5 & 1.1 \\
\hline Royaume-Uni & 4.2 & $(0.4)$ & 10.0 & 0.6 & 19.4 & 0.7 & 26.9 & 0.9 & 23.7 & 0.8 & 15.9 & 0.8 \\
\hline États-Unis & 6.4 & $(1.2)$ & 11.9 & 1.2 & 21.0 & 1.0 & 27.1 & 1.2 & 21.5 & 1.6 & 12.1 & 1.4 \\
\hline Moyenne de l'OCDE & 6.0 & $(0.1)$ & 11.9 & 0.2 & 21.5 & 0.2 & 28.5 & 0.2 & 22.3 & 0.0 & 9.8 & 0.1 \\
\hline Brésil & 20.3 & $(1.3)$ & 30.1 & 1.1 & 28.8 & 1.1 & 15.5 & 1.0 & 4.7 & 0.7 & 0.6 & 0.2 \\
\hline Lettonie & 11.9 & $(1.3)$ & 16.9 & 1.3 & 26.1 & 1.0 & 25.6 & 1.0 & 14.5 & 1.1 & 5.0 & 0.8 \\
\hline Liechtenstein & 8.2 & $(1.6)$ & 14.8 & 2.2 & 21.7 & 3.1 & 30.2 & 3.1 & 19.9 & 2.1 & 5.2 & 1.3 \\
\hline Fédération de Russie & 8.5 & $(0.9)$ & 17.6 & 0.9 & 29.0 & 1.0 & 27.7 & 1.0 & 13.9 & 1.0 & 3.5 & 0.5 \\
\hline
\end{tabular}


Tableau 4.14

Pourcentage d'élèves situés à chaque niveau de compétence sur la sous-échelle «Textes non continus »

\begin{tabular}{|c|c|c|c|c|c|c|c|c|c|c|c|c|}
\hline & \multicolumn{12}{|c|}{ Niveaux de compétence } \\
\hline & \multicolumn{2}{|c|}{$\begin{array}{c}\text { En deçà du niveau } 1 \\
\text { (moins de 335) }\end{array}$} & \multicolumn{2}{|c|}{$\begin{array}{c}\text { Niveau } 1 \\
\text { (entre } 335 \text { et } 407 \text { ) }\end{array}$} & \multicolumn{2}{|c|}{$\begin{array}{c}\text { Niveau } 2 \\
\text { (entre } 408 \text { et 480) }\end{array}$} & \multicolumn{2}{|c|}{$\begin{array}{c}\text { Niveau } 3 \\
\text { (entre } 481 \text { et } 552 \text { ) }\end{array}$} & \multicolumn{2}{|c|}{$\begin{array}{c}\text { Niveau } 4 \\
\text { (entre } 553 \text { et } 626 \text { ) }\end{array}$} & \multicolumn{2}{|c|}{$\begin{array}{l}\text { Niveau } 5 \\
\text { (plus de 626) }\end{array}$} \\
\hline & $\%$ & Er.T & $\%$ & Er. T & $\%$ & Er. T & $\%$ & Er.T & $\%$ & Er.T & $\%$ & Er.T \\
\hline Australie & 3.2 & 0.4 & 7.9 & 0.7 & 16.5 & 0.9 & 25.5 & 1.0 & 26.3 & 1.0 & 20.6 & 1.1 \\
\hline Autriche & 5.1 & 0.5 & 10.7 & 0.7 & 21.6 & 1.2 & 27.8 & 1.4 & 24.0 & 1.1 & 10.9 & 0.8 \\
\hline Belgique & 7.9 & 0.8 & 10.0 & 0.7 & 15.9 & 0.7 & 23.7 & 0.9 & 26.1 & 1.0 & 16.4 & 0.8 \\
\hline Canada & 2.6 & 0.3 & 7.4 & 0.3 & 17.4 & 0.4 & 27.3 & 0.5 & 27.2 & 0.5 & 18.1 & 0.5 \\
\hline République tchèque & 7.8 & 0.6 & 11.1 & 0.6 & 21.9 & 0.9 & 27.4 & 1.0 & 19.8 & 0.8 & 12.0 & 0.8 \\
\hline Danemark & 7.3 & 0.6 & 12.3 & 0.6 & 20.7 & 0.9 & 27.3 & 0.8 & 21.1 & 1.0 & 11.3 & 0.8 \\
\hline Finlande & 1.7 & 0.4 & 5.5 & 0.4 & 13.5 & 0.6 & 26.4 & 0.7 & 30.0 & 0.9 & 22.8 & 1.0 \\
\hline France & 3.9 & 0.5 & 9.3 & 0.7 & 20.0 & 0.9 & 28.5 & 1.2 & 25.4 & 1.0 & 13.0 & 0.9 \\
\hline Allemagne & 10.0 & 0.7 & 12.6 & 0.7 & 21.7 & 0.9 & 26.6 & 0.9 & 19.5 & 0.8 & 9.6 & 0.5 \\
\hline Grèce & 12.7 & 1.4 & 18.3 & 1.3 & 26.0 & 1.1 & 25.1 & 1.3 & 13.8 & 1.0 & 4.1 & 0.6 \\
\hline Hongrie & 9.9 & 1.0 & 15.9 & 1.2 & 22.7 & 1.1 & 25.0 & 1.2 & 18.3 & 1.2 & 8.2 & 0.8 \\
\hline Islande & 5.5 & 0.5 & 11.3 & 0.6 & 21.3 & 1.3 & 28.6 & 1.3 & 22.6 & 1.0 & 10.7 & 0.6 \\
\hline Irlande & 3.6 & 0.5 & 8.1 & 0.8 & 18.0 & 1.2 & 26.8 & 1.1 & 26.5 & 1.0 & 17.1 & 0.9 \\
\hline Italie & 8.0 & 0.8 & 14.7 & 1.0 & 25.2 & 1.1 & 28.1 & 1.0 & 17.6 & 0.8 & 6.5 & 0.6 \\
\hline Japon & 4.0 & 0.8 & 8.9 & 1.2 & 18.4 & 1.0 & 29.6 & 1.1 & 25.8 & 1.3 & 13.3 & 1.2 \\
\hline Corée & 2.0 & 0.3 & 7.2 & 0.6 & 21.4 & 0.9 & 35.4 & 1.0 & 26.6 & 1.1 & 7.5 & 0.7 \\
\hline Luxembourg & 17.2 & 0.7 & 18.9 & 0.8 & 24.6 & 1.1 & 23.1 & 1.0 & 12.2 & 0.6 & 3.9 & 0.3 \\
\hline Mexique & 25.7 & 1.3 & 27.5 & 1.2 & 25.4 & 0.9 & 15.1 & 1.1 & 5.5 & 0.8 & 0.9 & 0.2 \\
\hline Nouvelle-Zélande & 4.7 & 0.5 & 8.0 & 0.6 & 14.8 & 0.7 & 23.5 & 0.9 & 26.8 & 1.2 & 22.1 & 1.1 \\
\hline Norvège & 7.5 & 0.6 & 11.3 & 0.8 & 18.8 & 0.8 & 25.2 & 0.8 & 22.7 & 0.9 & 14.5 & 0.8 \\
\hline Pologne & 10.9 & 1.2 & 15.3 & 1.2 & 24.0 & 1.3 & 25.9 & 1.1 & 17.0 & 1.4 & 6.9 & 1.1 \\
\hline Portugal & 12.1 & 1.1 & 17.8 & 1.0 & 25.2 & 1.0 & 25.3 & 1.1 & 15.7 & 1.0 & 4.0 & 0.5 \\
\hline Espagne & 6.1 & 0.6 & 12.9 & 0.7 & 23.5 & 1.0 & 29.0 & 1.0 & 20.9 & 0.9 & 7.5 & 0.5 \\
\hline Suède & 4.1 & 0.4 & 9.7 & 0.7 & 18.5 & 0.7 & 27.5 & 0.8 & 25.6 & 0.9 & 14.6 & 0.7 \\
\hline Suisse & 8.1 & 0.8 & 12.7 & 0.8 & 20.8 & 1.1 & 26.2 & 1.2 & 21.0 & 0.9 & 11.3 & 1.1 \\
\hline Royaume-Uni & 3.1 & 0.3 & 8.2 & 0.5 & 17.9 & 0.8 & 26.8 & 0.8 & 25.9 & 0.9 & 18.2 & 0.9 \\
\hline États-Unis & 6.9 & 1.2 & 11.1 & 1.1 & 20.4 & 1.0 & 26.4 & 1.3 & 22.0 & 1.3 & 13.3 & 1.5 \\
\hline Moyenne de l'OCDE & 7.5 & 0.1 & 12.0 & 0.2 & 20.6 & 0.2 & 26.4 & 0.2 & 21.7 & 0.2 & 11.8 & 0.2 \\
\hline Brésil & 37.3 & 1.6 & 29.3 & 1.1 & 21.7 & 1.3 & 9.1 & 0.8 & 2.2 & 0.4 & 0.4 & 0.2 \\
\hline Lettonie & 15.5 & 1.5 & 19.3 & 1.1 & 25.6 & 1.1 & 22.2 & 1.1 & 13.1 & 1.2 & 4.2 & 0.7 \\
\hline Liechtenstein & 8.2 & 1.6 & 14.4 & 2.4 & 24.3 & 2.8 & 27.6 & 3.0 & 19.2 & 2.3 & 6.5 & 1.4 \\
\hline Féd.de Russie & 13.3 & 1.2 & 19.7 & 0.9 & 27.6 & 0.8 & 23.8 & 1.1 & 11.9 & 0.8 & 3.8 & 0.5 \\
\hline
\end{tabular}


Tableau 4.15

Différences de score entre le $10^{\mathrm{e}}$ et le $90^{\mathrm{e}}$ percentile sur les sous-échelles et l'échelle combinée de compréhension de l'écrit




Tableau 4.16

Variation de la performance des élèves sur l'échelle combinée de compréhension de l'écrit

\begin{tabular}{|c|c|c|c|c|c|c|}
\hline & \multirow{2}{*}{\multicolumn{2}{|c|}{ Écart type }} & \multicolumn{4}{|c|}{ Percentiles } \\
\hline & & & \multicolumn{2}{|c|}{$10^{\mathrm{e}}$} & \multicolumn{2}{|c|}{$90^{\mathrm{e}}$} \\
\hline & Éc.T. & Er.T & Score & Er.T & Score & Er.T \\
\hline Australie & 102 & $(1.6)$ & 394 & $(4.4)$ & 656 & $(4.2)$ \\
\hline Autriche & 93 & $(1.6)$ & 383 & $(4.2)$ & 621 & $(3.2)$ \\
\hline Belgique & 107 & $(2.4)$ & 354 & $(8.9)$ & 634 & $(2.5)$ \\
\hline Canada & 95 & $(1.1)$ & 410 & $(2.4)$ & 652 & (1.9) \\
\hline République tchèque & 96 & $(1.9)$ & 368 & $(4.9)$ & 610 & $(3.2)$ \\
\hline Danemark & 98 & $(1.8)$ & 367 & $(5.0)$ & 617 & (2.9) \\
\hline Finlande & 89 & $(2.6)$ & 429 & $(5.1)$ & 654 & $(2.8)$ \\
\hline France & 92 & $(1.7)$ & 381 & $(5.2)$ & 619 & $(2.9)$ \\
\hline Allemagne & 111 & $(1.9)$ & 335 & $(6.3)$ & 619 & $(2.8)$ \\
\hline Grèce & 97 & $(2.7)$ & 342 & $(8.4)$ & 595 & $(5.1)$ \\
\hline Hongrie & 94 & $(2.1)$ & 354 & $(5.5)$ & 598 & $(4.4)$ \\
\hline Islande & 92 & $(1.4)$ & 383 & $(3.6)$ & 621 & $(3.5)$ \\
\hline Irlande & 94 & $(1.7)$ & 401 & $(6.4)$ & 641 & $(4.0)$ \\
\hline Italie & 91 & $(2.7)$ & 368 & $(5.8)$ & 601 & $(2.7)$ \\
\hline Japon & 86 & $(3.0)$ & 407 & $(9.8)$ & 625 & $(4.6)$ \\
\hline Corée & 70 & $(1.6)$ & 433 & $(4.4)$ & 608 & $(2.9)$ \\
\hline Luxembourg & 100 & $(1.5)$ & 311 & $(4.4)$ & 564 & $(2.8)$ \\
\hline Mexique & 86 & $(2.1)$ & 311 & (3.4) & 535 & $(5.5)$ \\
\hline Nouvelle-Zélande & 108 & $(2.0)$ & 382 & $(5.2)$ & 661 & $(4.4)$ \\
\hline Norvège & 104 & $(1.7)$ & 364 & $(5.5)$ & 631 & (3.1) \\
\hline Pologne & 100 & $(3.1)$ & 343 & $(6.8)$ & 603 & $(6.6)$ \\
\hline Portugal & 97 & $(1.8)$ & 337 & $(6.2)$ & 592 & $(4.2)$ \\
\hline Espagne & 85 & $(1.2)$ & 379 & $(5.0)$ & 597 & $(2.6)$ \\
\hline Suède & 92 & $(1.2)$ & 392 & $(4.0)$ & 630 & $(2.9)$ \\
\hline Suisse & 102 & $(2.0)$ & 355 & $(5.8)$ & 621 & (5.5) \\
\hline Royaume-Uni & 100 & $(1.5)$ & 391 & $(4.1)$ & 651 & $(4.3)$ \\
\hline États-Unis & 105 & $(2.7)$ & 363 & (11.4) & 636 & $(6.5)$ \\
\hline Moyenne de l'OCDE & 100 & $(0.4)$ & 366 & $(1.1)$ & 623 & $(0.8)$ \\
\hline Brésil & 86 & (1.9) & 288 & $(4.5)$ & 507 & $(4.2)$ \\
\hline Lettonie & 102 & $(2.3)$ & 322 & $(8.2)$ & 586 & $(5.8)$ \\
\hline Liechtenstein & 96 & (3.9) & 350 & $(11.8)$ & 601 & (7.1) \\
\hline Fédération de Russie & 92 & $(1.8)$ & 340 & $(5.4)$ & 579 & $(4.4)$ \\
\hline
\end{tabular}


Tableau 4.17

Variation de la performance des élèves sur la sous-échelle «Trouver l’information »

\begin{tabular}{|c|c|c|c|c|c|c|}
\hline & \multirow{2}{*}{\multicolumn{2}{|c|}{ Écart type }} & \multicolumn{4}{|c|}{ Percentiles } \\
\hline & & & \multicolumn{2}{|c|}{$10^{\mathrm{e}}$} & \multicolumn{2}{|c|}{$90^{\mathrm{e}}$} \\
\hline & Éc.T. & Er.T & Score & Er.T & Score & Er.T \\
\hline Australie & 108 & $(1.6)$ & 393 & $(4.7)$ & 671 & $(5.0)$ \\
\hline Autriche & 96 & (1.5) & 374 & $(4.6)$ & 619 & $(3.1)$ \\
\hline Belgique & 120 & (2.7) & 343 & $(8.5)$ & 656 & $(2.6)$ \\
\hline Canada & 102 & $(1.2)$ & 397 & $(2.9)$ & 657 & $(2.4)$ \\
\hline République tchèque & 107 & $(1.9)$ & 343 & $(5.6)$ & 614 & $(3.9)$ \\
\hline Danemark & 105 & (1.9) & 359 & $(5.9)$ & 626 & (3.3) \\
\hline Finlande & 102 & (2.1) & 423 & $(4.7)$ & 682 & $(3.2)$ \\
\hline France & 101 & (2.1) & 376 & $(6.4)$ & 638 & $(4.0)$ \\
\hline Allemagne & 114 & $(2.0)$ & 331 & $(6.2)$ & 621 & $(3.1)$ \\
\hline Grèce & 109 & $(3.0)$ & 306 & $(9.2)$ & 585 & $(5.0)$ \\
\hline Hongrie & 107 & $(2.2)$ & 333 & $(6.2)$ & 613 & $(4.9)$ \\
\hline Islande & 103 & (1.3) & 362 & $(4.2)$ & 628 & $(2.9)$ \\
\hline Irlande & 100 & (1.7) & 392 & $(6.5)$ & 647 & (3.3) \\
\hline Italie & 104 & (3.0) & 352 & $(5.8)$ & 617 & $(4.0)$ \\
\hline Japon & 97 & (3.1) & 397 & $(10.2)$ & 644 & $(4.7)$ \\
\hline Corée & 82 & (1.6) & 421 & $(4.3)$ & 631 & (3.4) \\
\hline Luxembourg & 109 & (1.4) & 290 & $(4.3)$ & 567 & (2.6) \\
\hline Mexique & 101 & $(2.2)$ & 270 & $(4.5)$ & 533 & (6.0) \\
\hline Nouvelle-Zélande & 116 & (2.1) & 377 & $(6.3)$ & 677 & $(3.9)$ \\
\hline Norvège & 110 & (1.9) & 356 & $(6.5)$ & 637 & (3.3) \\
\hline Pologne & 112 & (3.3) & 324 & $(8.6)$ & 615 & (7.1) \\
\hline Portugal & 107 & $(2.2)$ & 311 & (7.9) & 588 & $(4.3)$ \\
\hline Espagne & 92 & $(1.2)$ & 361 & $(4.9)$ & 597 & $(2.8)$ \\
\hline Suède & 104 & (1.5) & 378 & $(4.3)$ & 645 & $(2.7)$ \\
\hline Suisse & 113 & (2.1) & 344 & (6.4) & 636 & $(5.2)$ \\
\hline Royaume-Uni & 105 & (1.5) & 384 & $(4.5)$ & 656 & $(4.3)$ \\
\hline États-Unis & 112 & (2.7) & 348 & $(12.0)$ & 638 & $(6.0)$ \\
\hline Moyenne de l'OCDE & 111 & $(0.4)$ & 349 & $(1.3)$ & 634 & $(0.9)$ \\
\hline Brésil & 97 & (2.1) & 239 & $(5.2)$ & 489 & (3.5) \\
\hline Lettonie & 117 & (2.4) & 296 & $(8.5)$ & 599 & $(5.7)$ \\
\hline Liechtenstein & 106 & $(4.7)$ & 345 & $(13.9)$ & 620 & (7.7) \\
\hline Fédération de Russie & 108 & $(2.1)$ & 309 & $(7.1)$ & 587 & $(5.6)$ \\
\hline
\end{tabular}


Tableau 4.18

Variation de la performance des élèves sur la sous-échelle « Développer une interprétation »

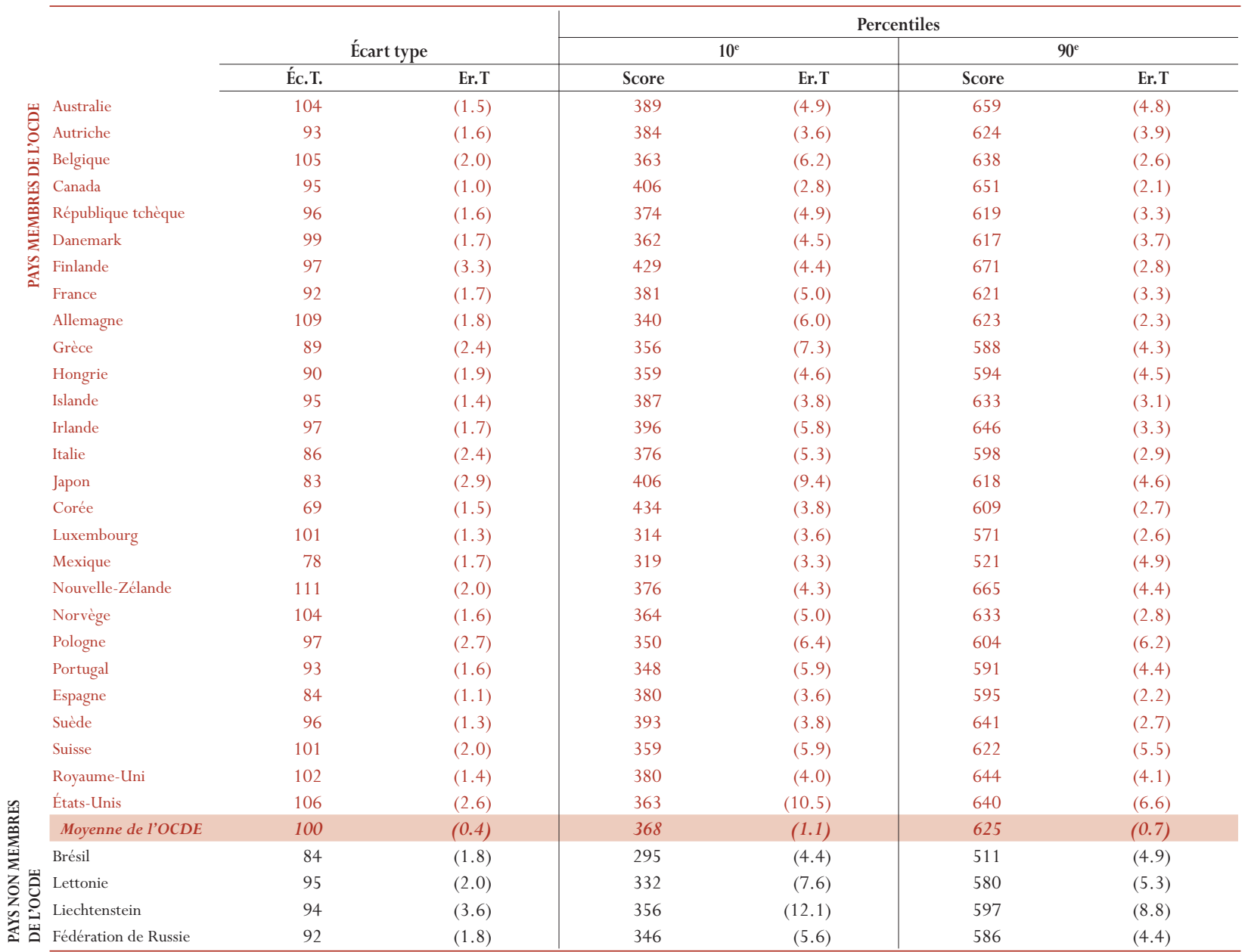


ANNEXE B

Tableau 4.19

Variation de la performance des élèves sur la sous-échelle «Réfléchir et évaluer»

\begin{tabular}{|c|c|c|c|c|c|c|}
\hline & \multirow{2}{*}{\multicolumn{2}{|c|}{ Écart type }} & \multicolumn{4}{|c|}{ Percentiles } \\
\hline & & & & & & \\
\hline & Éc.T. & Er.T & Score & Er.T & Score & Er.T \\
\hline Iy Australie & 100 & $(1.5)$ & 393 & $(5.3)$ & 651 & $(4.7)$ \\
\hline Autriche & 100 & $(1.8)$ & 379 & $(5.0)$ & 633 & $(4.6)$ \\
\hline Belgique & 114 & $(4.1)$ & 336 & $(9.4)$ & 629 & (2.4) \\
\hline त्र Canada & 96 & $(1.0)$ & 416 & (3.1) & 661 & $(1.8)$ \\
\hline République tchèque & 103 & $(1.8)$ & 354 & $(5.0)$ & 611 & $(3.9)$ \\
\hline Danemark & 102 & $(2.1)$ & 365 & $(5.5)$ & 625 & $(4.0)$ \\
\hline Finlande & 91 & (3.9) & 415 & $(5.0)$ & 640 & $(2.5)$ \\
\hline France & 98 & $(1.8)$ & 365 & $(6.1)$ & 618 & $(3.5)$ \\
\hline Allemagne & 124 & $(1.8)$ & 311 & (7.4) & 627 & $(3.1)$ \\
\hline Grèce & 115 & $(3.1)$ & 343 & $(9.3)$ & 638 & $(5.8)$ \\
\hline Hongrie & 100 & $(2.2)$ & 347 & $(5.6)$ & 606 & $(4.5)$ \\
\hline Islande & 93 & $(1.3)$ & 378 & $(3.8)$ & 616 & $(2.5)$ \\
\hline Irlande & 90 & $(1.7)$ & 414 & $(6.3)$ & 642 & $(3.3)$ \\
\hline Italie & 101 & $(2.9)$ & 348 & $(6.3)$ & 607 & $(3.1)$ \\
\hline Japon & 100 & $(3.3)$ & 397 & $(9.1)$ & 651 & $(4.7)$ \\
\hline Corée & 76 & (1.7) & 428 & $(4.5)$ & 619 & $(3.0)$ \\
\hline Luxembourg & 115 & $(1.8)$ & 293 & $(4.9)$ & 581 & (3.6) \\
\hline Mexique & 109 & $(2.2)$ & 303 & $(4.4)$ & 586 & $(6.5)$ \\
\hline Nouvelle-Zélande & 107 & $(1.8)$ & 387 & $(5.1)$ & 662 & $(4.7)$ \\
\hline Norvège & 108 & $(1.8)$ & 357 & $(5.2)$ & 636 & $(3.1)$ \\
\hline Pologne & 110 & $(3.2)$ & 328 & $(8.0)$ & 613 & (6.4) \\
\hline Portugal & 101 & (1.7) & 342 & $(6.8)$ & 607 & $(3.8)$ \\
\hline Espagne & 91 & $(1.2)$ & 383 & $(4.3)$ & 618 & $(2.7)$ \\
\hline Suède & 95 & $(1.2)$ & 382 & $(4.1)$ & 626 & $(4.0)$ \\
\hline Suisse & 113 & $(2.2)$ & 336 & $(6.5)$ & 629 & $(6.0)$ \\
\hline Royaume-Uni & 99 & $(1.6)$ & 408 & $(4.5)$ & 664 & (3.5) \\
\hline États-Unis & 105 & $(2.7)$ & 367 & (11.9) & 638 & (6.3) \\
\hline Moyenne de l'OCDE & 106 & $(0.4)$ & 361 & (1.4) & 630 & $(0.9)$ \\
\hline Brésil & 93 & $(2.2)$ & 298 & $(5.2)$ & 536 & (5.6) \\
\hline Lettonie & 113 & $(2.3)$ & 305 & (7.3) & 598 & (7.1) \\
\hline Liechtenstein & 108 & $(4.3)$ & 323 & $(12.9)$ & 603 & $(9.6)$ \\
\hline Fédération de Russie & 98 & $(1.7)$ & 326 & $(6.2)$ & 580 & $(4.2)$ \\
\hline
\end{tabular}


Tableau 4.20

Variation de la performance des élèves sur la sous-échelle «Textes continus »




ANNEXE B

Tableau 4.21

Variation de la performance des élèves sur la sous-échelle «Textes non continus »

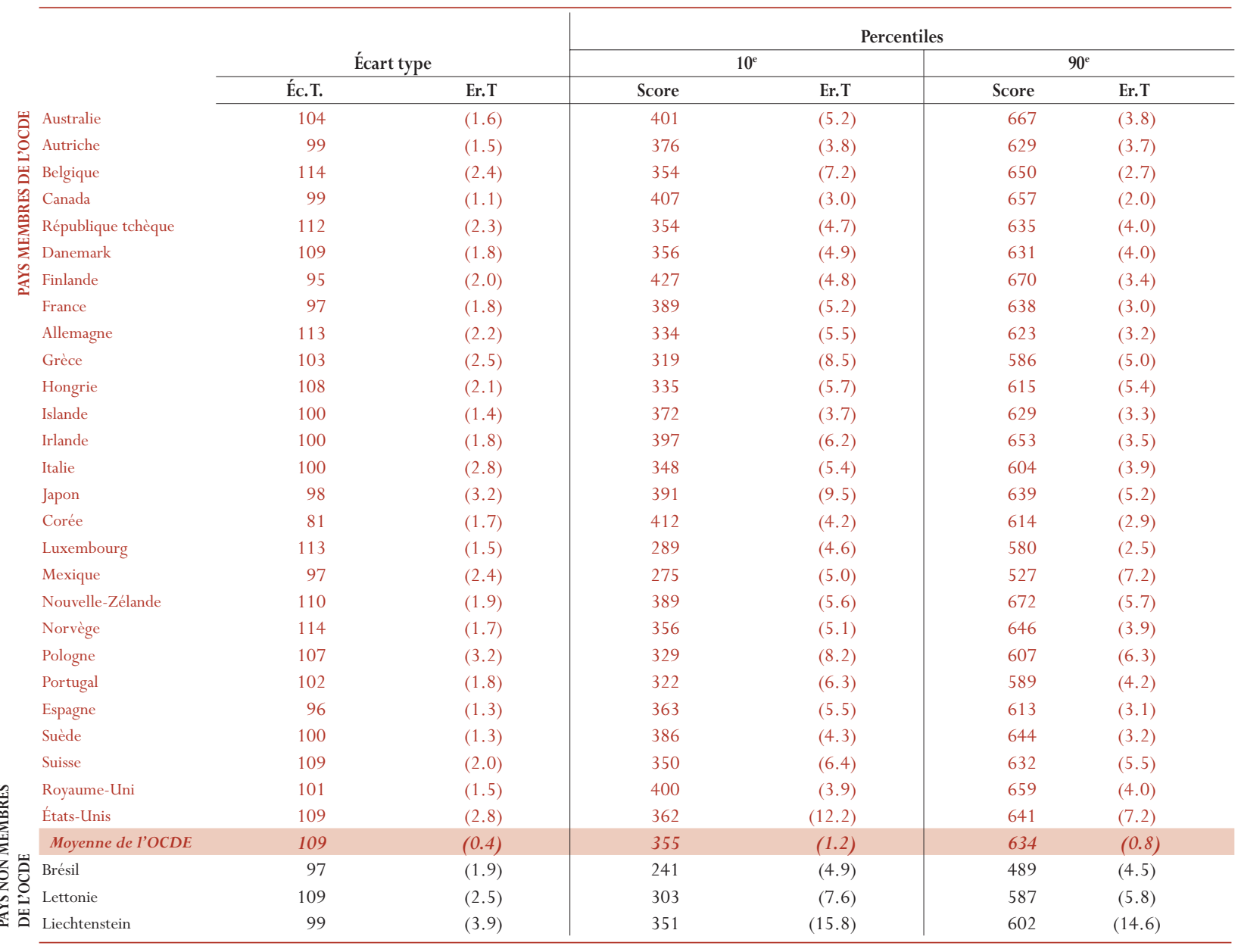


Tableau 5.1

Pourcentage d'élèves lisant chaque type d'écrit par profil de lecteur

\begin{tabular}{|c|c|c|c|}
\hline & Pas de lecture (\%) & Lecture occasionnelle (\%) & Lecture fréquente $(\%)$ \\
\hline \multicolumn{4}{|l|}{ Lectures peu diversifiées } \\
\hline Revues & 15.6 & 46.0 & 38.4 \\
\hline Bandes dessinées & 49.6 & 37.4 & 12.9 \\
\hline Livres de fiction & 40.6 & 47.1 & 12.3 \\
\hline Ouvrages documentaires & 53.7 & 40.7 & 5.7 \\
\hline Journaux & 47.6 & 52.2 & 0.2 \\
\hline \multicolumn{4}{|c|}{ Lectures modérément diversifiées } \\
\hline Revues & 4.6 & 25.4 & 69.9 \\
\hline Bandes dessinées & 60.7 & 38.9 & 0.4 \\
\hline Livres de fiction & 45.3 & 51.9 & 2.7 \\
\hline Ouvrages documentaires & 51.7 & 45.3 & 3.1 \\
\hline Journaux & - & 11.4 & 88.6 \\
\hline \multicolumn{4}{|c|}{$\begin{array}{l}\text { Lectures diversifiées de textes } \\
\text { courts }\end{array}$} \\
\hline Revues & 1.8 & 13.5 & 84.7 \\
\hline Bandes dessinées & - & 10.6 & 89.4 \\
\hline Livres de fiction & 18.2 & 51.3 & 30.5 \\
\hline Ouvrages documentaires & 24.5 & 54.4 & 21.0 \\
\hline Journaux & 2.4 & 16.1 & 81.4 \\
\hline \multicolumn{4}{|c|}{ Lectures diversifiées de textes longs } \\
\hline Revues & 3.2 & 26.3 & 70.5 \\
\hline Bandes dessinées & 46.0 & 48.4 & 5.6 \\
\hline Livres de fiction & 0.7 & 27.4 & 71.9 \\
\hline Ouvrages documentaires & 4.3 & 47.5 & 48.3 \\
\hline Journaux & 2.2 & 21.7 & 76.1 \\
\hline
\end{tabular}


Tableau 5.2

Pourcentages d'élèves par profil de lecteur et performance sur l'échelle combinée de compréhension de l'écrit

\begin{tabular}{|c|c|c|c|c|c|c|c|c|c|c|c|c|c|c|c|c|}
\hline & \multicolumn{4}{|c|}{$\begin{array}{l}\text { Lectures peu } \\
\text { diversifiées }\end{array}$} & \multicolumn{4}{|c|}{$\begin{array}{c}\text { Lectures modérément } \\
\text { diversifiées }\end{array}$} & \multicolumn{4}{|c|}{$\begin{array}{l}\text { Lectures diversifiées } \\
\text { de textes courts }\end{array}$} & \multicolumn{4}{|c|}{$\begin{array}{l}\text { Lectures diversifiées } \\
\text { de textes longs }\end{array}$} \\
\hline & $\begin{array}{c}\text { Score } \\
\text { moyen }\end{array}$ & Er.T & $\%$ & Er. T & $\begin{array}{c}\text { Score } \\
\text { moyen }\end{array}$ & Er.T & $\%$ & Er.T & $\begin{array}{c}\text { Score } \\
\text { moyen }\end{array}$ & Er.T & $\%$ & Er.T & $\begin{array}{c}\text { Score } \\
\text { moyen }\end{array}$ & Er.T & $\%$ & Er. T \\
\hline Australie & 494 & $(4.9)$ & 18.5 & $(0.9)$ & 514 & (3.7) & 35.0 & (1.1) & 522 & (6.3) & 10.8 & $(0.6)$ & 569 & $(4.4)$ & 35.7 & $(1.2)$ \\
\hline Autriche & 474 & $(4.6)$ & 16.6 & $(0.7)$ & 503 & (2.4) & 41.9 & (0.9) & 509 & (3.5) & 17.0 & $(0.5)$ & 545 & (3.6) & 24.6 & $(0.8)$ \\
\hline Belgique & 487 & $(4.4)$ & 36.3 & $(0.6)$ & 503 & (5.4) & 19.6 & $(0.6)$ & 537 & (3.4) & 31.8 & $(0.7)$ & 556 & (5.5) & 12.3 & $(0.5)$ \\
\hline Canada & 507 & $(2.3)$ & 24.3 & $(0.4)$ & 528 & (1.7) & 30.8 & $(0.5)$ & 531 & (2.5) & 16.2 & $(0.3)$ & 572 & (1.9) & 28.7 & $(0.5)$ \\
\hline Rép. tchèque & 482 & $(3.5)$ & 22.0 & $(0.7)$ & 492 & (2.8) & 35.6 & $(0.9)$ & 494 & (3.4) & 18.7 & (0.6) & 543 & (2.9) & 23.8 & $(0.7)$ \\
\hline Danemark & 453 & $(5.0)$ & 17.5 & $(0.8)$ & 464 & $(6.0)$ & 10.1 & $(0.6)$ & 511 & (2.3) & 56.2 & $(1.0)$ & 541 & $(5.2)$ & 16.2 & $(0.6)$ \\
\hline Finlande & 485 & (14.6) & 6.9 & $(0.5)$ & 522 & (4.4) & 14.2 & (0.6) & 550 & $(2.2)$ & 66.6 & $(0.9)$ & 597 & (3.5) & 12.3 & $(0.5)$ \\
\hline France & 488 & $(4.1)$ & 32.6 & $(0.9)$ & 503 & (3.4) & 19.2 & $(0.7)$ & 528 & (2.9) & 31.3 & $(0.9)$ & 534 & (4.1) & 16.8 & $(0.7)$ \\
\hline Allemagne & 464 & $(4.2)$ & 24.1 & $(0.8)$ & 485 & (2.8) & 38.0 & $(0.8)$ & 499 & (5.9) & 11.6 & $(0.6)$ & 541 & (3.1) & 26.3 & $(0.7)$ \\
\hline Grèce & 464 & $(5.3)$ & 35.4 & $(0.9)$ & 474 & (6.6) & 21.3 & $(0.8)$ & 478 & (5.8) & 21.5 & $(0.7)$ & 505 & $(5.2)$ & 21.8 & $(0.9)$ \\
\hline Hongrie & 450 & $(4.8)$ & 25.1 & (1.0) & 479 & $(4.3)$ & 25.1 & $(0.8)$ & 470 & (4.7) & 20.1 & $(0.7)$ & 525 & $(4.7)$ & 29.6 & $(1.0)$ \\
\hline Islande & 449 & $(6.5)$ & 6.6 & $(0.5)$ & 492 & (2.6) & 28.6 & $(0.7)$ & 520 & (2.1) & 49.7 & $(0.8)$ & 537 & (4.3) & 15.1 & $(0.6)$ \\
\hline Irlande & 510 & $(5.9)$ & 16.3 & $(0.7)$ & 515 & (3.3) & 47.0 & $(0.8)$ & 507 & (5.9) & 8.9 & $(0.6)$ & 571 & (3.6) & 27.8 & $(1.0)$ \\
\hline Italie & 469 & $(4.7)$ & 25.8 & $(0.9)$ & 485 & (3.3) & 27.9 & $(0.7)$ & 505 & (3.3) & 26.5 & $(0.8)$ & 503 & (4.1) & 19.8 & $(0.7)$ \\
\hline Japon & 482 & $(8.2)$ & 14.5 & $(0.9)$ & 514 & $(7.2)$ & 8.1 & $(0.5)$ & 532 & (4.6) & 74.4 & $(0.9)$ & 573 & (7.7) & 3.0 & $(0.3)$ \\
\hline Corée & 495 & (3.9) & 18.8 & $(0.6)$ & 525 & (3.7) & 14.6 & (0.6) & 531 & (2.4) & 53.1 & (1.1) & 545 & (3.8) & 13.6 & $(0.7)$ \\
\hline Luxembourg & 434 & $(2.5)$ & 39.4 & $(0.8)$ & 454 & $(4.3)$ & 21.3 & $(0.6)$ & 461 & $(4.0)$ & 19.2 & $(0.7)$ & 486 & (3.8) & 20.0 & $(0.6)$ \\
\hline Mexique & 403 & $(3.6)$ & 37.5 & (1.3) & 426 & (5.9) & 15.6 & $(0.8)$ & 438 & (4.3) & 22.2 & (5.9) & 443 & $(4.9)$ & 24.7 & $(0.7)$ \\
\hline Nouvelle-Zélande & 499 & $(4.8)$ & 18.0 & $(0.7)$ & 529 & (3.1) & 30.1 & $(0.9)$ & 500 & (6.4) & 12.4 & $(0.6)$ & 564 & (3.7) & 39.4 & $(1.0)$ \\
\hline Norvège & 433 & $(7.1)$ & 8.5 & $(0.6)$ & 492 & $(4.2)$ & 20.2 & $(0.7)$ & 520 & (2.7) & 58.0 & $(0.9)$ & 546 & (4.3) & 13.3 & $(0.5)$ \\
\hline Pologne & 445 & $(7.0)$ & 16.7 & $(0.9)$ & 491 & $(4.2)$ & 48.0 & $(1.1)$ & 474 & (6.6) & 11.4 & $(0.7)$ & 511 & $(6.3)$ & 24.0 & $(1.1)$ \\
\hline Portugal & 449 & $(5.8)$ & 29.8 & $(0.9)$ & 477 & $(4.1)$ & 25.9 & $(0.7)$ & 487 & (5.8) & 24.4 & $(0.6)$ & 489 & $(5.9)$ & 19.8 & $(0.6)$ \\
\hline Espagne & 474 & $(3.4)$ & 36.2 & (1.1) & 492 & (3.6) & 23.0 & $(0.7)$ & 503 & (3.4) & 17.5 & $(0.7)$ & 526 & (2.9) & 23.3 & $(0.7)$ \\
\hline Suède & 469 & $(4.8)$ & 11.1 & $(0.5)$ & 502 & $(2.8)$ & 30.3 & $(0.8)$ & 518 & $(2.8)$ & 37.3 & $(0.8)$ & 564 & (3.6) & 21.3 & $(0.7)$ \\
\hline Suisse & 455 & $(4.6)$ & 22.1 & $(0.9)$ & 487 & (4.3) & 30.3 & $(0.8)$ & 519 & (5.1) & 25.4 & $(0.8)$ & 534 & (5.2) & 22.2 & $(0.8)$ \\
\hline Royaume-Uni & 503 & $(4.3)$ & 17.1 & $(0.6)$ & 512 & $(2.7)$ & 39.4 & $(0.9)$ & 488 & (5.3) & 8.4 & $(0.5)$ & 566 & (3.7) & 35.1 & $(1.0)$ \\
\hline États-Unis & 478 & $(7.6)$ & 28.4 & (1.3) & 520 & $(5.8)$ & 32.1 & $(1.5)$ & 482 & $(10.9)$ & 10.8 & $(1.1)$ & 544 & $(6.0)$ & 28.7 & $(1.5)$ \\
\hline Moyenne de l'OCDE & 68 & $(1.0)$ & 22.4 & $(0.2)$ & 498 & $(0.7)$ & 27.1 & $(0.1)$ & 514 & $(0.9)$ & 28.3 & $(0.2)$ & 539 & $(0.9)$ & 22.2 & $(0.2)$ \\
\hline Brésil & 370 & $(4.4)$ & 29.5 & $(1.1)$ & 407 & $(5.1)$ & 15.1 & $(0.8)$ & 413 & $(4.3)$ & 27.5 & $(1.0)$ & 418 & (3.6) & 27.9 & $(1.1)$ \\
\hline Lettonie & 412 & $(8.2)$ & 13.8 & $(0.8)$ & 464 & $(5.3)$ & 39.9 & $(1.3)$ & 433 & $(8.7)$ & 15.2 & $(0.9)$ & 499 & $(5.7)$ & 31.1 & $(1.4)$ \\
\hline Liechtenstein & 442 & $(11.0)$ & 21.9 & $(2.1)$ & 478 & $(8.0)$ & 40.7 & $(2.5)$ & 524 & $(12.6)$ & 14.3 & $(2.1)$ & 526 & $(11.7)$ & 23.2 & $(2.5)$ \\
\hline Féd. de Russie & 426 & $(6.3)$ & 11.5 & $(0.5)$ & 451 & $(5.1)$ & 17.1 & $(0.6)$ & 432 & $(4.8)$ & 21.6 & $(1.2)$ & 495 & (3.9) & 49.7 & $(1.1)$ \\
\hline Pays-Bas* & 494 & $(5.4)$ & 24.7 & (1.3) & 530 & $(4.5)$ & 25.8 & $(1.0)$ & 544 & $(4.0)$ & 33.1 & $(1.2)$ & 573 & $(4.9)$ & 16.5 & $(0.9)$ \\
\hline
\end{tabular}

* Taux de participation insuffisant pour permettre des comparaisons.

Tableau 5.3

Pourcentage d'élèves dans chaque profil de lecteur par niveau de compétence sur l'échelle combinée de compréhension de l'écrit

\begin{tabular}{lc|c|c|c|c|c}
\hline & En deçà du niveau 1 (\%) & Niveau 1 (\%) & Niveau 2 (\%) & Niveau 3 (\%) & Niveau 4 (\%) & Niveau 5 (\%) \\
\cline { 2 - 7 } Lectures peu diversifiées & 9.6 & 17.8 & 25.7 & 26.4 & 15.7 & 4.8 \\
Lectures modérément diversifiées & 4.3 & 11.8 & 24.3 & 31.3 & 21.1 & 7.1 \\
Lectures diversifiées de textes courts & 3.4 & 9.4 & 20.5 & 31.2 & 25.4 \\
Lectures diversifiées de textes longs & 2.8 & 6.9 & 15.6 & 27.3 & 10.0 & 29.4 \\
\hline
\end{tabular}


Tableau 5.4

Scores moyens sur l'échelle combinée de compréhension de l'écrit et sur les sous-échelles par profil de lecteur

\begin{tabular}{|c|c|c|c|c|c|c|c|c|c|c|c|c|}
\hline & \multicolumn{2}{|c|}{$\left|\begin{array}{c}\text { Échelle combinée } \\
\text { de compréhen- } \\
\text { sion de l'écrit }\end{array}\right|$} & \multicolumn{2}{|c|}{$\begin{array}{c}\text { Trouver } \\
\text { l'information }\end{array}$} & \multicolumn{2}{|c|}{$\begin{array}{l}\text { Développer une } \\
\text { interprétation }\end{array}$} & \multicolumn{2}{|c|}{$\begin{array}{c}\text { Réflection et } \\
\text { évaluation }\end{array}$} & \multicolumn{2}{|c|}{$\begin{array}{c}\text { Textes } \\
\text { continus }\end{array}$} & \multicolumn{2}{|c|}{$\begin{array}{c}\text { Textes non } \\
\text { continus }\end{array}$} \\
\hline & $\begin{array}{l}\text { Score } \\
\text { moyen }\end{array}$ & Er.T & $\begin{array}{l}\text { Score } \\
\text { moyen }\end{array}$ & Er.T & $\begin{array}{l}\text { Score } \\
\text { moyen }\end{array}$ & Er.T & $\begin{array}{c}\text { Score } \\
\text { moyen }\end{array}$ & Er.T & $\begin{array}{l}\text { Score } \\
\text { moyen }\end{array}$ & Er.T & $\begin{array}{c}\text { Score } \\
\text { moyen }\end{array}$ & Er.T \\
\hline Lectures peu diversifiées & 468 & $(1.01)$ & 461 & $(1.10)$ & 470 & $(0.99)$ & 472 & $(1.05)$ & 470 & $(1.00)$ & 464 & $(1.08)$ \\
\hline Lectures modérément diversifiées & 498 & $(0.72)$ & 497 & $(0.78)$ & 498 & $(0.69)$ & 499 & $(0.81)$ & 497 & $(0.72)$ & 501 & $(0.79)$ \\
\hline Lectures diversifiées de textes courts & 514 & $(0.85)$ & 516 & $(0.92)$ & 515 & $(0.84)$ & 513 & $(0.93)$ & 514 & $(0.85)$ & 517 & $(0.95)$ \\
\hline Lectures diversifiées de textes longs & 539 & $(0.88)$ & 535 & $(0.96)$ & 539 & $(0.88)$ & 544 & $(0.88)$ & 541 & $(0.94)$ & 537 & $(0.98)$ \\
\hline
\end{tabular}

Tableau 5.5

Pourcentage d'élèves dans chaque profil de lecteur, par sexe

\begin{tabular}{|c|c|c|c|c|c|c|c|c|}
\hline & \multicolumn{2}{|c|}{ Lectures peu diversifiées } & \multicolumn{2}{|c|}{ Lectures modérément diversifiées } & \multicolumn{2}{|c|}{ Lectures diversifiées de textes courts } & \multicolumn{2}{|c|}{ Lectures diversifiées de textes longs } \\
\hline & Garçons (\%) & Filles (\%) & Garçons (\%) & Filles $(\%)$ & Garçons (\%) & Filles $(\%)$ & Garçons (\%) & Filles $(\%)$ \\
\hline Australie & 17.4 & 19.7 & 39.0 & 30.7 & 15.5 & 5.7 & 28.2 & 43.9 \\
\hline Autriche & 16.9 & 16.0 & 42.1 & 42.0 & 23.6 & 10.9 & 17.4 & 31.2 \\
\hline Belgique & 34.2 & 38.5 & 22.1 & 16.9 & 36.3 & 26.9 & 7.4 & 17.6 \\
\hline Canada & 24.7 & 23.9 & 34.3 & 27.2 & 19.4 & 13.1 & 21.6 & 35.8 \\
\hline Rép. tchèque & 19.4 & 24.3 & 44.8 & 27.3 & 22.9 & 14.9 & 12.9 & 33.5 \\
\hline Danemark & 18.2 & 16.8 & 11.7 & 8.4 & 60.3 & 52.1 & 9.7 & 22.8 \\
\hline Finlande & 8.1 & 5.8 & 12.2 & 15.9 & 74.1 & 59.7 & 5.6 & 18.6 \\
\hline France & 31.7 & 33.5 & 16.7 & 21.6 & 41.2 & 22.2 & 10.4 & 22.8 \\
\hline Allemagne & 23.3 & 24.8 & 42.6 & 33.3 & 16.7 & 6.7 & 17.4 & 35.2 \\
\hline Grèce & 24.7 & 46.0 & 29.6 & 12.9 & 27.4 & 15.7 & 18.3 & 25.3 \\
\hline Hongrie & 25.8 & 24.3 & 28.3 & 22.0 & 21.6 & 18.7 & 24.3 & 35.0 \\
\hline Islande & 6.5 & 6.8 & 29.0 & 28.2 & 55.2 & 44.3 & 9.4 & 20.7 \\
\hline Irlande & 15.7 & 16.9 & 53.7 & 40.6 & 11.2 & 6.7 & 19.5 & 35.8 \\
\hline Italie & 23.4 & 28.0 & 30.0 & 25.9 & 31.0 & 21.9 & 15.5 & 24.3 \\
\hline Japon & 12.2 & 16.7 & 6.4 & 9.7 & 79.5 & 69.5 & 1.9 & 4.0 \\
\hline Corée & 16.6 & 21.5 & 13.1 & 16.4 & 60.3 & 44.1 & 10.0 & 18.1 \\
\hline Luxembourg & 36.2 & 42.5 & 23.6 & 19.1 & 27.4 & 11.3 & 12.8 & 27.1 \\
\hline Mexique & 36.9 & 38.0 & 15.4 & 15.8 & 26.8 & 17.7 & 20.9 & 28.5 \\
\hline Nouvelle-Zélande & 18.2 & 17.9 & 33.9 & 26.5 & 17.6 & 7.2 & 30.4 & 48.4 \\
\hline Norvège & 8.6 & 8.3 & 19.6 & 20.7 & 66.0 & 49.9 & 5.8 & 21.0 \\
\hline Pologne & 21.0 & 12.3 & 48.1 & 48.0 & 14.6 & 8.2 & 16.3 & 31.6 \\
\hline Portugal & 22.9 & 36.0 & 37.2 & 15.7 & 27.8 & 21.4 & 12.1 & 26.9 \\
\hline Espagne & 30.7 & 41.5 & 27.9 & 18.4 & 25.1 & 10.4 & 16.4 & 29.8 \\
\hline Suède & 11.9 & 10.2 & 29.5 & 31.1 & 45.0 & 29.5 & 13.6 & 29.2 \\
\hline Suisse & 20.2 & 23.9 & 34.2 & 26.5 & 32.7 & 18.2 & 13.0 & 31.3 \\
\hline Royaume-Uni & 13.9 & 20.2 & 46.0 & 33.1 & 12.5 & 4.4 & 27.6 & 42.3 \\
\hline États-Unis & 30.4 & 26.5 & 33.2 & 31.1 & 15.0 & 7.0 & 21.4 & 35.4 \\
\hline Moyenne de l'OCDE & 20.9 & 23.6 & 29.8 & 24.7 & 33.8 & 22.9 & 15.5 & 28.8 \\
\hline Brésil & 33.4 & 26.2 & 19.5 & 11.4 & 29.4 & 25.9 & 17.7 & 36.5 \\
\hline Lettonie & 17.0 & 10.8 & 42.3 & 37.5 & 16.7 & 13.8 & 23.9 & 37.8 \\
\hline Liechtenstein & 17.3 & 24.8 & 51.1 & 31.1 & 17.9 & 10.9 & 13.7 & 33.2 \\
\hline Fédération de Russie & 15.4 & 7.7 & 21.4 & 13.0 & 22.1 & 21.3 & 41.0 & 58.1 \\
\hline Pays-Bas $^{1}$ & 24.5 & 25.0 & 27.2 & 24.3 & 41.4 & 24.3 & 6.9 & 26.3 \\
\hline
\end{tabular}

1. Taux de participation insuffisant pour permettre les comparaisons. 
Tableau 5.6

Milieu socio-économique ${ }^{1}$ par profil de lecteur

\begin{tabular}{|c|c|c|c|c|c|c|c|c|}
\hline & \multicolumn{2}{|c|}{$\begin{array}{l}\text { Lectures peu } \\
\text { diversifiées }\end{array}$} & \multicolumn{2}{|c|}{$\begin{array}{c}\text { Lectures modérément } \\
\text { diversifiées }\end{array}$} & \multicolumn{2}{|c|}{$\begin{array}{c}\text { Lectures diversifiées de textes } \\
\text { courts }\end{array}$} & \multicolumn{2}{|c|}{$\begin{array}{c}\text { Lectures diversifiées de textes } \\
\text { longs }\end{array}$} \\
\hline & Indice moyen & Er.T & Indice moyen & Er.T & Indice moyen & Er.T & Indice moyen & Er.T \\
\hline Australie & 50.7 & $(0.7)$ & 50.3 & $(0.6)$ & 51.8 & $(1.0)$ & 55.3 & $(0.7)$ \\
\hline Autriche & 47.7 & $(0.5)$ & 48.6 & $(0.3)$ & 50.3 & $(0.6)$ & 52.8 & $(0.5)$ \\
\hline Belgique & 47.6 & $(0.5)$ & 47.4 & $(0.6)$ & 51.4 & $(0.5)$ & 51.0 & $(0.8)$ \\
\hline Canada & 50.8 & $(0.3)$ & 52.6 & $(0.3)$ & 53.0 & $(0.4)$ & 54.8 & $(0.4)$ \\
\hline République tchèque & 47.3 & $(0.4)$ & 48.5 & $(0.4)$ & 48.1 & $(0.5)$ & 51.2 & $(0.5)$ \\
\hline Danemark & 47.3 & $(0.8)$ & 47.4 & $(0.9)$ & 50.0 & $(0.5)$ & 53.2 & $(0.9)$ \\
\hline Finlande & 48.1 & $(1.2)$ & 48.7 & $(0.7)$ & 49.9 & $(0.4)$ & 53.1 & $(0.9)$ \\
\hline France & 47.3 & $(0.5)$ & 45.9 & $(0.8)$ & 51.0 & $(0.6)$ & 47.3 & $(0.5)$ \\
\hline Allemagne & 45.7 & $(0.6)$ & 48.9 & $(0.4)$ & 50.4 & $(0.7)$ & 53.2 & $(0.6)$ \\
\hline Grèce & 45.1 & $(0.7)$ & 47.1 & $(0.9)$ & 49.9 & $(0.9)$ & 50.8 & $(0.8)$ \\
\hline Hongrie & 47.3 & $(0.6)$ & 49.4 & $(0.6)$ & 47.7 & $(0.6)$ & 53.2 & $(0.8)$ \\
\hline Islande & 49.5 & $(1.2)$ & 52.2 & $(0.6)$ & 53.1 & $(0.4)$ & 53.8 & $(0.7)$ \\
\hline Irlande & 50.1 & $(0.9)$ & 46.8 & $(0.5)$ & 46.2 & $(0.9)$ & 51.3 & $(0.6)$ \\
\hline Italie & 45.2 & $(0.5)$ & 46.1 & $(0.5)$ & 49.6 & $(0.6)$ & 47.8 & $(0.6)$ \\
\hline Japon $^{2}$ & $\mathrm{~m}$ & $\mathrm{~m}$ & $\mathrm{~m}$ & $\mathrm{~m}$ & $\mathrm{~m}$ & $\mathrm{~m}$ & $\mathrm{~m}$ & $\mathrm{~m}$ \\
\hline Corée & 38.2 & $(0.7)$ & 43.6 & $(0.7)$ & 43.8 & $(0.5)$ & 44.7 & $(0.8)$ \\
\hline Luxembourg & 42.9 & $(0.5)$ & 45.6 & $(0.7)$ & 46.9 & $(0.7)$ & 48.0 & $(0.7)$ \\
\hline Mexique & 39.1 & $(0.6)$ & 44.5 & $(1.2)$ & 45.2 & $(0.9)$ & 44.5 & $(0.9)$ \\
\hline Nouvelle-Zélande & 51.5 & $(0.7)$ & 51.5 & $(0.4)$ & 51.7 & $(0.9)$ & 53.8 & $(0.6)$ \\
\hline Norvège & 50.9 & $(0.9)$ & 52.7 & $(0.7)$ & 54.3 & $(0.4)$ & 55.9 & $(0.8)$ \\
\hline Pologne & 44.1 & $(1.0)$ & 46.2 & $(0.6)$ & 46.7 & $(0.9)$ & 47.9 & $(0.8)$ \\
\hline Portugal & 41.2 & $(0.7)$ & 44.6 & $(0.8)$ & 45.6 & $(0.8)$ & 45.7 & $(0.8)$ \\
\hline Espagne & 42.5 & $(0.7)$ & 46.1 & $(0.9)$ & 46.3 & $(0.9)$ & 47.0 & $(0.8)$ \\
\hline Suède & 48.4 & $(0.9)$ & 49.7 & $(0.5)$ & 50.1 & $(0.5)$ & 54.0 & $(0.6)$ \\
\hline Suisse & 46.1 & $(0.6)$ & 47.8 & $(0.6)$ & 51.6 & $(0.9)$ & 52.0 & $(0.7)$ \\
\hline Royaume-Uni & 51.0 & $(0.5)$ & 50.1 & $(0.4)$ & 48.5 & $(0.8)$ & 53.8 & $(0.6)$ \\
\hline États-Unis & 49.4 & $(1.0)$ & 54.7 & $(1.0)$ & 50.8 & $(1.4)$ & 54.0 & $(0.9)$ \\
\hline Moyenne de l'OCDE & 46.0 & $(0.1)$ & 48.6 & $(0.1)$ & 49.7 & $(0.1)$ & 51.6 & $(0.2)$ \\
\hline Brésil & 41.1 & $(0.7)$ & 45.6 & $(1.0)$ & 45.5 & $(0.9)$ & 45.5 & $(0.7)$ \\
\hline Lettonie & 47.9 & $(1.1)$ & 51.3 & $(0.8)$ & 47.1 & $(1.1)$ & 51.8 & $(0.7)$ \\
\hline Liechtenstein & 47.2 & $(1.9)$ & 46.2 & $(1.3)$ & 47.9 & $(3.2)$ & 51.0 & $(1.9)$ \\
\hline Fédération de Russie & 46.9 & $(0.9)$ & 48.2 & $(0.7)$ & 45.5 & $(0.6)$ & 52.4 & $(0.5)$ \\
\hline Pays-Bas ${ }^{3}$ & 47.7 & $(1.0)$ & 50.2 & $(0.8)$ & 52.5 & $(0.6)$ & 53.3 & $(0.9)$ \\
\hline
\end{tabular}

1. Le milieu socio-économique est évalue à l'aide de l'indice socio-économique de statut professionnel des parents (voir l'annexe A3 pour la définition). 2. Le Japon est exclu du tableau en raison de la quantité importante de données manquantes.

3. Taux de participation insuffisant pour permettre les comparaisons. 
Tableau 5.7

Accès à l'écrit ${ }^{1}$ par profil de lecteur

\begin{tabular}{|c|c|c|c|c|c|c|c|c|}
\hline & \multicolumn{2}{|c|}{$\begin{array}{l}\text { Lectures peu } \\
\text { diversifiées }\end{array}$} & \multicolumn{2}{|c|}{$\begin{array}{c}\text { Lectures modérément } \\
\text { diversifiées }\end{array}$} & \multicolumn{2}{|c|}{$\begin{array}{l}\text { Lectures diversifiées } \\
\text { de textes courts }\end{array}$} & \multicolumn{2}{|c|}{$\begin{array}{c}\text { Lectures diversifiées } \\
\text { de textes longs }\end{array}$} \\
\hline & Indice moyen & Er.T & Indice moyen & Er.T & Indice moyen & Er.T & Indice moyen & Er.T \\
\hline Australie & -0.39 & $(0.04)$ & -0.30 & $(0.04)$ & -0.03 & $(0.05)$ & 0.31 & $(0.03)$ \\
\hline Autriche & -0.28 & $(0.05)$ & -0.20 & $(0.03)$ & 0.05 & $(0.04)$ & 0.43 & $(0.04)$ \\
\hline Belgique & -0.57 & $(0.02)$ & -0.64 & $(0.03)$ & -0.22 & $(0.03)$ & 0.01 & $(0.04)$ \\
\hline Canada & -0.45 & $(0.02)$ & -0.31 & $(0.02)$ & -0.06 & $(0.02)$ & 0.25 & $(0.02)$ \\
\hline République tchèque & 0.12 & $(0.03)$ & 0.26 & $(0.03)$ & 0.43 & $(0.03)$ & 0.67 & $(0.02)$ \\
\hline Danemark & -0.54 & $(0.04)$ & -0.53 & $(0.06)$ & -0.09 & $(0.03)$ & 0.14 & $(0.05)$ \\
\hline Finlande & -0.45 & $(0.06)$ & -0.42 & $(0.04)$ & -0.02 & $(0.03)$ & -0.45 & $(0.06)$ \\
\hline France & -0.31 & $(0.03)$ & -0.28 & $(0.04)$ & 0.16 & $(0.03)$ & 0.29 & $(0.04)$ \\
\hline Allemagne & -0.36 & $(0.03)$ & -0.18 & $(0.03)$ & 0.18 & $(0.04)$ & 0.48 & $(0.03)$ \\
\hline Grèce & -0.10 & $(0.03)$ & -0.04 & $(0.04)$ & 0.11 & $(0.05)$ & 0.34 & $(0.03)$ \\
\hline Hongrie & 0.26 & $(0.04)$ & 0.45 & $(0.03)$ & 0.54 & $(0.04)$ & 0.86 & $(0.03)$ \\
\hline Islande & 0.23 & $(0.06)$ & 0.42 & $(0.03)$ & 0.64 & $(0.02)$ & 0.70 & $(0.03)$ \\
\hline Irlande & -0.23 & $(0.05)$ & -0.30 & $(0.03)$ & -0.02 & $(0.07)$ & 0.34 & $(0.03)$ \\
\hline Italie & 0.02 & $(0.03)$ & 0.09 & $(0.03)$ & 0.41 & $(0.03)$ & 0.49 & $(0.03)$ \\
\hline Japon & -0.59 & $(0.05)$ & -0.41 & $(0.06)$ & -0.13 & $(0.02)$ & 0.40 & $(0.07)$ \\
\hline Corée & -0.10 & $(0.03)$ & 0.32 & $(0.04)$ & 0.40 & $(0.02)$ & 0.64 & $(0.03)$ \\
\hline Luxembourg & -0.37 & $(0.03)$ & -0.18 & $(0.04)$ & 0.06 & $(0.04)$ & 0.32 & $(0.04)$ \\
\hline Mexique & -0.77 & $(0.03)$ & -0.64 & $(0.06)$ & -0.44 & $(0.05)$ & -0.32 & $(0.05)$ \\
\hline Nouvelle-Zélande & -0.51 & $(0.04)$ & -0.39 & $(0.03)$ & -0.19 & $(0.06)$ & 0.03 & $(0.03)$ \\
\hline Norvège & -0.33 & $(0.06)$ & -0.14 & $(0.04)$ & 0.19 & $(0.03)$ & 0.36 & $(0.04)$ \\
\hline Pologne & -0.02 & $(0.05)$ & 0.26 & $(0.02)$ & 0.37 & $(0.06)$ & 0.60 & $(0.04)$ \\
\hline Portugal & -0.35 & $(0.04)$ & -0.16 & $(0.04)$ & 0.16 & $(0.04)$ & 0.26 & $(0.04)$ \\
\hline Espagne & -0.01 & $(0.04)$ & 0.19 & $(0.04)$ & 0.47 & $(0.03)$ & 0.61 & $(0.03)$ \\
\hline Suède & -0.36 & $(0.04)$ & -0.19 & $(0.03)$ & 0.04 & $(0.03)$ & 0.39 & $(0.03)$ \\
\hline Suisse & -0.43 & $(0.03)$ & -0.35 & $(0.04)$ & 0.10 & $(0.04)$ & 0.28 & $(0.03)$ \\
\hline Royaume-Uni & -0.26 & $(0.04)$ & -0.32 & $(0.03)$ & -0.09 & $(0.04)$ & 0.40 & $(0.03)$ \\
\hline États-Unis & -0.48 & $(0.05)$ & -0.23 & $(0.05)$ & -0.18 & $(0.09)$ & 0.27 & $(0.04)$ \\
\hline Moyenne de l'OCDE & -0.30 & $(0.01)$ & -0.13 & $(0.01)$ & 0.11 & $(0.01)$ & 0.36 & $(0.01)$ \\
\hline Brésil & -0.78 & $(0.02)$ & -0.62 & $(0.05)$ & -0.33 & $(0.04)$ & -0.22 & $(0.04)$ \\
\hline Lettonie & 0.37 & $(0.06)$ & 0.66 & $(0.03)$ & 0.46 & $(0.05)$ & 0.89 & $(0.03)$ \\
\hline Liechtenstein & -0.22 & $(0.11)$ & -0.37 & $(0.09)$ & -0.02 & $(0.13)$ & 0.32 & $(0.10)$ \\
\hline Fédération de Russie & 0.38 & $(0.05)$ & 0.45 & $(0.04)$ & 0.50 & $(0.04)$ & 0.76 & $(0.03)$ \\
\hline Pays-Bas ${ }^{2}$ & -0.76 & $(0.03)$ & -0.62 & $(0.05)$ & -0.41 & $(0.04)$ & -0.16 & $(0.06)$ \\
\hline
\end{tabular}

1. Voir la remarque 9 du chapitre 5 pour la définition de l'indice.

2. Taux de participation insuffisant pour permettre les comparaisons. 
Tableau 5.8

Différence d'engagement à l'égard de la lecture entre les filles et les garçons

\begin{tabular}{|c|c|c|c|c|c|}
\hline & Tous les élèves & Er.T & Filles & Garçons & $\begin{array}{c}\text { Différence entre } \\
\text { les filles et les garçons }\end{array}$ \\
\hline Australie & -0.04 & $(0.03)$ & 0.11 & -0.18 & 0.28 \\
\hline Autriche & -0.08 & $(0.03)$ & 0.17 & -0.35 & 0.52 \\
\hline Belgique & -0.28 & $(0.02)$ & -0.07 & -0.48 & 0.41 \\
\hline Canada & 0.01 & $(0.01)$ & 0.24 & -0.23 & 0.47 \\
\hline République tchèque & 0.02 & $(0.02)$ & 0.29 & -0.29 & 0.57 \\
\hline Danemark & 0.26 & $(0.02)$ & 0.50 & 0.02 & 0.48 \\
\hline Finlande & 0.46 & $(0.02)$ & 0.82 & 0.08 & 0.74 \\
\hline France & -0.18 & $(0.02)$ & -0.03 & -0.33 & 0.30 \\
\hline Allemagne & -0.26 & $(0.02)$ & 0.01 & -0.53 & 0.55 \\
\hline Grèce & -0.09 & $(0.02)$ & 0.00 & -0.17 & 0.17 \\
\hline Hongrie & 0.03 & $(0.02)$ & 0.21 & -0.15 & 0.36 \\
\hline Islande & 0.27 & $(0.01)$ & 0.46 & 0.08 & 0.39 \\
\hline Irlande & -0.20 & $(0.02)$ & 0.03 & -0.43 & 0.46 \\
\hline Italie & -0.08 & $(0.02)$ & 0.10 & -0.27 & 0.37 \\
\hline Japon & 0.20 & $(0.03)$ & 0.28 & 0.11 & 0.17 \\
\hline Corée & 0.21 & $(0.02)$ & 0.23 & 0.19 & 0.04 \\
\hline Luxembourg & -0.19 & $(0.02)$ & 0.01 & -0.39 & 0.40 \\
\hline Mexique & 0.07 & $(0.01)$ & 0.17 & -0.03 & 0.20 \\
\hline Nouvelle-Zélande & 0.05 & $(0.02)$ & 0.20 & -0.09 & 0.29 \\
\hline Norvège & 0.09 & $(0.02)$ & 0.35 & -0.16 & 0.51 \\
\hline Pologne & -0.10 & $(0.02)$ & 0.09 & -0.28 & 0.36 \\
\hline Portugal & 0.13 & $(0.02)$ & 0.36 & -0.11 & 0.47 \\
\hline Espagne & -0.23 & $(0.02)$ & -0.09 & -0.38 & 0.29 \\
\hline Suède & 0.14 & $(0.02)$ & 0.37 & -0.08 & 0.45 \\
\hline Suisse & 0.00 & $(0.01)$ & 0.31 & -0.31 & 0.62 \\
\hline Royaume-Uni & -0.10 & $(0.02)$ & 0.03 & -0.24 & 0.26 \\
\hline États-Unis & -0.14 & $(0.03)$ & 0.04 & -0.32 & 0.36 \\
\hline Moyenne de I'OCDE & 0.00 & $\sim$ & 0.19 & -0.19 & 0.38 \\
\hline Brésil & 0.11 & $(0.02)$ & 0.36 & -0.17 & 0.53 \\
\hline Lettonie & -0.04 & $(0.02)$ & 0.17 & -0.27 & 0.44 \\
\hline Liechtenstein & -0.13 & $(0.05)$ & 0.13 & -0.36 & 0.49 \\
\hline Fédération de Russie & 0.17 & $(0.02)$ & 0.37 & -0.02 & 0.39 \\
\hline Pays-Bas ${ }^{1}$ & -0.17 & $(0.04)$ & 0.04 & -0.38 & 0.42 \\
\hline
\end{tabular}

1. Taux de participation insuffisant pour permettre les comparaisons.

Tableau 5.9

Performance sur l'échelle combinée de compréhension de l'écrit en fonction du milieu socio-économique ${ }^{1}$ et du degré d'engagement à l'égard de la lecture

\begin{tabular}{lccc}
\hline & $\begin{array}{c}\text { Degré faible d'engagement } \\
\text { à l'égard de la lecture }\end{array}$ & $\begin{array}{c}\text { Degré moyen d'engagement } \\
\text { à l'égard de la lecture }\end{array}$ & $\begin{array}{c}\text { Degré élevé d'engagement } \\
\text { à l'égard de la lecture }\end{array}$ \\
\hline Milieu socio-économique inférieur & 423 & 467 & 540 \\
Milieu socio-économique moyen & 463 & 506 & 548 \\
Milieu socio-économique supérieur & 491 & 540 & 583 \\
\hline
\end{tabular}

1. Voir l'annexe A3 pour la définition de l'indice. 
Tableau 6.1

Corrélation entre les caractéristiques individuelles des élèves et la performance sur l'échelle combinée de compréhension de l'écrit

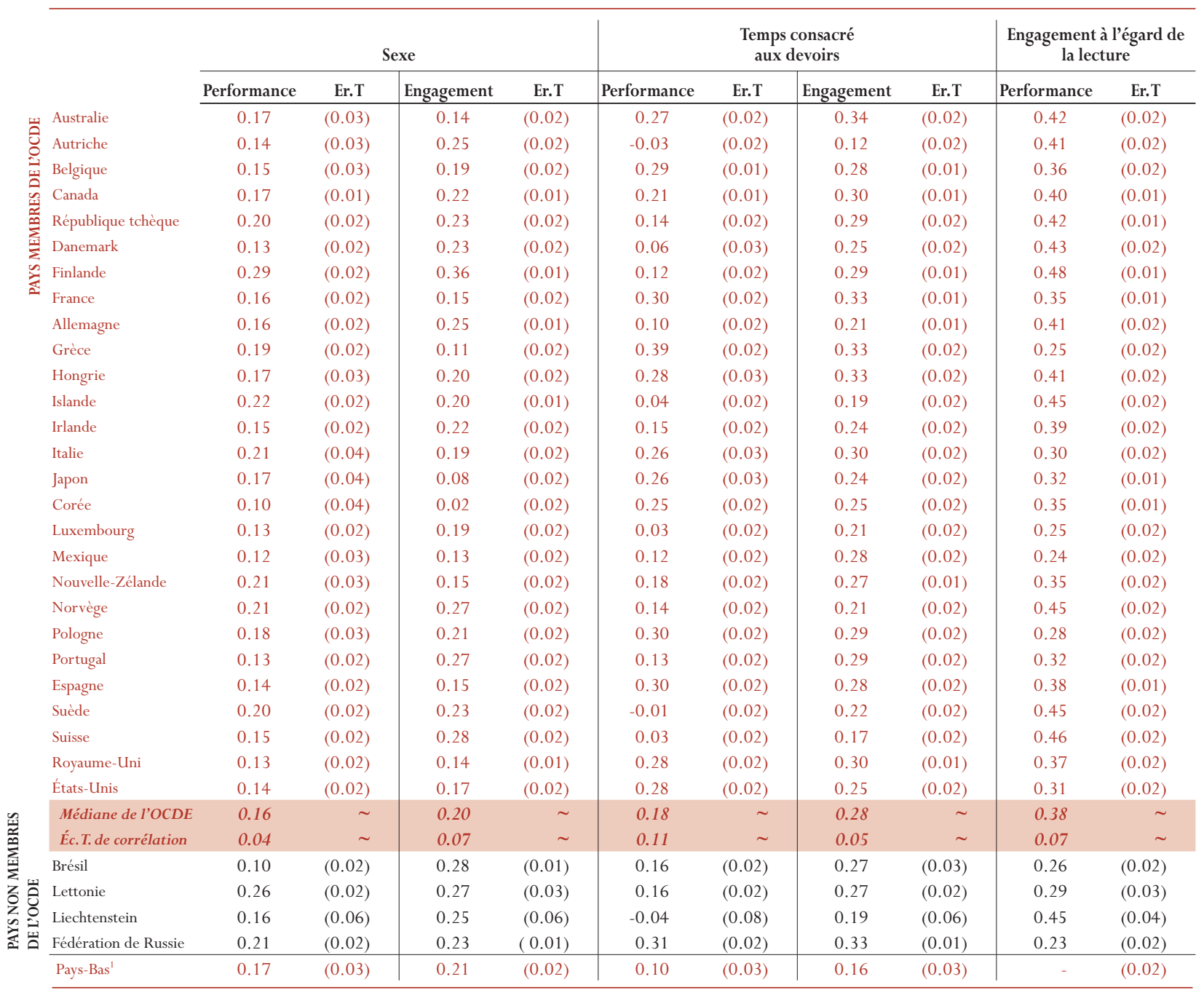

1. Taux de participation insuffisant pour permettre les comparaisons. 
Tableau 6.2a

Corrélation entre le sexe et la performance en compréhension de l'écrit

\begin{tabular}{|c|c|c|c|c|c|c|}
\hline & $\begin{array}{c}\text { Échelle combinée } \\
\text { de compréhension } \\
\text { de l'écrit }\end{array}$ & $\begin{array}{c}\text { Trouver } \\
\text { l'information }\end{array}$ & $\begin{array}{l}\text { Développer une } \\
\text { interprétation }\end{array}$ & $\begin{array}{c}\text { Réfléchir et } \\
\text { évaluer }\end{array}$ & $\begin{array}{c}\text { Textes } \\
\text { continus }\end{array}$ & $\begin{array}{c}\text { Textes non } \\
\text { continus }\end{array}$ \\
\hline Australie & 0.17 & 0.13 & 0.16 & 0.21 & 0.19 & 0.10 \\
\hline Autriche & 0.14 & 0.08 & 0.12 & 0.20 & 0.18 & 0.04 \\
\hline Belgique & 0.15 & 0.11 & 0.15 & 0.20 & 0.19 & 0.09 \\
\hline Canada & 0.17 & 0.12 & 0.15 & 0.24 & 0.20 & 0.09 \\
\hline République tchèque & 0.20 & 0.13 & 0.18 & 0.26 & 0.24 & 0.11 \\
\hline Danemark & 0.13 & 0.07 & 0.11 & 0.21 & 0.16 & 0.04 \\
\hline Finlande & 0.29 & 0.22 & 0.26 & 0.35 & 0.33 & 0.18 \\
\hline France & 0.16 & 0.11 & 0.15 & 0.20 & 0.19 & 0.07 \\
\hline Allemagne & 0.16 & 0.11 & 0.15 & 0.19 & 0.19 & 0.07 \\
\hline Grèce & 0.19 & 0.15 & 0.18 & 0.23 & 0.23 & 0.10 \\
\hline Hongrie & 0.17 & 0.12 & 0.16 & 0.22 & 0.20 & 0.09 \\
\hline Islande & 0.22 & 0.16 & 0.20 & 0.29 & 0.24 & 0.14 \\
\hline Irlande & 0.15 & 0.11 & 0.14 & 0.21 & 0.18 & 0.08 \\
\hline Italie & 0.21 & 0.15 & 0.23 & 0.24 & 0.24 & 0.10 \\
\hline Japon & 0.17 & 0.14 & 0.15 & 0.21 & 0.20 & 0.10 \\
\hline Corée & 0.10 & 0.04 & 0.06 & 0.18 & 0.13 & 0.03 \\
\hline Luxembourg & 0.13 & 0.09 & 0.13 & 0.18 & 0.18 & 0.06 \\
\hline Mexique & 0.12 & 0.06 & 0.11 & 0.16 & 0.15 & 0.05 \\
\hline Nouvelle-Zélande & 0.21 & 0.17 & 0.20 & 0.27 & 0.24 & 0.16 \\
\hline Norvège & 0.21 & 0.15 & 0.20 & 0.28 & 0.26 & 0.10 \\
\hline Pologne & 0.18 & 0.13 & 0.18 & 0.24 & 0.23 & 0.07 \\
\hline Portugal & 0.13 & 0.08 & 0.13 & 0.18 & 0.16 & 0.06 \\
\hline Espagne & 0.14 & 0.09 & 0.13 & 0.21 & 0.18 & 0.06 \\
\hline Suède & 0.20 & 0.15 & 0.18 & 0.27 & 0.24 & 0.10 \\
\hline Suisse & 0.15 & 0.10 & 0.13 & 0.20 & 0.19 & 0.04 \\
\hline Royaume-Uni & 0.13 & 0.09 & 0.12 & 0.18 & 0.15 & 0.06 \\
\hline États-Unis & 0.14 & 0.12 & 0.13 & 0.17 & 0.16 & 0.10 \\
\hline Médiane de l'OCDE & 0.16 & 0.12 & 0.15 & 0.21 & 0.19 & 0.09 \\
\hline Brésil & 0.10 & 0.05 & 0.08 & 0.13 & 0.12 & 0.03 \\
\hline Lettonie & 0.26 & 0.20 & 0.27 & 0.31 & 0.29 & 0.20 \\
\hline Liechtenstein & 0.16 & 0.10 & 0.12 & 0.21 & 0.19 & 0.04 \\
\hline Fédération de Russie & 0.21 & 0.16 & 0.19 & 0.25 & 0.24 & 0.13 \\
\hline Pays-Bas $^{1}$ & 0.17 & 0.11 & 0.17 & 0.21 & 0.20 & 0.07 \\
\hline
\end{tabular}

Régression de la performance en compréhension de l'écrit sur le sexe ${ }^{2}$




Tableau 6.2b

Corrélation entre le temps consacré aux devoirs et la performance en compréhension de l'écrit

\begin{tabular}{|c|c|c|c|c|c|c|}
\hline & $\begin{array}{c}\text { Échelle combinée de } \\
\text { compréhension de } \\
\text { l'écrit }\end{array}$ & $\begin{array}{c}\text { Trouver } \\
\text { l'information }\end{array}$ & $\begin{array}{c}\text { Développer une } \\
\text { interprétation }\end{array}$ & $\begin{array}{l}\text { Réfléchir } \\
\text { et évaluer }\end{array}$ & $\begin{array}{c}\text { Textes } \\
\text { continus }\end{array}$ & $\begin{array}{c}\text { Textes non } \\
\text { continus }\end{array}$ \\
\hline Australie & 0.27 & 0.26 & 0.26 & 0.28 & 0.26 & 0.27 \\
\hline Autriche & -0.03 & -0.04 & -0.04 & 0.00 & -0.02 & -0.03 \\
\hline Belgique & 0.29 & 0.28 & 0.28 & 0.32 & 0.30 & 0.28 \\
\hline Canada & 0.21 & 0.19 & 0.19 & 0.24 & 0.21 & 0.19 \\
\hline République tchèque & 0.14 & 0.10 & 0.13 & 0.16 & 0.15 & 0.11 \\
\hline Danemark & 0.06 & 0.06 & 0.05 & 0.08 & 0.07 & 0.06 \\
\hline Finlande & 0.12 & 0.09 & 0.10 & 0.15 & 0.13 & 0.10 \\
\hline France & 0.30 & 0.29 & 0.29 & 0.29 & 0.30 & 0.28 \\
\hline Allemagne & 0.10 & 0.08 & 0.09 & 0.11 & 0.10 & 0.07 \\
\hline Grèce & 0.39 & 0.36 & 0.39 & 0.38 & 0.39 & 0.36 \\
\hline Hongrie & 0.28 & 0.26 & 0.27 & 0.30 & 0.28 & 0.25 \\
\hline Islande & 0.04 & 0.02 & 0.03 & 0.08 & 0.05 & 0.01 \\
\hline Irlande & 0.15 & 0.14 & 0.14 & 0.18 & 0.15 & 0.14 \\
\hline Italie & 0.26 & 0.25 & 0.26 & 0.28 & 0.27 & 0.23 \\
\hline Japon & 0.26 & 0.23 & 0.24 & 0.25 & 0.25 & 0.23 \\
\hline Corée & 0.25 & 0.24 & 0.25 & 0.23 & 0.24 & 0.25 \\
\hline Luxembourg & 0.03 & -0.01 & 0.03 & 0.03 & 0.05 & 0.02 \\
\hline Mexique & 0.12 & 0.11 & 0.12 & 0.10 & 0.12 & 0.10 \\
\hline Nouvelle-Zélande & 0.18 & 0.18 & 0.16 & 0.19 & 0.17 & 0.19 \\
\hline Norvège & 0.14 & 0.12 & 0.11 & 0.17 & 0.15 & 0.12 \\
\hline Pologne & 0.30 & 0.25 & 0.29 & 0.33 & 0.31 & 0.25 \\
\hline Portugal & 0.13 & 0.13 & 0.11 & 0.16 & 0.13 & 0.12 \\
\hline Espagne & 0.30 & 0.30 & 0.27 & 0.30 & 0.29 & 0.29 \\
\hline Suède & -0.01 & -0.03 & -0.03 & 0.04 & 0.00 & -0.03 \\
\hline Suisse & 0.03 & 0.02 & 0.03 & 0.04 & 0.04 & 0.01 \\
\hline Royaume-Uni & 0.28 & 0.28 & 0.26 & 0.31 & 0.28 & 0.27 \\
\hline États-Unis & 0.28 & 0.26 & 0.27 & 0.30 & 0.28 & 0.25 \\
\hline Médiane de l'OCDE & 0.18 & 0.18 & 0.16 & 0.19 & 0.17 & 0.19 \\
\hline Brésil & 0.16 & 0.13 & 0.15 & 0.18 & 0.16 & 0.13 \\
\hline Lettonie & 0.16 & 0.15 & 0.17 & 0.15 & 0.16 & 0.14 \\
\hline Liechtenstein & -0.04 & -0.05 & -0.02 & 0.00 & -0.02 & -0.03 \\
\hline Fédération de Russie & 0.31 & 0.30 & 0.31 & 0.30 & 0.31 & 0.29 \\
\hline Pays-Bas $^{1}$ & 0.10 & 0.11 & 0.08 & 0.10 & 0.10 & 0.08 \\
\hline
\end{tabular}

Régression de la performance en compréhension de l'écrit sur le temps consacré aux devoirs²

\begin{tabular}{|c|c|c|c|c|c|c|}
\hline & $\begin{array}{c}\text { Échelle combinée de } \\
\text { compréhension } \\
\text { de l'écrit }\end{array}$ & $\begin{array}{c}\text { Trouver } \\
\text { l'information }\end{array}$ & $\begin{array}{l}\text { Développer une } \\
\text { interprétation }\end{array}$ & $\begin{array}{l}\text { Réfléchir } \\
\text { et évaluer }\end{array}$ & $\begin{array}{c}\text { Textes } \\
\text { continus }\end{array}$ & $\begin{array}{c}\text { Textes non } \\
\text { continus }\end{array}$ \\
\hline Australie & 27.31 & 27.82 & 26.96 & 28.19 & 27.42 & 27.99 \\
\hline Autriche & -2.85 & -4.15 & -4.19 & -0.32 & -2.43 & -3.62 \\
\hline Belgique & 32.94 & 35.04 & 30.63 & 37.93 & 34.16 & 33.06 \\
\hline Canada & 18.99 & 18.45 & 17.56 & 22.15 & 19.48 & 17.54 \\
\hline République tchèque & 12.58 & 10.13 & 11.96 & 15.42 & 13.33 & 11.24 \\
\hline Danemark & 7.55 & 8.05 & 5.65 & 9.44 & 7.90 & 7.18 \\
\hline Finlande & 12.97 & 11.24 & 11.68 & 15.91 & 13.47 & 11.23 \\
\hline France & 31.58 & 33.26 & 30.82 & 32.75 & 32.13 & 30.80 \\
\hline Allemagne & 11.57 & 9.75 & 10.39 & 14.80 & 12.74 & 8.85 \\
\hline Grèce & 34.28 & 35.52 & 31.30 & 39.23 & 35.09 & 33.37 \\
\hline Hongrie & 32.67 & 34.55 & 30.02 & 36.82 & 32.28 & 33.77 \\
\hline Islande & 4.17 & 2.33 & 2.98 & 8.83 & 5.46 & 1.27 \\
\hline Irlande & 13.05 & 13.44 & 12.80 & 14.74 & 12.96 & 12.92 \\
\hline Italie & 26.61 & 28.21 & 24.69 & 30.63 & 26.57 & 25.79 \\
\hline Japon & 17.70 & 18.18 & 16.14 & 20.05 & 17.27 & 18.51 \\
\hline Corée & 13.24 & 14.86 & 13.12 & 13.24 & 12.73 & 15.51 \\
\hline Luxembourg & 2.58 & -1.12 & 3.05 & 3.97 & 5.87 & 2.77 \\
\hline Mexique & 11.27 & 11.65 & 10.27 & 12.29 & 11.51 & 10.46 \\
\hline Nouvelle-Zélande & 20.07 & 20.64 & 18.48 & 20.50 & 18.49 & 21.46 \\
\hline Norvège & 15.05 & 13.86 & 12.22 & 19.73 & 15.92 & 14.64 \\
\hline Pologne & 30.28 & 28.64 & 29.04 & 36.82 & 32.32 & 27.64 \\
\hline Portugal & 12.28 & 13.44 & 10.07 & 15.66 & 13.04 & 11.97 \\
\hline Espagne & 24.65 & 26.62 & 22.18 & 26.70 & 23.31 & 27.58 \\
\hline Suède & -1.01 & -2.98 & -3.15 & 3.88 & -0.35 & -3.34 \\
\hline Suisse & 3.38 & 2.05 & 3.15 & 4.89 & 4.59 & 0.75 \\
\hline Royaume-Uni & 31.92 & 32.91 & 30.61 & 34.96 & 33.22 & 30.44 \\
\hline États-Unis & 26.53 & 26.46 & 26.49 & 28.96 & 26.99 & 24.96 \\
\hline Médiane de l'OCDE & 15.05 & 14.86 & 13.12 & 19.73 & 15.92 & 15.51 \\
\hline Brésil & 14.13 & 12.28 & 12.48 & 17.08 & 14.53 & 12.80 \\
\hline Lettonie & 16.91 & 18.42 & 17.07 & 18.22 & 17.69 & 16.17 \\
\hline Liechtenstein & -4.94 & -6.73 & -2.06 & 0.36 & -2.15 & -3.28 \\
\hline Fédération de Russie & 25.93 & 28.55 & 25.44 & 26.16 & 25.45 & 26.63 \\
\hline Pays-Bas ${ }^{1}$ & 10.38 & 13.36 & 8.99 & 9.29 & 10.92 & 8.70 \\
\hline
\end{tabular}


Tableau 6.2c

Corrélation entre le degré d'engagement à l'égard de la lecture et la performance en compréhension de l'écrit

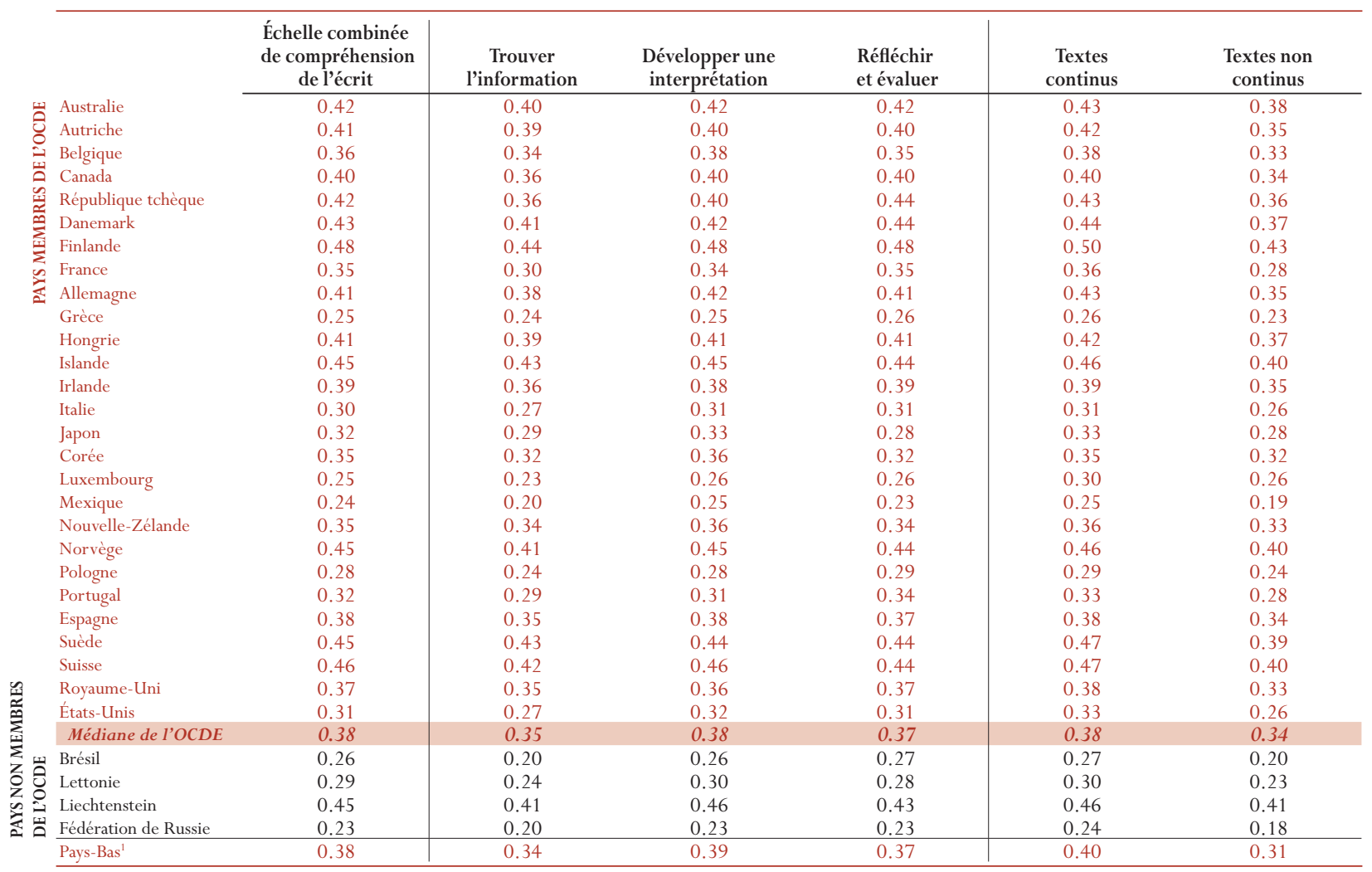

Régression de la performance en compréhension de l'écrit sur le degré d'engagement à l'égard de la lecture²

\begin{tabular}{|c|c|c|c|c|c|c|}
\hline 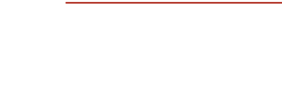 & $\begin{array}{c}\text { Échelle combinée de } \\
\text { compréhension de } \\
\text { l'écrit }\end{array}$ & $\begin{array}{c}\text { Trouver } \\
\text { l'information }\end{array}$ & $\begin{array}{l}\text { Développer une } \\
\text { interprétation }\end{array}$ & $\begin{array}{l}\text { Réfléchir } \\
\text { et évaluer }\end{array}$ & $\begin{array}{c}\text { Textes } \\
\text { continus }\end{array}$ & $\begin{array}{c}\text { Textes non } \\
\text { continus }\end{array}$ \\
\hline \multirow{33}{*}{ 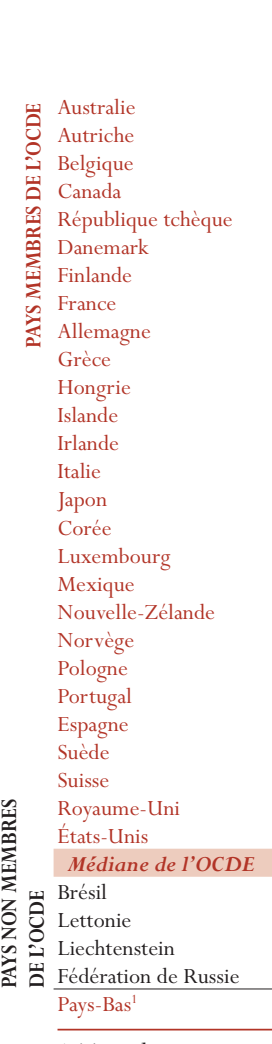 } & 42.52 & 42.76 & $\frac{1}{43.65}$ & 41.72 & 44.73 & 39.59 \\
\hline & 36.76 & 36.23 & 36.44 & 38.51 & 38.45 & 33.64 \\
\hline & 35.83 & 37.06 & 37.36 & 36.75 & 37.58 & 34.26 \\
\hline & 35.14 & 34.36 & 35.85 & 35.40 & 36.86 & 31.65 \\
\hline & 38.10 & 37.18 & 37.36 & 42.89 & 38.48 & 37.99 \\
\hline & 40.99 & 41.64 & 40.66 & 43.91 & 42.27 & 39.09 \\
\hline & 42.48 & 43.60 & 45.51 & 43.07 & 43.67 & 40.62 \\
\hline & 31.18 & 30.27 & 31.14 & 33.18 & 32.85 & 26.96 \\
\hline & 37.57 & 35.91 & 38.56 & 41.41 & 40.41 & 33.13 \\
\hline & 33.31 & 34.62 & 29.86 & 40.26 & 34.63 & 32.35 \\
\hline & 43.71 & 46.79 & 41.12 & 46.44 & 43.20 & 45.45 \\
\hline & 42.11 & 44.52 & 43.06 & 41.08 & 43.70 & 39.75 \\
\hline & 35.18 & 34.83 & 36.12 & 33.29 & 35.67 & 33.36 \\
\hline & 28.54 & 29.18 & 27.39 & 32.10 & 29.17 & 27.12 \\
\hline & 26.87 & 27.33 & 27.00 & 27.65 & 27.75 & 26.73 \\
\hline & 24.96 & 26.80 & 25.19 & 24.64 & 24.32 & 25.87 \\
\hline & 22.02 & 21.98 & 22.67 & 25.90 & 28.86 & 26.57 \\
\hline & 26.93 & 26.77 & 25.00 & 32.36 & 28.48 & 23.57 \\
\hline & 39.75 & 40.24 & 41.15 & 37.61 & 40.81 & 37.20 \\
\hline & 47.25 & 46.32 & 48.06 & 48.20 & 48.56 & 46.41 \\
\hline & 30.84 & 29.78 & 30.97 & 35.39 & 33.06 & 28.44 \\
\hline & 35.14 & 35.82 & 32.90 & 38.92 & 36.59 & 32.59 \\
\hline & 32.38 & 32.82 & 31.95 & 34.09 & 32.16 & 32.78 \\
\hline & 41.53 & 44.59 & 42.30 & 41.70 & 43.74 & 38.70 \\
\hline & 41.23 & 41.78 & 41.61 & 44.34 & 43.23 & 38.66 \\
\hline & 37.95 & 36.67 & 37.88 & 37.55 & 39.89 & 33.57 \\
\hline & 29.32 & 27.43 & 30.87 & 29.52 & 31.43 & 25.77 \\
\hline & 35.83 & 35.91 & 36.44 & 37.61 & 37.58 & 33.36 \\
\hline & 23.76 & 20.44 & 23.05 & 26.43 & 24.95 & 20.08 \\
\hline & 36.19 & 34.85 & 35.60 & 38.90 & 38.89 & 31.40 \\
\hline & 43.81 & 44.59 & 43.84 & 47.42 & 45.40 & 41.01 \\
\hline & 24.82 & 24.83 & 25.06 & 26.39 & 26.20 & 21.30 \\
\hline & 34.09 & 34.85 & 36.75 & 31.61 & 36.34 & 28.43 \\
\hline
\end{tabular}

1. Taux de participation insuffisant pour permettre les comparaisons.

2. Ces chiffres indiquent de combien varie la performance en compréhension de l'écrit si on augmente d'une unité l'indice de l'engagement à l'égard de la lecture. 
Tableau 6.3

Corrélation entre le milieu familial des élèves et la performance en compréhension de l'écrit d'une part, l'engagement à l'égard de la lecture d'autre part

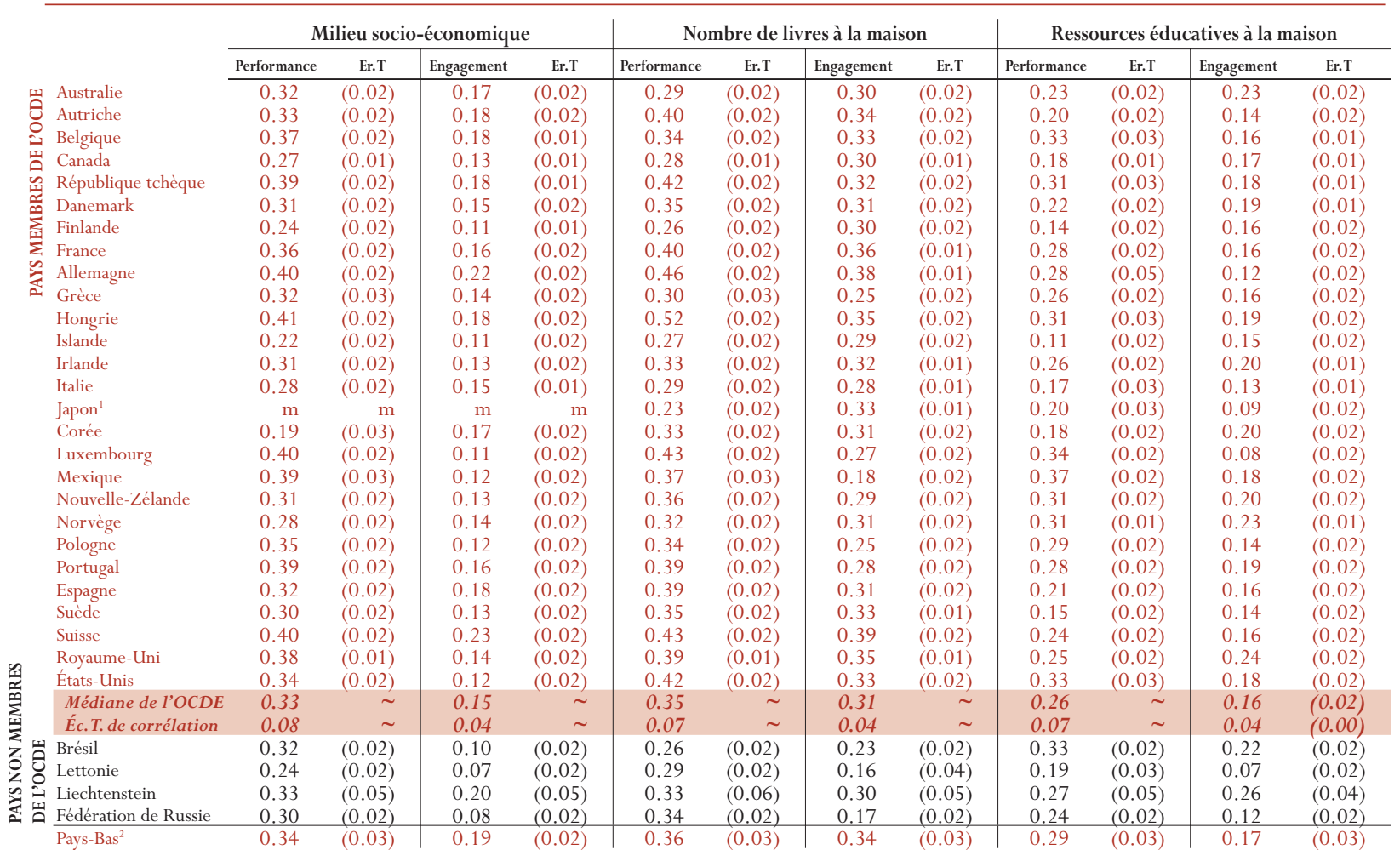

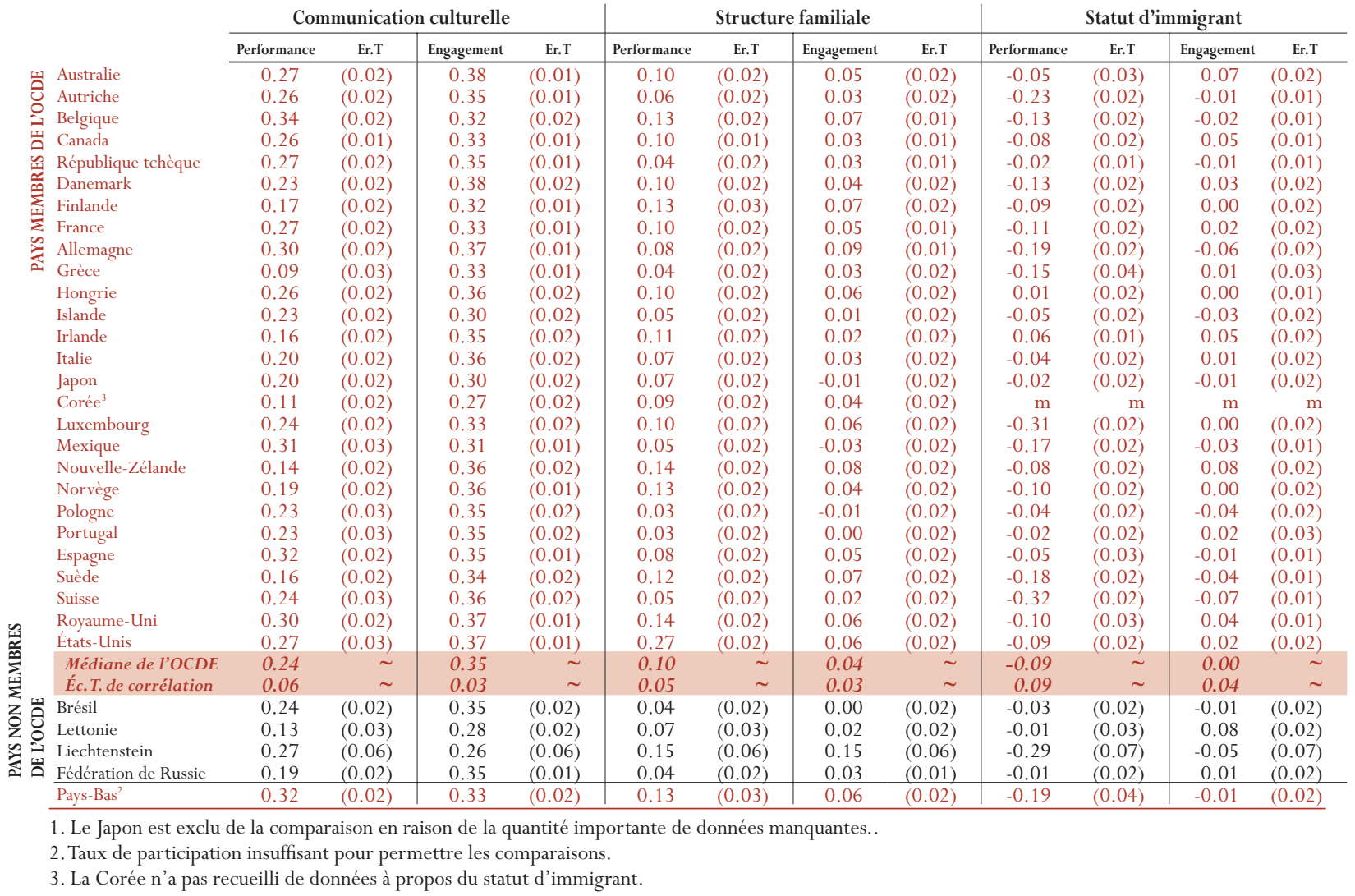


Tableau 6.4a

Corrélation entre le milieu socio-économique et la performance en compréhension de l'écrit

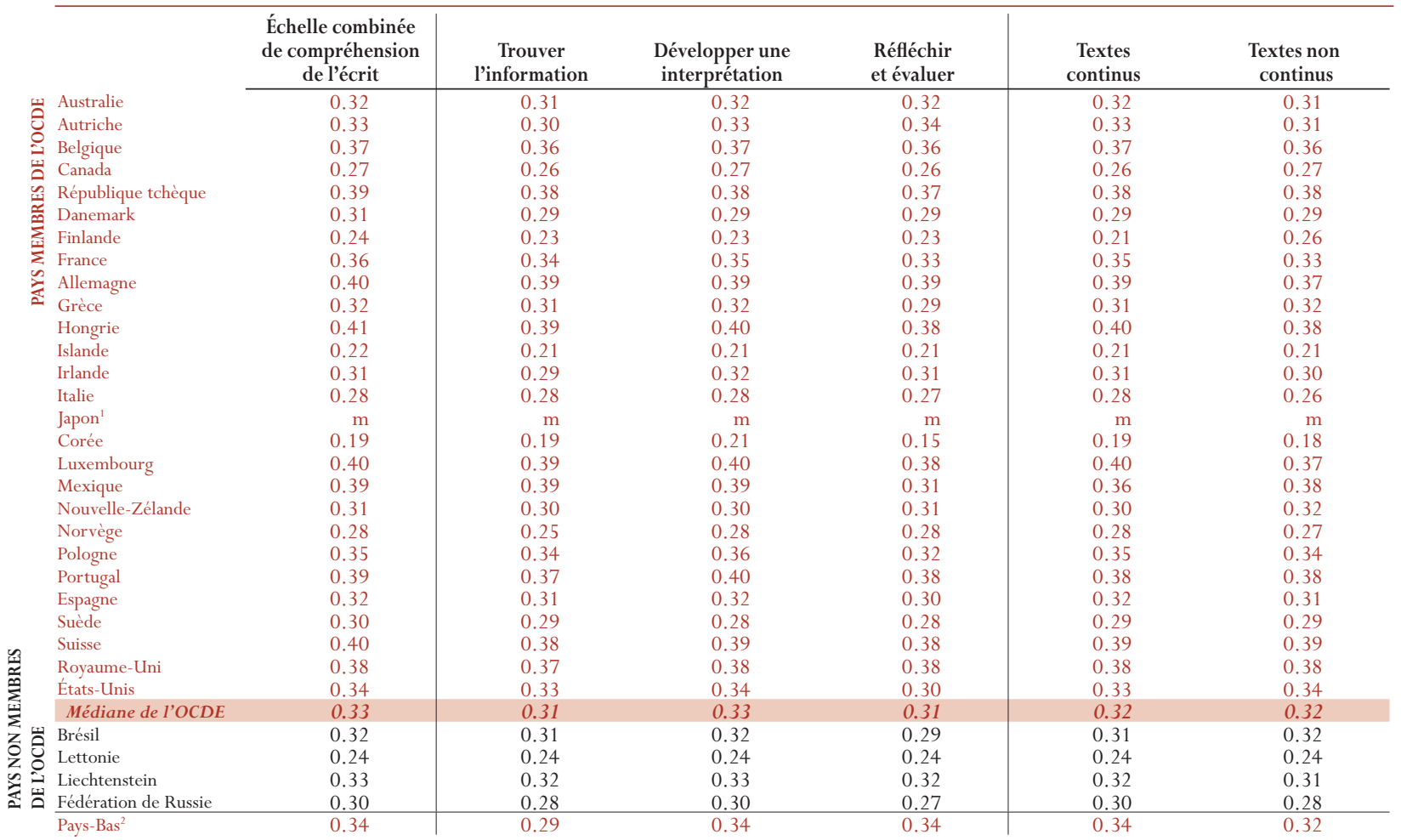

Régression de la performance en compréhension de l'écrit sur le milieu socio-économique ${ }^{3}$

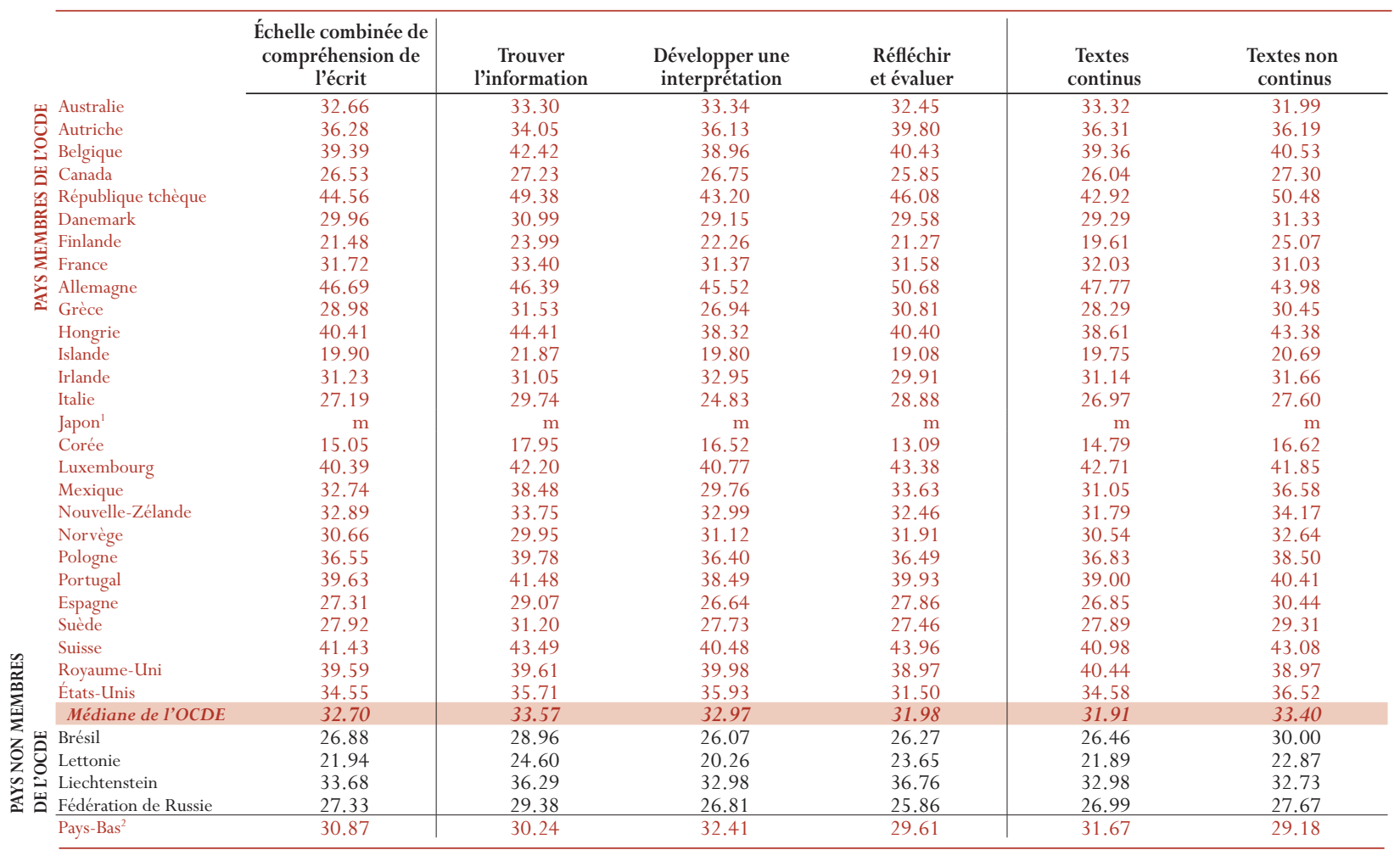

1. Le Japon est exclu de la comparaison en raison de la quantité importante de données manquantes.

2. Taux de participation insuffisant pour permettre les comparaisons.

3. Ces chiffres indiquent de combien varie la performance en compréhension de l'écrit si on augmente d'une unité l'indice socio-économique international. 
ANNEXE B

Tableau 6.4b

Corrélation entre le nombre de livres à la maison et la performance en compréhension de l'écrit



Régression du degré d'engagement à l'égard de la lecture et de la performance en compréhension de l'écrit sur le nombre de livres à la maison ${ }^{2}$

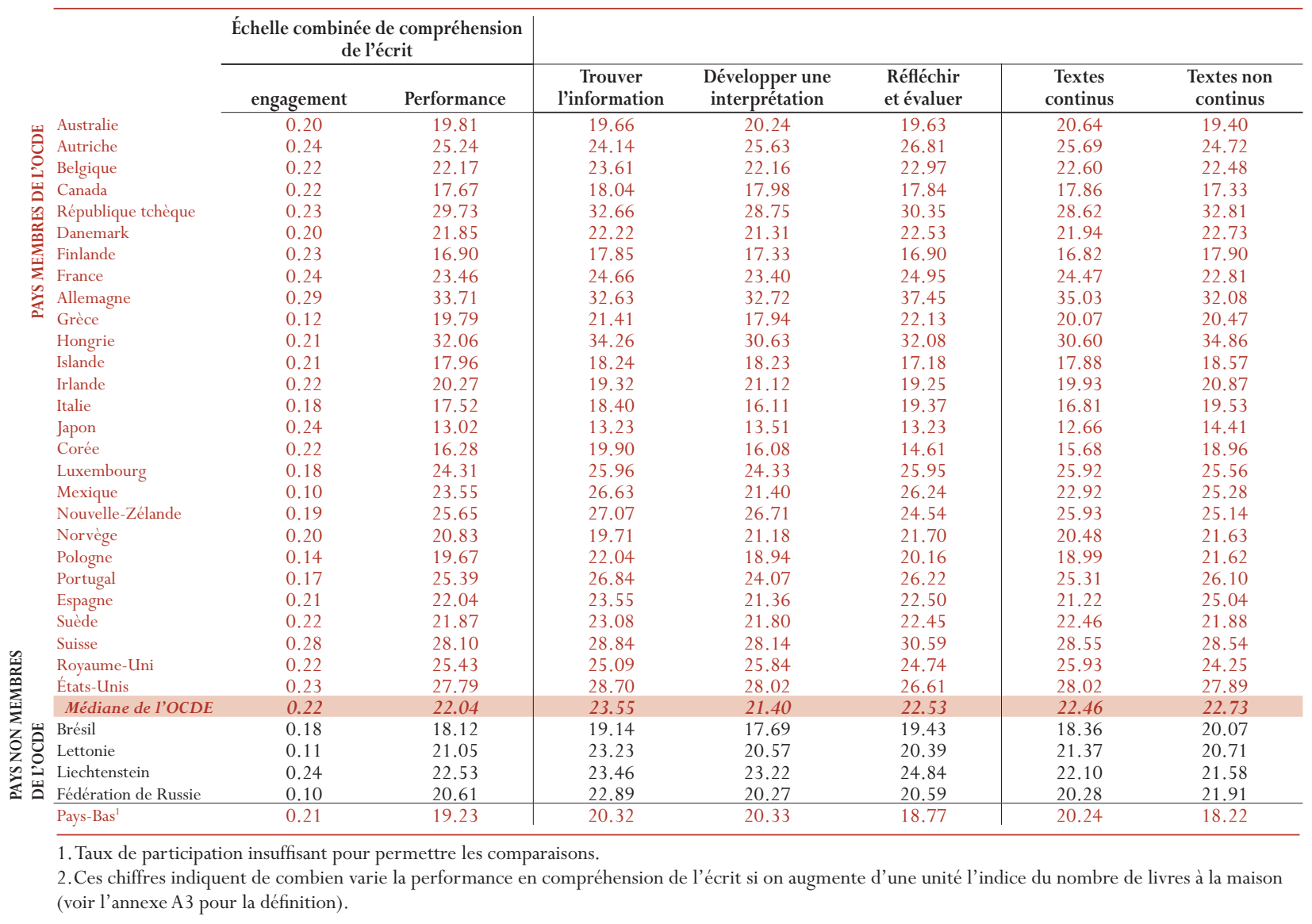


Tableau $6.4 \mathrm{c}$

Corrélation entre les ressources éducatives à la maison et la performance en compréhension de l'écrit

\begin{tabular}{|c|c|c|c|c|c|c|}
\hline & $\begin{array}{c}\text { Échelle combinée de } \\
\text { compréhension } \\
\text { de l'écrit }\end{array}$ & $\begin{array}{c}\text { Trouver } \\
\text { l'information }\end{array}$ & $\begin{array}{l}\text { Développer une } \\
\text { interprétation }\end{array}$ & $\begin{array}{l}\text { Réfléchir } \\
\text { et évaluer }\end{array}$ & $\begin{array}{l}\text { Textes } \\
\text { continus }\end{array}$ & $\begin{array}{c}\text { Textes non } \\
\text { continus }\end{array}$ \\
\hline Australie & 0.23 & 0.23 & 0.22 & 0.24 & 0.22 & 0.24 \\
\hline Оิ Autriche & 0.20 & 0.20 & 0.19 & 0.19 & 0.19 & 0.21 \\
\hline Belgique & 0.33 & 0.33 & 0.31 & 0.35 & 0.33 & 0.33 \\
\hline Canada & 0.18 & 0.18 & 0.16 & 0.19 & 0.17 & 0.18 \\
\hline République tchèque & 0.31 & 0.32 & 0.30 & 0.29 & 0.31 & 0.32 \\
\hline है Danemark & 0.22 & 0.21 & 0.21 & 0.21 & 0.22 & 0.22 \\
\hline 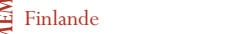 & 0.14 & 0.14 & 0.12 & 0.14 & 0.12 & 0.15 \\
\hline$\sum_{\infty}$ France & 0.28 & 0.29 & 0.27 & 0.26 & 0.28 & 0.26 \\
\hline Allemagne & 0.28 & 0.29 & 0.27 & 0.27 & 0.27 & 0.28 \\
\hline Grèce & 0.26 & 0.29 & 0.25 & 0.23 & 0.24 & 0.27 \\
\hline Hongrie & 0.31 & 0.31 & 0.30 & 0.29 & 0.29 & 0.31 \\
\hline Islande & 0.11 & 0.11 & 0.10 & 0.11 & 0.11 & 0.10 \\
\hline Irlande & 0.26 & 0.25 & 0.25 & 0.26 & 0.25 & 0.26 \\
\hline Italie & 0.17 & 0.16 & 0.15 & 0.18 & 0.16 & 0.17 \\
\hline Japon & 0.20 & 0.19 & 0.19 & 0.16 & 0.19 & 0.18 \\
\hline Corée & 0.18 & 0.18 & 0.18 & 0.15 & 0.17 & 0.18 \\
\hline Luxembourg & 0.34 & 0.33 & 0.33 & 0.34 & 0.30 & 0.31 \\
\hline Mexique & 0.37 & 0.37 & 0.37 & 0.30 & 0.35 & 0.37 \\
\hline Nouvelle-Zélande & 0.31 & 0.32 & 0.30 & 0.31 & 0.30 & 0.34 \\
\hline Norvège & 0.31 & 0.30 & 0.30 & 0.31 & 0.30 & 0.31 \\
\hline Pologne & 0.29 & 0.30 & 0.28 & 0.26 & 0.27 & 0.30 \\
\hline Portugal & 0.28 & 0.28 & 0.28 & 0.27 & 0.28 & 0.27 \\
\hline Espagne & 0.21 & 0.21 & 0.21 & 0.20 & 0.21 & 0.20 \\
\hline Suède & 0.15 & 0.14 & 0.12 & 0.16 & 0.14 & 0.15 \\
\hline Suisse & 0.24 & 0.23 & 0.24 & 0.26 & 0.24 & 0.22 \\
\hline Royaume-Uni & 0.25 & 0.25 & 0.24 & 0.27 & 0.25 & 0.25 \\
\hline États-Unis & 0.33 & 0.33 & 0.33 & 0.32 & 0.33 & 0.34 \\
\hline Médiane de l'OCDE & 0.26 & 0.25 & 0.25 & 0.26 & 0.25 & 0.26 \\
\hline Brésil & 0.33 & 0.31 & 0.32 & 0.32 & 0.32 & 0.33 \\
\hline ర్ Lettonie & 0.19 & 0.21 & 0.19 & 0.17 & 0.18 & 0.19 \\
\hline Liechtenstein & 0.27 & 0.27 & 0.27 & 0.25 & 0.28 & 0.24 \\
\hline Fédération de Russie & 0.24 & 0.24 & 0.23 & 0.22 & 0.23 & 0.24 \\
\hline Pays-Bas $^{1}$ & 0.29 & 0.29 & 0.28 & 0.28 & 0.28 & 0.30 \\
\hline
\end{tabular}

Régression de la performance en compréhension de l'écrit sur les ressources éducatives à la maison ${ }^{2}$

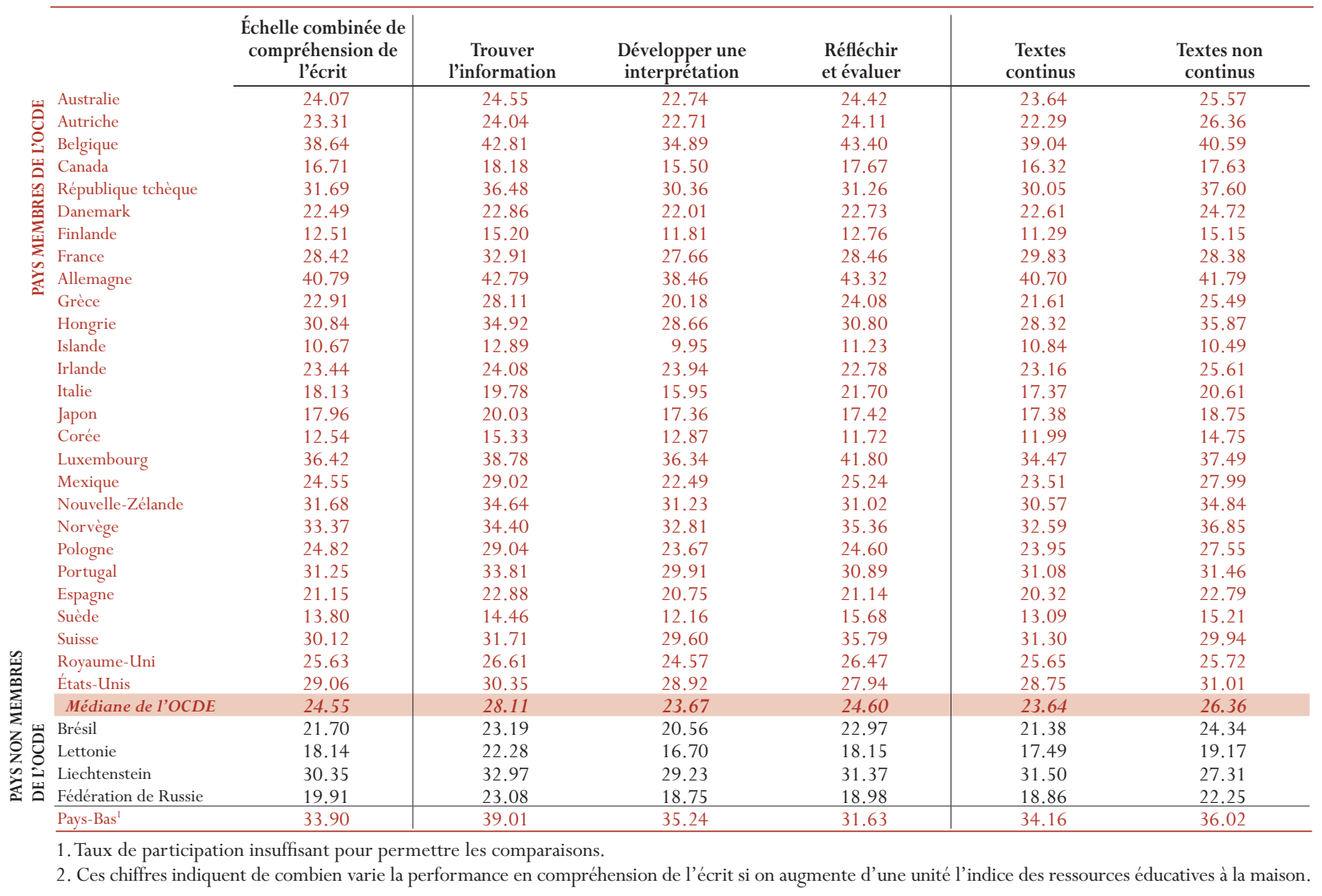


Tableau 6.4d

Corrélation entre la communication culturelle à la maison et la performance en compréhension de l'écrit



Régression de la performance en compréhension de l'écrit sur la communication culturelle à la maison ${ }^{2}$

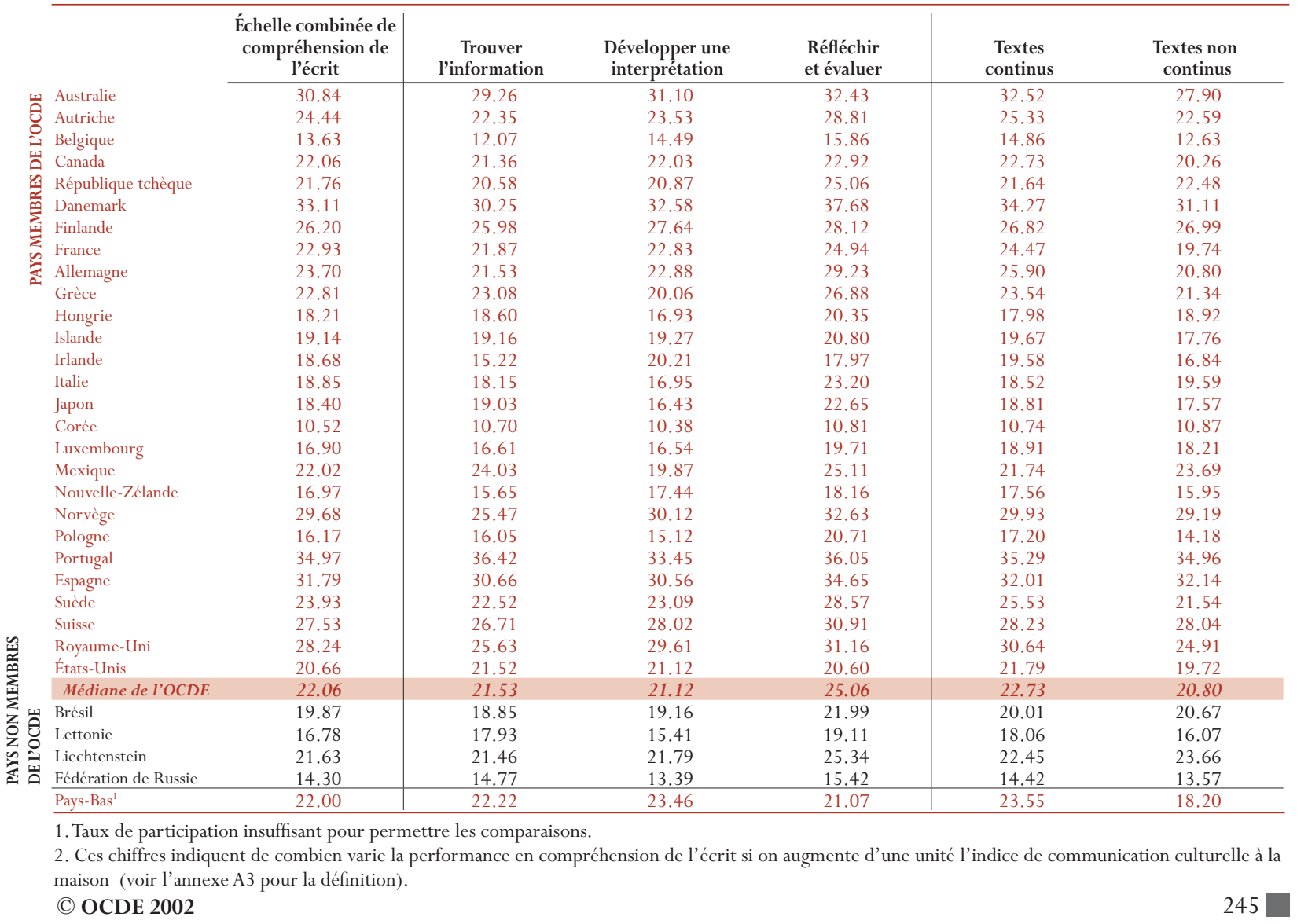


Tableau $6.4 \mathrm{e}$

Corrélation entre la structure familiale et la performance en compréhension de l'écrit

\begin{tabular}{|c|c|c|c|c|c|c|}
\hline & $\begin{array}{c}\text { Échelle combinée de } \\
\text { compréhension de } \\
\text { l'écrit }\end{array}$ & $\begin{array}{c}\text { Trouver } \\
\text { l'information }\end{array}$ & $\begin{array}{l}\text { Développer une } \\
\text { interprétation }\end{array}$ & $\begin{array}{l}\text { Réfléchir } \\
\text { et évaluer }\end{array}$ & $\begin{array}{c}\text { Textes } \\
\text { continus }\end{array}$ & $\begin{array}{c}\text { Textes non } \\
\text { continus }\end{array}$ \\
\hline Australie & 0.10 & 0.10 & 0.10 & 0.11 & 0.10 & 0.10 \\
\hline Autriche & 0.06 & 0.07 & 0.05 & 0.05 & 0.05 & 0.08 \\
\hline Belgique & 0.13 & 0.13 & 0.13 & 0.13 & 0.13 & 0.14 \\
\hline Canada & 0.10 & 0.10 & 0.09 & 0.10 & 0.09 & 0.10 \\
\hline République tchèque & 0.04 & 0.05 & 0.04 & 0.03 & 0.03 & 0.04 \\
\hline Danemark & 0.10 & 0.09 & 0.09 & 0.11 & 0.09 & 0.11 \\
\hline Finlande & 0.13 & 0.11 & 0.12 & 0.15 & 0.12 & 0.14 \\
\hline $\bar{z}$ France & 0.10 & 0.11 & 0.10 & 0.09 & 0.10 & 0.11 \\
\hline Allemagne & 0.08 & 0.09 & 0.09 & 0.08 & 0.09 & 0.09 \\
\hline Grèce & 0.04 & 0.05 & 0.03 & 0.03 & 0.04 & 0.03 \\
\hline Hongrie & 0.10 & 0.11 & 0.09 & 0.10 & 0.10 & 0.10 \\
\hline Islande & 0.05 & 0.06 & 0.04 & 0.06 & 0.04 & 0.07 \\
\hline Irlande & 0.11 & 0.10 & 0.10 & 0.12 & 0.10 & 0.12 \\
\hline Italie & 0.07 & 0.08 & 0.07 & 0.06 & 0.06 & 0.08 \\
\hline Japon & 0.07 & 0.07 & 0.07 & 0.05 & 0.07 & 0.08 \\
\hline Corée & 0.09 & 0.09 & 0.07 & 0.07 & 0.08 & 0.09 \\
\hline Luxembourg & 0.10 & 0.10 & 0.09 & 0.10 & 0.10 & 0.10 \\
\hline Mexique & 0.05 & 0.05 & 0.03 & 0.05 & 0.05 & 0.04 \\
\hline Nouvelle-Zélande & 0.14 & 0.16 & 0.14 & 0.13 & 0.14 & 0.16 \\
\hline Norvège & 0.13 & 0.14 & 0.12 & 0.13 & 0.12 & 0.13 \\
\hline Pologne & 0.03 & 0.03 & 0.04 & 0.02 & 0.03 & 0.04 \\
\hline Portugal & 0.03 & 0.04 & 0.03 & 0.03 & 0.04 & 0.02 \\
\hline Espagne & 0.08 & 0.08 & 0.07 & 0.07 & 0.07 & 0.07 \\
\hline Suède & 0.05 & 0.06 & 0.05 & 0.05 & 0.05 & 0.06 \\
\hline Suisse & 0.05 & 0.06 & 0.05 & 0.05 & 0.05 & 0.06 \\
\hline Royaume-Uni & 0.14 & 0.13 & 0.14 & 0.14 & 0.14 & 0.14 \\
\hline États-Unis & 0.27 & 0.27 & 0.26 & 0.26 & 0.26 & 0.28 \\
\hline Médiane de l'OCDE & 0.10 & 0.09 & 0.09 & 0.09 & 0.09 & 0.10 \\
\hline Brésil & 0.04 & 0.04 & 0.04 & 0.03 & 0.04 & 0.05 \\
\hline Lettonie & 0.07 & 0.06 & 0.08 & 0.05 & 0.06 & 0.07 \\
\hline Liechtenstein & 0.15 & 0.18 & 0.14 & 0.14 & 0.15 & 0.15 \\
\hline Fédération de Russie & 0.04 & 0.04 & 0.04 & 0.02 & 0.03 & 0.05 \\
\hline Pays-Bas ${ }^{1}$ & 0.13 & 0.13 & 0.12 & 0.13 & 0.12 & 0.13 \\
\hline
\end{tabular}

Régression de la performance en compréhension de l'écrit sur la structure familiale ${ }^{2}$

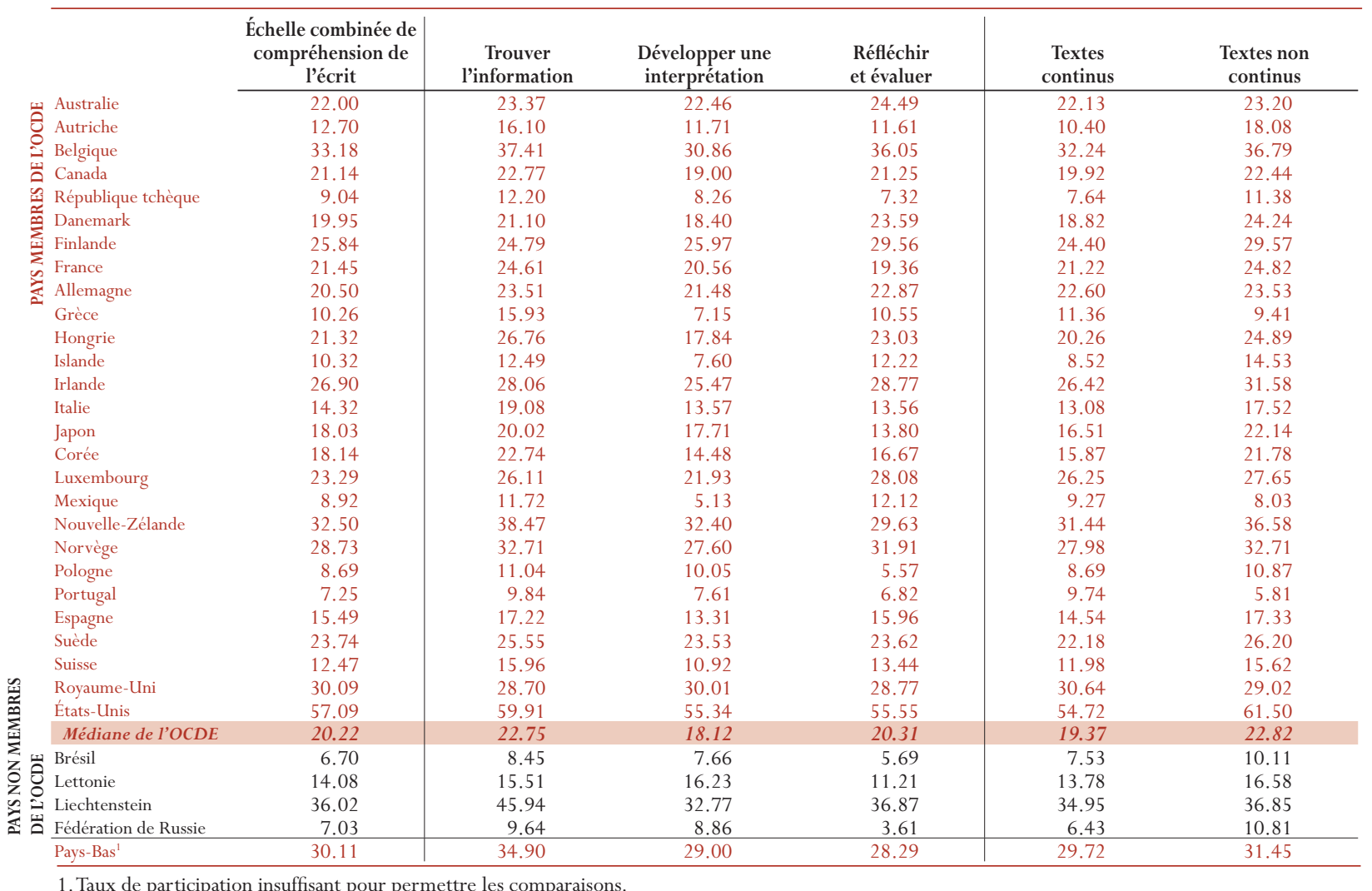

2. Ces chiffres indiquent de combien varie la performance en compréhension de l'écrit si on augmente d'une unité l'indice de la structure familiale. 
Tableau $6.4 \mathrm{f}$

Corrélation entre le statut d'immigrant et la performance en compréhension de l'écrit

\begin{tabular}{|c|c|c|c|c|c|c|}
\hline & $\begin{array}{c}\text { Échelle combinée de } \\
\text { compréhension } \\
\text { de l'écrit }\end{array}$ & $\begin{array}{c}\text { Trouver } \\
\text { l'information }\end{array}$ & $\begin{array}{l}\text { Développer une } \\
\text { interprétation }\end{array}$ & $\begin{array}{l}\text { Réfléchir } \\
\text { et évaluer }\end{array}$ & $\begin{array}{c}\text { Textes } \\
\text { continus }\end{array}$ & $\begin{array}{c}\text { Textes non } \\
\text { continus }\end{array}$ \\
\hline Australie & -0.05 & -0.05 & -0.06 & -0.03 & -0.04 & -0.04 \\
\hline Autriche & -0.23 & -0.22 & -0.22 & -0.22 & -0.22 & -0.22 \\
\hline Belgique & -0.13 & -0.13 & -0.12 & -0.13 & -0.13 & -0.13 \\
\hline Canada & -0.08 & -0.08 & -0.09 & -0.07 & -0.08 & -0.06 \\
\hline République tchèque & -0.02 & -0.02 & -0.02 & -0.01 & -0.01 & -0.02 \\
\hline Danemark & -0.13 & -0.11 & -0.13 & -0.13 & -0.13 & -0.11 \\
\hline Finlande & -0.09 & -0.09 & -0.08 & -0.08 & -0.08 & -0.11 \\
\hline France & -0.11 & -0.12 & -0.11 & -0.10 & -0.11 & -0.11 \\
\hline Allemagne & -0.19 & -0.20 & -0.19 & -0.20 & -0.18 & -0.19 \\
\hline Grèce ${ }^{\circ}$ & -0.15 & -0.16 & -0.16 & -0.13 & -0.16 & -0.13 \\
\hline Hongrie & 0.01 & 0.01 & 0.00 & 0.02 & 0.01 & 0.01 \\
\hline Islande & -0.05 & -0.06 & -0.05 & -0.05 & -0.06 & -0.04 \\
\hline Irlande & 0.06 & 0.06 & 0.06 & 0.05 & 0.05 & 0.07 \\
\hline Italie & -0.04 & -0.05 & -0.05 & -0.05 & -0.05 & -0.03 \\
\hline Japon & -0.02 & -0.02 & -0.01 & -0.02 & -0.01 & -0.01 \\
\hline Corée $^{1}$ & $\mathrm{~m}$ & $\mathrm{~m}$ & $\mathrm{~m}$ & $\mathrm{~m}$ & $\mathrm{~m}$ & $\mathrm{~m}$ \\
\hline Luxembourg & -0.31 & -0.30 & -0.31 & -0.31 & -0.27 & -0.27 \\
\hline Mexique & -0.17 & -0.15 & -0.16 & -0.15 & -0.17 & -0.15 \\
\hline Nouvelle-Zélande & -0.08 & -0.07 & -0.09 & -0.05 & -0.08 & -0.05 \\
\hline Norvège & -0.10 & -0.10 & -0.10 & -0.09 & -0.10 & -0.10 \\
\hline Pologne & -0.04 & -0.06 & -0.05 & -0.04 & -0.05 & -0.03 \\
\hline Portugal & -0.02 & -0.03 & -0.02 & -0.03 & -0.02 & -0.04 \\
\hline Espagne & -0.05 & -0.05 & -0.04 & -0.05 & -0.04 & -0.05 \\
\hline Suède & -0.18 & -0.19 & -0.17 & -0.15 & -0.17 & -0.17 \\
\hline Suisse & -0.32 & -0.33 & -0.31 & -0.29 & -0.30 & -0.32 \\
\hline Royaume-Uni & -0.10 & -0.10 & -0.10 & -0.10 & -0.10 & -0.10 \\
\hline États-Unis & -0.09 & -0.10 & -0.09 & -0.08 & -0.09 & -0.10 \\
\hline Médiane de l'OCDE & -0.09 & -0.10 & -0.09 & -0.08 & -0.09 & -0.10 \\
\hline Brésil & -0.03 & -0.02 & -0.02 & -0.01 & -0.02 & -0.03 \\
\hline Lettonie & -0.01 & -0.03 & 0.01 & -0.01 & 0.01 & -0.03 \\
\hline Liechtenstein & -0.29 & -0.30 & -0.28 & -0.25 & -0.26 & -0.27 \\
\hline Fédération de Russie & -0.01 & -0.02 & 0.00 & -0.01 & 0.00 & -0.01 \\
\hline Pays-Bas ${ }^{2}$ & -0.19 & -0.21 & -0.17 & -0.18 & -0.18 & -0.20 \\
\hline
\end{tabular}

Régression de la performance en compréhension de l'écrit sur le statut d'immigrant ${ }^{3}$

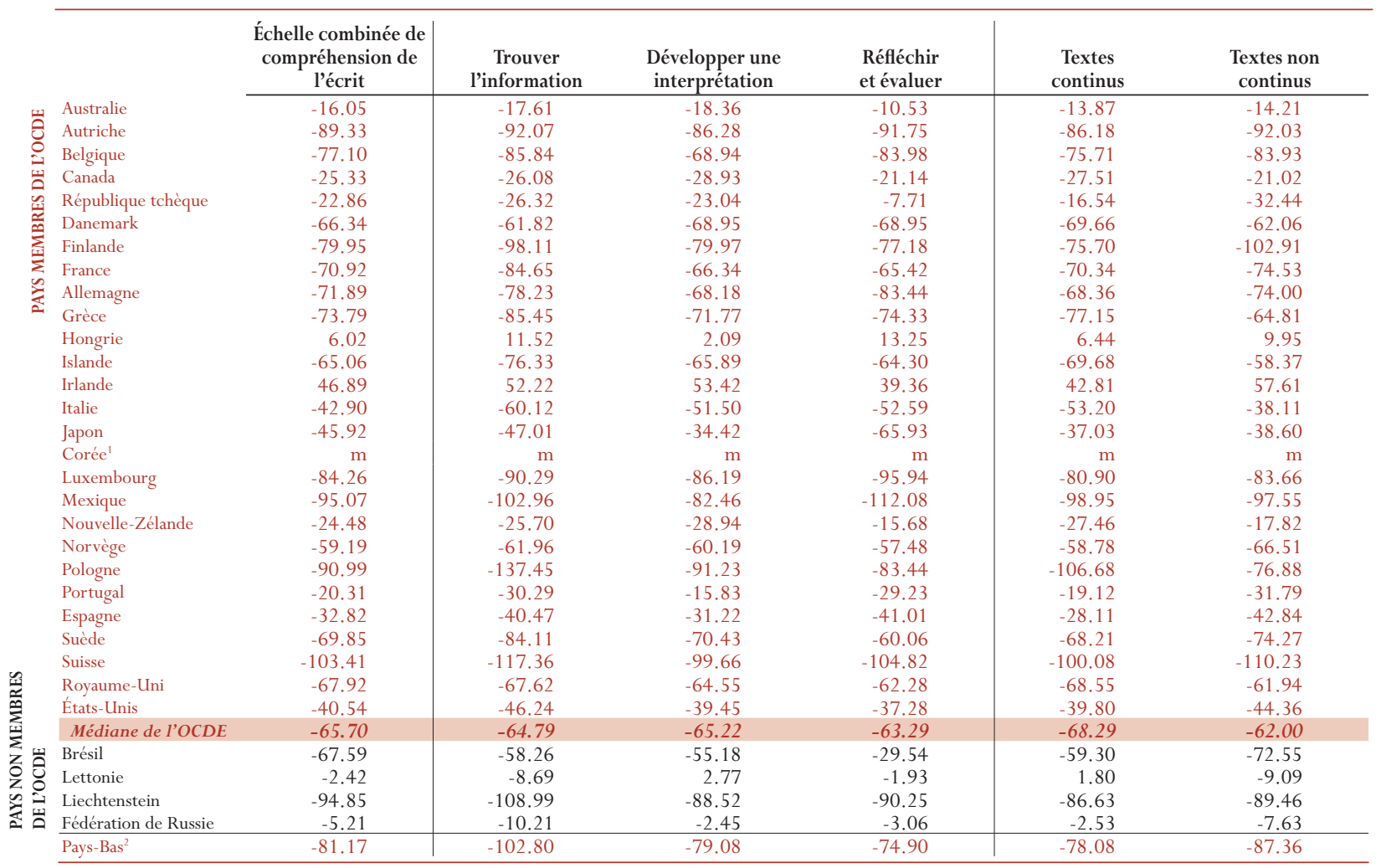

1. La Corée n'a pas recueilli de données à propos du statut d'immigrant.

2. Taux de participation insuffisant pour permettre les comparaisons.

3. Ces chiffres indiquent la variation du niveau de compétence en compréhension de l'écrit selon que les élèves sont autochtones ou allochtones.

(C) OCDE 2002 
Tableau 6.5

Corrélation entre les caractéristiques contextuelles de la classe et la performance sur l'échelle combinée de compréhension de l'écrit d'une part, le degré d'engagement à l'égard de la lecture d'autre part

\begin{tabular}{|c|c|c|c|c|c|c|c|c|c|c|c|c|c|c|c|c|}
\hline & \multicolumn{4}{|c|}{ Incitation au rendement } & \multicolumn{4}{|c|}{ Climat de discipline } & \multicolumn{4}{|c|}{ Sentiment d'appartenance } & \multicolumn{4}{|c|}{$\begin{array}{c}\text { Relations entre élèves } \\
\text { et enseignants }\end{array}$} \\
\hline & $\begin{array}{c}\text { Perfor- } \\
\text { mance }\end{array}$ & Er.T & $\begin{array}{c}\text { Engage- } \\
\text { ment }\end{array}$ & Er.T & $\begin{array}{c}\text { Perfor- } \\
\text { mance }\end{array}$ & Er.T & $\begin{array}{c}\text { Engage- } \\
\text { ment }\end{array}$ & Er.T & $\begin{array}{l}\text { Perfor- } \\
\text { mance }\end{array}$ & Er.T & $\begin{array}{c}\text { Engage- } \\
\text { ment }\end{array}$ & Er.T & $\begin{array}{l}\begin{array}{c}\text { Perfor- } \\
\text { mance }\end{array} \\
\end{array}$ & Er.T & $\begin{array}{l}\text { Engage- } \\
\text { ment }\end{array}$ & Er.T \\
\hline Australie & -0.09 & $(0.02)$ & 0.00 & $(0.02)$ & 0.15 & $(0.02)$ & 0.16 & $(0.02)$ & 0.04 & $(0.02)$ & -0.01 & $(0.02)$ & 0.16 & $(0.02)$ & 0.26 & $(0.02)$ \\
\hline Autriche & -0.08 & $(0.02)$ & -0.04 & $(0.02)$ & 0.06 & $(0.02)$ & 0.09 & $(0.02)$ & 0.06 & $(0.02)$ & 0.00 & $(0.02)$ & 0.03 & $(0.02)$ & 0.10 & $(0.02)$ \\
\hline Belgique & 0.02 & $(0.02)$ & -0.03 & $(0.01)$ & 0.03 & $(0.02)$ & 0.06 & $(0.02)$ & 0.08 & $(0.02)$ & 0.08 & $(0.01)$ & -0.02 & $(0.02)$ & 0.11 & $(0.01)$ \\
\hline Canada & -0.10 & $(0.01)$ & -0.02 & $(0.01)$ & 0.13 & $(0.01)$ & 0.15 & $(0.01)$ & 0.04 & $(0.01)$ & 0.00 & $(0.01)$ & 0.15 & $(0.01)$ & 0.20 & $(0.01)$ \\
\hline République tchèque & -0.09 & $(0.02)$ & -0.06 & $(0.02)$ & 0.10 & $(0.02)$ & 0.09 & $(0.02)$ & 0.13 & $(0.02)$ & 0.04 & $(0.02)$ & 0.03 & $(0.02)$ & 0.14 & $(0.02)$ \\
\hline Danemark & 0.02 & $(0.02)$ & 0.03 & $(0.01)$ & 0.08 & $(0.02)$ & 0.09 & $(0.02)$ & 0.06 & $(0.02)$ & 0.00 & $(0.02)$ & 0.17 & $(0.02)$ & 0.21 & $(0.02)$ \\
\hline Finlande & -0.15 & $(0.02)$ & -0.13 & $(0.02)$ & 0.10 & $(0.02)$ & 0.15 & $(0.02)$ & -0.03 & $(0.02)$ & 0.00 & $(0.02)$ & 0.12 & $(0.03)$ & 0.17 & $(0.02)$ \\
\hline France & -0.06 & $(0.02)$ & -0.06 & $(0.02)$ & 0.02 & $(0.02)$ & 0.07 & $(0.02)$ & 0.06 & $(0.02)$ & 0.08 & $(0.02)$ & -0.02 & $(0.02)$ & 0.12 & $(0.02)$ \\
\hline Allemagne & -0.11 & $(0.02)$ & -0.08 & $(0.02)$ & 0.10 & $(0.02)$ & 0.14 & $(0.02)$ & 0.08 & $(0.02)$ & 0.00 & $(0.02)$ & 0.03 & $(0.02)$ & 0.14 & $(0.02)$ \\
\hline Grèce & 0.12 & $(0.03)$ & 0.08 & $(0.02)$ & 0.03 & $(0.03)$ & 0.10 & $(0.02)$ & 0.11 & $(0.02)$ & 0.18 & $(0.02)$ & -0.02 & $(0.03)$ & 0.16 & $(0.02)$ \\
\hline Hongrie & -0.02 & $(0.02)$ & 0.00 & $(0.02)$ & 0.17 & $(0.04)$ & 0.17 & $(0.03)$ & 0.18 & $(0.02)$ & 0.15 & $(0.02)$ & -0.10 & $(0.03)$ & 0.15 & $(0.02)$ \\
\hline Islande & -0.14 & $(0.02)$ & -0.03 & $(0.02)$ & 0.09 & $(0.02)$ & 0.09 & $(0.02)$ & 0.03 & $(0.02)$ & 0.02 & $(0.02)$ & 0.19 & $(0.02)$ & 0.19 & $(0.02)$ \\
\hline Irlande & -0.07 & $(0.02)$ & -0.01 & $(0.02)$ & 0.19 & $(0.02)$ & 0.16 & $(0.02)$ & 0.00 & $(0.02)$ & 0.03 & $(0.02)$ & 0.12 & $(0.02)$ & 0.24 & $(0.01)$ \\
\hline Italie & -0.06 & $(0.02)$ & -0.04 & $(0.01)$ & 0.15 & $(0.02)$ & 0.21 & $(0.02)$ & -0.01 & $(0.02)$ & 0.07 & $(0.02)$ & -0.05 & $(0.02)$ & 0.12 & $(0.02)$ \\
\hline Japon & 0.05 & $(0.03)$ & 0.05 & $(0.02)$ & 0.22 & $(0.04)$ & 0.08 & $(0.02)$ & 0.09 & $(0.02)$ & 0.03 & $(0.02)$ & 0.20 & $(0.02)$ & 0.14 & $(0.02)$ \\
\hline Corée & 0.19 & $(0.02)$ & 0.12 & $(0.02)$ & 0.10 & $(0.02)$ & 0.10 & $(0.02)$ & 0.11 & $(0.02)$ & 0.11 & $(0.02)$ & 0.09 & $(0.02)$ & 0.14 & $(0.01)$ \\
\hline Luxembourg & 0.02 & $(0.02)$ & 0.00 & $(0.02)$ & 0.03 & $(0.02)$ & 0.07 & $(0.02)$ & 0.20 & $(0.02)$ & 0.06 & $(0.02)$ & -0.04 & $(0.02)$ & 0.17 & $(0.02)$ \\
\hline Mexique & 0.00 & $(0.02)$ & 0.13 & $(0.02)$ & 0.02 & $(0.03)$ & 0.08 & $(0.02)$ & 0.24 & $(0.02)$ & 0.22 & $(0.02)$ & 0.03 & $(0.02)$ & 0.18 & $(0.02)$ \\
\hline Nouvelle-Zélande & -0.15 & $(0.02)$ & -0.01 & $(0.02)$ & 0.11 & $(0.02)$ & 0.17 & $(0.02)$ & 0.05 & $(0.02)$ & 0.04 & $(0.02)$ & 0.12 & $(0.02)$ & 0.22 & $(0.02)$ \\
\hline Norvège & -0.11 & $(0.02)$ & -0.05 & $(0.02)$ & 0.06 & $(0.02)$ & 0.09 & $(0.02)$ & 0.07 & $(0.02)$ & 0.04 & $(0.02)$ & 0.18 & $(0.02)$ & 0.20 & $(0.02)$ \\
\hline Pologne & 0.03 & $(0.03)$ & -0.01 & $(0.02)$ & 0.23 & $(0.03)$ & 0.18 & $(0.02)$ & 0.19 & $(0.02)$ & 0.15 & $(0.02)$ & 0.04 & $(0.02)$ & 0.22 & $(0.02)$ \\
\hline Portugal & -0.05 & $(0.02)$ & 0.06 & $(0.02)$ & 0.09 & $(0.02)$ & 0.10 & $(0.02)$ & 0.27 & $(0.02)$ & 0.19 & $(0.02)$ & -0.06 & $(0.03)$ & 0.15 & $(0.02)$ \\
\hline Espagne & 0.03 & $(0.02)$ & 0.05 & $(0.02)$ & 0.14 & $(0.02)$ & 0.10 & $(0.02)$ & 0.06 & $(0.02)$ & 0.06 & $(0.02)$ & 0.08 & $(0.02)$ & 0.12 & $(0.02)$ \\
\hline Suède & -0.15 & $(0.02)$ & -0.08 & $(0.02)$ & 0.12 & $(0.02)$ & 0.10 & $(0.02)$ & -0.02 & $(0.02)$ & -0.03 & $(0.02)$ & 0.13 & $(0.02)$ & 0.18 & $(0.02)$ \\
\hline Suisse & -0.04 & $(0.03)$ & -0.07 & $(0.02)$ & 0.15 & $(0.02)$ & 0.12 & $(0.02)$ & 0.16 & $(0.02)$ & 0.14 & $(0.02)$ & 0.05 & $(0.02)$ & 0.16 & $(0.02)$ \\
\hline Royaume-Uni & -0.11 & $(0.02)$ & -0.03 & $(0.01)$ & 0.21 & $(0.02)$ & 0.19 & $(0.02)$ & 0.06 & $(0.02)$ & 0.04 & $(0.02)$ & 0.17 & $(0.02)$ & 0.22 & $(0.02)$ \\
\hline États-Unis & -0.02 & $(0.02)$ & 0.10 & $(0.02)$ & 0.13 & $(0.02)$ & 0.12 & $(0.02)$ & 0.14 & $(0.03)$ & 0.05 & $(0.02)$ & 0.19 & $(0.02)$ & 0.24 & $(0.03)$ \\
\hline Médiane de l'OCDE & -0.06 & $\sim$ & -0.01 & $\sim$ & 0.10 & $\sim$ & 0.10 & $\sim$ & 0.07 & $\sim$ & 0.04 & $\sim$ & 0.08 & $\sim$ & 0.17 & $\sim$ \\
\hline Éc.T. de corrélation & 0.08 & $\sim$ & 0.06 & $\sim$ & -0.06 & $\sim$ & -0.04 & $\sim$ & 0.08 & $\sim$ & 0.07 & $\sim$ & 0.09 & $\sim$ & 0.04 & - \\
\hline Brésil & 0.10 & $(0.02)$ & 0.15 & $(0.02)$ & -0.06 & $(0.02)$ & 0.10 & $(0.03)$ & 0.15 & $(0.02)$ & 0.21 & $(0.02)$ & -0.03 & $(0.03)$ & 0.20 & $(0.02)$ \\
\hline Lettonie & 0.02 & $(0.03)$ & 0.02 & $(0.02)$ & 0.09 & $(0.02)$ & 0.09 & $(0.03)$ & 0.13 & $(0.02)$ & 0.08 & $(0.03)$ & 0.08 & $(0.03)$ & 0.16 & $(0.03)$ \\
\hline Liechtenstein & -0.12 & $(0.05)$ & 0.02 & $(0.06)$ & -0.03 & $(0.06)$ & -0.02 & $(0.06)$ & 0.16 & $(0.05)$ & 0.04 & $(0.05)$ & -0.01 & $(0.05)$ & 0.17 & $(0.06)$ \\
\hline Fédération de Russie & -0.06 & $(0.02)$ & 0.03 & $(0.02)$ & 0.11 & $(0.02)$ & 0.18 & $(0.02)$ & 0.13 & $(0.02)$ & 0.16 & $(0.02)$ & 0.03 & $(0.02)$ & 0.22 & $(0.01)$ \\
\hline Pays-Bas ${ }^{1}$ & -0.10 & $(0.02)$ & -0.03 & $(0.02)$ & 0.03 & $(0.04)$ & 0.07 & $(0.02)$ & 0.13 & $(0.03)$ & 0.04 & $(0.02)$ & 0.07 & $(0.03)$ & 0.14 & $(0.02)$ \\
\hline
\end{tabular}

1. Taux de participation insuffisant pour permettre les comparaisons. 
Tableau 6.6a

Corrélation entre l'incitation au rendement et la performance en compréhension de l'écrit

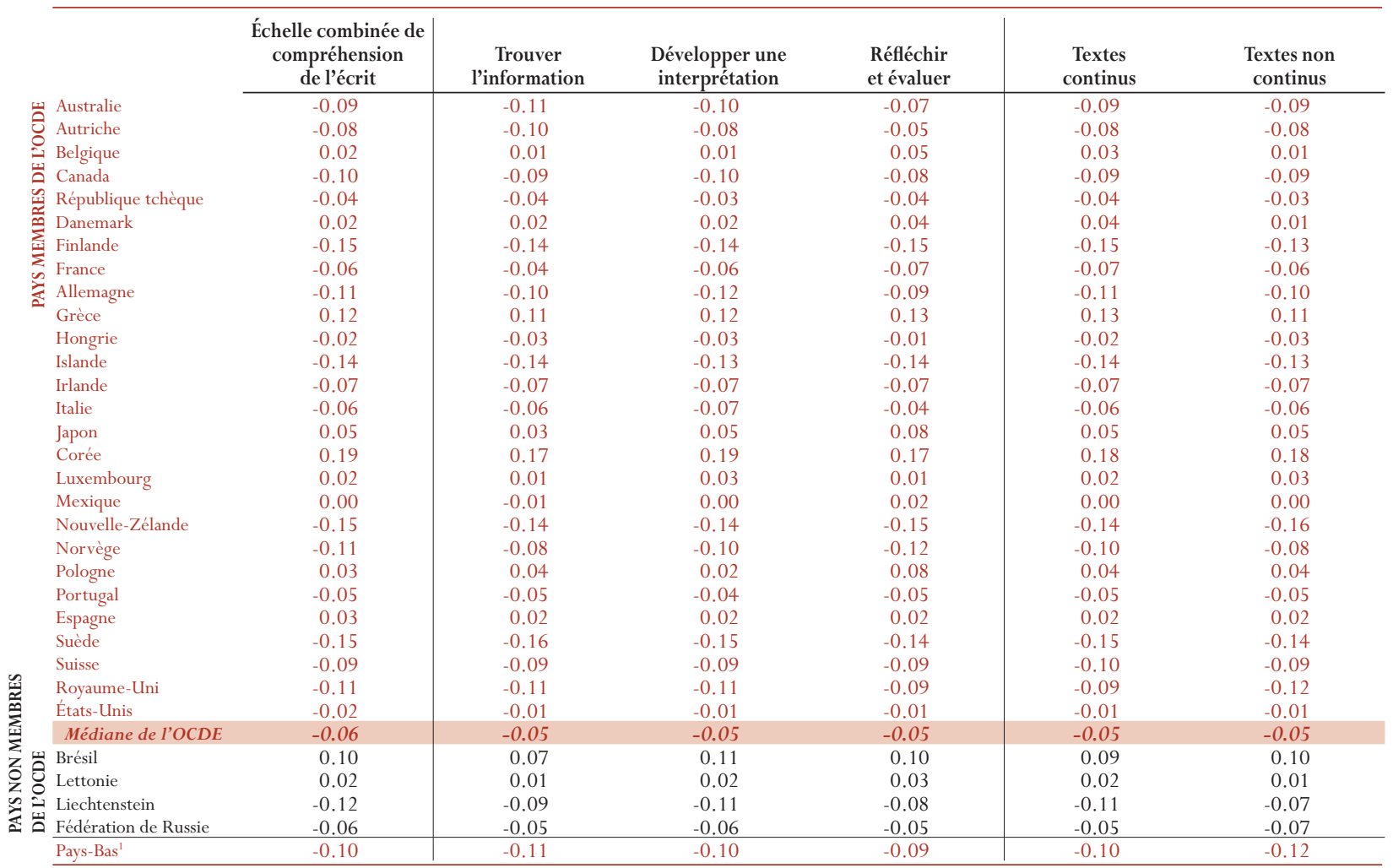

Régression de la performance en compréhension de l'écrit sur l'incitation au rendement ${ }^{2}$

\begin{tabular}{|c|c|c|c|c|c|c|}
\hline & $\begin{array}{c}\text { Échelle combinée de } \\
\text { compréhension de } \\
\text { l'écrit }\end{array}$ & $\begin{array}{c}\text { Trouver } \\
\text { l'information }\end{array}$ & $\begin{array}{l}\text { Développer une } \\
\text { interprétation }\end{array}$ & $\begin{array}{l}\text { Réfléchir } \\
\text { et évaluer }\end{array}$ & $\begin{array}{c}\text { Textes } \\
\text { continus }\end{array}$ & $\begin{array}{c}\text { Textes non } \\
\text { continus }\end{array}$ \\
\hline Australie & -10.06 & -13.27 & -11.40 & -7.72 & -10.64 & -10.66 \\
\hline Autriche & -6.39 & -8.25 & -6.37 & -4.52 & -6.44 & -7.28 \\
\hline Belgique & 1.95 & 1.58 & 0.89 & 5.31 & 3.23 & 1.24 \\
\hline Canada & -9.71 & -9.64 & -10.33 & -8.63 & -9.40 & -9.94 \\
\hline République tchèque & -3.41 & -3.40 & -2.71 & -3.78 & -3.22 & -3.23 \\
\hline Danemark & 2.97 & 3.01 & 2.49 & 5.05 & 4.85 & 0.84 \\
\hline Finlande & -14.69 & -16.24 & -15.43 & -15.16 & -15.01 & -13.68 \\
\hline France & -5.84 & -4.46 & -5.88 & -6.92 & -6.60 & -5.55 \\
\hline Allemagne & -11.07 & -10.03 & -12.43 & -10.60 & -11.24 & -10.55 \\
\hline Grèce & 11.73 & 11.25 & 10.66 & 14.91 & 12.58 & 11.22 \\
\hline Hongrie & -2.31 & -3.69 & -2.55 & -0.58 & -1.55 & -3.08 \\
\hline Islande & -13.21 & -14.93 & -12.62 & -13.64 & -13.49 & -13.05 \\
\hline Irlande & -7.32 & -7.50 & -7.16 & -6.43 & -7.40 & -7.82 \\
\hline Italie & -6.31 & -7.72 & -6.77 & -4.47 & -6.06 & -7.01 \\
\hline Japon & 4.36 & 2.80 & 3.64 & 7.82 & 4.36 & 4.30 \\
\hline Corée & 13.27 & 14.24 & 13.02 & 13.24 & 12.37 & 14.43 \\
\hline Luxembourg & 1.48 & 1.28 & 2.30 & 1.18 & 1.47 & 2.83 \\
\hline Mexique & -0.37 & -1.24 & -0.29 & 1.73 & 0.04 & -0.41 \\
\hline Nouvelle-Zélande & -18.04 & -18.09 & -17.73 & -17.51 & -16.89 & -19.45 \\
\hline Norvège & -11.93 & -9.21 & -11.74 & -13.95 & -11.77 & -10.41 \\
\hline Pologne & 3.37 & 4.57 & 1.89 & 8.25 & 3.83 & 4.08 \\
\hline Portugal & -5.10 & -5.03 & -4.04 & -5.15 & -5.03 & -4.94 \\
\hline Espagne & 2.04 & 1.88 & 1.52 & 1.66 & 1.43 & 2.08 \\
\hline Suède & -9.58 & -10.66 & -8.76 & -10.13 & -10.15 & -9.40 \\
\hline Suisse & -3.41 & -3.40 & -2.71 & -3.78 & -3.22 & -3.23 \\
\hline Royaume-Uni & -12.88 & -13.79 & -13.13 & -11.07 & -11.72 & -14.64 \\
\hline États-Unis & -1.51 & -0.91 & -1.39 & -1.11 & -1.11 & -1.45 \\
\hline Médiane de l'OCDE & -5.84 & -5.03 & -5.88 & -4.52 & -6.06 & -5.55 \\
\hline Brésil & 8.02 & 6.47 & 8.56 & 8.59 & 7.58 & 8.92 \\
\hline Lettonie & 1.55 & 0.60 & 2.05 & 3.48 & 2.34 & 1.04 \\
\hline Liechtenstein & -12.31 & -10.75 & -11.15 & -9.89 & -11.33 & -7.09 \\
\hline Fédération de Russie & -4.99 & -5.04 & -5.42 & -4.33 & -4.49 & -6.27 \\
\hline Pays-Bas $^{1}$ & -10.38 & -12.40 & -10.21 & -8.54 & -9.86 & -11.66 \\
\hline
\end{tabular}

1. Taux de participation insuffisant pour permettre les comparaisons.

2. Ces chiffres indiquent de combien varie la performance en compréhension de l'écrit si on augmente d'une unité l'indice d'incitation au rendement. 
Tableau 6.6b

Corrélation entre le climat de discipline et la performance en compréhension de l'écrit



Régression de la performance en compréhension de l'écrit sur le climat de discipline ${ }^{2}$

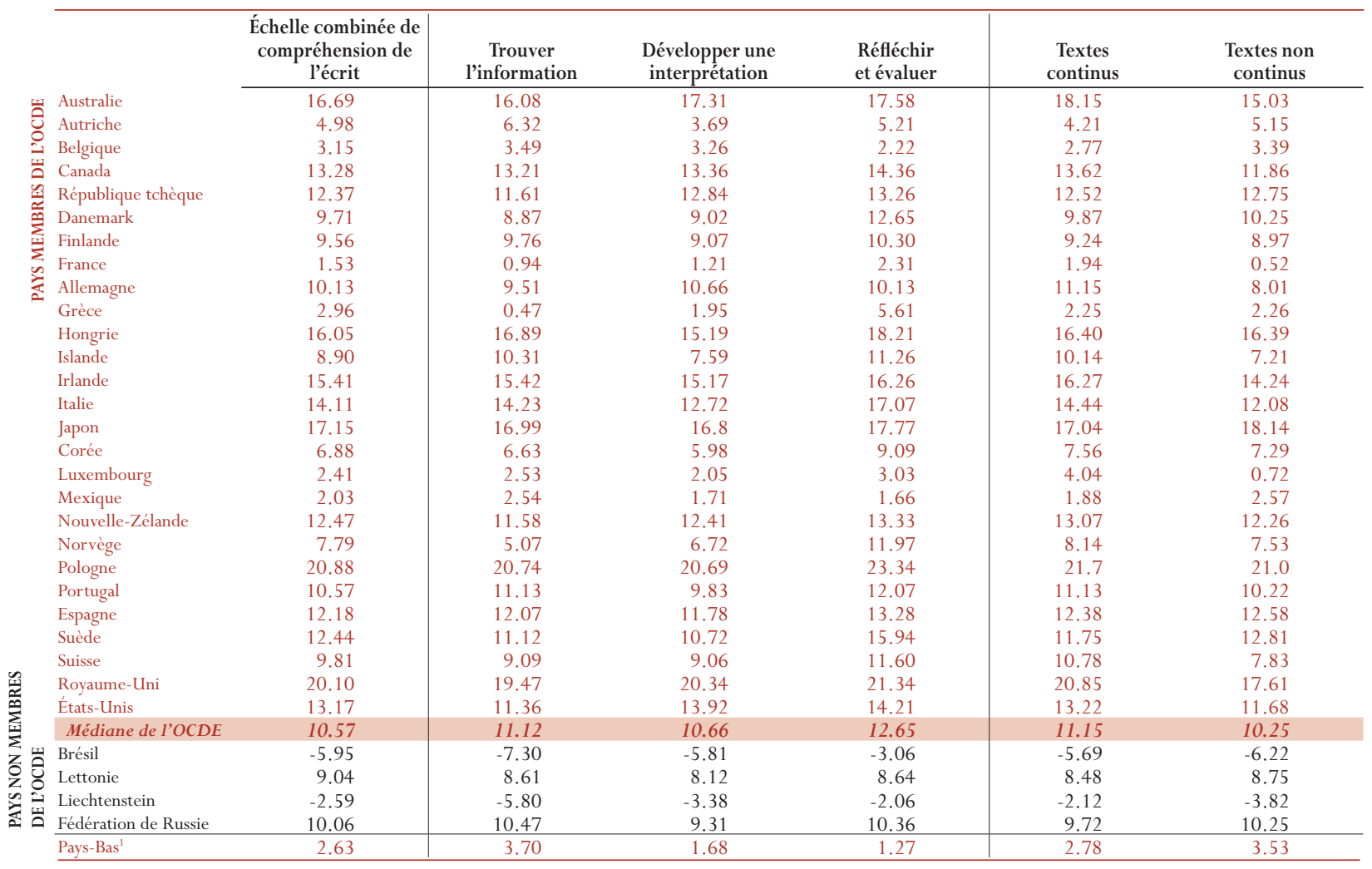

1. Taux de participation insuffisant pour permettre les comparaisons.

2. Ces chiffres indiquent de combien varie la performance en compréhension de l'écrit si on augmente d'une unité l'indice du climat de discipline (voir l'annexe A3 pour la définition). 
Tableau 6.6c

Corrélation entre le sentiment d'appartenance et la performance en compréhension de l'écrit

\begin{tabular}{|c|c|c|c|c|c|c|}
\hline & $\begin{array}{c}\text { Échelle combinée de } \\
\text { compréhension } \\
\text { de l'écrit }\end{array}$ & $\begin{array}{c}\text { Trouver } \\
\text { l'information }\end{array}$ & $\begin{array}{l}\text { Développer une } \\
\text { interprétation }\end{array}$ & $\begin{array}{l}\text { Réfléchir } \\
\text { et évaluer }\end{array}$ & $\begin{array}{c}\text { Textes } \\
\text { continus }\end{array}$ & $\begin{array}{c}\text { Textes non } \\
\text { continus }\end{array}$ \\
\hline Autriche & 0.06 & 0.08 & 0.05 & 0.06 & 0.04 & 0.08 \\
\hline Belgique & 0.08 & 0.08 & 0.07 & 0.09 & 0.08 & 0.08 \\
\hline Canada & 0.04 & 0.04 & 0.03 & 0.06 & 0.04 & 0.04 \\
\hline République tchèque & 0.16 & 0.15 & 0.14 & 0.16 & 0.15 & 0.15 \\
\hline Danemark & 0.06 & 0.06 & 0.06 & 0.08 & 0.06 & 0.06 \\
\hline Finlande & -0.03 & -0.02 & -0.05 & -0.01 & -0.04 & -0.01 \\
\hline France & 0.06 & 0.06 & 0.05 & 0.06 & 0.06 & 0.07 \\
\hline Allemagne & 0.08 & 0.08 & 0.08 & 0.08 & 0.08 & 0.07 \\
\hline Grèce & 0.11 & 0.10 & 0.11 & 0.12 & 0.11 & 0.11 \\
\hline Hongrie & 0.18 & 0.17 & 0.16 & 0.18 & 0.17 & 0.18 \\
\hline Islande & 0.03 & 0.04 & 0.03 & 0.02 & 0.02 & 0.04 \\
\hline Irlande & 0.00 & 0.00 & -0.01 & 0.01 & -0.01 & 0.00 \\
\hline Italie & -0.01 & -0.02 & -0.02 & 0.01 & -0.01 & -0.01 \\
\hline Japon & 0.09 & 0.08 & 0.07 & 0.10 & 0.08 & 0.09 \\
\hline Corée & 0.11 & 0.11 & 0.12 & 0.10 & 0.10 & 0.12 \\
\hline Luxembourg & 0.20 & 0.19 & 0.21 & 0.19 & 0.20 & 0.19 \\
\hline Mexique & 0.24 & 0.22 & 0.22 & 0.23 & 0.23 & 0.22 \\
\hline Nouvelle-Zélande & 0.05 & 0.06 & 0.03 & 0.06 & 0.05 & 0.06 \\
\hline Norvège & 0.07 & 0.05 & 0.06 & 0.09 & 0.07 & 0.07 \\
\hline Pologne & 0.19 & 0.20 & 0.18 & 0.16 & 0.18 & 0.18 \\
\hline Portugal & 0.27 & 0.28 & 0.26 & 0.26 & 0.26 & 0.27 \\
\hline Espagne & 0.06 & 0.06 & 0.05 & 0.06 & 0.06 & 0.05 \\
\hline Suède & -0.02 & -0.03 & -0.03 & 0.00 & -0.02 & -0.01 \\
\hline Suisse & 0.13 & 0.13 & 0.12 & 0.14 & 0.13 & 0.12 \\
\hline Royaume-Uni & 0.06 & 0.07 & 0.05 & 0.08 & 0.06 & 0.06 \\
\hline États-Unis & 0.14 & 0.14 & 0.12 & 0.14 & 0.12 & 0.14 \\
\hline Médiane de l'OCDE & 0.07 & 0.08 & 0.06 & 0.08 & 0.07 & 0.07 \\
\hline Brésil & 0.15 & 0.16 & 0.14 & 0.15 & 0.14 & 0.14 \\
\hline Lettonie & 0.13 & 0.12 & 0.13 & 0.13 & 0.13 & 0.13 \\
\hline Liechtenstein & 0.16 & 0.15 & 0.15 & 0.15 & 0.15 & 0.17 \\
\hline Fédération de Russie & 0.13 & 0.11 & 0.12 & 0.14 & 0.13 & 0.11 \\
\hline Pays-Bas ${ }^{1}$ & 0.13 & 0.12 & 0.13 & 0.13 & 0.13 & 0.13 \\
\hline
\end{tabular}

Régression de la performance en compréhension de l'écrit sur le sentiment d'appartenance²

\begin{tabular}{|c|c|c|c|c|c|c|}
\hline & $\begin{array}{c}\text { Échelle combinée de } \\
\text { compréhension } \\
\text { de l'écrit }\end{array}$ & $\begin{array}{c}\text { Trouver } \\
\text { l'information }\end{array}$ & $\begin{array}{l}\text { Développer une } \\
\text { interprétation }\end{array}$ & $\begin{array}{l}\text { Réfléchir } \\
\text { et évaluer }\end{array}$ & $\begin{array}{c}\text { Textes } \\
\text { continus }\end{array}$ & $\begin{array}{c}\text { Textes non } \\
\text { continus }\end{array}$ \\
\hline Autriche & 5.10 & 6.55 & 3.86 & 5.20 & 3.75 & 7.09 \\
\hline Belgique & 9.83 & 11.00 & 8.24 & 11.51 & 9.46 & 10.34 \\
\hline Canada & 3.27 & 3.64 & 2.44 & 4.85 & 3.28 & 3.39 \\
\hline République tchèque & 19.28 & 20.67 & 17.57 & 21.09 & 18.22 & 21.10 \\
\hline Danemark & 5.79 & 5.92 & 5.21 & 7.72 & 5.71 & 6.53 \\
\hline Finlande & -2.54 & -2.24 & -4.87 & -1.26 & -3.41 & -1.33 \\
\hline France & 5.70 & 6.45 & 5.04 & 6.58 & 5.63 & 6.87 \\
\hline Allemagne & 8.33 & 8.36 & 7.75 & 9.33 & 8.60 & 7.64 \\
\hline Grèce & 11.15 & 11.32 & 10.14 & 14.48 & 11.46 & 11.73 \\
\hline Hongrie & 16.82 & 18.83 & 15.01 & 18.70 & 16.02 & 19.53 \\
\hline Islande & 2.06 & 3.81 & 2.14 & 1.76 & 1.68 & 3.38 \\
\hline Irlande & -0.21 & 0.28 & -1.15 & 1.21 & -0.62 & 0.22 \\
\hline Italie & -0.74 & -1.96 & -1.42 & 1.57 & -0.47 & -1.31 \\
\hline Japon & 8.35 & 9.07 & 6.36 & 11.04 & 7.24 & 10.01 \\
\hline Corée & 9.42 & 11.01 & 10.07 & 8.91 & 8.55 & 12.37 \\
\hline Luxembourg & 16.64 & 17.49 & 18.10 & 18.26 & 19.34 & 18.81 \\
\hline Mexique & 20.75 & 22.95 & 17.69 & 25.44 & 20.39 & 22.14 \\
\hline Nouvelle-Zélande & 5.58 & 7.28 & 3.54 & 6.50 & 5.16 & 7.03 \\
\hline Norvège & 6.58 & 4.74 & 5.91 & 9.46 & 6.55 & 7.31 \\
\hline Pologne & 21.44 & 25.28 & 20.37 & 20.04 & 21.17 & 22.88 \\
\hline Portugal & 29.41 & 33.23 & 26.85 & 29.85 & 28.90 & 30.61 \\
\hline Espagne & 5.37 & 5.67 & 4.26 & 6.06 & 5.05 & 5.52 \\
\hline Suède & -2.09 & -2.59 & -3.00 & -0.06 & -2.07 & -1.15 \\
\hline Suisse & 12.31 & 13.51 & 11.19 & 14.82 & 12.20 & 12.17 \\
\hline Royaume-Uni & 6.26 & 7.09 & 4.86 & 7.63 & 6.21 & 6.11 \\
\hline États-Unis & 12.83 & 13.82 & 11.11 & 12.99 & 11.60 & 13.29 \\
\hline Médiane de l'OCDE & 6.58 & 7.28 & 5.91 & 8.91 & 6.55 & 7.31 \\
\hline Brésil & 12.50 & 14.80 & 11.08 & 13.64 & 12.19 & 13.32 \\
\hline Lettonie & 16.65 & 17.96 & 15.45 & 18.40 & 16.98 & 17.90 \\
\hline Liechtenstein & 13.35 & 14.36 & 12.19 & 14.00 & 13.27 & 14.49 \\
\hline Fédération de Russie & 13.81 & 13.34 & 13.15 & 15.79 & 13.73 & 13.47 \\
\hline Pays-Bas ${ }^{1}$ & 13.60 & 14.65 & 14.16 & 13.12 & 13.68 & 13.86 \\
\hline
\end{tabular}

1. Taux de participation insuffisant pour permettre les comparaisons.

2. Ces chiffres indiquent de combien varie la performance en compréhension de l'écrit si on augmente d'une unité l'indice du sentiment d'appartenance (voir l'annexe A3 pour la définition). 
Tableau 6.6d

Corrélation entre les relations élèves-enseignants et la performance en compréhension de l'écrit

\begin{tabular}{|c|c|c|c|c|c|c|}
\hline & $\begin{array}{c}\text { Échelle combinée de } \\
\text { compréhension } \\
\text { de l'écrit }\end{array}$ & $\begin{array}{c}\text { Trouver } \\
\text { l'information }\end{array}$ & $\begin{array}{l}\text { Développer une } \\
\text { interprétation }\end{array}$ & $\begin{array}{l}\text { Réfléchir } \\
\text { et évaluer }\end{array}$ & $\begin{array}{c}\text { Textes } \\
\text { continus }\end{array}$ & $\begin{array}{c}\text { Textes non } \\
\text { continus }\end{array}$ \\
\hline Australie & 0.16 & 0.14 & 0.16 & 0.18 & 0.17 & 0.15 \\
\hline Autriche & 0.03 & 0.06 & 0.02 & 0.03 & 0.02 & 0.04 \\
\hline Belgique & -0.02 & -0.02 & -0.02 & -0.02 & -0.02 & -0.02 \\
\hline Canada & 0.15 & 0.14 & 0.14 & 0.16 & 0.14 & 0.14 \\
\hline République tchèque & 0.05 & 0.06 & 0.05 & 0.07 & 0.05 & 0.06 \\
\hline Danemark & 0.17 & 0.16 & 0.16 & 0.18 & 0.17 & 0.15 \\
\hline Finlande & 0.12 & 0.12 & 0.10 & 0.13 & 0.12 & 0.11 \\
\hline France & -0.02 & -0.01 & -0.03 & -0.01 & -0.01 & -0.03 \\
\hline Allemagne & 0.03 & 0.02 & 0.02 & 0.04 & 0.03 & 0.02 \\
\hline Grèce & -0.02 & -0.03 & -0.02 & 0.00 & -0.01 & -0.02 \\
\hline Hongrie & -0.10 & -0.08 & -0.11 & -0.10 & -0.10 & -0.10 \\
\hline Islande & 0.19 & 0.17 & 0.17 & 0.21 & 0.20 & 0.16 \\
\hline Irlande & 0.12 & 0.12 & 0.10 & 0.13 & 0.11 & 0.12 \\
\hline Italie & -0.05 & -0.06 & -0.05 & -0.04 & -0.04 & -0.06 \\
\hline Japon & 0.20 & 0.18 & 0.18 & 0.19 & 0.19 & 0.18 \\
\hline Corée & 0.09 & 0.09 & 0.09 & 0.09 & 0.09 & 0.09 \\
\hline Luxembourg & -0.04 & -0.05 & -0.03 & -0.05 & -0.05 & -0.04 \\
\hline Mexique & 0.03 & 0.02 & 0.02 & 0.05 & 0.04 & 0.02 \\
\hline Nouvelle-Zélande & 0.12 & 0.11 & 0.12 & 0.14 & 0.12 & 0.13 \\
\hline Norvège & 0.18 & 0.16 & 0.16 & 0.19 & 0.18 & 0.15 \\
\hline Pologne & 0.04 & 0.04 & 0.05 & 0.04 & 0.04 & 0.03 \\
\hline Portugal & -0.06 & -0.05 & -0.06 & -0.03 & -0.04 & -0.06 \\
\hline Espagne & 0.08 & 0.08 & 0.05 & 0.10 & 0.08 & 0.06 \\
\hline Suède & 0.13 & 0.13 & 0.11 & 0.14 & 0.13 & 0.12 \\
\hline Suisse & 0.03 & 0.02 & 0.02 & 0.05 & 0.04 & 0.01 \\
\hline Royaume-Uni & 0.17 & 0.17 & 0.15 & 0.19 & 0.17 & 0.16 \\
\hline États-Unis & 0.19 & 0.18 & 0.19 & 0.20 & 0.18 & 0.18 \\
\hline Médiane de l'OCDE & 0.08 & 0.08 & 0.05 & 0.09 & 0.08 & 0.06 \\
\hline Brésil & -0.03 & -0.02 & -0.05 & -0.03 & -0.04 & -0.03 \\
\hline Lettonie & 0.08 & 0.06 & 0.08 & 0.09 & 0.09 & 0.06 \\
\hline Liechtenstein & -0.01 & -0.01 & -0.02 & -0.01 & -0.02 & 0.01 \\
\hline Fédération de Russie & 0.03 & 0.02 & 0.02 & 0.04 & 0.02 & 0.01 \\
\hline Pays-Bas $^{1}$ & 0.07 & 0.07 & 0.06 & 0.07 & 0.07 & 0.07 \\
\hline
\end{tabular}

Régression de la performance en compréhension de l'écrit sur les relations élèves-enseignants ${ }^{2}$

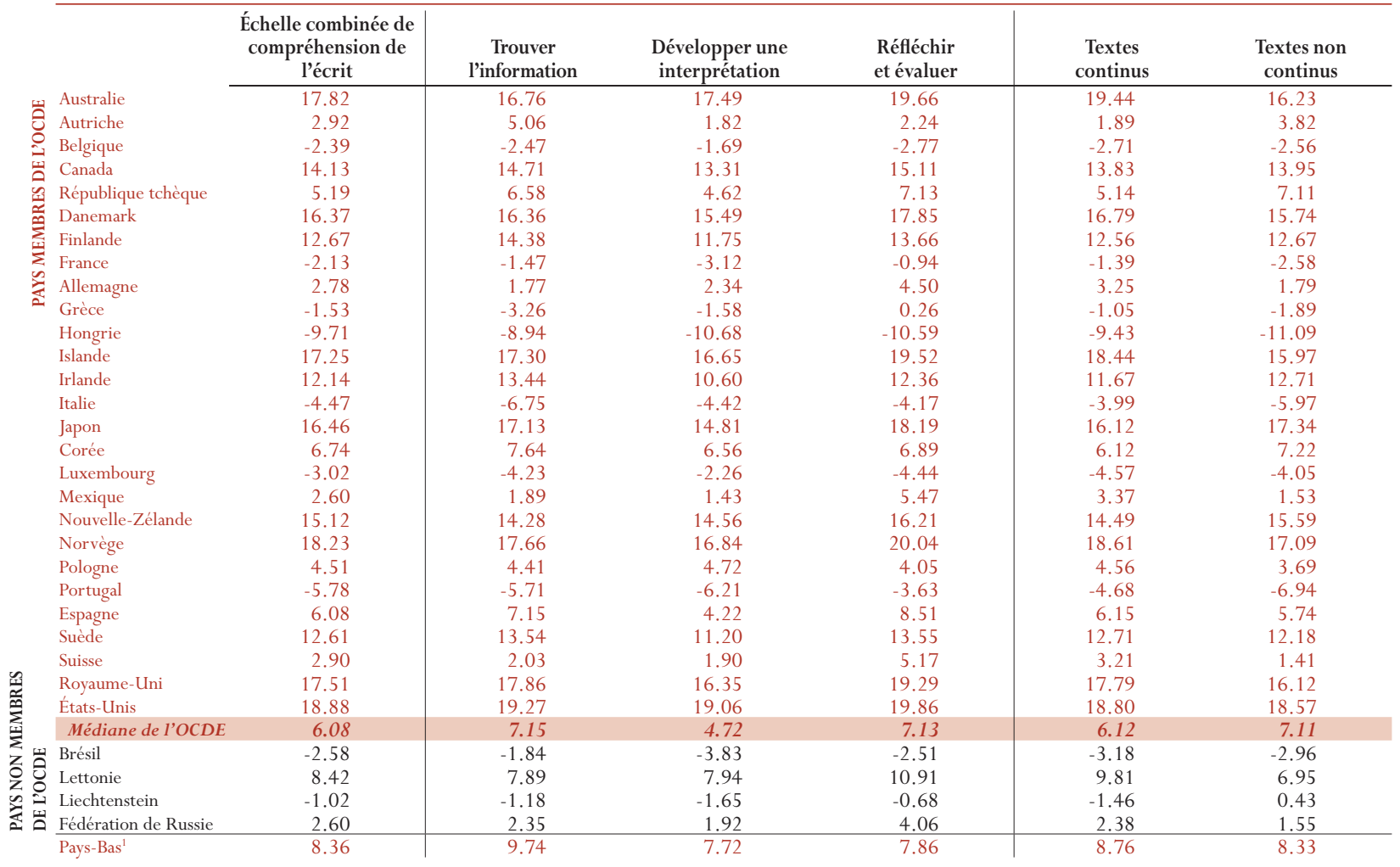

1. Taux de participation insuffisant pour permettre les comparaisons.

2. Ces chiffres indiquent de combien varie la performance en compréhension de l'écrit si on augmente d'une unité l'indice des relations entre élèves et enseignants (voir l'annexe A3 pour la définition).

252 
Tableau 6.7

Régression du degré d'engagement à l'égard de la lecture sur les caractéristiques contextuelles sélectionnées

\begin{tabular}{|c|c|c|c|c|c|c|c|c|c|c|c|c|c|}
\hline \multirow{9}{*}{ 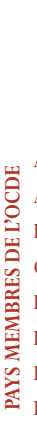 } & & $\begin{array}{c}\text { Statut } \\
\text { d'immigrant } \\
\end{array}$ & Sexe & $\begin{array}{l}\text { Structure } \\
\text { familiale }\end{array}$ & $\begin{array}{l}\text { Milieu } \\
\text { socio- } \\
\text { écono- } \\
\text { mique }\end{array}$ & $\begin{array}{l}\text { Nombre } \\
\text { de livres à } \\
\text { la maison }\end{array}$ & $\begin{array}{l}\text { Commu- } \\
\text { nication } \\
\text { culturelle }\end{array}$ & $\begin{array}{l}\text { Res- } \\
\text { sources } \\
\text { éduca- } \\
\text { tives à la } \\
\text { maison }\end{array}$ & $\begin{array}{c}\text { Incitation } \\
\text { au rende- } \\
\text { ment }\end{array}$ & $\begin{array}{l}\text { Climat } \\
\text { de disci- } \\
\text { pline }\end{array}$ & $\begin{array}{c}\text { Relations } \\
\text { élèves- } \\
\text { ensei- } \\
\text { gnants }\end{array}$ & $\begin{array}{c}\text { Sentiment } \\
\text { d'apparte- } \\
\text { nance }\end{array}$ & $\begin{array}{c}\text { Temps } \\
\text { consacré } \\
\text { aux } \\
\text { devoirs }\end{array}$ \\
\hline & Australie & 0.19 & 0.28 & 0.11 & 0.17 & 0.20 & 0.37 & 0.23 & -0.01 & 0.18 & 0.28 & -0.01 & 0.34 \\
\hline & Autriche & -0.01 & 0.52 & 0.07 & 0.21 & 0.24 & 0.38 & 0.17 & -0.04 & 0.09 & 0.10 & 0.00 & 0.15 \\
\hline & Belgique & -0.13 & 0.41 & 0.17 & 0.19 & 0.22 & 0.34 & 0.19 & -0.04 & 0.07 & 0.14 & 0.09 & 0.32 \\
\hline & Canada & 0.18 & 0.47 & 0.08 & 0.14 & 0.22 & 0.37 & 0.18 & -0.03 & 0.17 & 0.22 & 0.00 & 0.30 \\
\hline & République tchèque & -0.24 & 0.62 & 0.06 & 0.25 & 0.28 & 0.43 & 0.23 & -0.07 & 0.10 & 0.14 & 0.04 & 0.21 \\
\hline & Danemark & 0.18 & 0.48 & 0.07 & 0.15 & 0.20 & 0.41 & 0.22 & 0.03 & 0.13 & 0.21 & -0.01 & 0.31 \\
\hline & Finlande & 0.02 & 0.73 & 0.15 & 0.11 & 0.23 & 0.39 & 0.16 & -0.14 & 0.16 & 0.21 & -0.01 & 0.35 \\
\hline & France & 0.06 & 0.29 & 0.11 & 0.16 & 0.24 & 0.38 & 0.22 & -0.06 & 0.07 & 0.12 & 0.09 & 0.40 \\
\hline & Allemagne & -0.15 & 0.55 & 0.23 & 0.26 & 0.29 & 0.43 & 0.17 & -0.10 & 0.14 & 0.15 & -0.01 & 0.26 \\
\hline & Grèce & 0.04 & 0.15 & 0.06 & 0.10 & 0.12 & 0.29 & 0.09 & 0.05 & 0.09 & 0.12 & 0.13 & 0.22 \\
\hline & Hongrie & 0.04 & 0.36 & 0.10 & 0.16 & 0.21 & 0.37 & 0.18 & -0.01 & 0.15 & 0.14 & 0.14 & 0.36 \\
\hline & Islande & -0.26 & 0.38 & 0.03 & 0.10 & 0.21 & 0.29 & 0.15 & -0.03 & 0.09 & 0.19 & 0.02 & 0.20 \\
\hline & Irlande & 0.43 & 0.46 & 0.08 & 0.14 & 0.22 & 0.38 & 0.21 & -0.01 & 0.15 & 0.28 & 0.02 & 0.23 \\
\hline & Italie & 0.14 & 0.36 & 0.07 & 0.15 & 0.18 & 0.36 & 0.15 & -0.05 & 0.20 & 0.12 & 0.08 & 0.32 \\
\hline & Japon $^{1}$ & -0.86 & 0.24 & -0.08 & $\mathrm{~m}$ & 0.24 & 0.25 & 0.10 & 0.02 & 0.08 & 0.13 & -0.01 & 0.21 \\
\hline & Corée $^{2}$ & $\mathrm{~m}$ & 0.03 & 0.11 & 0.19 & 0.22 & 0.21 & 0.20 & 0.12 & 0.09 & 0.14 & 0.12 & 0.19 \\
\hline & Luxembourg & 0.02 & 0.42 & 0.17 & 0.12 & 0.18 & 0.36 & 0.08 & -0.02 & 0.07 & 0.15 & 0.06 & 0.24 \\
\hline & Mexique & -0.09 & 0.20 & -0.05 & 0.09 & 0.10 & 0.24 & 0.10 & 0.09 & 0.07 & 0.13 & 0.18 & 0.24 \\
\hline & Nouvelle-Zélande & 0.23 & 0.27 & 0.16 & 0.12 & 0.19 & 0.35 & 0.17 & -0.01 & 0.17 & 0.23 & 0.04 & 0.25 \\
\hline & Norvège & 0.08 & 0.50 & 0.09 & 0.13 & 0.20 & 0.34 & 0.25 & -0.07 & 0.13 & 0.20 & 0.04 & 0.22 \\
\hline & Pologne & -0.99 & 0.36 & -0.04 & 0.11 & 0.14 & 0.32 & 0.11 & -0.01 & 0.15 & 0.19 & 0.15 & 0.28 \\
\hline & Portugal & 0.26 & 0.45 & -0.03 & 0.15 & 0.17 & 0.32 & 0.19 & 0.05 & 0.11 & 0.14 & 0.18 & 0.25 \\
\hline & Espagne & -0.10 & 0.28 & 0.10 & 0.17 & 0.21 & 0.40 & 0.19 & 0.05 & 0.09 & 0.12 & 0.06 & 0.27 \\
\hline & Suède & -0.12 & 0.44 & 0.14 & 0.14 & 0.22 & 0.38 & 0.14 & -0.09 & 0.11 & 0.18 & -0.04 & 0.24 \\
\hline & Suisse & -0.07 & 0.56 & 0.06 & 0.19 & 0.23 & 0.36 & 0.19 & -0.06 & 0.11 & 0.16 & 0.17 & 0.28 \\
\hline & Royaume-Uni & 0.32 & 0.25 & 0.11 & 0.13 & 0.22 & 0.40 & 0.23 & -0.03 & 0.18 & 0.22 & 0.03 & 0.33 \\
\hline & États-Unis & 0.13 & 0.35 & 0.11 & 0.13 & 0.23 & 0.37 & 0.17 & 0.07 & 0.13 & 0.24 & 0.05 & 0.25 \\
\hline & Médiane de l'OCDE & 0.02 & 0.38 & 0.09 & 0.14 & 0.22 & 0.37 & 0.18 & -0.02 & 0.11 & 0.15 & 0.04 & 0.25 \\
\hline & Éc.T. de corrélation & 0.31 & 0.15 & 0.07 & 0.05 & 0.04 & 0.06 & 0.05 & 0.06 & -0.04 & 0.05 & 0.07 & 0.06 \\
\hline & Brésil & -0.52 & 0.52 & 0.00 & 0.09 & 0.18 & 0.30 & 0.16 & 0.13 & 0.11 & 0.18 & 0.20 & 0.27 \\
\hline & Lettonie & 0.17 & 0.45 & 0.01 & 0.04 & 0.11 & 0.23 & 0.06 & 0.01 & 0.09 & 0.15 & 0.09 & 0.22 \\
\hline & Liechtenstein & -0.02 & 0.46 & 0.25 & 0.21 & 0.24 & 0.29 & 0.31 & 0.04 & -0.06 & 0.13 & 0.04 & 0.28 \\
\hline & Fédération de Russie & 0.09 & 0.40 & 0.06 & 0.07 & 0.10 & 0.28 & 0.09 & 0.03 & 0.15 & 0.21 & 0.16 & 0.26 \\
\hline & Pays-Bas ${ }^{3}$ & 0.02 & 0.43 & 0.15 & 0.20 & 0.21 & 0.30 & 0.21 & -0.02 & 0.07 & 0.19 & 0.03 & 0.18 \\
\hline
\end{tabular}

1. Le Japon a été exclu de la comparaison en raison de la quantité importante de données manquantes.

2. La Corée n'a pas recueilli de données à propos du statut d'immigrant.

3. Taux de participation insuffisant pour permettre les comparaisons. 
Tableau 7.1

Coefficients de régression pour le milieu socio-économique des élèves selon trois modèles différents

\begin{tabular}{|c|c|c|c|c|}
\hline & \multicolumn{2}{|c|}{ Régression linéaire } & \multicolumn{2}{|c|}{ Régression multi-niveaux } \\
\hline & Facteur unique & Parmi d'autres facteurs & Intra-établissement & Inter-établissements \\
\hline Australie & 31.7 & 20.0 & 12.2 & 42.7 \\
\hline Autriche & 35.2 & 12.7 & 2.3 & 46.0 \\
\hline Belgique & 38.3 & 15.9 & 6.5 & 61.1 \\
\hline Canada & 25.8 & 16.2 & 11.8 & 28.1 \\
\hline République tchèque & 43.3 & 18.9 & 8.0 & 50.4 \\
\hline Danemark & 29.1 & 15.3 & 12.4 & 21.3 \\
\hline Finlande & 20.9 & 13.5 & 11.8 & 11.1 \\
\hline France & 30.8 & 10.7 & 7.4 & 19.2 \\
\hline Allemagne & 45.3 & 16.7 & 3.7 & 63.7 \\
\hline Grèce & 28.1 & 17.0 & 7.5 & 46.8 \\
\hline Hongrie & 39.2 & 16.8 & 0.7 & 61.8 \\
\hline Islande & 19.3 & 12.6 & 10.5 & 7.5 \\
\hline Irlande & 30.3 & 18.6 & 13.3 & 40.5 \\
\hline Italie & 26.4 & 14.4 & 3.0 & 54.2 \\
\hline Japon & $\mathrm{m}$ & $\mathrm{m}$ & $\mathrm{m}$ & $\mathrm{m}$ \\
\hline Corée & 14.6 & 4.4 & -1.1 & 17.1 \\
\hline Luxembourg & 39.2 & 17.4 & 10.7 & 53.2 \\
\hline Mexique & 31.8 & 13.1 & 3.4 & 45.8 \\
\hline Nouvelle-Zélande & 31.9 & 20.9 & 15.6 & 42.6 \\
\hline Norvège & 29.8 & 14.4 & 12.9 & 10.0 \\
\hline Pologne & 35.5 & 20.1 & 1.5 & 82.6 \\
\hline Portugal & 38.5 & 11.3 & 7.3 & 20.9 \\
\hline Espagne & 26.5 & 9.2 & 3.7 & 18.8 \\
\hline Suède & 27.1 & 16.9 & 14.1 & 20.6 \\
\hline Suisse & 40.2 & 16.8 & 9.0 & 19.1 \\
\hline Royaume-Uni & 38.5 & 24.6 & 16.7 & 44.5 \\
\hline États-Unis & 33.6 & 16.5 & 9.9 & 52.8 \\
\hline Brésil & 26.1 & 12.6 & 3.3 & 40.7 \\
\hline Lettonie & 21.3 & 13.6 & 8.4 & 45.0 \\
\hline Liechtenstein $^{2}$ & $\mathrm{~m}$ & $\mathrm{~m}$ & $\mathrm{~m}$ & $\mathrm{~m}$ \\
\hline Fédération de Russie & 26.5 & 15.1 & 9.2 & 48.3 \\
\hline Pays-Bas ${ }^{3}$ & 30.0 & 15.1 & 6.5 & 76.4 \\
\hline
\end{tabular}

1. Le Japon est exclu de l'analyse en raison d'une quantité importante de données manquantes.

2. Le Liechtenstein est exclu de l'analyse, car le nombre d'établissements de ce pays a été jugé insuffisant pour les analyses concernant la décomposition de la variance.

3. Taux de participation insuffisant pour permettre les comparaisons. 
Tableau 7.2

Valeurs moyennes OCDE des coefficients de régression multi-niveaux intra et inter-établissements

\begin{tabular}{|c|c|c|c|c|c|c|}
\hline & $\begin{array}{c}\text { Échelle combinée } \\
\text { de compréhen- } \\
\text { sion de l'écrit }\end{array}$ & $\begin{array}{c}\text { Trouver } \\
\text { l'information }\end{array}$ & $\begin{array}{l}\text { Développer une } \\
\text { interprétation }\end{array}$ & $\begin{array}{l}\text { Réfléchir et } \\
\text { évaluer }\end{array}$ & $\begin{array}{l}\text { Textes } \\
\text { continus }\end{array}$ & $\begin{array}{c}\text { Textes non } \\
\text { continus }\end{array}$ \\
\hline \multicolumn{7}{|l|}{ Variables personnelles des élèves } \\
\hline Incitation au rendement & -3.1 & -3.8 & -3.1 & -2.3 & -2.9 & -3.7 \\
\hline Sentiment d'appartenance & 1.0 & 1.3 & 0.4 & 1.9 & 0.9 & 1.6 \\
\hline Climat de discipline & 1.4 & 0.7 & 1.3 & 2.3 & 1.8 & 0.8 \\
\hline Relations élèves-enseignants & 1.0 & 1.4 & 0.5 & 1.2 & 0.8 & 0.8 \\
\hline Communication culturelle & 2.9 & 1.4 & 2.8 & 4.5 & 3.3 & 2.1 \\
\hline Engagement à l'égard de la lecture & 18.5 & 19.0 & 19.5 & 17.7 & 19.5 & 17.3 \\
\hline Temps consacré aux devoirs & 0.2 & 0.0 & -0.5 & 1.3 & 0.1 & 0.5 \\
\hline Ressources éducatives & 3.0 & 4.2 & 2.6 & 2.8 & 2.4 & 4.4 \\
\hline Milieu socio-économique & 8.1 & 8.2 & 8.3 & 7.5 & 7.9 & 7.9 \\
\hline Nombre de livres à la maison & 5.0 & 5.2 & 5.2 & 5.0 & 5.0 & 5.5 \\
\hline Structure familiale & 2.3 & 3.3 & 1.8 & 2.2 & 2.0 & 3.6 \\
\hline Sexe & 11.9 & 3.5 & 10.2 & 23.9 & 18.6 & -2.5 \\
\hline Année d'études & 37.3 & 42.1 & 36.3 & 36.1 & 36.3 & 41.0 \\
\hline Statut d'immigrant & -21.8 & -24.3 & -21.1 & -20.4 & -21.4 & -20.8 \\
\hline \multicolumn{7}{|l|}{ Variables collectives des établissements } \\
\hline Engagement à l'égard de la lecture & 22.6 & 24.2 & 21.4 & 24.0 & 22.7 & 22.3 \\
\hline Milieu socio-économique & 39.8 & 42.9 & 38.7 & 41.0 & 40.4 & 41.8 \\
\hline
\end{tabular}


ANNEXE B

Tableau 7.3

Coefficients de régression multi-niveaux pour les caractéristiques individuelles des élèves

\begin{tabular}{|c|c|c|c|c|}
\hline & Sexe & Er.T & Engagement à l'égard de la lecture & Er.T \\
\hline Australie & 17.9 & $(2.8)$ & 24.7 & $(2.0)$ \\
\hline Autriche & 6.3 & $(3.2)$ & 18.1 & $(1.3)$ \\
\hline Belgique & 4.5 & $(2.2)$ & 18.0 & $(1.0)$ \\
\hline Canada & 14.8 & $(1.6)$ & 24.5 & $(0.8)$ \\
\hline République tchèque & 7.7 & $(2.4)$ & 17.9 & $(1.2)$ \\
\hline Danemark & 6.6 & $(2.7)$ & 29.1 & $(1.6)$ \\
\hline Finlande & 26.0 & $(2.2)$ & 30.5 & $(1.3)$ \\
\hline France & 10.3 & $(2.0)$ & 12.3 & $(1.1)$ \\
\hline Allemagne & 4.3 & $(2.3)$ & 17.9 & $(1.6)$ \\
\hline Grèce & 18.1 & $(2.5)$ & 8.9 & $(1.5)$ \\
\hline Hongrie & 7.4 & $(2.3)$ & 14.7 & $(1.0)$ \\
\hline Islande & 22.3 & $(2.9)$ & 31.4 & $(1.8)$ \\
\hline Irlande & 6.6 & (3.6) & 24.5 & $(1.5)$ \\
\hline Italie & 11.2 & (2.6) & 12.4 & $(1.0)$ \\
\hline Japon $^{1}$ & $\mathrm{~m}$ & $\mathrm{~m}$ & $\mathbf{m}$ & $\mathrm{m}$ \\
\hline Corée & 18.3 & $(2.6)$ & 12.6 & $(1.1)$ \\
\hline Luxembourg & 13.6 & $(2.7)$ & 11.8 & $(1.9)$ \\
\hline Mexique & 7.6 & $(2.3)$ & 9.4 & $(1.2)$ \\
\hline Nouvelle-Zélande & 28.6 & (3.4) & 24.6 & $(1.8)$ \\
\hline Norvège & 19.1 & (3.7) & 28.9 & $(2.0)$ \\
\hline Pologne & -0.5 & $(2.9)$ & 11.2 & $(1.8)$ \\
\hline Portugal & 2.0 & $(2.7)$ & 13.3 & $(1.5)$ \\
\hline Espagne & 7.7 & $(1.9)$ & 14.2 & $(1.3)$ \\
\hline Suède & 19.7 & $(2.5)$ & 30.3 & $(1.8)$ \\
\hline Suisse & 6.7 & $(2.1)$ & 23.4 & $(1.5)$ \\
\hline Royaume-Uni & 13.8 & $(2.3)$ & 19.8 & $(1.2)$ \\
\hline États-Unis & 10.7 & (3.2) & 16.8 & $(2.7)$ \\
\hline Brésil & 0.5 & (2.7) & 14.1 & (1.4) \\
\hline Lettonie & 25.3 & (3.2) & 14.7 & $(2.1)$ \\
\hline Liechtenstein ${ }^{2}$ & $\mathrm{~m}$ & $\mathrm{~m}$ & $\mathbf{m}$ & $\mathrm{m}$ \\
\hline Fédération de Russie & 16.7 & $(2.3)$ & 10.3 & $(1.2)$ \\
\hline Pays-Bas $^{3}$ & 2.9 & $(2.2)$ & 15.5 & $(1.7)$ \\
\hline
\end{tabular}


Tableau 7.4

Coefficients de régression multi-niveaux pour la structure familiale, le milieu socio-économique et le statut d'immigrant

\begin{tabular}{|c|c|c|c|c|c|c|}
\hline & $\begin{array}{r}\text { Structure } \\
\text { familiale }\end{array}$ & Er.T & $\begin{array}{l}\text { Milieu socio- } \\
\text { économique }\end{array}$ & Er.T & $\begin{array}{c}\text { Statut } \\
\text { d'immigrant } \\
\end{array}$ & Er.T \\
\hline Australie & 5.0 & $(3.9)$ & 12.2 & $(1.9)$ & -29.5 & $(4.8)$ \\
\hline Autriche & -2.8 & $(3.1)$ & 2.3 & (1.3) & -30.9 & $(5.5)$ \\
\hline Belgique & -4.5 & $(2.1)$ & 6.5 & (1.3) & -4.7 & $(6.0)$ \\
\hline Canada & 7.0 & (1.6) & 11.8 & $(0.9)$ & -25.1 & (3.6) \\
\hline République tchèque & -4.0 & (2.6) & 8.0 & (1.1) & -1.4 & $(18.1)$ \\
\hline Danemark & 5.1 & $(2.9)$ & 12.4 & (1.9) & -44.7 & $(7.6)$ \\
\hline Finlande & 10.2 & (2.9) & 11.8 & $(1.2)$ & -61.8 & $(10.3)$ \\
\hline France & -0.6 & $(2.3)$ & 7.4 & (1.4) & -15.7 & (7.9) \\
\hline Allemagne & -4.6 & (2.6) & 3.7 & $(1.5)$ & -23.5 & $(6.3)$ \\
\hline Grèce & 0.8 & $(3.5)$ & 7.5 & (1.6) & 2.1 & $(8.8)$ \\
\hline Hongrie & -4.0 & (2.3) & 0.7 & (1.1) & 3.0 & $(7.0)$ \\
\hline Islande & 4.3 & (3.4) & 10.5 & (1.5) & -36.5 & $(22.5)$ \\
\hline Irlande & 8.8 & (3.3) & 13.3 & (1.5) & 8.7 & $(10.2)$ \\
\hline Italie & 4.9 & $(2.1)$ & 3.0 & $(1.2)$ & -6.2 & $(17.7)$ \\
\hline Japon $^{1}$ & $\mathrm{~m}$ & $\mathrm{~m}$ & $\mathrm{~m}$ & $\mathrm{~m}$ & $\mathrm{~m}$ & $\mathrm{~m}$ \\
\hline Corée ${ }^{2}$ & -0.5 & (2.9) & -1.1 & $(1.2)$ & $\mathrm{m}$ & $\mathrm{m}$ \\
\hline Luxembourg & 2.4 & (3.1) & 10.7 & $(1.7)$ & -21.5 & $(4.0)$ \\
\hline Mexique & -5.0 & $(2.2)$ & 3.4 & (1.3) & -32.3 & $(8.6)$ \\
\hline Nouvelle-Zélande & 6.8 & (3.1) & 15.6 & (1.7) & -35.8 & $(4.8)$ \\
\hline Norvège & 11.9 & (3.7) & 12.9 & (1.6) & -41.2 & $(10.2)$ \\
\hline Pologne & -0.2 & (3.9) & 1.5 & (1.7) & -29.8 & $(29.0)$ \\
\hline Portugal & -4.6 & $(2.5)$ & 7.3 & (1.4) & -15.8 & $(10.7)$ \\
\hline Espagne & -2.3 & (2.5) & 3.7 & (1.1) & -9.3 & $(9.2)$ \\
\hline Suède & 11.9 & $(2.6)$ & 14.1 & $(1.5)$ & -27.1 & $(6.5)$ \\
\hline Suisse & -1.6 & $(2.6)$ & 9.0 & (1.4) & -43.1 & $(4.1)$ \\
\hline Royaume-Uni & 13.6 & (2.6) & 16.7 & (1.3) & -31.1 & $(8.9)$ \\
\hline États-Unis & 21.5 & (3.9) & 9.9 & (2.0) & -6.4 & $(8.1)$ \\
\hline Brésil & -4.7 & (3.3) & 3.3 & (1.6) & -59.0 & $(40.4)$ \\
\hline Lettonie & -3.9 & (3.7) & 8.4 & $(1.5)$ & -5.8 & $(5.6)$ \\
\hline Liechtenstein ${ }^{3}$ & $\mathrm{~m}$ & $\mathrm{~m}$ & $\mathrm{~m}$ & $\mathrm{~m}$ & $\mathrm{~m}$ & $\mathrm{~m}$ \\
\hline Fédération de Russie & -3.0 & $(2.1)$ & 9.2 & $(1.3)$ & 2.5 & $(5.2)$ \\
\hline Pays-Bas ${ }^{4}$ & -0.6 & $(4.2)$ & 6.5 & (1.6) & -32.0 & $(8.2)$ \\
\hline
\end{tabular}

1. Le Japon est exclu de l'analyse en raison d'une quantité importante de données manquantes.

2. Le Liechtenstein est exclu de l'analyse, car le nombre d'établissements de ce pays a été jugé insuffisant pour les analyses concernant la décomposition de la variance.

3. Taux de participation insuffisant pour permettre les comparaisons.

Note: Les valeurs en caractère gras sont statistiquement significatives. 
Tableau 7.5

Coefficients de régression multi-niveaux pour la communication culturelle avec les parents, les ressources éducatives et le nombre de livres à la maison

\begin{tabular}{|c|c|c|c|c|c|c|}
\hline & $\begin{array}{l}\text { Communication } \\
\text { culturelle }\end{array}$ & Er.T & $\begin{array}{l}\text { Ressources éduca- } \\
\text { tives à la maison }\end{array}$ & Er.T & $\begin{array}{c}\text { Nombre de livres à la } \\
\text { maison }\end{array}$ & Er.T \\
\hline Australie & 9.2 & $(1.8)$ & 3.0 & $(1.7)$ & 4.4 & $(1.2)$ \\
\hline Autriche & 1.7 & $(1.5)$ & 2.2 & $(1.6)$ & 4.6 & $(1.0)$ \\
\hline Belgique & 1.0 & $(1.0)$ & 2.0 & $(1.0)$ & 3.5 & $(0.7)$ \\
\hline Canada & 3.8 & $(0.8)$ & 0.5 & $(0.8)$ & 4.9 & $(0.6)$ \\
\hline République tchèque & 3.1 & $(1.1)$ & 3.3 & $(1.3)$ & 6.9 & $(0.9)$ \\
\hline Danemark & 10.9 & $(1.6)$ & 0.7 & (1.4) & 6.5 & $(1.2)$ \\
\hline Finlande & 5.2 & $(1.3)$ & 0.3 & $(1.3)$ & 4.1 & $(0.9)$ \\
\hline France & 1.3 & $(1.2)$ & 4.6 & $(1.0)$ & 5.2 & $(0.8)$ \\
\hline Allemagne & -0.1 & $(1.3)$ & 5.0 & $(2.2)$ & 3.9 & (1.1) \\
\hline Grèce & 1.7 & $(1.3)$ & 6.1 & $(1.2)$ & 3.0 & (1.1) \\
\hline Hongrie & -3.1 & $(0.9)$ & -0.3 & $(1.0)$ & 6.8 & $(0.8)$ \\
\hline Islande & 5.8 & $(1.6)$ & -1.8 & $(1.5)$ & 7.0 & $(1.1)$ \\
\hline Irlande & 1.7 & $(1.4)$ & 4.8 & $(1.5)$ & 6.7 & (1.1) \\
\hline Italie & 2.8 & $(1.2)$ & 0.6 & $(1.1)$ & 1.1 & $(0.7)$ \\
\hline Japon $^{1}$ & $\mathrm{~m}$ & $\mathrm{~m}$ & $\mathrm{~m}$ & $\mathrm{~m}$ & $\mathrm{~m}$ & $\mathrm{~m}$ \\
\hline Corée & 0.0 & $(1.1)$ & -0.5 & $(1.1)$ & 5.7 & $(0.7)$ \\
\hline Luxembourg & 1.3 & $(1.8)$ & 8.5 & $(1.3)$ & 6.3 & $(1.0)$ \\
\hline Mexique & 1.4 & $(1.5)$ & 1.4 & $(1.2)$ & 4.6 & $(1.2)$ \\
\hline Nouvelle-Zélande & -1.4 & $(1.6)$ & 9.6 & $(1.8)$ & 7.9 & $(1.2)$ \\
\hline Norvège & 7.2 & $(1.7)$ & 10.3 & $(1.7)$ & 5.1 & (1.3) \\
\hline Pologne & -1.9 & $(1.3)$ & 2.1 & (1.3) & 1.8 & $(1.0)$ \\
\hline Portugal & 5.4 & $(1.1)$ & 2.2 & $(1.2)$ & 3.5 & (1.1) \\
\hline Espagne & 4.6 & $(1.2)$ & 1.7 & $(1.2)$ & 6.6 & (1.1) \\
\hline Suède & 6.9 & $(1.4)$ & 1.3 & $(1.2)$ & 5.5 & (1.1) \\
\hline Suisse & 1.5 & $(1.2)$ & 4.8 & $(1.1)$ & 4.4 & $(0.9)$ \\
\hline Royaume-Uni & 3.3 & $(1.3)$ & 3.6 & (1.3) & 5.6 & $(1.0)$ \\
\hline États-Unis & 1.1 & $(2.0)$ & 0.2 & $(1.7)$ & 9.3 & $(1.2)$ \\
\hline Brésil & 2.1 & $(1.5)$ & 3.3 & $(1.8)$ & 0.6 & (1.4) \\
\hline Lettonie & 4.3 & $(1.8)$ & 2.5 & (1.8) & 7.2 & (1.4) \\
\hline Liechtenstein $^{2}$ & $\mathrm{~m}$ & $\mathrm{~m}$ & $\mathrm{~m}$ & $\mathrm{~m}$ & $\mathrm{~m}$ & $\mathrm{~m}$ \\
\hline Fédération de Russie & 0.3 & $(1.0)$ & 3.7 & (1.1) & 5.0 & $(0.9)$ \\
\hline Pays-Bas ${ }^{3}$ & 5.2 & $(1.6)$ & 3.3 & $(2.0)$ & 3.3 & $(1.2)$ \\
\hline
\end{tabular}

1. Le Japon est exclu de l'analyse en raison d'une quantité importante de données manquantes à propos du statut professionnel des parents.

2. Le Liechtenstein est exclu de l'analyse, car le nombre d'établissements de ce pays a été jugé insuffisant pour les analyses concernant la décomposition de la variance.

3. Taux de participation insuffisant pour permettre les comparaisons.

Note: Les valeurs en caractère gras sont statistiquement significatives. 
Tableau 7.6

Coefficients de régression multi-niveaux pour les variables d'enseignement et d'apprentissage

\begin{tabular}{|c|c|c|c|c|c|c|}
\hline & Encouragement & Er.T & $\begin{array}{c}\text { Temps consacré aux } \\
\text { devoirs }\end{array}$ & Er. T & Année d'études & Er. T \\
\hline Australie & -7.7 & (1.4) & 5.2 & (1.9) & 39.9 & (3.0) \\
\hline Autriche & -1.8 & $(1.3)$ & -7.3 & $(1.3)$ & 31.0 & $(1.8)$ \\
\hline Belgique & -1.5 & $(1.1)$ & 0.8 & $(1.2)$ & 51.7 & $(2.9)$ \\
\hline Canada & -6.7 & $(0.8)$ & 3.2 & $(0.8)$ & 46.1 & $(1.7)$ \\
\hline République tchèque & -4.0 & $(1.4)$ & -6.3 & $(1.1)$ & 31.4 & (2.6) \\
\hline Danemark & -1.2 & $(1.5)$ & -7.9 & $(1.6)$ & 32.8 & $(5.2)$ \\
\hline Finlande & -3.3 & $(1.4)$ & -4.9 & $(1.4)$ & 40.4 & $(4.2)$ \\
\hline France & -2.8 & $(1.1)$ & 3.3 & $(1.1)$ & 46.6 & (3.1) \\
\hline Allemagne & -1.5 & $(1.2)$ & -2.3 & $(1.2)$ & 35.0 & (1.8) \\
\hline Grèce & -1.3 & $(1.4)$ & 8.5 & $(1.6)$ & 18.3 & (3.9) \\
\hline Hongrie & -0.8 & $(0.9)$ & -0.3 & $(1.0)$ & 22.5 & $(1.9)$ \\
\hline Islande $^{1}$ & -7.9 & $(1.5)$ & -8.6 & $(1.6)$ & n.a. & n.a. \\
\hline Irlande & -4.0 & (1.3) & 2.1 & $(1.5)$ & 24.0 & (1.6) \\
\hline Italie & -3.5 & $(1.1)$ & 1.7 & $(1.2)$ & 32.2 & (3.0) \\
\hline Japon $^{2}$ & $\mathrm{~m}$ & $\mathrm{~m}$ & $\mathrm{~m}$ & $\mathrm{~m}$ & $\mathrm{~m}$ & $\mathrm{~m}$ \\
\hline Corée & 2.5 & $(1.0)$ & 0.7 & $(1.1)$ & 13.4 & (11.8) \\
\hline Luxembourg & -1.5 & (1.6) & -2.8 & $(1.3)$ & 30.7 & $(2.0)$ \\
\hline Mexique & -3.0 & $(1.3)$ & 2.2 & $(1.0)$ & 29.0 & (3.0) \\
\hline Nouvelle-Zélande & -8.1 & $(1.8)$ & 3.6 & $(1.6)$ & 54.0 & $(4.7)$ \\
\hline Norvège & -7.6 & (1.6) & -1.2 & $(1.7)$ & 42.6 & (22.0) \\
\hline Pologne ${ }^{1}$ & -3.2 & $(1.2)$ & 0.3 & $(1.6)$ & n.a. & n.a. \\
\hline Portugal & -3.7 & $(1.0)$ & -3.7 & $(1.2)$ & 53.3 & (1.7) \\
\hline Espagne & -1.4 & $(1.2)$ & 5.6 & $(1.3)$ & 70.7 & $(2.2)$ \\
\hline Suède & -7.6 & $(1.2)$ & -11.3 & $(1.4)$ & 70.3 & $(9.8)$ \\
\hline Suisse & 1.5 & $(1.3)$ & -1.4 & $(1.2)$ & 44.0 & $(2.9)$ \\
\hline Royaume-Uni & -4.3 & $(1.2)$ & 8.1 & $(1.5)$ & 10.5 & (2.1) \\
\hline États-Unis & -2.0 & (1.9) & 7.4 & $(1.5)$ & 37.6 & (2.9) \\
\hline Brésil & 2.9 & (1.6) & 0.8 & (1.4) & 33.8 & (1.7) \\
\hline Lettonie & -1.1 & $(1.4)$ & 2.7 & (1.9) & 30.8 & (3.1) \\
\hline Liechtenstein $^{3}$ & $\mathrm{~m}$ & $\mathrm{~m}$ & $\mathrm{~m}$ & $\mathrm{~m}$ & $\mathrm{~m}$ & $\mathrm{~m}$ \\
\hline Fédération de Russie & -3.2 & $(1.2)$ & 9.5 & $(1.2)$ & 34.6 & $(2.8)$ \\
\hline Pays-Bas ${ }^{4}$ & -5.8 & $(1.4)$ & -1.7 & $(1.2)$ & 35.9 & (3.7) \\
\hline
\end{tabular}

1. En Islande et en Pologne, les élèves inclus dans la base de données internationale fréquentaient la même année d'études. La variable relative à l'année d'études n'est donc pas applicable à ces pays.

2. Le Japon est exclu de l'analyse en raison d'une quantité importante de données manquantes.

3. Le Liechtenstein est exclu de l'analyse, car le nombre d'établissements de ce pays a été jugé insuffisant pour les analyses concernant la décomposition de la variance.

4. Taux de participation insuffisant pour permettre les comparaisons.

Note: Les valeurs en caractère gras sont statistiquement significatives. 
Tableau 7.7

Coefficients de régression multi-niveaux pour le sentiment d'appartenance et le climat d'apprentissage

\begin{tabular}{|c|c|c|c|c|c|c|}
\hline & Sens d'appartenance & Er.T & Climat de discipline & Er.T & $\begin{array}{c}\text { Relations élèves- } \\
\text { enseignants }\end{array}$ & Er. T \\
\hline Australie & -2.7 & $(1.5)$ & 2.7 & $(1.6)$ & 2.0 & $(1.7)$ \\
\hline Autriche & 1.8 & (1.4) & -2.0 & (1.3) & 2.9 & (1.3) \\
\hline Belgique & 2.0 & $(0.9)$ & 1.5 & $(1.1)$ & -3.0 & (1.1) \\
\hline Canada & -2.0 & $(0.7)$ & 2.2 & $(0.8)$ & 5.7 & $(0.8)$ \\
\hline République tchèque & 5.1 & $(1.0)$ & 1.0 & (1.1) & -1.7 & (1.1) \\
\hline Danemark & 2.0 & (1.3) & 2.5 & (1.4) & 4.7 & (1.4) \\
\hline Finlande & -4.3 & (1.3) & 1.0 & (1.3) & 5.7 & $(1.2)$ \\
\hline France & 0.4 & $(1.1)$ & -1.4 & $(1.2)$ & -4.9 & (1.3) \\
\hline Allemagne & -0.5 & $(1.2)$ & -0.5 & (1.4) & 0.6 & $(1.2)$ \\
\hline Grèce & 3.3 & $(1.3)$ & -0.5 & $(1.4)$ & -2.2 & $(1.2)$ \\
\hline Hongrie & 4.4 & $(0.9)$ & 0.2 & $(1.1)$ & -5.8 & (1.1) \\
\hline Islande & -1.0 & (1.4) & 2.1 & (1.4) & 7.9 & (1.6) \\
\hline Irlande & -2.0 & (1.4) & 7.8 & $(1.7)$ & 0.7 & (1.6) \\
\hline Italie & -0.5 & $(1.1)$ & -0.6 & $(1.2)$ & -1.4 & (1.4) \\
\hline Japon $^{1}$ & $\mathrm{~m}$ & $\mathrm{~m}$ & $\mathrm{~m}$ & $\mathrm{~m}$ & $\mathrm{~m}$ & $\mathrm{~m}$ \\
\hline Corée & -0.6 & (1.1) & 1.1 & (1.1) & 2.4 & $(0.9)$ \\
\hline Luxembourg & 5.3 & (1.4) & 0.1 & $(1.5)$ & -1.0 & (1.5) \\
\hline Mexique & 5.4 & (1.3) & 2.0 & $(1.2)$ & 0.1 & (1.1) \\
\hline Nouvelle-Zélande & -2.0 & $(1.8)$ & -0.4 & $(2.0)$ & 5.3 & (1.7) \\
\hline Norvège & -2.9 & (1.7) & -2.0 & $(1.7)$ & 8.7 & (1.9) \\
\hline Pologne & 3.0 & (1.3) & 5.3 & (1.6) & -3.2 & (1.6) \\
\hline Portugal & 6.3 & $(0.9)$ & 0.9 & $(1.2)$ & -1.4 & $(1.2)$ \\
\hline Espagne & -0.3 & $(1.0)$ & -0.0 & (1.4) & 1.0 & $(1.0)$ \\
\hline Suède & -2.8 & (1.3) & 2.8 & $(1.3)$ & 5.5 & (1.6) \\
\hline Suisse & 5.2 & (1.3) & 4.8 & (1.3) & 0.1 & (1.1) \\
\hline Royaume-Uni & -1.8 & $(1.2)$ & 7.4 & $(1.5)$ & 3.2 & $(1.3)$ \\
\hline États-Unis & 2.4 & $(1.8)$ & 6.2 & $(1.5)$ & 2.3 & (1.8) \\
\hline Brésil & 2.2 & $(1.2)$ & -3.6 & $(1.4)$ & -2.9 & (1.4) \\
\hline Lettonie & 0.8 & (1.7) & 2.1 & $(1.8)$ & -0.1 & (2.3) \\
\hline Liechtenstein $^{2}$ & $\mathrm{~m}$ & $\mathrm{~m}$ & $\mathrm{~m}$ & $\mathrm{~m}$ & $\mathrm{~m}$ & $\mathrm{~m}$ \\
\hline Fédération de Russie & 1.0 & $(1.2)$ & -0.9 & $(1.1)$ & -0.5 & $(1.2)$ \\
\hline Pays-Bas ${ }^{3}$ & 1.9 & $(1.3)$ & -0.9 & $(1.5)$ & -0.4 & (1.6) \\
\hline
\end{tabular}

1. Le Japon est exclu de l'analyse en raison d'une quantité importante de données manquantes.

2. Le Liechtenstein est exclu de l'analyse, car le nombre d'établissements de ce pays a été jugé insuffisant pour les analyses concernant la décomposition de la variance.

3. Taux de participation insuffisant pour permettre les comparaisons.

Note: Les valeurs en caractère gras sont statistiquement significatives. 
Tableau 7.8

Coefficients de régression multi-niveaux pour les caractéristiques des établissements

\begin{tabular}{|c|c|c|c|c|}
\hline & \multicolumn{2}{|c|}{ Degré d'engagement à l'égard de la lecture } & \multicolumn{2}{|c|}{ Milieu socio-économique au niveau établissement } \\
\hline & Coefficient & Er.T & Coefficient & Er.T \\
\hline Australie & 4.4 & $(3.9)$ & 42.7 & $(3.2)$ \\
\hline Autriche & 44.4 & (3.6) & 46.0 & $(3.5)$ \\
\hline Belgique & 22.0 & $(2.3)$ & 61.1 & (2.6) \\
\hline Canada & 2.1 & $(2.1)$ & 28.1 & $(1.9)$ \\
\hline République tchèque & 37.2 & $(3.2)$ & 50.4 & $(2.5)$ \\
\hline Danemark & 15.2 & $(5.2)$ & 21.3 & $(4.0)$ \\
\hline Finlande & 17.5 & $(5.4)$ & 11.1 & $(2.5)$ \\
\hline France & 33.4 & $(6.0)$ & 19.2 & (3.6) \\
\hline Allemagne & 44.8 & (3.1) & 63.7 & $(2.7)$ \\
\hline Grèce & 65.3 & (3.9) & 46.8 & (2.6) \\
\hline Hongrie & 35.8 & (4.4) & 61.8 & (3.8) \\
\hline Islande & 8.3 & (6.5) & 7.5 & (3.9) \\
\hline Irlande & 12.6 & $(4.6)$ & 40.5 & $(2.8)$ \\
\hline Italie & 30.9 & (6.2) & 54.2 & (3.5) \\
\hline Japon $^{1}$ & $\mathrm{~m}$ & $\mathrm{~m}$ & $\mathrm{~m}$ & $\mathrm{~m}$ \\
\hline Corée & 64.3 & (3.0) & 17.1 & (2.6) \\
\hline Luxembourg & 9.4 & $(8.6)$ & 53.2 & $(4.8)$ \\
\hline Mexique & 9.8 & (6.9) & 45.8 & (3.2) \\
\hline Nouvelle-Zélande & -6.1 & $(4.8)$ & 42.6 & $(3.8)$ \\
\hline Norvège & 24.2 & (7.9) & 10.0 & $(4.1)$ \\
\hline Pologne & 58.9 & (3.9) & 82.6 & $(2.7)$ \\
\hline Portugal & 19.3 & $(4.5)$ & 20.9 & (2.4) \\
\hline Espagne & 25.0 & $(2.8)$ & 18.8 & $(1.7)$ \\
\hline Suède & -2.5 & (3.9) & 20.6 & (3.2) \\
\hline Suisse & 41.2 & $(4.5)$ & 19.1 & (4.4) \\
\hline Royaume-Uni & 11.0 & $(5.6)$ & 44.5 & (3.0) \\
\hline États-Unis & -7.6 & (6.1) & 52.8 & $(4.3)$ \\
\hline Brésil & -1.0 & (3.1) & 40.7 & $(2.7)$ \\
\hline Lettonie & 23.9 & (5.3) & 45.0 & $(4.2)$ \\
\hline Liechtenstein $^{2}$ & $\mathrm{~m}$ & $\mathrm{~m}$ & $\mathrm{~m}$ & $\mathrm{~m}$ \\
\hline Fédération de Russie & 13.7 & $(2.7)$ & 48.3 & $(3.6)$ \\
\hline Pays-Bas ${ }^{3}$ & 20.5 & (3.2) & 76.4 & $(3.1)$ \\
\hline
\end{tabular}

1. Le Japon est exclu de l'analyse en raison d'une quantité importante de données manquantes.

2. Le Liechtenstein est exclu de l'analyse, car le nombre d'établissements de ce pays a été jugé insuffisant pour les analyses concernant la décomposition de la variance.

3. Taux de participation insuffisant pour permettre les comparaisons.

Note: Les valeurs en caractère gras sont statistiquement significatives. 
ANNEXE B

Tableau 7.9

Part de la variance entre établissements dans la variance totale (ICC) des performances des élèves en compréhension de l'écrit

\begin{tabular}{|c|c|c|}
\hline & Corrélation intra-classe totale (ICC) & Er.T \\
\hline Australie & 0.18 & $(0.02)$ \\
\hline Autriche & 0.60 & $(0.01)$ \\
\hline Belgique & 0.60 & $(0.01)$ \\
\hline Canada & 0.18 & $(0.01)$ \\
\hline République tchèque & 0.53 & $(0.04)$ \\
\hline Danemark & 0.19 & $(0.01)$ \\
\hline Finlande & 0.12 & $(0.01)$ \\
\hline France & 0.50 & $(0.01)$ \\
\hline Allemagne & 0.59 & $(0.01)$ \\
\hline Grèce & 0.51 & $(0.01)$ \\
\hline Hongrie & 0.67 & $(0.01)$ \\
\hline Islande & 0.08 & $(0.02)$ \\
\hline Irlande & 0.18 & $(0.02)$ \\
\hline Italie & 0.55 & $(0.03)$ \\
\hline Japon $^{1}$ & $\mathrm{~m}$ & $\mathrm{~m}$ \\
\hline Corée & 0.37 & $(0.02)$ \\
\hline Luxembourg & 0.31 & $(0.01)$ \\
\hline Mexique & 0.53 & $(0.01)$ \\
\hline Nouvelle-Zélande & 0.16 & $(0.01)$ \\
\hline Norvège & 0.10 & $(0.02)$ \\
\hline Pologne & 0.62 & $(0.02)$ \\
\hline Portugal & 0.37 & $(0.01)$ \\
\hline Espagne & 0.20 & $(0.01)$ \\
\hline Suède & 0.09 & $(0.01)$ \\
\hline Suisse & 0.43 & $(0.01)$ \\
\hline Royaume-Uni & 0.22 & $(0.01)$ \\
\hline États-Unis & 0.29 & $(0.01)$ \\
\hline Brésil & 0.44 & $(0.01)$ \\
\hline Lettonie & 0.31 & $(0.02)$ \\
\hline Liechtenstein $^{2}$ & $\mathrm{~m}$ & $\mathrm{~m}$ \\
\hline Fédération de Russie & 0.37 & $(0.02)$ \\
\hline Pays-Bas ${ }^{3}$ & 0.50 & $(0.02)$ \\
\hline
\end{tabular}

1. En raison des méthodes d'échantillonnage appliquées au Japon, la variance entre établissements ne peut être séparée de la variance entre classes au sein des établissements.

2. Le Liechtenstein est exclu de l'analyse, car le nombre d'établissements de ce pays a été jugé insuffisant pour les analyses concernant la décomposition de la variance.

3. Taux de participation insuffisant pour permettre les comparaisons.

Remarque: les résultats présentés dans ce tableau sont basés sur tous les élèves inclus dans la base de données internationale PISA 2000. Les résultats présentés dans les autres tableaux sont basés sur les élèves inclus dans les analyses multi-niveaux. Pour davantage d'informations à cet égard, il y a lieu de consulter le tableau A3.1 à l'annexe A3. 
Tableau 7.10

Corrélation intra-classe dans PISA, dans IEA/RLS et dansTIMSS

\begin{tabular}{|c|c|c|c|}
\hline & PISA & IEA/RLS & TIMSS 95 \\
\hline Australie & 0.18 & n.a. & 0.45 \\
\hline Autriche & 0.60 & n.a. & 0.33 \\
\hline Belgique & 0.60 & n.a. & n.a. \\
\hline Belgique (Fl.) & n.a. & n.a. & 0.40 \\
\hline Belgique (Fr.) & n.a. & 0.40 & 0.47 \\
\hline Canada & 0.18 & n.a. & 0.18 \\
\hline République tchèque & 0.53 & n.a. & 0.21 \\
\hline Danemark & 0.19 & 0.09 & 0.08 \\
\hline Finlande & 0.12 & 0.03 & n.a. \\
\hline France & 0.50 & 0.35 & 0.25 \\
\hline Allemagne & 0.59 & n.a. & 0.46 \\
\hline Grèce & 0.51 & 0.22 & 0.14 \\
\hline Hongrie & 0.67 & n.a. & 0.18 \\
\hline Islande & 0.08 & 0.08 & 0.10 \\
\hline Irlande & 0.18 & 0.48 & 0.45 \\
\hline Italie & 0.55 & 0.28 & n.a. \\
\hline Corée & 0.37 & n.a. & 0.06 \\
\hline Luxembourg & 0.31 & n.a. & n.a. \\
\hline Mexique & 0.53 & n.a. & n.a. \\
\hline Nouvelle-Zélande & 0.16 & 0.41 & 0.36 \\
\hline Norvège & 0.10 & 0.06 & 0.06 \\
\hline Pologne & 0.62 & n.a. & n.a. \\
\hline Portugal & 0.37 & 0.27 & 0.15 \\
\hline Espagne & 0.20 & 0.22 & 0.16 \\
\hline Suède & 0.09 & 0.08 & 0.31 \\
\hline Suisse & 0.43 & 0.48 & 0.42 \\
\hline Royaume-Uni & 0.22 & n.a. & 0.19 \\
\hline États-Unis & 0.29 & 0.42 & 0.46 \\
\hline Brésil & 0.44 & n.a. & n.a. \\
\hline Lettonie & 0.31 & n.a. & 0.17 \\
\hline Liechtenstein $^{1}$ & $\mathrm{~m}$ & n.a. & n.a. \\
\hline Fédération de Russie & 0.37 & n.a. & 0.31 \\
\hline Pays-Bas² & 0.50 & 0.50 & 0.52 \\
\hline
\end{tabular}

1. Le Liechtenstein est exclu de l'analyse, car le nombre d'établissements de ce pays a été jugé insuffisant pour fonder les analyses concernant la décomposition de la variance.

2. Taux de participation insuffisant pour permettre les comparaisons.

Remarque: la variance entre classes calculée dans le cadre de l'IEA pourrait être inférieure à la variance entre établissements calculée dans PISA. Ce phénomène s'explique par plusieurs raisons. (i) Les populations cibles des deux enquêtes ne sont pas exactement identiques. Ainsi, en France, la variance PISA entre établissements est affectée par la structure du système d'éducation qui différencie les deux cycles de l'enseignement secondaire: le premier cycle est dispensé dans les collèges et le deuxième cycle, dans les lycées. (ii) Erreurs d'échantillonnage. (iii) La performance des élèves qui ont participé à PISA a été calculée à l'aide de valeurs plausibles générées après conditionnement selon l'appartenance aux écoles. En revanche, dans l'étude IEA/RLS, on a utilisé des estimations par maximum de vraisemblance qui peuvent surestimer la variance intra-établissement et, donc, sous-estimer la corrélation intra-classe. Remarque: n.a. signifie non administré. 
Tableau 7.11

Parts de variances inexpliquées au niveau des établissements et des élèves et pourcentages de variance expliquée entre et au sein des établissements

\begin{tabular}{|c|c|c|c|c|}
\hline & \multicolumn{2}{|c|}{ Variance inexpliquée } & \multicolumn{2}{|c|}{ Variance expliquée (en \%) } \\
\hline & Élèves & Établissements & Élèves & Établissements \\
\hline Australie & 5742.5 & 371.0 & 0.27 & 0.77 \\
\hline Autriche & 3487.9 & 794.1 & 0.18 & 0.84 \\
\hline Belgique & 3415.7 & 1545.4 & 0.25 & 0.75 \\
\hline Canada & 5186.1 & 649.2 & 0.28 & 0.55 \\
\hline République tchèque & 3343.8 & 691.7 & 0.20 & 0.78 \\
\hline Danemark & 5347.3 & 404.0 & 0.29 & 0.65 \\
\hline Finlande & 4514.2 & 703.9 & 0.34 & 0.29 \\
\hline France & 3210.7 & 419.0 & 0.19 & 0.89 \\
\hline Allemagne & 3536.5 & 895.7 & 0.22 & 0.83 \\
\hline Grèce & 4250.2 & 1340.2 & 0.10 & 0.70 \\
\hline Hongrie & 2631.9 & 1038.7 & 0.15 & 0.83 \\
\hline Islande & 5373.7 & 313.9 & 0.27 & 0.51 \\
\hline Irlande & 4959.9 & 397.0 & 0.28 & 0.71 \\
\hline Italie & 3385.3 & 1762.9 & 0.12 & 0.61 \\
\hline Japon $^{1}$ & $\mathrm{~m}$ & $\mathrm{~m}$ & $\mathrm{~m}$ & $\mathrm{~m}$ \\
\hline Corée & 2723.0 & 512.5 & 0.09 & 0.71 \\
\hline Luxembourg & 4067.4 & 205.1 & 0.26 & 0.91 \\
\hline Mexique & 3126.4 & 650.8 & 0.09 & 0.83 \\
\hline Nouvelle-Zélande & 6332.1 & 375.2 & 0.28 & 0.76 \\
\hline Norvège & 6218.1 & 370.4 & 0.29 & 0.59 \\
\hline Pologne & 3366.3 & 1439.5 & 0.05 & 0.74 \\
\hline Portugal & 3364.9 & 212.2 & 0.39 & 0.93 \\
\hline Espagne & 3323.2 & 340.2 & 0.40 & 0.75 \\
\hline Suède & 5008.6 & 143.2 & 0.32 & 0.78 \\
\hline Suisse & 3761.6 & 1276.9 & 0.31 & 0.68 \\
\hline Royaume-Uni & 5350.8 & 396.4 & 0.24 & 0.78 \\
\hline États-Unis & 5452.3 & 425.0 & 0.24 & 0.83 \\
\hline Brésil & 3448.1 & 735.9 & 0.17 & 0.75 \\
\hline Lettonie & 5577.9 & 1232.9 & 0.18 & 0.59 \\
\hline Liechtenstein $^{2}$ & $\mathrm{~m}$ & $\mathrm{~m}$ & $\mathrm{~m}$ & $\mathrm{~m}$ \\
\hline Fédération de Russie & 4198.1 & 1515.1 & 0.18 & 0.48 \\
\hline Pays-Bas ${ }^{3}$ & 2789.3 & 785.3 & 0.28 & 0.79 \\
\hline
\end{tabular}

1. En raison des méthodes d'échantillonnage appliquées au Japon, la variance intra-établissement ne peut être séparée de la variance entre classes au sein des établissements.

2. Le Liechtenstein est exclu de l'analyse, car le nombre d'établissements de ce pays a été jugé insuffisant pour les analyses concernant la décomposition de la variance.

3. Taux de participation insuffisant pour permettre les comparaisons. 
Tableau 8.1

Taux de participation à la vie active de la population âgée de 25 à 65 ans, selon le niveau de compétence en compréhension de textes suivis de IALS

\begin{tabular}{|c|c|c|c|c|}
\hline & Niveaux 1 et $2(\%)$ & Er.T & Niveaux 3, 4 et $5(\%)$ & Er. T \\
\hline Australie & 67 & $(1.1)$ & 84 & $(0.6)$ \\
\hline Belgique (Fl.) & 63 & $(1.7)$ & 83 & $(1.3)$ \\
\hline Canada & 67 & $(4.2)$ & 82 & $(4.6)$ \\
\hline Chili & 65 & $(1.4)$ & 81 & $(2.3)$ \\
\hline République tchèque & 71 & $(1.4)$ & 85 & (1.4) \\
\hline Danemark & 75 & $(0.9)$ & 86 & $(1.3)$ \\
\hline Finlande & 69 & $(1.5)$ & 86 & $(0.8)$ \\
\hline Allemagne & 61 & $(3.1)$ & 72 & $(1.3)$ \\
\hline Hongrie & 63 & $(1.1)$ & 85 & $(2.2)$ \\
\hline Irlande & 56 & $(1.7)$ & 73 & (1.3) \\
\hline Pays-Bas & 56 & $(1.6)$ & 77 & (1.3) \\
\hline Nouvelle-Zélande & 70 & $(1.9)$ & 81 & (1.1) \\
\hline Norvège & 73 & $(1.5)$ & 89 & $(0.8)$ \\
\hline Pologne & 67 & $(0.7)$ & 82 & $(2.2)$ \\
\hline Portugal & 71 & $(2.9)$ & 90 & $(3.6)$ \\
\hline Slovénie & 72 & $(0.7)$ & 92 & (1.3) \\
\hline Suède & 71 & $(1.7)$ & 87 & (1.1) \\
\hline Suisse & 76 & $(2.8)$ & 84 & (2.0) \\
\hline Royaume-Uni & 68 & $(1.3)$ & 87 & $(0.9)$ \\
\hline États-Unis & 74 & $(1.7)$ & 84 & (1.3) \\
\hline
\end{tabular}

Source: Enquête internationale sur la littératie des adultes, tableau 3.6 (OCDE et Statistique Canada, 2000). 
Tableau 8.2

Taux de chômage dans la population âgée de 16 à 65 ans, selon le niveau de compétence sur l'échelle de textes suivis de IALS

\begin{tabular}{|c|c|c|c|c|}
\hline & Niveaux 1 et $2(\%)$ & Er.T & Niveaux 3, 4 et $5(\%)$ & Er.T \\
\hline Australie & 11 & $(0.8)$ & 5 & $(0.5)$ \\
\hline Belgique (Fl.) & 17 & $(2.7)$ & 7 & $(1.0)$ \\
\hline Canada & 16 & $(3.9)$ & 8 & $(1.1)$ \\
\hline Chili & 14 & $(1.0)$ & $8^{1}$ & $(1.8)$ \\
\hline République tchèque & 8 & $(0.9)$ & 4 & $(0.7)$ \\
\hline Danemark & 9 & $(1.1)$ & 5 & $(0.7)$ \\
\hline Finlande & 21 & $(1.7)$ & 9 & $(0.7)$ \\
\hline Allemagne & 14 & $(1.7)$ & 8 & $(1.4)$ \\
\hline Hongrie & 15 & $(1.2)$ & 13 & $(2.2)$ \\
\hline Irlande & 23 & $(2.9)$ & 11 & $(1.9)$ \\
\hline Pays-Bas & 9 & $(1.2)$ & 5 & $(0.7)$ \\
\hline Nouvelle-Zélande & 16 & $(1.7)$ & 4 & $(0.7)$ \\
\hline Norvège & 6 & $(0.8)$ & 3 & $(0.4)$ \\
\hline Pologne & 17 & $(1.1)$ & 11 & $(1.9)$ \\
\hline Portugal & 15 & $(2.3)$ & 9 & $(1.3)$ \\
\hline Slovénie & 14 & $(1.0)$ & 8 & $(1.5)$ \\
\hline Suède & 11 & $(1.2)$ & 7 & (0.6) \\
\hline Suisse & 5 & $(0.9)$ & 3 & $(0.8)$ \\
\hline Royaume-Uni & 16 & $(1.2)$ & 9 & (0.9) \\
\hline États-Unis & 7 & $(1.2)$ & 4 & $(0.8)$ \\
\hline
\end{tabular}

1. Le chiffre de cette cellule n'a pas pu être estimé correctement.

Source: Enquête internationale sur la littératie des adultes, tableau 3.7 (OCDE et Statistique Canada, 2000). 
Tableau 8.3

Pourcentage de la population âgée de 25 à 65 ans faisant partie des 60 pour cent de salariés les mieux rémunérés, selon le niveau de compétence sur l'échelle de textes suivis de IALS

\begin{tabular}{|c|c|c|c|c|c|c|c|c|}
\hline & $\begin{array}{c}\text { Niveau } 1 \\
(\%)\end{array}$ & Er.T & $\begin{array}{c}\text { Niveau } 2 \\
(\%)\end{array}$ & Er.T & $\begin{array}{c}\text { Niveau } 3 \\
(\%)\end{array}$ & Er.T & $\begin{array}{c}\text { Niveaux } 4 \\
\text { et } 5(\%)\end{array}$ & Er.T \\
\hline Australie & 26 & $(1.8)$ & 44 & $(1.4)$ & 51 & $(1.2)$ & 58 & (1.7) \\
\hline Belgique (Fl.) & $6^{1}$ & $(1.2)$ & 13 & $(2.1)$ & 23 & $(1.9)$ & 34 & $(3.7)$ \\
\hline Canada & 24 & $(6.4)$ & 45 & $(8.4)$ & 57 & $(3.0)$ & 69 & $(7.5)$ \\
\hline Chili & 26 & $(1.8)$ & 44 & $(2.0)$ & 61 & $(3.2)$ & 72 & $(9.2)$ \\
\hline République tchèque & 17 & $(2.6)$ & 28 & $(2.0)$ & 42 & $(2.7)$ & 55 & $(4.1)$ \\
\hline Danemark & 37 & $(3.6)$ & 57 & $(1.5)$ & 70 & $(1.5)$ & 67 & $(5.2)$ \\
\hline Finlande & 26 & $(2.6)$ & 52 & $(2.0)$ & 67 & $(1.5)$ & 74 & $(2.4)$ \\
\hline Allemagne & 35 & $(4.2)$ & 44 & $(2.5)$ & 50 & $(3.5)$ & 56 & $(3.1)$ \\
\hline Hongrie & 21 & $(1.4)$ & 41 & $(2.2)$ & 62 & $(3.7)$ & $55^{1}$ & $(9.8)$ \\
\hline Irlande & 24 & $(2.6)$ & 45 & $(3.8)$ & 60 & $(3.0)$ & 75 & $(4.0)$ \\
\hline Pays-Bas & 33 & $(4.3)$ & 48 & $(1.7)$ & 61 & $(1.6)$ & 63 & $(3.1)$ \\
\hline Nouvelle-Zélande & 34 & $(3.0)$ & 54 & $(3.1)$ & 63 & $(2.3)$ & 73 & $(2.3)$ \\
\hline Norvège & 38 & $(4.6)$ & 57 & (2.3) & 74 & $(1.2)$ & 70 & $(2.3)$ \\
\hline Pologne & 57 & $(2.1)$ & 65 & $(1.5)$ & 75 & $(2.6)$ & 87 & $(4.2)$ \\
\hline Portugal & 52 & $(3.8)$ & 59 & $(4.3)$ & 75 & $(4.4)$ & 74 & $(6.1)$ \\
\hline Slovénie & 23 & $(1.3)$ & 52 & $(2.6)$ & 72 & $(2.8)$ & $67^{1}$ & $(8.5)$ \\
\hline Suède & 72 & $(6.1)$ & 79 & $(2.4)$ & 81 & $(1.5)$ & 83 & $(1.9)$ \\
\hline Suisse & 46 & $(6.0)$ & 59 & $(4.3)$ & 72 & $(3.9)$ & 71 & $(5.1)$ \\
\hline Royaume-Uni & 25 & $(1.9)$ & 43 & $(2.2)$ & 57 & $(1.7)$ & 72 & $(3.0)$ \\
\hline États-Unis & 13 & $(1.9)$ & 32 & $(2.8)$ & 47 & $(2.6)$ & 60 & $(2.6)$ \\
\hline Moyenne de l'OCDE & 26 & $(1.0)$ & 41 & $(1.2)$ & 53 & $(1.4)$ & 62 & $(1.5)$ \\
\hline
\end{tabular}

2. Le chiffre de cette cellule n'a pas pu être estimé correctement.

Source: Enquête internationale sur la littératie des adultes, tableau 4.9 (OCDE et Statistique Canada, 2000). 
Tableau 8.4

Pourcentage de la population âgée de 16 à 65 ans ayant participé à une formation pour adultes au cours de l'année précédant l'interview, selon le niveau de compétence sur l'échelle de textes schématiques ${ }^{1}$

\begin{tabular}{|c|c|c|c|c|c|c|c|c|c|c|}
\hline & $\begin{array}{c}\text { Taux de par- } \\
\text { ticipation }\end{array}$ & Er.T & $\begin{array}{c}\text { Niveau } 1 \\
(\%)\end{array}$ & Er.T & $\begin{array}{c}\text { Niveau } 2 \\
(\%)\end{array}$ & Er.T & $\begin{array}{c}\text { Niveau } 3 \\
(\%)\end{array}$ & Er.T & $\begin{array}{c}\text { Niveaux } 4 \\
\text { et } 5(\%)\end{array}$ & Er.T \\
\hline Australie & 39 & $(0.7)$ & 14 & $(1.2)$ & 29 & $(1.2)$ & 47 & $(1.2)$ & 62 & $(1.5)$ \\
\hline Belgique (Fl.) & 21 & $(1.1)$ & $4^{2}$ & $(1.3)$ & 15 & $(2.4)$ & 26 & (1.6) & 37 & $(3.2)$ \\
\hline Canada & 38 & $(1.0)$ & 17 & $(6.2)$ & 29 & $(2.4)$ & 40 & $(2.9)$ & 60 & $(2.2)$ \\
\hline Chili & 19 & $(1.1)$ & 11 & $(1.1)$ & 24 & $(2.1)$ & 39 & (3.7) & $51^{2}$ & (11.1) \\
\hline République tchèque & 26 & $(0.9)$ & 11 & (1.9) & 23 & $(1.2)$ & 29 & (1.9) & 35 & $(2.0)$ \\
\hline Danemark & 56 & $(0.7)$ & 25 & (3.3) & 44 & $(1.8)$ & 60 & (1.4) & 70 & $(1.5)$ \\
\hline Finlande & 57 & $(0.9)$ & 19 & $(1.8)$ & 44 & $(2.5)$ & 66 & $(1.6)$ & 78 & (1.7) \\
\hline Allemagne $^{3}$ & $\mathrm{~m}$ & $\mathrm{~m}$ & $\mathrm{~m}$ & $\mathrm{~m}$ & $\mathrm{~m}$ & $\mathrm{~m}$ & $\mathrm{~m}$ & $\mathrm{~m}$ & $\mathrm{~m}$ & $\mathrm{~m}$ \\
\hline Hongrie & 19 & $(0.7)$ & 8 & $(1.1)$ & 17 & $(1.7)$ & 31 & (1.7) & 44 & $(4.7)$ \\
\hline Irlande & 24 & $(2.3)$ & 10 & $(2.0)$ & 20 & $(2.3)$ & 34 & $(2.8)$ & 47 & $(4.2)$ \\
\hline Pays-Bas & 37 & $(1.2)$ & 17 & $(2.3)$ & 27 & $(1.6)$ & 42 & (1.8) & 53 & (3.1) \\
\hline Nouvelle-Zélande & 48 & $(1.2)$ & 29 & $(2.3)$ & 41 & $(2.3)$ & 55 & $(2.0)$ & 68 & (1.9) \\
\hline Norvège & 48 & $(1.5)$ & 18 & $(2.3)$ & 36 & $(2.8)$ & 51 & (1.6) & 63 & $(2.0)$ \\
\hline Pologne & 14 & $(0.9)$ & 8 & $(1.0)$ & 15 & $(1.8)$ & 23 & (2.4) & 32 & $(6.2)$ \\
\hline Portugal & 14 & $(1.0)$ & 5 & $(1.4)$ & 19 & $(2.6)$ & 33 & (3.4) & 53 & $(7.0)$ \\
\hline Slovénie & 32 & (1.1) & 14 & $(1.3)$ & 37 & $(1.7)$ & 59 & $(2.1)$ & 61 & $(5.2)$ \\
\hline Suède & 53 & $(1.1)$ & 29 & $(5.2)$ & 40 & $(2.2)$ & 55 & $(1.8)$ & 62 & $(1.3)$ \\
\hline Suisse & 42 & (1.1) & 20 & $(2.7)$ & 34 & $(2.2)$ & 48 & (1.4) & 64 & (3.4) \\
\hline Royaume-Uni & 44 & $(0.9)$ & 22 & (1.7) & 34 & $(1.8)$ & 54 & $(1.8)$ & 71 & $(2.0)$ \\
\hline États-Unis & 40 & (1.4) & 17 & $(2.1)$ & 32 & $(1.8)$ & 49 & $(1.8)$ & 59 & $(3.0)$ \\
\hline Moyennenne I'OCDE & 35 & $(0.6)$ & 14 & $(0.9)$ & 29 & $(0.9)$ & 46 & $(0.9)$ & 59 & (1.4) \\
\hline
\end{tabular}

1. Les étudiants à temps plein et les adultes ayant suivi moins de six heures de formation ont été exclus de ces estimations.

2. Le chiffre de cette cellule n'a pas pu être estimé correctement.

3. L'Allemagne est exclue de cette analyse, car les questions sur la formation des adultes n'ont pas été posées de la même manière.

Source: Enquête internationale sur la littératie des adultes, tableau 3.12 (OCDE et Statistique Canada, 2000). 
Tableau 8.5

Performance moyenne et pourcentage des élèves de PISA à chaque niveau de compétence de l'échelle de textes suivis de IALS

\begin{tabular}{|c|c|c|c|c|c|c|c|c|c|c|}
\hline & $\begin{array}{l}\text { Score } \\
\text { moyen }\end{array}$ & Er.T & $\begin{array}{l}\text { Niveau } 1 \\
(\%)\end{array}$ & Er.T & $\begin{array}{l}\text { Niveau } 2 \\
(\%)\end{array}$ & Er.T & $\begin{array}{l}\text { Niveau } 3 \\
(\%)\end{array}$ & Er.T & $\begin{array}{l}\text { Niveaux } 4 \\
\text { et } 5(\%)\end{array}$ & Er.T \\
\hline Australie & 281.3 & $(1.2)$ & 9.3 & $(0.5)$ & 35.3 & $(0.9)$ & 41.4 & $(0.7)$ & 13.9 & $(0.7)$ \\
\hline Autriche & 262.1 & $(0.9)$ & 19.8 & $(0.6)$ & 41.4 & $(0.8)$ & 33.0 & $(0.8)$ & 5.8 & $(0.3)$ \\
\hline Belgique & 277.7 & $(1.1)$ & 11.7 & $(0.8)$ & 33.0 & $(0.6)$ & 44.4 & $(0.9)$ & 10.9 & $(0.4)$ \\
\hline Canada & 285.6 & $(0.5)$ & 5.4 & $(0.2)$ & 33.9 & $(0.4)$ & 47.0 & $(0.3)$ & 13.7 & $(0.3)$ \\
\hline République tchèque & 268.0 & $(0.8)$ & 15.3 & $(0.6)$ & 41.1 & $(0.6)$ & 36.6 & $(0.6)$ & 6.9 & $(0.3)$ \\
\hline Danemark & 266.1 & $(0.7)$ & 15.5 & $(0.6)$ & 43.0 & $(0.5)$ & 35.5 & $(0.6)$ & 5.9 & $(0.3)$ \\
\hline Finlande & 301.9 & $(0.7)$ & 4.2 & $(0.2)$ & 22.8 & $(0.5)$ & 43.3 & $(0.5)$ & 29.7 & $(0.6)$ \\
\hline France & 272.5 & $(1.0)$ & 12.5 & $(0.7)$ & 40.3 & $(0.7)$ & 38.1 & $(0.8)$ & 9.0 & $(0.4)$ \\
\hline Allemagne & 264.1 & $(1.0)$ & 20.6 & $(0.7)$ & 37.8 & $(0.6)$ & 32.7 & $(0.6)$ & 8.9 & $(0.3)$ \\
\hline Grèce & 267.7 & $(2.0)$ & 18.7 & $(1.4)$ & 36.5 & $(0.9)$ & 34.3 & $(1.2)$ & 10.4 & $(0.7)$ \\
\hline Hongrie & 267.1 & $(1.5)$ & 15.7 & $(1.1)$ & 41.5 & $(0.8)$ & 36.5 & $(1.2)$ & 6.3 & $(0.4)$ \\
\hline Islande & 276.3 & $(0.6)$ & 12.2 & $(0.4)$ & 36.8 & $(0.5)$ & 38.8 & $(0.5)$ & 12.2 & $(0.4)$ \\
\hline Irlande & 281.2 & (1.1) & 8.6 & $(0.6)$ & 35.4 & $(0.7)$ & 43.0 & $(0.7)$ & 13.0 & $(0.5)$ \\
\hline Italie & 271.1 & $(1.0)$ & 13.2 & $(0.7)$ & 40.0 & $(0.8)$ & 38.8 & $(0.8)$ & 8.0 & $(0.4)$ \\
\hline Japon & 287.7 & $(1.3)$ & 3.2 & $(0.4)$ & 31.5 & $(1.3)$ & 54.0 & $(1.1)$ & 11.2 & $(0.7)$ \\
\hline Corée & 285.1 & $(0.7)$ & 2.5 & $(0.2)$ & 33.7 & $(0.8)$ & 56.3 & $(0.7)$ & 7.4 & $(0.4)$ \\
\hline Luxembourg & 243.7 & $(0.5)$ & 33.2 & $(0.5)$ & 42.9 & $(0.5)$ & 21.4 & $(0.4)$ & 2.5 & $(0.2)$ \\
\hline Mexique & 248.4 & $(1.1)$ & 28.1 & $(1.0)$ & 46.9 & $(0.7)$ & 22.9 & $(0.9)$ & 2.0 & $(0.0)$ \\
\hline Nouvelle-Zélande & 279.7 & $(1.0)$ & 10.2 & $(0.6)$ & 34.9 & $(0.6)$ & 41.6 & $(0.7)$ & 13.3 & $(0.6)$ \\
\hline Norvège & 266.2 & $(0.9)$ & 16.8 & $(0.7)$ & 41.0 & $(0.6)$ & 35.5 & $(0.7)$ & 6.8 & $(0.3)$ \\
\hline Pologne & 268.1 & $(1.3)$ & 16.5 & $(0.9)$ & 39.9 & $(0.8)$ & 35.1 & $(1.0)$ & 8.5 & $(0.6)$ \\
\hline Portugal & 258.5 & $(1.5)$ & 20.2 & $(1.2)$ & 45.1 & $(0.7)$ & 31.7 & $(1.3)$ & 2.9 & $(0.3)$ \\
\hline Espagne & 270.1 & $(0.8)$ & 9.2 & $(0.5)$ & 45.7 & $(0.7)$ & 42.3 & $(0.9)$ & 2.8 & $(0.2)$ \\
\hline Suède & 269.6 & $(0.7)$ & 11.7 & $(0.5)$ & 43.3 & $(0.6)$ & 39.8 & $(0.7)$ & 5.2 & $(0.2)$ \\
\hline Suisse & 273.3 & $(1.4)$ & 12.8 & $(0.7)$ & 38.9 & $(0.9)$ & 38.4 & $(0.8)$ & 9.8 & $(0.8)$ \\
\hline Royaume-Uni & 275.2 & $(0.9)$ & 10.9 & $(0.5)$ & 39.3 & $(0.7)$ & 40.0 & $(0.5)$ & 9.8 & $(0.6)$ \\
\hline États-Unis & 272.5 & $(2.1)$ & 11.1 & $(1.2)$ & 41.8 & $(1.2)$ & 39.7 & $(1.5)$ & 7.5 & $(0.8)$ \\
\hline Brésil & 249.8 & $(1.1)$ & 25.6 & $(1.0)$ & 50.8 & $(0.8)$ & 22.2 & $(1.0)$ & 1.4 & $(0.2)$ \\
\hline Lettonie $^{1}$ & 261.3 & (1.6) & 22.1 & $(1.2)$ & 39.1 & $(0.6)$ & 30.8 & $(1.0)$ & 8.0 & $(0.4)$ \\
\hline Liechenstein & 275.1 & $(1.7)$ & 10.3 & $(1.4)$ & 36.7 & $(1.6)$ & 44.9 & $(1.5)$ & 8.1 & $(1.0)$ \\
\hline Fédération de Russie & 265.8 & $(1.0)$ & 16.4 & $(0.6)$ & 42.3 & $(0.5)$ & 34.6 & $(0.7)$ & 6.7 & $(0.3)$ \\
\hline Pays-Bas $^{2}$ & 288.5 & $(1.3)$ & 6.1 & $(0.7)$ & 29.9 & $(1.0)$ & 47.0 & $(1.1)$ & 16.9 & $(0.9)$ \\
\hline $\begin{array}{l}\text { Moyenne des pays } \\
\text { ayant participé à PISA }\end{array}$ & 267.8 & $(0.4)$ & 15.2 & $(0.3)$ & 41.7 & $(0.3)$ & 36.3 & $(0.4)$ & 6.9 & $(0.0)$ \\
\hline
\end{tabular}

1. Dans ce pays, la taille de l'échantillon des élèves qui ont répondu aux items de IALS était insuffisante pour permettre de calculer des estimations fiables.

2. Taux de participation insuffisant pour permettre les comparaisons. 
Tableau 8.6

Performance moyenne et pourcentage des filles dans PISA à chaque niveau de compétence de l'échelle de textes suivis de IALS

\begin{tabular}{|c|c|c|c|c|c|c|c|c|c|c|}
\hline & Score moyen & Er.T & $\begin{array}{c}\text { Niveau } 1 \\
(\%)\end{array}$ & Er.T & $\begin{array}{c}\text { Niveau } 2 \\
(\%)\end{array}$ & Er.T & $\begin{array}{c}\text { Niveau } 3 \\
(\%)\end{array}$ & Er.T & $\begin{array}{l}\text { Niveaux } 4 \\
\text { et } 5(\%)\end{array}$ & Er.T \\
\hline Australie & 289.0 & $(1.5)$ & 5.8 & $(0.5)$ & 31.4 & $(1.2)$ & 45.1 & $(0.9)$ & 17.7 & (1.1) \\
\hline Autriche & 269.7 & $(1.3)$ & 14.8 & $(0.8)$ & 39.4 & $(1.1)$ & 37.9 & $(1.2)$ & 7.9 & $(0.5)$ \\
\hline Belgique & 286.9 & $(1.5)$ & 7.6 & $(0.9)$ & 27.6 & $(1.0)$ & 49.8 & $(1.1)$ & 15.0 & $(0.7)$ \\
\hline Canada & 293.7 & $(0.5)$ & 3.0 & $(0.2)$ & 27.5 & $(0.4)$ & 51.0 & $(0.4)$ & 18.5 & $(0.4)$ \\
\hline République tchèque & 276.1 & $(0.9)$ & 10.1 & $(0.5)$ & 38.7 & $(0.9)$ & 42.0 & $(0.7)$ & 9.2 & $(0.5)$ \\
\hline Danemark & 273.7 & $(1.0)$ & 11.0 & $(0.8)$ & 39.9 & $(0.8)$ & 41.0 & $(0.9)$ & 8.2 & $(0.5)$ \\
\hline Finlande & 314.7 & $(0.8)$ & 1.8 & $(0.2)$ & 14.9 & $(0.6)$ & 43.3 & $(0.6)$ & 40.1 & $(0.8)$ \\
\hline France & 280.8 & $(1.0)$ & 8.0 & $(0.6)$ & 36.6 & $(0.9)$ & 43.1 & $(0.9)$ & 12.3 & $(0.5)$ \\
\hline Allemagne & 272.1 & $(1.2)$ & 16.0 & $(1.0)$ & 35.6 & $(0.9)$ & 37.0 & $(0.8)$ & 11.3 & $(0.5)$ \\
\hline Grèce & 278.3 & $(1.8)$ & 11.6 & $(1.1)$ & 35.2 & $(1.0)$ & 39.2 & $(1.2)$ & 14.0 & $(0.9)$ \\
\hline Hongrie & 275.5 & $(1.6)$ & 10.7 & $(1.0)$ & 38.1 & $(1.3)$ & 42.2 & (1.4) & 9.0 & $(0.7)$ \\
\hline Islande & 288.3 & $(0.8)$ & 6.3 & $(0.4)$ & 31.6 & $(0.7)$ & 44.4 & $(0.8)$ & 17.7 & $(0.6)$ \\
\hline Irlande & 288.9 & $(1.1)$ & 5.9 & $(0.6)$ & 30.1 & $(0.9)$ & 46.9 & $(0.9)$ & 17.2 & $(0.7)$ \\
\hline Italie & 282.2 & $(1.1)$ & 7.2 & $(0.6)$ & 35.0 & $(0.9)$ & 46.0 & $(1.0)$ & 11.8 & $(0.5)$ \\
\hline Japon & 294.0 & (1.4) & 1.9 & $(0.4)$ & 25.6 & $(1.4)$ & 57.7 & $(0.9)$ & 14.9 & (1.0) \\
\hline Corée & 287.9 & $(1.2)$ & 1.9 & $(0.2)$ & 31.3 & $(1.7)$ & 57.7 & $(1.2)$ & 9.1 & $(0.7)$ \\
\hline Luxembourg & 251.0 & $(0.9)$ & 27.4 & $(0.8)$ & 43.8 & $(0.7)$ & 25.3 & $(0.7)$ & 3.4 & $(0.3)$ \\
\hline Mexique & 254.0 & $(1.3)$ & 23.2 & $(1.1)$ & 47.5 & $(0.8)$ & 26.7 & $(1.0)$ & 2.6 & $(0.3)$ \\
\hline Nouvelle-Zélande & 289.7 & $(1.3)$ & 5.8 & $(0.5)$ & 30.5 & $(0.9)$ & 44.7 & $(0.8)$ & 19.0 & $(1.0)$ \\
\hline Norvège & 277.3 & $(0.9)$ & 9.6 & $(0.6)$ & 37.6 & $(0.9)$ & 43.1 & $(0.8)$ & 9.7 & $(0.5)$ \\
\hline Pologne & 276.8 & $(1.7)$ & 10.8 & $(1.0)$ & 37.9 & $(1.2)$ & 39.8 & $(1.2)$ & 11.4 & $(0.9)$ \\
\hline Portugal & 265.1 & $(1.6)$ & 15.3 & $(1.1)$ & 43.4 & $(1.0)$ & 37.5 & $(1.5)$ & 3.8 & (0.4) \\
\hline Espagne & 276.0 & $(0.9)$ & 5.6 & $(0.5)$ & 42.1 & $(1.2)$ & 48.6 & $(1.2)$ & 3.7 & $(0.3)$ \\
\hline Suède & 279.9 & $(0.7)$ & 5.8 & $(0.4)$ & 38.2 & $(0.7)$ & 48.0 & $(0.8)$ & 7.9 & $(0.4)$ \\
\hline Suisse & 281.4 & $(1.5)$ & 8.7 & $(0.6)$ & 34.8 & $(1.2)$ & 43.5 & $(0.9)$ & 13.0 & (1.1) \\
\hline Royaume-Uni & 282.6 & $(1.2)$ & 7.7 & $(0.5)$ & 34.9 & $(1.0)$ & 44.0 & $(0.8)$ & 13.4 & $(0.9)$ \\
\hline États-Unis & 279.1 & $(2.0)$ & 7.4 & $(1.0)$ & 38.8 & $(1.5)$ & 44.0 & $(1.6)$ & 9.8 & (1.0) \\
\hline Brésil & 255.5 & $(1.4)$ & 20.0 & $(1.2)$ & 51.5 & $(1.0)$ & 26.6 & $(1.4)$ & 1.9 & $(0.2)$ \\
\hline Lettonie $^{1}$ & 273.7 & $(1.5)$ & 13.5 & $(0.9)$ & 37.6 & $(0.9)$ & 37.7 & $(1.2)$ & 11.2 & $(0.5)$ \\
\hline Liechenstein & $282 ; 9$ & $(2.4)$ & 5.9 & $(1.6)$ & 34.3 & $(2.2)$ & 49.8 & $(2.1)$ & 10.1 & (1.6) \\
\hline Fédération de Russie & 275.5 & $(1.0)$ & 10.3 & $(0.6)$ & 39.3 & $(0.8)$ & 40.9 & $(0.8)$ & 9.5 & $(0.5)$ \\
\hline Pays-Bas ${ }^{2}$ & 296.8 & $(1.6)$ & 3.3 & $(0.6)$ & 25.5 & $(1.5)$ & 48.3 & $(1.5)$ & 22.9 & $(1.5)$ \\
\hline $\begin{array}{l}\text { Moyenne des pays } \\
\text { ayant participé àPISA }\end{array}$ & 274.7 & $(0.5)$ & 10.9 & $(0.3)$ & 39.3 & $(0.4)$ & 40.7 & $(0.4)$ & 9.1 & $(0.2)$ \\
\hline
\end{tabular}

1. Dans ce pays, la taille de l'échantillon des élèves qui ont répondu aux items de IALS était insuffisante pour permettre de calculer des estimations fiables. 2. Taux de participation insuffisant pour permettre les comparaisons. 
Tableau 8.7

Performance moyenne et pourcentage des garçons dans PISA à chaque niveau de compétence de l'échelle de textes suivis de IALS

\begin{tabular}{|c|c|c|c|c|c|c|c|c|c|c|}
\hline & $\begin{array}{c}\text { Score } \\
\text { moyen }\end{array}$ & Er.T & $\begin{array}{c}\text { Niveau } 1 \\
(\%)\end{array}$ & Er.T & $\begin{array}{c}\text { Niveau } 2 \\
(\%)\end{array}$ & Er.T & $\begin{array}{c}\text { Niveau } 3 \\
(\%)\end{array}$ & Er.T & $\begin{array}{l}\text { Niveaux } 4 \\
\text { et } 5(\%)\end{array}$ & Er.T \\
\hline Australie & 274.6 & $(1.4)$ & 12.5 & $(0.7)$ & 38.5 & $(1.1)$ & 38.3 & $(1.2$ & 10.7 & $(0.7)$ \\
\hline Autriche & 254.8 & $(1.3)$ & 24.5 & $(1.2)$ & 43.7 & $(0.8)$ & 28.2 & $(1.1$ & 3.6 & $(0.3)$ \\
\hline Belgique & 270.0 & $(1.3)$ & 15.1 & $(0.9)$ & 37.6 & $(1.0)$ & 40.0 & $(1.1$ & 7.3 & $(0.5)$ \\
\hline Canada & 277.8 & $(0.5)$ & 7.8 & $(0.3)$ & 39.8 & $(0.5)$ & 43.3 & $(0.5$ & 9.2 & $(0.3)$ \\
\hline République tchèque & 259.8 & $(1.4)$ & 20.5 & $(1.2)$ & 43.9 & $(0.9)$ & 31.0 & $(1.1$ & 4.6 & $(0.3)$ \\
\hline Danemark & 258.8 & $(0.9)$ & 20.0 & $(0.9)$ & 45.8 & $(0.8)$ & 30.5 & $(0.8$ & 3.8 & $(0.3)$ \\
\hline Finlande & 288.3 & $(1.0)$ & 6.8 & $(0.4)$ & 31.2 & $(0.8)$ & 43.3 & $(0.7$ & 18.7 & $(0.6)$ \\
\hline France & 263.8 & $(1.1)$ & 17.3 & $(0.9)$ & 44.1 & $(0.8)$ & 32.9 & $(0.9$ & 5.7 & $(0.4)$ \\
\hline Allemagne & 257.1 & $(1.2)$ & 24.5 & $(0.9)$ & 39.9 & $(0.7)$ & 28.9 & $(0.7$ & 6.6 & $(0.4)$ \\
\hline Grèce & 257.4 & $(2.5)$ & 25.8 & (1.9) & 37.6 & $(1.0)$ & 29.5 & $(1.4$ & 7.0 & $(0.8)$ \\
\hline Hongrie & 259.0 & $(2.0)$ & 20.6 & $(1.6)$ & 44.6 & $(1.1)$ & 31.1 & $(1.6$ & 3.7 & $(0.5)$ \\
\hline Islande & 265.2 & $(0.8)$ & 17.7 & $(0.6)$ & 41.7 & $(0.8)$ & 33.8 & $(0.7$ & 6.9 & $(0.4)$ \\
\hline Irlande & 273.9 & $(1.4)$ & 11.1 & $(0.8)$ & 40.6 & $(1.0)$ & 39.3 & $(1.0$ & 9.0 & $(0.7)$ \\
\hline Italie & 260.6 & $(1.5)$ & 18.9 & $(1.2)$ & 44.8 & $(1.0)$ & 32.0 & $(1.1$ & 4.4 & $(0.4)$ \\
\hline Japon & 281.4 & $(1.5)$ & 4.6 & $(0.6)$ & 37.6 & $(1.6)$ & 50.2 & $(1.5$ & 7.5 & $(0.7)$ \\
\hline Corée & 282.9 & $(1.1)$ & 3.1 & $(0.4)$ & 35.5 & $(1.3)$ & 55.2 & $(1.3$ & 6.2 & $(0.5)$ \\
\hline Luxembourg & 236.9 & $(0.9)$ & 38.7 & $(0.9)$ & 41.8 & $(0.8)$ & 18.0 & $(0.6$ & 1.5 & $(0.2)$ \\
\hline Mexique & 242.6 & $(1.4)$ & 33.7 & $(1.3)$ & 45.5 & $(0.8)$ & 19.4 & $(1.1$ & 1.5 & $(0.2)$ \\
\hline Nouvelle-Zélande & 270.4 & $(1.4)$ & 14.3 & $(1.0)$ & 39.1 & $(0.9)$ & 38.9 & $(1.1$ & 7.7 & $(0.5)$ \\
\hline Norvège & 256.2 & $(1.1)$ & 23.0 & $(1.0)$ & 44.1 & $(0.8)$ & 28.9 & $(1.0$ & 4.0 & $(0.3)$ \\
\hline Pologne & 259.6 & $(1.9)$ & 22.0 & $(1.5)$ & 41.8 & $(1.1)$ & 30.5 & $(1.7$ & 5.7 & $(0.5)$ \\
\hline Portugal & 251.6 & $(1.7)$ & 25.4 & $(1.5)$ & 46.8 & $(0.9)$ & 25.8 & $(1.3$ & 2.0 & $(0.3)$ \\
\hline Espagne & 264.5 & $(0.9)$ & 12.6 & $(0.6)$ & 49.0 & $(1.0)$ & 36.5 & $(1.2$ & 1.9 & $(0.2)$ \\
\hline Suède & 259.6 & $(1.6)$ & 17.5 & $(0.7)$ & 48.2 & $(0.8)$ & 31.8 & $(0.9$ & 2.6 & $(0.2)$ \\
\hline Suisse & 265.4 & $(1.6)$ & 16.8 & $(1.0)$ & 42.9 & $(1.1)$ & 33.7 & $(1.2$ & 6.7 & $(0.6)$ \\
\hline Royaume-Uni & 268.2 & $(0.9)$ & 14.0 & $(0.7)$ & 43.1 & $(0.8)$ & 36.5 & $(0.7$ & 6.4 & $(0.5)$ \\
\hline États-Unis & 265.4 & $(2.3)$ & 14.9 & $(1.6)$ & 45.0 & $(1.3)$ & 35.1 & $(1.7$ & 5.0 & $(0.7)$ \\
\hline Brésil & 243.7 & $(1.1)$ & 31.4 & $(1.3)$ & 50.2 & $(1.0)$ & 17.5 & $(0.9$ & 1.0 & $(0.2)$ \\
\hline Lettonie $^{1}$ & 248.4 & $(1.9)$ & 31.2 & $(1.6)$ & 40.5 & $(0.9)$ & 23.5 & $(1.0$ & 4.8 & $(0.4)$ \\
\hline Liechenstein & 269.9 & $(2.9)$ & 12.7 & $(2.1)$ & 39.7 & $(2.4)$ & 41.3 & $(2.4$ & 6.3 & (1.4) \\
\hline Fédération de Russie & 256.3 & $(1.2)$ & 22.5 & $(1.0)$ & 45.1 & $(0.5)$ & 28.4 & $(0.9$ & 3.9 & $(0.2)$ \\
\hline Pays-Bas ${ }^{2}$ & 280.4 & $(1.8)$ & 9.0 & $(1.2)$ & 34.0 & $(1.2)$ & 45.9 & $(1.6$ & 11.2 & $(0.9)$ \\
\hline $\begin{array}{l}\text { Moyenne des pays } \\
\text { ayant participé à PISA }\end{array}$ & 260.9 & $(0.5)$ & 19.3 & $(0.4)$ & 44.0 & $(0.3)$ & 32.1 & $(0.4$ & 4.6 & $(0.2)$ \\
\hline
\end{tabular}

1. Dans ce pays, la taille de l'échantillon des élèves qui ont répondu aux items de IALS était insuffisante pour permettre de calculer des estimations fiables. 2. Taux de participation insuffisant pour permettre les comparaisons. 
Tableau 8.8

Différences de scores moyens entre filles et garçons sur l'échelle de textes suivis de IALS

\begin{tabular}{|c|c|c|c|c|c|}
\hline & $\begin{array}{l}\text { Scores moyens de } \\
\text { tous les élèves }\end{array}$ & $\begin{array}{l}\text { Scores moyens } \\
\text { des filles }\end{array}$ & $\begin{array}{l}\text { Scores moyens } \\
\text { des garçons }\end{array}$ & $\begin{array}{c}\text { Différence entre } \\
\text { les filles et les garçons }\end{array}$ & $\begin{array}{l}\text { Écart exprimé } \\
\text { en \% de l'Éc.T. }\end{array}$ \\
\hline Australie & 281.3 & 289.0 & 274.6 & 14.4 & 0.36 \\
\hline Autriche & 262.1 & 269.7 & 254.8 & 14.9 & 0.37 \\
\hline Belgique & 277.7 & 286.9 & 270.0 & 16.9 & 0.42 \\
\hline Canada & 285.6 & 293.7 & 277.8 & 15.9 & 0.39 \\
\hline République tchèque & 268.0 & 276.1 & 259.8 & 16.3 & 0.40 \\
\hline Danemark & 266.1 & 273.7 & 258.8 & 14.9 & 0.37 \\
\hline Finlande & 301.9 & 314.7 & 288.3 & 26.4 & 0.66 \\
\hline France & 272.5 & 280.8 & 263.8 & 17.0 & 0.42 \\
\hline Allemagne & 264.1 & 272.1 & 257.1 & 15.0 & 0.37 \\
\hline Grèce & 267.7 & 278.3 & 257.4 & 20.9 & 0.52 \\
\hline Hongrie & 267.1 & 275.5 & 259.0 & 16.5 & 0.41 \\
\hline Islande & 276.3 & 288.3 & 265.2 & 23.1 & 0.57 \\
\hline Irlande & 281.2 & 288.9 & 273.9 & 15.0 & 0.37 \\
\hline Italie & 271.1 & 282.2 & 260.6 & 21.6 & 0.54 \\
\hline Japon & 287.7 & 294.0 & 281.4 & 12.6 & 0.31 \\
\hline Corée & 285.1 & 287.9 & 282.9 & 5.0 & 0.12 \\
\hline Luxembourg & 243.7 & 251.0 & 236.9 & 14.1 & 0.35 \\
\hline Mexique & 248.4 & 254.0 & 242.6 & 11.4 & 0.28 \\
\hline Nouvelle-Zélande & 279.7 & 289.7 & 270.4 & 19.3 & 0.48 \\
\hline Norvège & 266.2 & 277.3 & 256.2 & 21.1 & 0.52 \\
\hline Pologne & 268.1 & 276.8 & 259.6 & 17.2 & 0.43 \\
\hline Portugal & 258.5 & 265.1 & 251.6 & 13.5 & 0.33 \\
\hline Espagne & 270.1 & 276.0 & 264.5 & 11.5 & 0.29 \\
\hline Suède & 269.6 & 279.9 & 259.6 & 20.3 & 0.50 \\
\hline Suisse & 273.3 & 281.4 & 265.4 & 16.0 & 0.40 \\
\hline Royaume-Uni & 275.2 & 282.6 & 268.2 & 14.4 & 0.36 \\
\hline États-Unis & 272.5 & 279.1 & 265.4 & 13.7 & 0.34 \\
\hline Brésil & 249.8 & 255.5 & 243.7 & 11.8 & 0.29 \\
\hline Lettonie $^{1}$ & 261.3 & 273.7 & 248.4 & 25.3 & 0.50 \\
\hline Liechenstein & 275.1 & 282.9 & 269.9 & 13.0 & 0.32 \\
\hline Fédération de Russie & 265.8 & 275.5 & 256.3 & 19.2 & 0.48 \\
\hline${\text { Pays- }-B^{2}{ }^{2}}$ & 288.5 & 296.8 & 280.4 & 16.4 & 0.41 \\
\hline $\begin{array}{l}\text { Moyenne des pays } \\
\text { ayant participé àPISA }\end{array}$ & 267.8 & 274.7 & 260.9 & 13.8 & 0.34 \\
\hline
\end{tabular}

1. Dans ce pays, la taille de l'échantillon des élèves qui ont répondu aux items de IALS était insuffisante pour permettre de calculer des estimations fiables. 2. Taux de participation insuffisant pour permettre les comparaisons. 
Tableau 8.9

Comparaison de la performance des élèves de PISA et des jeunes de 16 à 25 ans de IALS sur l'échelle de textes suivis de IALS, en fonction du sexe

\begin{tabular}{|c|c|c|c|c|}
\hline & \multicolumn{2}{|c|}{ Jeunes de 15 ans - PISA } & \multicolumn{2}{|c|}{ Jeunes de 16 à 25 ans - IALS } \\
\hline & Hommes & Femmes & Hommes & Femmes \\
\hline & 275 & 289 & 278 & 290 \\
\hline Belgique $^{1}$ & 270 & 287 & 292 & 295 \\
\hline Canada & 278 & 294 & 287 & 287 \\
\hline Suisse & 265 & 281 & 280 & 288 \\
\hline République tchèque & 260 & 276 & 278 & 283 \\
\hline Allemagne & 257 & 272 & 284 & 283 \\
\hline Danemark & 259 & 274 & 279 & 288 \\
\hline Finlande & 288 & 315 & 304 & 322 \\
\hline Hongrie & 259 & 276 & 254 & 264 \\
\hline Irlande & 274 & 289 & 272 & 283 \\
\hline Italie & 261 & 282 & 272 & 278 \\
\hline Pays-Bas ${ }^{2}$ & 280 & 297 & 291 & 297 \\
\hline Norvège $^{1}$ & 256 & 277 & 295 & 306 \\
\hline Nouvelle-Zélande & 270 & 290 & 267 & 286 \\
\hline Pologne & 260 & 277 & 243 & 261 \\
\hline Portugal & 252 & 265 & 251 & 270 \\
\hline Suède & 260 & 280 & 310 & 314 \\
\hline Royaume-Uni & 268 & 283 & 270 & 277 \\
\hline États-Unis & 265 & 279 & 260 & 265 \\
\hline $\begin{array}{l}\text { Moyenne des pays } \\
\text { ayant participé à PISA } \\
\text { et à IALS }\end{array}$ & 266 & 283 & 279 & 287 \\
\hline
\end{tabular}

1. Les résultats ne sont pas directement comparables en raison des différences dans la représentation des groupes linguistiques dans les deux enquêtes. 


\title{
ANNEXE
}

$\mathrm{C}$

\author{
DÉVELOPPEMENT ET MISE \\ EN OEUVRE DE PISA \\ - UNE INITIATIVE CONCERTÉE
}


LES ÉDITIONS DE L'OCDE, 2, rue André-Pascal, 75775 PARIS CEDEX 16 IMPRIMÉ EN FRANCE (962002072 P) - n 527802003 


\section{Membres du Conseil des pays participants de PISA 2000}

Président : Eugene Owen

Allemagne : Jochen Schweitzer, Helga Hinke, Gudrun Stoltenberg

Australie : Wendy Whitham

Autriche : Friedrich Plank

Belgique : Dominique Barthélémy, Christiane Blondin, Dominique Lafontaine, Liselotte van de Perre

Brésil : Maria Helena Guimarães de Castro

Canada : Satya Brink, Patrick Bussière, Dianne Pennock,

Corée : Kooghyang Ro

Danemark : Birgitte Bovin

Espagne : Guillermo Gil

États-Unis : Mariann Lemke

Finlande : Ritva Jakku-Sihvonen

France : Gérard Bonnet

Grèce : Vassilis Koulaidis

Hongrie : Péter Vári

Irlande : Gerry Shiel

Islande : Einar Gudmundsson

Italie : Chiara Croce, Elisabetta Midena, Benedetto Vertecchi

Japon : Ryo Watanabe

Luxembourg : Jean-Paul Reeff

Mexique : Fernando Córdova Calderón

Norvège : Alette Schreiner

Nouvelle-Zélande : Lynne Whitney

Pays-Bas : Arnold Spee

Pologne : Kazimierz Korab

Portugal : Glória Ramalho

République tchèque : Jan Koucky, Jana Straková

Royaume-Uni : Lorna Bertrand, Brian Semple

Suède : Anders Auer, Birgitta Fredander, Anita Wester

Suisse : Heinz Gilomen

\section{Directeurs nationaux de projet pour PISA 2000}

Allemagne : Juergen Baumert, Petra Stanat

Australie : Jan Lokan

Autriche : Günter Haider

Belgique : Dominique Lafontaine, Luc van de Poele

Brésil : Tereza Cristina Cotta, Maria Lucia Guardia,

Maria Inês Pestana

Canada : Marc Lachance, Dianne Pennock

Corée : Kooghyang Ro

Danemark : Vita Bering Pruzan
Espagne : Guillermo Gil

États-Unis : Ghedam Bairu, Marilyn Binkley

Finlande : Jouni Välijärvi

France : Jean-Pierre Jeantheau

Grèce : Katerina Kassotakis

Hongrie : Péter Vári

Irlande : Judith Cosgrove

Islande : Julius Bjornsson, Ragna Benedikta Garðarsdóttir

Italie : Emma Nardi

Japon : Ryo Watanabe

Lettonie : Andris Kangro

Luxembourg : Iris Blanke, Jean-Paul Reeff

Mexique : Fernando Córdova Calderón

Norvège : Svein Lie

Nouvelle-Zélande : Steve May

Pays-Bas : Johan Wijnstra

Pologne : Michal Federowicz

Portugal : Glória Ramalho

République tchèque : Jana Straková

Royaume-Uni : Baljit Gill, Graham Thorpe

Suède : Bengt Olov Molander, Astrid Pettersson,

Karin Taube

Suisse : Huguette McCluskey

\section{Secrétariat de l'OCDE}

Andreas Schleicher (coordination générale du PISA et des relations entre pays Membres)

Claudia Tamassia (gestion du projet)

Eric Charbonnier (assistance statistique)

Hannah Cocks (assistance statistique)

Juliet Evans (assistance administrative)

Kooghyang Ro (analyses thématiques) 


\section{Groupes d'experts de PISA}

\section{Groupe d'experts chargé de la lecture}

Irwin Kirsch (Président) (Educational Testing Service, États-Unis)

Marilyn Binkley (National Center for Educational Statistics, États-Unis)

Alan Davies (Université d’Édimbourg, Royaume-Uni) Stan Jones (Statistique Canada, Canada)

John de Jong (Language Testing Services, Pays-Bas)

Dominique Lafontaine (Université de Liège, Belgique)

Pirjo Linnakylä (Université de Jyväskylä, Finlande)

Martine Rémond (Institut National de Recherche

Pédagogique, France)

Ryo Watanabe (National Institute for Educational

Policy Research, Japon)

Prof. Wolfgang Schneider (Université de Würzburg, Allemagne)

\section{Groupe de conseillers techniques de PISA}

Ray Adams (ACER, Australie)

Pierre Foy (Statistique Canada, Canada)

Aletta Grisay (Belgique)

Larry Hedges (Université de Chicago, États-Unis)

Eugene Johnson (American Institutes for Research, États-Unis)

John de Jong (Language Testing Services, Pays-Bas)

Geoff Masters (ACER, Australie)

Keith Rust (WESTAT, États-Unis)

Norman Verhelst (Citogroep, Pays-Bas)

J. Douglas Willms (Université du Nouveau-Brunswick, Canada)

\section{Consortium PISA}

\section{Australian Council for Educational Research}

Ray Adams (Directeur de projet du Consortium PISA) Alla Berezner (traitement et analyse des données)

Claus Carstensen (analyse des données)

Lynne Darkin (développement des tests de lecture)

Brian Doig (développement des tests de mathématiques)

Adrian Harvey-Beavis (contrôle de la qualité et développement des questionnaires)

Kathryn Hill (développement des tests de lecture)
John Lindsey (développement des tests de mathématiques)

Jan Lokan (contrôle de la qualité et développement des opérations sur le terrain)

Le Tu Luc (traitement des données)

Greg Macaskill (traitement des données)

Joy McQueen (développement des tests de lecture et rapport)

Gary Marks (développement des questionnaires)

Juliette Mendelovits (développement des tests de lecture et rapport)

Christian Monseur (Directeur du Consortium PISA

pour le traitement et l'analyse des données et pour le contrôle de la qualité)

Gayl O’Connor (développement des tests de sciences) Alla Routitsky (traitement des données)

Wolfram Schulz (analyse des données)

Ross Turner (analyse des tests et coordination des rapports)

Nikolai Volodin (traitement des données)

Craig Williams (traitement et analyse des données)

Margaret Wu (Directrice de projet adjointe du Consortium PISA)

\section{WESTAT}

Nancy Caldwell (Directrice du Consortium PISA pour les opérations sur le terrain et le contrôle de la qualité) Ming Chen (échantillonnage et pondération) Fran Cohen (échantillonnage et pondération) Susan Fuss (échantillonnage et pondération) Brice Hart (échantillonnage et pondération) Sharon Hirabayashi (échantillonnage et pondération) Sheila Krawchuk (échantillonnage et pondération) Dward Moore (opérations de terrain et contrôle de la qualité)

Phu Nguyen (échantillonnage et pondération)

Monika Peters (opérations sur le terrain et contrôle de la qualité)

Merl Robinson (opérations sur le terrain et contrôle de la qualité)

Keith Rust (Directeur du Consortium PISA pour

l'échantillonnage et la pondération)

Leslie Wallace (échantillonnage et pondération)

Dianne Walsh (opérations sur le terrain et contrôle de la qualité)

Trevor Williams (développement des questionnaires) 


\section{Citogroep}

Steven Bakker (développement des tests de sciences) Bart Bossers (développement des tests de lecture)

Truus Decker (développement des tests de mathématiques)

Erna van Hest (développement des tests de lecture et contrôle de la qualité)

Kees Lagerwaard (développement des tests de mathématiques)

Gerben van Lent (développement des tests de mathématiques)

Ico de Roo (développement des tests de sciences)

Maria van Toor (assistance administrative et contrôle de la qualité)

Norman Verhelst (conseil technique et analyse des données)

\section{Educational Testing Service}

Irwin Kirsch (développement des tests de lecture)

\section{Autres experts}

Marc Demeuse (contrôle de la qualité)

Harry Ganzeboom (développement des questionnaires) Aletta Grisay (conseil technique, analyse des données, traduction et développement des questionnaires) Donald Hirsch (rédaction et révision) Jules Peschar (développement des questionnaires) Erich Ramseier (développement des questionnaires) Marie-Andrée Somers (analyse des données et rapport) Peter Sutton (rédaction et révision)

Rich Tobin (développement des questionnaires et rapport)

J. Douglas Willms (développement des questionnaires, analyse des données et rapport) 
LES ÉDITIONS DE L'OCDE, 2, rue André-Pascal, 75775 PARIS CEDEX 16 IMPRIMÉ EN FRANCE

(96 2002072 P) - n 527802003 
OECD PUBLICATION, 2, rue André-Pascal, 75775 PARIS CEDEX 16

PRINTED IN FRANCE

(32 2003043 P) - No. 529832003 